\title{
Kinetics and Modeling of the Radical Polymerization of Acrylic Acid and of Methacrylic Acid in Aqueous Solution
}

\author{
Dissertation \\ zur Erlangung des mathematisch-naturwissenschaftlichen Doktorgrades \\ „Doctor rerum naturalium“ \\ der Georg-August-Universität Göttingen \\ im Promotionsprogramm Chemie \\ der Georg-August University School of Science (GAUSS)
}

vorgelegt von

Nils Friedrich Gunter Wittenberg

aus Hamburg

Göttingen, 2013 
Betreuungsausschuss

Prof. Dr. M. Buback, Technische und Makromolekulare Chemie, Institut für Physikalische Chemie, Georg-August-Universität Göttingen

Prof. Dr. P. Vana, MBA, Makromolekulare Chemie, Institut für Physikalische Chemie, Georg-August-Universität Göttingen

Mitglieder der Prüfungskommission

Referent: Prof. Dr. M. Buback, Technische und Makromolekulare Chemie, Institut für Physikalische Chemie, Georg-August-Universität Göttingen

Korreferent: Prof. Dr. P. Vana, MBA, Makromolekulare Chemie, Institut für Physikalische Chemie, Georg-August-Universität Göttingen

Weitere Mitglieder der Prüfungskommission:

Prof. Dr. G. Echold, Physikalische Chemie fester Körper, Institut für Physikalische Chemie, Georg-August-Universität Göttingen

Prof. Dr. B. Geil, Biophysikalische Chemie, Institut für Physikalische Chemie, Georg-AugustUniversität Göttingen

Jun.-Prof. Dr. R. Mata, Computerchemie und Biochemie,Institut für Physikalische Chemie, Georg-August-Universität Göttingen

Prof. Dr. A. Wodtke, Physikalische Chemie I / Humboldt-Professur, Institut für Physikalische Chemie, Georg-August-Universität Göttingen

Tag der mündlichen Prüfung: 24.10.2013 
Meiner Familie 



\section{Table of Contents}

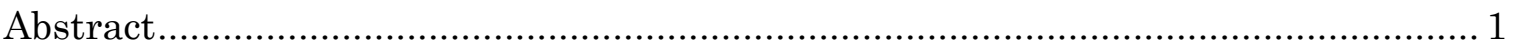

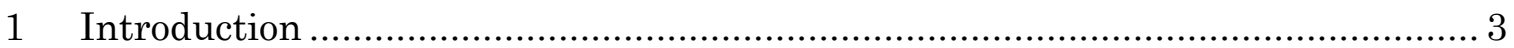

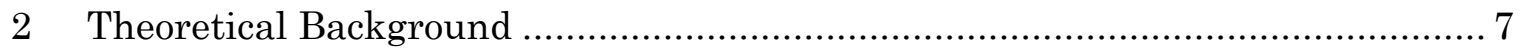

2.1 General Aspects of Radical Stability and Reactivity ................................. 7

2.2 Ideal Polymerization Kinetics of Radical Polymerization .......................... 9

2.2.1 Formation of Radicals and Initiation ............................................. 10

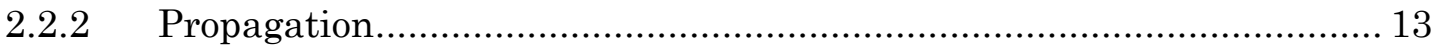

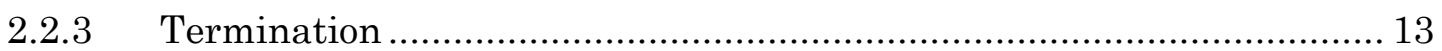

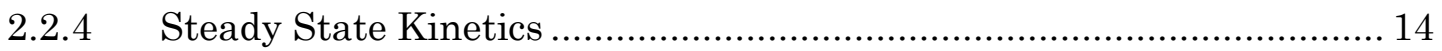

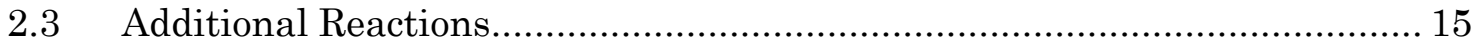

2.3.1 Transfer Reactions to Small Molecules................................................ 15

2.3.2 Intermolecular Transfer to Polymer .................................................. 19

2.3.3 Intramolecular Transfer to Polymer - Backbiting .............................. 20

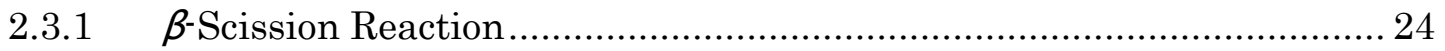

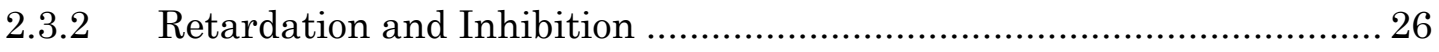

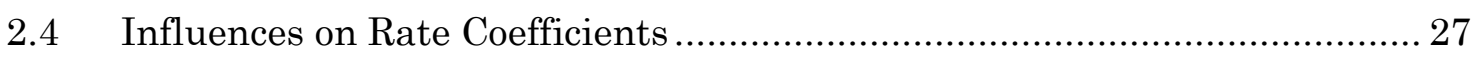

2.4.1 Temperature and Pressure (............................................................. 29

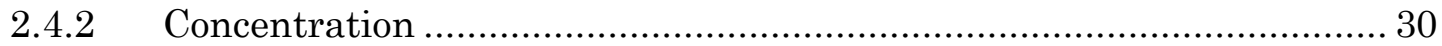

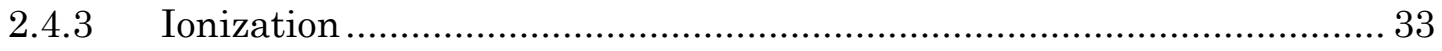

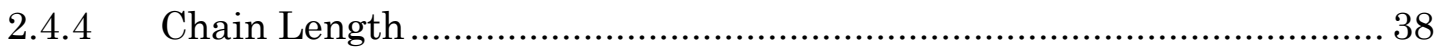

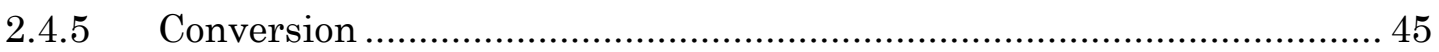

2.5 Computer Modeling of Polymerizations ................................................. 51

3 Materials, Experimental Procedures and Data Evaluation .............................. 55

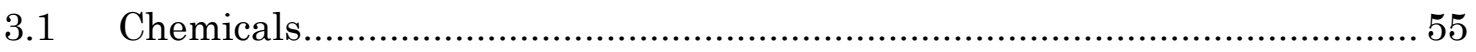




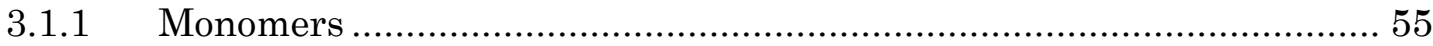

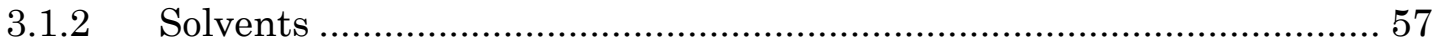

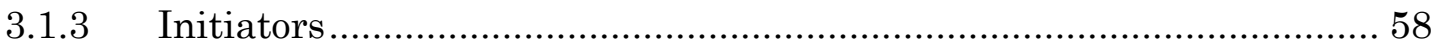

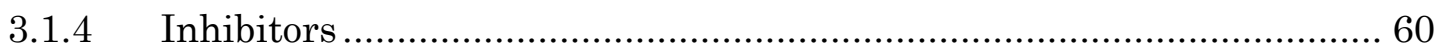

3.1.5 Substances used to prepare Buffer Solutions .................................... 60

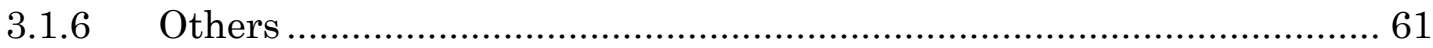

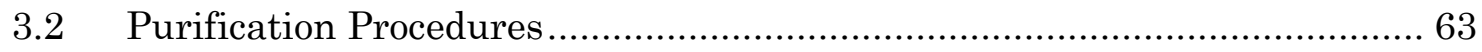

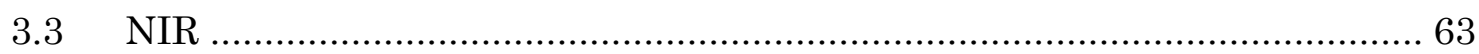

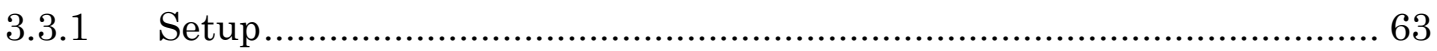

3.3.2 Thermally initiated Polymerization in a Cuvette............................... 64

3.3.3 Photoinitiated Polymerization in a Cuvette ...................................... 65

3.3.4 Degree of Monomer Conversion ……................................................. 65

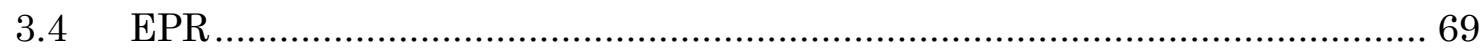

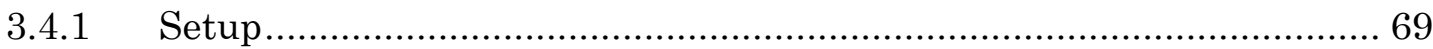

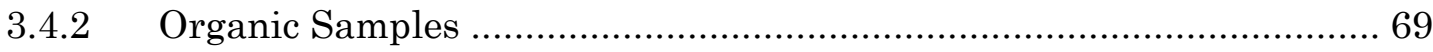

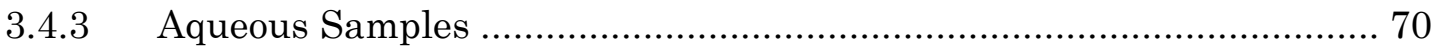

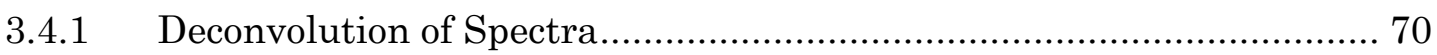

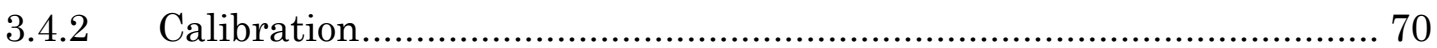

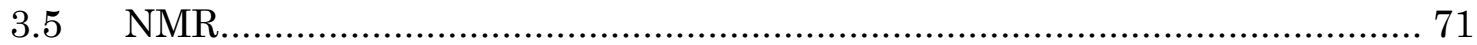

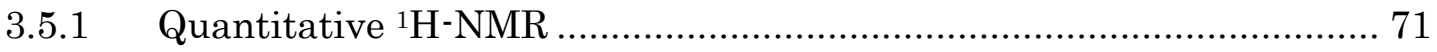

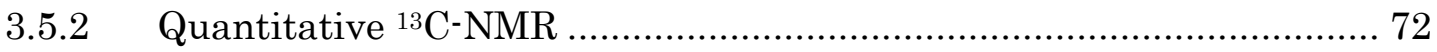

3.5.3 Polymerization in NMR Sample Tube ….......................................... 77

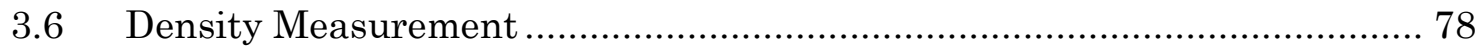

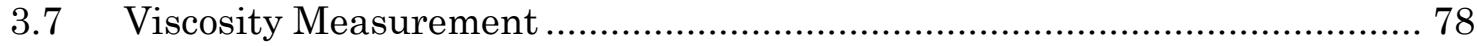

3.7.1 Important Features of Polymer Solutions .......................................... 79

3.7.2 Polymerization in Viscosity Measurement Capillary ........................... 80

3.8 Preparation of Buffer Solutions ............................................................. 80

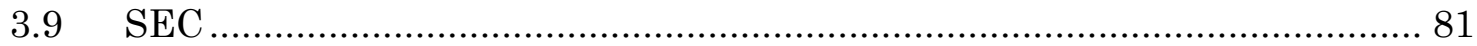




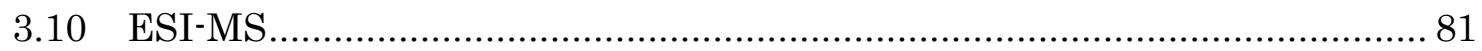

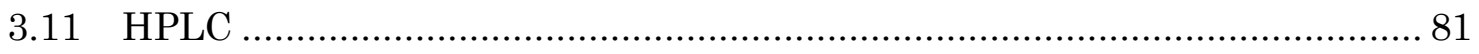

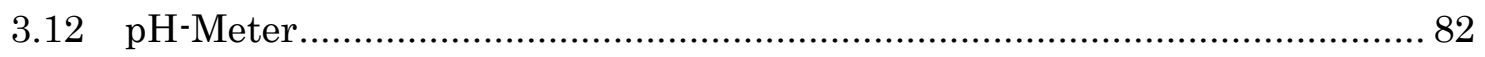

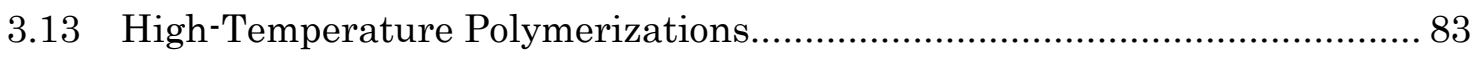

3.13.1 Stopped-Flow experiments in High-Pressure Cell................................ 84

3.13.2 Polymerization in a Tubular Reactor ............................................... 84

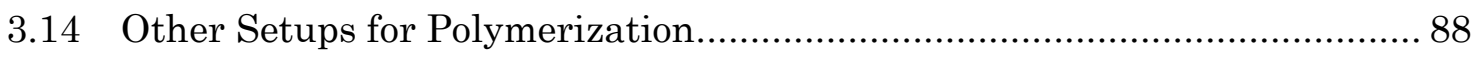

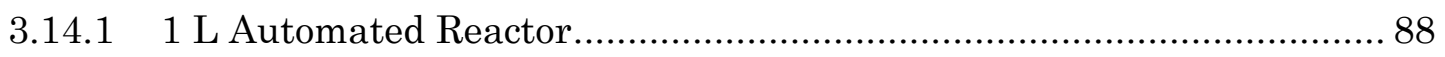

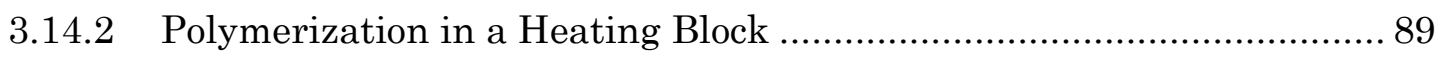

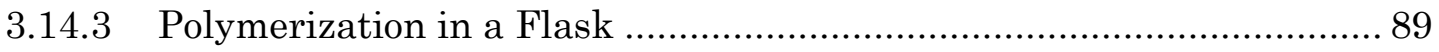

3.14.4 Polymerization in a Lined Flask ................................................. 90

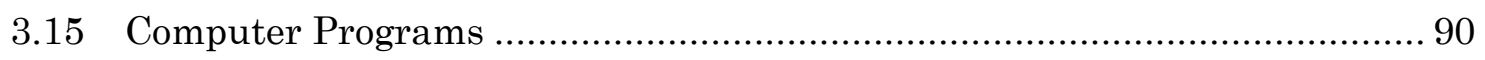

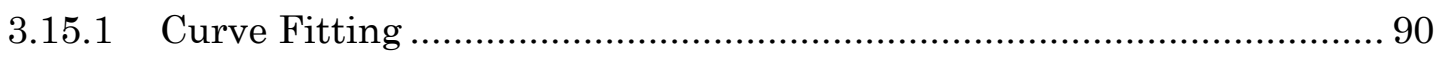

3.15.2 Determination of Joint Confidence Regions ....................................... 90

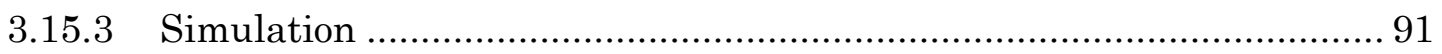

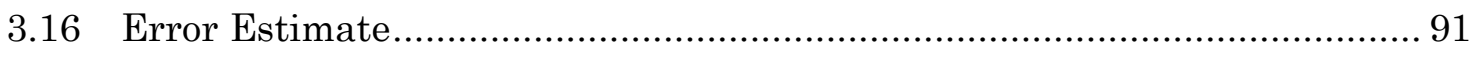

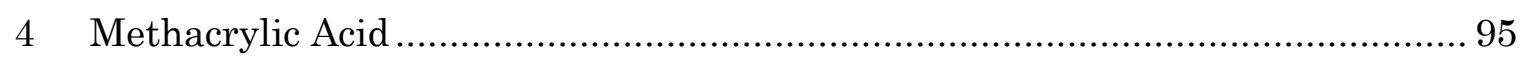

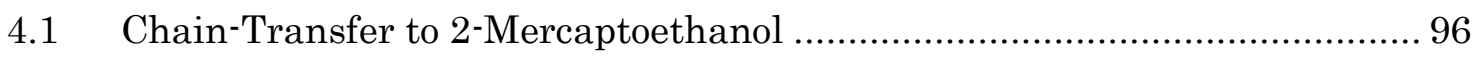

4.1.1 Chain Transfer Constants deduced by the Mayo Method .................... 97

4.1.2 Chain Transfer Constants deduced by the CLD Method .................... 101

4.1.3 Comparison of Mayo and CLD methods ......................................... 103

4.2 Model development for Non-ionized Methacrylic Acid ............................. 106

4.2.1 Modeling Polymerization at Medium initial Monomer Content ......... 110

4.2.2 Modeling Polymerization at Low initial Monomer Content ............... 123

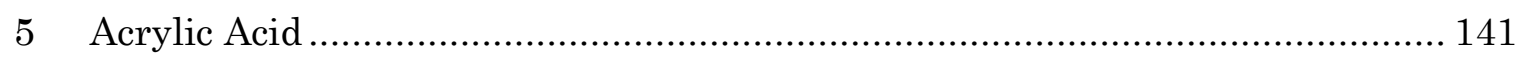

5.1 Model development for Non-ionized Acrylic Acid .................................. 142

5.1.1 Initiator Kinetics ....................................................................... 146

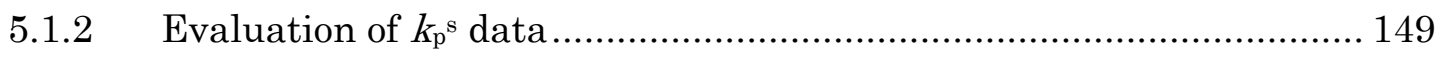


5.1.3 Evaluation of $k \mathrm{t}$ and Viscosity data ............................................. 155

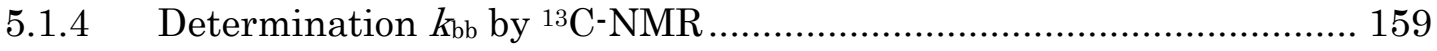

5.1.5 BA as a Model for AA to estimate $C_{\mathrm{CTA}}{ }^{\mathrm{t}}$ by EPR............................. 164

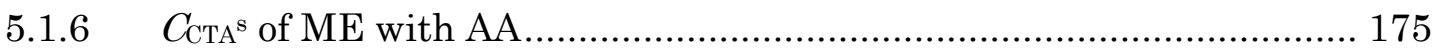

5.1.7 Determination of $k_{\mathrm{p}}$ for AA Macromonomers by ${ }^{1} \mathrm{H}-\mathrm{NMR} \ldots \ldots \ldots \ldots \ldots \ldots . . . . . . .178$

5.1.8 Modeling Polymerization at 35 to $80{ }^{\circ} \mathrm{C}$......................................... 181

5.1.9 Modeling Polymerization at High Temperature ............................... 188

5.2 Model Development for Ionized Acrylic Acid ......................................... 197

5.2.1 $\quad k_{\mathrm{p}}$ of Fully Ionized AA and dependence on Ionic Strength ................. 198

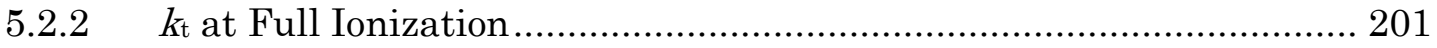

5.2.3 $k_{\mathrm{b} b}$ at Full Ionization and dependence on Ionic Strength.................. 202

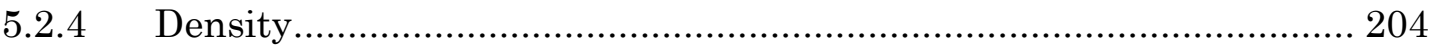

5.2.5 Modeling the Polymerization of Fully Ionized AA............................. 208

5.2.6 The dependence of $k_{\mathrm{p}}$ on the Degree of Ionization............................ 212

5.2.7 The dependence of $k_{\mathrm{t}}$ on the Degree of Ionization ........................... 214

5.2.8 The dependence of $k_{\mathrm{bb}}$ on the Degree of Ionization............................ 217

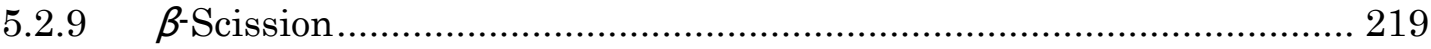

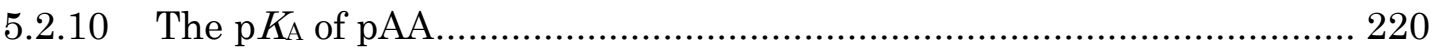

5.2.11 Modeling the Polymerization of Partly Ionized AA ............................ 224

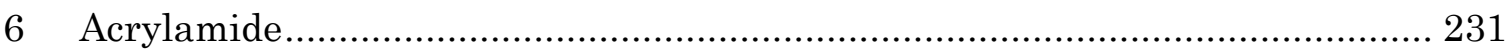

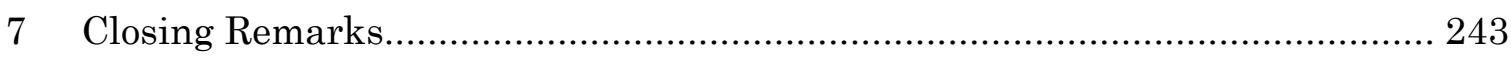

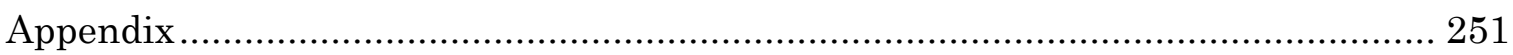

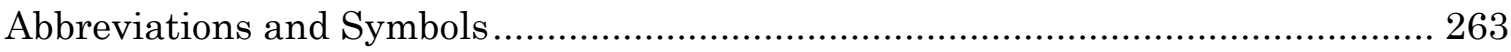

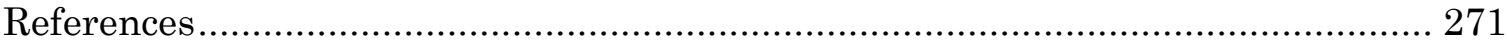




\section{Abstract}

The radical polymerization of methacrylic acid, acrylic acid and acrylamide in aqueous solution has been investigated. Detailed kinetic models for both acrylic acid, AA, and methacrylic acid, MAA, have been developed applying the program PREDICI $^{\mathrm{TM}}$. Good representation of experimental conversion vs. time profiles and molar mass distributions as well as, in case of AA, the branching level could be achieved.

The polymerization of MAA has been studied at 35 and $50{ }^{\circ} \mathrm{C}$ with focus on the influence of 2-mercaptoethanol, ME, as chain transfer agent, CTA, on reaction kinetics. The rate coefficient of transfer to CTA, $k_{\mathrm{tr}, \mathrm{CTA}}$, was measured for different monomer levels by the Mayo and the chain length distribution procedure. The ratio of $k_{\mathrm{tr}, \mathrm{CTA}}$ to the propagation rate coefficient, $k_{\mathrm{p}}$, is independent of monomer to water ratio while both rate coefficients increase by approximately one order of magnitude in passing from bulk to dilute aqueous solution.

It was found that addition of CTA reduces the rate of MAA polymerization by two effects on $k_{\mathrm{t}}$. At negligible monomer conversion, $k_{\mathrm{t}}$ increases towards higher content of CTA, because average chain length is reduced by the CTA. Chain-length dependent termination may be represented by adopting the composite model, which is a well-established theory to describe chain-length dependency of termination of macroradicals of identical size. The composite model could be applied to average chain length. The reduction of $k_{\mathrm{t}}$ towards higher degrees of monomer conversion (Norrish-Trommsdorff or gel effect) becomes weaker towards higher levels of CTA, which could be described by correlating the intensity of the gel effect to molar mass of polymer in solution. 
The polymerization of non-ionized AA in aqueous solution has been studied between 35 and $80^{\circ} \mathrm{C}$ with and without ME as CTA. Chain-length dependent termination was taken into account for modeling as for MAA. During AA polymerization a 1,5hydrogen shift (backbiting) takes place transforming the secondary propagating radical, SPR, into a tertiary midchain radical, MCR, the kinetics of which were included into the model. The backbiting reaction was quantified via ${ }^{13} \mathrm{C}-\mathrm{NMR}$, the other MCR reactions were estimated from conversion vs. time profiles. By measuring the MCR fraction during butyl acrylate, BA, polymerization via electron paramagnetic resonance, EPR, it could be shown that the transfer of MCRs to CTA is not an important reaction path. BA can be used as AA model compound so that the same finding should also apply for AA polymerizations in aqueous phase. Chain transfer of SPRs of AA was measured by the Mayo method.

The model was extended towards high-temperature polymerization of AA between 90 and $170{ }^{\circ} \mathrm{C}$, where $\beta$-scission and propagation of macromonomers need to be considered. Moreover, a model for the polymerization of ionized AA was developed, which takes numerous dependencies of rate coefficients on ionization and ionic strength into account, e.g., propagation is reduced by ionization of monomer, but to a higher extent for lower monomer concentration. Moreover, propagation of ionized monomer augments towards higher ionic strength. MCRs were found during acrylamide polymerization via EPR revealing the backbiting reaction to apply for this monomer as well. Thus, the kinetic scheme is the same as for AA polymerization.

Parts of this thesis have already been published:

Wittenberg, N. F. G.; Buback, M.; Stach, M.; Lacík, I. Macromol. Chem. Phys. 2012, $213,2653-2658$.

Wittenberg, N. F. G.; Buback, M.; Hutchinson, R. A. Macromol. React. Eng. 2013, 7, $267-276$. 


\section{1 \\ Introduction}

Polymer chemistry began with the pioneering research by Staudinger, ${ }^{[1,2]}$ who discovered the chain structure of polymers consisting of chemically bonded monomeric units. Baekeland's investigations leading to Bakelite ${ }^{\mathrm{TM}[3]}$ formed from an elimination reaction of phenol with formaldehyde started the age of commercial synthetic polymers over 100 years ago.

Since those early times, polymer production grew rapidly and became a major field of the chemical industry. In 2012, the polymer production in Germany had a production value of 27.7 billion euro, which is $19.5 \%$ of the chemical and pharmaceutical industry. ${ }^{[4]}$

Polymers may be synthesized via polycondensation, polyinsertion (catalytic), cationic, anionic or radical polymerization. All of these methods have special advantages and disadvantages and are used in industry to different extent. Radical polymerization is a robust and versatile technique, which is applied to produce e.g. polyethylene, polystyrene, polyacrylates, polymethacrylates, and corresponding copolymers in high quantities.

The physical properties of a polymer derive from the functionalities of its monomer units, but also from its molecular mass distribution (MMD) and microstructure. Thus, with the same monomer (composition) the production of quite different polymers is possible. Provided the structure-properties relationship is known, modeling of the polymerization process can be applied to simulate polymerization and predict the properties of the resulting polymer. Kinetic models are utilized as an 
additional tool for planning new industrial processes or improving established ones, e.g., reducing consumption of resources or enhancing product quality. They also find application for a more accurate process control (online use).

For precise models, accurate knowledge of all rate coefficients of the process including their various dependencies is essential. Rate coefficients are not easily determined and are often not known with sufficient accuracy.

The introduction of pulsed-laser polymerization, PLP, techniques led to a great advancement in knowledge of rate coefficients. The propagation rate coefficient can be measured precisely by the PLP-SEC method, invented by Olaj et al. ${ }^{[5]}$ based on the older rotating sector technique. PLP is combined with subsequent analysis of the formed polymer by size-exclusion chromatography, SEC. The termination rate coefficient including conversion dependence is accessible via the SP-PLP-NIR technique, introduced by Buback et al. ${ }^{[6]}$ The decline in monomer concentration after a single laser pulse, SP, initiation is monitored via time-resolved near infrared, NIR, spectroscopy. Electron paramagnetic resonance, EPR, spectroscopy allows for direct measurement of radical concentration; combination with pulsed laser polymerization led to the SP-PLP-EPR technique introduced by Buback et al. ${ }^{[7]}$ The technique provides access to chain-length dependence of the rate coefficient of termination and different types of radicals may be distinguished.

During polymerization of acrylate type monomers a 1,5-hydrogen shift (backbiting) takes place transforming the secondary propagating radical, SPR, into a tertiary midchain radical, MCR, the kinetics of which are quite different from SPR kinetics and have to be accounted for in a kinetic model.

The polymerization of water-soluble monomers is of industrial importance, as the associated polymers find various application as superabsorber material, e.g., part of hygiene and cosmetics products as well as in packaging and soil improvement, or as thickener, dispersant and emulsifier, e.g., applied in wastewater treatment, mining, textile, and paper industry.

Kinetics in aqueous solution are more complex due to the strong dependence of the rate coefficient of propagation on monomer concentration, and thus degree of monomer conversion. ${ }^{[8-10]}$ For monomers featuring ionizable moieties, kinetics are particularly challenging. The influences of ionization and ionic strength are not limited to effects on the structure of the polymer in solution; they have a great impact on polymerization kinetics as well, e.g., the rate coefficient of propagation of methacrylic acid at low monomer concentration in aqueous solution declines by 
about one order of magnitude from the non-ionized to ionized monomer. ${ }^{[11]}$ Addition of more ionizing agent, e.g., $\mathrm{NaOH}$, to fully ionized methacrylic acid, i.e., increasing ionic strength, leads to a pronounced enhancement of polymerization rate. ${ }^{[12]}$ 



\section{2 \\ Theoretical Background}

This chapter summarizes the theoretical background of the research presented in this thesis. Especially the general aspects were already presented in several other works and are therefore given briefly only. Afterwards particular aspects important, e.g., for the polymerization of acrylic monomers, effects of high temperature, and ionization of monomer are presented. Chain-length and conversion dependency are also important aspects for the modeling presented in this thesis and are consequently outlined in more detail.

\subsection{General Aspects of Radical Stability and Reactivity}

In order to understand reactivity in radical polymerization, one has to consider the factors that determine stability of organic radicals. The stability of one radical is interesting in absolute terms, but mostly relative to other radicals. At this, one has to consider how easily a radical is formed. It is equipollent to look upon the contribution of the strength of the bond, which has to be broken to form the radical, and the intrinsic stability of the radical.

First, the electronegativity of the atom where the radical is essentially located has to be considered. In general, carbon-centered radicals are more stable than nitrogencentered ones, which again are more stable than oxygen-centered ones. That is why carbon-centered radicals are most common in organic chemistry. Due to this factors transfer to carboxyl groups of acrylic acid and methacraylic acid (two monomers, on 
which this thesis focuses,) can be excluded. Furthermore, transfer of the radical function from a growing chain to the solvent water need not be considered. Nevertheless, this effect may be overcompensated by other factors, e.g., TEMPO (2,2,6,6-tetramethylpiperidine 1-oxyl) is a stable radical.

The bond strength between a carbon and a hydrogen atom is strongly influenced by hybridization: $\mathrm{sp}^{3}$ is more stable than $\mathrm{sp}^{2}$ which again is more stable than $\mathrm{sp}$. This can be explained, firstly, by an increasing s-character of the bond, which decreases bond length, and secondly, by stabilization of the radical by aliphatic substituents. At this, the radical is stabilized by hyperconjugation between the p-orbital of the radical and the $\mathrm{C}-\mathrm{H}$-bond of vicinal carbons. This effect is additive. Alkinyl and benzyl radicals are rather exotic. The only radical polymerization that features primary radicals is the polymerization of ethene (ethylene), which is only performed at high temperatures. A major part of this work is about the kinetics of secondary and tertiary radicals; their difference in reactivity originates from their difference in stability (subchapter 2.3.3).

Delocalization by conjugation to double bonds or aromatic rings causes especially strong stabilization of ca. $12 \mathrm{kcal} \mathrm{mol}^{-1}$ (vinyl and phenyl group). A good example for the impact of this stabilization is the propenyl radical formed by transfer to monomer during radical polymerization of propene. During this polymerization, transfer is so potent that only oligomeric product can be produced. For rare alkinyl radicals conjugation to only one $\pi$-bond is possible, because the other one is orthogonal. Heteroatoms can stabilize radicals by conjugation to a lone electron pair. In this case, the effect strengthens the more electron density can be transferred to the radical function. Amino groups stabilize more than hydroxyl groups because nitrogen has a lower electronegativity. A negative charge on the oxygen leads to a better stabilization. This is important for monomers with a carboxylate moiety, which are treated in subchapter 5.2.

Both donor and acceptor substituents stabilize radicals and for captodative radicals the effects (most often) add up instead of compensating each other, or yet cause an even more enhanced stabilization. Radicals show a tendency to compensate electron shortage and abundance, respectively, i.e. radicals with a prevailing influence of donors react rather with double bonds under the influence of acceptors and vice versa. This is very important for reactivity ratios in copolymerization, but also for the initiation step (see subchapter 2.2.1). 
Charges and polarity, respectively, have a strong influence on reactivity as they lower the energy of the transition state. They always reduce the entropy of the transition state thus acceleration the reaction. ${ }^{[13]}$

The formation of radical anions and cations is also possible. ${ }^{I}$ Solutions of radical anions are quite stable as long as they remain oxygen free and no protonation sources are available (Birch reduction). Radical anions are sometimes used as initiators, e.g., in BuNA (butadiene rubber) production. Radical cations are less stable and do not play a role in radical polymerization.

Furthermore, steric effects are important. Strong van-der-Waals repulsion by moieties next to the radical center stabilizes the radical function and reduce its reactivity. This is the reason why 1,2 substituted monomers are rather uncommon. Due to repulsion of moieties in the corresponding polymeric product, growth of the chain is slow and the ceiling temperature (the temperature, above which the polymer is thermodynamically less stable than the corresponding monomer) thereof is low.

Steric effects are also very important for regioselectivity. Radicals add to a 1,1substituted double bond at the C2 side; this even holds for monosubstituted double bonds. Only for a few monomer, e.g., vinyl acetate, head-head-propagation becomes significant at high temperature.

\subsection{Ideal Polymerization Kinetics of Radical Polymerization}

During radical polymerization, the reactive radical species can undergo various reactions. For a simple treatment some assumptions are made:

All reactions are irreversible.

All starting radicals are only generated by initiator and consumed by initiation.

Monomer is solely consumed by propagation.

Radicals exclusively stop growing by mutual deactivation.

All rate coefficients are independent of chain-length and concentrations.

These basic reactions and deductions are described in the following subchapters.

\footnotetext{
I Here, radical ion refers to compounds that carry a connected charge and radical function. This should not be mixed up with radicals that also have charges somewhere else. Under basic conditions, a growing chain of pAA is a polycation and a radical but not a radical cation, because the radical function is separated from the charge.
} 


\subsubsection{Formation of Radicals and Initiation}

In principle, all reactions generating radical species can be used for radical polymerization; this includes, e.g., ionizing radiation, supersonic and electrochemical reactions. Nevertheless, more common is the addition of an initiator. Initiator decomposition is induced either by UV-rays (photo initiation) or thermically (chemical initiation). Another commercially important initiation system is redox initiation, e.g., hydroperoxide and iron(II) react to hydroxide, hydroxyl radical and iron(III).

Common photoinitiators are ketones that undergo $\alpha$-cleavage after photoexcitation of the carbonyl function. This is the Norrish type I reaction. ${ }^{[14]}$ For photoinitiators, the rate of decomposition is usually independent of temperature. Due to higher costs, this method of initiation is used more often in research than in industrial production.

Common chemical initiators are peroxides and azo-compounds, because both the oxygen-oxygen-bond and the nitrogen-carbon-bond can undergo homolytic bond cleavage rather easily. The former are cheaper and thus of higher industrial importance. In order to reduce the activation energy of the decay reaction, peroxides are sometimes combined with a reducing agent forming a redox initiation system.

Initiation may also occur by reactions between components in the reaction mixture other than proper initiator. A well known example are two mechanisms of thermal auto initiation of monomer styrene. ${ }^{[15,16]}$ The thiol-ene reaction is another example of initiation by non-initiator compounds within the reaction mixture. ${ }^{[17]}$

The normal photo and chemical initiators follow the reaction scheme:

$$
\mathrm{I} \stackrel{k_{\mathrm{d}} \cdot f}{\longrightarrow} 2 \mathrm{R}_{0}^{\bullet}
$$

The initiator, I, decomposes into two growing chains of chain length zero. This ignores the initiator fragment completely. Sometimes the initiator fragment at the end of the chain is counted as one monomer unit leading to the following scheme instead: 


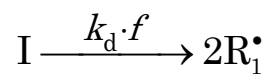

The decay of the initiator takes place as a first-order reaction with the rate coefficient $k_{\mathrm{d}}$.

Merely a fraction of initiator fragments is available to initiate radical polymerization. This fraction is given by the correction factor, $f$, which is the initiator efficiency. Its value depends on viscosity of solvent and effective size of the fragments; usually it varies between 0.4 and 0.9 . After the decay of the initiator the fragments both being radicals may recombine as long as they remain together in the solvent cage. Only after one of them has left it by diffusion, immediate recombination is prevented. In addition, side reactions of the initiator radicals may further reduce the share of radicals available for initiation decreasing $f$ even more.

The rate of formation of radicals, which describes the built up of the radical concentration, $c_{\mathrm{R}}$, from initiator concentration, $c_{\mathrm{I}}$, with time, $t$, can be expressed by eq. (2.1).

$$
\frac{\mathrm{d} c_{\mathrm{R}}}{\mathrm{d} t}=2 \cdot k_{\mathrm{d}} \cdot f \cdot c_{\mathrm{I}}
$$

These newly produced radicals react with a monomer molecule, M, to initiate chain growth with the rate coefficient $k_{\mathrm{i}}$. This step is usually very fast and therefore ignored, because in this case it is negligible for overall rate of polymerization.

$$
\mathrm{R}_{0}^{\bullet}+\mathrm{M} \stackrel{k_{\mathrm{i}}}{\longrightarrow} \mathrm{R}_{1}^{\bullet}
$$

Initiator decay reduces initiator concentration and it may happen that initiator is decomposed completely prior to complete monomer conversion, which is referred to 
as dead-end polymerization. In industrial practice, often mixtures (cocktails) of initiators with different rates of decomposition are used.

It is important to keep in mind that even in the more robust radical polymerizations not each initiator is able to initiate effectively. Depending on the stability of the initiating and the resulting radical, initiation can be slow and the corresponding initiator would be considered unsuitable for this polymerization.

In case of photochemically initiated polymerization induced by a short (a few ns) UVlaser pulse, as used in pulsed-laser-polymerization, PLP, techniques, creation of radicals can be considered as instantaneous, because the formation of radicals is fast in comparison to a subsequent reaction steps.

In principel, the radical concentration produced upon applying a laser pulse at time zero, $c_{\mathrm{R}}^{0}$ may be determined by eq. (2.2), which contains quantum yield (fraction of absorbed photons leading to decomposition), $\Phi$, initiator efficiency, quantity, $n$, of absorbed photons, and irradiated sample volume, $V$.

$$
c_{\mathrm{R}}^{0}=2 \cdot \Phi \cdot f \cdot \frac{n}{V}
$$

According to the Beer-Lambert-Bouguer law, ${ }^{[18-20]}$ the amount of absorbed photons can be calculated from the total amount of photons hitting the sample by eq. (2.3);

$$
n(\text { absorbed })=n(\text { total }) \cdot\left(1-\frac{\Phi}{\Phi_{0}}\right)=\frac{E_{\mathrm{p}}}{E_{\lambda}} \cdot\left(1-10^{-\varepsilon(\tilde{v}) \cdot c_{\mathrm{I}} \cdot 1}\right)
$$

$\Phi$ is the radiant power (intensity) at a certain wavenumber, $\tilde{v}$, the index 0 means: in front of the cell. $E$ denotes energy, at this, an index of p refers to laser pulse, an index of $\lambda$ to molar energy of photons at given laser wavelength. $\varepsilon$ is the molar decadic absorption coefficient, and $l$ the path length within the sample cell. In practice, determining all these values proves virtually impossible and $c_{\mathrm{R}}^{0}$ is measured directly (see subchapter 3.4). 


\subsubsection{Propagation}

Polymer chains grow by adding monomer, $\mathrm{M}$, thus increasing chain length, $i$, by one. This process is called propagation.

$$
\mathrm{R}_{i}^{\bullet}+\mathrm{M} \stackrel{k_{\mathrm{p}}}{\longrightarrow} \mathrm{R}_{i+1}^{\bullet}
$$

The rate of monomer consumption by propagation is described by eq. (2.4). The corresponding rate coefficient is $k_{\mathrm{p}}$.

$$
-\frac{\mathrm{d} c_{\mathrm{M}}}{\mathrm{d} t}=k_{\mathrm{p}} \cdot c_{\mathrm{M}} \cdot c_{\mathrm{R}}
$$

\subsubsection{Termination}

The process of chain growth ends with the termination of the radical (or with transfer, v.i.). Chain termination is characterized by the reaction of two radicals eliminating both radical functions. It proceeds either by disproportionation, the transfer of a $\beta$-hydrogen from one radical to the other forming an unsaturated chainend, or by combination, i.e., a formation of a covalent bond between the active centers of propagating radicals.

In case of combination, the degree of polymerization, $i+j$, of the resulting macromolecule, $\mathrm{P}_{i+j}$, is the sum of the degrees of polymerization $i$ and $j$ of the two primordial growing chains, while disproportionation does not change the degrees of polymerization of the reactants.

$$
\begin{aligned}
& \mathrm{R}_{i}^{\bullet}+\mathrm{R}_{j}^{\bullet} \stackrel{k_{\mathrm{t}, \text { comb }}}{\longrightarrow} \mathrm{P}_{i+j} \\
& \mathrm{R}_{i}^{\bullet}+\mathrm{R}_{j}^{\bullet} \stackrel{k_{\mathrm{t}, \text { disp }}}{\longrightarrow} \mathrm{P}_{i}+\mathrm{P}_{j}
\end{aligned}
$$


The overall termination rate coefficient is the sum of the rate coefficients of combination, $k_{\mathrm{t}, \mathrm{comb}}$, and of disproportionation, $k_{\mathrm{t}, \mathrm{disp}}$. Which of the mechanisms prevails is mostly determined by the structure of the monomer, steric hindrance favoring disproportionation. To some degree, higher temperature supports disproportionation. The fraction of disproportionation is given by $\kappa$.

The rate of consumption of radicals is described by a second-order rate equation. To describe the process eq. (2.5) including a factor of 2 is used throughout this work, as recommended by IUPAC. ${ }^{[21]}$

$$
-\frac{\mathrm{d} c_{\mathrm{R}}}{\mathrm{d} t}=2 \cdot k_{\mathrm{t}} \cdot c_{\mathrm{R}}^{2}
$$

\subsubsection{Steady State Kinetics}

Under continuous initiation, a quasi-stationary state (Bodenstein principle) is reached quickly. Thus, the rates of generation and consumption of radicals are equal, hence eq. (2.1) and eq. (2.5) can be combined to eq. (2.6). Further combination with eq. (2.4) leads to eq. (2.7), which gives the rate of polymerization, $r_{\mathrm{P}}$.

$$
\begin{gathered}
k_{\mathrm{d}} \cdot f \cdot c_{\mathrm{I}}=k_{\mathrm{t}} \cdot c_{\mathrm{R}}^{2} \\
r_{\text {polym }}=-\frac{\mathrm{d} c_{\mathrm{M}}}{\mathrm{d} t}=c_{\mathrm{M}} \cdot \frac{k_{\mathrm{p}}}{\sqrt{k_{\mathrm{t}}}} \cdot \sqrt{k_{\mathrm{d}} \cdot f \cdot c_{\mathrm{I}}}
\end{gathered}
$$

Likewise considerations allow for calculating the average number of monomer units added to an initiating radical until it terminates. This is called the kinetic chain length, $\delta$, and can be calculated according to eq. (2.8) as the rate of the overall reaction divided by the rate of the initiation reaction. 


$$
\delta=\frac{c_{\mathrm{M}} \cdot k_{\mathrm{p}}}{\sqrt{k_{\mathrm{d}} \cdot f \cdot c_{\mathrm{I}} \cdot k_{\mathrm{t}}}}
$$

\subsection{Additional Reactions}

The reactions given in subchapter 2.2 are generally considered to be the most important ones, but depending on reaction conditions and desired accuracy of the description of the process other reactions need to be taken into account. They are described in the following subchapters. The growing radicals are very reactive and can basically react with all other substances in the reaction mixture.

The so-obtained radicals may reinitiate quickly. This process is called transfer. It can occur with small molecules as described in subchapter 2.3.1. Transfer to polymer has different aspects and is treated separately. Intermolecular (see subchapter 2.3.2) and intramolecular transfer (see subchapter 2.3.3) are different in kinetics and in their impact on produced polymer. At higher temperature, $\beta$-scission becomes important for polymerization, especially as a follow-up process of transfer to polymer.

If the small molecular transfer product initiates slowly or not at all, this process is called retardation or inhibition, respectively (see subchapter 2.3.2).

\subsubsection{Transfer Reactions to Small Molecules}

In the context of radical polymerization transfer reaction always means transfer of the radical function. The following schemes illustrate possible reactions.

Transfer reaction: 


$$
\mathrm{R}_{i}^{\cdot}+\mathrm{X} \stackrel{k_{\mathrm{tr}, \mathrm{X}}}{\longrightarrow} \mathrm{P}_{i}+\mathrm{X}^{\cdot}
$$

The radical function is transferred from the growing chain, $\mathrm{R}_{i}^{\cdot}$, with chain length $i$ to an arbitrary species, $\mathrm{X}$, forming the new radical $\mathrm{X}^{\bullet}$ and dead polymer, $\mathrm{P}_{i}$. The corresponding rate coefficient $k_{\mathrm{tr}, \mathrm{X}}$ is correlated with the ratio of stabilities of $\mathrm{R}_{i}^{\cdot}$ and $\mathrm{X}^{\bullet}$.

Reinitiation:

$$
\mathrm{X}^{\bullet}+\mathrm{M} \stackrel{k_{\mathrm{i}, \mathrm{X}}}{\longrightarrow} \mathrm{R}_{1}^{\bullet}
$$

By adding monomer, $\mathrm{M}$, the newly formed radical produces another growing chain, $\mathrm{R}_{1}^{\cdot}$, of chain length unity. This takes place with the rate coefficient of reinitiation by $\mathrm{X}^{\bullet}, k_{\mathrm{i}, \mathrm{X}}$.

Termination:

$$
\mathrm{R}_{i}^{\cdot}+\mathrm{X}^{\cdot} \stackrel{k_{\mathrm{t}, \mathrm{X}}}{\longrightarrow} \mathrm{P}_{i}
$$

Instead of initiating, $\mathrm{X}^{\bullet}$ can also undergo termination reactions. The corresponding rate coefficient is $k_{\mathrm{t}, \mathrm{X}}$. If this process is of importance, it reduces radical concentration and thus rate.

Usually, the only transfer rate coefficients that is of interest is $k_{\mathrm{tr}, \mathrm{X}}$. Typically, not the rate coefficient itself but its ratio to $k_{\mathrm{p}}$, called chain transfer constant of transfer to $\mathrm{X}, C_{\mathrm{X}}$, is considered, see eq. (2.9). Strictly speaking, it is not a real constant, but mostly the two coefficients change in the same way under different conditions, e.g., upon change of temperature, thus leaving $C_{\mathrm{x}}$ untouched. The activation energy (compare subchapter 2.4.1) of $C_{\mathrm{X}}$ is typically rather small $(10 \mathrm{~kJ} / \mathrm{mol})$ or imperceptible, respectively. ${ }^{[22,23]}$ Overall, there are surprisingly few studies about the activation energy of chain transfer. Nevertheless, this assumption of $C_{\mathrm{x}}$ being a 
constant may not hold under all conditions, e.g., the value can change with solvent composition. ${ }^{[24]}$

$$
C_{\mathrm{X}}=\frac{k_{\mathrm{tr}, \mathrm{X}}}{k_{\mathrm{p}}}
$$

Chain transfer can occur to all species in a reaction mixture, e.g., initiator, monomer, solvent. Every chain-transfer event reduces molar mass of produced polymer. Thus, the kinetic-chain length does not give the degree of polymerization. A transfer term has to be added yielding eq. (2.10).

$$
\langle i\rangle=\frac{c_{\mathrm{M}} \cdot k_{\mathrm{p}}}{\sqrt{k_{\mathrm{d}} \cdot f \cdot c_{\mathrm{I}} \cdot k_{\mathrm{t}}}+\sum_{i} k_{\mathrm{tr}, i} \cdot c_{i}}
$$

Components that easily undergo transfer may be added to a polymerization system in order to control molar mass. They are called chain-transfer agents, CTAs. If the rate of chain transfer is so high that only oligomer is produced, the process is called telomerization and instead of CTA the additive is called telogen. Typically, halogenated alkanes or thiols are used as CTAs with high chain transfer constants and aldehydes or alcohols are used as weaker CTAs.

The facile cleavage of the S-H bond in thiols is associated with large chain-transfer rate coefficients. ${ }^{[25]}$ The sulfur-centered radical produced by hydrogen transfer may add to monomer rapidly.

Transfer reduces chain length but does not influence radical concentration directly. Thus, the CTA should not influence polymerization kinetics. Later in this work, it will be shown that this assumption has only limited validity (see subchapter 4.2) 


\section{Determination of Chain-Transfer Constants}

The most widespread technique for determing $C_{\mathrm{X}}$ is the Mayo method. ${ }^{[26]}$ A more recently developed technique is referred to as CLD method. ${ }^{[27]} \mathrm{In}$ addition, there is a third scarcely used method: O'Brien and Gornick ${ }^{[28]}$ showed, based on considerations of Mayo, ${ }^{[26]}$ a way to determine chain transfer-constants without the necessity to measure molecular masses.

In principle, the Mayo and CLD technique should work equally well. Nonetheless, there has been quite some dispute about the method of choice. ${ }^{[22,23,29,30]}$ Both methods require polymer from reaction to low conversion under steady-state conditions, which is subsequently analyzed for molar-mass distribution, MMD. Under particular conditions $C_{\mathrm{X}}$ may also be deduced from pulsed laser polymerization. ${ }^{[22,31]}$

The Mayo procedure refers to eq. (2.11). If only one chain transfer process is of interest, eq. (2.12) can be used. The inverse of the number-average degree of polymerization, $\left\langle i_{\mathrm{n}}\right\rangle$, is plotted vs. the ratio of CTA to monomer concentrations. The slope to a straight-line fit yields $C_{\mathrm{X}}$. Commercial SEC control programs directly yield the number and weight averages, $M_{\mathrm{n}}$ and $M_{\mathrm{w}}$, respectively. From $M_{\mathrm{n}},\left\langle i_{\mathrm{n}}\right\rangle$ is simply obtained by dividing by monomer mass, which makes the Mayo method easily applicable.

Eq. (2.11) is transformed into eq. (2.12) defining $\left\langle i_{\mathrm{n}}^{0}\right\rangle$ as the degree of polymerization in the absence of the CTA.

$$
\begin{gathered}
\frac{1}{\left\langle i_{\mathrm{n}}\right\rangle}=\frac{(1-\alpha) \cdot\left\langle k_{\mathrm{t}}\right\rangle \cdot c_{\mathrm{R}}}{k_{\mathrm{p}} \cdot c_{\mathrm{M}}}+\sum_{j} \frac{k_{\mathrm{tr}, j} \cdot c_{j}}{k_{\mathrm{p}} \cdot c_{\mathrm{M}}} \\
\frac{1}{\left\langle i_{\mathrm{n}}\right\rangle}=C_{\mathrm{CTA}} \frac{c_{\mathrm{CTA}}}{c_{\mathrm{M}}}+\frac{1}{\left\langle i_{\mathrm{n}}^{0}\right\rangle}
\end{gathered}
$$

$c_{\mathrm{R}}$ and $c_{\mathrm{M}}$ refer to concentration of radical and monomer, respectively. Guillemets indicate: chain-length averaged. $c_{j}$ is the concentration of an arbitrary species $j$, to which transfer occurs with the rate coefficient $k_{\mathrm{tr}, j}$. 
The CLD method uses eq. (2.13) and (2.14). ${ }^{[23,29]}$ Plotting the logarithm of polymer mass distribution, $P_{m}$, as a function of mass, $m$, should yield a straight line with slope $\lambda$ for large molar masses, i.e., for $m$ approaching infinity. Within a second step, the product of $\lambda$ and negative molar mass of the monomer, $M_{\mathrm{M}}$, is plotted vs. the ratio of CTA to monomer concentrations, $c_{\mathrm{CTA}} / c_{\mathrm{M}}$. According to eq. (2.14), the slope to the so-obtained straight line yields the transfer constant, $C_{\mathrm{CTA}}$.

$$
\begin{gathered}
\lim _{m \rightarrow \infty} \frac{\mathrm{d} \ln \left(P_{m}\right)}{\mathrm{d} m}=-\frac{1}{M_{\mathrm{M}}}\left(\frac{\left\langle k_{\mathrm{t}}\right\rangle \cdot c_{\mathrm{R}}}{k_{\mathrm{p}} \cdot c_{\mathrm{M}}}+\sum_{i} \frac{k_{\mathrm{tr}, i} \cdot c_{i}}{k_{\mathrm{p}} \cdot c_{\mathrm{M}}}\right)=\lambda \\
\lambda \cdot\left(-M_{\mathrm{M}}\right)=C_{\text {СТА }} \frac{c_{\text {СTA }}}{c_{\mathrm{M}}}+\lambda^{0} \cdot\left(-M_{\mathrm{M}}\right)
\end{gathered}
$$

It has been articulated that the CLD method is less sensitive towards problems with SEC calibration and signal analysis. ${ }^{[23]}$

The method of O'Brien and Gornick ${ }^{[28]}$ employs eq. (2.15). A double logarithmic plot of the ratio of initial concentration to concentration of CTA and monomer at any conversion should give a straight line, the slope of which is $C_{\mathrm{CTA}}$. The technique works with non-catalytic CTAs only. For end-group analysis usually ${ }^{1} \mathrm{H}-\mathrm{NMR}$ or titration is used. This method may also used to measure CTA concentration.

$$
\ln \left(\frac{c_{\mathrm{CTA}}^{0}}{c_{\mathrm{CTA}}}\right)=C_{\mathrm{CTA}} \ln \left(\frac{c_{\mathrm{M}}^{0}}{c_{\mathrm{M}}}\right)
$$

\subsubsection{Intermolecular Transfer to Polymer}

Instead of transfer of the radical function to a small molecule, it can also be transferred to polymer in the reaction mixture, following this scheme: 


$$
\mathrm{R}_{i}^{\bullet}+\mathrm{P}_{j} \stackrel{k_{\mathrm{tr}, \mathrm{P}}}{\longrightarrow} \mathrm{R}_{j}^{\bullet}+\mathrm{P}_{i}
$$

Commonly, the newly formed radical is not of the same type as the original one, because the rate coefficient of transfer to polymer is higher if the newly formed radical is more stable. Naturally, the reactivity of the more stable radical is smaller. During polymerization of acrylate-type monomers the secondary propagating radical, SPR, may react to the more stable tertiary radical midchain radical, MCR, by a transfer process.

The transfer constant varies a lot with the monomer type. Under most conditions, the effective rate of intermolecular transfer to polymer is too low to have a notable kinetic effect.

Transfer to polymer can have a strong effect on polymer properties. Long-chain branching points are formed by transfer to polymer and subsequent addition of polymer, or subsequent termination. Already a small number of long chain branching points has a strong effect on the physical properties of the polymer. Transfer to polymer often becomes important at high conversion when the concentration of polymer is elevated. This reaction broadens the MMD. If the polymerization temperature is sufficiently high, $\beta$-scission (see subchapter 2.3.1) becomes an important follow-up reaction.

\subsubsection{Intramolecular Transfer to Polymer - Backbiting}

A growing polymer chain may transfer the radical function backwards along the chain. This reaction is called backbiting. The rate coefficient of backbiting, $k_{\mathrm{bb}}$, is higher, in case that more stable radicals are formed. It was first described for ethene polymerization. ${ }^{[32]}$ Here a 1,4-, 1,5-, and 1,6-hydrogen shift takes place. During polymerization of acrylic monomers, only backbiting via a 1-5-hydrogen shift is significant. ${ }^{[33]}$ As for intermolecular transfer, this shift transforms a secondary into a tertiary radical, also called MCR. The higher stability of the tertiary radical makes backbiting an enthalpically-driven process. In Figure 2-1 the mechanism of backbiting is depicted, which occurs via an intermediate six-membered ring. 


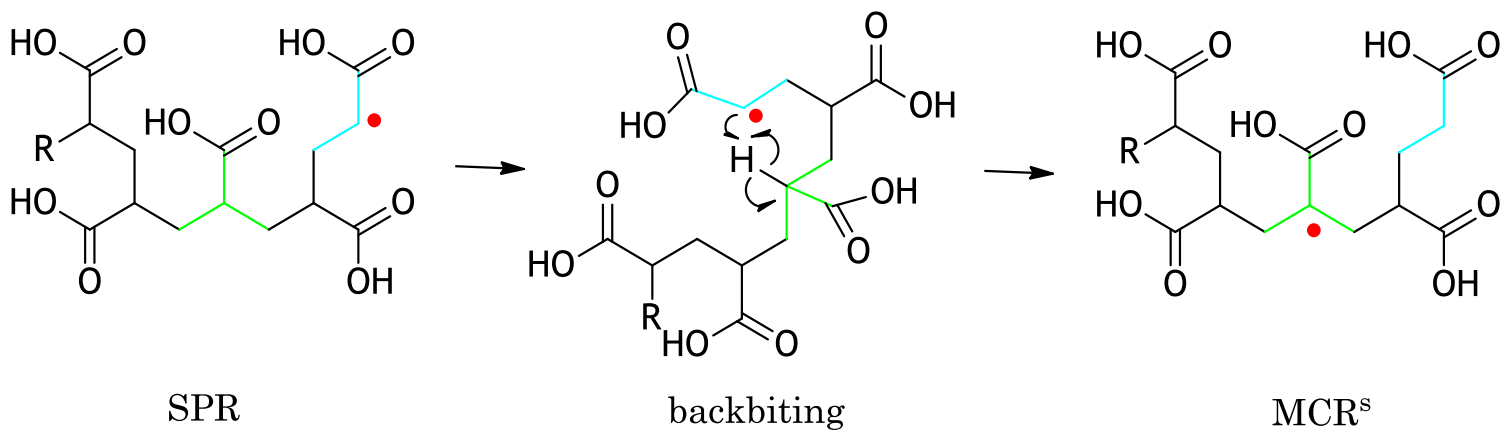

Figure 2-1 The mechanism of backbiting is shown for a growing chain in acrylic acid polymerization. First, the radical function (marked red) is located at the end of the chain (marked turquoise). Then a six-membered ring is formed and one electron from the bond of the hydrogen atom attached to the carbon atom five bonds back in the chain (marked green) forms together with the electron of the original radical function a new bond between the hydrogen atom and the end of the chain. By this process a new radical function is formed at the position of the primordial hydrogen bond.

The only difference between MCRs formed by inter- and intramolecular chain transfer is the position in the chain, to which the radical function is transferred. If necessary to specify, in this work, $\mathrm{MCR}^{\mathrm{s}}$ denotes an MCR formed by an 1,5-hydrogen shift and $\mathrm{MCR}^{1}$ those with the radical function somewhere in the chain.

MCRs can add monomer and thus be retransformed into SPRs. This is shown in Figure 2-2. Note that it was calculated for BA, that the newly formed SPR reacts with different rate coefficients as "normal" SPRs. ${ }^{[34]}$ This should be true for all acrylate-type monomers.
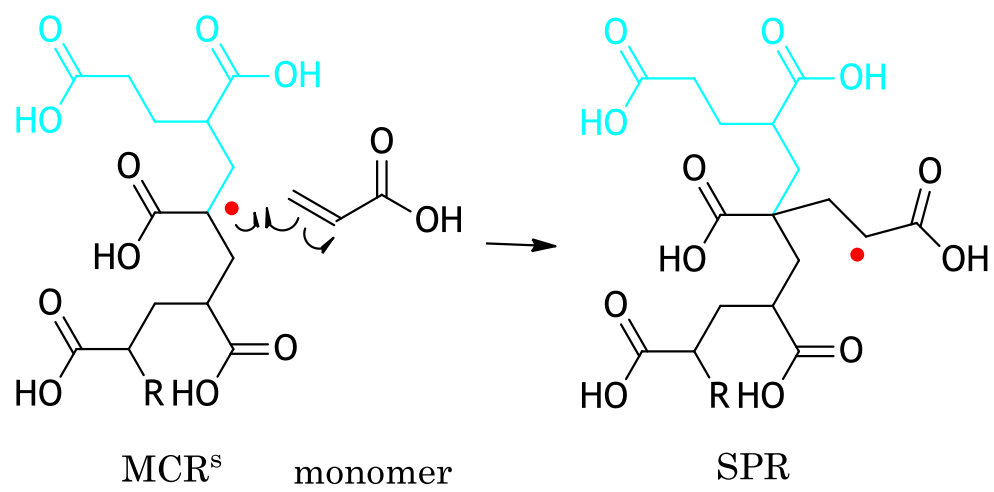

Figure 2-2 The mechanism of MCR-propagation is shown for an MCR of acrylic acid. By adding monomer, an MCR is transformed back into an SPR, which has an additional short branch (marked turquoise). 
Significant backbiting makes reaction kinetics more complicated. First, the backbiting itself has to be considered:

$$
\mathrm{R}_{\mathrm{SPR}, i}^{\cdot \stackrel{k_{\mathrm{bb}}}{\longrightarrow}} \mathrm{R}_{\mathrm{MCR}, i}
$$

Additionally, propagation has to be distinguished:

$$
\begin{aligned}
& \mathrm{R}_{\mathrm{SPR}, i}^{\bullet}+\mathrm{M} \stackrel{k_{\mathrm{p}}^{\mathrm{s}}}{\longrightarrow} \mathrm{R}_{\mathrm{SPR}, i+1}^{\bullet} \\
& \mathrm{R}_{\mathrm{MCR}, i}^{\bullet}+\mathrm{M} \stackrel{k_{\mathrm{p}}^{\mathrm{t}}}{\longrightarrow} \mathrm{R}_{\mathrm{SPR}, i+1}^{\bullet}
\end{aligned}
$$

And the same applies to termination:

$$
\begin{aligned}
& \mathrm{R}_{\mathrm{SPR}, i}^{\cdot}+\mathrm{R}_{\mathrm{SPR}, j}^{\cdot} \stackrel{k_{\mathrm{t}}^{\mathrm{ss}}}{\longrightarrow} \mathrm{P}_{i+j} \dot{\vee} \mathrm{P}_{i}+\mathrm{P}_{j} \\
& \mathrm{R}_{\mathrm{SPR}, i}^{\cdot}+\mathrm{R}_{\mathrm{MCR}, j}^{\cdot \stackrel{k_{\mathrm{t}}^{\mathrm{st}}}{\longrightarrow}} \mathrm{P}_{i+j} \dot{\vee} \mathrm{P}_{i}+\mathrm{P}_{j} \\
& \mathrm{R}_{\mathrm{MCR}, i}^{\cdot}+\mathrm{R}_{\mathrm{MCR}, j}^{\cdot} \stackrel{k_{\mathrm{t}}^{\mathrm{tt}}}{\longrightarrow} \mathrm{P}_{i+j} \dot{\vee} \mathrm{P}_{i}+\mathrm{P}_{j}
\end{aligned}
$$

Backbiting has a strong effect on rate of polymerization and product properties. The latter effect led to its discovery. ${ }^{[32,35]}$ MCRs are more stable than SPRs and thus propagate much slower, e.g., in AA polymerization at $50{ }^{\circ} \mathrm{C}$ the ratio $k_{\mathrm{p}}^{\mathrm{t}}$ to $k_{\mathrm{p}}^{s}$ is $5.33 \cdot 10^{-4} \cdot{ }^{[36,37]}$ This means $R_{\mathrm{P}}$ is slowed down by the backbiting reaction and eq. (2.4) has to be transformed into eq. (2.16), which results in an effective $k_{\mathrm{p}}$ value defined by eq. (2.17). 


$$
-\frac{\mathrm{d} c_{\mathrm{M}}}{\mathrm{d} t}=k_{\mathrm{p}}^{\mathrm{s}} \cdot c_{\mathrm{M}} \cdot c_{\mathrm{R}} \cdot \boldsymbol{x}_{\mathrm{SPR}}+k_{\mathrm{p}}^{\mathrm{t}} \cdot c_{\mathrm{M}} \cdot c_{\mathrm{R}} \cdot \boldsymbol{X}_{\mathrm{MCR}}=k_{\mathrm{p}}^{\mathrm{s}} \cdot c_{\mathrm{M}} \cdot c_{\mathrm{SPR}}+k_{\mathrm{p}}^{\mathrm{t}} \cdot c_{\mathrm{M}} \cdot c_{\mathrm{MCR}}
$$

$$
k_{\mathrm{p}}(\text { effective })=k_{\mathrm{p}}^{\mathrm{s}} \cdot \boldsymbol{x}_{\mathrm{SPR}}
$$

The reduction of effective propagation leads to lower polymer molecular mass.

If a steady-state assumption is made: $\mathrm{d} c_{\mathrm{MCR}} / \mathrm{dt}=$ const. (compare subchapter 2.2.4) and transfer to monomer plus $\beta$-scission (see subchapter 2.3.1) is ignored, the fraction of MCRs may be estimated by eq. (2.18). [38]

$$
x_{\mathrm{MCR}}=\frac{c_{\mathrm{MCR}}}{c_{\mathrm{SPR}}+c_{\mathrm{MCR}}}=\frac{k_{\mathrm{bb}}}{k_{\mathrm{p}}^{\mathrm{t}} \cdot c_{\mathrm{M}}+2 \cdot k_{\mathrm{t}}^{\mathrm{tt}} \cdot c_{\mathrm{MCR}}+2 \cdot k_{\mathrm{t}}^{\mathrm{st}} \cdot c_{\mathrm{SPR}}+k_{\mathrm{bb}}}
$$

Major simplification may be achieved with the so-called long-chain hypothesis, i.e., it is much more probable for an MCR to add to a monomer molecule than to terminate or undergo transfer reactions $\left(k_{\mathrm{p}}^{\mathrm{t}} \cdot c_{\mathrm{M}} \gg k_{\mathrm{tr}, \mathrm{M}}^{\mathrm{t}} \cdot c_{\mathrm{M}}+2 \cdot k_{\mathrm{t}}^{\mathrm{tt}} \cdot c_{\mathrm{MCR}}+2 \cdot k_{\mathrm{t}}^{\mathrm{st}} \cdot c_{\mathrm{SPR}}\right)$ :

$$
x_{\mathrm{MCR}}=\frac{k_{\mathrm{bb}}}{k_{\mathrm{p}}^{\mathrm{t}} \cdot c_{\mathrm{M}}+k_{\mathrm{bb}}}
$$

There is some indication that the radical function of an MCR formed by backbiting can move further back along the chain, transforming into an MCR, which is similar to those formed by intermolecular transfer. ${ }^{[34,39]}$ There is no enthalpical gain by this process, but activation energy has been calculated to be rather low for BA, making it a relevant mechanism. ${ }^{[34]}$ It was calculated that an $\mathrm{MCR}^{\mathrm{s}}$ is likely to undergo backbiting again, because its geometry favors it. ${ }^{[34]}$ 
Hutchinson et al. ${ }^{[40,41]}$ found that backbiting can be influenced by the choice of solvent. They hypothesize that hydrogen bond interactions between the growing chain and solvent molecules stiffen the chain hence hindering its backward movements reducing the rate of backbiting.

Short-chain branching has consequences for polymer properties that differ from the ones of long-chain branching.

\subsection{1 $\quad \beta$-Scission Reaction}

The $\beta$-scission means the breakage of the $\mathrm{C}$ - $\mathrm{C}$-bond in 2-position to the carbon atom bearing the radical function. Therefore, a scission of the carbon backbone of the polymer chain takes place.

If this happens to an SPR, the reaction is the reverse of propagation, forming a monomer and a polymer chain shortened by one; this is why it is called depropagation. Depropagation has a higher activation energy than propagation. The temperature, at which the rate of depropagation becomes as fast as the rate of propagation, is called ceiling temperature. As the rate of propagation depends on monomer concentration, the ceiling temperature also depends on it. Above the ceiling temperature, polymerization is no longer possible.

If the split comes about for an MCR, it is converted into an SPR and a dead polymer chain with an unsaturated end-group. With its terminal double-bond it can function as a monomer, thus it is called macromonomer, MM. $\beta$-scission can go to both sides. This is especially important for $\mathrm{MCR}^{\mathrm{s}}$, because here, depending on the side of scission, either a "real" MM or a three-monomer-unit-MM can be built. Labeling the latter macromonomer may be actually misleading. Yet, in this work, they are still called MM for reasons of continuity. 


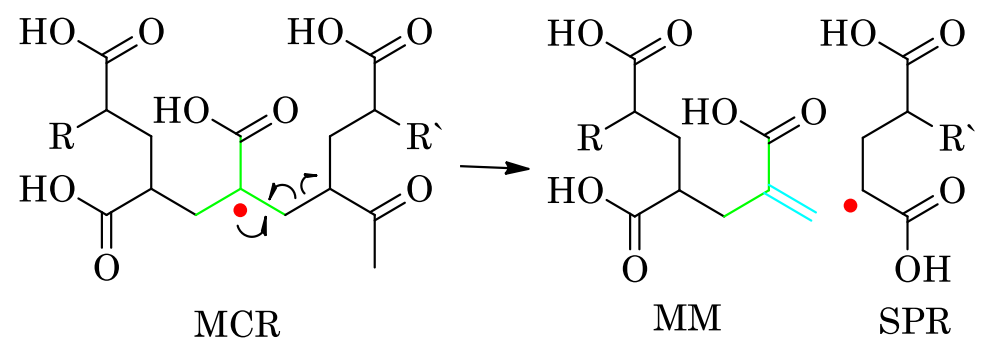

Figure 2-3 The mechanism of $\beta$-scission is shown for an MCR of acrylic acid.

$\beta$-scission of MCRs can have a strong effect on reaction kinetics and product properties. Follow-up reactions are as follows.

An MCR formed by backbiting may undergo $\beta$-scission in either direction. $k_{\beta}$ denotes the rate coefficient of $\beta$-scission:

$$
\mathrm{R}_{\mathrm{MCR}^{\mathrm{s}}, i}^{\bullet} \stackrel{k_{\beta}}{\longrightarrow} \mathrm{R}_{\mathrm{SPR}, i-2}+\mathrm{MM}_{2} \dot{\vee} \mathrm{R}_{\mathrm{SPR}, 3}+\mathrm{MM}_{i-3}
$$

An SPR can add to an MM and form an MCR with the radical function somewhere on the chain:

$$
\mathrm{R}_{i}+\mathrm{MM}_{j} \stackrel{k_{\mathrm{p}, \mathrm{MM}}}{\longrightarrow} \mathrm{R}_{\mathrm{MCR}^{1}, i+j}^{\cdot}
$$

This radical can afterwards add monomer (or terminate) consequently forming a long-chain branching point:

$$
\mathrm{R}_{\mathrm{MCR}^{1}, i+j}^{\bullet}+\mathrm{M} \stackrel{k_{\mathrm{p}}^{\mathrm{t}}}{\longrightarrow} \mathrm{R}_{\mathrm{SPR}, i+j+1}^{\bullet}
$$

But it can also undergo $\beta$-scission again: 


$$
\mathrm{R}_{\mathrm{MCR}^{1}, i+j} \stackrel{k_{\beta}}{\longrightarrow} \mathrm{R}_{\mathrm{SPR}, i}+\mathrm{MM}_{j} \dot{\vee} \mathrm{R}_{\mathrm{SPR}, j}+\mathrm{MM}_{i}
$$

Polymerization kinetics of BA at high temperature including $\beta$-scission and follow-up reactions have been modeled successfully. ${ }^{[42,43]}$

\subsubsection{Retardation and Inhibition}

If the radical function is transferred to a small molecule and the product reinitiates very slowly or not at all, the former process is called retardation and the latter inhibition. The chemical species are called retardant and inhibitor, respectively. Retardants decrease the rate of polymerization. Inhibitors prevent the polymerization from taking place until they are used up (induction period). It should be noted that this designation is not handled very consequently. Transfer to polymer which can slow down the rate of polymerization a lot (vide supra) is called transfer nonetheless.

There are not only transfer-type retardants and inhibitors, but also addition-type retardants and inhibitors.

A transfer-type inhibition:

$$
\mathrm{R}_{i}^{\bullet}+\mathrm{X} \stackrel{k_{\mathrm{tr}, \mathrm{X}}}{\longrightarrow} \mathrm{P}+\mathrm{X}_{i}^{\cdot}
$$

An addition-type inhibition:

$$
\mathrm{R}_{i}^{\bullet}+\mathrm{X} \stackrel{k_{\mathrm{tr}, \mathrm{X}}}{\longrightarrow} \mathrm{P}-\mathrm{X}_{i}^{\bullet}
$$

Both radicals formed in these reactions do neither propagate nor initiate. Often they still terminate. 
Kinetics may become very complicated, because under different conditions chemical species may play different roles. From the earliest days of polymerization research it is known that oxygen initiates polymerization. ${ }^{[44]}$ On the other hand it is the most abundant of the addition-type inhibitors. It adds to growing chains rapidly. This reaction is probably diffusion controlled. ${ }^{[45,46]}$ The so-formed peroxide radical does not propagate. Peroxides or hydroperoxides formed by this process dissociate at high temperature forming radicals, which can initiate radical polymerization. For that reason, there is even a second mechanism of initiation. Peroxides that do not decompose during the polymerization process remain in the product reducing its quality. Hence oxygen plays an ambiguous role in polymerization kinetics. ${ }^{[47]}$ Usually, it is attempted to remove it completely from the reaction mixture.

Often unwanted impurities function as inhibitors or retardants.

Inhibitors are added to all monomers to keep them from polymerizing during storage and transport. In this context, they are sometimes called stabilizers. In industrial practice, inhibitors are usually not removed but just compensated for by additional initiator.

Common inhibitors are, e.g., quinone, hydroquinone, which is oxidized to quinone by oxygen, and hydrochinone monomethyl ether. The latter is only effective in combination with oxygen.

\subsection{Influences on Rate Coefficients}

In this subchapter different influences on rate coefficients are discussed. Like all chemical reactions the sub-steps of radical polymerization depend on temperature and pressure (see subchapter 2.4.1).

For some chemically controlled reactions, there is a distinct dependence of rate coefficients on concentration. This is above all true for aqueous systems (see subchapter 2.4.2). In general, these systems exhibit more complicated polymerization kinetics than organic systems. By ionizing or protonating components their electronic structure and thus chemical reactivity is altered; moreover, diffusion rate is modified as well (see subchapter 2.4.3). 
Some sub-steps of radical polymerization are not governed by the chemical reaction itself. To understand this it has be to be taken into account that all chemical reactions with molecularity other than unity are preceded by mutual approach of the reactants by diffusion. This way the rate coefficient can be split into a diffusiondependent term and a chemical-reaction term as given by eq. (2.20).

$$
\frac{1}{k}=\frac{1}{k(\text { diffusion })}+\frac{1}{k(\text { chemical reaction })}
$$

If the first term of eq. (2.20) RHS predominates, the reaction is considered to be diffusion controlled. If the second term predominates, the reaction is considered to be chemically controlled. Termination, initiator efficiency ${ }^{[48]}$, inhibition and catalyzed chain transfer ${ }^{[49]}$ are generally considered to be diffusion controlled, while initiator decay, initiation, propagation and transfer are generally considered to be chemically controlled.

The diffusion step may be described by the Smoluchowski equation:[50]

$$
k=4 \cdot \pi \cdot N_{\mathrm{A}} \cdot\left(D^{\mathrm{X}}+D^{\mathrm{Y}}\right) \cdot\left(r_{\mathrm{c}, \mathrm{X}}+r_{\mathrm{c}, \mathrm{Y}}\right)
$$

Here $N_{\mathrm{A}}$ denotes the Avogadro constant, $D^{\mathrm{X}}$ and $D^{\mathrm{Y}}$ are the diffusion coefficients of the reacting species $\mathrm{X}$ and $\mathrm{Y}$, and $r_{\mathrm{c}, \mathrm{X}}$ and $r_{\mathrm{c}, \mathrm{Y}}$ are the capture radii of $\mathrm{X}$ and $\mathrm{Y}$, respectively. Therefore, the corresponding rate coefficient of the diffusive step is proportional to the sum of the diffusion coefficients of the two reacting molecular species.

Under the assumption of negligible ionic interaction, the individual diffusion coefficients may be approximated by the Stokes-Einstein equation:[51] 


$$
D_{\mathrm{S}}=\frac{k_{\mathrm{B}} \cdot T}{6 \cdot \pi \cdot r_{\mathrm{h}, X} \cdot \eta}
$$

$k_{\mathrm{B}}$ stands for the Boltzmann constant, $T$ for the thermodynamic temperature, $r_{\mathrm{h}, X}$ for the hydrodynamic radius of $\mathrm{X}, \eta$ is the dynamic viscosity of the solution. Diffusion rate is decreased towards larger size and towards higher viscosity of medium.

Often in chemistry capture radii and hydrodynamic radii are of similar size. Thus canceling out each other after combining eq. (2.21) and eq. (2.22).However, this is not true for growing polymer chains, which have one distinct centre of reactivity, the radical function, that does not change in size, while the rest of the molecule vary a lot. This chain-length dependence is discussed in subchapter 2.4.4.

Eq. (2.22) contains viscosity as well, which in many cases augments dramatically during the course of polymerization. Thus, rate coefficients will not stay constant with increasing conversion. Effects of varying concentration and ionization also matter with the treatment of conversion dependence. This is addressed in subchapter 2.4.5.

There are a lot of phenomena that influence $k_{\mathrm{p}}$ and depending on solvent different ones are of importance. A good overview, also on aspects not important in this work, is given elsewhere. ${ }^{[10]}$

\subsubsection{Temperature and Pressure}

The most widespread method to describe the temperature dependence of rate coefficients is eq. (2.23), the Arrhenius equation, derived by van't Hoff ${ }^{[52]}$ and Arrhenius ${ }^{[53]}$ based on thermodynamic theory:

$$
k=A \cdot \exp \left(\frac{-E_{\mathrm{A}}}{R \cdot T}\right)
$$


The rate coefficient depends on a temperature independent pre-exponential factor, $A$, the activation energy, $E_{\mathrm{A}}$, the gas constant, $R$, and the absolute temperature, $T$.

For diffusion-controlled reactions, $E_{\mathrm{A}}$ is the same as for fluidity, the reverse of viscosity (compare eq: (2.21) and eq. (2.22)). The latter is assumed to be a fraction of the energy of vaporization. For molecules possessing spherical symmetry, it is $1 / 3$, for nonspherical molecules it is less, usually $1 / 4 .{ }^{[54]}$ If hydrogen bonds are present in the solvent, the activation energy decreases towards higher temperature due to reduced strength of the hydrogen bonds. ${ }^{[55]}$

In fact activation energy is pressure depended, but as a convention, pressure dependence is put into the pre-exponential factor, A. Following this, a pressureindependent pre-exponential factor, $A^{\prime}$, can be defined (eq. (2.24))

At low isothermal compressibility or in case of first-order reactions both temperature and pressure dependence may be represented by the rather simple eq. (2.25), which is an extension of eq. (2.23).

$$
\begin{gathered}
A=A^{\prime} \cdot \exp \left(\frac{\Delta^{\ddagger} V \cdot p}{R \cdot T}\right) \\
k=A^{\prime} \cdot \exp \left(-\frac{E_{\mathrm{A}}+\Delta^{\ddagger} V \cdot p}{R \cdot T}\right)
\end{gathered}
$$

At ambient pressure, $\Delta^{\ddagger} V \cdot p$ is normally lower than the error of measurement of activation energy and can be neglected.

\subsubsection{Concentration}

For ideal polymerization kinetics, rate coefficients are considered to be independent of the concentrations of compunds. Often, this is assumed for real polymerizations as well, but for both diffusion-controlled and chemically-controlled polymerization reactions, the rate coefficients may vary significantly with concentration. 
A different composition obviously leads to a different viscosity. Hence, all diffusion-controlled rates (termination, initiator efficiency, inhibition and catalyzed chain transfer) are affected. Following eq. (2.22) and eq. (2.21) their rate coefficients increase and decrease with fluidity. Sometimes a small change of one component has a large impact on viscosity.

Less obvious is the concentration dependence of chemically controlled rate coefficients. Initiator decay can be influenced a lot by other components in a hardly predictable way, e.g., rate of decomposition of sodium persulfate is increased by a factor of up to seven in the presence of acrylic acid, but depending on concentration and ionization of monomer it can also be decreased. ${ }^{[56]}$

A special case, which will be discussed in greater detail, is the rate coefficient of propagation, $k_{\mathrm{p}}$. In the late 90 ies it was begun to measure propagation rate coefficients for polymerizations in aqueous solution by PLP-SEC (pulsed laser polymerization size exclusion chromatogrophy) - a method superior over the older rotating sector technique. It has been found that $k_{\mathrm{p}}$ depends on monomer concentration. Several explanatory approaches were made for these astonishing results:

First, water-soluble monomers like acrylic acid and methacrylic acid (two of the earliest examined monomers) tend to associate with each other forming a variety of different dimers up to oligomers. Changes in the solvent to monomer ratio necessarily lead to different amounts of the various associations of monomer. Under the assumption that these monomer associations show different reactivities, the rate has to depend on monomer concentration. ${ }^{[57]}$ This would mean that reactivity in polar organic solvents, e.g., ethanol or dimethyl sulfoxide changed in a similar way as in water, but this is not the case. ${ }^{[58]}$ This theory has been discarded.

Second, the "local" concentration may be differ from overall concentration. Usually, it is assumed that overall monomer concentration is identical to the "local" monomer concentration in close proximity to the radical centre. If overall and "local" monomer concentrations are different, following eq. (2.4) $k_{\mathrm{p}}$ will appear higher than the same factor as the "local" concentration is higher as the overall concentration. However, in case of polymerizations in aqueous solution, this assumption requires an enormously large difference - a factor of ten. At low monomer concentrations almost all monomer molecules would have to be situated in the direct vicinity of macroradicals. As a consequence, the reaction solution consists of a few radicals with associated monomer molecules dissolved in almost pure water. ${ }^{[59]}$ In addition polymer in the reaction mixture does not influence $k_{\mathrm{p}}$. ${ }^{[9]}$ If the polymer collected monomer from the 
solution to achieve the elevated "local" concentration, additional polymer would reduce the measured $k_{\mathrm{p}}$. This theory is now considered dismissed.

Third, the corresponding reaction is chemically controlled and thus the rate coefficient can be described by the Eyring equation, eq. (2.26), which assumes the reactants to go through a transition state (TS) as the highest point of the "pass". ${ }^{[60,61]}$ If it is a genuine kinetic effect, it can be explained by this equation.

$$
k_{\mathrm{p}}=\sigma \cdot \frac{k_{\mathrm{B}} \cdot T}{h} \cdot \frac{Q^{\ddagger}}{Q_{\mathrm{M}} \cdot Q_{\mathrm{R}} \cdot} \cdot \exp \left(-\frac{E_{0}}{k_{\mathrm{B}} \cdot T}\right)
$$

$\mathrm{Q}$ stands for the partition functions of species, ${ }^{\ddagger}$ denotes the transition state. $\sigma$ is the transmission coefficient ( 1 or less), $h$ the Planck constant, and $E_{0}$ the zero-point energy difference between educts and transition state.

The transmission coefficient is independent of the concentrations of the components in the reaction mixture. Thus, there remain only two possibilities. Either the partition functions are influenced consequently shifting the Arrhenius prefactor (compare eq. (2.23)) or the zero-point energy difference and the activation energy (compare eq. (2.23)), respectively. Detailed examination of the temperature dependence of $k_{\mathrm{p}}$ of MAA has shown that $E_{\mathrm{A}}$ is almost in

sensitive towards a variation of monomer content within a large concentration range and it is primarily $A$ that varies. ${ }^{[59]}$ Consequently, the partition functions have to be influenced by the solvent environment. Gilbert et al. ${ }^{[62]}$ calculated that the effect is due to different extents of hindrance to internal rotation (vibration with an activation energy in the order of magnitude of a rotation) in the transition state (TS) structure for propagation. The solvent molecules in the surrounding area of the activated complex may impose a hindrance to the internal rotation of the activated complex depending on how strong they are attached and how big they are. The stronger intermolecular interactions of the activated complex with an environment that basically consists of monomer molecules result in a lower mobility of side groups and thus lead to a reduced pre-exponential factor towards higher monomer content. ${ }^{[9,59]}$.

The same group ${ }^{[63]}$ has found in a newer investigation again through calculation that a different solvent field causes a different activation energy. They found for the 
propagation of AA that the activation energy in toluene representing non-polar solvents is as in the gas phase while it is considerably reduced in a water environment. The level of accuracy, however, was not sufficient for quantitative accuracy. The variation of $E_{\mathrm{A}}$ was ascribed to better resonance stabilization of the TS in the polar solvent, and better mixing of the molecular orbitals of the reactants, assisting in the transfer of electrons from the monomer to the growing chain.

In another calculation of the polymerization of MAA and AA the experimental finding was confirmed that the rate acceleration of both polymers in water is mainly due to entropic rather than electrostatic effects. Degirmenci et al. also calculated the difference of the $k_{\mathrm{p}} \mathrm{s}$ of MAA and AA arises mainly from steric hindrance of the methyl group and not from difference in electronic structure. ${ }^{[64]}$

For AAm, calculations that compare propagation in gas phase with those in aqueous phase conclude that activation energy is reduced. ${ }^{[65]}$ Experimental results for 1vinylpyrrolidin-2-one ${ }^{[66]}$ and N-vinylformamide, ${ }^{[67]}$ suggest as well that $E_{\mathrm{A}}$ varies with solvent content, although not in a way that could explain the dependency of $k_{\mathrm{p}}$ on $c_{\mathrm{M}}$.

Overall, the influence on $k_{\mathrm{p}}$ in aqueous solution is mostly based on an alteration of the entropy of the transition state, but there is an also a smaller effect on $E_{\mathrm{A}}$. In subchapter 5.1.2, this is discussed in detail including new results for $k_{\mathrm{p}}$.

\subsubsection{Ionization}

Shifting the $\mathrm{pH}$ of a reaction mixture away from its "natural" value by addition of acid or base can have an enormous influence on reaction kinetics. Both diffusion and chemically controlled reactions are affected by various mechanisms. The effect of ionization on the rate of polymerization has been investigated by several groups. The best investigated monomers are acrylic acid and methacrylic acid. ${ }^{[11,12,68-80]}$ Although, most groups did not know at the time of publication that acrylic acid undergoes backbiting (see 2.3.3) during polymerization.

To characterize the ionization of monomer and polymer, the degree of ionization, $\alpha$, is defined by eq. (2.27). 


$$
\alpha=\frac{n(\text { ionizing agent })}{n(\text { ionizable groups })}
$$

First, density changes with ionization. Therefore, a different concentration applies for the same mole ratio of monomer to solvent. Ionized molecules are preferably located near to contrary charged ions, thus the local concentration of charged monomer near to charged monomer or a charged growing chain is lower than the overall concentration. If the monomer has ionizable functionalities, the corresponding polymer has them as well. The $\mathrm{p} K_{\mathrm{A}}$ and $\mathrm{p} K_{\mathrm{B}}$ value of the polymer are different from the values of the monomer. ${ }^{\left[{ }^{[1,82]}\right.}$ Thus, polymerizing with initially partly ionized monomer, the degree of ionization of monomer and polymer is different for the same $\mathrm{pH}$ and change during polymerization.

In dealing with polyions, in addition to $\mathrm{pH}$, another factor has to be taken into account, the ionic strength, $I$, given by eq. (2.28).

$$
I=\frac{1}{2} \sum_{i} c_{i} \cdot z_{i}^{2}
$$

$z$ is the charge number of the ion.

The degree of ionization of polymer and ionic strength have an enormous effect on the structure of polymer. ${ }^{[82-87]}$ As more and more side groups become ionized, Coulomb repulsion leads to a widening of the polymer coil. However, higher ionic strength, thus more counter ions, weakens this effect. Screening by counter ions may even lead to a polymer structure of the ionized polymer like the one of the non-ionized polymer. ${ }^{[84,85,87]}$

Addition of salts most often increases viscosity, which can be calculated rather easily. ${ }^{[88]}$ However, ionized monomer and polymer make the prediction of solution viscosity more complicated. The equations used in the previous subchapters to calculate the influence of viscosity on diffusion-controlled reactions (eq: (2.22) and eq. (2.21)) are insufficient in this case, because diffusion of charged species cannot be described ignoring Coulomb interaction. 
The investigation into the polymerization of ionizable monomers has been focused on the two commercially most important ones: acrylic acid and methacrylic acid. All works agree in that the initial rate of polymerization decreases towards higher degree of ionization of monomer (AA and MAA) and increases again with even higher degree of ionization, although the increase by overtitration is higher for AA. ${ }^{\mathrm{II}[12,68-}$ 75,78-80] The same trend was found for molar masses of polymer product. Most groups found this minimum at full ionization, but Cutié et al. discovered that the minimum of rate of polymerization is shifted towards lower degrees of ionization with higher temperature. ${ }^{[80]}$ In addition, the different experimental studies differ a lot with respect to the magnitude of the decrease of the rate of polymerization with higher $\alpha$. The strongest effect was found by Kabanov et al. ${ }^{[12]}$ who observed a 50-fold decrease in overall rate from polymerizing non-ionized AA to polymerizing fully-ionized AA. ${ }^{\text {III }}$

Comparison of rates of polymerization without understanding the dependencies of individual reactions can be problematic. The $\mathrm{pH}$ value or the ionization of monomer can have surprising effects on chemically controlled reactions like the initiator decay. One example that may illustrate the problems, which initiators can cause, shall be given here: In an early work, Katchalsky and Blauer ${ }^{[83]}$ reported that monomers with a carboxyl group did not polymerize if this function were ionized. This way they explained the decline in rate of polymerization they found with higher pH. Later it was pointed out by Pinner ${ }^{[72]}$ that their initiator of choice (hydrogen peroxide) does not work under too basic conditions. After this was published, Blauer performed more experiments with a different initiator (AIBN and $4 \%$ ethanol to make it soluble) and polymerized fully ionized MAA successfully up to $\mathrm{pH} 12 .{ }^{[73]}$

Only a few authors tried to understand the polymerization kinetics in detail. An outstanding exception is the path breaking work by the group of Kabanov. ${ }^{[12,69]}$ They explained both the reduction of overall rate and the decrease of molar masses of polymer with increasing $\alpha$ by a reduction of $k_{\mathrm{p}}$ through Coulomb repulsion of the ionized growing chain and the ionized monomer. The finding of increasing rate and molar mass with "overtitration" was explained as a $k_{\mathrm{p}}$-effect as well. They concluded that an ion pair mechanism increases propagation. A counter ion,, e.g., a sodium cation, can bring a monomer anion and the end of the polyanion, i.e. the growing chain, together having one of them at each side so they may react. As the number of

II The reader should note that the degree of ionization is defined here in a way that a value of more than one is possible. Additional neutralizing agent after full ionization is counted as well.

III Their overview graph (Fig.1 in the paper cited) of initial rate of polymerization for AA and MAA as a function of $\mathrm{pH}$ between 1 and 14 is reproduced in many other works. Unfortunately, in the English translation from the Russian original the labels in the graph were swapped (No.1 is AA and not MAA). 
counter ions rises, this effect becomes more and more important enhancing $k_{\mathrm{p}}$. This is supported by their finding that additional salt has qualitatively the same effect as overtitration. The quantity of the effect depends on the nature of the counter ion (v.i.).

Furthermore, they found an increase of tacticity of the newly produced polymer in the same way as they found an increase of rate with overtitration, i.e. more salt had a stronger effect on fully ionized monomers and different counter ions varied in their effectiveness, e.g., 2-methylpropan-1-aminium lead to a higher percentage of syndiotactic triads in pMAA than ammonium (up to $87 \%$ ). This was explained by van-der-Waals interaction between methyl groups. Moreover, lower temperature led to higher tacticity. By polymerizing at the highest ionic strength, they even produced crystalline pAA that had the same interplanary distances as pAA produced by hydrolysis of syndiotactic poly (isopropyl acrylate).

Measuring $k_{\mathrm{p}}$ has gained precision in comparison to the rotating sector method by the development of the PLP-SEC method (pulsed laser polymerization carried out in conjunction with size-exclusion chromatographic), put forward by Olaj et al. ${ }^{[5,89]}$ SEC of polyacids may be performed after esterfication ${ }^{[57,90]}$ or directly by aqueous phase SEC. ${ }^{[91]}$ The latter being the preferred method. This method has been employed to measure $k_{\mathrm{p}}$ values of MAA and AA as a function of concentration and degree of ionization directly. ${ }^{[11,76,92]}$ The best investigated monomer considering the influence of degree of ionization and concentration is MAA. As the influence of ionization seems to be the same for both MAA and AA, ${ }^{[11]}$ only MAA is discussed here. The rate varies enormously as a function of both monomer concentration and degree of ionization, ${ }^{[9,11,58,59,91,92]}$ e.g., decrease by about one order of magnitude in passing from dilute aqueous solution of non-ionized MAA to either bulk polymerization of nonionized MAA (as discussed in subchapter 2.4.2) or to fully ionized MAA in dilute solution. Lacík et al. ${ }^{[1]}$ have developed an empirical equation, eq. (2.29), that incorporates both influences over a broad range and covers a wide temperature range as well.

$$
\begin{aligned}
& k_{p}=4.1 \cdot 10^{6} \exp \left(\frac{-1.88 \cdot 10^{3}}{T / \mathrm{K}}\right) \cdot(0.08+(1-0.08) \cdot \exp (-5.3 \cdot W)) \\
& \left((-0.202 \cdot W-0.553) \alpha^{2}+(-2.283 \cdot W-0.475) \alpha+1\right) \\
& 0 \leq \alpha \leq 1 ; 0.05 \leq W \leq 0.40 ; 6 \leq \vartheta \leq 80{ }^{\circ} \mathrm{C}
\end{aligned}
$$


Their findings for MAA polymerization should be transferable to the polymerization of other monomers (not only AA) in ionized or partly ionized form in aqueous solution - at least for similar monomers. The plot of eq. (2.29) is given in Figure 2-4 to visualize the complex relationship. The variation of $k_{\mathrm{p}}$ with monomer content is weaker for partially ionized MAA and may even be reversed with fully ionized MAA. A broader range than the strict validity of the equation $(0.05<w<0.4)$ is plotted.

In addition, for MAA polymerizations at different degrees of ionization (0-1) no effect by addition of sodium chloride (increasing ionic strength and number of counter ions, respectively, without increasing the degree of ionization) was found. ${ }^{[11]}$

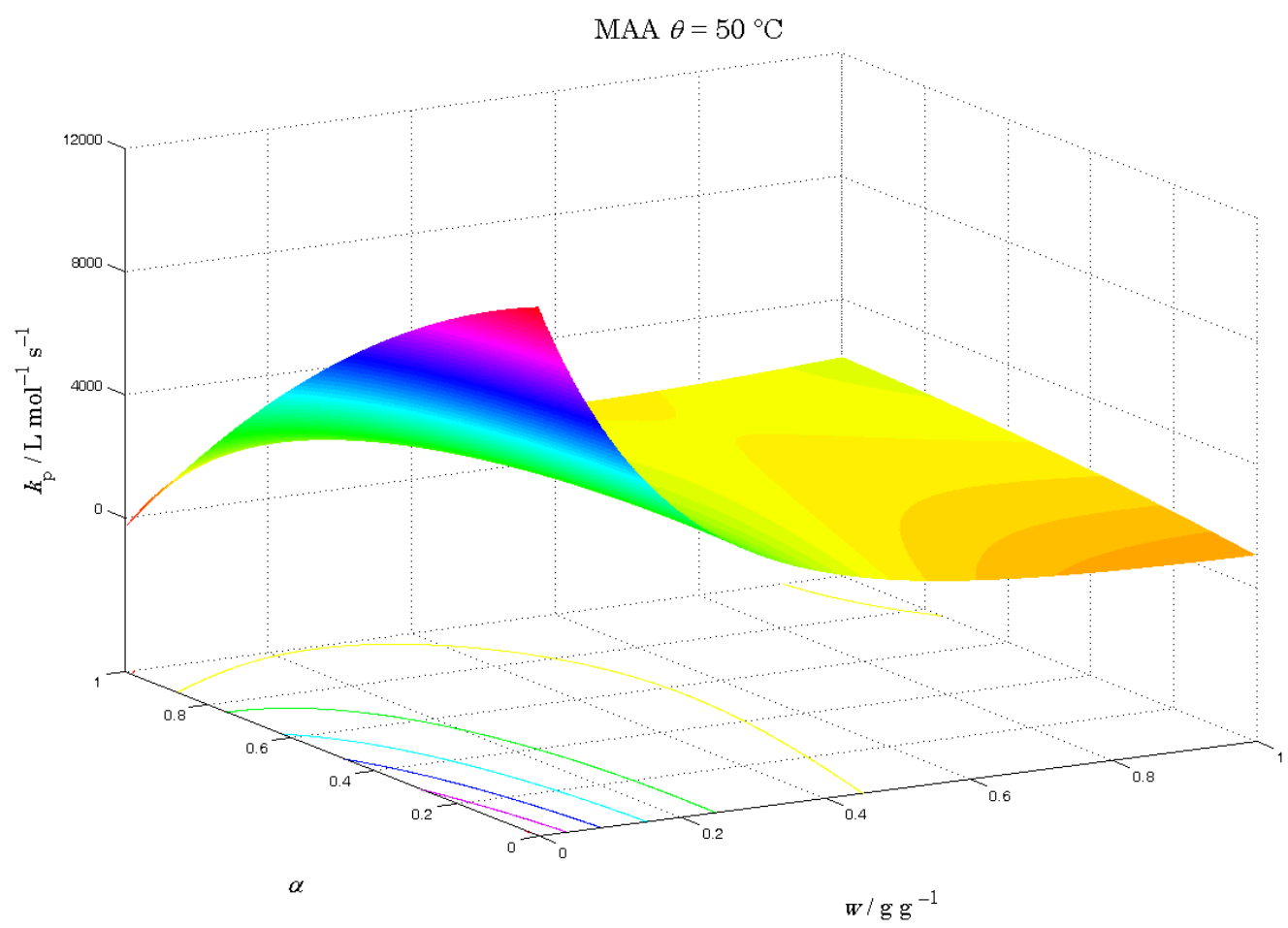

Figure 2-4 The rate coefficient of propagation of MAA as a function of both weight fraction of monomer and degree of ionization as given by eq. (2.29). Please note that although the full range of $w$ is plotted the equation was only derived for $0.05<w<0.4$.

There is scarce experimental data on the influence of degree of ionization on the rate of termination. Because of viscosity increase and additional repulsion, a reduction of the rate coefficient must be assumed. Based on the observation that screening of counter ions can lead to a structure like the non-ionized polymer chain, one may 
assume that screening of counter ions can increase $k_{\mathrm{t}}$ of ionized growing chains may be even up to the point that the reduction by Coulomb repulsion becomes compensated. Experimental data, e.g., by Kabanov ${ }^{[12]}$ et al. did not present this effect. Later, Anseth et al. ${ }^{[78]}$ found a reduction of $k_{\mathrm{t}}$ with ionization of polymer, which could be partly compensated by an increase of the number of counter ions. However, as their measurement of $k_{\mathrm{p}} / k_{\mathrm{t}}$ was done after 20-30 s of dark time, one should be rather cautious with these experiments. In a more recent set of experiments ${ }^{[36]}$ it was shown for non-ionized AA that radical concentration has faded out completely after $0.003 \mathrm{~s}$ (SPR) and $0.6 \mathrm{~s}$ (MCRs) dark time, respectively. In a new study, radicals during polymerization of fully ionized AA were investigated directly by EPR. ${ }^{[77]} k_{\mathrm{t}}$ was found to decrease by about a factor of 15 from $10 \mathrm{cg} \mathrm{g}^{-1} \mathrm{AA}$ to $20 \mathrm{cg} \mathrm{g}^{-1}$ sodium acrylate $(\mathrm{NaA})$.

\subsubsection{Chain Length}

The influence of chain length is ignored in ideal polymerization kinetics. Notwithstanding, it would be more correct to write the rate coefficients for propagation and termination as $\left\langle k_{\mathrm{p}}\right\rangle$ and $\left\langle k_{\mathrm{t}}\right\rangle$, respectively. The guillemet indicates that the rate coefficients are chain-length averaged. Often it is omitted for reasons of convenience. Chain-length dependence of propagation and termination is of completely different nature, as the former coefficient is chemically controlled while the latter is diffusion controlled.

Chain-length dependency of termination results from an increase of the hydrodynamic radius (see eq. (2.22)) while the capture radius stays the same or changes slightly only (see eq. (2.21)). This is not at all trivial. The hydrodynamic radius is a function of polymer type and chain length as well as solvent and temperature. The capture radius may be influenced by more or less effective shielding by the long unreactive chain attached to the reactive radical center. Diffusion coefficients of polymers usually follow a power-law expression. ${ }^{[93,94]}$ To illustrate this, one example is given in Figure 2-5. The diffusion coefficients of pAA are plotted for different molar masses ( $M_{\mathrm{w}}$, weight average molecular mass) and are best represented by the expression: $D=2.1 \cdot 10^{-4} \cdot M_{\mathrm{w}}{ }^{-0.58} \mathrm{~cm}^{2} \mathrm{~s}^{-1}$. The reader may note the similarity to eq. (2.30) which will be introduced next. 
If just one chain length, $i$, is treated, the chain-length-dependent termination (CLDT) may be given by a power.law function, which follows the form found for diffusion of polymers through a solution

$$
k_{\mathrm{t}}^{i, i}=k_{\mathrm{t}}^{0} \cdot i^{-\alpha_{1}}
$$

$k_{\mathrm{t}}^{i, i}$ denotes the rate coefficient of termination of two radicals of identical chain length $i$. Here, $k_{t}^{0}$ is the rate coefficient of termination directly extrapolated from long-chain regime to both chain lengths being unity. $\alpha_{1}$ describes chain-length dependency.

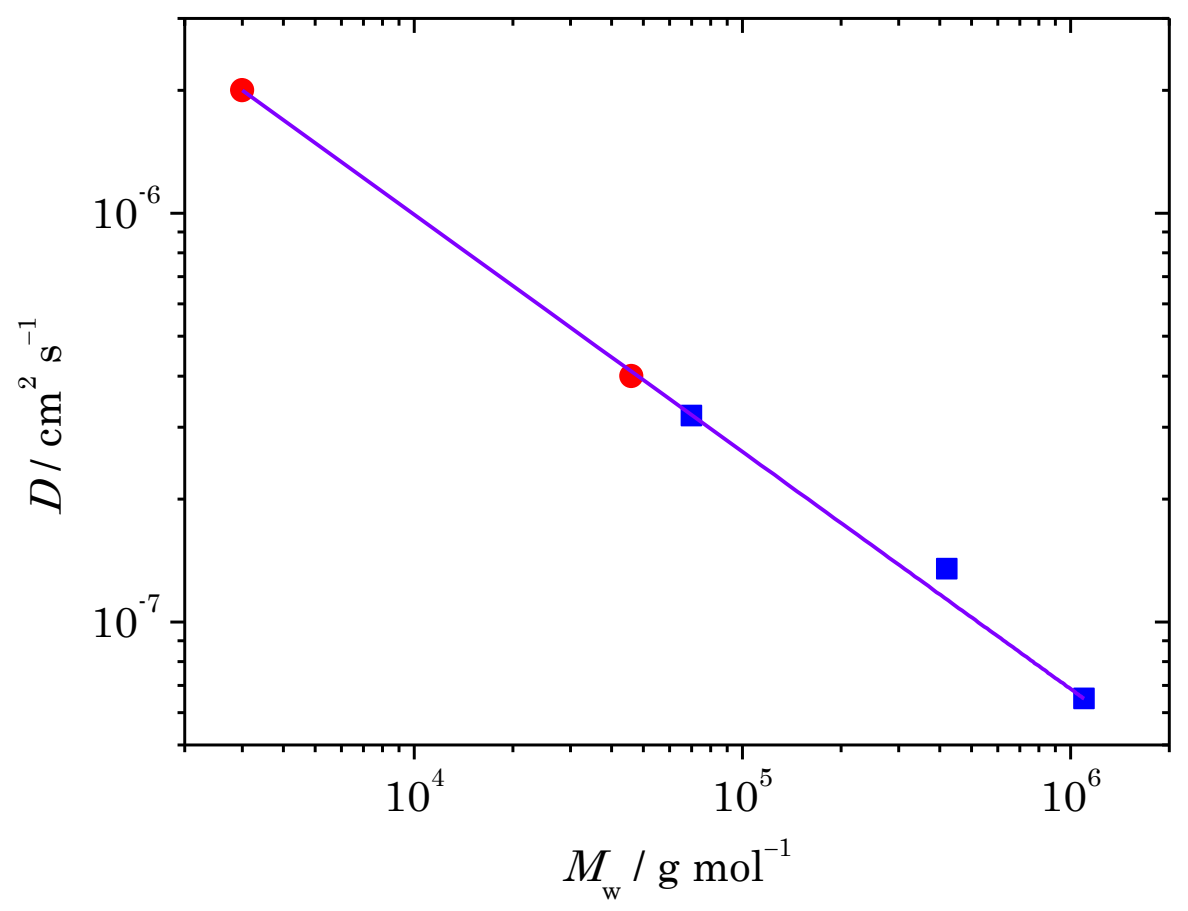

Figure 2-5 The selfdiffusion coefficients of pAA for different molar masses are plotted. pAA is fully ionized and $0.01 \mathrm{~mol} \mathrm{~L}^{-1} \mathrm{NaCl}$ is added. The solid violet line is the best power-law fit. The exponent is -0.58 . Data is taken from literature: red circles, ${ }^{[82]}$ blue squares. ${ }^{[95]}$

There is usually a distribution of chain-lengths and two chains terminating mostly will have different chain-lengths. There are three ways under discussion, by which termination of radicals of arbitrary size is described best. These are the diffusion mean, eq. (2.31), the geometric mean, eq. (2.32), and the harmonic mean, eq. (2.33). 


$$
\begin{gathered}
k_{\mathrm{t}}^{\mathrm{i}, \mathrm{j}}=\frac{k_{\mathrm{t}}^{0}}{2} \cdot\left(i^{-\alpha_{1}}+j^{-\alpha_{1}}\right) \\
k_{\mathrm{t}}^{\mathrm{i}, \mathrm{j}}=k_{\mathrm{t}}^{0} \cdot(i j)^{-\frac{\alpha_{1}}{2}} \\
k_{\mathrm{t}}^{\mathrm{i}, \mathrm{j}}=k_{\mathrm{t}}^{0} \cdot\left(\frac{2 \cdot i \cdot j}{i+j}\right)^{-\alpha_{1}}
\end{gathered}
$$

In case of $i=j$ all three equations are transformed back into eq. (2.30). Under many conditions, the results from eq. (2.31), eq. (2.32), and eq. (2.33) are very similar and at conditions, under which the expressions predict quite different $k_{\mathrm{t}}$-values, the rate coefficient is difficult to measure with the required accuracy. ${ }^{[96]}$ The geometric mean model is computationally less demanding. The diffusion mean is the physicochemically most plausible.

If eq. (2.30) represented the chain-length dependence of $k_{\mathrm{t}}$ correctly, it would be possible to measure chain length dependency in the domain of long chains and extrapolate back to chain length unity or vice versa - as this can be done with diffusion coefficients. It was found that such extrapolation does not work. In order to explain this, the composite model was developed. ${ }^{[97]}$ It states that termination follows pure centre-of-mass diffusion only up to a certain chain-length - the crossover chainlength, $i_{\mathrm{c}}$, after which entanglement becomes important. In this second region segmental diffusion is dominant. Thus, two different equations are needed depending on the chain length of the terminating radicals. These are given as eq. (2.34). For chains with $i$ below $i_{\mathrm{c}}$, a different exponent applies than for chains with $i$ above than $i_{\mathrm{c}}$.

$$
\begin{array}{ll}
k_{\mathrm{t}}^{i, i}=k_{\mathrm{t}}^{1,1} \cdot i^{-\alpha_{\mathrm{s}}} & i \leq i_{\mathrm{c}} \\
k_{\mathrm{t}}^{i, i}=k_{\mathrm{t}}^{1,1} \cdot i_{\mathrm{c}}^{-\alpha_{\mathrm{s}}+\alpha_{1}} \cdot i^{-\alpha_{1}}=k_{\mathrm{t}}^{0} \cdot i^{-\alpha_{1}} & i>i_{\mathrm{c}}
\end{array}
$$


$k_{\mathrm{t}}^{1,1}$ represents the coefficient for two chains with chain length unity. The essential message is that $k_{\mathrm{t}}^{1,1}$ is not $k_{\mathrm{t}}^{0}$, the coefficient from direct extrapolation from long chain to chain length unity. $\alpha_{\mathrm{s}}$ is the exponent of chain-length dependency in the region of short chains. It is in the range of 0.5 to 1.0. For larger radicals with chain lengths above the crossover chain length, $i_{\mathrm{c}}$, which is in the range of 30 to 100 monomer units, the decrease of $k_{\mathrm{t}}^{i, i}$ with $i$ is less pronounced with the exponent, $\alpha_{1}$, being in the range of 0.16 to 0.24 . This model was confirmed experimentially for all monomers studied so far by different techniques. Most notable of these is single pulse - pulsed laser polymerization in conjunction with electron paramagnetic resonance spectroscopy (SP-PLP-EPR). The majority of existing SP-PLP-EPR studies has been carried out on methacrylate-type monomers. ${ }^{[7,98-103]}$

$k_{\mathrm{t}}$ as given by the composite model is plotted in Figure 2-6 with typical values.

0.16 is the theoretical value of $\alpha_{1}$ for two radical centers located at the end of the chain in good solvents. ${ }^{[104-106]}$ If the two radical centers are not located at the end of their chain with one being situated somewhere in the middle, the theoretical value of $\alpha_{1}$ will become 0.27 , and if both of them are somewhere in the chain, it will become 0.43. ${ }^{[106]}$ So far, there is no experimental data to support the latter two coefficients, while the first is in close agreement with values found experimentally. ${ }^{[7,99,100,107,108]}$

A good overview of measured values for $k_{\mathrm{t}}$ in general and also of values for chainlength dependency is given elsewhere. ${ }^{[107]}$ 


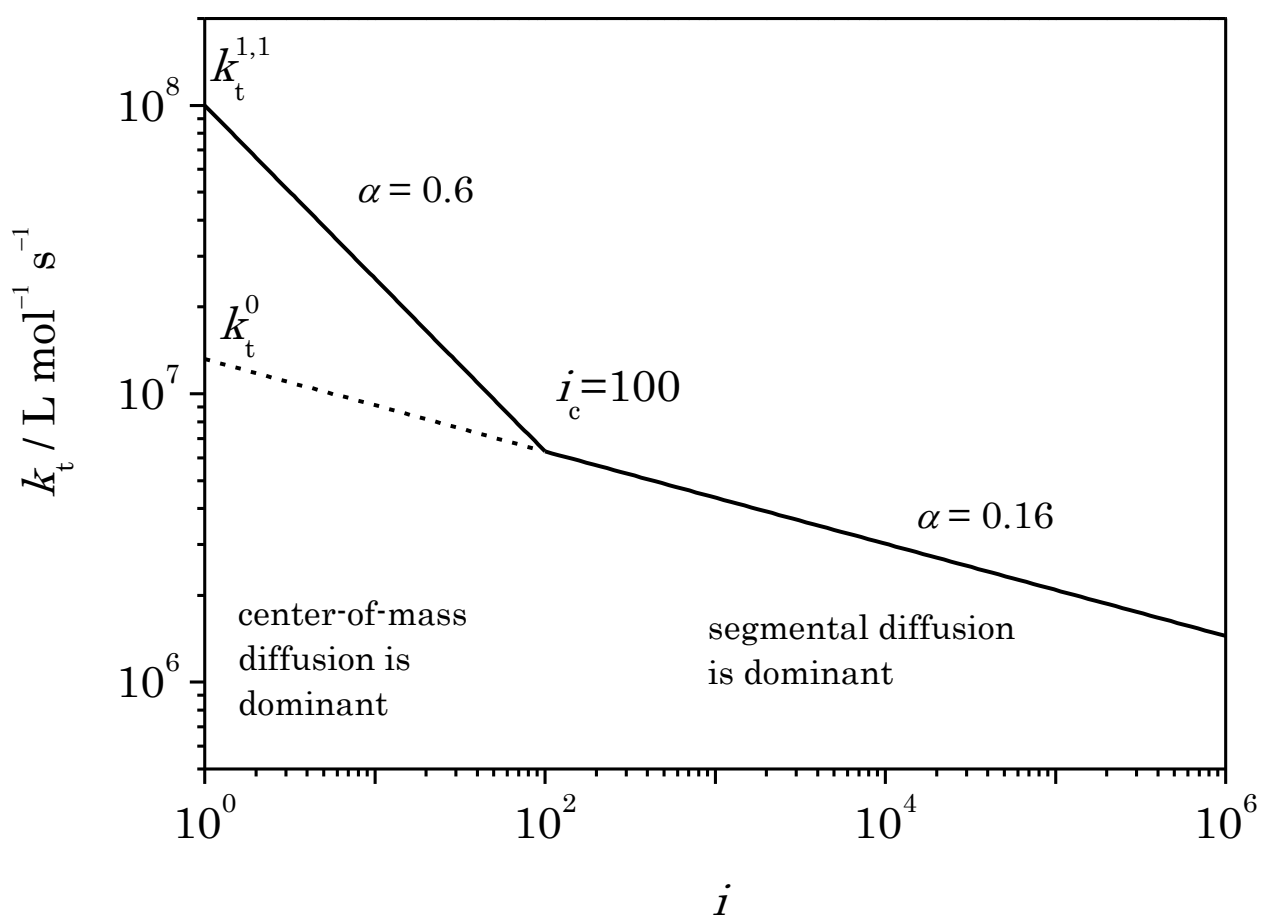

Figure 2-6 The rate of termination for two chains of identical length following the composite model (eq. (2.34)) is plotted as solid line and the back extrapolation from long-chain region (eq. (2.30)) is plotted as dashed line. The two lines merge at the crossover chain length, $i_{\mathrm{c}}$. Typical parameters for a good solvent are chosen.

The composite model may explain the ratio of long-chain $k_{\mathrm{t}}$ of styrene to long-chain $k_{\mathrm{t}}$ of methyl methacrylate (MMA). ${ }^{[109]}$ The diffusion coefficient of MMA is slightly above the one of styrene. Thus, one would expect that $k_{\mathrm{t}}$ of MMA were higher as well. Surprisingly, comparing the long chain behavior, $\left\langle k_{\mathrm{t}}\right\rangle$ of styrene is higher by more than a factor of two. Detailed kinetic analysis ot the short-chain region has revealed that MMA exhibits a higher $i_{\mathrm{c}}$ and a higher $\alpha_{\mathrm{s}}$. The combination thereof leads to lower long-chain $k_{\mathrm{t}}$-values even with a higher $k_{\mathrm{t}}^{1,1}$. On the other hand, the two monomers exhibit very different $k_{\mathrm{p}}$ values. Hence, the comparison of long-chain $k_{\mathrm{t}}$-values is conducted between two quite different chain lengths, this might as well explain the difference. This question will only be solved finally, after $k_{\mathrm{t}}^{1,1}, i_{\mathrm{c}}$ and $\alpha_{\mathrm{s}}$ of styrene will have been measured with sufficient precision.

The diffusion-controlled nature of $k_{\mathrm{t}}$ allows for an estimate of the maximum possible value for $k_{\mathrm{t}}^{1,1}$ given by eq. (2.21) and eq. (2.22), which is called the diffusion limit. At this, one more aspect has to be considered: When the two radical centers 
react, the affected two radicals combine, which results in one of three possible triplet states or one possible singlet state. ${ }^{[110]}$ Only the singlet leads to a stable molecule. It is possible (but unlikely) that inter-system crossing takes place and it is possible that spins flip through strokes to neighboring molecules while the two radical centers are still together in the solvent cage. They hit each other and the surrounding molecules around 100 to 1000 times before they leave the solvent cage again. Thus, the value of from the diffusion limit has to be corrected by a factor between 0.25 and 1 . The actual values of $k_{\mathrm{t}}$ are always even lower than the diffusion limit, because steric hindrance may reduce the effective capture radii.

CLDT has been troughfully studied at low degrees of monomer conversion. No clear picture on CLDT at moderate and high conversion has emerged so far. Work about chain-length dependency varying with conversion is scarce. Applying the SP-PLPNIR method, it was found for butyl methacrylate and tert butyl methacrylate, that $\alpha$ stays constant up to a certain degree of monomer conversion (ca. 0.3 in case of tert butyl methacrylate), then increases linearly by about a factor of 4 up $X=0.5$; afterwards both $k_{t}^{0}$, which has stayed constant that long, and $\alpha$ decrease linearly; $\alpha$ reaches 0 at about $X=0.7 .{ }^{[108]}$ However, SP-PLP-NIR does not provide direct access to CLDT. Moreover, the procedure is tedious. ${ }^{[111]}$ The combined dependence on chainlength and conversion was also investigated by the RAFT-CLD-T method. It was found that chain-length dependence becomes more and more pronounced towards higher conversion and that it declines wavelike. ${ }^{[112]}$ An extended composite model was proposed, in which there is in addition to the crossover chain-length another turnover point for even higher chain length. This chain-length marks the onset of the gel point. At this point, $\alpha$ jumps from a value of about 0.16 to about unity. ${ }^{[113-115]}$ The RAFT-CLD-T method suffers from the inherent problem that the RAFT agent influences the kinetics. The results found for low conversion, which region is quite well investigated, are in some cases contradictory to experiments without RAFT agent and thus the results for higher conversion may be wrong as well. Preferable would be a more direct measurement, e.g., by EPR, but this has not been attempted so far.

The temperature dependence of $k_{\mathrm{t}}^{1,1}$ scales with the temperature dependence of fluidity (inverse dynamic viscosity); $i_{\mathrm{c}}, \alpha_{\mathrm{s}}$, and $\alpha_{1}$ have experimentally been found to be temperature independent. ${ }^{[7,98-100]}$ As $i_{c}$ is assumed to depending on chain flexibility, its lack of temperature dependence is rather surprising and future experiments covering an even broader temperature range are required.

Experimental proof for chain-length-dependent propagation (CLDP) has been found indirectly by pulsed laser polymerization size exclusion chromatography (PLP-SEC) 
experiments $^{[116]}$ and pulsed laser polymerization matrix-assisted laser desorption ionization time-of-flight mass spectrometry (PLP-MALDI-ToF-MS) ${ }^{[117]}$ thus excluding SEC artifacts. The basic idea is that the reactive center at the end of the chain cannot see the rest of the chain; accordingly, there cannot be a chain length dependence for chemically controlled reactions except for very short chains. In case of high chain transfer or very high rate of initiation, this situation may apply.

It has been proposed by Smith et al. ${ }^{[18]}$ to represent CLDP by this equation:

$$
k_{\mathrm{p}}^{i}=k_{\mathrm{p}}^{\infty} \cdot\left(1+\frac{k_{\mathrm{p}}^{1}-k_{\mathrm{p}}^{\infty}}{k_{\mathrm{p}}^{\infty}} \cdot \exp \left(\frac{\ln (1 / 2)}{i_{1 / 2}} \cdot(i-1)\right)\right)
$$

The rate coefficient of propagation at chain-length $i, k_{\mathrm{p}}^{i}$, decreases from the highest value at chain length unity, $k_{\mathrm{p}}^{1}$, with a "half-life" chain length, $i_{1 / 2}$, to reach the "normal" value i.e. the value of the rate coefficient of propagation for log chains, $k_{\mathrm{p}}^{\infty}$.

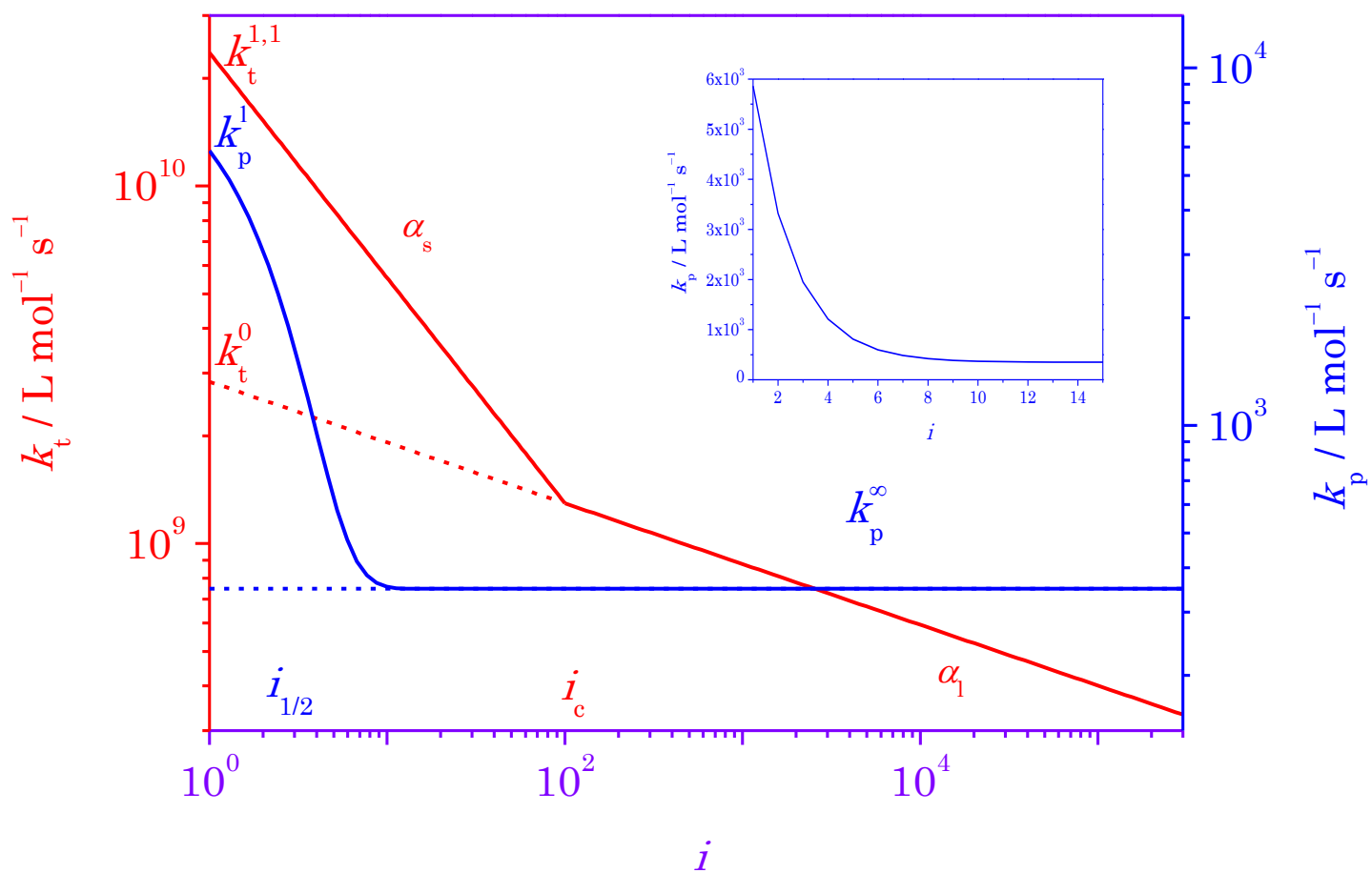

Figure 2-7 In order to illustrate the difference between CLDT (red) and CLDP (blue) both have been plotted for methyl methacrylate bulk polymerization at $25^{\circ} \mathrm{C}$. Both ordinates are 
scaled to cover two orders of magnitude to make the curves more comparable. In the region of very short chains, $k_{t}$ decreases less with chain length than $k_{\mathrm{p}}$ but it keeps declining, while $k_{\mathrm{p}}$ stays constant after a few additions. The rate coefficient of termination has been plotted according to eq. (2.30) (simple exponential, short dash) and eq. (2.34) (composite model, solid line). The values were taken from literature:[103,119] $\boldsymbol{k}_{\mathrm{t}}^{1,1}=2.3610^{10} \mathrm{~L} \mathrm{~mol}^{-1} \mathrm{~s}^{-1} ; \quad \alpha_{\mathrm{s}}=0.63 ; i_{\mathrm{c}}=200 ; \quad \alpha=0.17$. The rate coefficient of propagation has been plotted as constant (value at high chain length, short dash) and according to eq. (2.34) (exponential decay, solid line). The values were taken from literature:[116,118] $k_{\mathrm{p}}^{\infty}=3.4910^{2} \mathrm{~L} \mathrm{~mol}^{-1} \mathrm{~s}^{-1} ; \quad k_{\mathrm{p}}{ }^{1}=15.8 k_{\mathrm{p}}^{\infty} ; \quad \alpha_{\mathrm{s}}=0.63$; $i_{1 / 2}=1.12$. Eq. (2.34) is plotted in the insert with axes scaled linearly.

In order to compare the effects of CLDT and CLDP both $k_{\mathrm{p}}$ and $k_{\mathrm{t}}$ were plotted together for MMA bulk polymerization at $25^{\circ} \mathrm{C}$ in Figure 2-7.

It should be noted that not all calculations support a higher $k_{\mathrm{p}}$ for the first addition steps, e.g., for butyl acrylate and vinyl chloride even a slight increase or a to-and-fro over the first four steps was computed, respectively. ${ }^{[34,39]}$

\subsubsection{Conversion}

During the course of conversion, all effects of concentration as discussed in subchapter 2.4.2 apply as the concentration of monomer declines with conversion and in case of partially ionized or protonized monomer the degree of ionization of monomer may change with conversion, which has consequences on the kinetics as discussed in subchapter 2.4.3. Apart from that, viscosity increases during the course of polymerization, which is dealt with in this subchapter. As all chemical reactions with molecularity other than unity are preceded by mutual approach of the reactants by diffusion and diffusion rate is decreased towards higher viscosity of medium, diffusion controlled reactions become slower and non-diffusion-controlled reactions may run under diffusion control. This can be understood by combining the Smulochwski (eq. (2.20)), and the Stokes-Einstein equation (eq. (2.21)) as discussed previously in this work (introduction 2.4).

It is important to understand that macroscopic viscosity and the effective viscosity which applies for the growing radicals are not necessarily the same. $k_{\mathrm{t}}^{1,1}$ at negligible conversion scales with dynamic viscosity, $\eta$, i.e. if the value in one solution 
is known, the value in a different solvent can be predicted easily by scaling of $k_{\mathrm{t}}^{1,1}$ with the ratio of reciprocal solution viscosities:[102]

$$
\frac{k_{\mathrm{t}}^{1,1}(\text { solution } \mathrm{A})}{k_{\mathrm{t}}^{1,1}(\text { solution } \mathrm{B})}=\frac{\eta(\text { solution } \mathrm{B})}{\eta(\text { solution } \mathrm{A})}
$$

But $k_{\mathrm{t}}^{1,1}$ does not decrease by addition of polymer to the same degree as fluidity. This is demonstrated in Figure 2-8 (purple triangles). The same is true for $k_{\mathrm{t}}$ (red diamonds, black squares, and magenta triangles).

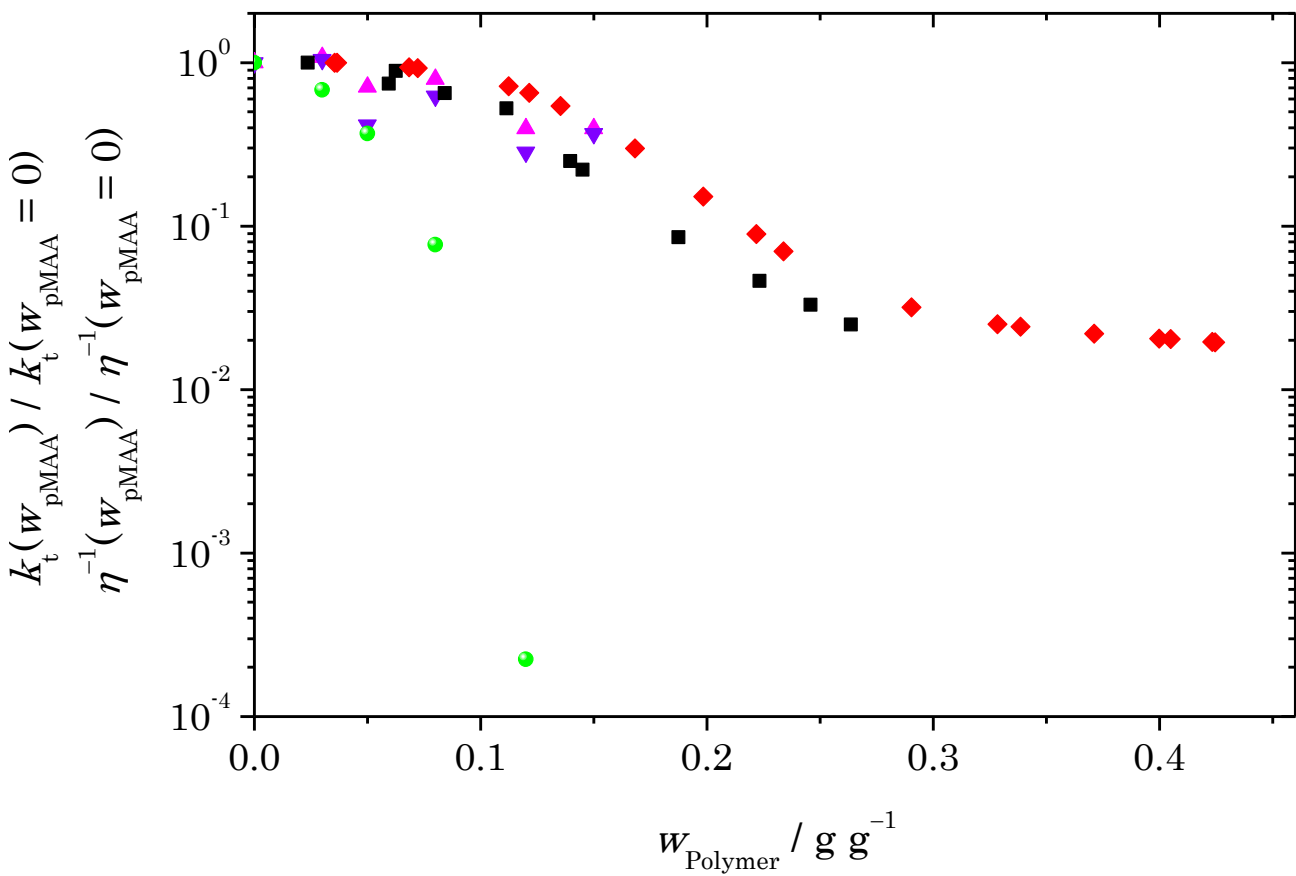

Figure 2-8 To compare the influence of polymer contained in the reaction mixture on $k_{\mathrm{t}}$ and $\eta^{-1}$, experimental data for MAA/pMAA is plotted. Values are given as ratio to the value at zero polymer content. Green spheres: relative fluidity ${ }^{[120]}$, red diamonds: $<k_{t}>$ SP-PLPNIR technique, WMAA $^{0}=0.6 ;{ }^{[121]}$ black squares: $\left\langle k_{\mathrm{t}}>\right.$ SP-PLP-NIR technique, WMAA ${ }^{0}=$ $0.6 ;{ }^{[121]}$ purple triangles: $\boldsymbol{k}_{\mathrm{t}}^{1,1}$ SP-PLP-EPR technique, wMAA $=0.1$ polymer-premix; ${ }^{[120]}$ magenta triangles: $\left\langle k_{t}>\right.$ SP-PLP-EPR technique, wMAA $=0.1$, polymer-premix. ${ }^{[120]}$ It should be noted that the polymer produced during laser experiments is of smaller size than the polymer used for premix and viscosity measurements. 
In the following section, viscosity and its influence on termination are discussed. It turns out that $k_{\mathrm{t}}$ and $\eta^{-1}$ are influenced by the presence of polymer to different extent (Figure 2-8). The growing chains are not influenced by polymer coils around them in the same way as with the solvent, with which they stand in direct contact. The exact relationship is not known.

In order to characterize the change in viscosity over conversion, relative viscosity, $\eta_{\mathrm{r}}$, is defined as the ratio of viscosity at a certain conversion $X, \eta^{X}$, to viscosity at zero conversion $\eta^{0}$ :

$$
\eta_{\mathrm{r}}=\frac{\eta^{X}}{\eta^{0}}
$$

The termination reaction of two macroradicals proceeds in a three-stage mechanism according to Benson and North. ${ }^{[122,123]}$ First, both macroradicals have to come into contact by translational (center-of-mass) diffusion (TD). Subsequently, the radical functionalities have to come into immidiate proximity (a few $\AA$ ) by segmental diffusion (SD). The third and final step is the chemical reaction (CR) proceeding either by combination or disproportionation. Hence the rate coefficient of diffusion-controlled termination, $k_{\mathrm{t}, \mathrm{D}}$, is given by:

$$
\frac{1}{k_{\mathrm{t}, \mathrm{D}}}=\frac{1}{k_{\mathrm{t}, \mathrm{SD}}}+\frac{1}{k_{\mathrm{t}, \mathrm{TD}}}+\frac{1}{k_{\mathrm{t}, \mathrm{CR}}}
$$

Another mechanism, by which two radical centers of growing chains can approach each other is reaction diffusion ( $\mathrm{RD}$ ), where radical sites advance towards each other not by movement of the polymer chain, but by growing in the direction of the other radical center. This term has to be added: 


$$
k_{\mathrm{t}}=k_{\mathrm{t}, \mathrm{D}}+k_{\mathrm{t}, \mathrm{RD}}
$$

At low degree of monomer conversion, segmental diffusion mostly dominates. The associated rate coefficient $k_{\mathrm{t}, \mathrm{SD}}$ is controlled by the type of polymer and the viscosity of the monomer-solvent mixture. As the former is conversion-independent and the latter does not change much, $k_{\mathrm{t}, \mathrm{SD}}$ remains more or less constant, which results in a plateau value of $k_{\mathrm{t}}$ up to moderate degree of monomer conversion.

At higher conversion, $k_{\mathrm{t}}$ starts to decrease notably, when TD becomes slower than SD and constitutes the bottle neck thus controlling the mechanism. The corresponding rate coefficient, $k_{\mathrm{t}, \mathrm{TD}}$, scales with the inverse viscosity of the polymerizing medium. $k_{\mathrm{t}, \mathrm{TD}}$ can be expressed relative to $k_{\mathrm{TD}}^{0}$, the theoretical termination rate coefficient under translational diffusion control at conversion zero, and relative viscosity. The stark decrease of $k_{\mathrm{t}}$ with $k_{\mathrm{p}}$ staying constant leads to an augment of both rate of polymerization and molar mass of polymer produced. This is called the Norrish-Trommsdorff or gel effect. ${ }^{[124,125]}$

Towards even higher conversion, center-of-mass diffusion of macroradicals essentially ceases and termination runs under RD control. Termination under RD conditions scales with $k_{\mathrm{p}}$ via the reaction-diffusion constant, $C_{\mathrm{RD}}$, which is enhanced by chain flexibility. Studies into the termination kinetics of ethene indicated that the reaction-diffusion constant may be estimated with the help of the volume-swept-out model which considers the diameter of the macroradical and the jump distance. ${ }^{[126-}$ 128] Typically, $C_{\mathrm{RD}}$ is independent of temperature, but decreases towards higher pressure. ${ }^{[129,130]}$ It should be noted that, in this work, $C_{\mathrm{RD}}$ is defined differently from some other publications, where bulk polymerizations have been analyzed and RD was correlated with conversion, $X$, via a constant $C_{\mathrm{RD}}^{*}$ (see eq. (2.40)). The $k_{\mathrm{t}, \mathrm{TD}}$ expression of the present work into solution polymerization uses monomer concentration (eq. (2.41)).

$$
\begin{gathered}
k_{\mathrm{t}, \mathrm{RD}}=C_{\mathrm{RD}}^{*} \cdot(1-X) \cdot k_{\mathrm{p}} \\
k_{\mathrm{t}, \mathrm{RD}}=C_{\mathrm{RD}} \cdot c_{\mathrm{M}} \cdot k_{\mathrm{p}}
\end{gathered}
$$


At very high conversion and thus high viscosity even propagation may run under diffusion control. This is especially the case for bulk polymerizations. As termination by reaction diffusion is proportional to propagation, from this point on both $k_{\mathrm{t}}$ and $k_{\mathrm{p}}$ begin to decline rapidly and radicals remain "frozen" in the polymer matrix. This is called the glass effect.

Diffusion controlled $k_{\mathrm{p}, \mathrm{D}}$ may be scaled to viscosity applying a diffusion controlled rate coefficient of propagation at zero conversion, $k_{\mathrm{p}, \mathrm{D}}^{0}$, and relative viscosity, $\eta_{\mathrm{r}}$, which changes with conversion. This leads to modification of eq. (2.20) into eq. (2.42).

$$
\frac{1}{k_{\mathrm{p}}}=\frac{1}{k_{\mathrm{p}, 0}^{0}}+\frac{\eta_{\mathrm{r}}}{k_{\mathrm{p}, \mathrm{D}}^{0}}
$$

Including diffusion-controlled propagation and assuming translation-diffusion controlled and reaction-diffusion-controlled termination to occur in parallel, yields Equation (8) for the overall termination rate coefficient of bulk polymerization. ${ }^{[123,130]}$

$$
k_{\mathrm{t}}=\frac{1}{\frac{1}{k_{\mathrm{t}, \mathrm{SD}}}+\frac{\eta_{\mathrm{r}}}{k_{\mathrm{t}, \mathrm{TD}}^{0}}}+\frac{C_{\mathrm{RD}}^{*} \cdot(1-X)}{\frac{1}{k_{\mathrm{p}, 0}^{0}}+\frac{\eta_{\mathrm{r}}}{k_{\mathrm{p}, \mathrm{D}}^{0}}}
$$

Information about the effective reduced viscosity in polymer solution is hardly available (v.s.). The variation of relative viscosity has been approximated by an exponential relation containing one single parameter $C_{\eta}:[90]$

$$
\eta_{\mathrm{r}}^{X}=\exp \left(C_{\eta} \cdot X\right)
$$

Combining eq. (2.42) with eq. (2.44) yields: 


$$
\frac{1}{k_{\mathrm{p}}}=\frac{1}{k_{\mathrm{p}, 0}^{0}}+\frac{\exp \left(C_{\eta} \cdot X\right)}{k_{\mathrm{p}, \mathrm{D}}^{0}}
$$

Combining eq. (2.43)(2.42) with eq. (2.44) yields:

$$
k_{\mathrm{t}}=\frac{1}{\frac{1}{k_{\mathrm{t}, \mathrm{SD}}}+\frac{\exp \left(C_{\eta} \cdot X\right)}{k_{\mathrm{t}, \mathrm{TD}}^{0}}}+\frac{C_{\mathrm{RD}}^{*} \cdot(1-X)}{\frac{1}{k_{\mathrm{p}, 0}^{0}}+\frac{\exp \left(C_{\eta} \cdot X\right)}{k_{\mathrm{p}, \mathrm{D}}^{0}}}
$$

These two equations are plotted in Figure 2-9.

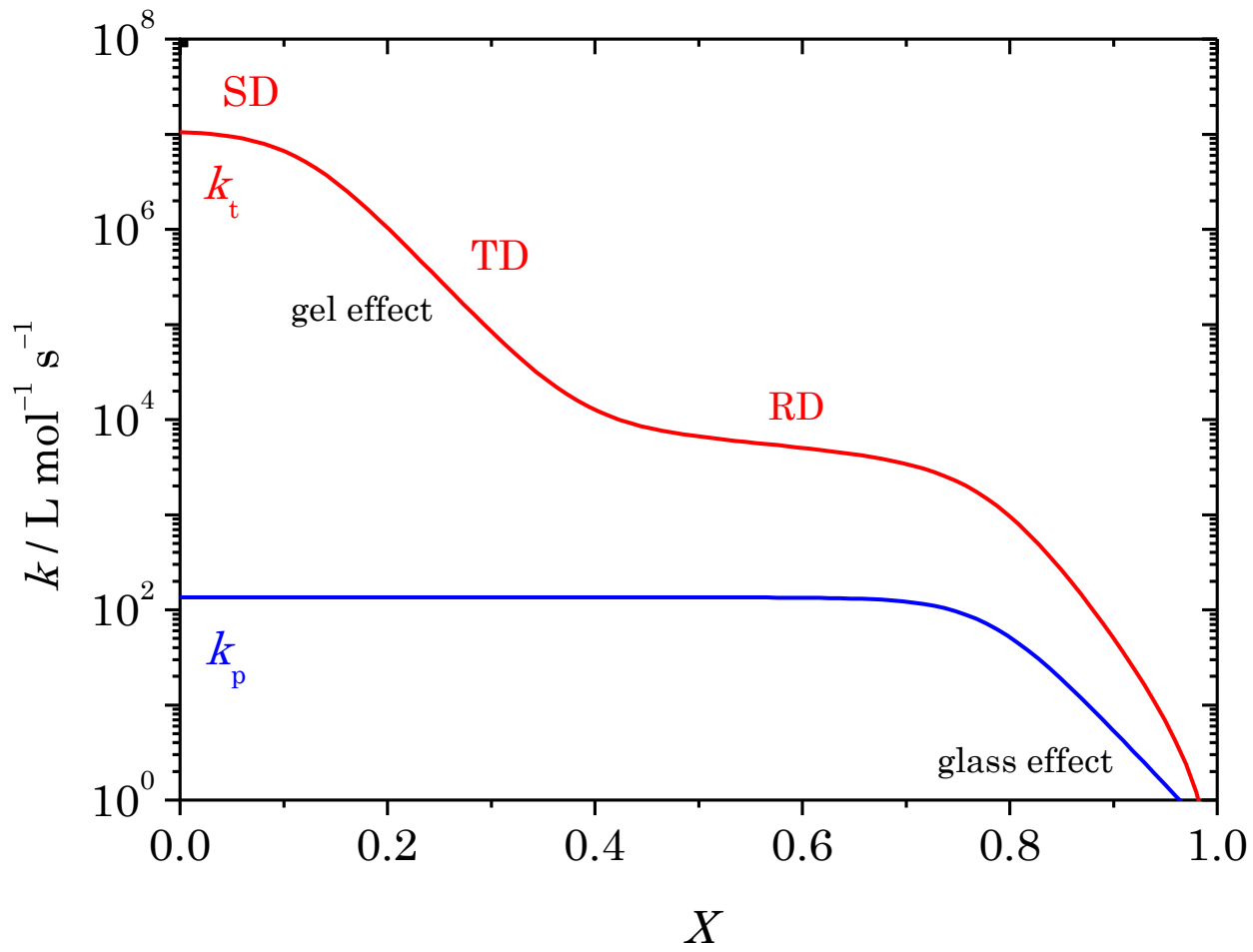

Figure 2-9 The conversion-dependence of $k_{\mathrm{t}}$ and $k_{\mathrm{p}}$ plotted for MMA bulk polymerization according to eq. (2.46) and eq. (2.45). The regions of dominant segmental diffusion, translational diffusion, and reaction diffusion are marked by $\mathrm{SD}, \mathrm{TD}$, and $\mathrm{RD}$, respectively. Rate coefficients are taken from literature. ${ }^{[130,136]}$ 
If initiator decay is a truly unimolecular reaction, it should not be influenced by viscosity. An initiator system with higher molecularity, e.g., a redox initiator can become diffusion controlled. Initiator efficiency decreases as the viscosity increases. The two newly formed radicals remain near one another, i.e. in the solvent cage, for a longer period, so they have more time to terminate or undergo side reactions. The time span between addition of two monomer molecules also increases with conversion, because at very high conversion there is less monomer available. Experimental confirmation for a pronounced decrease of $f$ at high conversions has been obtained from comparison of bulk and emulsion polymerization of MMA. ${ }^{[131]}$ Furthermore EPR studies found this effect. ${ }^{[132-135]}$

\subsection{Computer Modeling of Polymerizations}

The physical properties of a polymer derive from the functionalities of its monomer units, but also from its molecular mass distribution (MMD) and microstructure. Thus, with the same monomer (composition) the production of quite different polymers is possible. This is one of the reasons why even though lots of new monomers have been developed over the last decades, predominantly the same monomers as in the beginning of large-scale industrial application of polymerization are used. New requirements on products were rather met by modification of production processes of existing monomers than by application of new monomers. This may demonstrate the importance of meticulous optimization of industrial polymerization processes.

Modeling can be used to simulate polymerization, e.g., conversion and thereby heat production, but also all properties of the resulting polymer as they are determined by the process. It is a more sophisticated approach than just doing experiments to see how modification of one parameter, e.g., temperature, affects others, e.g., $M_{w}$. As far as possible, all relevant reactions with all their individual dependencies are regarded separately. Hence, special experiments should be carried out to yield individual rate coefficients.

Modeling can be used to test hypothesis about mechanisms, i.e. checking if they lead to correct predictions, or to plan experiments. In addition, a model may be used for evaluation and interpretation of a complex experiment. Thus, modeling may lead to a better understanding of the underlying kinetics. 
Kinetic models are applied as an additional tool for planning new industrial processes or improving established ones, e.g., reducing consumption of resources or enhancing product quality. They also find application in process control (online use). If it is known, how the system will react to alteration of parameters, due to a working model, steering also considers the development to come. This makes operation more precise thus enhancing product quality and safety of the plant. The use of detailed kinetic models for intelligent process control becomes more and more widespread.

Mathematically the model consists of a set of differential equations and is integrated numerically. For a very accurate picture, it is possible to use Monte Carlo calculations. Monte Carlo means figuratively throwing dice as the numerical integration works with a random number generator. It usually requires much computation time and enormous memory capacities. These calculations yield information about individual chains with their specific structure are calculated. Monte Carlo is used, if these special information is desired. In contrast to very slow Monte Carlo, the use of moments equations is the quickest way to solve the problem, but in this case it is not possible to calculate distributions and use chain-length dependent rate coefficients. Calculating including distributions gives more information and allows for chain-length dependency, but needs more computational effort. There are several methods to achieve this. The most wide spread software package is PREDICI ${ }^{\mathrm{TM}}$, which is also the one used in this work. It utilizes discrete Galerkin methods and allows for modeling, which involves an arbitrary number of species and chain-length distributions and any number of chain-length dependent reaction steps; moreover there are no restrictions on the form of MWD and equilibria may be involved. ${ }^{[137]}$ These factors slow computation down and the limitations of calculation arise either from time-consumption or from the instability of the program. 



\section{Materials, Experimental Procedures and Data} Evaluation

\subsection{Chemicals}

\subsubsection{Monomers}

Acrylamide<smiles>C=CC(N)=O</smiles>

Acrylamide (AAm, IUPAC: prop-2-enamide, Fluka, purum, $\geq 98.0 \%$, stabilized with $\mathrm{Cu}^{2+}$, IV CASRN: 79-06-1, $M=71.08 \mathrm{~g} \mathrm{~mol}^{-1}$ ) was used as received or recrystallized from acetone where remarked. purum

\footnotetext{
IV technical information given by Sigma-Aldrich GmbH on inquiry
} 
Acrylic acid<smiles>C=CC(=O)O</smiles>

Acrylic acid (AA, IUPAC: prop-2-enoic acid, Merck, $\geq 99 \%$, anhydrous, stabilized with hydroquinone monomethyl ether, CASRN: 79-10-7, $M=72.06 \mathrm{~g} \mathrm{~mol}^{-1}$ ) was used as received or purified by distillation (v.s.) where remarked.

AA has a Michael system and undergoes 1,4-Michael addition with itself. Diacrylic acid (DiAA, 2-(acryloyloxy)acetic acid) is formed. Water contamination and higher temperature promote this reaction. ${ }^{[138,139]}$ Thus, storage stability of AA is limited and ${ }^{1} \mathrm{H}-\mathrm{NMR}$ was done in regular intervals to ensure DiAA concentration being below $0.01 \mathrm{~mol} \mathrm{~mol}^{-1}$.

Butyl acrylate<smiles>C=CC(=O)OCCCC</smiles>

Butyl acrylate (BA, IUPAC: prop-2-enoic acid butyl ester, Fluka, purum, $\geq 99 \%$, stabilized with hydroquinone monomethyl ether, CASRN: 205-480-7, $M=127.17 \mathrm{~g} \mathrm{~mol}^{-1}$ ) was purified by passing the monomer through a column filled with inhibitor remover (v.i.).

Methacrylic acid<smiles>C=C(C)C(=O)O</smiles>

Methacrylic acid (MAA, IUPAC: 2-methylprop-2-enoic acid, Merck, $\geq 99 \%$, anhydrous, stabilized with hydroquinone monomethyl ether, CASRN: 79-41-4, $M=100.12 \mathrm{~g} \mathrm{~mol}^{-1}$ ) was used as received or purified by distillation (v.s.) where remarked. 
Sodium acrylate<smiles>C=CC(=O)O[Hg]</smiles>

Sodium acrylate (NaA, IUPAC: sodium prop-2-enoate, Aldrich, purum, $97 \%$, stabilized with $140 \mathrm{ppm}$ hydroquinone monomethyl ether, ${ }^{\mathrm{C}}$ CASRN: 7446-81-3, $M=94.04 \mathrm{~g} \mathrm{~mol}^{-1}$ ) was used as received. Polymerization during longer storage of several months was observed. ${ }^{1} \mathrm{H}-\mathrm{NMR}$ was carried out before a set of experiments to ensure that monomer is not contaminated by polymer.

\subsubsection{Solvents}

Demineralized water

Demineralized water (CASRN: $7732-18-5, \quad M=18.02 \mathrm{~g} \mathrm{~mol}^{-1}$ ) was taken from an internal cycle.

Deuterium oxide

Deuterium oxide (Aldrich or Deutero, 99.9\%, CASRN: 7789-20-0, $M=20.03 \mathrm{~g} \mathrm{~mol}^{-1}$ ) was used without further purification.

Dimethyl sulfoxide

Dimethyl sulfoxide (DMSO, Grüssing, für analytische Zwecke 99.5\%, CASRN: 67-68-5, $M=78.13 \mathrm{~g} \mathrm{~mol}^{-1}$ ) was used without further purification.

$o$-Xylene

$o$-Xylene (IUPAC: 1,2-dimethylbenzene, Sigma-Aldrich, puriss, > $99.0 \%$,CASRN: 95-47-6, $M=106.17 \mathrm{~g} \mathrm{~mol}^{-1}$ ) was used as received.

\footnotetext{
$\mathrm{v}$ technical information given by Sigma-Aldrich GmbH on inquiry
} 
Toluene

Toluene (IUPAC: methylbenzene, Fluka, puriss, >99.7 \%, CASRN: 108-88-3, $M=92.14 \mathrm{~g} \mathrm{~mol}^{-1}$ ) was used without further purification.

\subsubsection{Initiators}

2-[(E)-2-(1-carbamimidoyl-1-methylethyl)diazen-1-yl]-2-methylpropanimidamide dihydrochloride<smiles>CC(C)(C)C(=N)NC(C)(C)C(N)=[NH2+]</smiles>

2-[(E)-2-(1-carbamimidoyl-1-methylethyl)diazen1-yl]-2-methylpropanimidamide dihydrochloride, alternatively labeled 2,2'-azobis-(2-methyl propionamidine) dihydrochloride (V-50, Aldrich, $97 \%$, CASRN: 2997-92-4, $M=271.19 \mathrm{~g} \mathrm{~mol}^{-1}$ ) was used as received.

2,2'-Azobis[2-methyl- $N$-(2-hydroxyethyl)propionamide]<smiles>CC(C)(N=NC(C)(C)C(=O)NCCO)C(=O)NCCO</smiles>

2,2'-Azobis[2-methyl- $N$-(2-hydroxyethyl)propionamide] (VA-086, Wako, 1st grade, $>98 \%$, CASRN: $61551-69-7, M=288.35 \mathrm{~g} \mathrm{~mol}^{-1}$ ) was used as received. 
2-Hydroxy-2-methyl-1-phenylpropan-1-one<smiles>CC(C)(O)C(=O)c1ccccc1</smiles>

2-Hydroxy-2-methyl-1-phenylpropan-1-one （D1173， Aldrich, >97\%, CASRN: 7473-98-5, $M=164.20 \mathrm{~g} \mathrm{~mol}^{-1}$ ) was used as received.

2-Methyl-4'-(methylthio)-2-morpholinopropiophenone<smiles>CSc1ccc(C(=O)C(C)(C)N2CCOCC2)cc1</smiles>

2-Methyl-4-(methylthio)-2-morpholino-propiophenone (MMMP, Aldrich, > $98 \%$, CASRN: 71868-10-5, $M=279.40 \mathrm{~g} \mathrm{~mol}^{-1}$ ) was used without further purification.

Di-tert-butyl peroxide<smiles>CC(C)(C)OOC(C)(C)C</smiles>

Di-tert-butyl peroxide (DTBP, IUPAC: 2-(tert-butylperoxy)-2-methylpropane, CASRN: $\left.110-05-4, M=146.23 \mathrm{~g} \mathrm{~mol}^{-1}\right)$ was used without further purification.

Sodium persulfate<smiles>O=S(=O)([O-])OOS(=O)(=O)[O-]</smiles>

Sodium persulfate (NaPS, IUPAC: disodium $O$-[(sulfonatoperoxy)sulfonyl $]$ oxidanidolate, CASRN: $7775-27-1, M=238.11 \mathrm{~g} \mathrm{~mol}^{-1}$ ) was used without further purification. 


\subsubsection{Inhibitors}

Hydroquinone monomethyl ether<smiles>COc1ccc(O)cc1</smiles>

Hydroquinone monomethyl ether (MeHQ, IUPAC: 4-Methoxyphenol, Fluka, CASRN: 150-76-5, $M=124.14 \mathrm{~g} \mathrm{~mol}^{-1}$ ) was used as received.

$N, N$-Di-sec-butyl- $p$-phenylenediamine<smiles>CCC(C)Nc1ccc(NC(C)CC)cc1</smiles>

$N, N$-Di-sec-butyl- $p$-phenylenediamine （IUPAC: $\quad \mathrm{N}^{1}, \mathrm{~N}^{4-}$-di-(2-butyl)benzene-1,4diamine, Aldrich $\geq 94.0 \%$, CASRN: $101-96-2, M=220.35 \mathrm{~g} \mathrm{~mol}^{-1}$ ) was used without further purification.

\subsubsection{Substances used to prepare Buffer Solutions}

Citric acid<smiles>O=C(O)CC(O)(CC(=O)O)C(=O)O</smiles>

Citric acid (IUPAC: 2-hydroxypropane-1,2,3-tricarboxylic acid, Sigma-Aldrich, 99 \%, CASRN: 201-069-51, $M=192.12 \mathrm{~g} \mathrm{~mol}^{-1}$ ) was used as received.

Hydrochloric acid standard solution

Hydrochloric acid standard solution ( $1 \mathrm{~mol} \mathrm{~L}^{-1}$, Fluka) was used as received. 
Potassium dihydrogen phosphate

Potassium dihydrogen phosphate (potassium phosphate monobasic powder, suitable for cell culture, suitable for insect cell culture, suitable for plant cell culture, Sigma, $\geq 99.0 \%$, CASRN: 7778-77-0, $M=136.09 \mathrm{~g} \mathrm{~mol}^{-1}$ ) was used as received.

Potassium chloride

Potassium chloride (for molecular biology, Sigma, $\geq 99.0 \%$, CASRN: 7447-40-7, $M=74.55 \mathrm{~g} \mathrm{~mol}^{-1}$ ) was used as received.

Sodium hydroxide volumetric solution

Sodium hydroxide volumetric solution ( $1 \mathrm{~mol} \mathrm{~L}^{-1}$, Fluka $)$ was used as received.

\subsubsection{Others}

2,2,6,6-Tetramethylpiperidine 1־oxyl

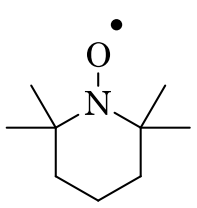

2,2,6,6-Tetramethylpiperidine 1-oxyl (TEMPO, Aldrich, > 99 \%, CASRN: 2564-83-2, $M=156.26 \mathrm{~g} \mathrm{~mol}^{-1}$ ) was used for ESR calibration without further purification.

2-Mercaptoethanol<smiles>OCCS</smiles>

2-Mercaptoethanol (ME, IUPAC: 2-sulfanylethan-1-ol, Aldrich, $\geq 99 \%$, CASRN: 60-24-2, $M=78.13 \mathrm{~g} \mathrm{~mol}^{-1}$ ) was used as CTA without further purification. 
Argon

Argon $(\geq 99.999 \%$, CASRN: 7440-37-1) was used as received.

Formic acid

$\stackrel{\mathrm{O}}{\mathrm{OH}}$

Formic acid (Fluka, eluent additive for LC-MS, 98 \% CASRN: 64-18-6, $M=46.03 \mathrm{~g} \mathrm{~mol}^{-1}$ ) was used as protonating agent without further purification.

Inhibitor remover

Inhibitor remover (Aldrich, "replacement packing for removing hydroquinone and monomethyl ether hydroquinone") was used to free monomer from MeHQ.

$I_{S O}$-butyric acid<smiles>CC(C)C(=O)O</smiles>

Iso-butyric acid (IBA, IUPAC: 2-methylpropanoic acid, Fluka, p.a., > 99.5 \%, CASRN: 79-31-2, $M=88.11 \mathrm{~g} \mathrm{~mol}^{-1}$ ) was used as saturated monomer analog without further purification.

Nitrogen

Nitrogen $(\geq 99.999 \%$, CASRN: 7727-37-9) was used as received.

Polyvinylpyrrolidone

Polyvinylpyrrolidone (pVP, Aldrich, CASRN: 90093-39-8, $M_{\mathrm{w}}=3.6 \cdot 10^{5} \mathrm{~g} \mathrm{~mol}^{-1}$, $\left.M_{\mathrm{n}}=2.4 \cdot 10^{4} \mathrm{~g} \mathrm{~mol}^{-1}\right)$ was used as received. 
Sodium chloride

Sodium chloride (Merck, p.a., > 99.5\%) was used as received.

\subsection{Purification Procedures}

Monomers may come into contact with ground glass joints during purification and storage. In order to reduce impurities, PTFE rings (Dr. Dietmar Glindemann, Glindemann ${ }^{\mathrm{TM}}$ PTFE sealing rings) were used instead of grease to seal ground glass joints and PTFE stopcocks were used for column chromatography. The chemical substances were stored according to instructions of supplier. Monomer free of inhibitor was stored at $-20^{\circ} \mathrm{C}$.

\section{Inhibitor Remover}

Inhibitors that are more polar than the monomer can be removed by passing the mixture through a column filled with inhibitor remover. By this procedure MeHQfree BA of was obtained.

\section{Distillation}

In order to remove impurities, e.g., inhibitor, monomer was distilled over a $20 \mathrm{~cm}$ Vigreux column at 8 mbar. Fine copper wire was placed in the still pot and the lower parts of the column to prevent polymerization. This is especially important for AA.

\section{$3.3 \quad$ NIR}

\subsubsection{Setup}

Spectra were recorded with the FTIR spectrometer IFSTM 88 (Bruker Optik). A water-cooled cell holder was used to reduce heat transfer from the cell to spectrometer components. The optical system was permanently purged with compressed air, dried by an adsorption dryer (ZANDER Aufbereitungstechnik). The 
optical configuration consisted of a tungsten halogen lamp (Gilway Technical Lamp, L7417A, $12 \mathrm{~V}$, $50 \mathrm{~W}$ ), a silicon-coated calcium difluoride beam splitter (model T8401), and a liquid-nitrogen-cooled InSb detector (InfraRed Associates, model $\mathrm{D} 413,1 \mathrm{~mm}$ in diameter). The sample compartment was separated from the optical system by calcium difluoride windows.

Cuvettes were connected to the heated/refrigerated circulating bath F3-K (Haake) operated with an aqueous ethane-1,2-diol solution (50 percent by volume) used as heat transfer fluid.

Data acquisition and data analysis were performed using the software OPUS (Bruker Optik, version 4.2 or 6.0).

\subsubsection{Thermally initiated Polymerization in a Cuvette}

All components were mixed and filled into a cylindrical, thermostated optical cell (65.14/Q, optical path length, if not stated otherwise $10 \mathrm{~mm}$, Spectrosil, Starna) and purged with argon for 4 minutes. The cell was positioned into the spectrometer (v.s.) and brought to the desired temperature. The time required for heating to polymerization temperature was in most cases shorter than the inhibition period. This initial range of negligible degree of monomer conversion was cut off in the conversion-time profiles, which were subjected to modeling. A few reaction mixtures led to significant degree of monomer conversion before the final temperature had been reached. Change in temperature was checked via the shape of the broad water peak (v.i. Figure 3-2.). In these particular cases the data points from before final temperature were removed and the remaining profile was extrapolated to correct the time. These profiles were not used for modeling, but only to compare with the predictions of the final model. NIR spectra were taken in short intervals (of 3 to $60 \mathrm{~s}$ ) until monomer conversion was complete, which took up to several hours. The absence of residual monomer was additionally checked by ${ }^{1} \mathrm{H}-\mathrm{NMR}$.

Cleaning of the cell can become tedious, especially for measurements without CTA. Leaving the devices for extended time periods (several hours to days) in saturated $\mathrm{NaHCO}_{3}$ solution turned out to be the best method for precleaning them. 


\subsubsection{Photoinitiated Polymerization in a Cuvette}

All components were mixed and filled into a cylindrical, thermostated optical cell (Starna 65.14/Q/ optical path length, if not stated otherwise $10 \mathrm{~mm}$, Spectrosil ${ }^{\mathrm{TM}}$, Starna). D1173 was used as photoinitiator. The initial mixture was purged with argon for 4 minutes. After heating to reaction temperature, the polymerizing mixture was irradiated for 40 to $80 \mathrm{~s}$ with an Osram Ultra-Vitalux ${ }^{\mathrm{TM}} 300 \mathrm{~W}$ lamp positioned at a distance of $18 \mathrm{~cm}$, until a degree of monomer conversion of a few percent had been reached, which was monitored via NIR spectroscopy (v.s.). Polymerization was stopped by adding MeHQ to the reaction mixture, which was afterwards cooled to $-20^{\circ} \mathrm{C}$.

\subsubsection{Degree of Monomer Conversion}

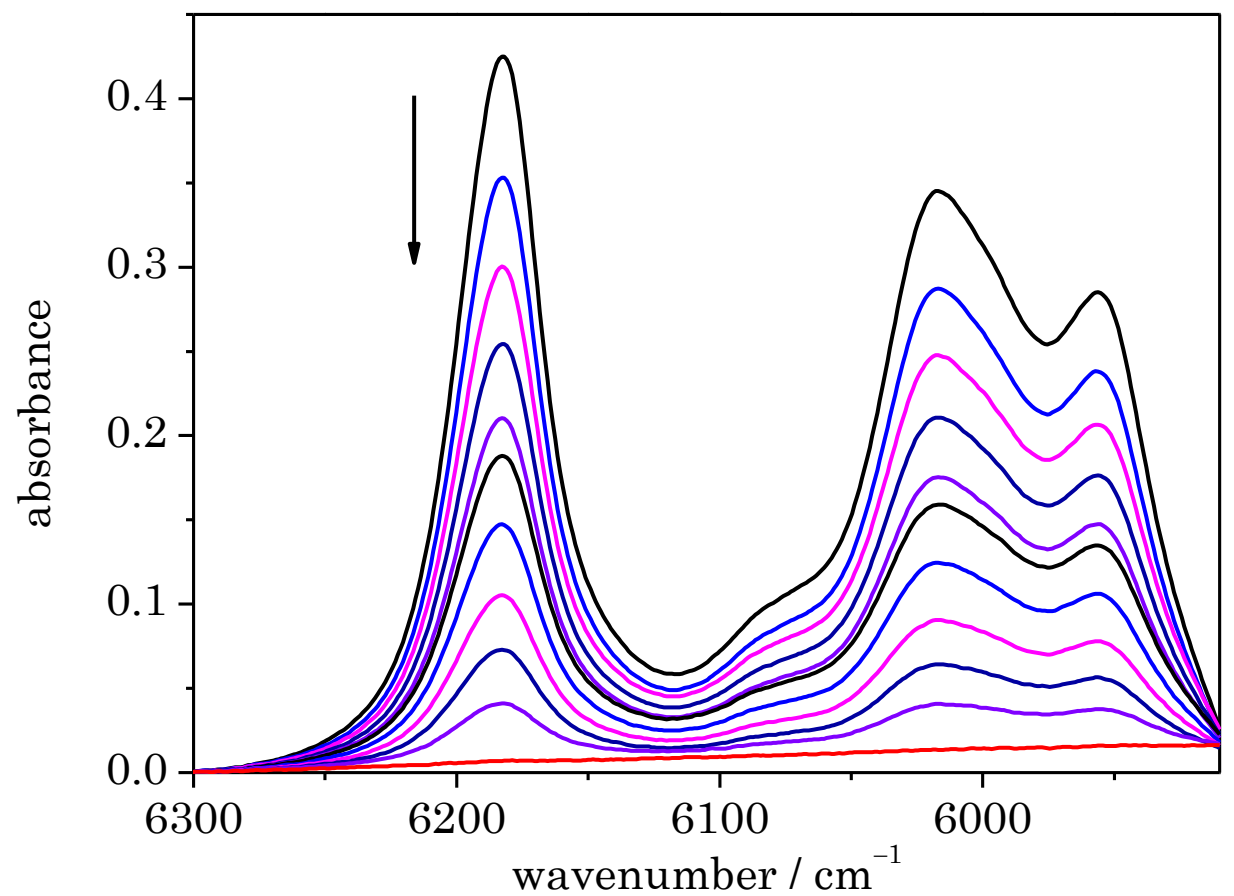

Figure 3-1 NIR absorbance spectrum showing the signal of the first overtone of the C-H stretching recorded during a polymerization of $0.11 \mathrm{~g} \mathrm{~g}^{-1} \mathrm{AA}$ in $\mathrm{D}_{2} \mathrm{O}$. The arrow indicates the direction of change with reaction time. Only the peak around $1175 \mathrm{~cm}^{-1}$ is used for calculation of conversion. 
The first overtone of the $\mathrm{C}-\mathrm{H}$-stretching mode at the $\mathrm{C}=\mathrm{C}$ double bond was used to quantitatively measure degree of monomer conversion as a function of polymerization time. This method works in both organic and aqueous solutions. ${ }^{[130,140,141]}$ A typical set of lines for different degrees of monomer conversion (from zero to full) during AA polymerization is depicted in Figure 3-1.

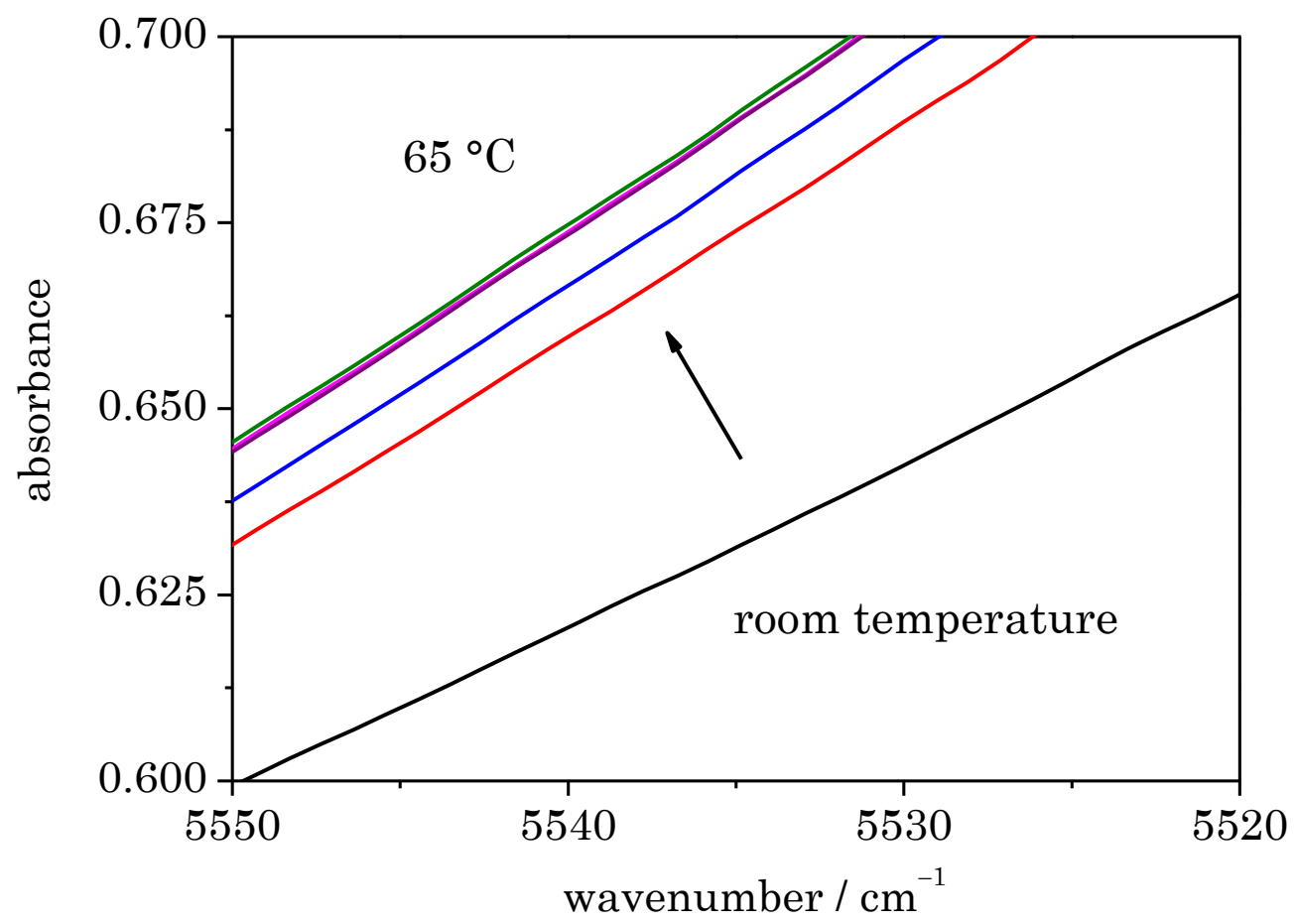

Figure 3-2 Shown is a part of the broad water signal also depicted in Figure 3-3 for a series of spectra taken every $15 \mathrm{~s}$ during the initial period of a polymerization of $0.11 \mathrm{~g} \mathrm{~g}^{-1} \mathrm{AA}$ in $\mathrm{D}_{2} \mathrm{O}$. The arrow gives the direction of change with time. Final temperature is reached approximately after the fourth spectrum.

For AA, the absorbance of the $\mathrm{C}-\mathrm{H}$ mode was integrated between 6250 and $6120 \mathrm{~cm}^{-1}$ against a baseline fitted via a polynomial passing through the absorbance data points at $6370,6280,6113$ and $6085 \mathrm{~cm}^{-1}$. Ionization of monomer shifted the signal. For NaA, the absorbance of the $\mathrm{C}-\mathrm{H}$ mode was integrated between 6261 and $6053 \mathrm{~cm}^{-1}$ against a baseline fitted via a polynomial passing through the absorbance data points at $6284,6255,6062$ and $6051 \mathrm{~cm}^{-1}$. For MAA, the absorbance of the $\mathrm{C}-\mathrm{H}$ mode was integrated between 6275 and $6092 \mathrm{~cm}^{-1}$ against a baseline fitted via a polynomial passing through the absorbance data points at 6310, 6274, 6088 and $6065 \mathrm{~cm}^{-1}$. As the shift resulting from ionization is not very strong and other 
influences might interfere, the NIR signal was not used to calculate the degree of ionization of monomer for different degrees of conversion.

The polymer signal (C-H-stretching mode at the saturated carbon atom) is much weaker and positioned at smaller wavenuber (Figure 3-3 c) compared to the corresponding monomer signal. The low wavenuber region of monomer peak overlaps to a small extent with high wavenuber region of the polymer peak (Figure 3-3 d). As not the full but rather the high wavenuber part of the signal was used, this did not affect the data evaluation described above.

As shown in Figure 3-2, the shape of the broad peak associated with water is especially sensitive towards temperature. Such being the case, the water peak was used to check temperature and ensure that only data points were used after final temperature was reached. Moreover, it was secured that reaction enthalpy of polymerization did not lead to significant temperature increase and the whole process remained isothermal.

For the purpose of improving signal-to-noise quality, $\mathrm{D}_{2} \mathrm{O}$ was used as the solvent instead of $\mathrm{H}_{2} \mathrm{O} .{ }^{[141]}$ For a typical monomer weight fraction of $0.1 \mathrm{~g} \mathrm{~g}^{-1}$ the monomer signal is just a small peak on the flank of the $\mathrm{H}_{2} \mathrm{O}$ peak (Figure 3-3 a), whereas the background absorption of $\mathrm{D}_{2} \mathrm{O}$ is at smaller wave numbers than the one of $\mathrm{H}_{2} \mathrm{O}$ in the $=\mathrm{C}-\mathrm{H}$ region under investigation (Figure 3-3 b).

No notable solvent isotope effect on the polymerization kinetics is expected to occur. ${ }^{[141]}$ This assumption has been verified by comparison with reported conversion vs. time profiles for $\mathrm{H}_{2} \mathrm{O}$ being the solvent. ${ }^{[90]}$ In recipes of PREDICI ${ }^{\mathrm{TM}}$ simulation weight was corrected by ration of densities taken from ref. ${ }^{[142]}$ 

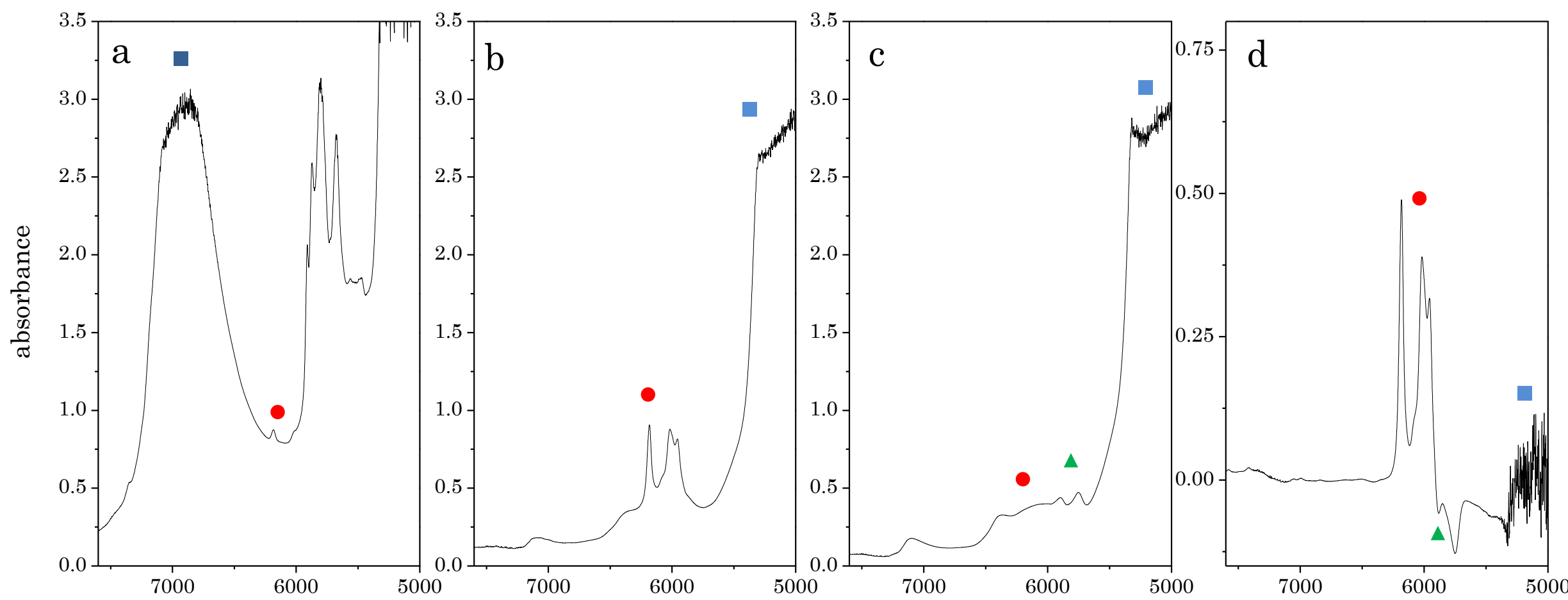

wavenumber $/ \mathrm{cm}^{-1}$

Typical NIR spectra.with important lines being marked: dark blue square: $\mathrm{H}_{2} \mathrm{O}$; medium blue square: $\mathrm{D}_{2} \mathrm{O}$; red circle: monomer; green triangle: polymer; conditions: a $: 0.10 \mathrm{~g} \mathrm{~g}^{-1} \mathrm{AA}$ in $\mathrm{H}_{2} \mathrm{O}$, optical path $2 \mathrm{~mm} ; \underline{\mathrm{b}}: 0.11 \mathrm{~g} \mathrm{~g}^{-1} \mathrm{AA}$ in $\mathrm{D}_{2} \mathrm{O}$, low conversion, optical path $10 \mathrm{~mm}$; : same as b, but full conversion; $\underline{d}$ : difference between $\mathrm{b}$ and $\mathrm{c}$ 


\section{$3.4 \quad$ EPR}

\subsubsection{Setup}

EPR spectra were recorded on an EPR CW/Transient Spectrometer System ElexsysII $^{\mathrm{TM}}$ 500T (Bruker Biospin) or an Elexsys ${ }^{\mathrm{TM}}$ E500 (Bruker Biospin) both operating with the X-band. The resonator, Optical Transmission Cavity (Bruker), was open or equipped with a grid, to allow for irradiation. Temperature was set by a 4131VT unit (Bruker) purging the sample cavity with nitrogen.

Samples were irradiated inside the cavity by a $500 \mathrm{~W}$ mercury arc lamp (LAX 1450, Müller Elektronik; HBO 500 W/2, Osram). It was checked that spectra taken with a cut-off filter WG335 (Schott) had the same ratio of EPR line intensities. As the weaker UV radiation with filter impaired S/N ratio, samples from measurements without filter were used for data processing.

\subsubsection{Organic Samples}

Monomer in solvent was by several freeze-pump-thaw cycles and CTA in solvent was degassed by several freeze-pump-thaw cycles as well .The following preparation was carried out in a glove-box under an argon atmosphere. Then the two solutions were mixed giving a monomer concentration of $1.52 \mathrm{~mol} \mathrm{~L}^{-1}$ with either $0.02 \mathrm{~mol} \mathrm{~mol}^{-1}$, i.e. $3.04 \mathrm{mmol} \mathrm{L}^{-1}$, or $0.05 \mathrm{~mol} \mathrm{~mol}^{-1}$, i.e. $7.60 \mathrm{mmol} \mathrm{L}^{-1}$, and an initiator concentration of $16 \mathrm{mmol} \mathrm{L}^{-1}$. An EPR quartz tube of $5 \mathrm{~mm}$ outer, and $4 \mathrm{~mm}$ inner diameter (Bruker) was filled with $200 \mu \mathrm{L}$ reaction mixture. The sample tube was closed with a plastic cap and sealed with PARAFILM ${ }^{\mathrm{TM}}$. The samples were measured directly afterwards to avoid side reactions, e.g., the thiol-ene reaction occurred.

Optimized measuring conditions were: single scan: $83.9 \mathrm{~s}$, width: $100 \mathrm{G}$; time constant: $10.24 \mathrm{~ms}$; attenuation: 13 ; receiver gain: 60; microwave power: $10 \mathrm{~mW}$; modulation amplitude: $3 \mathrm{G}$. 


\subsubsection{Aqueous Samples}

Measuring EPR in water is especially challenging. A special EPR-cell constructed for measurements in water was used, the ER 165FCVT-S-Q, also called WG-808-S-Q, (WilmadLabGlass/Rototec-Spintec) made of Suprasil ${ }^{\mathrm{TM}}$ to guarantee a clean background. The measuring section is between two planar plates maximizing the filling factor. It has a volume of $150 \mu \mathrm{L}$ and is filled completely. No liquid is left above this section as this would reduce S/N-ratio. D1173 (initiator) weight fraction was $4.7 \cdot 10^{-3} \mathrm{~g} \mathrm{~g}^{-1}$.

Optimized measuring conditions were: single scan: $10.0 \mathrm{~s}$, width: $100 \mathrm{G}$; time constant: $0.01 \mathrm{~ms}$; attenuation: 13; receiver gain: 70; microwave power: $10 \mathrm{~mW}$; modulation amplitude: $3 \mathrm{G}$.

\subsubsection{Deconvolution of Spectra}

Spectra were simulated using Simfonia ${ }^{\mathrm{TM}}$ v.1.25 (Bruker). Coupling constants and line broadening were first adjusted for spectra measured at temperatures where one radical species dominates, i.e. SPRs at $-50{ }^{\circ} \mathrm{C}$ and MCRs at $70{ }^{\circ} \mathrm{C}$. Simulation of the spectra was carried out by Dr. Tatiana Sergeeva. For details see ref.[101]

The simulated spectra were used to fit the complex spectra applying Excel ${ }^{\mathrm{TM}}$ solver and gain fraction of different radical species.

\subsubsection{Calibration}

Radical concentrations were obtained by calibration with TEMPO. The double integral of the signal and radical concentration are correlated linearly. The double integral of spectra at different concentrations of TEMPO are plotted against radical concentration and fitted to a straight line, the slope of which is the calibration constant. It has to be measured for each composition of the reaction mixture and temperature. This is only possible for BA-toluene mixtures, because ME and TEMPO undergo a redox reaction. Calibration with TEMPO was carried out by Dr. Johannes Barth. For details see ref. ${ }^{[143,144]}$ 
Spectra were subjected to double integration to obtain absolute radical concentration by multiplying with the calibration constant. In principle, both the measured spectrum and the fitted spectrum should yield the same result. However, the baseline has to be corrected. It can be straight and just tilted, but also slightly curved. The more lines in a spectrum the more difficult it is to correct the baseline without erasing signal.

Analysis of SPR dominated spectra (few lines) gave the same result for original and simulated spectra. Analysis of spectra with many lines from both SPR and MCR gave lower radical concentration for measured (and baseline-corrected) spectra. As this could be traced back to signal being cut off, simulated spectra were used for analysis.

\subsection{NMR}

${ }^{1}$ H-NMR spectra were recorded in thin-walled tubes (e.g. Schott S, Norell ${ }^{\text {TM }}$ 508-up) at solute mass concentrations of about $0.06 \mathrm{~g} \mathrm{~g}^{-1}$. Spectra for qualitative analysis were determined at room temperature with an Avance ${ }^{\mathrm{TM}}$ III (300 MHz, Bruker). In order to improve S/N-ratio, 16 or 32 FIDs were co-added.

Spectra for quantitative ${ }^{1} \mathrm{H}-\mathrm{NMR}$ analysis were determined by a Unity ${ }^{\mathrm{TM}} 300$ or Mercury ${ }^{\mathrm{TM}} 300(300 \mathrm{MHz}$, Varian).

\subsubsection{Quantitative ${ }^{1} \mathrm{H}-\mathrm{NMR}$}

Optimum ${ }^{1} \mathrm{H}-\mathrm{NMR}$ measuring conditions for quantitative analysis were taken from Schrooten. ${ }^{[145]}$ The relaxation of the hydrogen atom attached to the $\alpha$-carbon is unusually slow. Baseline (Bernstein polynomial fit) and phase (manual) were corrected. 


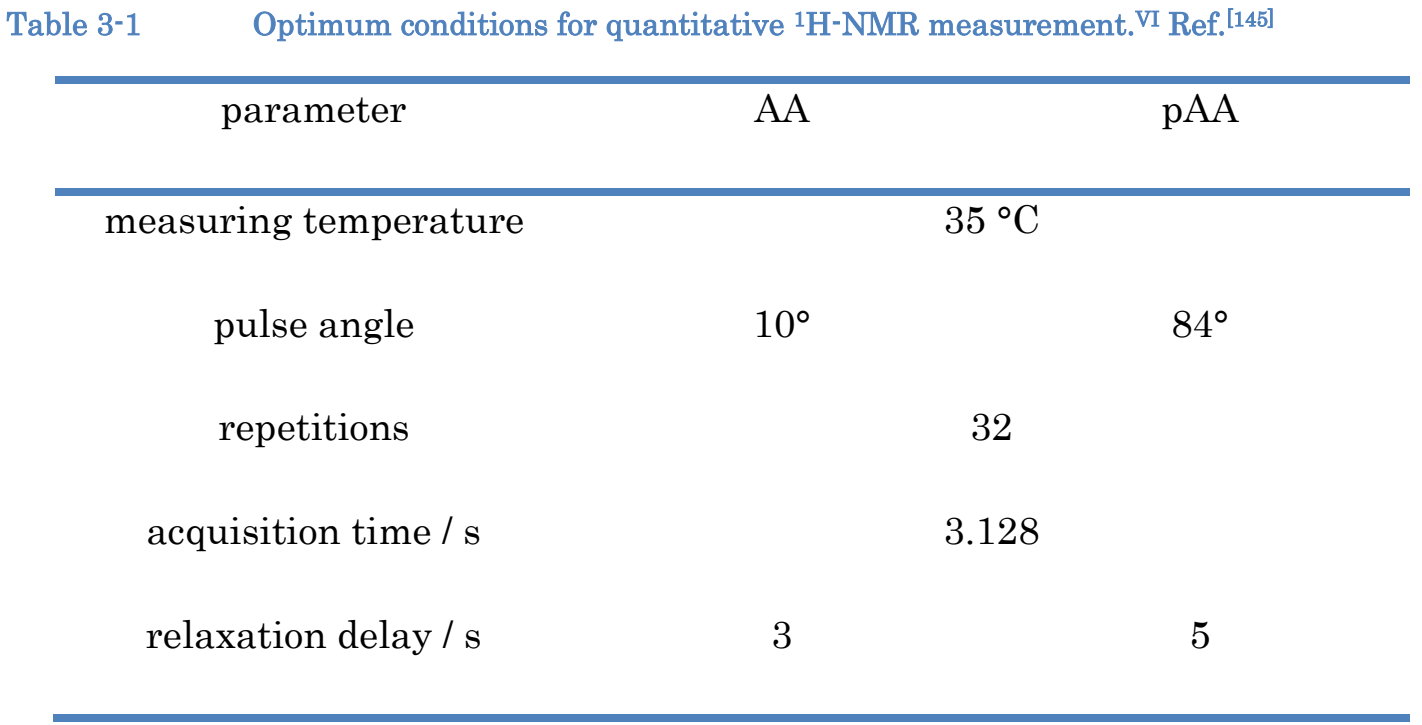

\subsubsection{Quantitative ${ }^{13} \mathrm{C}-\mathrm{NMR}$}

FIDs were processed by the MestReNova ${ }^{\mathrm{TM}}$ software package. Baseline (Bernstein polynomial fit or multipoint baseline correction) and phase (manual) were corrected. Exponential apodization of the FID was done to improve S/N. It was checked for the samples that exhibit a rather intense quaternary carbon peak that apodization does not influence the results. ${ }^{\text {VII }}$

Spectra for quantitative ${ }^{13} \mathrm{C}-\mathrm{NMR}$ analysis were determined with an Inova 500 equipped with a cryo-probe (500 MHz, Varian). Because of the cryo-probe, as it is oversaturated in the very beginning of recording, backward linear prediction (up to 22 points, Toeplitz) was necessary.

VI NMR measurements were performed at the Institut für Organische und Biomolekulare Chemie (Georg-August-Universität Göttingen) by R. Machinek.

VII Apodization may cause higher intensity for peaks of atoms with a shorter relaxation time. 


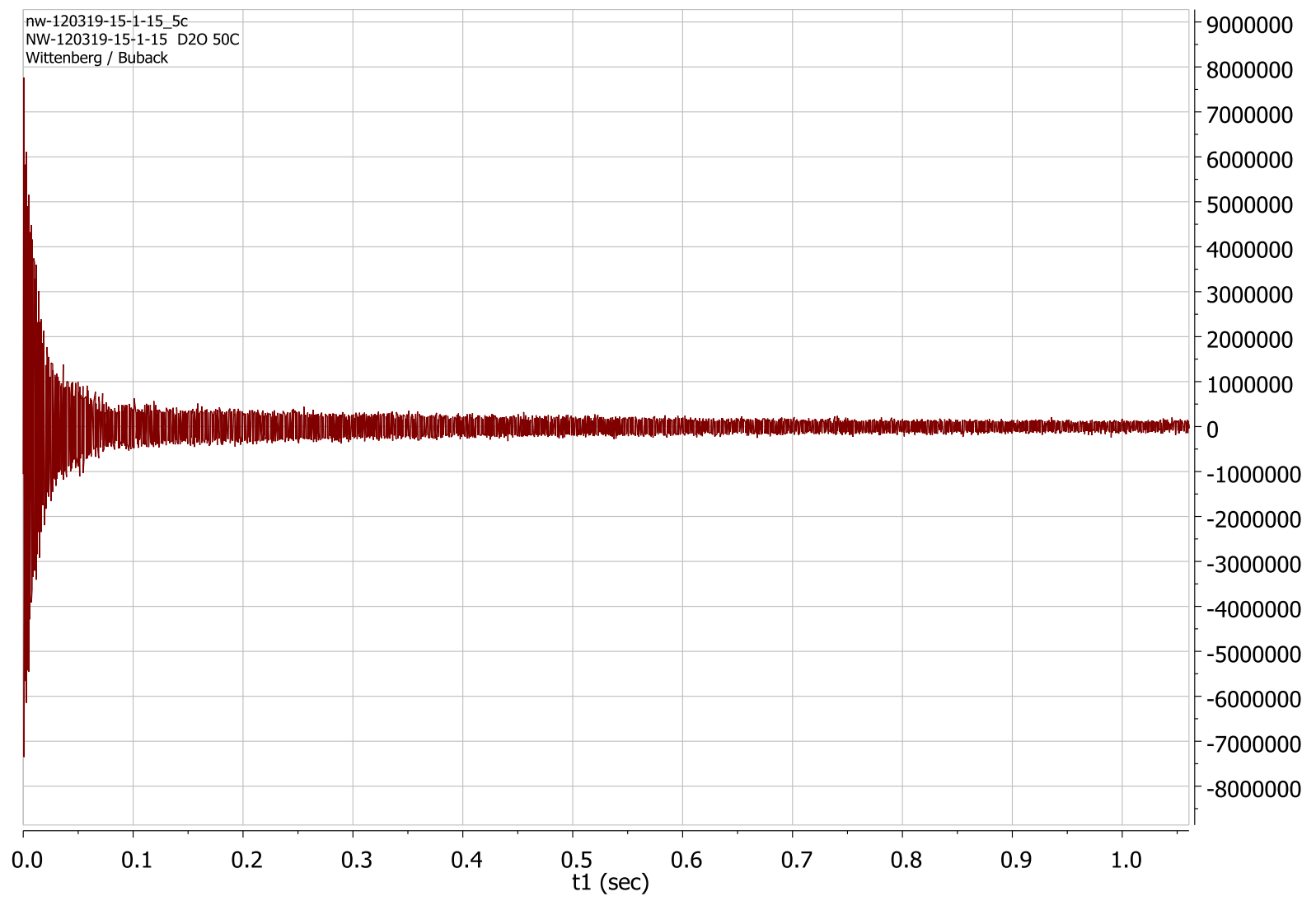

Figure 3-4 Typical FID of a ${ }^{13} \mathrm{C}-\mathrm{NMR}$ of pAA.

Optimum ${ }^{13} \mathrm{C}$-NMR measuring conditions for quantitative analysis were determined in this work. Different measuring parameters were tested. A sample with very high branching was chosen (polymerization conditions: $0.1 \mathrm{~g} \mathrm{~g}^{-1} \mathrm{AA}, 0.02 \mathrm{~g} \mathrm{~g}^{-1} \mathrm{VA}-086$, in water, $\left.170{ }^{\circ} \mathrm{C}, X=85 \%\right)$ to abtain a good $\mathrm{S} / \mathrm{N}$-ratio.

The two questions were, which delay is needed to get complete relaxation of all crucial nuclei, and which delay is necessary for the nuclear Overhauser effect, NOE, built up during acquisition, to recede. In order to minimize NOE, measurement was carried out with inverse gated decoupling, i.e. it was only decoupled during acquisition. Different combinations of acquisition time, at, and relaxation delay, rd, were tried. Acquisition should not be too long because of NOE built-up, on the other hand not too short, because otherwise signal might be cut off and this to a different extent for different nuclei. Thus, looking at the FID, shown in Figure 3-4, the value should not be smaller than $0.5 \mathrm{~s}$ and not bigger than $1.0 \mathrm{~s}$. The relaxation delay should be so long further extension thereof has no more influence. To allow for shorter delays, the pulse angle was reduced. 
Baseline correction and subsequent integration were carried out with the program Origin. The spectra were taken with the same spectrometer and sample straightaway one after another and thus should be directly comparable. Figure 3-5 shows the signal of the quaternary carbon of branching points, Figure 3-6 the corresponding tertiary one of monomer units that do not have a branching point. The integrals are given in Table 3-2; the ratio thereof in Table 3-3.

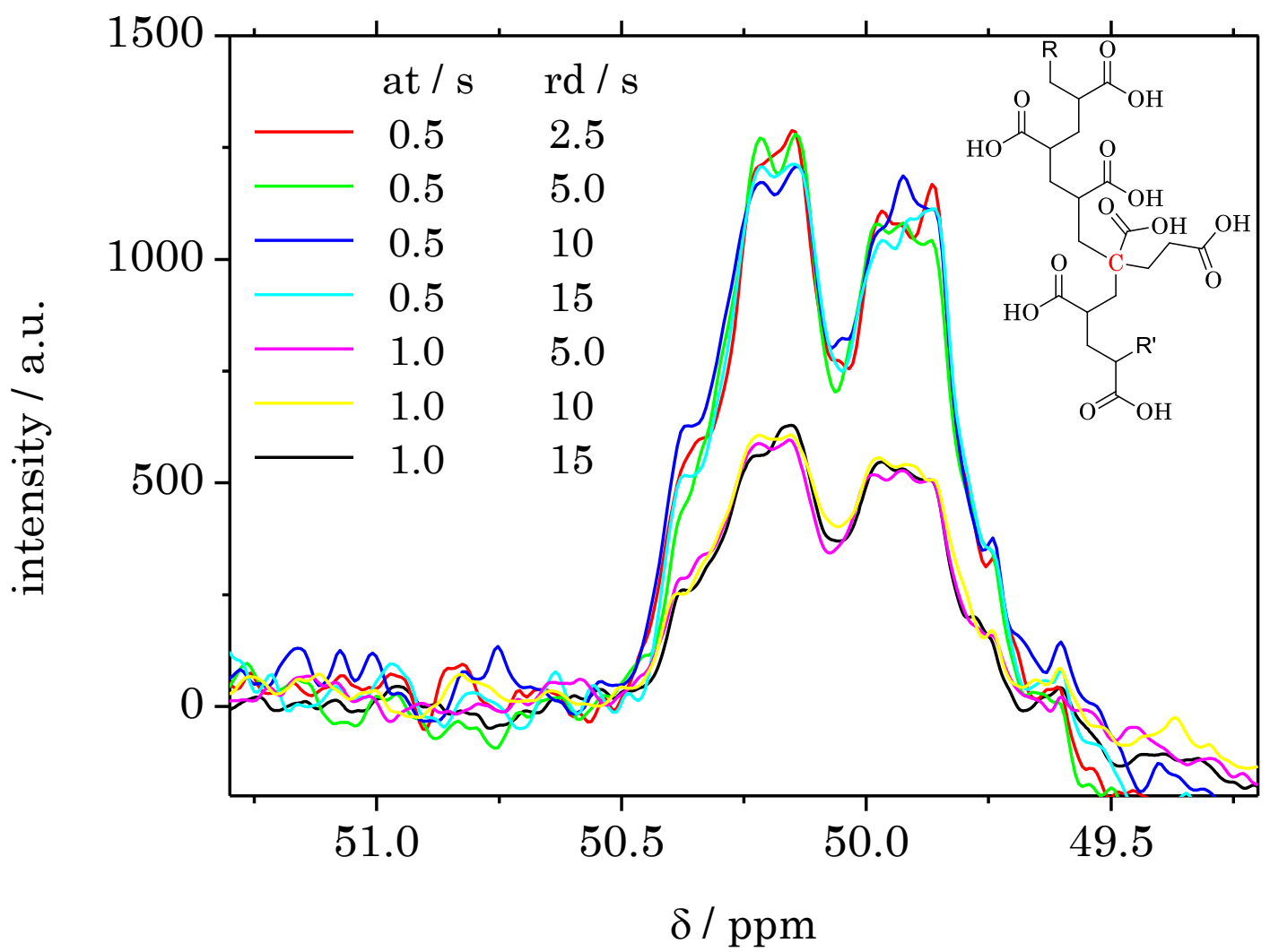

Figure 3-5 The line of the quaternary carbon (marked red in the pAA structure) at branching points as measured with different settings. at: acquisition time; rd: relaxation delay.

As it turns out, NOE is a bigger problem than relaxation time-a longer acquisition time leads to the signal of the $\mathrm{C}_{\mathrm{q}}$ being reduced relative to the $\mathrm{C}_{\mathrm{t}}$. The $\mathrm{S} / \mathrm{N}$-ratio is clearly better for the shorter acquisition time while the result (ratio) is consistent within an error range of $3.1 \%$. In addition with $1.0 \mathrm{~s}$ acquisition time a longer delay seems to be required, which goes with expectation. 
Summing up, an acquisition time of $0.5 \mathrm{~s}$ gives a better signal and requires only a relaxation delay of $2.5 \mathrm{~s}$. Therefore, these measuring conditions are the optimal ones, summarized in Table 3-4.

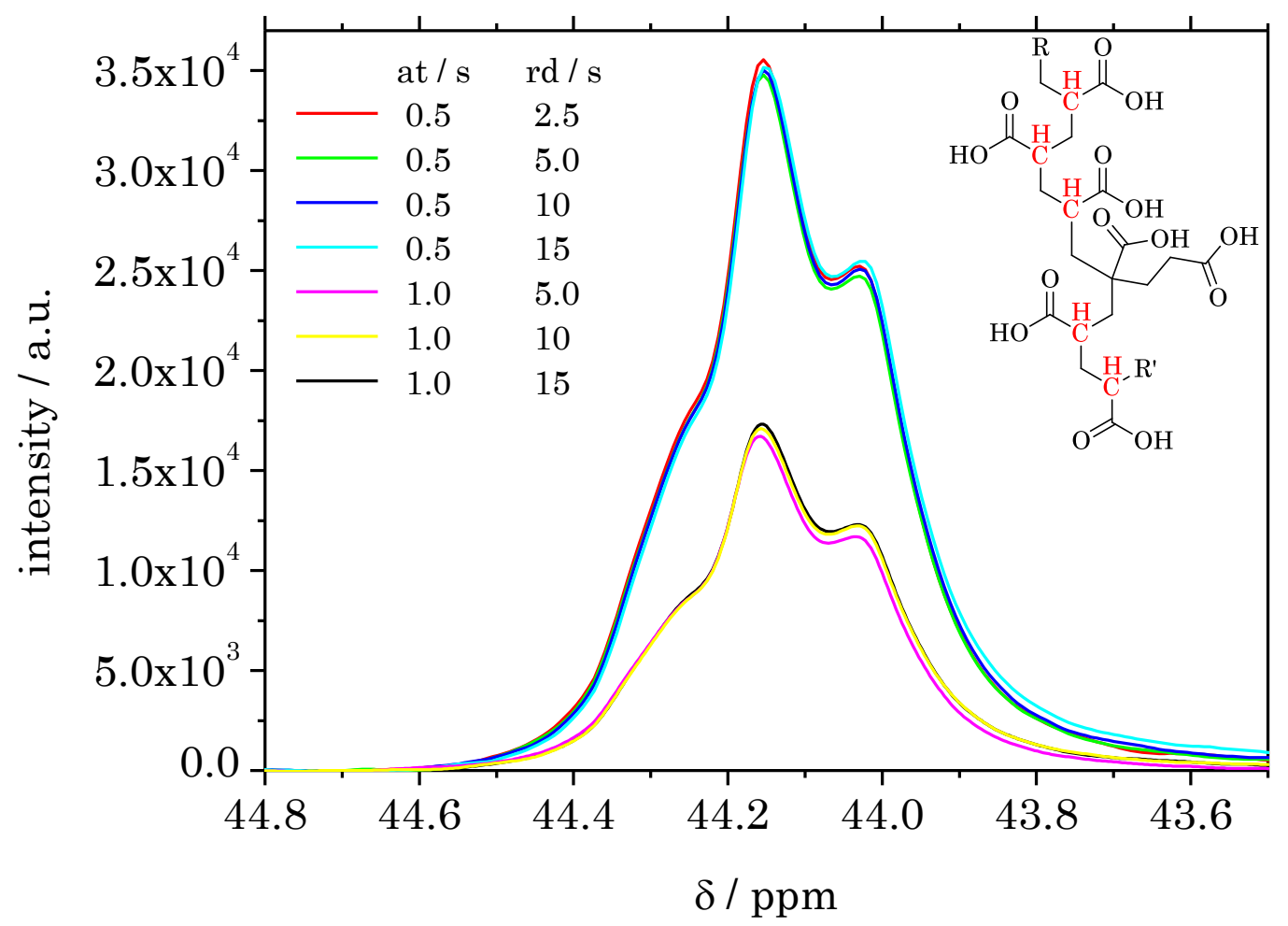

Figure 3-6 The line of the tertiary carbon (marked red in the pAA structure), at which branching points can occur, as measured with different settings. at: acquisition time; rd: relaxation delay.

Table 3-2 Integrals of the peaks shown in Figure 3-5 and Figure 3-6.

\begin{tabular}{llll}
\hline measuring condition & range / ppm & Integral / a.u. & Deviation / \% \\
\hline at 0.5 rd 2.5 & $50.50-49.60$ & 612.1 & 0.99 \\
at 0.5 rd 5.0 & $50.50-49.60$ & 599.8 & -1.04 \\
at 0.5 rd 10 & $50.50-49.60$ & 639.1 & 5.44 \\
at 0.5 rd 15 & $50.50-49.60$ & 606.1 & 0.00 \\
\hline
\end{tabular}




\begin{tabular}{llll}
\hline at 1.0 rd 5.0 & $50.50-49.60$ & 320.2 & 5.82 \\
at 1.0 rd 10 & $50.50-49.60$ & 345.1 & 14.05 \\
at 1.0 rd 15 & $50.50-49.60$ & 302.6 & 0.00 \\
\hline at 0.5 rd 2.5 & $44.60-43.60$ & 10436.2 & -0.87 \\
at 0.5 rd 5.0 & $44.60-43.60$ & 10236.1 & -2.77 \\
at 0.5 rd 10 & $44.60-43.60$ & 10368.6 & -1.51 \\
at 0.5 rd 15 & $44.60-43.60$ & 10527.9 & 0.00 \\
\hline at $1.0 \mathrm{rd} 5.0$ & $44.60-43.60$ & 4847.0 & -4.74 \\
at $1.0 \mathrm{rd} 10$ & $44.60-43.60$ & 5036.9 & -1.01 \\
at $1.0 \mathrm{rd} 15$ & $44.60-43.60$ & 5088.2 & 0.00 \\
\hline
\end{tabular}

Table 3-3 Ratio of the integrals of the peaks shown in Figure 3-2 and Figure 3-3.

\begin{tabular}{lll}
\hline measuring condition & $\begin{array}{l}\text { ratio quaternary to } \\
\text { tertiary line }\end{array}$ & $\begin{array}{l}\text { deviation } \\
\text { ratio of } 0.5 \mathrm{rd} 15 \mathrm{~s} / \%\end{array}$ \\
\hline at $0.5 \mathrm{rd} 2.5$ & 0.055 & 1.8 \\
at $0.5 \mathrm{rd} 5.0$ & 0.055 & 1.7 \\
at $0.5 \mathrm{rd} 10$ & 0.058 & 6.6 \\
at $0.5 \mathrm{rd} 15$ & 0.054 & 0.0 \\
at $1.0 \mathrm{rd} 5.0$ & 0.062 & 13.8 \\
at $1.0 \mathrm{rd} 10$ & 0.064 & 17.8 \\
at $1.0 \mathrm{rd} 15$ & 0.056 & 3.1 \\
\hline
\end{tabular}


Table 3-4 Optimum conditions for quantitative ${ }^{13} \mathrm{C}-\mathrm{NMR}$ measurement of pAA

$W_{\mathrm{P}}$

0.3

measuring

temperature

decoupling

pulse angle

repetitions often not possible due to viscosity and

homogeneity issues

$$
50{ }^{\circ} \mathrm{C} \quad \text { or higher }
$$

n n y decoupling with an NOE as low as possible

compromise between maximum intensity and a measuring time as short as possible

for highly branched polymer

for sample with minimal branching

acquisition time $\quad 0.5 \mathrm{~s}$

relaxation delay $\quad 2.5 \mathrm{~s}$

\subsubsection{Polymerization in NMR Sample Tube}

The reaction mixture was filled into an NMR tube and purged with argon through a hollow needle for 6 minutes. The sample tube was closed with a plastic cap and sealed with PARAFIM ${ }^{\mathrm{TM}}$. The tube was kept in the heated/refrigerated circulating bath F3-K (Haake) operated with an aqueous ethane-1,2-diol solution (50 percent by volume) used as heat transfer fluid for the desired time. The polymerization was quenched by addition of $\mathrm{MeHQ}$ and oxygen (air) plus cooling to $0{ }^{\circ} \mathrm{C}$ (ice bath).

VIII NMR measurements were performed at the Institut für Organische und Biomolekulare Chemie (Georg-August-Universität Göttingen) by R. Machinek. 


\subsection{Density Measurement}

A density meter based on the oscillating U-tube principle was used. It consists of a data acquisition unit, DMA 60 (Anton Paar), a measuring unit, DMA 602TP (Anton Paar), and a high-temperature cell, DMA $602 \mathrm{H}$ (DURAN ${ }^{\mathrm{TM}} 50,-10 \leq \theta \leq 150{ }^{\circ} \mathrm{C}$, Anton Paar). Temperature monitoring inside the U-tube was carried out by means of a digital thermometer (Voltcraft $302 \mathrm{~K} / \mathrm{J}$ Thermometer). Heating was performed by a water bath circulator (Haake, D2-L). To reduce overheating, the water bath circulator was connected to a water circulation cooler (Lauda Dr. R. Wobser, WK 500).

The measurement is only correct, if the U-tube is filled completely and there are no bubbles inside it. This became a problem especially for measurements a higher temperature, because solubility of gases decreases. Therefore, solutions were first heated to desired temperature and filled into the capillary afterwards.

\subsection{Viscosity Measurement}

If not stated otherwise, viscosity was measured by an $\mathrm{AMVn}{ }^{\mathrm{TM}}$ instrument (Anton Paar $\mathrm{GmbH}$ ) using Rheoplus ${ }^{\mathrm{TM}}$ (Anton Paar $\mathrm{GmbH}$ ) as the analysis software. It is a falling/rolling sphere viscosimeter, which uses four different capillaries to cover a range from 0.3 to $20000 \mathrm{mPa}$ s. The capillaries were filled by sucking in the solution into it with a syringe from the other end. Ix

In a few experiments, kinematic viscosity, $v$, was measured with an Ubbelohde viscometer (SCHOTT, 0a, inside diameter of $0.53 \mathrm{~mm}$ ) and corrected via the Hagenbach-Couette procedure. The viscometer was mounted inside a clear-view thermostat (Lauda Dr. R. Wobser, D40) filled with water. It remained inside for at least $900 \mathrm{~s}$ before measurement to allow for thermal equilibration. The water temperature was determined by a Pt100 connected to a PID controller (Eurotherm, 2460). The PID controller regulated a universal relay box (Lauda Dr. R. Wobser, R3), which controlled the power supply of an immersion heater.

Ix Some of the viscosity and density measurements were carried out by Roman Kremring during his bachelor thesis. 
Dynamic viscosities, $\eta$, were determined via combination of the measured kinematic viscosity and density, $\rho$, by:

$$
\eta=v \cdot \rho
$$

\subsubsection{Important Features of Polymer Solutions}

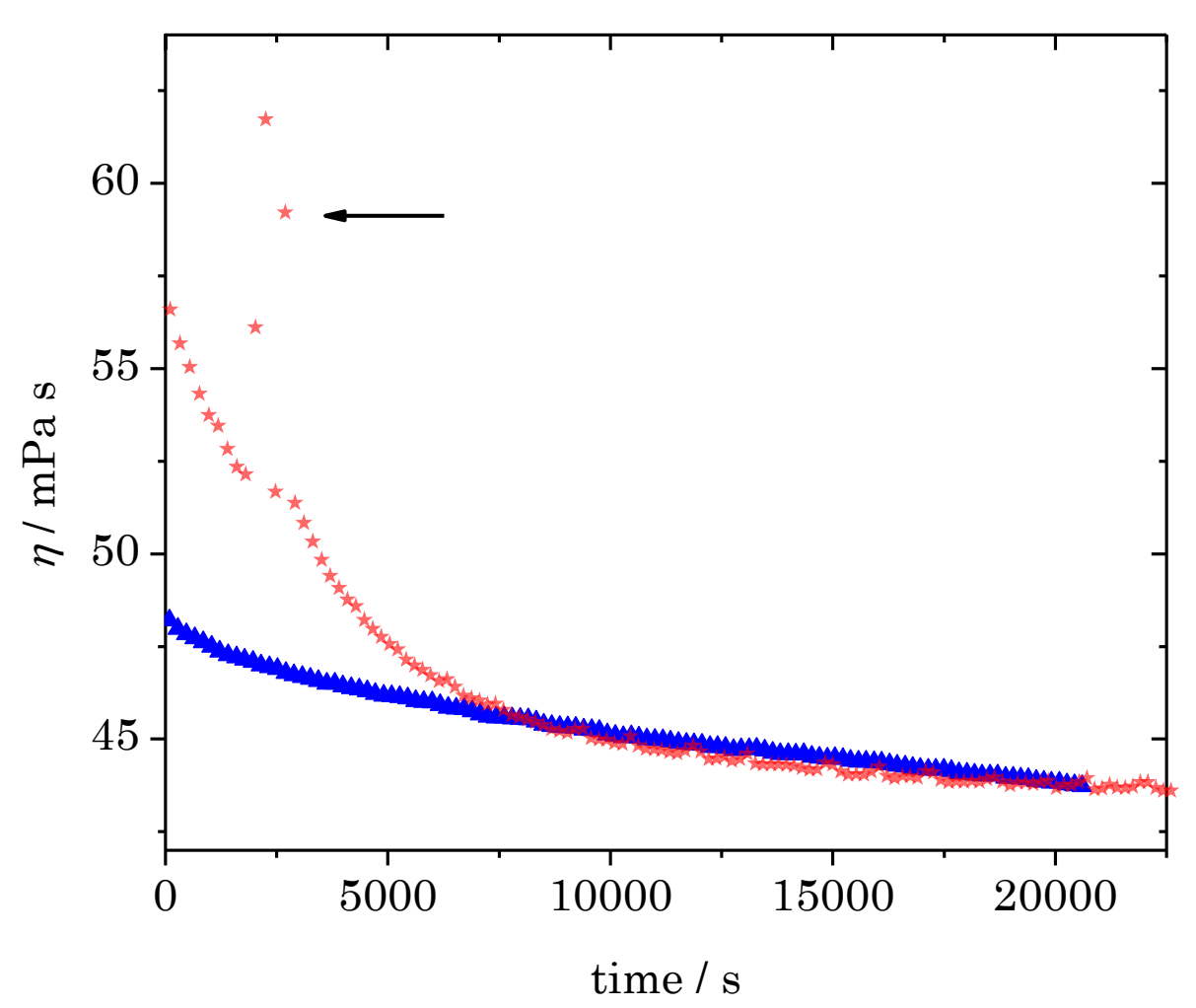

Figure 3-7 A sample of $0.06 \mathrm{~g} \mathrm{~g}^{-1} \mathrm{pMAA}$ was measured at $25^{\circ} \mathrm{C}$ by an $\mathrm{AMVn}$ (falling/rolling sphere viscosimeter) two times. First, the capillary was filled quickly and with high sheer force (red stars), second, the capillary was filled gingerly with lower sheer force (blue triangles). The arrow indicates three singular measurements, during which a tiny bubble in the capillary slowed the sphere down hence giving a too high value of $\eta$.

Polymer solutions are non-Newtonian fluids, viz., when stressed they become either less viscous over time (thixotropy), or they become more viscous over time 
(rheopecty). Both the Ubbelohde viscometer and the falling/rolling sphere viscosimeter cause sheer forces on the sample during filling and measuring. For the latter these can be minimized by choosing a small angle, thus slowing down the sphere. If not stated otherwise, viscosity was measured at an angle of $20^{\circ}$. Figure 3-7 shows how the filling procedure increases viscosity and it slowly decreases afterwards. Therefore, the sheer forces during measurement are very small, if the right conditions have been chosen. Filling the capillary gingerly reduces sheer-induced rise in viscosity. Nevertheless, it takes time for viscosity to decrease. Samples were filled and left for 18000 to $25200 \mathrm{~s}$ before measurement. Another problem is occurrence of bubbles as they slow down or even block the movement of the sphere. Measurements that gave indication of bubbles or other interferences were discarded. Bubbles became a problem especially for measurements a higher temperature, because solubility of gases decreases. For that reason, solutions were first heated to desired temperature and filled into the capillary afterwards.

\subsubsection{Polymerization in Viscosity Measurement Capillary}

All components were mixed, purged with argon for 8 minutes, and directly sucked into the measuring capillary by a syringe at the other end. The capillary was positioned into the viscosimeter (v.s.) and brought to the desired temperature. Initiators that produce gas, e.g., azo-initiators are unsuitable for this experiment. A $0.05 \mathrm{~g} \mathrm{~g}^{-1}$ monomer solution changes density by less than $1 \%$, this effect was ignored.

\subsection{Preparation of Buffer Solutions}

Buffer solutions were prepared based on ref. ${ }^{[146]}$ Main components, e.g., citric acid, were chosen following the recipe given. Aqueous solutions $\left(1.0 \mathrm{~mol} \mathrm{~L}^{-1}\right)$ of sodium hydroxide or hydrochloric acid, respectively, were added under stirring until the desired $\mathrm{pH}$ value had been reached. During this process, the $\mathrm{pH}$ value was monitored online. 


\subsection{SEC}

The entire set of size-exclusion chromatography, SEC, analysis was carried out in aqueous phase by I. Lacík, DSc, and M. Stach, $\mathrm{PhD}$, and co-workers at the Polymer Institute of the Slovak Academy of Sciences according to procedures detailed elsewhere. ${ }^{[59,91,147]}$

\subsection{ESI-MS}

The polymer was dissolved, e.g., in water/ethanol to give a solution of ca. $0.1 \mathrm{~g} \mathrm{~L}^{-1}$. Two spectrometers were used to measure ESI-MS.X

An Ion-trap LCQ ${ }^{\mathrm{TM}}$ (Thermo Finnigan) connected to an HPLC setup (v.i.) was operated with an electrospray voltage of $4.5 \mathrm{kV}$ and a capillary temperature of $200{ }^{\circ} \mathrm{C}$. It had a measuring range of $200-2000 \mathrm{~m} / \mathrm{z}$. In order to improve S/N-ratio ca. 200 spectra were coadded.

A micrOTOF ${ }^{\mathrm{TM}}$ (Bruker) was operated with an electrospray voltage of $3.8 \mathrm{kV}$ and a capillary temperature of $180^{\circ} \mathrm{C}$. It had a measuring range of $30-3000 \mathrm{~m} / \mathrm{z}$. In order to improve $\mathrm{S} / \mathrm{N}$-ratio ca. 200 spectra were coadded.

Spectra were analyzed by OriginPro ${ }^{\mathrm{TM}} 8.5$ (OriginLab).

\subsection{HPLC}

Setup

The high-performance liquid chromatography, HPLC, setup consisted of an AS $1555^{\mathrm{TM}}$ autosampler (Jasco), degasser 3492 (Kontron), a 57 Synergi ${ }^{\mathrm{TM}}$ MAX RP column (Phenomenex), and a Surveyor ${ }^{\mathrm{TM}}$ PDA UV-detector (Finnigan) operating

\footnotetext{
x ESI-MS analysis was performed at the Institut für Organische und Biomolekulare Chemie (Georg-August-Universität Göttingen) by Dr. H. Frauendorf.
} 
from 200 to $800 \mathrm{~nm}$. ${ }^{\text {XI }}$ The HPLC setup was connected to an ESI-MS spectromter (Finnigan, Ion Trap LCQ ${ }^{\mathrm{TM}}$ ), v.i.

Sample preparation

Aqueous samples with $1 \mathrm{mg} \mathrm{g}^{-1} \mathrm{XII}$ initiator were stirred until dissolution was complete. They were kept at the desired temperature for a defined time analogously to either polymerization in a heating block or polymerization in a tubular reactor (v.i.). In the latter case, residence time was corrected analogously to polymerization (v.i.). Afterwards samples were cooled down rapidly in an ice bath.

Measurement

The injection volume was $5 \mu \mathrm{L}$, the flow rate $5 \mu \mathrm{L} \mathrm{s}^{-1}$. The starting eluent was water with $5 \cdot 10^{-4} \mathrm{~g} \mathrm{~g}^{-1}$ formic acid. Within $900 \mathrm{~s}$ the eluent changed gradually to methanol with $5 \cdot 10^{-4} \mathrm{~g} \mathrm{~g}^{-1}$ formic acid. Eluation was continued with the latter for $420 \mathrm{~s}$.

The signal appertaining to non-decomposed initiator could be assigned to a line found after $360 \mathrm{~s}$ via ESI-MS. Integration of the corresponding UV signal gave the concentration of the initiator.

\section{$3.12 \mathrm{pH}-$ Meter}

A S47 SevenMulti ${ }^{\mathrm{TM}}$ dual meter $\mathrm{pH} /$ conductivity (Mettler-Toledo, $-2.000 \leq \mathrm{pH} \leq 20.000,0.001 \mu \mathrm{S} \mathrm{cm}^{-1} \leq \kappa_{\mathrm{c}} \leq 1000 \mathrm{mS} \mathrm{cm}^{-1}$ ) was used to determine as $\mathrm{pH}$ values.

XI HPLC analysis was performed at the Institut für Organische und Biomolekulare Chemie (Georg-August-Universität Göttingen) by Dr. H. Frauendorf.

XII For some samples a higher initial weight fraction was chosen and the samples were diluted prior to HPLC measurement. 


\subsection{High-Temperature Polymerizations}

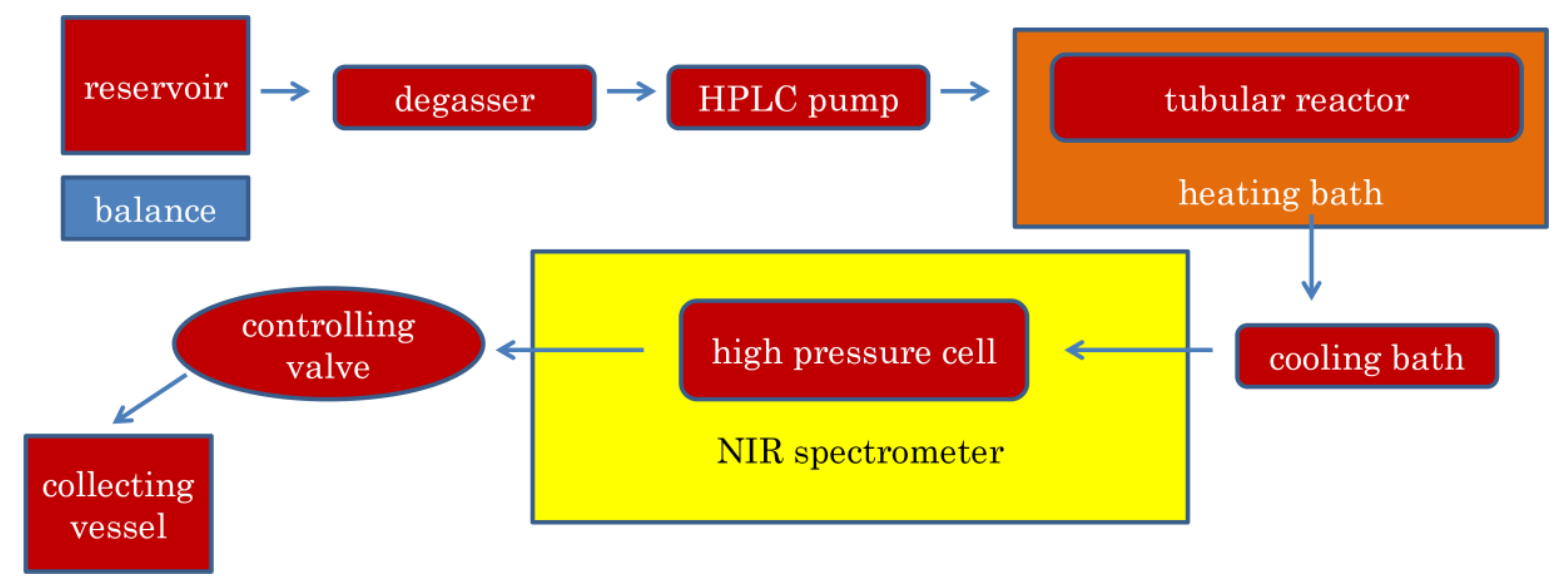

Figure 3-8 The setup for high temperature experiments is shown schematically. It could be operated in two modes.

Figure 3-8 depicts the setup schematically. XIII The reaction mixture started from the reservoir container located on a balance (Omnilab, OL 3100-P), which was connected to a computer to calculate mass flow in $1 \mathrm{~s}$ intervals. The reaction mixture flowed via a PTFE tube (inner diameter $1 \mathrm{~mm}$ ) to the degasser (Ercatech AG, ERC-3215a) and further to an HPLC pump (built by the mechanical workshop of the Institut für Physikalische Chemie, Georg-August-Universität Göttingen). The pump gave a pressure of 200 bar and from here on connection was by a stainless steel capillary (AD: 1/16") with an inner diameter of $0.5 \mathrm{~mm}$. Then it entered a double line tube $(1 \mathrm{~m})$ to get a temperature of $50{ }^{\circ} \mathrm{C}$., which was not enough to induce significant monomer conversion but reduced temperature difference to reaction temperature. In the next step the reaction mixture flowed into the tubular reactor located in a heating bath (Haake, N3) filled with silicone oil. The stainless steel, tubular reactor consisted of two 510.1156 (Nova Swiss, AD: 1/16", ID: $0.5 \mathrm{~mm}$ ). It was spiral-shaped, had a length of $1050 \mathrm{~cm}$, an inner diameter of $0.5 \mathrm{~mm} \pm 0.05 \mathrm{~mm}$, and thus a geometric volume of $2.06 \mathrm{~mL} \pm 0.41 \mathrm{~mL}$. Temperature was measured by CIA S250 Chromel/Alumel thermocouple and pressure by a P3MB (HBM). Afterwards the reaction mixture was cooled to $50{ }^{\circ} \mathrm{C}$ in a double line tube $(1 \mathrm{~m})$. Then it flowed into a high-pressure cell (described in more detail elsewhere ${ }^{[145,148]}$ ) heated electrically to $50{ }^{\circ} \mathrm{C}$ and located in the FTIR spectrometer IFS 88 (Bruker Optik). A controlling

XIII The high-temperature polymerizations were all carried out by Daniel Weiß.[148] under cosupervision of Dr. H.-P. Voegele. 
valve followed to set the velocity of the flow. The last part of the setup was a collecting vessel.

The setup allowed for two different modes of operation described in what follows.

\subsubsection{Stopped-Flow experiments in High-Pressure Cell}

The tubular reactor was bypassed and polymerization took place in the high pressure cell. The reaction mixture flowed without prior warming through the cell until stationary condition was reached. Then the valves of the high-pressure cell were closed and the cell was used as a batch reactor. Polymerization was followed by NIR as described in subchapter 3.3. The upper temperature limit for measurements with this mode of operation was set by initiator decay. At temperatures above $140{ }^{\circ} \mathrm{C}$, VA-086 decayed so fast that significant $X$ had already been reached at the starting point.

With this setup, it was not possible to draw samples during polymerization.

The procedure is described in more detail here. ${ }^{[148]}$.

\subsubsection{Polymerization in a Tubular Reactor}

Polymerization was carried out inside the tubular reactor. Residence time, $\tau$, was set by flow rate and was calculated from mass flow, density of the reaction mixture and geometric volume of the reactor. This theoretical value had to be corrected (v.i.).

The lower and upper temperature limits for measurements with this mode of operation were set by initiator decay. At temperatures above $170{ }^{\circ} \mathrm{C}$, VA-086 decayed so fast that high final monomer conversion was reached even at the highest flow rate. Measuring a conversion vs. time profile is not possible under these conditions. At temperatures below $130{ }^{\circ} \mathrm{C}$, VA-086 decayed so slowly even for the lowest flow rate, which still allowed for turbulent flow, high conversion could not be reached.

Samples were taken at each flow rate. They were dried in vacuo at temperatures up to $90^{\circ} \mathrm{C}$ and measured by NMR (subchapter 3.5.1 and 3.5.2). 


\section{Correction of residence time in the tubular reactor}

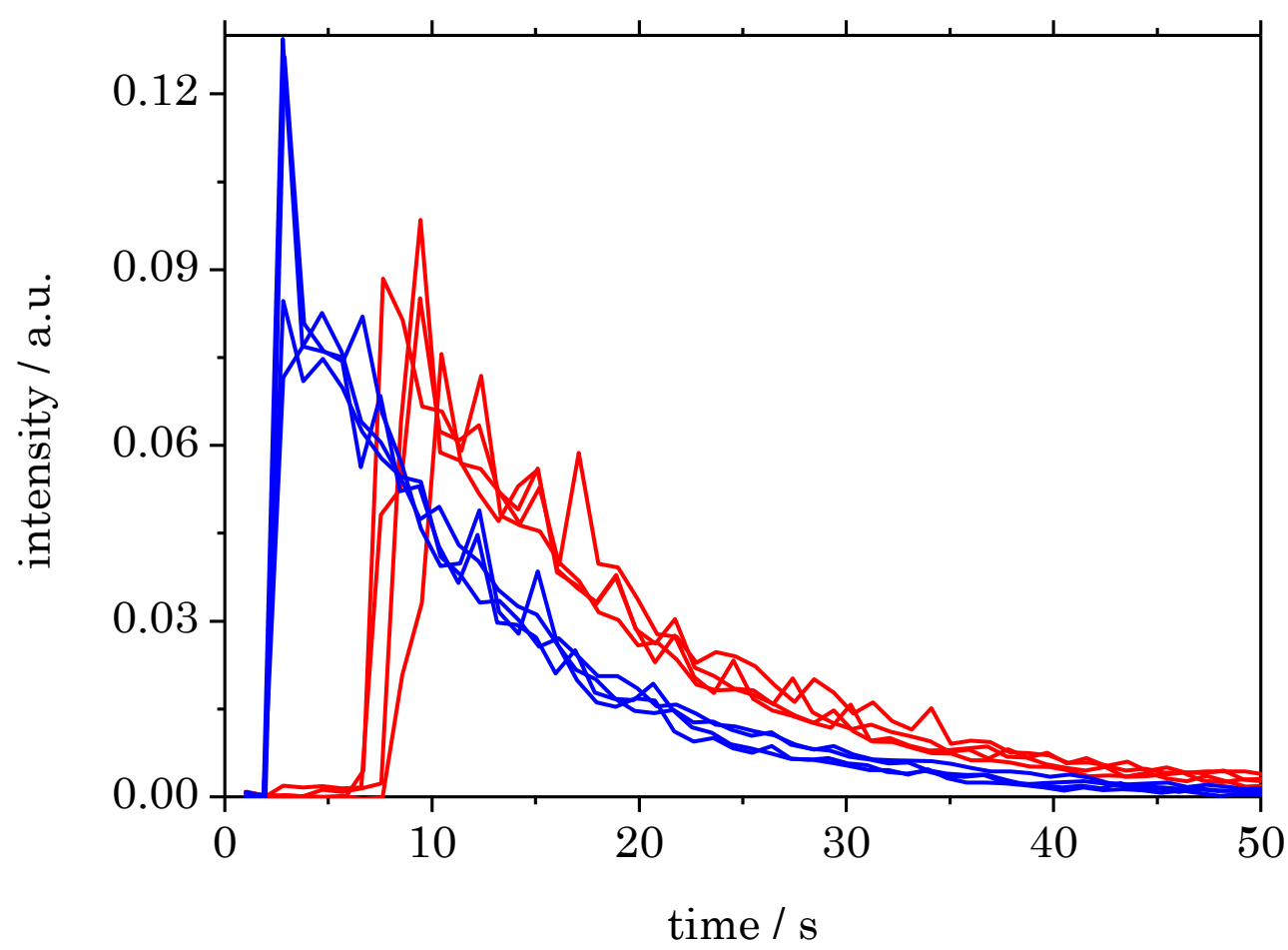

Figure 3-9 Residence time distribution of the whole setup (red) and without the tubular reactor (blue) measured as time-dependent signal intensity (pulse-response) for residence time experiments applying marker (AA) as approximate delta function.

Residence time of the tubular reactor was measured by flowing water through the apparatus with and without the reactor being included. AA was injected as marking substance as an approximate delta function. Increase of the center peak of the interferogram (to increase time resolution) was used to measure the arrival of marking substance. The result is shown in Figure 3-9.

The curves from experiments with the tubular reactor have, within experimental accuracy, the same shape as the curves from experiments without the tubular reactor-there is only a time offset. Thus, no significant broadening by the highpressure cell can be observed.

The curves were integrated to obtain $\tau$, see Figure 3-10. The true residence time of the tubular reactor was calculated as the difference between residence time including the tubular reactor and residence time not including the reactor. 


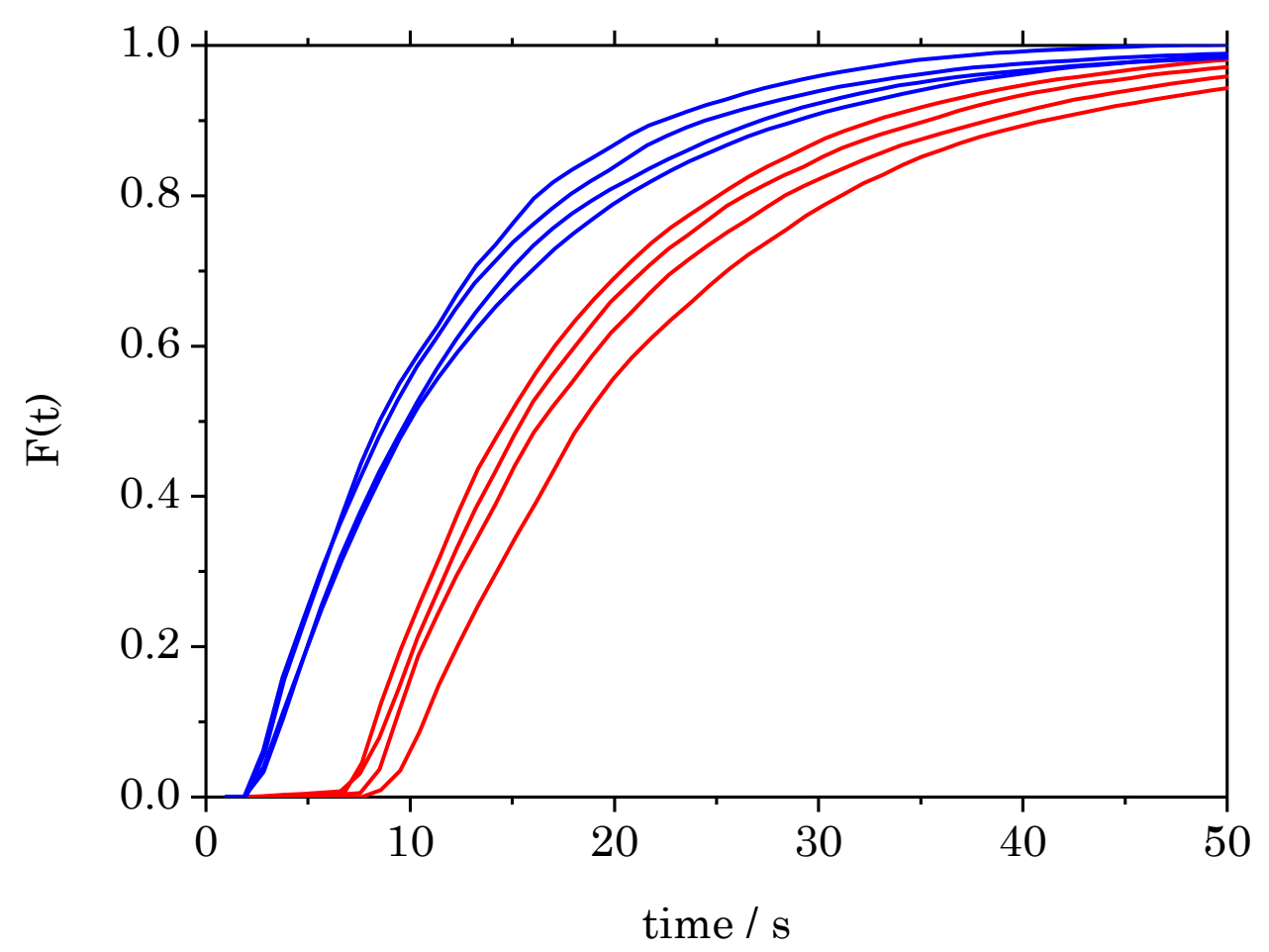

Figure 3-10 Sum functions of the curves shown in Figure 3-9. This is used to compute the true residence time of the tubular reactor as the difference between residence time including the tubular reactor (red) and residence time not including the reactor (blue).

This was done for different flow rates and the residence time calculated from mass flow, density of the reaction mixture and geometric volume of the reactor. This theoretical value, $\tau_{\text {calc }}$, was plotted against the true residence time, $\tau$. This is shown in Figure 3-11. A linear relationship was found and an empirical correction function was determined, eq. (3.2), with which all experimental results given later have been corrected.

$$
\tau / \mathrm{s}=\tau_{\text {calc }} / \mathrm{s} \cdot 2.38-4.58
$$

The procedure is described in more detail elsewhere. ${ }^{[148]}$ 


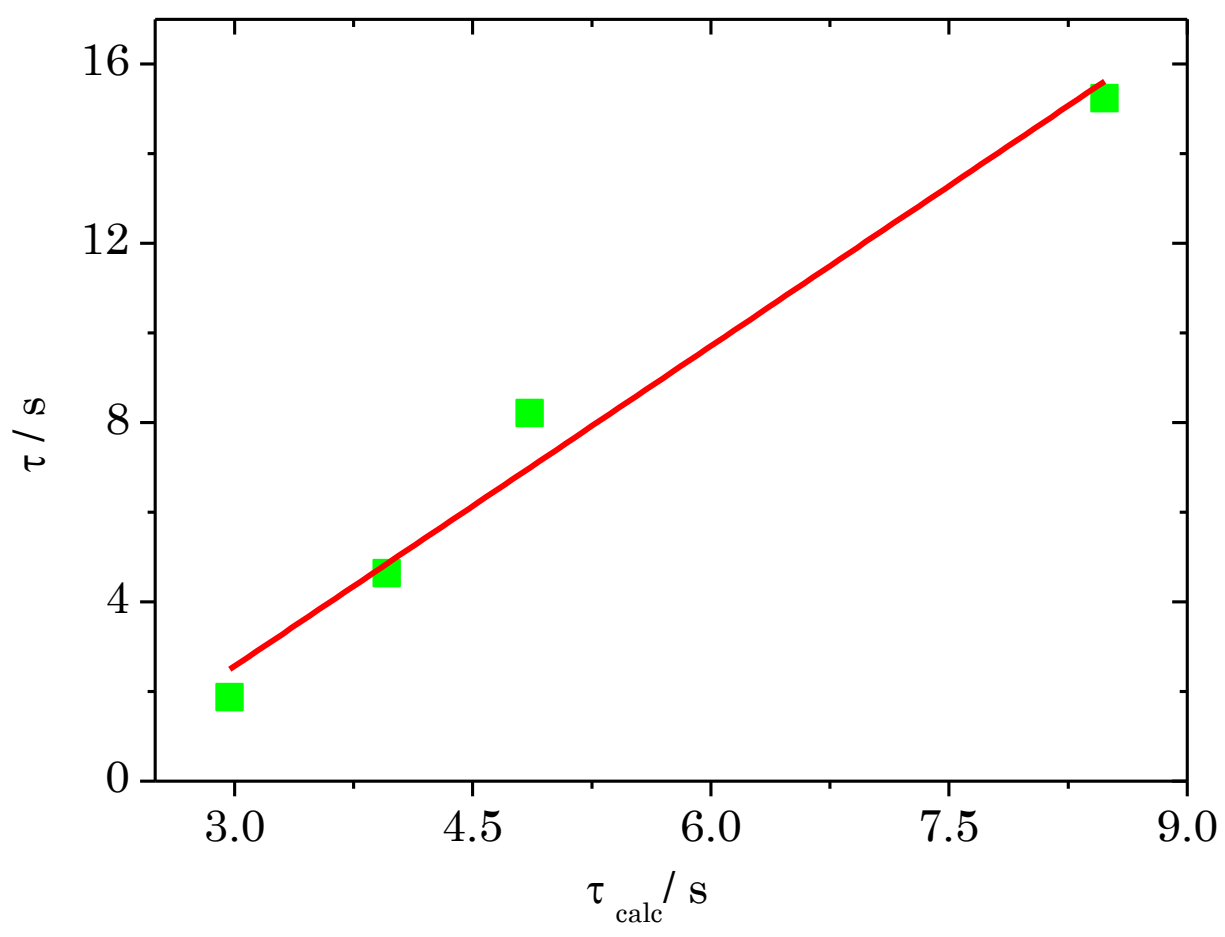

Figure 3-11 Calculated and directly measured residence times are compared (green squares). These values are fitted to a straight line (red, eq. (3.2)).

As it was only possible to measure $\tau$ for high flow rates, for lower flow rates, and thus higher conversion, residence time was corrected via linear extrapolation, eq. (3.2). The time has to be more precise for the beginning of the polymerization, as the rate of polymerization is highest here. The comparison of the conversion-time profiles of polymerization with the stopped-flow operation to conversion-time profiles from polymerization in the tubular reactor before and after correction suggest that the correction and even the extrapolation works well. An example is given in Figure 3-12. Small symbols give the result from batch polymerization (stopped-floe operation) used as reference here. Big open symbols belong to a polymerization of the same reaction mixture in the tubular reactor. The rate of polymerization seems to be smaller for the tubular reactor. Correction of residence time of the tubular reactor via eq. (3.2) yields the big, solid symbols. The corrected conversion-time profile of the polymerization in the tubular reactor agrees with the one of the batch reactor, thus the rates of polymerization are the same. 


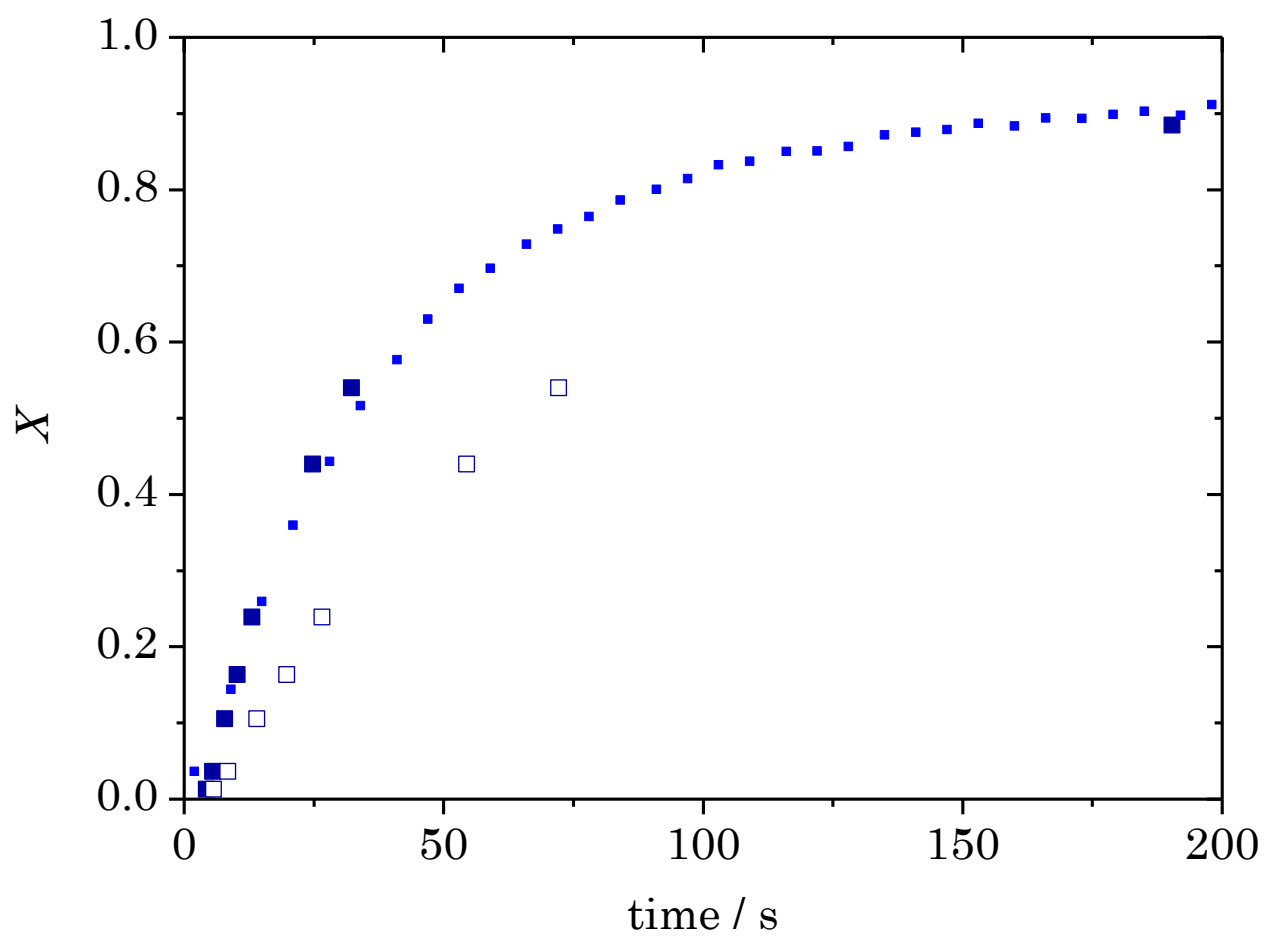

Figure 3-12 Conversion-time profiles of polymerization with the stopped flow operation (small symbols) is compared to conversion-time profiles from polymerization in the tubular reactor before correction (big open symbols) and after correction (big filled symbols) are compared. $0.1 \mathrm{~g} \mathrm{~g}^{-1} \mathrm{AA}$ was polymerized with $0.002 \mathrm{~g} \mathrm{~g}^{-1} \mathrm{VA}-086$ in $\mathrm{H}_{2} \mathrm{O}$ at $140{ }^{\circ} \mathrm{C}$.

\subsection{Other Setups for Polymerization}

\subsection{1 $1 \mathrm{~L}$ Automated Reactor}

A LabMax ${ }^{\mathrm{TM}} 1 \mathrm{~L}$ automated, stirred reactor (Mettler-Toledo) was connected to a nitrogen cylinder and operated by iC Software (Mettler-Toledo).

A mixture of water and monomer was stirred under a nitrogen blanket for $1800 \mathrm{~s}$ and heated to desired temperature. Then initiator and, if required, CTA were added in countercurrent. Samples were drawn in increasing time intervals. They were cooled immediately after having been taken from the polymerizing mixture and both MeHQ and oxygen (air) were added. The decay of monomer and CTA concentrations was monitored by ${ }^{1} \mathrm{H}-\mathrm{NMR}$ and by gravimetric analysis. 


\subsubsection{Polymerization in a Heating Block}

The reaction mixture was filled into a dark (protection from UV light) twist-off-glass with a septum integrated in the cap. Argon was purged through the reaction mixture by a hollow needle. The sample was kept in the Block Heater 250 (Rotilab) for the desired time. In order to ensure good mixing, the heater was standing on a KS-15 shaker (Edmund Bühler GmbH)

Polymerization was stopped either by cooling the sample rapidly with liquid nitrogen, which sometimes caused the glass to break, or by adding MeHQ and oxygen (air) and cooling the sample in an ice bath.

\subsubsection{Polymerization in a Flask}

The reactor was a flask connected to a Schlenk line. A mixture of water and solvent was either purged with argon for 30 minutes or degassed by several freeze-pumpthaw circles and flooded with argon afterwards. It was brought to desired temperature. Then dissolved initiator and, if required, CTA were added by a syringe through a septum. Samples were drawn in increasing intervals. They were cooled immediately after having been taken from the polymerizing mixture and both MeHQ and oxygen (air) were added.

Later, this procedure was replaced by polymerization in a lined flask, which allows for better temperature control.

\section{$\underline{\text { Synthesis of arylic acid macromonomer }}$}

The above described procedure was modified to synthesize arylic acid macromonomer, MM(AA).

$150 \mathrm{~mL}$ DMSO were purged with argon and brought to $138^{\circ} \mathrm{C}$. $0.0116 \mathrm{~g}$ VA-086 was dissolved in $5.3 \mathrm{~g}$ DMSO. Half of the initiator solution was added through a syringe. $10.72 \mathrm{~g}$ AA was added dropwise. The rest of the initiator was added slowly. After $2400 \mathrm{~s}$ the reaction mixture was cooled in an ice bath. No inhibitor was added. The product of extraction from water was dried in vacuo. 
$190 \mathrm{~mL} \sigma$-Xylol were purged with argon and brought to $140{ }^{\circ} \mathrm{C} .0 .116 \mathrm{~g}$ DTBP was added through a syringe, then $10.79 \mathrm{~g}$ AA. After $8700 \mathrm{~s}$ the reaction mixture was cooled in an ice bath. No inhibitor was added. The reaction product was dried in vacuo.

\subsubsection{Polymerization in a Lined Flask}

The reactor was a lined flask with its hose barbs connected to a heated/refrigerated circulating bath (Haake, F3-K) with ethanol used as heat transfer fluid. The flask was completely covered with aluminum foil. The reaction mixture was purged with argon for $2400 \mathrm{~s}$ and brought to the desired temperature. The foil was opened on one side and the flask was irradiated by a $100 \mathrm{~W}$ mercury arc lamp (LAX 100, Müller Elektronik; HBO $100 \mathrm{~W} / 2$, Osram) from ca. $40 \mathrm{~cm}$ distance. Afterwards a sample was drawn by a wide, hollow needle, cooled immediately to $-20^{\circ} \mathrm{C}$, and stored in darkness. This procedure was repeated until final conversion was reached.

\subsection{Computer Programs}

\subsubsection{Curve Fitting}

Fitting of mathematical functions to experimental data was conducted by the software OriginPro ${ }^{\mathrm{TM}}$ 8.5 (OriginLab), except for curve fitting described in 3.15.2. Linear fitting is carried out applying the method of least squares. For non-linear curve fitting the Levenberg-Marquardt algorithm is used for iterative adjustment of parameters.

\subsubsection{Determination of Joint Confidence Regions}

The program Contour (A. M. van Herk, version 1.8) was used to generate ellipses corresponding to joint confidence regions for Arrhenius activation energy and Arrhenius pre-exponential factor of $k_{\mathrm{p}}$. The details of the program are given elsewhere. ${ }^{[149,150]}$ 


\subsubsection{Simulation}

Simulations were performed by the program Predici ${ }^{\mathrm{TM}}$ v6.4.8 (Dr. Michael Wulkow Computing in Technology GmbH).

\subsection{Error Estimate}

\section{Density}

The density meter is specified to be accurate to $\pm 1.5 \cdot 10^{-6} \mathrm{~g} \mathrm{~cm}^{-3}$.

\section{ESI-MS}

Results are from the $\mathrm{LCQ}^{\mathrm{TM}}$ are precise by $0.1 \mathrm{~m} / z$ and results from the micrOTOF ${ }^{\mathrm{TM}}$ are precise by $0.005 \mathrm{~m} / \mathrm{z}$.

Monomer Concentration from FT-NIR

Repeated recording of spectra of the same sample shows slightly different results. Errors may be introduced by apodization and phase correction, but the main source of error seems to arise from uncertainties of the baseline.

The error of the integrated absorbance is estimated to be less than $\pm 2 \%$, but rises for very low concentrations.

\section{NMR}

As correct measuring conditions were checked and results from different data processing were compared, values derived by ${ }^{1} \mathrm{H}-\mathrm{NMR}$ are estimated to have at least two significant digits and those from ${ }^{13} \mathrm{C}-\mathrm{NMR}$ to have at least one significant digit.

$\mathrm{pH}$

The inaccuracy of the $\mathrm{pH}$ values is given by the manufacturer to be \pm 0.002 .

Statistical Error

The errors of parameters which are deduced by curve fitting are determined using statistical methods. They are mostly given together with the respective value in the text. 
Temperature

For experiments in cuvettes as well as for viscosity determination, an error of $\pm 0.5^{\circ} \mathrm{C}$ is estimated on the basis of manufacturer's information. The temperature in the $\mathrm{pH}$ measurements is precise to $\pm 0.1^{\circ} \mathrm{C}$. The temperature in density measurements is accurate within $\pm 0.1^{\circ} \mathrm{C}$. The temperature in the nitrogen flow to thermostat EPR tubes is precise to $\pm 0.1^{\circ} \mathrm{C}$.

Viscosity

The error given by manufacturer of the AMVn is: accuracy (trueness) $<0.7 \%$, precision $<0.35 \%$. The accuracy of the other viscosity measurements is slightly lower.

Weight

For preparing the reaction solutions and gravimetric analysis, an analytical balance (CPA 3245, Sartorius) with an accuracy of $\pm 0.1 \mathrm{mg}$ was used. 



\section{4 \\ Methacrylic Acid}

The polymerization of MAA (methacrylic acid, IUPAC: 2-methylpropenoic acid) is of industrial interest as the product is widely used, most often as comonomer for polymers applied, e.g., in hygiene and cosmetics. These polymers are produced by radical polymerization in aqueous phase. In addition to solution polymerization, emulsion polymerizations are performed in which carboxylic monomers such as methacrylic acid may act as in-situ stabilizer. Moreover, MAA is similar to AA, but the kinetics are simpler due to lack of backbiting. Knowledge about MAA kinetics may help to better understand AA kinetics. For modeling polymerization in aqueous solution, detailed kinetic and mechanistic knowledge is required.

The propagation rate coefficient is known from PLP-SEC analysis. ${ }^{[5,89,151]}$ SEC on pMAA may be performed after quantitative methylation of the carboxyl moieties yielding $\mathrm{PMMA}^{[57,90]}$ or directly on pMAA via aqueous-phase SEC. ${ }^{[91]}$ The $k_{\mathrm{p}}$ value of MAA varies enormously as a function of both monomer concentration and degree of ionization, ${ }^{[9,11,58,59,91,92]}$ e.g., decreases by about one order of magnitude in passing from dilute aqueous solution of non-ionized MAA to either bulk polymerization of non-ionized MAA or to fully ionized MAA in dilute solution. These effects are not independent of each other, i.e., the variation of $k_{\mathrm{p}}$ with monomer content is weaker with partially ionized MAA and may even be reversed with fully ionized MAA. ${ }^{[11]}$ It has been attempted to model solution and emulsion polymerization from $\alpha=0$ to $\alpha=1 .{ }^{[79]}$ PLP-SEC studies on samples, to which pMAA had been added, thus mimicking situations of different degrees of monomer conversion, establish that it is 
essentially the ratio of MAA-to-water concentrations which is responsible for the characteristic dependence of $k_{\mathrm{p}}$ in aqueous-solution. ${ }^{[9]}$

For non-ionized MAA, both the SP-PLP-NIR ${ }^{[121]}$ and the SP-PLP-EPR techniques ${ }^{[120]}$ have been applied in aqueous solution. The experimental $k_{\mathrm{p}}$ and $k_{\mathrm{t}}$ values turned out to be well suited for modeling aqueous-phase MAA polymerization kinetics and polymer molar mass distribution in the absence of efficient chain transfer. ${ }^{[90]}$

So far, modeling of MAA polymerization in aqueous solution has not been addressed at technically relevant CTA concentrations. This chaper describes polymerization kinetics and modeling of MAA with a special focus on chain transfer. Unlike to preceding investigations, ${ }^{[90]}$ the chain-length dependence of $k_{\mathrm{t}}$ has to be explicitly taken into account and the impact of CTA concentration on the variation of termination rate with degree of monomer conversion has to be accounted for as well.

Parts of this chapter have already been published:

Wittenberg, N. F. G.; Buback, M.; Stach, M.; Lacík, I. Macromol. Chem. Phys. 2012, 213, 2653-2658.

Wittenberg, N. F. G.; Buback, M.; Hutchinson, R. A. Macromol. React. Eng. 2013, 7, 267-276.

\subsection{Chain-Transfer to 2-Mercaptoethanol}

Modeling polymerization under the influence of chain-transfer requires knowledge of the corresponding rate coefficients. Therefore, the chain-transfer constant of 2 -mercaptoethanol for methacrylic acid polymerization in aqueous solution has been measured for varying MAA content from 0.05 to $0.3 \mathrm{~g} \mathrm{~g}^{-1}$ at $50{ }^{\circ} \mathrm{C}$. The analysis has been carried out via both the Mayo and the chain length distribution, CLD, method. XIV

Another question to be answered is, whether $k_{\text {tr }}$ in aqueous solution varies with MAA content as does $k_{\mathrm{p}}$ or stays constant as is the case with conventional

XIV CLD stands for chain length distribution, while CLDT stands for chain-length dependent termination. While a little confusing, these are the common abbreviations in literature. 
monomers in organic solvents. It turns out that $C_{\text {СТA }}$ is independent of monomer concentration. Thus, $k_{\text {tr, CTA }}$ exhibits the same strong decrease with monomer concentration as has been reported for $k_{\mathrm{p}}$.

The question was easier to answer with ME being the CTA, as the associated $C_{\mathrm{CTA}}$ is large and may be accurately measured. ${ }^{[28,152]}$ Non-ionized MAA was selected as monomer, because $k_{\mathrm{p}}$ is already known. ${ }^{[9,59]}$ Moreover, in contrast to acrylate-type monomers, where secondary chain-end radicals may undergo backbiting reactions to produce tertiary midchain radicals, in MAA polymerization only one type of propagating radical occurs. The simultaneous presence of these two types of radicals adds complexity to the kinetic analysis, ${ }^{[33,101,153]}$ as addressed in chapter 5 .

\subsubsection{Chain Transfer Constants deduced by the Mayo Method}

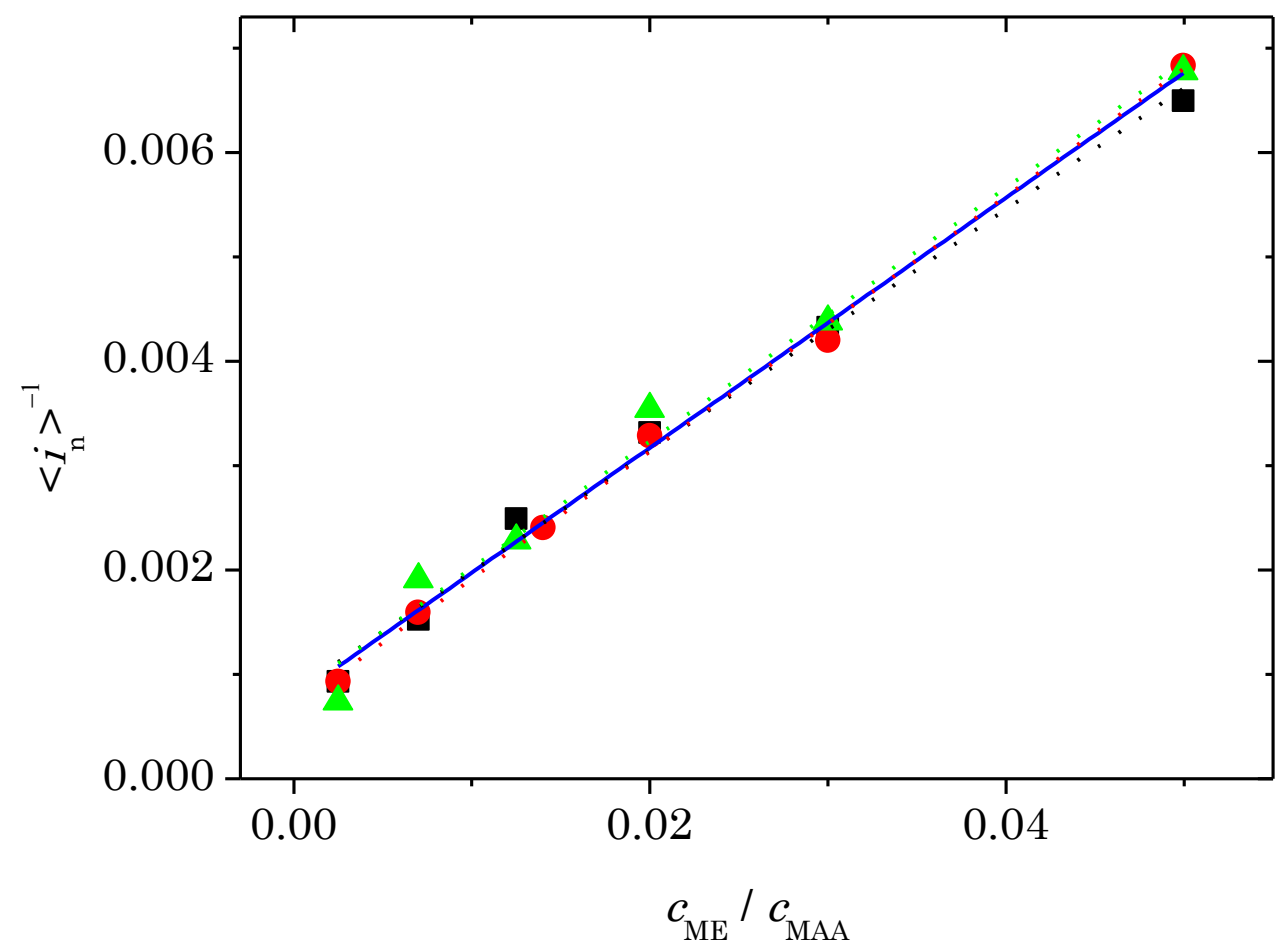

Figure 4-1 Mayo plot of product from polymerization of $0.30 \mathrm{~g} \mathrm{~g}^{-1} \mathrm{MAA}$ at $50^{\circ} \mathrm{C}$ in aqueous solution at different levels of photoinitiator (D1173): $1.31 \mathrm{mmol} \mathrm{L}^{-1}$ (red), $6.56 \mathrm{mmol} \mathrm{L}^{-1}$ (black), and $32.8 \mathrm{mmol} \mathrm{L}^{-1}$ (green). The corresponding best fits to a straight line are given as dotted lines and the concatenate fit as blue solid line. Due to dominance of chain transfer as chain stopping event, the individual slopes are almost identical. 
The Mayo method requires polymerizations to be dominated by chain transfer as stopping event. In order to demonstrate the fulfillment of the necessary conditions, polymerizations were carried out at different initiator concentrations, but otherwise identical conditions. The result of the Mayo procedure, eq. (2.12), yielding $C_{\text {CTA }}$ is shown in Figure 4-1 for the three different initiator concentrations. Five times more and five times less initiator content than used for the other experiments presented later resulted in almost the same slope of the Mayo plot.

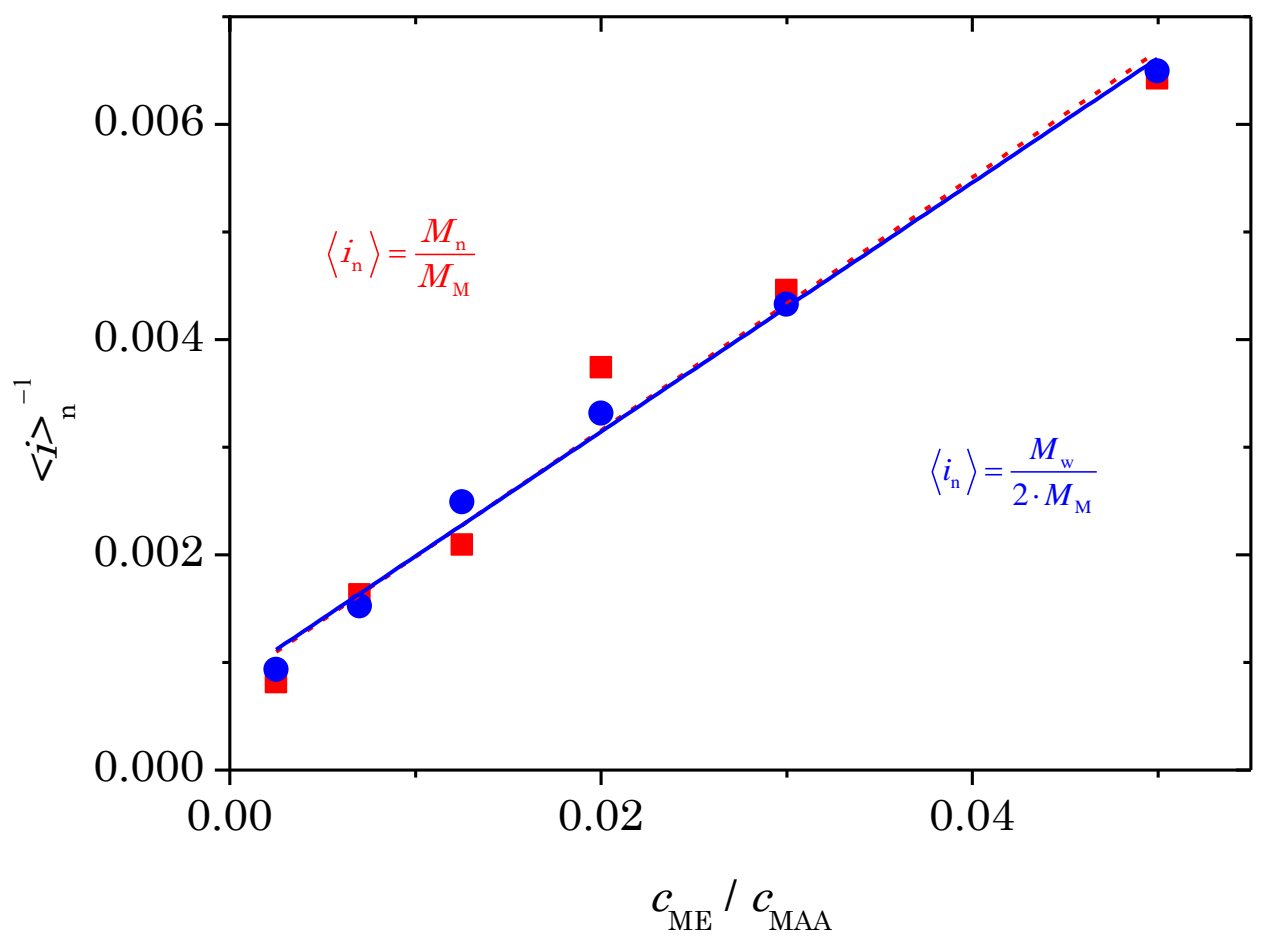

Figure 4-2 Inverse of the number average degree of polymerization for pMAA samples plotted as a function of the ratio of CTA (here ME) to monomer (here MAA) content. Polymer was produced by reaction to low degrees of monomer conversion for $0.30 \mathrm{~g} \mathrm{~g}^{-1}$ MAA in aqueous solution at $50{ }^{\circ} \mathrm{C}$ and $6.56 \mathrm{mmol} \mathrm{L}^{-1} \mathrm{D} 1173$ added as photoinitiator; data points are either deduced from $M_{\mathrm{n}}$ (red squares) or from $M_{\mathrm{w}}$ (blue circles). The dotted, red line is fitted to the data from $M_{\mathrm{n}}$ (eq. (4.1)) and the straight, blue line is fitted to the data from $M_{\mathrm{W}}$ (eq. (4.2)).

The number-average degree of polymerization, $\left\langle i_{\mathrm{n}}\right\rangle$, may be deduced either from $M_{\mathrm{n}}$ or $M_{\mathrm{w}}$. The quality of $M_{\mathrm{n}}$ determination may be affected by uncertainties of SEC analysis at lower molar masses. It thus appeared recommendable to determine $M_{\mathrm{n}}$ from $M_{\mathrm{w}}$, the value of which is accessible with better accuracy than $M_{\mathrm{n}}$. In case of 
transfer-controlled polymerization, as with ME being the CTA, dispersity $M_{\mathrm{w}} / M_{\mathrm{n}}$ is close to 2 , which allows for an estimate of $M_{\mathrm{n}}$ from $M_{\mathrm{w}} \cdot{ }^{[23,29]}$

Figure 4-2 shows a typical Mayo plot for MAA polymerization in aqueous phase with $\left\langle i_{\mathrm{n}}\right\rangle$ being deduced from SEC curves either via eq. (4.1) or via eq. (4.2).

$$
\left\langle i_{\mathrm{n}}\right\rangle=\frac{M_{\mathrm{n}}}{M_{\mathrm{M}}}
$$

$$
\left\langle i_{\mathrm{n}}\right\rangle=\frac{M_{\mathrm{w}}}{2 M_{\mathrm{M}}}
$$

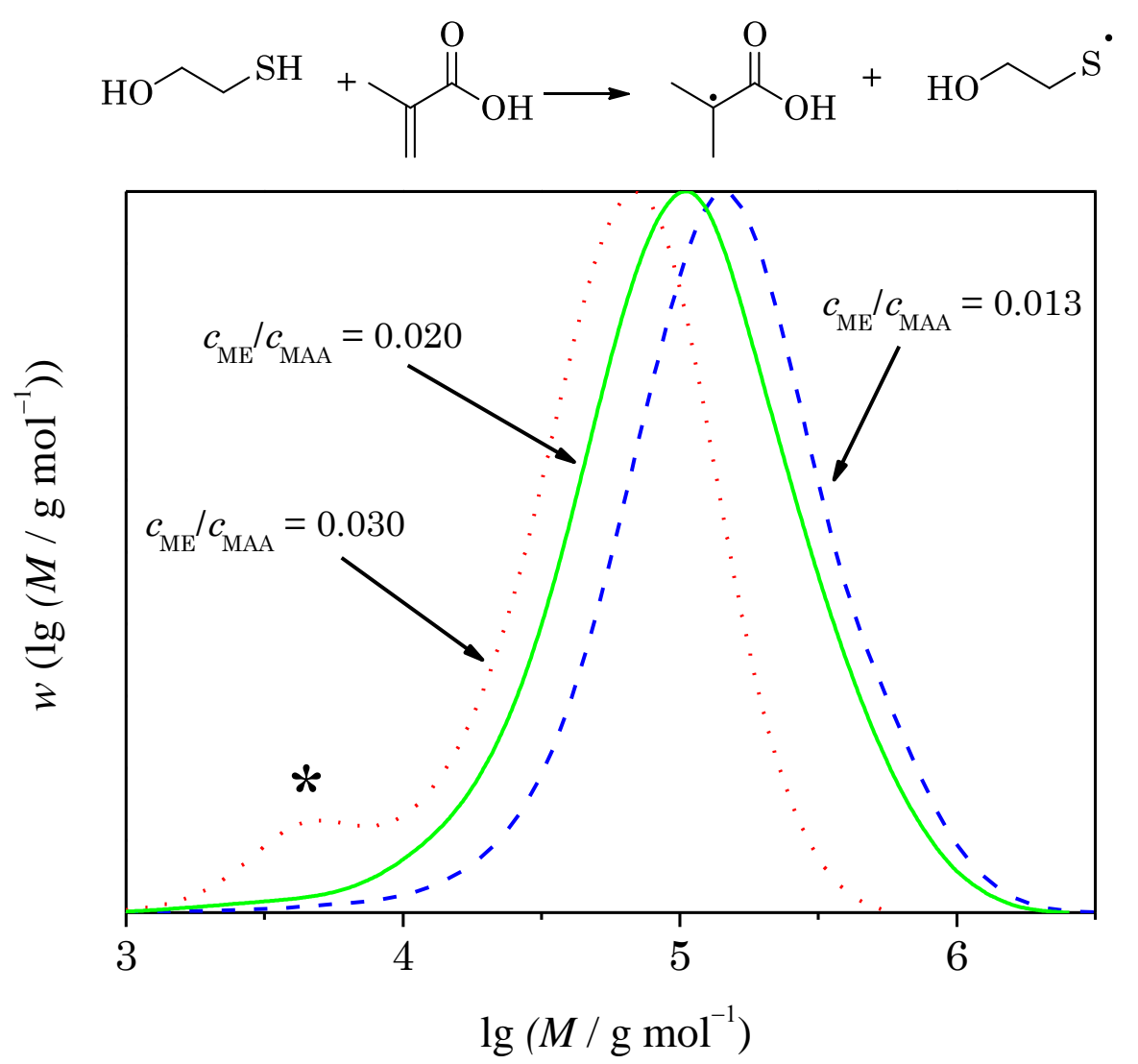

Figure 4-3 Shown are three typical MMDs of polymer produced by reaction to low degrees of monomer conversion for $0.15 \mathrm{~g} \mathrm{~g}^{-1} \mathrm{MAA}$ in aqueous solution at $50{ }^{\circ} \mathrm{C}, \mathrm{CME}_{\mathrm{ME}} / \mathrm{CMA}_{\mathrm{MA}}=0.013$ (dashed line) 0.020 (solid line) 0.030 (dotted line), and $6.56 \mathrm{mmol} \mathrm{L}^{-1} \mathrm{D} 1173$. The MMD represented by a dotted line has a second maximum marked by an asterisk. This occurred only for a few samples of polymerizations with high ME content and was probably caused by posterior initiation by ME. The thiol-ene reaction as a possible mechanism of initiation is shown at the top. Analysis was still possible (see text). 
The data from both $M_{\mathrm{n}}$ and $M_{\mathrm{w}}$ are in close agreement, which demonstrates the quality of SEC analysis and also suggests that $M_{\mathrm{w}} / M_{\mathrm{n}}$ is close to 2 , as is expected in case of chain transfer to ME being the dominant chain-terminating event. As the scatter on the data from $M_{\mathrm{w}}$ is smaller, only these numbers have been fitted by the straight line. From the slope to this line $C_{\mathrm{CTA}}=0.116 \pm 0.010$ is obtained. According to the same procedure, $C_{\mathrm{CTA}}$ has been determined for other MAA weight fractions and photoinitiator concentrations. The so-obtained numbers are listed in Table 4-1.

Using $M_{\mathrm{w}}$ for analysis has an additional advantage. Among the Mayo data a few results of polymerizations with high $\mathrm{ME}$ concentration are included where the MMDs of the pMAA samples were bimodal probably from subsequent initiation by ME (see Figure 4-3). As water evaporates faster than MAA and ME, the concentration of both latter species increase during drying and the thiol-ene reaction (Figure 4-3 top) may become important. For these MMDs, the data points obtained from $M_{\mathrm{n}}$ (eq. (4.1)) deviate from a straight line deduced by fitting the values of monomodal MMDs only. This does not hold true for fitting via eq. (4.2). The soobtained $\left\langle i_{\mathrm{n}}\right\rangle^{-1}$ values all closely fit to a straight line. This is shown in Figure 4-4.

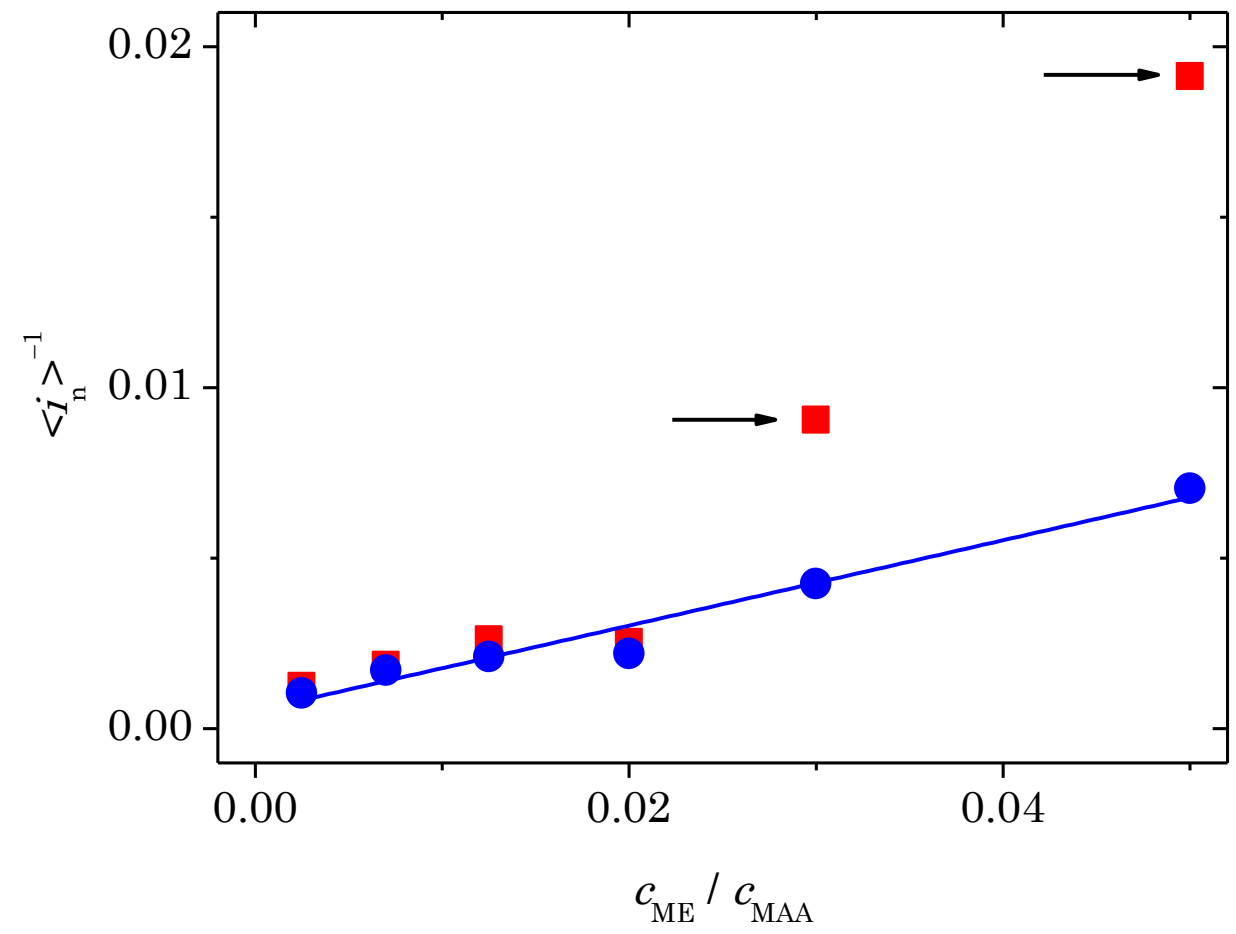

Figure 4-4 Mayo plot of polymer produced by reaction to low degrees of monomer conversion for $0.15 \mathrm{~g} \mathrm{~g}^{-1} \mathrm{MAA}$ in aqueous solution at $50^{\circ} \mathrm{C}$. Data points are either deduced from $M_{\mathrm{n}}$ (red squares) or from $M_{\mathrm{w}}$ (blue circles); the blue line is fitted to the data from $M_{\mathrm{W}}$ (eq. (4.2)). Data points corresponding to bimodal MMDs are marked by an arrow. 


\subsubsection{Chain Transfer Constants deduced by the CLD Method}

The CLD method estimates $C_{\mathrm{CTA}}$ according to eq. (2.14), which requires the slope of the $\ln \left(P_{M}\right)$ vs. $M$ plot, eq. (2.13), to be known. SEC yields the weight fraction of polymer of molar mass $M, w_{M}$. From $w_{M}$ the quantity $P_{M}$ is obtained via eq. (4.3). ${ }^{[154]}$

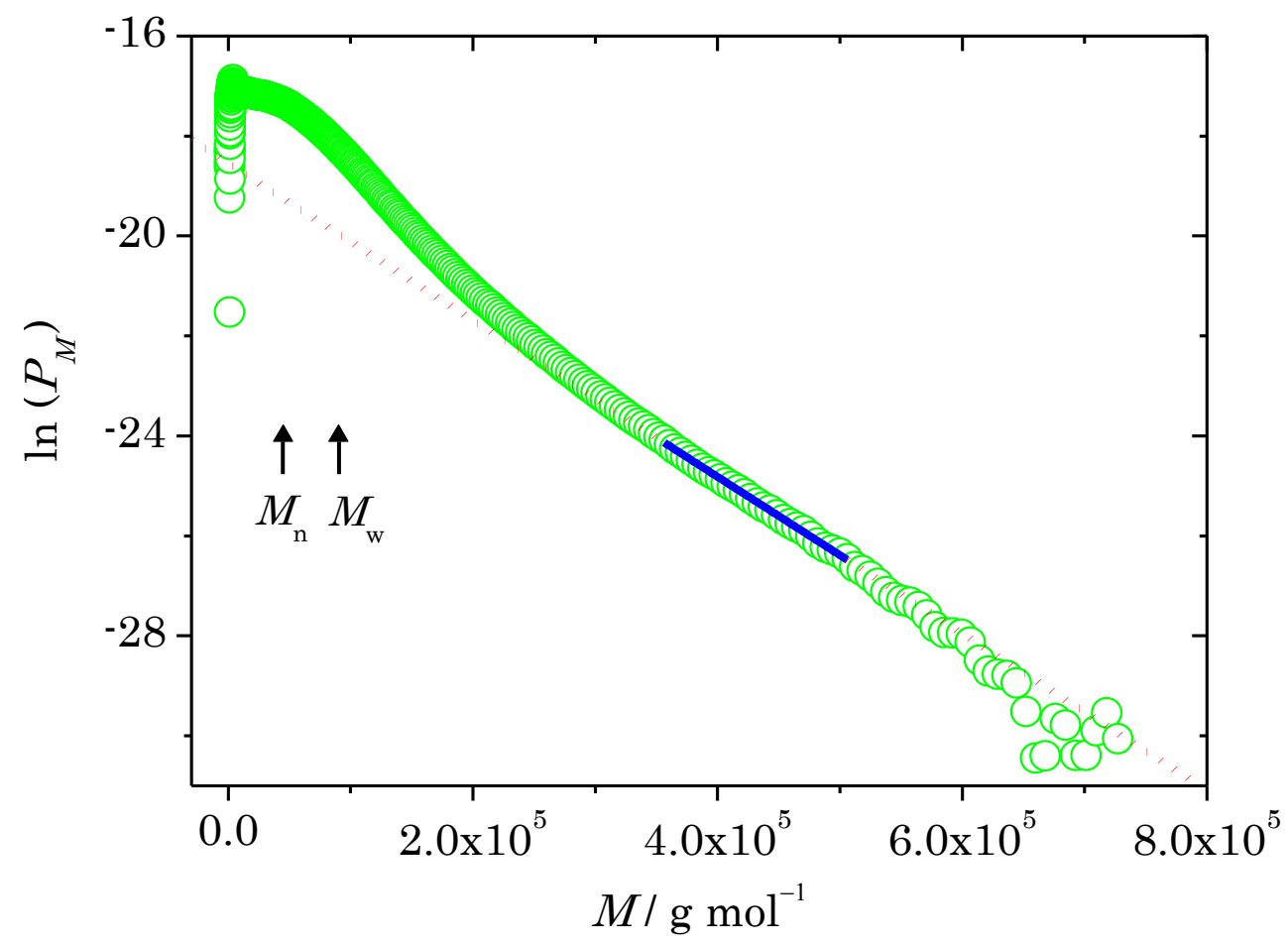

Figure 4-5 CLD plot for determination of the chain-transfer constant according to eq. (4.3) and (4.4); the data refers to polymer produced by reaction to low degrees of monomer conversion for $0.30 \mathrm{~g} \mathrm{~g}^{-1} \mathrm{MAA}$ in aqueous solution at $50{ }^{\circ} \mathrm{C}, \mathrm{c}_{\mathrm{ME}} / \mathrm{C}_{\mathrm{MAA}}=0.007$, and $6.56 \mathrm{mmol} \mathrm{L}{ }^{-1} \mathrm{D} 1173$ being added as the photoinitiator; the slope to the straight line is used for determination of the chain-transfer constant $C_{\text {СTA }}$ (see Figure 4-6). The region of fitting is indicated by solid line. $M_{\mathrm{n}}$ and $M_{\mathrm{w}}$ are given for orientation.

$$
P_{M} \propto \frac{W_{M}}{M^{2}}
$$




$$
\ln \left(\frac{W_{M}}{M^{2}}\right)=\lambda \cdot M
$$

The so-obtained $\ln \left(P_{M}\right)$ vs. $M$ correlation for pMAA from polymerization of $0.30 \mathrm{~g} \mathrm{~g}^{-1} \mathrm{MAA}$ in aqueous solution at $50^{\circ} \mathrm{C}$ is shown in Figure $4-5$. Straight-line behavior is not seen over the entire range of pMAA masses. According to eq. (2.13), the data for very high molar mass should be considered for deducing the slope $\lambda$, in particular in cases where termination becomes significant. ${ }^{[23,29]}$ The resulting dilemma is illustrated in Figure 4-5 by indicating the positions of $M_{\mathrm{n}}$ and $M_{\mathrm{w}}$, which are in the region of maximum signal intensity. In this region, molar mass is not sufficiently high as to allow for determination of the chain-transfer constant. On the other hand, the very high molar mass region, e.g., above $500000 \mathrm{~g} \mathrm{~mol}^{-1}$, is too poor in signal quality as to provide a reliable estimate of the slope $\lambda$. The CLD method has thus been applied in this example to molar masses around $400000 \mathrm{~g} \mathrm{~mol}^{-1}$ (Figure 4-5, solid line), to which the straight line is fitted. In general, the region used was a compromise between choosing the highest molar masses, and still having sufficient signal intensity. The region of maximum RI intensity is not suitable for CLD analysis in this example.

An example of a final plot yielding $C_{\mathrm{CTA}}$ from the CLD method is depicted in Figure 4-6. 


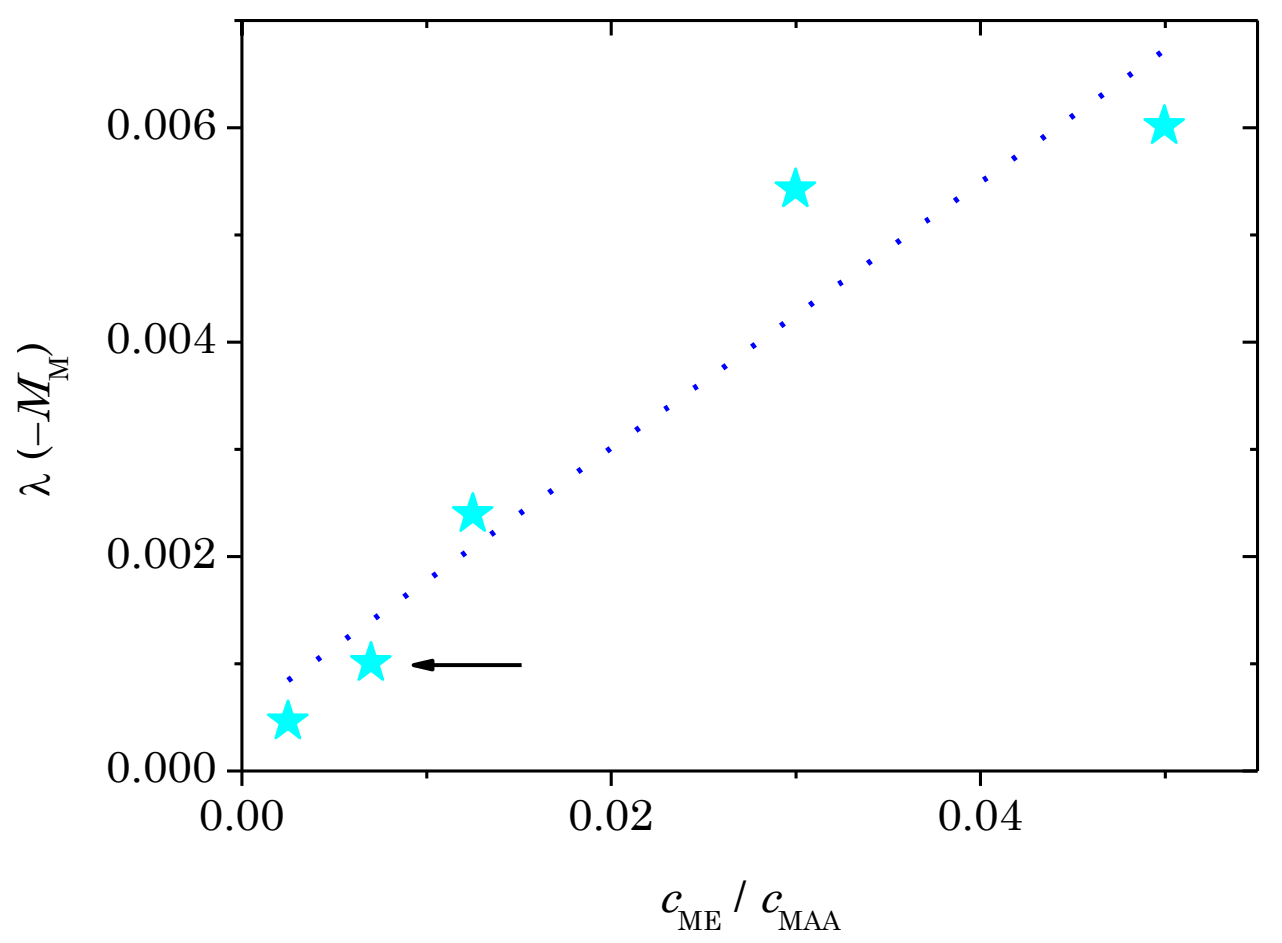

Figure 4-6 The asterisk symbols represent the results from CLD fitting according to eq. (4.4). The dotted line represents the slope taken from the Mayo plot in Figure 4-2 for the same experimental conditions: $0.30 \mathrm{~g} \mathrm{~g}^{-1} \mathrm{MAA}$ in aqueous solution at $50^{\circ} \mathrm{C}$, and $6.56 \mathrm{mmol} \mathrm{L}^{-1} \mathrm{D} 1173$. This line has almost identical slope as one from fitting the asterisk symbols to a straight line, which yields $C_{\text {CTA }}$ from CLD method (Table 4-1). The data point marked by the arrow is the one deduced from the slope to the fitted line in Figure 4-5.

\subsubsection{Comparison of Mayo and CLD methods}

Data obtained for $C_{\mathrm{CTA}}$ from the CLD method are represented by the asterisk symbols in Figure 4-6. The results from the Mayo procedure (see Figure 4-2) are given by the dotted line. The CLD results exhibit a significantly larger scatter than the Mayo ones. The $C_{\text {СтА }}$ values from the two procedures are compared in Table 4-1.

Within the larger scatter of the CLD data, good agreement between the Mayo and CLD method is found. The uncertainty is slightly larger at the lowest MAA content $\left(0.05 \mathrm{~g} \mathrm{~g}^{-1}\right)$. It should further be noted from Table 4-1 that the CLD method yields unusually high $C_{\text {СTA }}$ at the largest photoinitiator concentration, which is most likely due to the occurrence of smaller radicals and thus to a larger impact of termination 
affecting CLD analysis. With the exception of this particular $C_{\text {СTA }}$ value, the data in Table 4-1 indicate that $C_{\text {СTA }}$ at $50{ }^{\circ} \mathrm{C}$ is more or less independent of both MAA and photoinitiator concentration. The bottom entry in Table 4-1, lists the result from an experiment at $90{ }^{\circ} \mathrm{C}$. Comparison with the associated experiment at $50{ }^{\circ} \mathrm{C}$ tells that the Mayo and CLD methods yield opposite trends for the temperature effect on $C_{\mathrm{CTA}}$, which may be understood as an indication of $C_{\mathrm{CTA}}$ not being dependent on temperature to a significant extent, as expected (see subchapter 2.3.1). The Mayo method appears to provide simple but accurate access to the transfer constant in case of high CTA activity with polymerization in aqueous solution.

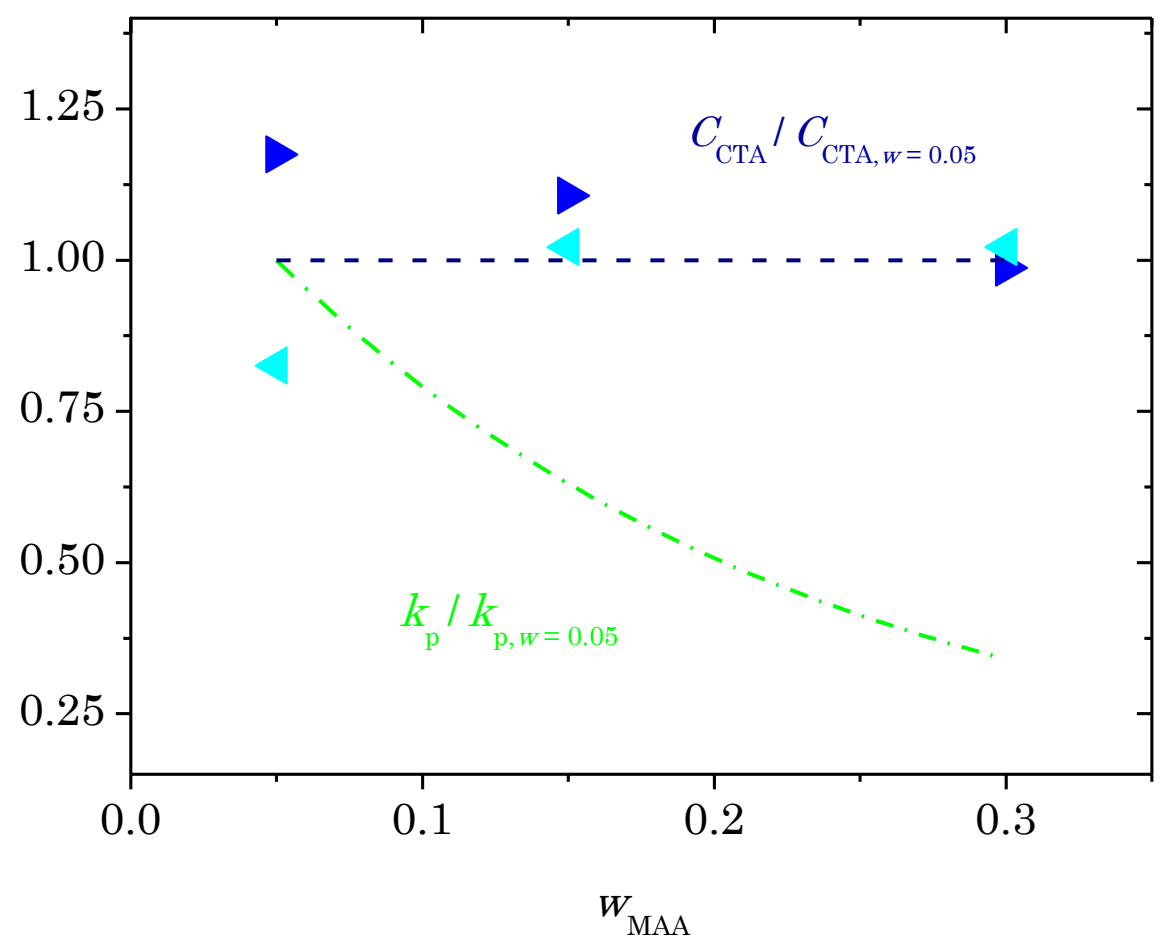

Figure 4-7 Relative $C_{\mathrm{CTA}}$ and $k_{\mathrm{p}}$ (with respect to the associated value for wMAA $=0.05$ ). The $C_{\mathrm{CTA}} / C_{\mathrm{CTA}}($ WMAA $=0.05)$ data refers to $\mathrm{MAA}$ polymerization at $50^{\circ} \mathrm{C}$, blue triangles pointing right: Mayo method, aqua triangles pointing left: CLD method. The data points scatter more for lower concentrations. The $k_{\mathrm{p}}$ data is taken from ref. ${ }^{[59,121]}$

The essential result of the present study is illustrated in Figure 4-7, in which both $C_{\text {CTA }}$ and $k_{\mathrm{p}}$ are plotted relative to the respective values at $W_{M A A}=0.95 \mathrm{~g} \mathrm{~g}^{-1}$. Whereas $C_{\mathrm{CTA}}$ is independent of monomer content, $k_{\mathrm{p}}$ exhibits the well-known strong decrease upon enhancing MAA concentration. This observation says that the genuine kinetic effect associated with hindrance to rotational motion in the 
transition state structure by the molecular environment is primarily due to characteristics of the radical chain-end and thus is more or less identical for propagation and for transfer to the CTA. The important consequence of this finding is that the known enormous variation of $k_{\mathrm{p}}$ with monomer concentration, and thus with conversion, also applies to chain transfer, which may easily be taken into account by adopting constant $C_{\text {CTA }}$ for a wide range of monomer concentration. This finding probably holds for other transfer reactions, e.g., transfer to monomer, as well. It was found for modeling MAA polymerization in aqueous solution that transfer to monomer had to be included and both the $k_{\mathrm{tr}} / k_{\mathrm{p}}=$ constant and the $k_{\text {tr }}=$ constant approach were tested. [90] The first approach gave a slightly better representation of the experimental MMDs, although, within experimental accuracy it was not possible to decide whether the rate coefficient of transfer is affected in the same way by the monomer-to-water ratio as is $k_{\mathrm{p}}$. The findings of the present work indicates that the $k_{\mathrm{tr}} / k_{\mathrm{p}}=$ constant approach is correct.

Table 4-1 Chain-transfer constants, $C$ CTA, of ME in MAA polymerizations in aqueous solution at different MAA concentrations, CMAA, and initiator concentrations, CD1173, at 50 and $90^{\circ} \mathrm{C}$ deduced via the Mayo and the CLD method. The uncertainties are estimated as twice the statistical error of each underlying experimental series. The weighted mean value were calculated.

$$
C_{\text {СтА }} \text { (Mayo) } \quad C_{\text {СТА }} \text { (CLD) }
$$

variation of $c_{\mathrm{MAA}} / \mathrm{mol} \mathrm{L}^{-1}$

$\left(50{ }^{\circ} \mathrm{C}, c_{\mathrm{D} 1173} / \mathrm{mmol} \mathrm{L}^{-1}=6.56\right)$
0.600 (5 wt.\%)
$0.138 \pm 0.015$
$0.10 \pm 0.03$
$1.80(15$ wt.\%)
$0.125 \pm 0.024$
$0.12 \pm 0.04$
3.60 (30 wt.\%)
$0.116 \pm 0.010$
$0.12 \pm 0.05$
mean value
$0.12 \pm 0.01$
$0.11 \pm 0.02$

variation of $c_{\mathrm{D} 1173} / \mathrm{mmol} \mathrm{L}^{-1}$

$\left(50{ }^{\circ} \mathrm{C}, c_{\mathrm{MAA}} / \mathrm{mol} \mathrm{L}^{-1}=3.60\right)$
1.31
$0.122 \pm 0.006$
$0.13 \pm 0.75$
6.56
$0.116 \pm 0.010$
$0.12 \pm 0.05$
32.8
$0.121 \pm 0.014$
$0.19 \pm 0.02$
$0.12 \pm 0.01$
$0.18 \pm 0.02$

mean value

variation of temperature $/{ }^{\circ} \mathrm{C}$

$$
\begin{gathered}
\left(c_{\mathrm{MAA}} / \mathrm{mol} \mathrm{L}^{-1}=0.600,\right. \\
\left.c_{\mathrm{D} 1173} / \mathrm{mmol} \mathrm{L}^{-1}=6.56\right)
\end{gathered}
$$




\subsection{Model development for Non-ionized Methacrylic Acid}

Batch radical polymerization of non-ionized methacrylic acid, $0.1-0.3 \mathrm{~g} \mathrm{~g}^{-1}$ in aqueous solution, has been studied between 35 and $50{ }^{\circ} \mathrm{C}$ at ambient pressure with 2 -mercaptoethanol as the chain-transfer agent. Initial polymerization rate decreases with CTA concentration, which has been varied up to $n_{\mathrm{CTA}} / n_{\mathrm{M}}=0.24$. In order to illustrate the influence of CTA, three conversion vs. time profiles of polymerizations with different level of CTA content (but otherwise identical conditions) are depicted in Figure 4-8.

Kinetic modeling is presented, which includes chain-length-dependent termination and uses an empirical function to account for the dependence of termination rate on both monomer conversion and molar mass of the polymeric product. In conjunction with PREDICI ${ }^{\mathrm{TM}}$ simulation, these models afford for an adequate representation of the measured monomer conversion vs. time profiles and MMDs.

The kinetic models consider initiation, propagation, termination, transfer to monomer and transfer to a CTA. Using a highly efficient CTA, such as ME, reduces chain length to an extent that requires the dependence of $k_{\mathrm{t}}$ on radical chain length and on the molar mass (distribution) of the produced polymer to be taken into account.

The reaction scheme is presented in Table 4-2, where $I_{2}$ represents the initiator, which decays thermally with the rate coefficient $k_{\mathrm{d}}$ and exhibits a growth efficiency, $f$, of primary radicals $\mathrm{I}^{\bullet}$. Within a first addition step, a growing chain $\mathrm{P}$ of length unity is formed from $\mathrm{I}^{\bullet}$ and one monomer molecule $\mathrm{M}$. The radical chain grows by addition of monomer molecules with the rate coefficient $k_{\mathrm{p}}$. Termination of two radicals may occur via combination, with rate coefficient $k_{\text {tc }}$, producing a (dead) macromolecule $\mathrm{D}$, or via disproportionation, which proceeds with rate coefficient $k_{\mathrm{td}}$ by hydrogen transfer and results in the formation of two macromolecules. Chain growth also ceases upon transfer to monomer or to a CTA. The chain length of polymeric species is given by the indices $i, j$, or 1 . Radical species are marked by a dot, e.g., $\mathrm{P}_{i}^{\cdot}$. The values of the individual rate coefficients, are summarized in Table 4-3 and Table 4-4, respectively 


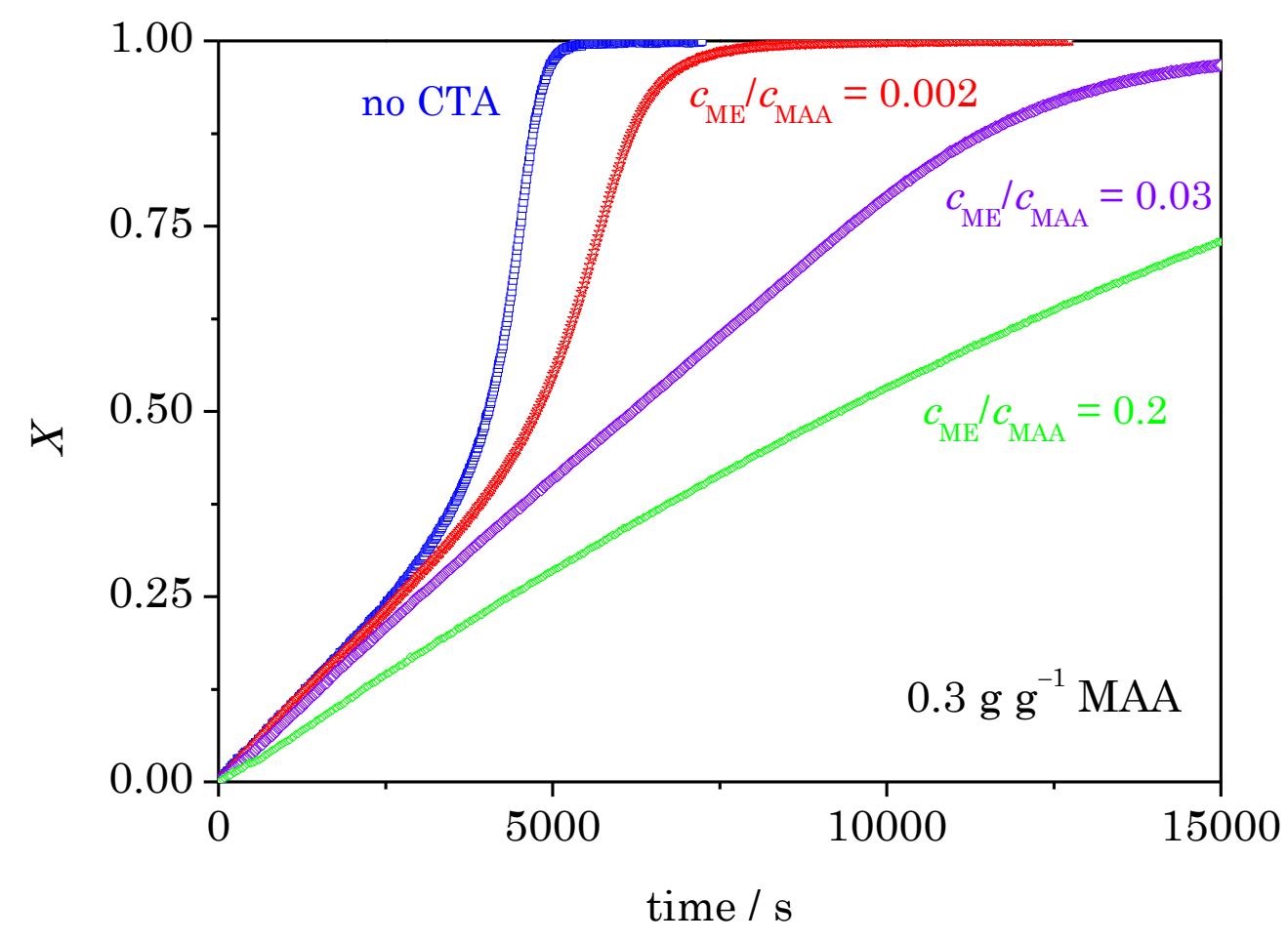

Figure 4-8 Polymerization of $0.30 \mathrm{~g} \mathrm{~g}^{-1} \mathrm{MAA}$ in aqueous solution with $0.001 \mathrm{~g} \mathrm{~g}^{-1} \mathrm{~V}-50$ as initiator at $50{ }^{\circ} \mathrm{C}$. The relative CTA content is given for each profile in the graph.

The azo-initiator V-50 was used, as peroxides and thiols may form a redox initiation system. ${ }^{[155]}$ Moreover, initiator decay should be independent of $\mathrm{pH}$, which is the case with V-50. ${ }^{[156]} \mathrm{V}-50$ decomposes according to a first-order rate law with a half-life of about 10 hours at $50{ }^{\circ} \mathrm{C}$. ${ }^{[156]}$ This slow initiator decay ensures almost constant initiator content during the reaction and avoids dead-end polymerization. Initiator efficiency was assumed to be $f=0.8$, which is a typical value that has successfully been used for modeling. ${ }^{[90]}$ The variation of density was considered in the simulation by assuming ideal mixing. The temperature dependence of the density of monomer and solvent was fitted by a third-order and a second-order polynomial, respectively. ${ }^{[157]}$

It was checked that the thiol-ene reaction does not play a significant role for the systems under investigation. 
Table 4-2 Individual reaction steps used for modeling radical polymerization of non-ionized methacrylic acid in aqueous solution.

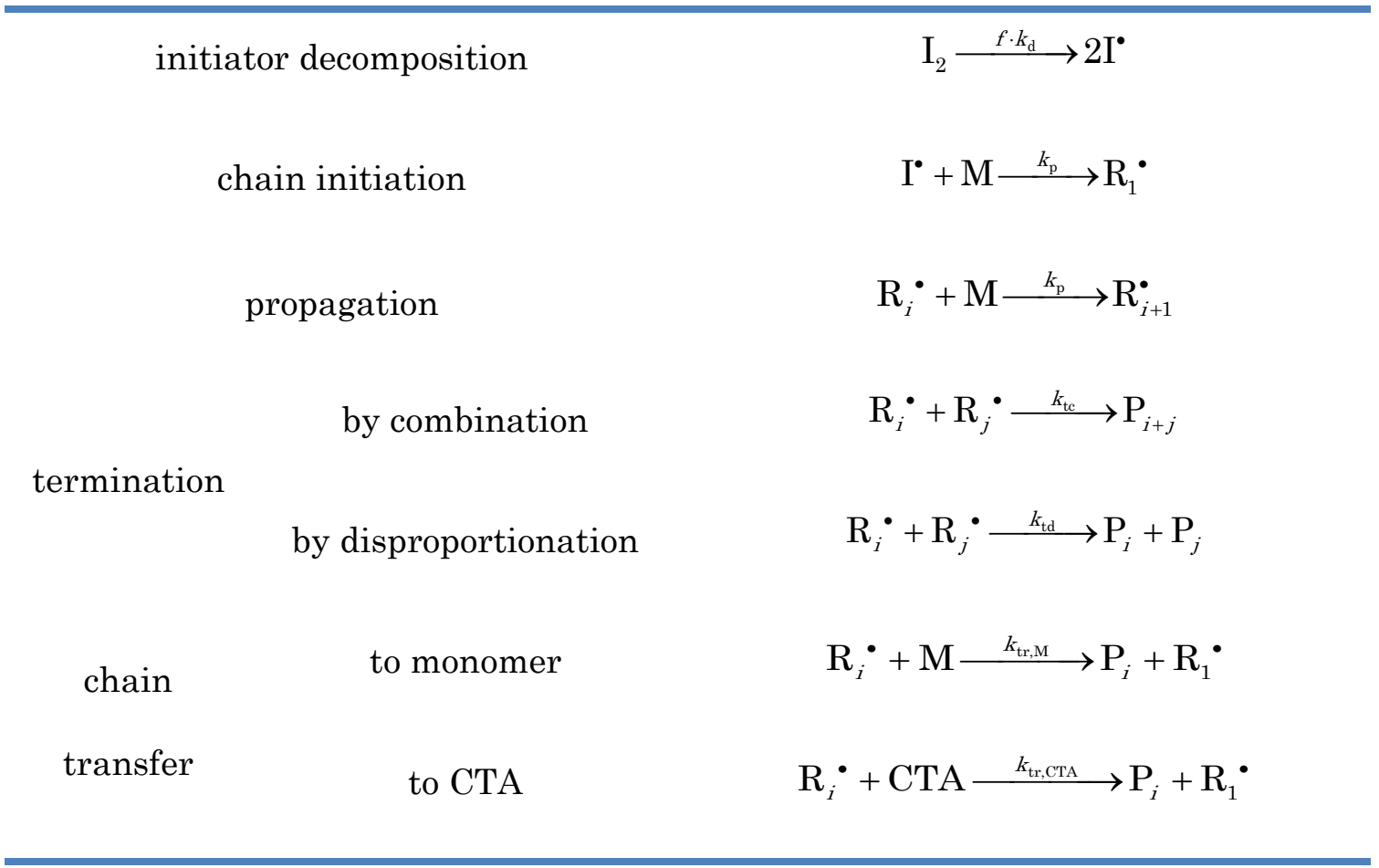

The variation of $k_{\mathrm{p}}$ as a function of initial MAA weight fraction, $W_{\text {MAA }}^{0}$, of degree of monomer conversion, $X$, and of temperature, $T$, is known from literature; $k_{\mathrm{p}, \max }$ in Table 4-3 refers to propagation in highly diluted aqueous solution. ${ }^{[121]}$ There are indications of $k_{\mathrm{p}}$ being chain-length dependent up to $i=10 .{ }^{[118]}$ This dependence needs to be considered under conditions of extreme chain transfer, as with catalytic chain-transfer agents. ${ }^{[49]}$ For the present system no need for incorporation of this effect was found.

$C_{\mathrm{CTA}}$ for $\mathrm{ME}$ is 0.122 , with this number being insensitive towards MAA-to-water content and temperature (4.1). Transfer to monomer is known from literature ${ }^{[90]}$ and as $C_{\text {CTA }}$ was found to be constant, the same was assumed for $C_{\mathrm{M}}$.

$\mathrm{xx}$ The fraction of termination by disproportionation, $\kappa$, was assumed to be 0.8 , which is a typical value for methacrylates. ${ }^{[90]}$ The composite model is used for representation of chain-length-dependent termination. Information about $k_{\mathrm{t}}$ as a function of MAA concentration and of radical chain length is available from SPPLP-EPR experiments. ${ }^{[120]}$ To avoid the high computational effort associated with considering individual reactions between two radicals of chain lengths $i$ and $j$, respectively, termination is scaled via the number-average radical size. The chainlength dependence of $k_{\mathrm{t}}$ has been implemented into PREDICI ${ }^{\mathrm{TM}}$ via the composite 
model with the relevant chain length being given by the number-average size. This procedure is associated with only modest additional computational effort.

This procedure is based on the fact that $k_{\mathrm{t}}$ averaged over all chain lengths, $\left\langle k_{\mathrm{t}}\right\rangle$, has been shown to be equal to the $k_{\mathrm{t}}$ of the average chain length of that distribution, $\left\langle i_{n}\right\rangle$, times a correction factor, $\zeta_{d}$, which is usually close to unity as given in eq. (4.5). ${ }^{[107,158-161]}$ The correction factor results from the fact that shorter chains contribute more to overall termination. Eq. (4.5) is valid in case of termination being dominant.

$$
\zeta_{\mathrm{d}}=\left[\Gamma\left(\frac{2}{2-\alpha_{1}}\right)\right]^{\alpha_{1}-2} \cdot\left(\frac{2}{2-\alpha_{1}} \cdot \frac{2}{1+\kappa}\right)^{\alpha_{1}}
$$

In case of transfer being dominant eq. (4.5) transforms into eq. (4.6) yielding almost the same result. ${ }^{[161]}$

$$
\begin{gathered}
\zeta_{\mathrm{d}}=\left[\Gamma\left(1-\alpha_{1}\right)\right]^{2} \\
\left\langle k_{\mathrm{t}}\right\rangle=k_{\mathrm{t}}^{0} \cdot\left\langle i_{n}\right\rangle^{-\alpha_{1}} \cdot \zeta_{\mathrm{d}}
\end{gathered}
$$

Beyond chain-length dependence, the variation of $k_{\mathrm{t}}$ with monomer conversion has to be incorporated. Such information is available from SP-PLP-NIR measurements. Within the present study, the effects of chain length and conversion on termination rate are both taken into account to allow for simulation of MAA polymerization in aqueous solution within a wide range of CTA concentrations and up to complete degrees of monomer conversion. 


\subsubsection{Modeling Polymerization at Medium initial Monomer Content}

The entries in Table 4-3 demonstrate that a large body of kinetic information on MAA radical polymerization in aqueous MAA solution is available from independent experiments. It is essentially termination kinetics at different levels of $\mathrm{ME}$ concentration, which remains to be determined via simulation of the experimental monomer conversion vs. polymerization time data. Under conditions of highly efficient chain transfer, e.g., with ME, the distributions of radical size and of polymer molar mass are controlled by propagation and transfer rates.

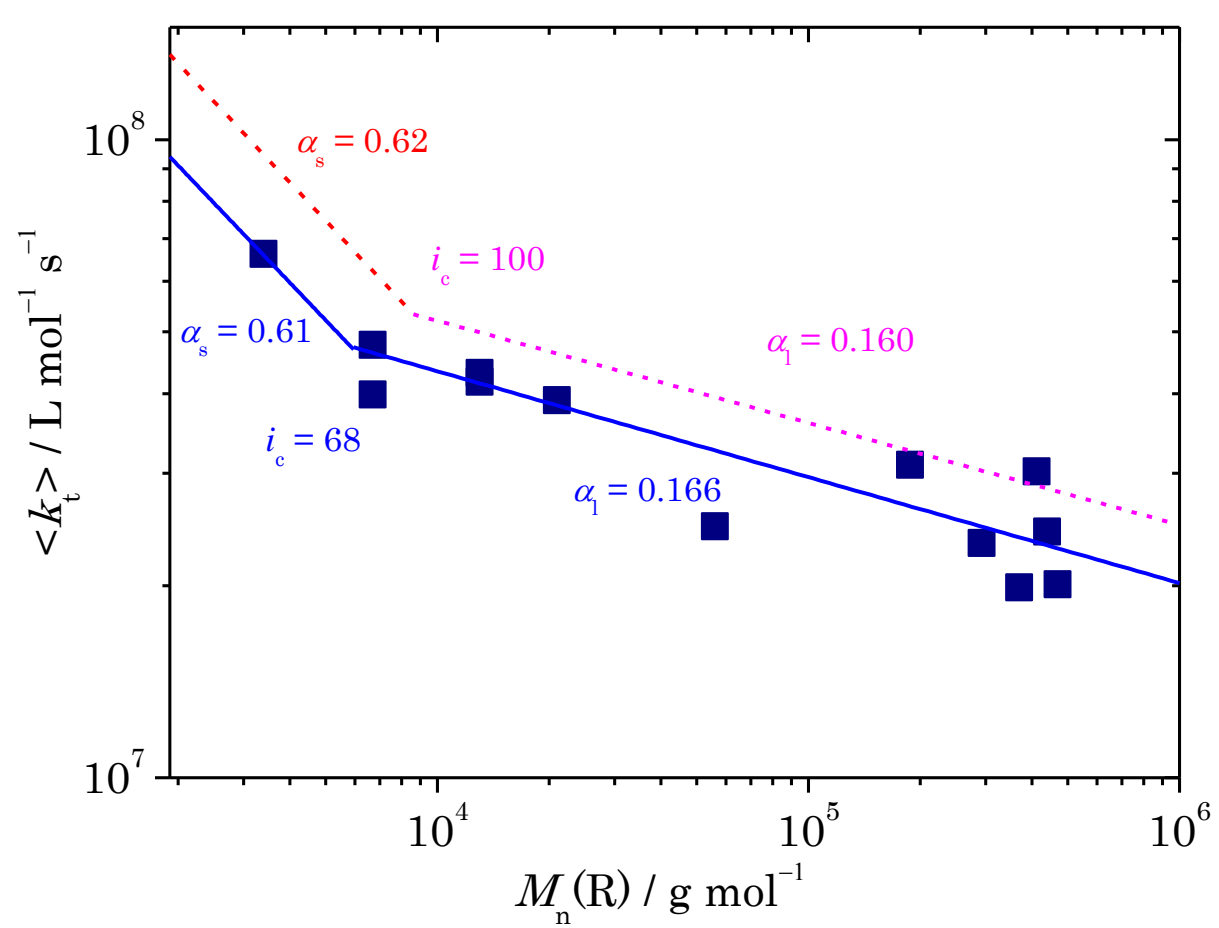

Figure 4-9 Dark blue squares: Initial $k$ values deduced as mean values from the conversion range $0<X<0.025$ of MAA polymerizations $\left(0.30 \mathrm{~g} \mathrm{~g}^{-1}\right.$ in aqueous solution) at varying $\mathrm{cmE}_{\mathrm{ME}} \mathrm{c \textrm {M }}$ up to 0.2 . The abscissa values are simulated number-average values of growing polymer chains. Solid blue line: fitting according to the composite model; data points for $M_{\mathrm{n}}$ below $5852 \mathrm{~g} \mathrm{~mol}^{-1}$ were used for fitting the short-chain behavior, whereas data at higher average molar masses are subjected to fitting the long-chain behavior. Red dashed line: $k_{\mathrm{t}}^{i, i}$ and $\alpha_{\mathrm{s}}$ from SP-PLP-EPR work on MAA;[120] pink dashed line: extrapolation with $i_{\mathrm{c}}$ of MAA and $\alpha_{1}$ from theoretical considerations. ${ }^{[106,119]}$

To determine the effect of radical chain length on $k_{\mathrm{t}}$ without interference of contributions from polymer-induced viscosity changes, rates at different $\mathrm{ME}$ 
concentrations and low degrees of monomer conversion, i.e., below 2.5 percent were estimated under the assumption of $k_{\mathrm{t}}$ being independent of monomer conversion. The so-obtained $k_{\mathrm{t}}$ values are plotted as a function of the number-average size of growing polymer chains, with these latter values being deduced from simulation. A double-log plot of the resulting $k_{\mathrm{t}}$ vs. the number-average radical size is shown in Figure 4-9. CTA concentration has been widely varied which results in the broad range of radical sizes.

In addition to $\left\langle k_{\mathrm{t}}\right\rangle$ from simulation of measured MAA conversion vs. time traces (open square symbols), values of $k_{\mathrm{t}}^{i, i}$ deduced from SP-PLP-EPR experiments ${ }^{[120]}$ are represented by the dashed line in Figure 4-9. The EPR experiment is highly instationary, but refers to a narrow distribution of radical chain lengths. Termination occurs between two radicals of approximately the same size, which increases linearly with time $t$ after pulsing. The square symbols refer to termination under stationary conditions with the reacting radicals exhibiting a broad distribution of chain lengths. The number-average size of radicals, $M_{\mathrm{n}}$, is obtained as the mean value over a broad distribution of radical sizes, whereas the abscissa value for the SP-PLP-EPR data refers to a narrow distribution of radical chain lengths. In view of this fundamental difference, the data from the two experiments are remarkably close to each other in slope, thus in $\alpha_{\mathrm{s}}$, but also in absolute value. The close comparison also holds for $k_{\mathrm{t}}^{i, i}$ extrapolated to longer radical chain lengths, which values are illustrated by the pink dashed line in Figure 4-9. This line is constructed by adopting the cross-over chain length of methyl methacrylate (MMA) for MAA and taking the power-law exponent $\alpha_{1}$ from theory. ${ }^{[106,119]}$

From SP-PLP-EPR, the value of $\alpha_{\mathrm{s}}$ for MAA was determined to be $0.62 \pm 0.06,{ }^{[120]}$ which is close to $\alpha_{\mathrm{s}}=0.61$, the corresponding number from the present study. It should however be noted that the latter $\alpha_{\mathrm{s}}$ is based on only three data points. For MMA bulk polymerization, $\alpha_{\mathrm{s}}$ has been obtained from SP-PLP-EPR measurements to be $0.63^{[103]}$ and from RAFT-CLDT to be $0.65 .^{[119]}$ So far, no value of either $i_{\mathrm{c}}$ or $\alpha_{1}$ has been measured for MAA by the SP-PLP-EPR technique because of poor signal intensity. ${ }^{[120]}$ The values for MMA are close to $i_{\mathrm{c}}=100^{[107]}$ and $\alpha_{1}=0.16$. $^{[106]}$ The corresponding numbers deduced within the present study from the $\left\langle k_{t}\right\rangle$ vs. $P_{\mathrm{n}}$ correlation in Figure 1 are: $i_{\mathrm{c}}=68$ and $\alpha_{1}=0.17$.

In Figure 4-9, an offset is seen between the dashed and the solid line, which corresponds to $k_{\mathrm{t}}^{1,1}$ from stationary experiments being by a factor of 0.7 below the SP-PLP-EPR value. However, according to theory, ${ }^{[161]}$ this factor should be 1.27 (calculated from eq. (4.6), eq. (4.5) gives 1.12). Closer inspection tells that the two lines correspond to different systems, $0.1 \mathrm{~g} \mathrm{~g}^{-1} \mathrm{MAA}$ in $\mathrm{H}_{2} \mathrm{O}$ and $0.3 \mathrm{~g} \mathrm{~g}^{-1} \mathrm{MAA}$ in 
$\mathrm{D}_{2} \mathrm{O}$ both at $50{ }^{\circ} \mathrm{C}$. Viscosity measurements for these two solutions at ambient pressure resulted in the values 0.6681 and $1.2064 \mathrm{mPa} \mathrm{s}$, respectively. The associated ratio of viscosities is 0.554 . In conjunction with the theoretical factor of 1.27 the ratio of $k_{\mathrm{t}}^{1,1}$ values thus should be $0.554 \cdot 1.27=0.704$, which is in perfect agreement with the measured difference. The close comparison demonstrates the dominant effect of viscosity on $k_{\mathrm{t}}^{1,1}$.

The satisfactory agreement of the two types of $k_{\mathrm{t}}$ representations (Figure 4-9) provides support for applying the composite model to the correlation of the low conversion chain-length-averaged termination rate coefficient with average radical chain length, $\left\langle i_{n}\right\rangle$, according to the full line in Figure 1 . Below $M_{\mathrm{n}}=5852 \mathrm{~g} \mathrm{~mol}^{-1}$, which corresponds to $i_{\mathrm{c}}=68$, the expression for short-chain radicals, eq. (4.8), has been used, whereas eq. (4.9) has been applied for long-chain radicals. In what follows, $k_{\mathrm{t}}$ always refers to the chain length averaged rate coefficient; for reasons of convenience, chevrons are omitted.

$$
\begin{array}{ll}
k_{\mathrm{t}, \mathrm{CLD}}=k_{\mathrm{t}}^{1,1} \cdot\left\langle i_{\mathrm{n}}\right\rangle^{-\alpha_{\mathrm{s}}} & \left\langle i_{\mathrm{n}}\right\rangle \leq i_{\mathrm{c}} \\
k_{\mathrm{t}, \mathrm{CLD}}=k_{\mathrm{t}}^{1,1} \cdot i_{\mathrm{c}}^{-\alpha_{\mathrm{s}}+\alpha_{1}} \cdot\left\langle i_{\mathrm{n}}\right\rangle^{-\alpha_{1}}=k_{\mathrm{t}}^{0} \cdot\left\langle i_{\mathrm{n}}\right\rangle^{-\alpha_{1}} & \left\langle i_{\mathrm{n}}\right\rangle>i_{\mathrm{c}}
\end{array}
$$

After focusing on CLDT, the variation of termination rate with monomer conversion will now be addressed. At low degrees of monomer conversion, segmental diffusion (SD) operates, which is controlled by the viscosity of the monomer-solvent mixture. Over an initial range of monomer conversion, the associated coefficient $k_{\mathrm{t}, \mathrm{SD}}$ remains more or less constant which results in a plateau value of $k_{\mathrm{t}}$ up to moderate degrees of monomer conversion. At higher conversion, $k_{\mathrm{t}}$ starts to clearly decrease, as termination transitions to translational diffusion control with the associated rate coefficient $k_{\mathrm{t}, \mathrm{TD}}$ scaling with the inverse viscosity of the polymerizing medium. This type of bulk viscosity includes the impact of the concentration and of the type of polymer produced during the course of a particular polymerization. $k_{\mathrm{t}, \mathrm{TD}}$ is expressed in terms of $k_{\mathrm{t}, \mathrm{TD}}^{0}$, the hypothetical termination rate coefficient under translational diffusion control prior to polymerization, and of $\eta_{\mathrm{r}}$, the relative viscosity, $\eta_{\mathrm{r}}=\eta / \eta^{0}$ with $\eta^{0}$ referring to the viscosity of the initial solution prior to polymerization. Towards even higher conversion, center-of-mass diffusion of macroradicals 
essentially ceases and termination runs under reaction-diffusion (RD) control, which assumes two radical sites to approach each other by propagation of the chain ends in conjunction with mobility of chain segments. Termination under RD conditions thus scales with $k_{\mathrm{p}}$ via the reaction-diffusion constant, $C_{\mathrm{RD}}$, which is enhanced by chain flexibility. The $k_{\mathrm{t}, \mathrm{TD}}$ expression of the present study into solution polymerization uses monomer concentration, eq. (2.41). At very high conversion and thus high viscosity even propagation may run under diffusion control. (see subchapter 2.4.5)

The variation of relative viscosity has been described by an exponential relation containing one single parameter $C_{\eta}{ }^{.900]}$ Adopting this notation and assuming $k_{\mathrm{p}}$ not to run under diffusion control, turns eq. (2.43) into eq. (4.10), which has been successfully applied for modeling MAA polymerization in aqueous solution. ${ }^{[90]}$

$$
k_{\mathrm{t}}=\frac{1}{\frac{1}{k_{\mathrm{t}, \mathrm{SD}}}+\frac{\mathrm{e}^{X \cdot C_{\eta}}}{k_{\mathrm{t}, \mathrm{TD}}^{0}}}+C_{\mathrm{RD}}^{*} \cdot(1-X) \cdot k_{\mathrm{p}}
$$

For an initial MAA content of $0.6 \mathrm{~g} \mathrm{~g}^{-1}$, the plateau value of constant (but chainlength dependent) $k_{\mathrm{t}, \mathrm{SD}}$ holds up to about $X=0.1$. For an MAA content of $0.3 \mathrm{~g} \mathrm{~g}^{-1}$, the region of constant $k_{\mathrm{t}, \mathrm{SD}}$ is approximately twice as large and extends up to $X=0.2$ with these ranges, however, being affected by the size and the structure of produced pMAA. ${ }^{[121]}$

As no backbiting occurs during MAA polymerization, ideal polymerization kinetics, $\mathrm{XV}$ eq. (2.7), has been used for estimating $k_{\mathrm{t}}$ as a function of $X$ from experimental monomer conversion vs. time data of all experiments.

Figure 4-10 illustrates $k_{\mathrm{t}}$ data deduced via eq. (2.7) for two polymerizations in the absence of CTA. The different regions of diffusion control, i.e., by SD, TD, and RD are clearly seen. Also presented in Figure 4-10 are conversion vs. time profiles measured at two ME levels. The initial plateau region which is assigned to SD, increases with $\mathrm{ME}$ content and extends over the entire experimental conversion range at the highest $\mathrm{ME}$ concentration. At $c_{\mathrm{ME}} / c_{\mathrm{MAA}}=0.2$, the plateau value for termination under SD control is significantly above $k_{\mathrm{t}}$ at lower ME content. The $k_{\mathrm{t}}$ plateau value for MAA polymerization with $c_{\mathrm{ME}} / c_{\mathrm{MAA}}=0.002$ is close to the one for

$\mathrm{xv}$ Here, ideal refers to each single data point, i.e., overall constancy is not assumed. Moreover, CLD-T is not excluded. 
MAA polymerization without CTA. The conversion range of SD control is however larger than in the absence of ME. Moreover, the decline of $k_{\mathrm{t}}$ in the TD region is less pronounced. The data for chemically induced MAA polymerization with $c_{\mathrm{ME}} / c_{\mathrm{MAA}}=0.002$ is remarkably close to $k_{\mathrm{t}}$ deduced from SP-PLP-NIR experiments on $0.3 \mathrm{~g} \mathrm{~g}^{-1}$ MAA in aqueous solution without CTA. ${ }^{[121]}$ The reason behind this agreement probably is that the high radical concentration, which is instantaneously produced by the laser pulse, leads to similarly low molar masses as in chemically induced polymerizations with $c_{\mathrm{ME}} / c_{\mathrm{MAA}}=0.002$.

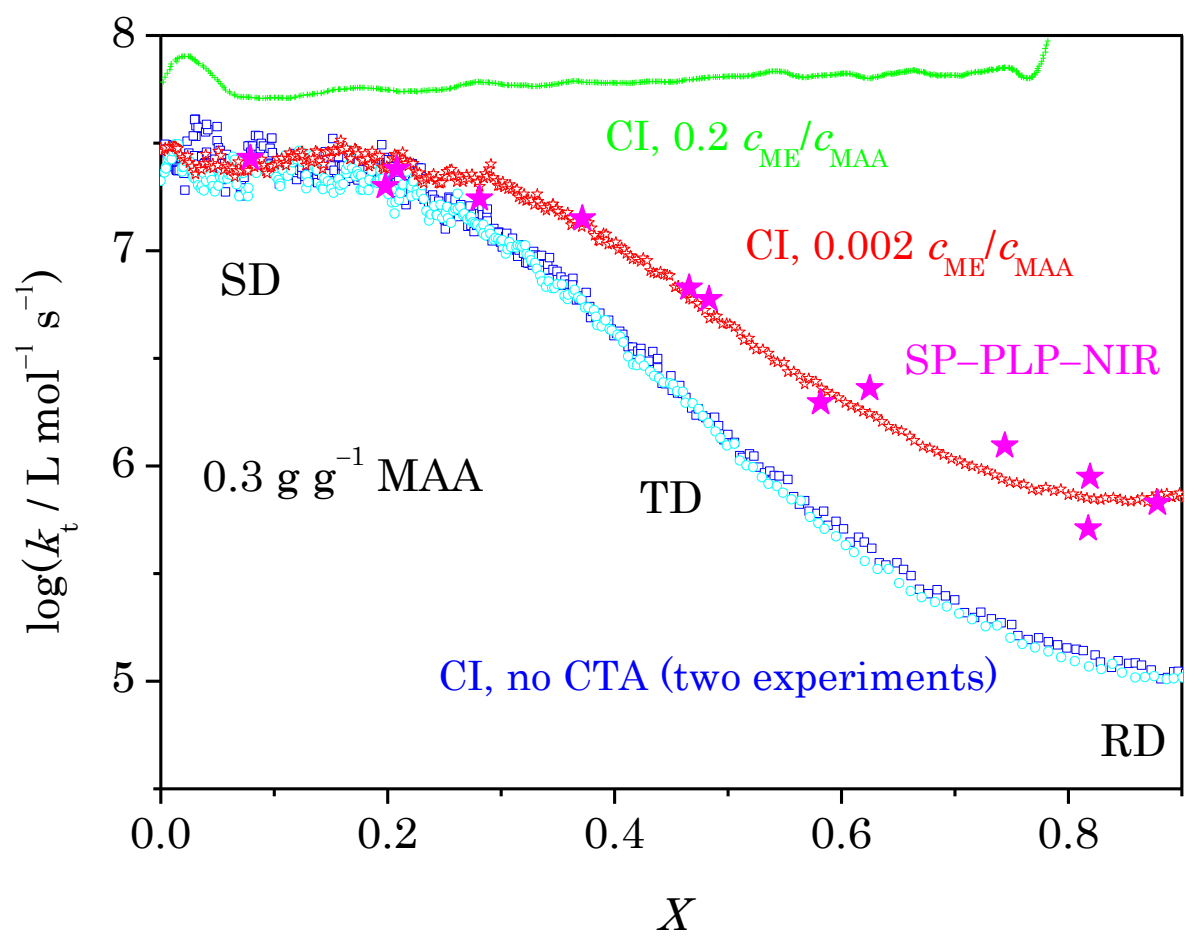

Figure 4-10 Dependence of the termination rate coefficient on monomer conversion as derived from ideal polymerization kinetics (eq. (2.7)) for four chemically initiated (CI) polymerizations $\left(0.30 \mathrm{~g} \mathrm{~g}^{-1}\right.$ in aqueous solution) at ambient pressure; data points were smoothed; the asterisk symbols are data from SP-PLP-NIR experiments $\left(\right.$ MMAA $\left.^{0}=0.30 \mathrm{~g} \mathrm{~g}^{-1}, 50{ }^{\circ} \mathrm{C}, 2000 \mathrm{bar}\right),{ }^{[121]}$ which were extrapolated to ambient pressure with eq. (2.25) via the activation volume of $12.4 \mathrm{~cm}^{3} \mathrm{~mol}^{-1}$. The ME concentrations are given in the Figure. The notations SD, TD, and, RD refer to control of termination by segmental, translational (centre-of-mass), and reaction diffusion, respectively.

Eq. (4.11) has been conceived as an expression for $k_{t}$, in which chain-length dependence and conversion dependence are merged together. Aspects of conversiondependent $\alpha_{\mathrm{s}}{ }^{[120]}$ and $\alpha_{1}{ }^{[108]}$ have already been discussed. Most studies into CLDT 
refer to low monomer conversion, whereas studies into the conversion dependence ignore CLDT. No clear indications for a potential dependence of $\alpha_{\mathrm{s}}$ and of $\alpha_{1}$ on monomer conversion were found. Hence, both power-law exponents are assumed to be insensitive towards monomer conversion. In eq. (4.11), the parameters $k_{\mathrm{t}, \mathrm{SD}}$ and $k_{\mathrm{t}, \mathrm{TD}}^{0}$ are replaced by $s d=k_{\mathrm{t}, \mathrm{CLD}} / k_{\mathrm{t}, \mathrm{SD}}$ and by $t d=k_{\mathrm{t}, \mathrm{CLD}} / k_{\mathrm{t}, \mathrm{TD}}^{0}$. The parameters $s d$ and $t d$ account for the relative contributions of segmental diffusion and translational diffusion:

$$
k_{\mathrm{t}}=k_{\mathrm{t}, \mathrm{CLD}} \cdot\left(s d+t d \cdot \mathrm{e}^{X \cdot C_{\mathrm{n}}}\right)^{-1}+C_{\mathrm{RD}} \cdot c_{\mathrm{M}} \cdot k_{\mathrm{p}}
$$

Transformation of eq. (4.11) into eq. (4.8) and eq. (4.9), respectively, requires: $s d+t d=1$ for $X=0$. Analysis of the individual monomer conversion vs. time profiles by fitting to eq. (4.11) via the Levenberg-Marquardt algorithm yields numbers for $s d$, $t d, C_{\eta}$, and $C_{\mathrm{RD}}$. The parameters $s d=0.96 \pm 0.01$ and $t d=0.04 \pm 0.01$ turned out to provide an adequate representation of $k_{\mathrm{t}}$ for the entire set $\left(0.3 \mathrm{~g} \mathrm{~g}^{-1}\right.$ MAA) of measured conversion vs. time traces. The conversion dependence of termination rate on ME concentration is exclusively assigned to $C_{\eta}$. The impact of ME content is thus contained in $k_{\mathrm{t}, \mathrm{CLD}}$ and in $C_{\eta}$. The parameter $C_{\mathrm{RD}}=20$ was deduced from polymerization rates measured in the absence of $\mathrm{ME}$. This value is in reasonable agreement with the one of MMA, for which $C_{\mathrm{RD}}^{*}=93.5$ was measured, which corresponds to $C_{\mathrm{RD}}=10.3 .{ }^{[130]}$ Assuming $k_{\mathrm{p}}$ to be independent of chain length results in $k_{\mathrm{t}, \mathrm{RD}}$ being also insensitive toward chain length.

Illustrated in Figure 4-11 are the individual contributions of the terms in eq. (4.11) to overall $k_{\mathrm{t}}$ for MAA polymerization $\left(0.3 \mathrm{~g} \mathrm{~g}^{-1}\right)$ in aqueous solution without CTA being present. $k_{\mathrm{t}, \mathrm{CLD}}$ remains essentially constant over the wide conversion range, in which average chain-length drops only by a factor of 2.6. Up to about $X=0.6$, the first term on the RHS eq. (4.11) dominates and RD plays no significant role. Above $0.74, k_{\mathrm{t}, \mathrm{RD}}$ exceeds the first term. 


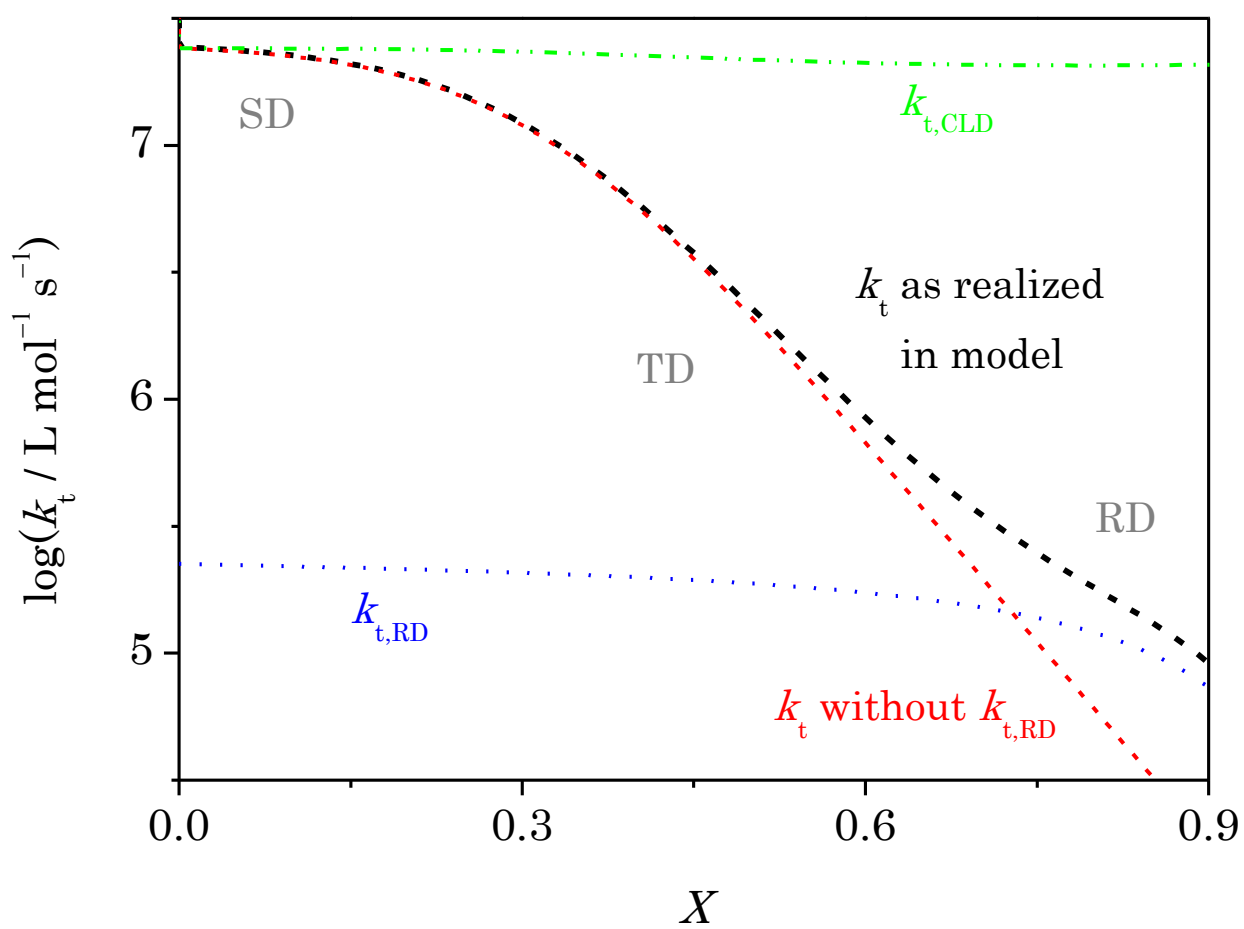

Figure 4-11 Contributions to overall $k_{\mathrm{t}}$ according to eq. (4.11) as a function of degree of monomer conversion. The notations $\mathrm{SD}, \mathrm{TD}$, and, $\mathrm{RD}$ refer to control of termination by segmental, translational (centre-of-mass), and reaction diffusion, respectively.

The parameter $C_{\eta}$ may be deduced by PREDICI ${ }^{\mathrm{TM}}$ fitting of the entire body of polymerization data for a wide range of molar masses, which may be achieved by variation of CTA content. In Figure 4-12 the so-obtained $C_{\eta}$ values are plotted as a function of the weight average molar mass at $X=0.5$, a value which is close to the mean molar mass taken over the entire conversion range. The entire set of experimental $C_{\eta}$ vs. $M_{w}$ data is fitted by eq. (4.12) which results in the parameter values $a=1.4$ and $b=0.15$. Interestingly, this value for $b$ is similar to the power-law exponent for self-diffusion of polymer in good solvents (see subchapter 2.4.4) and thus also similar to $\alpha_{1}$.

$$
C_{\eta}=a \cdot M_{\mathrm{w}}^{b}
$$


Combination of eq. (4.11) and eq. (4.12) replaces $C_{\eta}$ by $a$ and $b$. Thus, by using one additional parameter, an adequate representation of the measured conversion vs. time traces at widely different polymer molar masses is achieved.

The combined influence of $M_{\mathrm{w}}$ of dead polymer in solution and chain length of macroradicals (long-chain region) on $k_{\mathrm{t}}$ is depicted in Figure 4-13 top. The weight of the former is at $X=0.5$ much stronger. $M_{\mathrm{w}}$ and $X$ have the same influence on $k_{\mathrm{t}}$ shown in Figure 4-13 bottom.

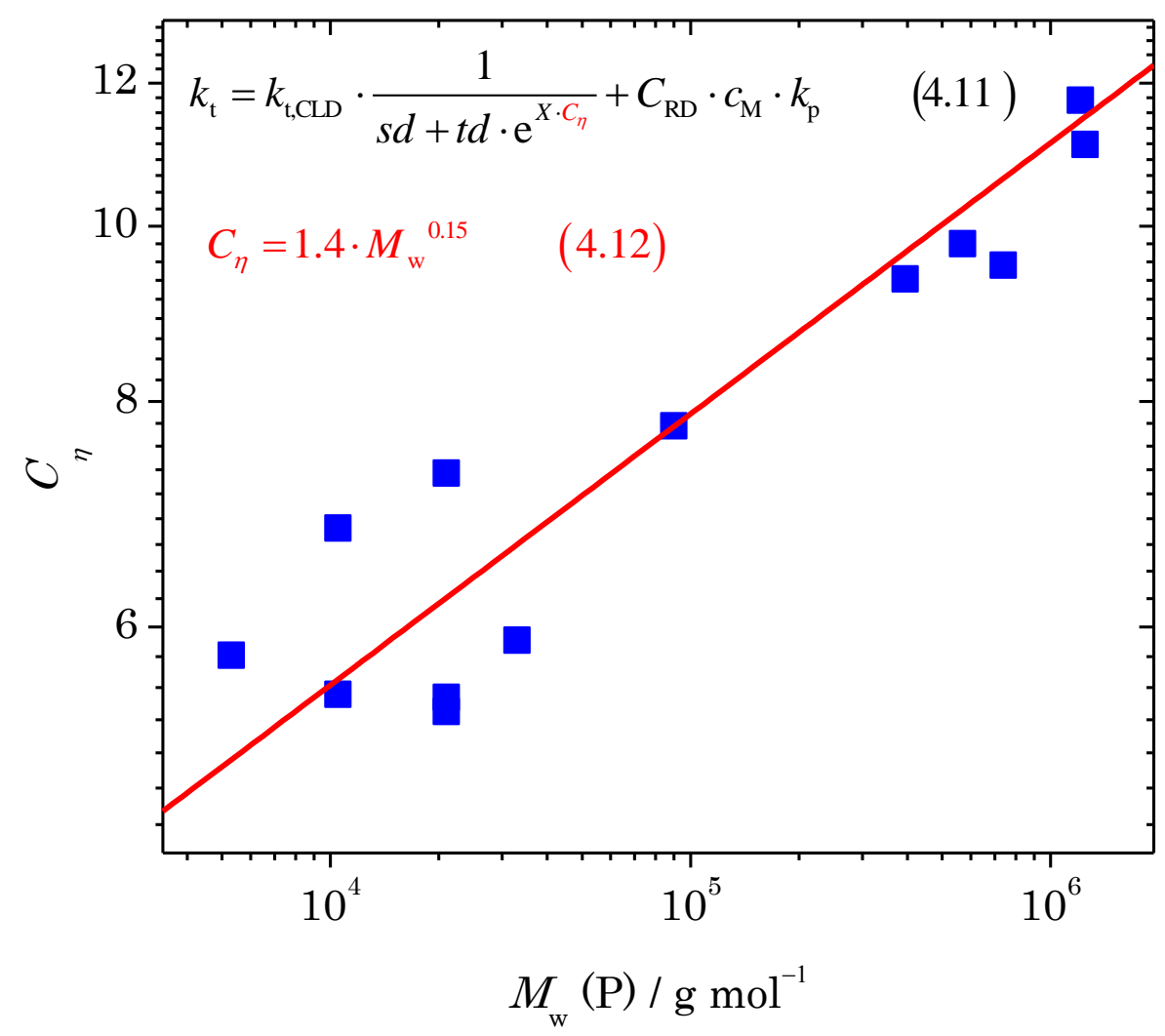

Figure 4-12 Correlation of $C_{\eta}$ with the weight-average molar mass of pMAA produced up to X=0.5 at different levels of chain-transfer agent ME. The symbols are deduced from PREDICI ${ }^{\mathrm{TM}}$ fitting of experimental conversion-time data. The obtained fit parameters refer to eq. (4.12), which extends eq. (4.11).

Illustrated in Figure 4-14 is the impact on $k_{\mathrm{t}}$ of $\mathrm{ME}$ concentrations up to $c_{\mathrm{ME}} / c_{\mathrm{MAA}}=0.2$ according to eq. (4.11) and eq. (4.12). The minor increase of $k_{\mathrm{t}}$ upon enhancing monomer conversion at the highest $\mathrm{ME}$ content results from a weak decrease of radical chain length towards lower MAA content, i.e., toward higher 
conversion. In addition to the logarithm of $k_{\mathrm{t}}$, the square root of $k_{\mathrm{t}}$ is plotted (in the lower part of Figure 4-14). The inverse of the latter quantity scales with both rate of polymerization and kinetic chain length.

The quality of the kinetic model associated with the rate coefficients summarized in Table 4-3 is illustrated by comparison of measured and simulated monomer conversion vs. time traces in Figure 4-15. The model turns out to adequately represent the polymerization kinetics of $0.3 \mathrm{~g} \mathrm{~g}^{-1} \mathrm{MAA}$ in aqueous solution at $50{ }^{\circ} \mathrm{C}$ for $\mathrm{ME}$ contents between zero and $c_{\mathrm{ME}} / c_{\mathrm{MAA}}=0.2$. For $0,0.05$ and $0.1 \mathrm{ME}$ content, repeat experiments are included. They demonstrate that the quality of simulation is close to experimental reproducibility. At high monomer conversion, the model slightly overestimates polymerization rate, which may be partly due to reduced initiator efficiency or to diffusion control of $k_{\mathrm{p}}$ at very high viscosity. These phenomena are not included in the model. 

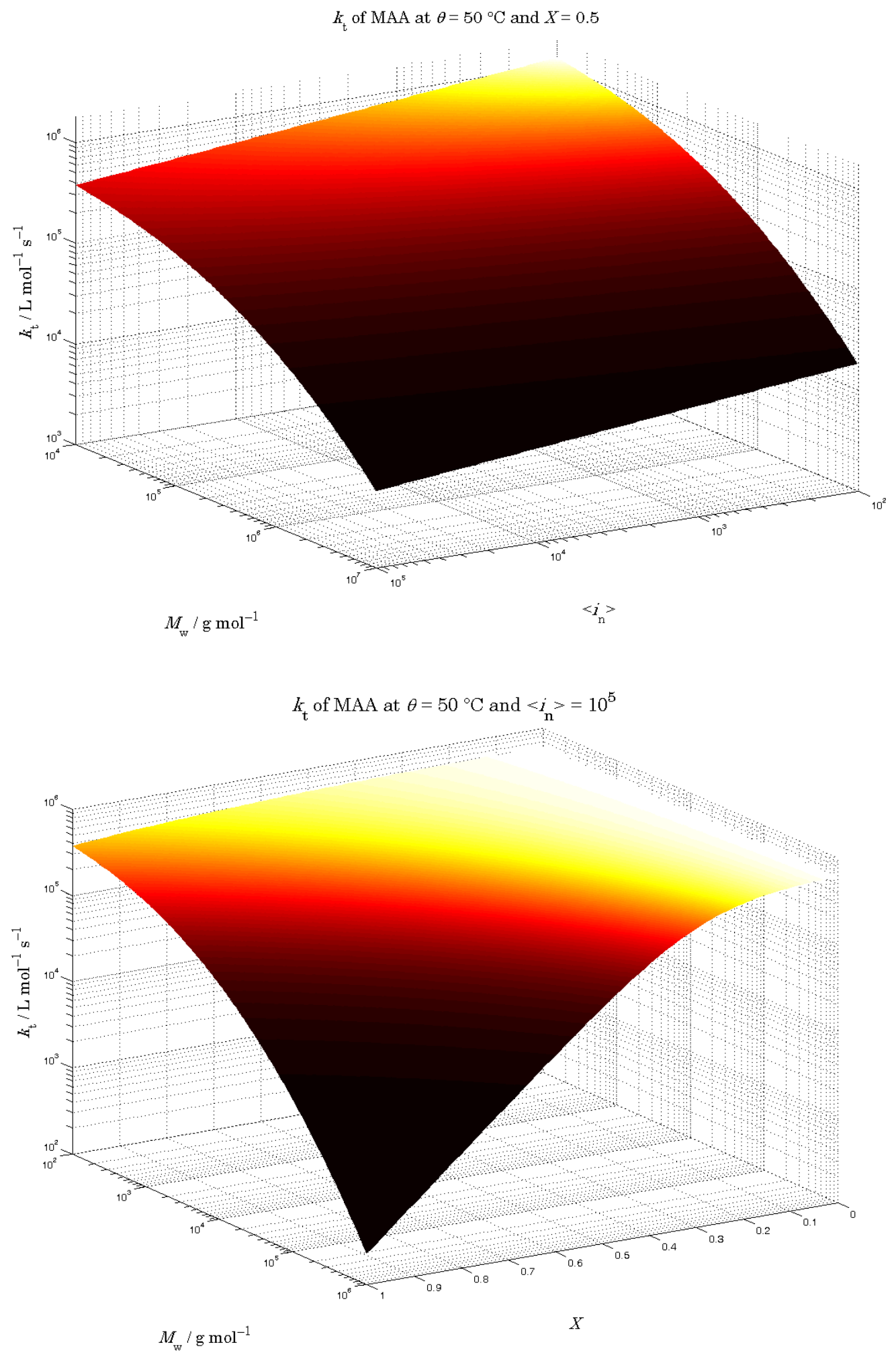

Figure 4-13 The dependence of $k_{\mathrm{t}}$ on molar mass of polymer in solution and chain length of macroradicals at $X=0.5$ is plotted at the top. The dependence of $k_{\mathrm{t}}$ on molar mass of polymer in solution and degree of monomer conversion at $\left\langle i_{n}\right\rangle=10^{5}$ is plotted at the bottom. 


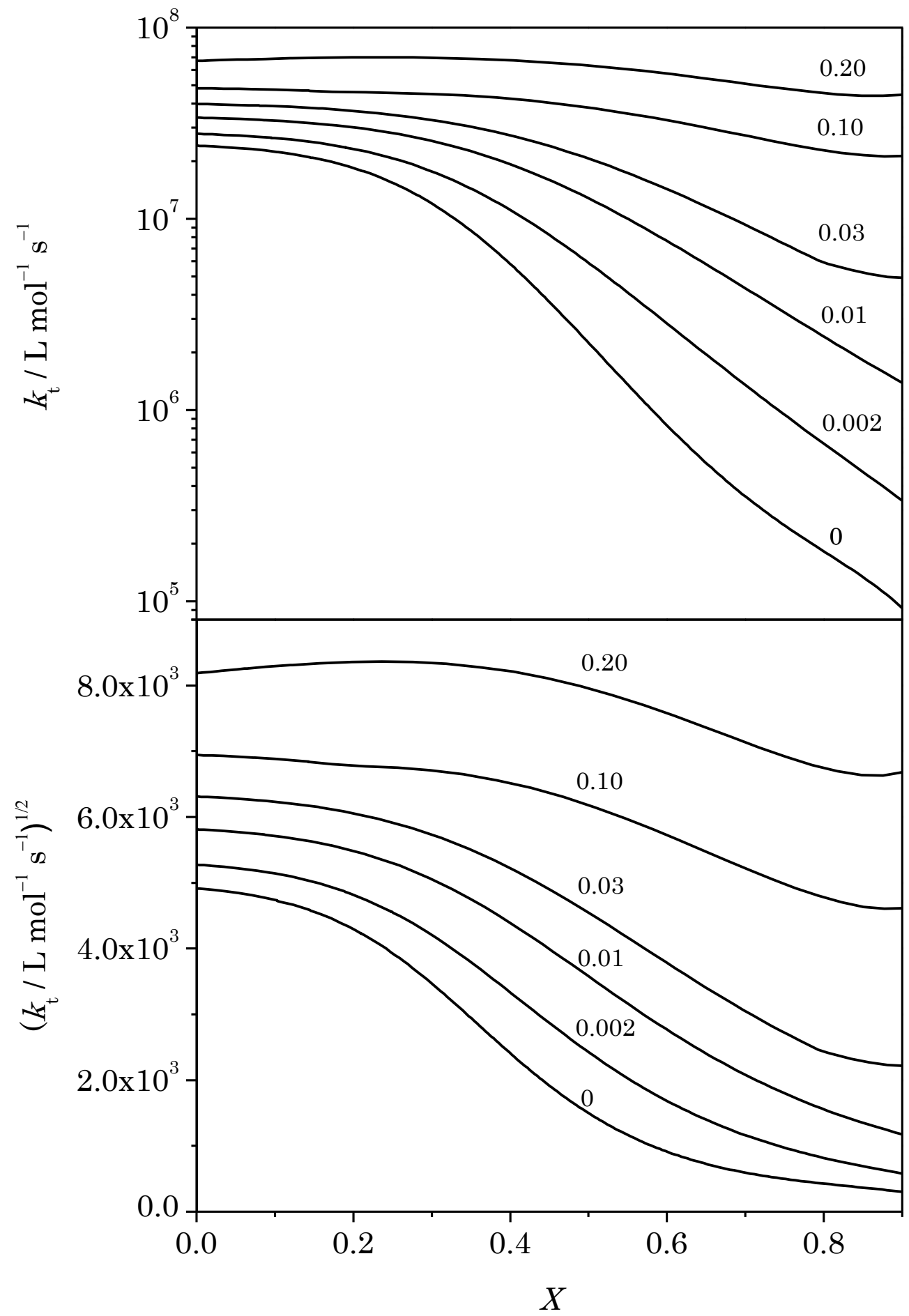

Figure 4-14 Dependence of overall termination rate coefficient, $k_{t}$, and of $k_{t}^{0.5}$ (lower figure) on monomer conversion, as estimated from eq. (4.11) and eq. (4.12) with the parameter values being deduced from PREDICI ${ }^{\mathrm{TM}}$ fitting of the experimental MAA conversion vs. time traces for $\mathrm{WMAA}^{0}=0.30 \mathrm{~g} \mathrm{~g}^{-1}$ and $50^{\circ} \mathrm{C}$. $\mathrm{CME}_{\mathrm{ME}} / \mathrm{CMA}_{\mathrm{MA}}$ is indicated at each curve. 

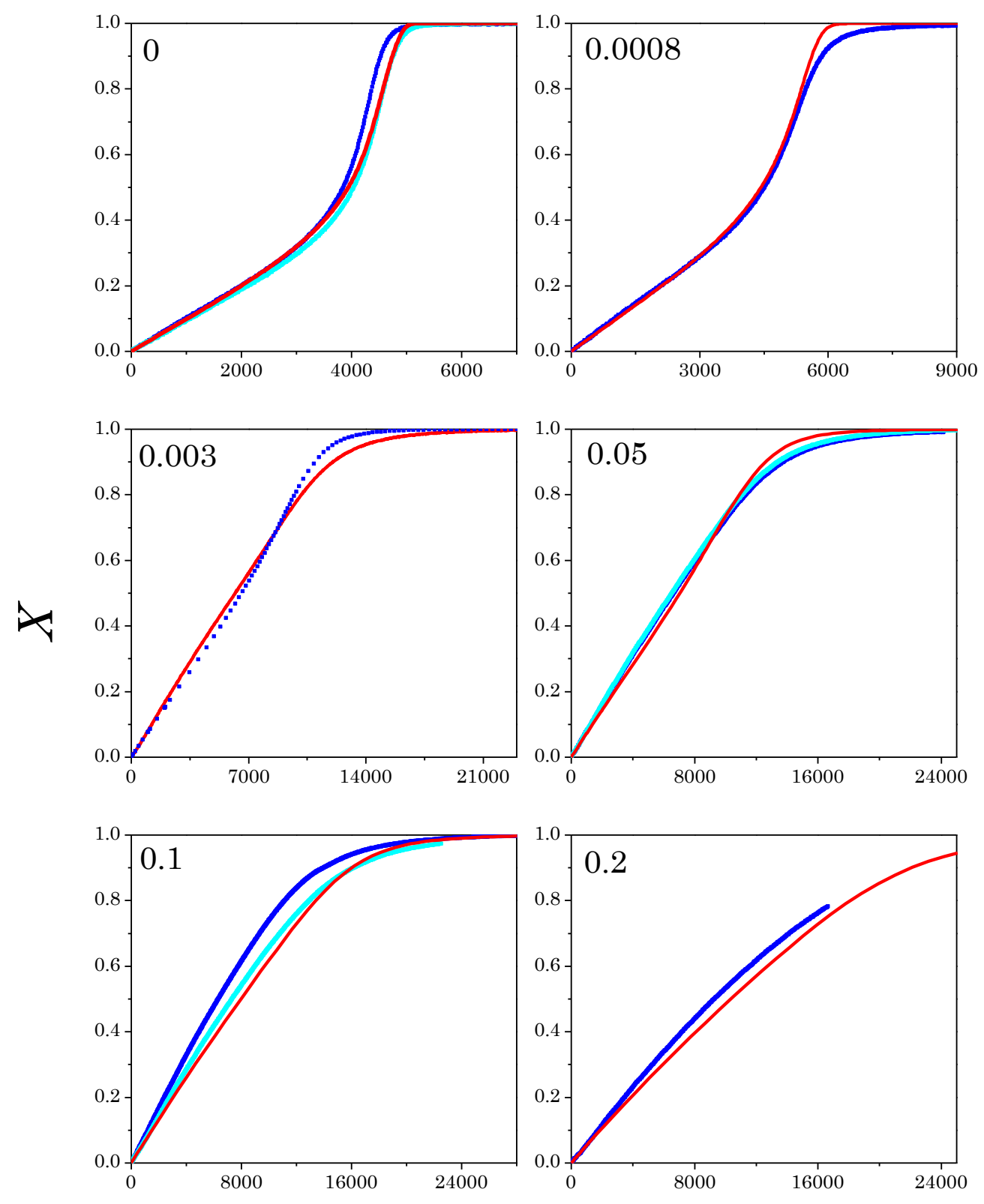

\section{time / s} CME to CMAA ratios as given for each graph) for MAA polymerizations (with $0.30 \mathrm{~g} \mathrm{~g}^{-1}$ MAA) at $50{ }^{\circ} \mathrm{C}$, ambient pressure, and $0.001 \mathrm{~g} \mathrm{~g}^{-1} \mathrm{~V}-50$ as the initiator. Depicted as squares in blue are the experiments and in cyan repeat experiments (for $c_{\mathrm{ME}} / \mathrm{c}_{\mathrm{MAA}}=0$, 0.05 and 0.10$)$. The simulations thereof are indicated by the red lines. 
Table 4-3 Summary of rate coefficients and other parameters used for modeling radical polymerization of $0.3 \mathrm{~g} \mathrm{~g}^{-1}$ non-ionized methacrylic acid in aqueous solution at $50{ }^{\circ} \mathrm{C}$.

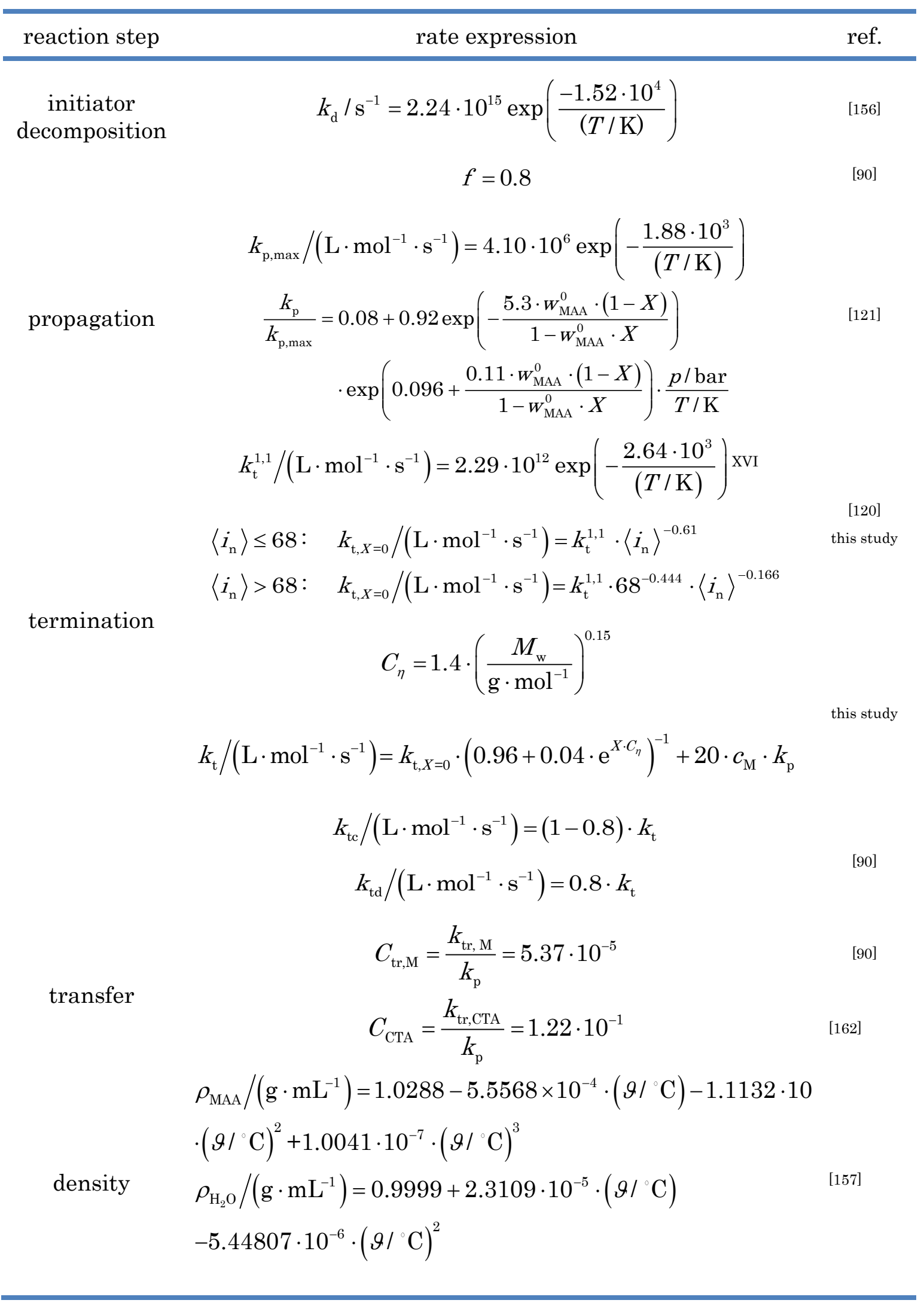

XVI The value is already corrected for a distribution of chain lengths. 


\subsubsection{Modeling Polymerization at Low initial Monomer Content}

In this subchapter the termination behavior of $0.1 \mathrm{~g} \mathrm{~g}^{-1} \mathrm{MAA}$ is dealt with and compared to the data for $0.3 \mathrm{~g} \mathrm{~g}^{-1}$ initial weight fraction of monomer. Under conditions of efficient chain transfer, as with $\mathrm{ME}$, the distributions of radical size and of polymer molar mass are controlled by propagation and transfer rates. Furthermore, it is demonstrated that the decline of $k_{\mathrm{t}}$ with conversion caused by the presence of polymer also depends on the amount of monomer being present at the same time.

At slow rate of initiation and in the absence of CTA, transfer to monomer becomes the essential chain stopping event. Modeling polymerization of MAA at lower initial monomer concentration was carried out analogously to the modeling procedure described in subchapter 4.2.1. XvII

The entries in Table 4-3 demonstrate that a large body of kinetic information on MAA radical polymerization in aqueous MAA solution is available from independent experiments. As with the previous modeling problem, it is essentially termination kinetics at different levels of ME concentration, which remains to be determined via simulation of experimental monomer conversion vs. polymerization time data.

In what follows, $k_{\mathrm{t}}$ refers to the chain-length averaged rate coefficient; for reasons of convenience, chevrons are omitted.

To determine the effect of radical chain length on $k_{\mathrm{t}}$ without interference of contributions from polymer-induced viscosity changes, rates at different $\mathrm{ME}$ concentrations and low degrees of monomer conversion, i.e., below 2.5 percent were estimated under the assumption of $k_{\mathrm{t}}$ remaining constant. The so-obtained $k_{\mathrm{t}}$ values are plotted as a function of the number-average size of growing polymer chains, with these latter values being deduced from simulation. With accurate values for $k_{\mathrm{p}}$ and $C_{\mathrm{CTA}}$ being available, these calculated values should be precise. A doublelog plot of the resulting $k_{\mathrm{t}}$ vs. the number-average radical size is shown in Figure 416. CTA concentration has been widely varied which results in the broad range of radical sizes. This was done for three sets of experiments: $0.3 \mathrm{~g} \mathrm{~g}^{-1}$ MAA at $50{ }^{\circ} \mathrm{C}$, $0.1 \mathrm{~g} \mathrm{~g}^{-1} \mathrm{MAA} 50^{\circ} \mathrm{C}$, and $0.1 \mathrm{gg}^{-1} \mathrm{MAA} 35^{\circ} \mathrm{C}$. The values for $35^{\circ} \mathrm{C}$ were extrapolated to $50{ }^{\circ} \mathrm{C}$ applying $E_{\mathrm{A}}=22 \mathrm{~kJ} \mathrm{~mol}^{-1}$ from lit. ${ }^{[120]}$

XVII Some of the conversion time and MMD data used for modeling in this subchapter were taken from previous work. ${ }^{[147]}$ 
Surprisingly, $k_{\mathrm{t}}^{1,1}$ is, within experimental uncertainty, the same for $0.3 \mathrm{~g} \mathrm{~g}^{-1}$ and $0.1 \mathrm{~g} \mathrm{~g}^{-1}$ MAA. Thus, the representation of $k_{\mathrm{t}}$ at negligible conversion, viz., the chain-length dependency of $k_{\mathrm{t}}$ can be adopted from the previous subchapter. The ratio of viscosity for $0.3 \mathrm{~g} \mathrm{~g}^{-1}$ and $0.1 \mathrm{~g} \mathrm{~g}^{-1}$ MAA content $\left(\eta_{W_{\mathrm{MAA}}=0.3} / \eta_{W_{\mathrm{MAA}}=0.1}=1.5\right)$ suggests that $k_{\mathrm{t}}$ should be higher for $0.1 \mathrm{~g} \mathrm{~g}^{-1}$ MAA content by a factor of 1.5 . This may indicate an influence of MAA content on the polymer coil dynamics.

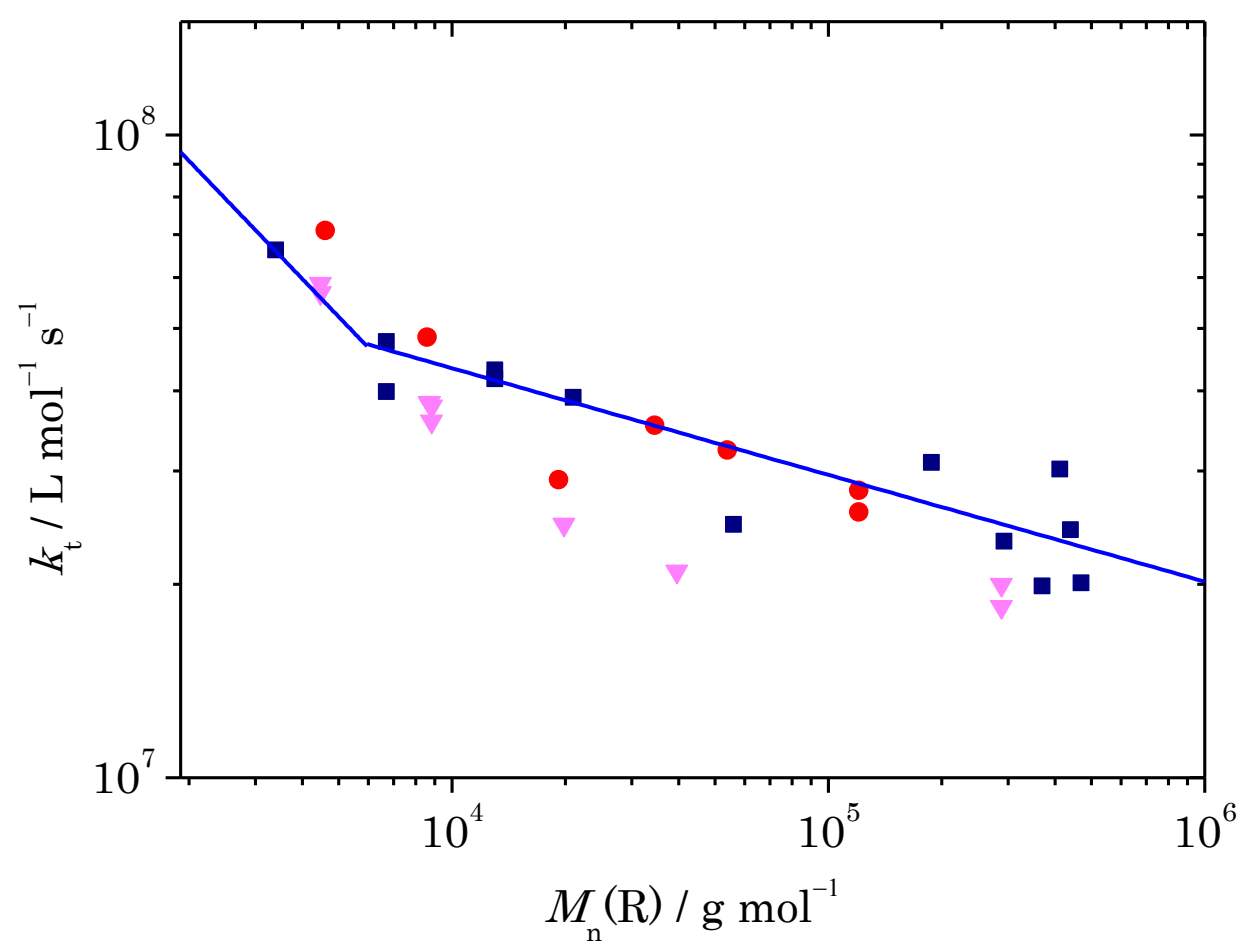

Figure 4-16 Symbols: Initial $k_{t}$ values deduced as mean values from the conversion range $0<X<0.025$ of MAA polymerizations in aqueous solution at varying $c_{\mathrm{ME}} / \mathrm{c}_{\mathrm{M}}$ up to 0.2 . The abscissa values are simulated number-average values of growing polymer chains. Blue squares: WMAA $=0.30 \mathrm{~g} \mathrm{~g}^{-1}$, W- $-50=0.001 \mathrm{~g} \mathrm{~g}^{-1}, 50{ }^{\circ} \mathrm{C}$; circles: WMAA $=0.10 \mathrm{~g} \mathrm{~g}^{-1}$, $W_{\mathrm{V}-50}=0.01 \mathrm{~g} \mathrm{~g}^{-1}, 50^{\circ} \mathrm{C}$; triangles: WMAA $=0.10 \mathrm{~g} \mathrm{~g}^{-1}, 35^{\circ} \mathrm{C}$ extrapolated to $50^{\circ} \mathrm{C}$ applying $E_{\mathrm{A}}$ from lit. ${ }^{[120]}$ Line: fitting of the $0.30 \mathrm{~g} \mathrm{~g}^{-1}$ data according to the composite model; data points for $M_{\mathrm{n}}$ below $5852 \mathrm{~g} \mathrm{~mol}^{-1}$ were used for fitting the short-chain behavior, whereas data at higher average molar masses are subjected to fitting the long-chain behavior. The chain-length dependency of $k_{\mathrm{t}}$ for $0.10 \mathrm{~g} \mathrm{~g}^{-1}$ and $0.30 \mathrm{~g} \mathrm{~g}^{-1}$ is in good agreement.

As no backbiting occurs during MAA polymerization, ideal polymerization kinetics, ${ }^{\text {XVIII }}$ eq. (2.7), has been used for estimating $k_{\mathrm{t}}$ as a function of $X$ from

XVIII Here, ideal refers to each single data point, i.e., overall constancy is not assumed. Moreover, CLD-T is not excluded. 
experimental monomer conversion vs. time data in the same way as for higher MAA concentration. The result for three CTA concentrations is shown in Figure 4-17. The most pronounced decrease of $k_{\mathrm{t}}$ occurs for polymerizations without CTA. The reproducibility is very good. The data points in blue and cyan, belonging to experiments under ostensibly the same conditions, show close agreement (see Figure 4-17).

The reduction of $k_{\mathrm{t}}$ towards higher degree of monomer conversion becomes less pronounced as the ME content increases. The initial value of $k_{\mathrm{t}}$ increases with higher ME content, as has been discussed for Figure 4-9 and Figure 4-10, respectively. At $c_{\mathrm{ME}} / c_{\mathrm{MAA}}=0.2, k_{\mathrm{t}}$ is significantly higher than at lower ME content and does not change with $X$.

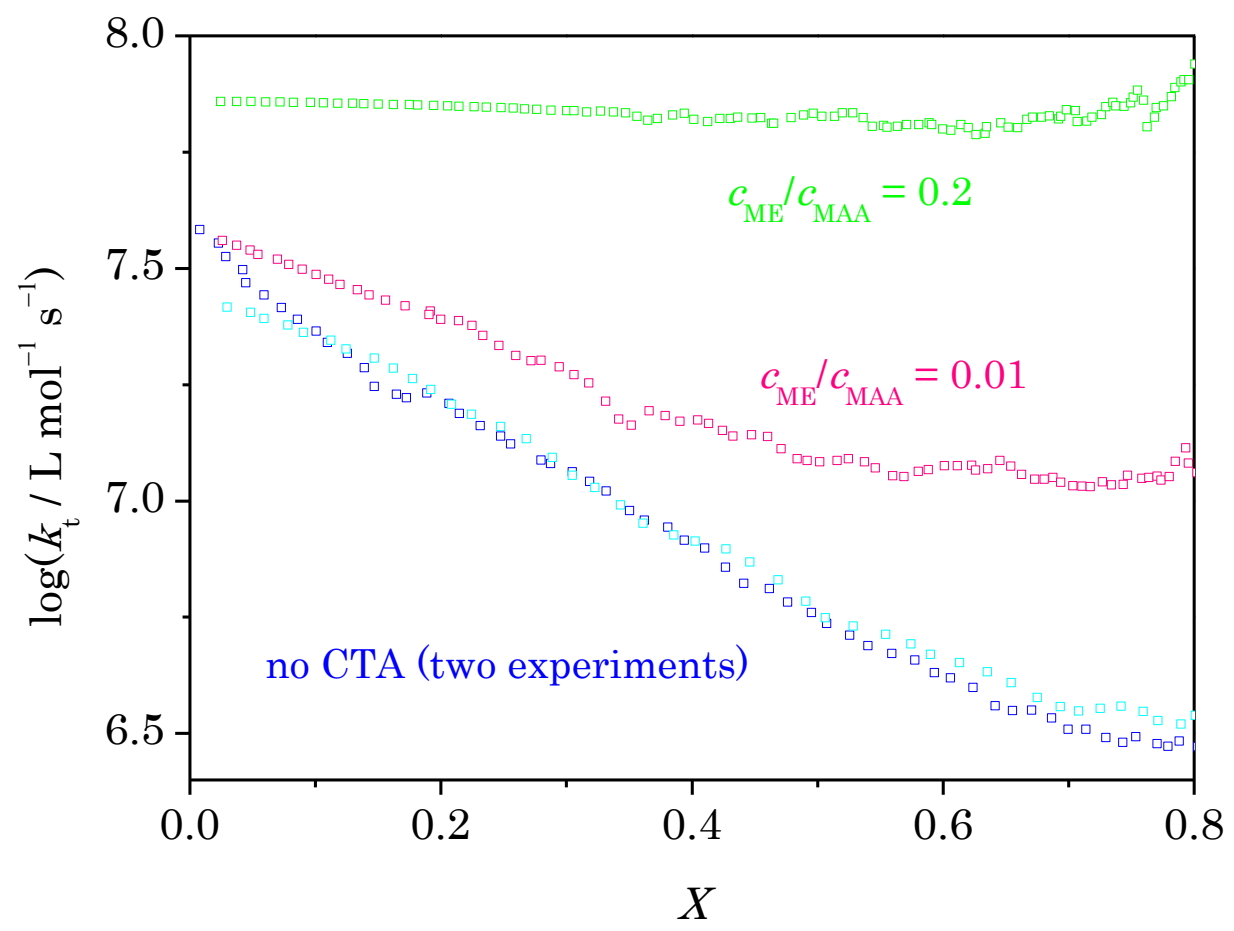

Figure 4-17 Dependence of the termination rate coefficient as deduced by adapting ideal polymerization kinetics (eq. (2.7)) for polymerization of $0.10 \mathrm{~g} \mathrm{~g}^{-1} \mathrm{MAA}$ in aqueous solution with $0.01 \mathrm{~g} \mathrm{~g}^{-1} \mathrm{~V}-50$ at ambient pressure. The realtive ME concentrations are given in the figure.

In Figure 4-18, the dependence of $k_{\mathrm{t}}$ on $\mathrm{ME}$ content and degree of monomer conversion is compared for $w_{\text {MAA }}^{0}=0.1 \mathrm{~g} \mathrm{~g}^{-1}$ and $w_{\text {MAA }}^{0}=0.3 \mathrm{~g} \mathrm{~g}^{-1}$. For the $0.3 \mathrm{~g} \mathrm{~g}^{-1}$ MAA the initial plateau region of $k_{\mathrm{t}}$, which is assigned to SD, increases with ME content 
and extends over the entire experimental conversion range at the highest $\mathrm{ME}$ concentration. The decline of $k_{\mathrm{t}}$ in the TD region is less pronounced, the higher the $\mathrm{ME}$ content. The initial value of $k_{\mathrm{t}}$ increase towards higher ME content, which becomes visible at high levels of CTA.

For the $0.1 \mathrm{~g} \mathrm{~g}^{-1}$ MAA, the Norrish-Trommsdorf effect is much weaker, due to the weak increase of polymer content towards higher monomer conversion. For CTA-free polymerization, the $k_{\mathrm{t}}$ value at $X=0$ is the same for the two initial MAA concentrations, but at $X=0.8$ the $k_{\mathrm{t}}$ value for $W_{\text {MAA }}^{0}=0.3 \mathrm{~g} \mathrm{~g}^{-1}$ is by one and a half orders of magnitude below the one for $W_{\text {MAA }}^{0}=0.1 \mathrm{~g} \mathrm{~g}^{-1}$. Aside from that, there are no pronounced SD and TD dominated regions for low MAA content. The form may be described as a "tilted plateau". Buback et al. ${ }^{[90]}$ also found for polymerizations without CTA and MAA content of $0.1 \mathrm{~g} \mathrm{~g}^{-1}, 0.2 \mathrm{~g} \mathrm{~g}^{-1}$, and $0.3 \mathrm{~g} \mathrm{~g}^{-1}$ that the NorrishTrommsdorf effect becomes weaker towards low initial monomer content. The data was more scattered and all polymerizations were modeled with $k_{\mathrm{t}}$ only varying with respect to reaction diffusion. In this work, a more fundamental treatment of termination kinetics is carried out.

The general effect of adding CTA is the same for both $0.1 \mathrm{~g} \mathrm{~g}^{-1}$ and $0.3 \mathrm{~g} \mathrm{~g}^{-1}$ MAA. Towards higher ME content, $k_{\mathrm{t}}$ is larger and varies less with conversion. At $c_{\mathrm{ME}} / c_{\mathrm{MAA}}=0.2$, the value for termination is the same for both initial weight fractions over the entire range of conversion (see Figure 4-18).

Analysis of the individual monomer conversion vs. time profiles of $0.1 \mathrm{~g} \mathrm{~g}^{-1}$ MAA (without addition of ME) by fitting to eq. (4.11), via the Levenberg-Marquardt algorithm, yields numbers for $s d, t d$ and, $C_{\eta}$. The procedure was the same as applied for $0.3 \mathrm{~g} \mathrm{~g}^{-1}$ MAA. The parameter $C_{\mathrm{RD}}$ is 20 , as for $0.3 \mathrm{~g} \mathrm{~g}^{-1}$ MAA. The parameters $s d=0.00 \pm 0.01$ and $t d=1.00 \pm 0.01$ turned out to provide the best representation of $k_{\mathrm{t}}$ for the entire set of measured MAA conversion vs. time traces at $0.1 \mathrm{~g} \mathrm{~g}^{-1}$ MAA. Eq. (4.11) is turned into eq. (4.13). Differences in the conversion dependence of termination rate on $\mathrm{ME}$ concentration are exclusively assigned to $C_{\eta}$. The impact of ME content is thus contained in $k_{\mathrm{t}, \mathrm{CLD}}$ (v.s.) and in $C_{\eta}$ (v.i.).

$$
k_{\mathrm{t}}=\frac{k_{\mathrm{t}, \mathrm{CLD}}}{\mathrm{e}^{X \cdot C_{\mathrm{n}}}}+C_{\mathrm{RD}} \cdot c_{\mathrm{M}} \cdot k_{\mathrm{p}}
$$




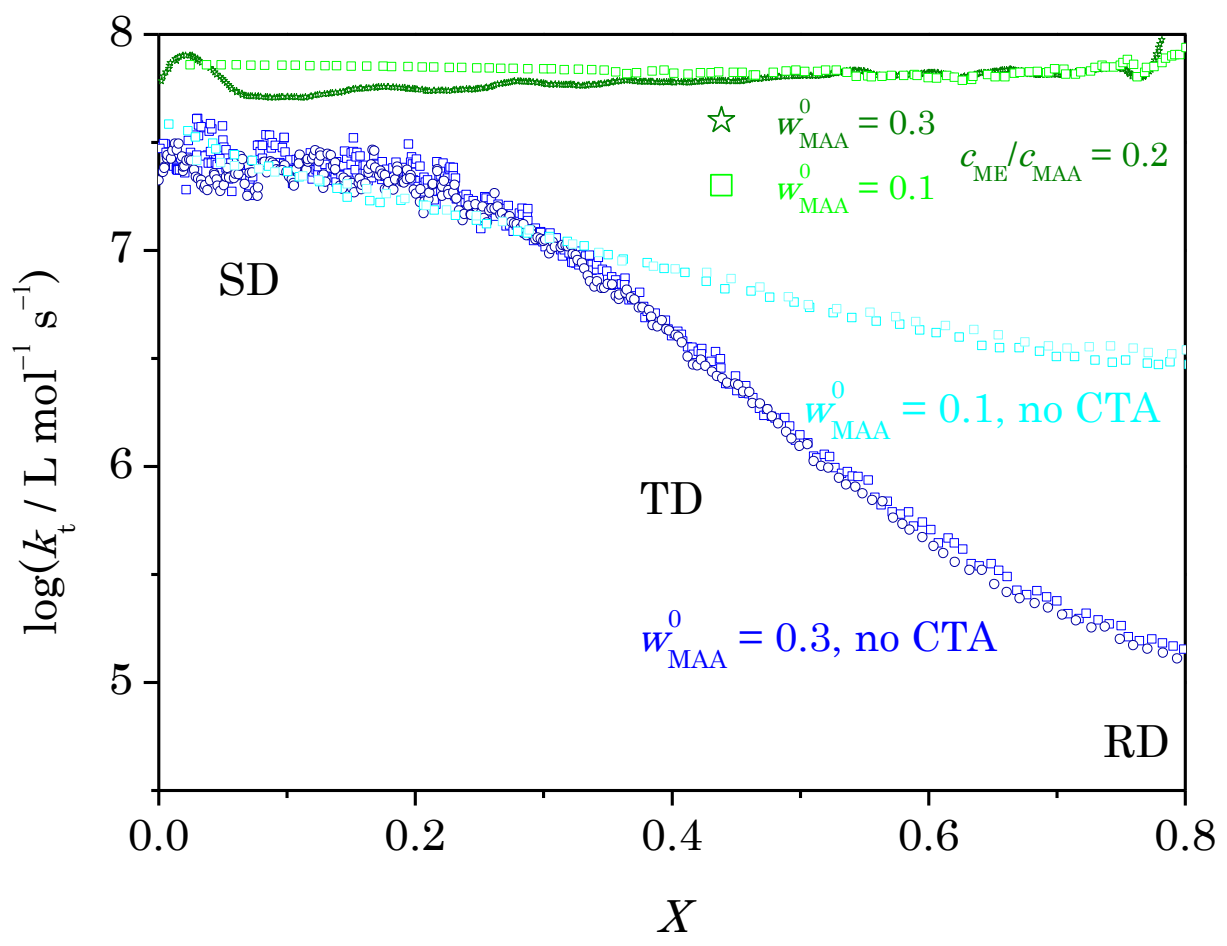

Figure 4-18 Dependence of the termination rate coefficient as derived from ideal polymerization kinetics (eq. (2.7)) for three polymerizations ( $0.30 \mathrm{~g} \mathrm{~g}^{-1} \mathrm{MAA}$ with $0.001 \mathrm{~g} \mathrm{~g}^{-1} \mathrm{~V}-50$, (blue/darkblue) and $0.10 \mathrm{~g} \mathrm{~g}^{-1} \mathrm{MAA}$ with $0.01 \mathrm{~g} \mathrm{~g}^{-1} \mathrm{~V}-50$ (cyan/pale cyan) in aqueous solution). The relative ME concentrations are given in the Figure. The notations SD, $\mathrm{TD}$, and, RD refer to control of termination by segmental, translational (centre-ofmass), and reaction diffusion, respectively.

Given the initial plateau of $k_{\mathrm{t}}$ for $W_{\mathrm{MAA}}^{0}=0.3 \mathrm{~g} \mathrm{~g}^{-1}$ and considering polymer content, one may expect constant $k_{\mathrm{t}}$ for $W_{\text {MAA }}^{0}=0.1 \mathrm{~g} \mathrm{~g}^{-1}$ over almost the whole conversion range. However, the polymer content for $W_{\text {MAA }}^{0}=0.1 \mathrm{~g} \mathrm{~g}^{-1}$ at $X=0.6$ is the same for $W_{\text {MAA }}^{0}=0.3 \mathrm{~g} \mathrm{~g}^{-1}$ at $X=0.2$, but the $k_{\mathrm{t}}$ value of the former is lower by a factor of five. This aspect will be addressed in what follows.

Impurities that function as retarders lead to an increased apparent $k_{\mathrm{t}}$. As they are consumed during the course of polymerization, their effect on apparent $k_{\mathrm{t}}$ decreases, which means that the apparent $k_{\mathrm{t}}$ decreases as well. Given the initial $k_{\mathrm{t}}$ values of the polymerization with $W_{\text {MAA }}^{0}=0.1 \mathrm{~g} \mathrm{~g}^{-1}$ being the same as the ones of the polymerization with $W_{\text {MAA }}^{0}=0.3 \mathrm{~g} \mathrm{~g}^{-1}$, a retardation of the former polymerization appears rather unlikely (see Figure 4-18).

In order to check whether such impurities affect the polymerization at $0.1 \mathrm{~g} \mathrm{~g}^{-1}$ initial weight fraction of MAA, a two-step experiment was carried out. The reaction mixture was prepared as before, but prior to chemically initiated polymerization 200 
UV-laser pulses were applied. ${ }^{\mathrm{XIX}}$ Azo-compounds can be used as both chemical and photoinitiators. The high energy laser pulses cause V-50 to decay and, with each pulse, high radical concentrations are achieved. They do not lead to significant monomer conversion, because of high termination rate. Components that react with growing chains faster than does the monomer are used up to a significant extent. After the "laser cleaning", within the second step, a chemically initiated polymerization was carried out as with the other samples. Due to a high initiator concentration in the beginning, high radical production by the laser pulses is combined with the initiator concentration being reduced slightly only.

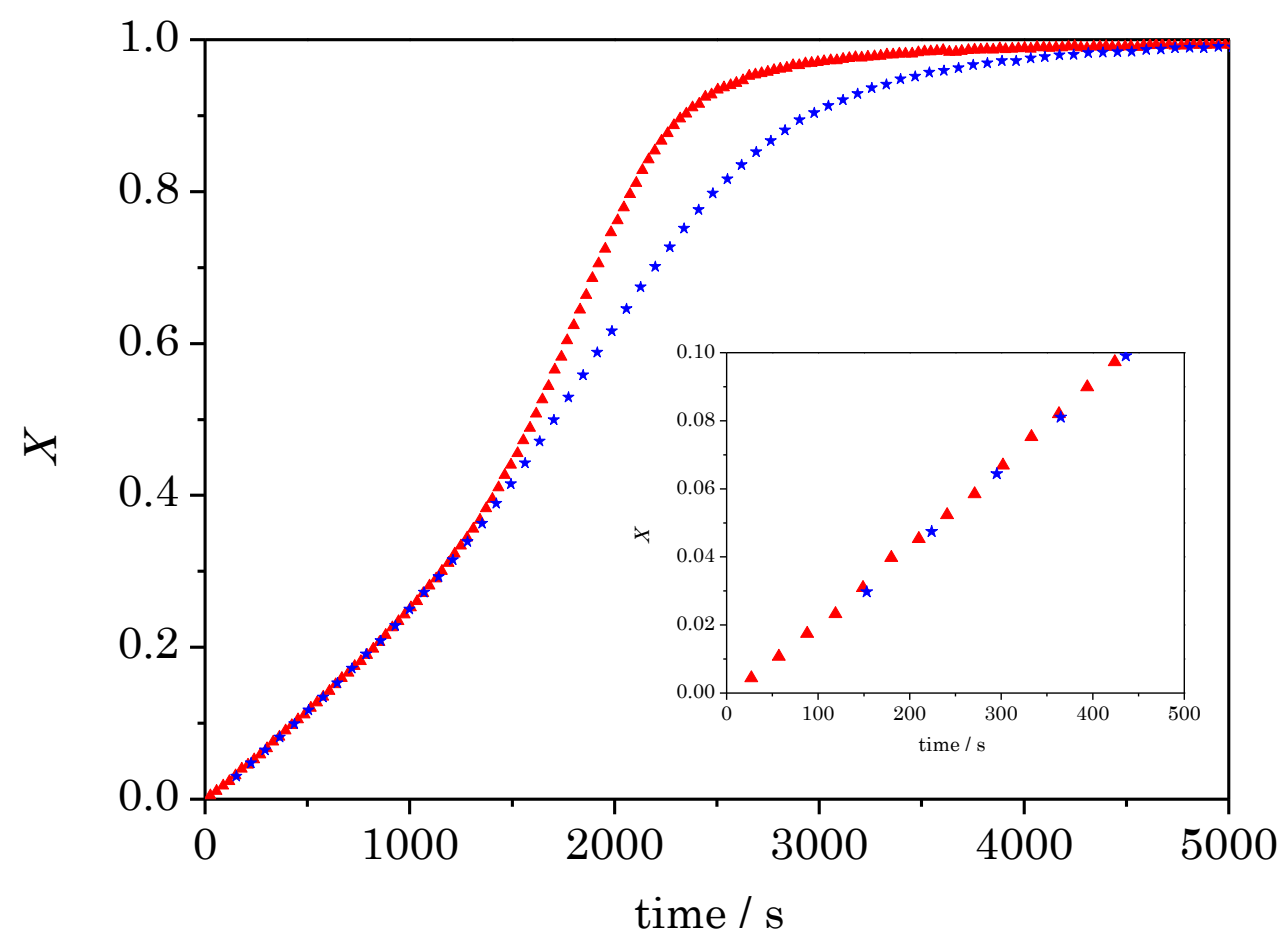

Figure 4-19 Comparison of two polymerizations of $0.10 \mathrm{~g} \mathrm{~g}^{-1} \mathrm{MAA}$ with $0.001 \mathrm{~g} \mathrm{~g}^{-1} \mathrm{~V}-50$ as the initiator in aqueous solution at $50{ }^{\circ} \mathrm{C}$ and ambient pressure. Red triangles: chemically initiated polymerization; Blue stars: First, photopolymerization leading to high radical concentration and to $X=0.03$. Second, chemically initiated polymerization; time zero was corrected. In the insert the region of low conversion is depicted enlarged. "Laser cleaning" was carried out by a LPXpro 240 (Coherent), 200 pulses were applied (100 Hz, $74.3 \mathrm{~mJ}$ per pulse)

XIX This step was carried out with the help of Dr. Jens Schrooten. 
The conversion-time profiles of a polymerization with "laser cleaning" is compared to a polymerization under the same condition, but without laser pulses being applied, in Figure 4-19. Laser initiation led to $X=0.03$; the time zero of the profiles was corrected accordingly. The two conversion-time profiles, with and without "laser cleaning", are in perfect agreement up to $X=0.4$. Hence, impurities functioning as retarders provide no explanation for the decrease of $k_{\mathrm{t}}$ with monomer conversion. At higher degree of monomer conversion, the Norrish-Trommsdorf effect is weaker for polymerization with laser pulses being applied in the beginning. The reason for this probably is that the oligomeric material produced by laser pulsing reduces viscosity.

Beyond the plateau region, the polymerization of $0.1 \mathrm{~g} \mathrm{~g}^{-1}$ and $0.3 \mathrm{~g} \mathrm{~g}^{-1}$ initial weight fraction of monomer, respectively, exhibit different $k_{\mathrm{t}}$ at the same level of polymer content. It should be noted that the monomer content for a given polymer content is three times higher for $0.3 \mathrm{~g} \mathrm{~g}^{-1}$ MAA. Thus, the monomer may affect the stability and permeability, respectively, of the polymer-water solution weakening the NorrishTrommsdorf effect. The data indicates that a higher MAA content in the reaction mixture, and thus in the solvent-swollen polymer coils, enhances segmental mobility. This observation would be in line with the unexpected finding of the same initial $k_{\mathrm{t}}$ values for $0.1 \mathrm{~g} \mathrm{~g}^{-1}$ and $0.3 \mathrm{~g} \mathrm{~g}^{-1}$ MAA (v.i.). It may also explain the different extend of the Norrish-Trommsdorf effect seen in Figure 4-19.

In order to verify this assumption, a polymerization was carried out with isobutyric acid, IBA, being added as non-polymerizing monomer analog. The effect of IBA addition on $k_{\mathrm{p}}$ is the same as monomer addition. ${ }^{[9]}$ The idea is that IBA, while not polymerizing, will have the same effect as monomer on the polymer-water matrix, and thus on $k_{\mathrm{t}}$. A reaction mixture of $0.1 \mathrm{~g} \mathrm{~g}^{-1}$ MAA and $0.2 \mathrm{~g} \mathrm{~g}^{-1}$ IBA corresponds over almost the entire range to the initial plateau region of $k_{\mathrm{t}}$ found for the polymerization of $0.3 \mathrm{~g} \mathrm{~g}^{-1}$ MAA. The $k_{\mathrm{t}}$ values derived by ideal polymerization kinetics are depicted in Figure 4-20. With $0.2 \mathrm{~g} \mathrm{~g}^{-1}$ IBA in the reaction mixture, $k_{\mathrm{t}}$ does not decrease with $X$. On the contrary, a slight enhancement is seen, which might result from $k_{\mathrm{p}}$ being a little higher with IBA replacing MAA. The abovementioned explanation for the decrease of $k_{\mathrm{t}}$ with conversion found for $W_{\text {MAA }}^{0}=0.1 \mathrm{~g} \mathrm{~g}^{-1}$ is supported by this experiment as well. This observed variation of $k_{\mathrm{t}}$ upon addition of IBA is not yet fully understood and should be subject to further research. It needs to be considered whenever species are added to MAA polymerization in aqueous phase. 


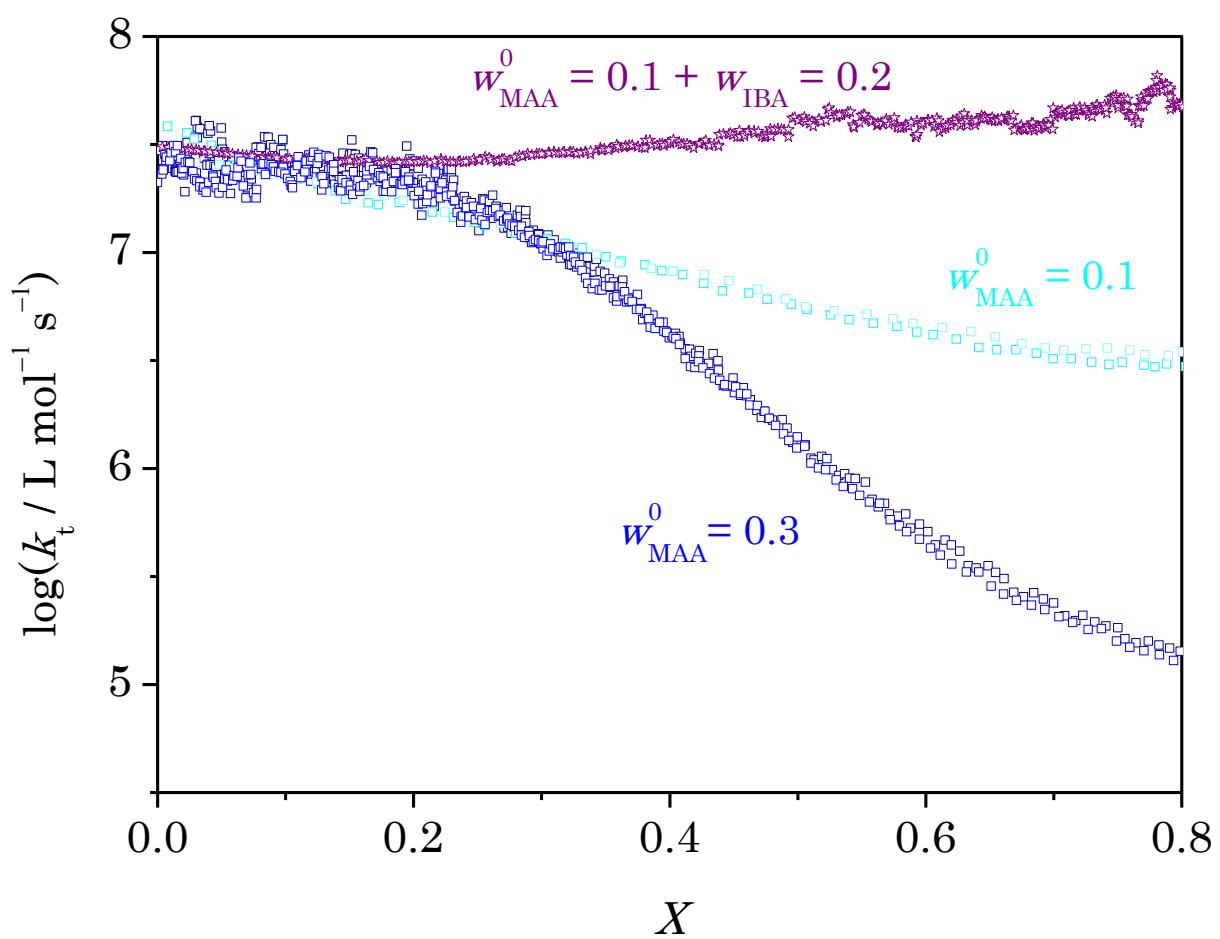

Figure 4-20 Dependence of the termination rate coefficient as derived from ideal polymerization kinetics, eq. (2.7), for three polymerizations: $0.30 \mathrm{~g} \mathrm{~g}^{-1} \mathrm{MAA}$ with $0.001 \mathrm{~g} \mathrm{~g}^{-1} \mathrm{~V}-50$, (blue/darkblue), $0.10 \mathrm{~g} \mathrm{~g}^{-1}$ MAA with $0.01 \mathrm{~g} \mathrm{~g}^{-1} \mathrm{~V}-50$ (cyan/pale cyan), and $0.10 \mathrm{~g} \mathrm{~g}^{-1}$ MAA with $0.01 \mathrm{~g} \mathrm{~g}^{-1} \mathrm{~V}-50$ and $0.20 \mathrm{~g} \mathrm{~g}^{-1}$ IBA as non-polymerizing monomer analog in aqueous solution.

Differences in the conversion dependence of termination rate on ME concentration are exclusively assigned to $C_{\eta}$. The impact of ME content is thus contained in $k_{\mathrm{t}, \mathrm{CLD}}$ and in $C_{\eta}$. The parameter $C_{\eta}$ was deduced by PREDICI ${ }^{\text {TM }}$ fitting of the entire body of polymerization data for a wide range of molar masses, which may be achieved by variation of CTA content. In Figure 4-21 the so-obtained $C_{\eta}$ values are plotted as a function of the weight average molar mass at 0.5 degree of monomer conversion, a value which is close to the mean molar mass taken over the entire conversion range. The $C_{\eta}$ values derived for $W_{\mathrm{MAA}}^{0}=0.1 \mathrm{~g} \mathrm{~g}^{-1}$ are smaller than the ones for $W_{\text {MAA }}^{0}=0.3 \mathrm{~g} \mathrm{~g}^{-1}$, because $t d$ is smaller for $W_{\text {MAA }}^{0}=0.3 \mathrm{~g} \mathrm{~g}^{-1}$. The entire set of experimental $C_{\eta}$ vs. $M_{w}$ data, including both temperatures and the theoretical data point of $(86.02 \mid 0)$ for a polymerization producing polymer of chain length unity only and thus exhibiting no conversion dependence, was fitted by eq. (4.12) which results in the parameter values $a=0.27$ and $b=0.19$. 


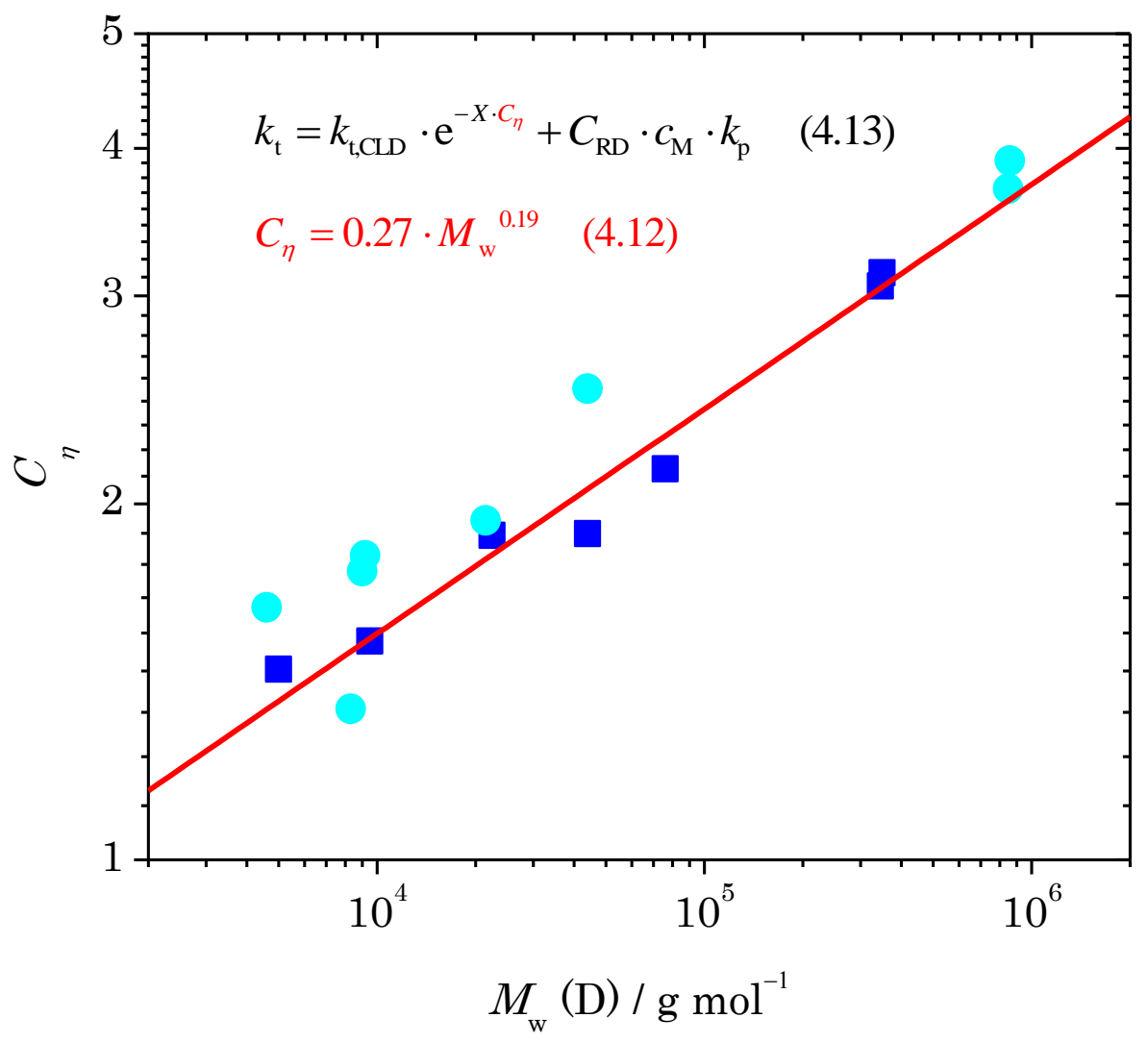

Figure 4-21 Correlation of $C_{\eta}$ with simulated weight-average molar mass of pMAA at $X=0.5$ for polymerization at different levels of chain-transfer agent ME. The symbols are deduced from PREDICI ${ }^{\mathrm{TM}}$ fitting of experimental conversion-time data. The obtained fit parameters refer to eq. (4.12), which complements eq. (4.11). Both equations are also given in the graph. Blue symbols refer to $50^{\circ} \mathrm{C}$ and cyan symbols refer to $35^{\circ} \mathrm{C}$. The theoretical data point for a polymerization producing polymer of chain length unity only and thus exhibiting no conversion dependence $(86.02 \mid 0)$ is not shown, but was included into the fitting of data.

Viscosity measurement were carried out, in order to investigate how the empirical exponential function featuring the parameter $C_{\eta}$ depending on $M_{\mathrm{w}}$ is related to actual conversion dependence of viscosity. The relative viscosity of premixed solutions of water, MAA, and pMAA simulating different degrees of monomer conversion are plotted in Figure 4-22. ${ }^{\mathrm{xx}}$ For comparison, the function used in the model is scaled and plotted as a line. Good agreement is observed, which, however, does not mean that $k_{\mathrm{t}}$ measurement may be replaced by viscosity measurements,

xx There are two problems to be kept in mind: Premixed solutions do not have exactly the same viscosity as the genuine reaction mixture at a given degree of conversion and the Ubbelohde method may cause problems with non-Newtonian liquids, even though low flow rates were chosen. 
but it is interesting to observe, as the exact relationship between $k_{\mathrm{t}}$ and viscosity of polymer solutions is unknown (see subchapter 2.4.5).

Furthermore, the relative viscosity shows the same temperature dependence at 35, 50,65 , and $80^{\circ} \mathrm{C}$, which supports the finding of $C_{\eta}$ being temperature independent.

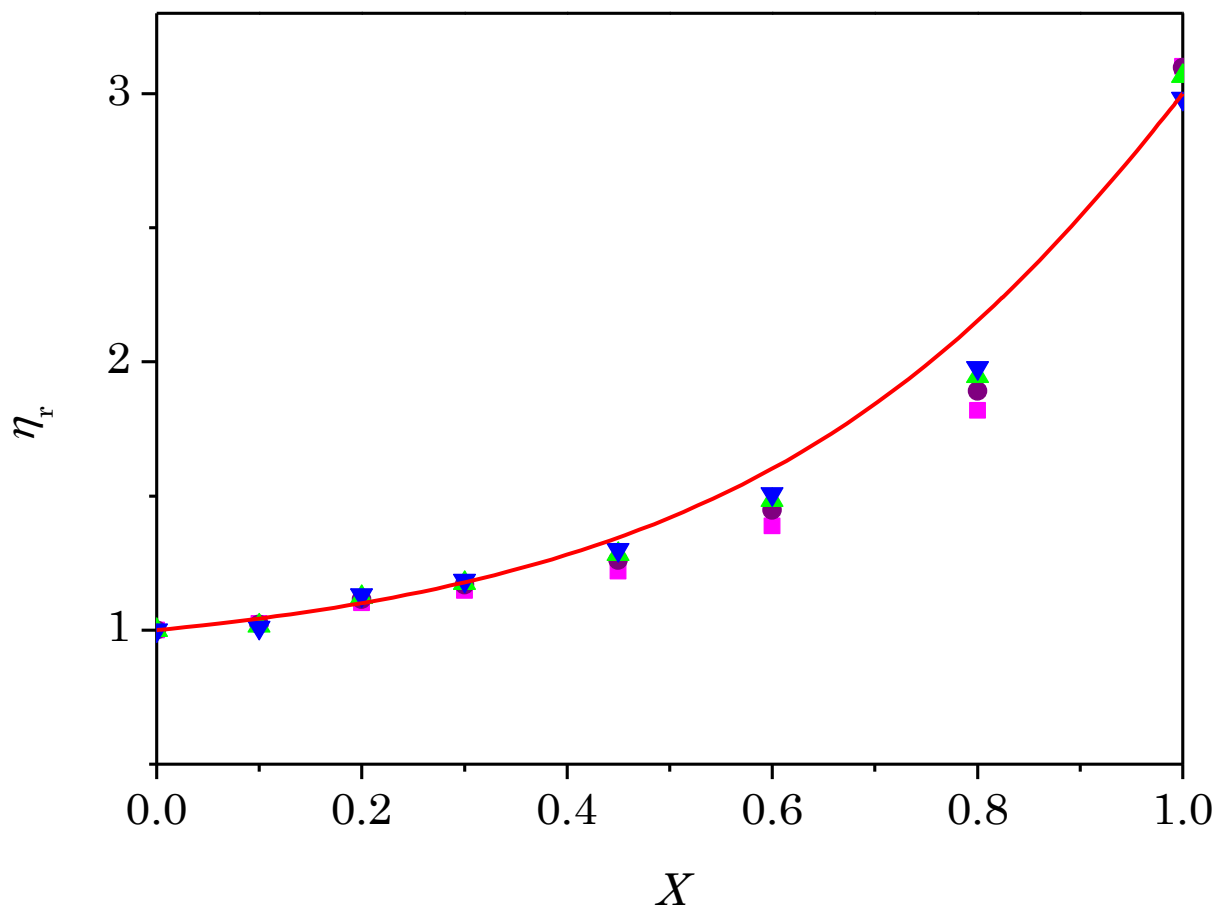

Figure 4-22 Relative viscosity of premixed solutions of water, MAA, and pMAA simulating different degrees of monomer conversion ( $\mathrm{WMAA}^{0}=0.1 \mathrm{~g} \mathrm{~g}^{-1}$ ) is given for $35^{\circ} \mathrm{C}$ (pink), $50^{\circ} \mathrm{C}$ (purple), $65^{\circ} \mathrm{C}$ (green), and $80^{\circ} \mathrm{C}$ (blue). The function used in the model (red line) is plotted for comparison. Samples were measured by Ubbelohde method.

The quality of the kinetic model associated with the rate coefficients summarized in Table 4-4 is illustrated by comparison of measured and simulated monomer conversion vs. time traces in Figure $4-23$ for $50{ }^{\circ} \mathrm{C}$ and in Figure $4-24$ for $35{ }^{\circ} \mathrm{C}$. The model turns out to satisfactorily represent the polymerization kinetics of $0.1 \mathrm{~g} \mathrm{~g}^{-1}$ MAA in aqueous solution at both 35 and $50{ }^{\circ} \mathrm{C}$ for $\mathrm{ME}$ contents between $c_{\mathrm{ME}} / c_{\mathrm{MAA}}=0$ and $c_{\mathrm{ME}} / c_{\mathrm{MAA}}=0.2$. For comparison, some repeat experiments are included. They demonstrate that the quality of simulation is close to experimental reproducibility. 
MMDs have been measured for different temperatures $\left(35,50\right.$, and $\left.65{ }^{\circ} \mathrm{C}\right)$ and for different levels of initiator concentration (0.01, 0.001, and $\left.0.0001 \mathrm{gg}^{-1}\right)$. With $C_{\mathrm{tr}, \mathrm{M}}=5.37 \cdot 10^{-5}$ taken from ref. ${ }^{[90]} \mathrm{MMDs}$ were predicted too high. The deviation was systematically higher towards lower rate of initiation, i.e., low initiator concentration and low temperature. Therefore, transfer to monomer had to be increased and $C_{\mathrm{tr}, \mathrm{M}}=1 \cdot 10^{-4}$ yields simulated MMDs being in satisfying agreement experimental ones.

In Figure 4-25 some MMDs are compared to the prediction of the PREDICI ${ }^{\mathrm{TM}}$ model. Measured MMDs exhibit a kink slightly below $10^{6} \mathrm{~g} \mathrm{~mol}^{-1}$. This is a calibration artifact. Measured and predicted MMDs are in good agreement within experimental uncertainty, which is higher in this case due to the problem of MMDs being partly out of calibration range. MMDs of polymerizations at high levels of CTA are not shown, as their prediction is rather trivial for known $k_{\mathrm{p}}$ and $C_{\mathrm{tr}, \mathrm{CTA}}$ being deduced from MMDs. 

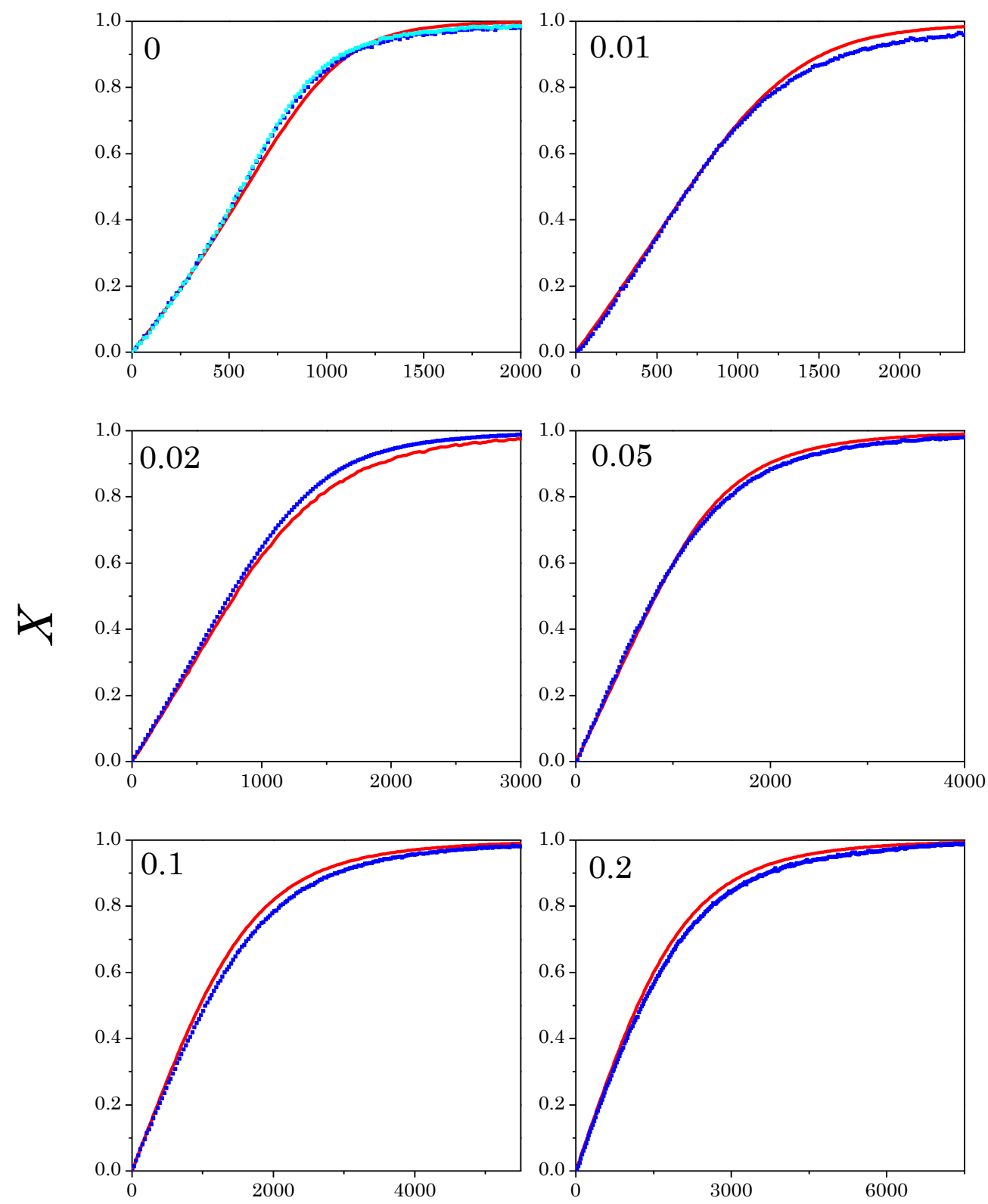

time / s

Figure 4-23 Comparison of measured and simulated MAA conversion vs. time plots. Blue lines: polymerization of $0.1 \mathrm{~g} \mathrm{~g}^{-1}$ MAA with $0.01 \mathrm{~g} \mathrm{~g}^{-1} \mathrm{~V}-50$ as the initiator at $50^{\circ} \mathrm{C}$ with different initial $C_{\mathrm{ME}}$ to CMAA ratios given for each graph; the simulations are indicated by the red lines; an independent repeat experiments for $\mathrm{CME}_{\mathrm{ME}}=0$ is included (cyan). 

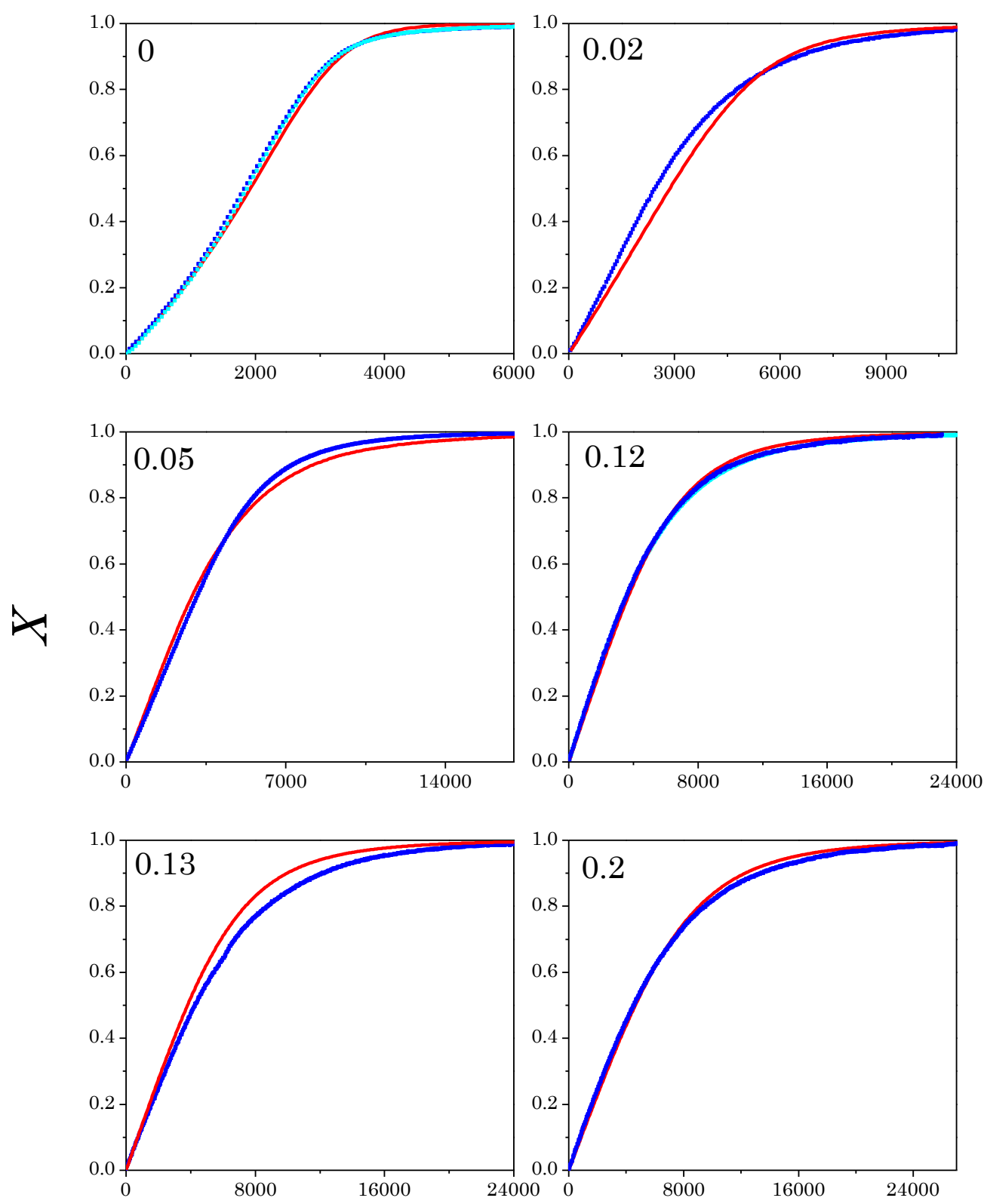

\section{time / s}

Figure 4-24 Comparison of measured and simulated MAA conversion vs. time plots. Blue lines: polymerization of $0.1 \mathrm{~g} \mathrm{~g}^{-1} \mathrm{MAA}$ with $0.01 \mathrm{~g} \mathrm{~g}^{-1} \mathrm{~V}-50$ as the initiator at $35^{\circ} \mathrm{C}$ with different initial $C_{M E}$ to CMAA ratios given for each graph; the simulations are indicated by the red lines; independent repeat experiments are included (cyan). 


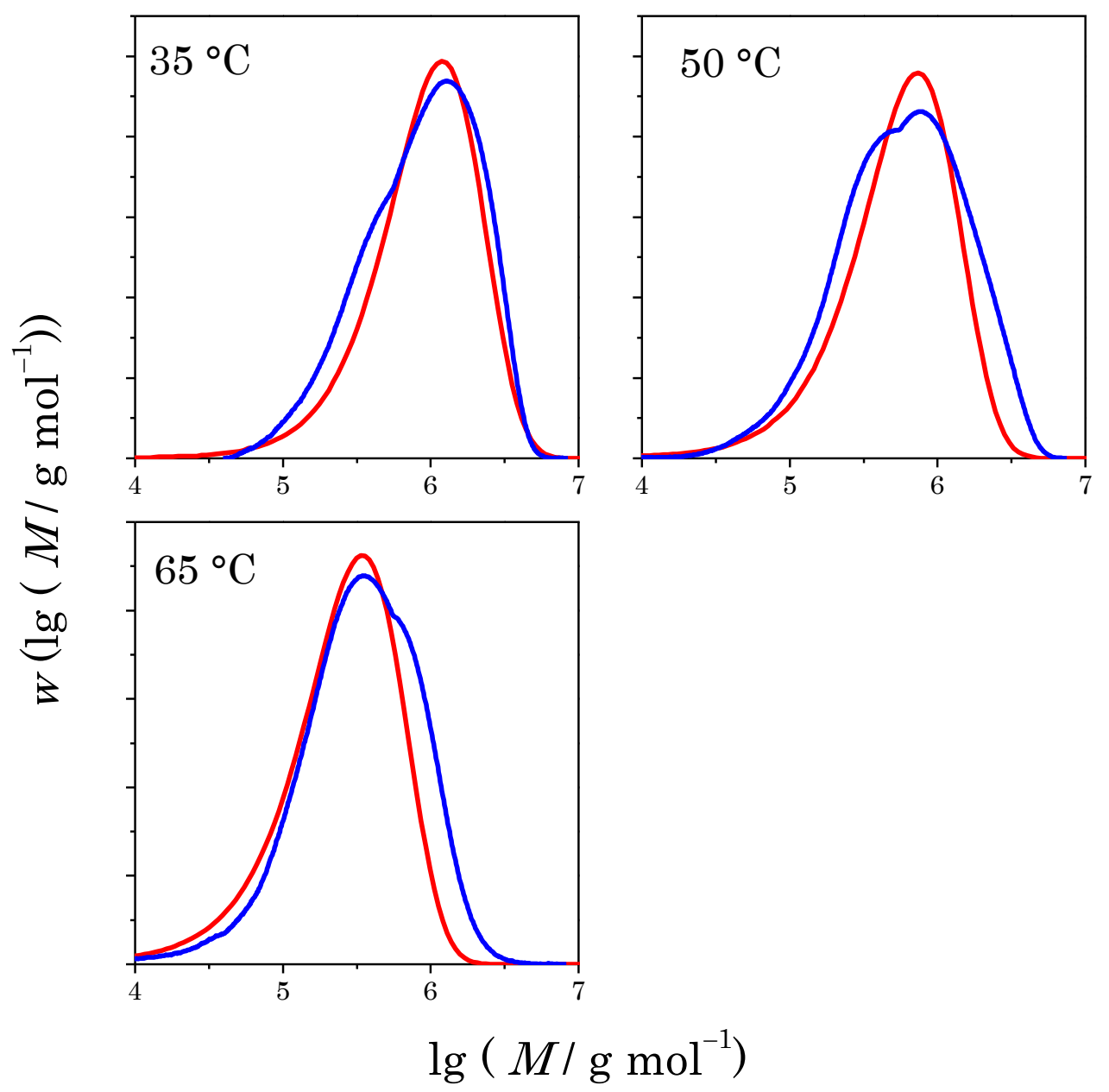

Figure 4-25 Comparison of measured and simulated MMDs. Blue line: polymerization of $0.1 \mathrm{~g} \mathrm{~g}^{-1}$ MAA to full conversion with $0.001 \mathrm{~g} \mathrm{~g}^{-1} \mathrm{~V}-50$ and at different temperatures given for each graph. Very high molar masses were reached and a significant amount of polymer was beyond the limits of SEC calibration. This resulted in a kink as a calibration artifact. Red line: simulation.

To test and further verify this model, polymerizations with a completely different setup and lower monomer concentration were carried out. As demonstrated by Figure 4-26, the model developed with conversion time data from experiments in small scale reactors and with deutered water as solvent is well suited for predictions of larger scale $(1 \mathrm{~L})$. In addition, the agreement shows the absence of isotope effects. Figure 4-26 also shows that the model is capable of predicting conversion of CTA correctly. 
$C_{\text {СТА }}$ of 2-mercaptoethanol for MAA polymerization in aqueous solution has been determined to be $0.12 \pm 0.01$ at $50{ }^{\circ} \mathrm{C}$. The Mayo and the CLD method were applied for analysis of the aqueous-phase SEC data. Both methods yield more or less identical results with the Mayo method being slightly more robust when applied to a system under conditions of strong chain-transfer activity and with SEC analysis being difficult. $C_{\mathrm{CTA}}$ turned out to be independent of MAA content, which means that the chain-transfer rate coefficient largely decreases from dilute to concentrated aqueous solution of MAA as does $k_{\mathrm{p}}$. The measured constancy of $C_{\text {CTA }}$ largely facilitates modeling of aqueous-solution radical polymerizations once the variation of $k_{\mathrm{p}}$ with monomer content has been mapped out and a reliable $C_{\text {СTA }}$ value is known.

Batch radical polymerization of $0.1 \mathrm{~g} \mathrm{~g}^{-1}$ non-ionized MAA in aqueous solution has been investigated between 35 and $65^{\circ} \mathrm{C}$ for a wide concentration range of 2 -mercaptoethanol, which acts as the chain-transfer agent. The measured monomer conversion vs. time profiles and MMDs may be adequately simulated via PREDICI ${ }^{\mathrm{TM}}$ using a physic-chemically based model which includes kinetic information from independent laser-induced experiments. Efficient chain transfer of ME affects both the initial rate of termination, due to the dependence of $k_{\mathrm{t}}$ on radical chain length, and termination up to high degrees of monomer conversion, due to bulk viscosity being tuned by the impact of ME content on polymer molar mass. Chain-length dependency of $k_{\mathrm{t}}$ could be well described by applying the composite model, which was originally developed for one predominant chain length. During polymerization of $0.3 \mathrm{~g} \mathrm{~g}^{-1}$ MAA without CTA $k_{\mathrm{t}}$ shows clearly defined regions of SD, TD, and RD. For $0.1 \mathrm{~g} \mathrm{~g}^{-1}$ MAA this is not the case, it is more a tilted plateau. The development of $k_{\mathrm{t}}$ with degree of monomer conversion can be described by three parameters $s d, t d$, and $C_{\eta}$. These parameters are different for $0.1 \mathrm{gg}^{-1}$ and $0.3 \mathrm{~g} \mathrm{~g}^{-1}$ MAA, while the low conversion $k_{\mathrm{t}}$ values were found to be the same for both concentrations. For modeling monomer concentrations in between, e.g., $0.2 \mathrm{gg}^{-1}$ MAA linearly interpolated values may be used, but this should be subject of further research. Polymerizations with an initial monomer content below $0.1 \mathrm{gg}^{-1}$ MAA can be predicted by the model quite well, as Figure 4-26 demonstrates. Nevertheless, the gel effect becomes weaker towards lower initial MAA content.

$C_{\eta}$ depends on molar mass of polymer in solution, but appears to be temperature independent between 35 and $80^{\circ} \mathrm{C}$. The model should predict conversion vs. time profiles and MMDs for polymerizations up to $80^{\circ} \mathrm{C}$ without problems. 


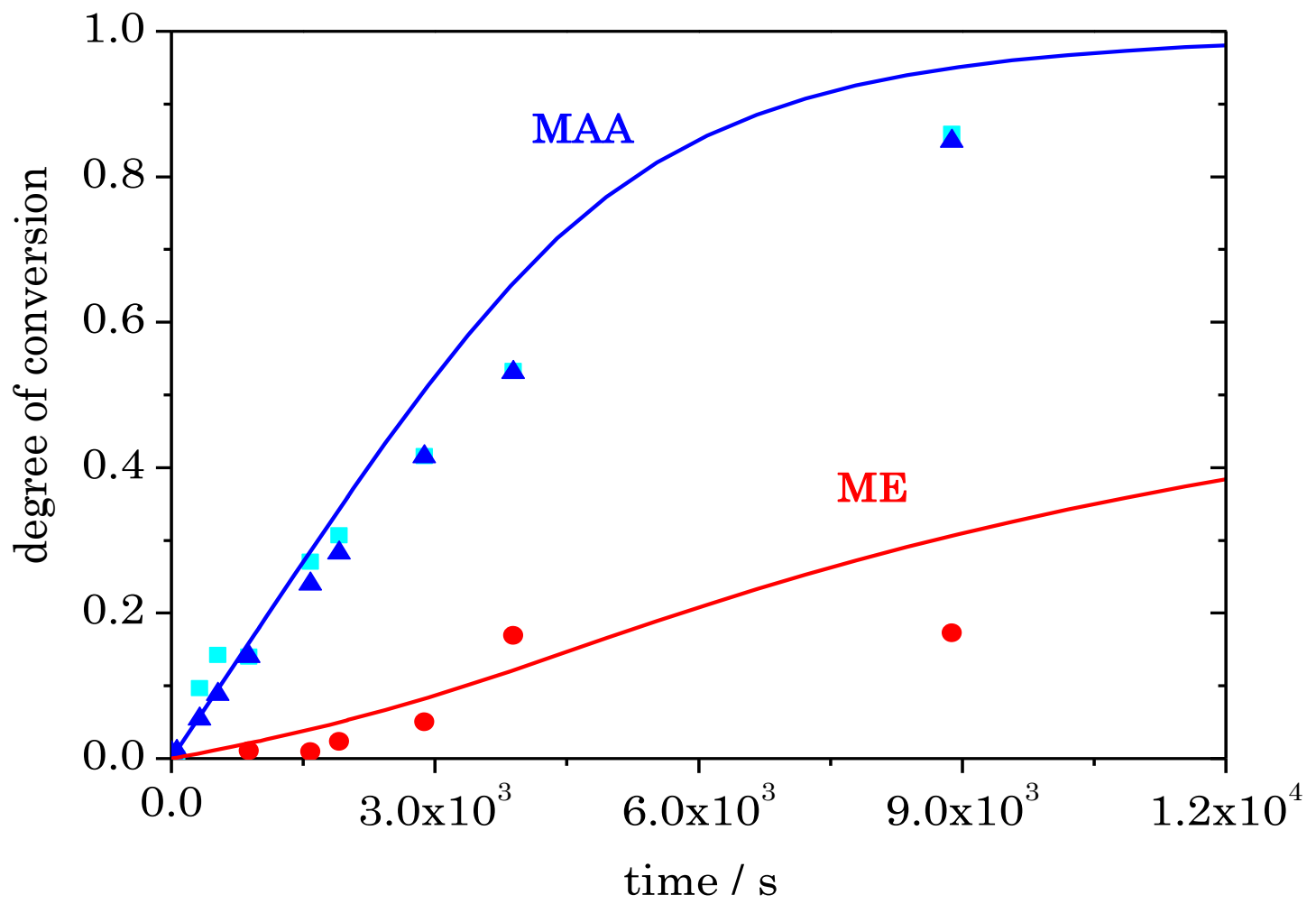

Figure 4-26 $\quad 0.032 \mathrm{~g} \mathrm{~g}^{-1} \mathrm{MAA}$ were polymerized with $0.05 \mathrm{~mol} \mathrm{~mol}^{-1} \mathrm{ME}$ and $0.007 \mathrm{~g} \mathrm{~g}^{-1} \mathrm{~V}-50$ in $\mathrm{H}_{2} \mathrm{O}$ at $50{ }^{\circ} \mathrm{C}$ inside a $1 \mathrm{~L}$ stirred reactor. Degree of monomer conversion was monitored by ${ }^{1} \mathrm{H}-\mathrm{NMR}$ (cyan squares) and gravimetrically (blue triangles); the simulation thereof is given as a blue, solid line. Degree of CTA conversion was monitored by ${ }^{1} \mathrm{H}-\mathrm{NMR}$ (red circles); the simulation thereof is given as a red, solid line.

Table 4-4 Summary of modified rate coefficients used for modeling radical polymerization of $0.1 \mathrm{~g} \mathrm{~g}^{-1}$ non-ionized methacrylic acid in aqueous solution from 35 to $50^{\circ} \mathrm{C}$ and $65^{\circ} \mathrm{C}$ for MMDs, respectively. This table complements Table 4-3.

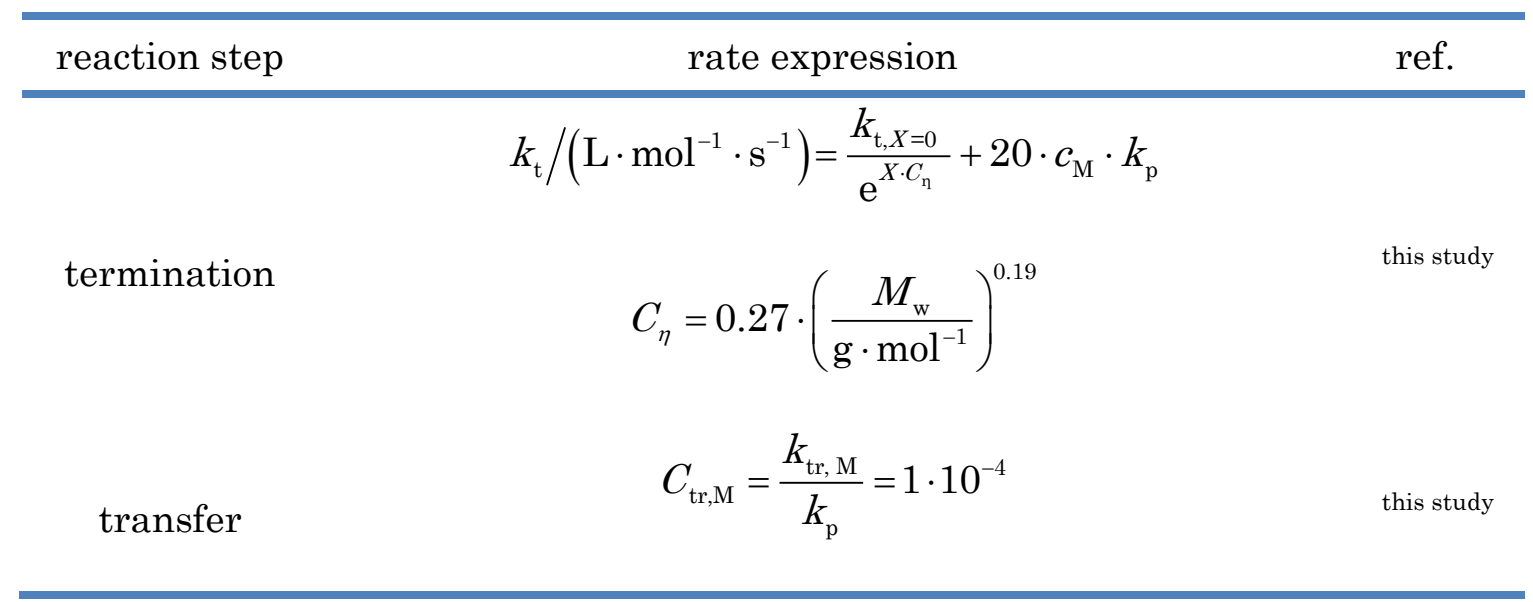





\section{5 \\ Acrylic Acid}

The polymerization of AA (acrylic acid, IUPAC: propenoic acid) is of great industrial importance. Polyacrylic acid, pAA, is the most important superabsorber material, e.g., part of hygiene and cosmetics products as well as in packaging and soil improvement. pAA is widely used as thickener, dispersant and emulsifier. It is applied, e.g., in wastewater treatment, mining, textile, and paper industry.

Each monomer unit of the polymer bears an ionizable moiety making pAA a polyelectrolyte. pAA is a weak electrolyte, thus its degree of ionization varies vigorously with $\mathrm{pH}$. Solutions of $\mathrm{pAA}$ have interesting properties as the structure of the polymer varies a great deal with degree of ionization and ionic strength as well as the nature of counterions. ${ }^{[82,84-87,163-166]}$

The influences of ionization and ionic strength are not limited to effects on the structure of the polymer in solution; they have a great impact on polymerization kinetics as well. In subchapter 5.1, polymerization kinetics and modeling of AA at its natural $\mathrm{pH}$ is discussed. Ionization and ionic strength are addressed in subchapter 5.2

The effect of ionization on overall polymerization kinetics of AA has been studied by several groups. ${ }^{[12,68,70,74,80,83,167]}$ Propagation has been examined separately by PLPSEC, for both non-ionized ${ }^{[37,57,91]}$ and partly as well as fully ionized monomer. ${ }^{[76]}$ Copolymerization, e.g., with AAm has been studied and a pronounced dependence on $\mathrm{pH}$ was found. Both reactivity ratios vary with degree of ionization, so does the rate of polymerization. ${ }^{[75,168]}$ Theoretical estimates of $k_{\mathrm{p}}$ for non-ionized monomer have been carried out to explain the concentration effect ${ }^{[63,64]}$ (see subchapter 2.4.2) as well 
as the influence of ionization. ${ }^{[169]}$ Termination, backbiting and propagation of MCRs have been studied by modeling radical concentration vs. time traces from SP-PLPEPR of both non-ionized ${ }^{[36]}$ and fully ionized ${ }^{[77]}$ AA.

\subsection{Model development for Non-ionized Acrylic Acid}

Batch radical polymerization of non-ionized acrylic acid, 0.1 and $0.3 \mathrm{~g} \mathrm{~g}^{-1}$ in aqueous solution, has been studied at ambient pressure between 35 and $80{ }^{\circ} \mathrm{C}$ under variation of initiator concentration and upon addition of different levels of 2-mercaptoethanol as CTA. Furthermore, high-temperature experiments were carried out between 90 and $170{ }^{\circ} \mathrm{C}$ at 200 bar at different levels of initiator concentration in a batch and in a tubular reactor. ${ }^{\mathrm{XXI}}$

Initial polymerization rate decreases with CTA concentration, which has been varied up to $n_{\mathrm{CTA}} / n_{\mathrm{M}}=0.28$. Accurate kinetic modeling of these polymerizations requires chain-length-dependent termination as has been shown for MAA (see subchapter 4.2).

An overview of the relevant reactions and associated rate coefficients is given in Table 5-1. The basic kinetic scheme given in the first part of Table 5-1 is similar to the one for MAA polymerization given in Table 4-2. Because of backbiting taking place with AA, the scheme has to be extended by MCR kinetics presented in Table 5-1 second part. The MCR related reactions have been described in subchapter 2.3.3. Modeling at high temperature requires even more reactions taken into account. They are listed in the last part of Table 5-1. The associated reactions are discussed in subchapter 2.3.1.

xxI The high-temperature polymerizations in a tubular reactor device have been carried out by Daniel Weiß.[148] 
Table 5-1 Individual reaction steps used for modeling radical polymerization of non-ionized acrylic acid in aqueous solution.

\section{Basic kinetics}

initiator decomposition

chain initiation

propagation of SPRs

by combination

SPR-SPR termination

by disproportionation

to monomer

to CTA

$$
\mathrm{I} \stackrel{k_{\mathrm{d}} \cdot f}{\longrightarrow} 2 \mathrm{R}_{0}
$$

$$
\mathrm{R}_{0}^{\bullet}+\mathrm{M} \stackrel{k_{\mathrm{i}}}{\longrightarrow} \mathrm{R}_{\mathrm{SPR}, 1}^{\bullet}
$$

$$
\mathrm{R}_{\mathrm{SPR}, i}^{\cdot}+\mathrm{M} \stackrel{k_{\mathrm{p}}^{\mathrm{s}}}{\longrightarrow} \mathrm{R}_{\mathrm{SPR}, i+1}^{\cdot}
$$

$$
\mathrm{R}_{\mathrm{SPR}, i}^{\cdot}+\mathrm{R}_{\mathrm{SPR}, j}^{\cdot} \stackrel{k_{\mathrm{t}}^{\mathrm{ss}} \cdot\left(1-\kappa^{\mathrm{ss}}\right)}{\longrightarrow} \mathrm{P}_{i+j}
$$

$$
\mathrm{R}_{\mathrm{SPR}, i}^{\cdot}+\mathrm{R}_{\mathrm{SPR}, j}^{\cdot} \stackrel{k_{\mathrm{t}}^{\mathrm{ss}} \cdot \kappa^{\mathrm{ss}}}{\longrightarrow} \mathrm{P}_{i}+\mathrm{P}_{j}
$$

$$
\mathrm{R}_{\mathrm{SPR}, i}^{\cdot}+\mathrm{M} \stackrel{k_{\mathrm{tr}, \mathrm{M}}^{\mathrm{s}}}{\longrightarrow} \mathrm{P}_{i}+\mathrm{R}_{\mathrm{SPR}, 1}^{\cdot}
$$

$$
\mathrm{R}_{\mathrm{SPR}, i}^{\cdot}+\mathrm{CTA} \stackrel{k_{\mathrm{tr}, \mathrm{CTA}}^{\mathrm{s}}}{\longrightarrow} \mathrm{P}_{i}+\mathrm{R}_{\mathrm{SPR}, 1}^{\bullet}
$$

\section{MCR kinetics}

backbiting

propagation of MCRs

SPR-MCR termination

MCR-MCR

termination

chain transfer of

MCRs by disproportionation

by combination

by disproportionation

$$
\text { by combination }
$$

to monomer

to CTA

$$
\mathrm{R}_{\mathrm{SPR}, i}^{\bullet} \stackrel{k_{\mathrm{bb}}}{\longrightarrow} \mathrm{R}_{\mathrm{MCR}, i}^{\bullet}
$$

$$
\mathrm{R}_{\mathrm{MCR}, i}^{\cdot}+\mathrm{M} \stackrel{k_{\mathrm{p}}^{\mathrm{t}}}{\longrightarrow} \mathrm{R}_{\mathrm{SPR}, i+1}^{\cdot}
$$

$$
\mathrm{R}_{\mathrm{SPR}, i}^{\cdot}+\mathrm{R}_{\mathrm{MCR}, j}^{\cdot} \stackrel{k_{\mathrm{t}}^{\mathrm{st}} \cdot\left(1-\kappa^{\mathrm{st}}\right)}{\longrightarrow} \mathrm{P}_{i+j}
$$

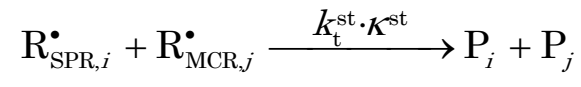

$$
\mathrm{R}_{\mathrm{MCR}, i}^{\cdot}+\mathrm{R}_{\mathrm{MCR}, j} \stackrel{k_{\mathrm{t}}^{\mathrm{tt}} \cdot\left(1-\kappa^{\mathrm{tt}}\right)}{\longrightarrow} \mathrm{P}_{i+j}
$$

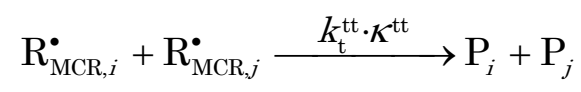

$$
\mathrm{R}_{\mathrm{MCR}, i}^{\cdot}+\mathrm{M} \stackrel{k_{\mathrm{tr}, \mathrm{M}}^{\mathrm{t}}}{\longrightarrow} \mathrm{P}_{i}+\mathrm{R}_{\mathrm{SPR}, 1}^{\cdot}
$$


Additional high temperature reactions

\begin{tabular}{cc}
\hline \multicolumn{1}{c}{$\mathrm{MCR}^{\mathrm{s}}$} & $\mathrm{R}_{\mathrm{MCR}^{\mathrm{s}}, i}^{\cdot} \stackrel{k_{\beta}}{\longrightarrow} \mathrm{R}_{\mathrm{SPR}, i-2}+\mathrm{MM}_{2} \dot{\vee} \mathrm{R}_{\mathrm{SPR}, 3}+\mathrm{MM}_{i-3}$ \\
SPR adding MM & $\mathrm{R}_{\mathrm{MCR}^{1}, i+j}^{\cdot \stackrel{k_{\beta}}{\longrightarrow}} \mathrm{R}_{\mathrm{SPR}, i}+\mathrm{MM}_{j} \dot{\vee} \mathrm{R}_{\mathrm{SPR}, j}+\mathrm{MM}_{i}$ \\
propagation of $\mathrm{MCR}^{1}$ & $\mathrm{R}_{\mathrm{SPR}, i}^{\cdot}+\mathrm{MM}_{j} \stackrel{k_{\mathrm{p}, \mathrm{MM}}^{\mathrm{s}}}{\longrightarrow} \mathrm{R}_{\mathrm{MCR}^{1}, i+j}^{\cdot}$ \\
& $\mathrm{R}_{\mathrm{MCR}^{1}, i+j}^{\cdot}+\mathrm{M} \stackrel{k_{\mathrm{p}}^{\mathrm{t}}}{\longrightarrow} \mathrm{R}_{\mathrm{SPR}, i+j+1}^{\cdot}$ \\
\hline
\end{tabular}

Table 5-2 summarizes the numerous rate coefficients, almost all of which have been determined or modified in this work. Initiator decay, propagation of SPRs, termination of SPRs, transfer to CTA, and backbiting are available from independent experiments. Chain-length dependency of termination rate was included, because polymerization under addition of CTA was modeled as well. Transfer to monomer was obtained rather independently from the MMDs of the product of polymerization with low rate of initiation.

MCR kinetics is less certain and was subject to fitting. As simulation results are rather insensitive towards MCR termination the main fitting factor was $k_{\mathrm{p}}^{\mathrm{t}} / k_{\mathrm{p}}^{\mathrm{s}}$.

Table 5-2 Summary of rate coefficients and of other parameters used for modeling radical polymerization of non-ionized acrylic acid in aqueous solution up to $90^{\circ} \mathrm{C}$ at ambient pressure.

\begin{tabular}{ccc}
\hline reaction step & rate expression & ref. \\
\hline decay of $\mathrm{V}-50$ & $f=0.8$ & [156] \\
& $k_{\mathrm{d}} / \mathrm{s}^{-1}=2.24 \cdot 10^{15} \exp \left(\frac{-1.52 \cdot 10^{4}}{(T / \mathrm{K})}\right)$ & [90] \\
decay of & $k_{\mathrm{d}} / \mathrm{s}^{-1}=1.24 \cdot 10^{13} \exp \left(\frac{-1.48 \cdot 10^{4}}{(T / \mathrm{K})}\right)$ & this study \\
VA-086 & $f=0.38$ & [170]
\end{tabular}




$$
\begin{aligned}
& k_{\mathrm{p}}^{\mathrm{s}} / \mathrm{L} \mathrm{mol}^{-1} \mathrm{~s}^{-1}=\left(1.2 \cdot 10^{8}(0.063+(1-0.063) \cdot \exp (-17 \cdot W))\right) \\
& \text { propagation } \\
& \cdot \exp \left(\frac{-\left(6.7 \cdot \exp (-W \cdot 8.6)+\frac{2.6}{1+50 \cdot \exp (-W \cdot 9.9)}+10.4\right)}{R /\left(\mathrm{J} \mathrm{mol}^{-1} \mathrm{~K}^{-1}\right) \cdot T / \mathrm{K}}\right){ }_{\text {this study }} \\
& k_{\mathrm{p}}^{\mathrm{t}} / k_{\mathrm{p}}^{\mathrm{s}}=2.41 \cdot \exp \left(-\frac{2314}{T / \mathrm{K}}\right) \\
& k_{\mathrm{t}}^{1,1, \mathrm{ss}} /\left(\mathrm{L} \cdot \mathrm{mol}^{-1} \cdot \mathrm{s}^{-1}\right)=1.9 \cdot 10^{12} \exp \left(-\frac{1.86 \cdot 10^{3}}{(T / \mathrm{K})}\right) \text { xxII } \\
& \left\langle i_{\mathrm{n}}\right\rangle \leq 30 \text { : } \\
& k_{\mathrm{t}, X=0} /\left(\mathrm{L} \cdot \mathrm{mol}^{-1} \cdot \mathrm{s}^{-1}\right)=k_{\mathrm{t}}^{1,1} \cdot\left\langle i_{\mathrm{n}}\right\rangle^{-0.8} \\
& \left\langle i_{\mathrm{n}}\right\rangle>30 \text { : } \\
& k_{\mathrm{t}, X=0} /\left(\mathrm{L} \cdot \mathrm{mol}^{-1} \cdot \mathrm{s}^{-1}\right)=k_{\mathrm{t}}^{1,1} \cdot 30^{-0.64} \cdot\left\langle i_{\mathrm{n}}\right\rangle^{-0.16} \\
& k_{\mathrm{t}}^{1,1}=k_{\mathrm{t}}^{1,1}\left(0.1 \mathrm{~g} \mathrm{~g}^{-1}\right) \cdot \zeta_{\eta W} \\
& \zeta_{\eta w}=1.13-1.22 \cdot W_{\mathrm{AA}}-0.968 \cdot{W_{\mathrm{AA}}}^{2}+1.76 \cdot{W_{\mathrm{AA}}}^{3} \\
& k_{\mathrm{t}}^{1,1, \mathrm{tt}} / k_{\mathrm{t}}^{1,1, \mathrm{ss}}=0.1 \\
& k_{\mathrm{t}}^{\mathrm{st}}=0.5 \cdot\left(k_{\mathrm{t}}^{\mathrm{tt}}+k_{\mathrm{t}}^{\mathrm{ss}}\right)
\end{aligned}
$$

termination

$$
\begin{gathered}
k_{\mathrm{tc}}^{\mathrm{ss}} /\left(\mathrm{L} \cdot \mathrm{mol}^{-1} \cdot \mathrm{s}^{-1}\right)=(1-0.05) \cdot k_{\mathrm{t}}^{\mathrm{ss}} \\
k_{\mathrm{td}}^{\mathrm{ss}} /\left(\mathrm{L} \cdot \mathrm{mol}^{-1} \cdot \mathrm{s}^{-1}\right)=0.05 \cdot k_{\mathrm{t}}^{\mathrm{ss}} \\
k_{\mathrm{tc}}^{\mathrm{st}} /\left(\mathrm{L} \cdot \mathrm{mol}^{-1} \cdot \mathrm{s}^{-1}\right)=(1-0.4) \cdot k_{\mathrm{t}}^{\mathrm{st}} \\
k_{\mathrm{td}}^{\mathrm{st}} /\left(\mathrm{L} \cdot \mathrm{mol}^{-1} \cdot \mathrm{s}^{-1}\right)=0.4 \cdot k_{\mathrm{t}}^{\mathrm{st}} \\
k_{\mathrm{tc}}^{\mathrm{tt}} /\left(\mathrm{L} \cdot \mathrm{mol}^{-1} \cdot \mathrm{s}^{-1}\right)=(1-0.8) \cdot k_{\mathrm{t}}^{\mathrm{tt}} \\
k_{\mathrm{td}}^{\mathrm{tt}} /\left(\mathrm{L} \cdot \mathrm{mol}^{-1} \cdot \mathrm{s}^{-1}\right)=0.8 \cdot k_{\mathrm{t}}^{\mathrm{tt}} \\
k_{\mathrm{t}} /\left(\mathrm{L} \cdot \mathrm{mol}^{-1} \cdot \mathrm{s}^{-1}\right)=\frac{k_{\mathrm{t}, X=0}}{\mathrm{e}^{X \cdot C_{\mathrm{n}}}}+20 \cdot c_{\mathrm{M}} \cdot k_{\mathrm{p}} \\
C_{\eta}=360 \cdot W^{3.7} \\
0 \leq W_{\mathrm{AA}}^{0} \leq 0.3
\end{gathered}
$$

backbiting

$$
k_{\mathrm{bb}} / \mathrm{s}^{-1}=4.25 \cdot 10^{9} \cdot \exp \left(\frac{-5020}{T / \mathrm{K}}\right)
$$

$$
C_{\mathrm{tr}, \mathrm{M}}^{\mathrm{s}}=\frac{k_{\mathrm{tr}, \mathrm{M}}^{\mathrm{s}}}{k_{\mathrm{p}}^{\mathrm{s}}}=7.5 \cdot 10^{-5}
$$

this study

xxII The value is already corrected for a distribution of chain lengths (compare subchapter 4.2). 


$$
\begin{gathered}
C_{\mathrm{tr}, \mathrm{M}}^{\mathrm{t}}=\frac{k_{\mathrm{tr}, \mathrm{M}}^{\mathrm{t}}}{k_{\mathrm{p}}^{\mathrm{t}}} \simeq 7.5 \cdot 10^{-5} \\
C_{\mathrm{CTA}}^{\mathrm{s}}=\frac{k_{\mathrm{tr}, \mathrm{CTA}}^{\mathrm{s}}}{k_{\mathrm{p}}^{\mathrm{s}}}=0.28 \pm 0.04 \\
\begin{array}{c}
\text { transfer to } \\
\mathrm{ME}
\end{array} \\
C_{\mathrm{CTA}}^{\mathrm{t}}=\frac{k_{\mathrm{tr}, \mathrm{CTA}}^{\mathrm{t}} \simeq 0.28}{k_{\mathrm{p}}^{\mathrm{t}}} \\
\rho_{\mathrm{AA}} /\left(\mathrm{g} \cdot \mathrm{mL}^{-1}\right)=1.0731-1.0826 \cdot 10^{-3} \cdot\left(\vartheta /{ }^{\circ} \mathrm{C}\right) \\
-7.237910^{-7} \cdot\left(\vartheta /{ }^{\circ} \mathrm{C}\right)^{2} \\
\rho_{\mathrm{H}_{2} \mathrm{O}} /\left(\mathrm{g} \cdot \mathrm{mL}^{-1}\right)=0.9999+2.3109 \cdot 10^{-5} \cdot\left(\vartheta /{ }^{\circ} \mathrm{C}\right) \\
-5.44807 \cdot 10^{-6} \cdot\left(\vartheta /{ }^{\circ} \mathrm{C}\right)^{2}
\end{gathered} \quad \text { this study }
$$

\subsubsection{Initiator Kinetics}

According to ideal polymerization kinetics (see subchapter 2.2), rate of initiation enhances the rate of polymerization and lowers molar mass by a square root dependence. The simple kinetic scheme does not strictly apply for AA polymerization, but should be valid to a good approximation.

Even though initiator decay is generally understood as a first order reaction, it is often influenced by the concentration of other compounds (see subchapters 2.2.1 and 2.4 especially 2.4.3).

The initiators used for chemical initiation of polymerizations presented here are the azo compounds V-50 and VA-086. The slow initiator decay ensures almost constant initiator content during most of the reactions and avoids dead-end polymerization. Furthermore, a slow rate of polymerization is important in that the temperature of the polymerization mixture may be kept constant.

Azo compounds were selected for initiation, as thiols and peroxides may form a redox initiation system. ${ }^{[155]}$ Moreover, initiator decay should be independent of $\mathrm{pH}$, which requirement is more or less fulfilled by $\mathrm{V}-50^{[156]}$ and perfectly holds for VA-086, as will be presented below.

The initiator efficiency of V-50 was assumed to be $f=0.8$, which is a typical value, that had been successfully used for modeling before. ${ }^{[90]}$ The initiator efficiency of VA-086 has been reported to be $0.38 .{ }^{[170]}$ 


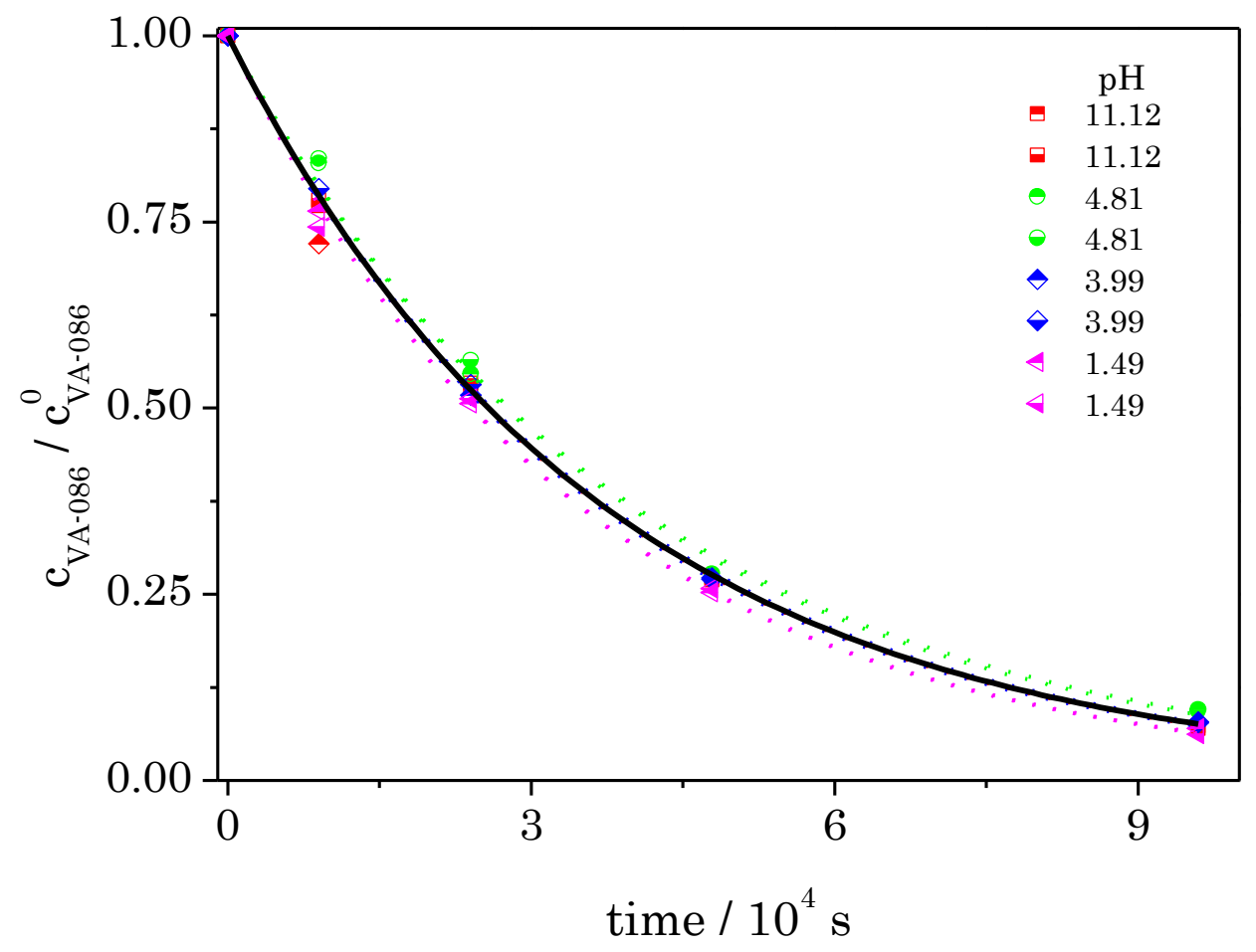

Figure 5-1 Exponential fit via eq. (5.1), of time-dependent relative initiator concentration at $90^{\circ} \mathrm{C}$. Buffer solutions have been prepared to simulate different degrees of ionization of monomer; $\mathrm{pH}=11.12$ (square), 4.81 (circle), 3.99 (diamond), 1.49 (triangle). Symbols: measured data, dashed line: fit; solid line: concatenate fit.

The decay of VA-086 has been measured for different $\mathrm{pH}$ in buffer solutions (see subchapter 3.8) prepared to simulate different degrees of ionization of monomer. Samples were heated in a heating block and analyzed by HPLC subsequently (see subchapter 3.11). The resulting relative concentrations as a function of time are shown in Figure 5-1. The exponential fit, eq. (5.1), yields $k_{\mathrm{d}}$. Independent fits for each $\mathrm{pH}$ value $(11.12,4.81,3.99$, and 1.49) yield identical results of good reproducibility. The concatenate fit gives: $k_{\mathrm{d}, 90^{\circ} \mathrm{C}}=(2.69 \pm 0.03) \cdot 10^{-5} \mathrm{~s}^{-1}$, which is in fair agreement with $k_{\mathrm{d}, 90^{\circ} \mathrm{C}}=2.90 \cdot 10^{-5} \mathrm{~s}^{-1}$, which is the value provided by the manufacturer. ${ }^{[156]}$

$$
c / c^{0}=\exp \left(-k_{\mathrm{d}} \cdot t\right)
$$




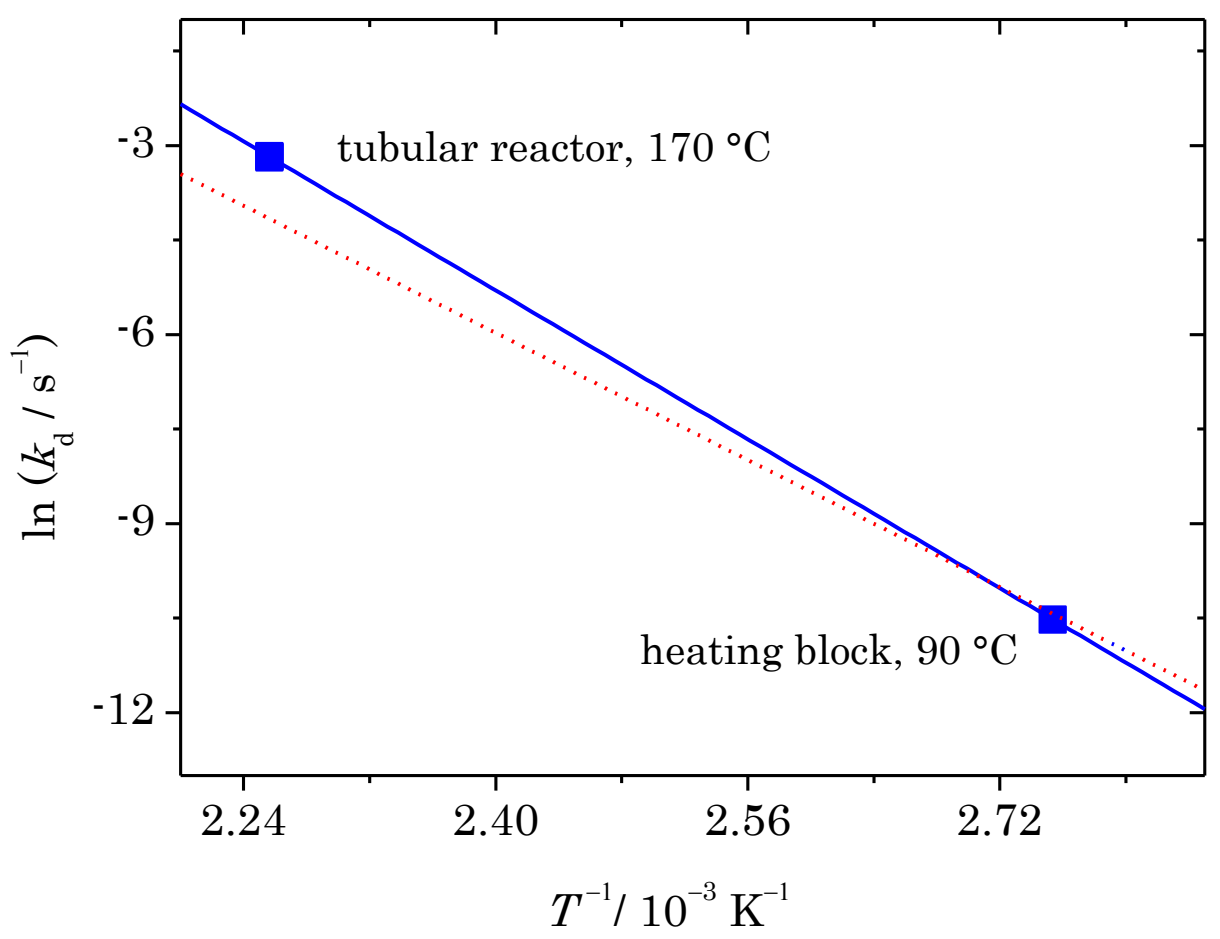

Figure 5-2 Squares: measured $k_{\mathrm{d}}$; solid line: best fit to straight line yielding eq. (5.3), red, dotted line: plot with Arrhenius parameter provided by the supplier, eq. (5.2).[156]

$k_{\mathrm{d}}$ was also measured at the highest temperature of the tubular reactor experiments, $170{ }^{\circ} \mathrm{C}$. Analogously to the polymerization procedure, an initiator solution was heated at different flow rates and the solution was measured by HPLC afterwards. Eq. (5.1) yields: $k_{\mathrm{d}, 170^{\circ} \mathrm{C}}=4.2 \cdot 10^{-2} \mathrm{~s}^{-1}$.

The measured $k_{\mathrm{d}}$ values and the Arrhenius lines according to equation, eq. (2.23), with values from the supplier, eq. (5.2), as well as the best fit to the measured data points, eq. (5.3), are plotted in Figure 5-2. The latter equation is used for modeling. The activation energy of $123 \mathrm{~kJ} \mathrm{~mol}^{-1}$ associated with eq. (5.3) appears to be more realistic than the supplier value of $105 \mathrm{~kJ} \mathrm{~mol}^{-1}$.

$$
k_{\mathrm{d}} / \mathrm{s}^{-1}=3.54 \cdot 10^{10} \exp \left(\frac{-1.26 \cdot 10^{4}}{(T / \mathrm{K})}\right)
$$




$$
k_{\mathrm{d}} / \mathrm{s}^{-1}=1.24 \cdot 10^{13} \exp \left(\frac{-1.48 \cdot 10^{4}}{(T / \mathrm{K})}\right)
$$

\subsubsection{Evaluation of $k_{\mathrm{p}}^{\mathrm{s}}$ data}

Both rate of polymerization and molar mass scale with $k_{\mathrm{p}}$, therefore accurate values of $k_{\mathrm{p}}$ are essential for modeling. In this subchapter, the evaluation of both published $^{[37]}$ and unpublished PLP-SEC results from Lacík et al. for non-ionized AA is presented.XxIII Moreover, the monomer concentration effect on $k_{\mathrm{p}}$ and $k_{\mathrm{p}}^{\mathrm{s}}$, respectively, in aqueous solution is discussed.

The $k_{\mathrm{p}}^{\mathrm{s}}$ values discussed here refer to negligible conversion, while modeling was carried out for polymerizations up to full conversion. Polymer in the reaction mixture is not included for calculation of $w$ in the model, as it has been shown for MAA polymerization that $k_{\mathrm{p}}$ only refers to the monomer to water ratio. ${ }^{[9]}$

The most probable combination of $A$ and $E_{\mathrm{A}}$ is given in Figure 5-3 for different weight fractions of monomer. Joint $95 \%$ confidence intervals are presented for the high monomer contents $\left(W \geq 01 \mathrm{~g} \mathrm{~g}^{-1}\right)$, whereas for the less accurate data at low AA content joint $75 \%$ confidence intervals are given. A strong dependence of $A$ on monomer content can be observed. A smaller dependence of $E_{\mathrm{A}}$ on monomer content cannot be ruled out.

Two approaches have been considered to fit an expression for $k_{\mathrm{p}}^{\mathrm{s}}$ as a function of temperature and weight fraction of monomer. Depending on the desired accuracy, the dependence of $E_{\mathrm{A}}$ on AA content may be ignored. In this case, the maximum of $k_{\mathrm{p}}^{\mathrm{s}}$ around $W=0.03 \mathrm{~g} \mathrm{~g}^{-1}$ can be ascribed entirely to $A$ and an averaged $E_{\mathrm{A}}$, $11.6 \mathrm{~kJ} \mathrm{~mol}^{-1}$, deduced from the data for $0.2 \mathrm{~g} \mathrm{~g}^{-1}$ and $0.4 \mathrm{~g} \mathrm{~g}^{-1}$ AA may be used, as the polymerization of this amount of monomer is of highest interest. $A$ values may be calculated and fitted to an equation of the same form as has been used before to obtain a good representation of $k_{\mathrm{p}}^{\mathrm{s}}$ without maximum. ${ }^{[121]}$ This procedure yields the first term of eq. (5.4) RHS. Calculating the difference between $A$ values from

XxIII Thus, all $k_{\mathrm{p}}^{\mathrm{s}}$ measurements were carried out the Lacík group. 
measured $k_{\mathrm{p}}^{\mathrm{s}}$ and the first term of eq. (5.4) RHS and fitting those exponentially yields the second term of eq. (5.4) RHS. Eq. (5.4) provides a good representation of $k_{\mathrm{p}}^{\mathrm{s}}$ between 10 and $60^{\circ} \mathrm{C}$. However, for low AA content $\left(w=0.06 \mathrm{~g} \mathrm{~g}^{-1}\right)$, eq. (5.4) RHS $1^{\text {st }}$ term underestimates $k_{\mathrm{p}}^{\mathrm{s}}$ at $10^{\circ} \mathrm{C}$ and overestimates it at $60{ }^{\circ} \mathrm{C}$. If the value of $k_{\mathrm{p}}^{\mathrm{s}}$ at low monomer content $\left(W \leq 0.06 \mathrm{~g} \mathrm{~g}^{-1}\right)$ is not of interest, the first term of eq. (5.4) RHS will be sufficient for an adequate estimation of $k_{\mathrm{p}}^{\mathrm{s}}$.

$$
\begin{aligned}
k_{\mathrm{p}}^{\mathrm{s}} / \mathrm{L} \mathrm{mol}^{-1} \mathrm{~s}^{-1}= & 1.94 \cdot 10^{7} \cdot \exp \left(-\frac{1395}{T / \mathrm{K}^{-1}}\right) \cdot(0.16+(1-0.16) \cdot \exp (-3.7 \cdot W)) \\
& -6.5 \cdot 10^{6} \cdot \exp \left(-\frac{1395}{T / \mathrm{K}^{-1}}\right) \cdot \exp (-59 \cdot W)
\end{aligned}
$$

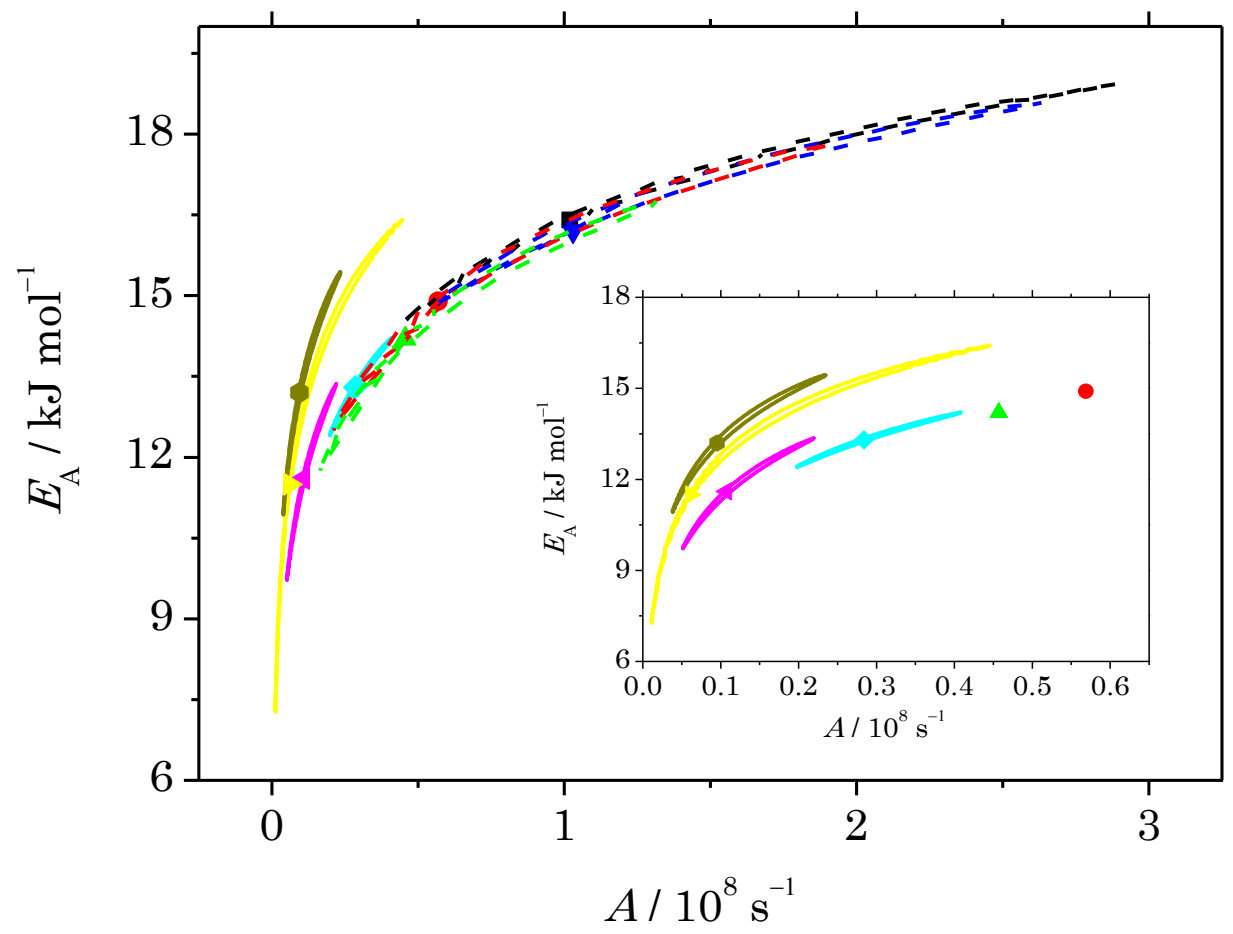

Figure 5-3 Joint confidence intervals of the Arrhenius parameters for $k_{\mathrm{p}}$ of non-ionized AA (lines) and the most probable combination (symbols) for different weight fractions of AA. Joint $95 \%$ confidence intervals (solid lines) are given for monomer concentrations up to $w=0.1$ and, as accuracy of the data of lower concentration is notably lower, joint $75 \%$ confidence intervals (dashed line) are depicted for lower AA content. $w=0.01$ (black), 0.02 (red), 0.03 (green), 0.05 (blue), 0.1 (aqua), 0.2 (magenta), 0.4 (yellow), 0.6 (brown); symbols: best values. Data for high $w$ is also shown enlarged. 
It is highly desirable to have an adequate representation for the entire range of concentrations and temperatures under investigation. Moreover, for extrapolation towards high temperature, e.g., the modeling presented in subchapter 5.1.9, it is essential to use an activation energy as precise as possible. Given this, dependency of $E_{\mathrm{A}}$ on monomer content was included in the modeling presented.

The $A$ values taken from Figure 5-3 are depicted as a function of weight fraction in Figure 5-4, with the accuracy of values corresponding to lower monomer content being lower as has been shown before. As the effect of monomer content on $k_{\mathrm{p}}^{\mathrm{s}}$ arises mostly from a difference in entropy of the respective transition states, the course of $A$ is very similar to the one of $k_{\mathrm{p}}^{\mathrm{s}}$, yet, it does not exhibit a maximum. Accordingly, the $A$ values are fitted in the same form as $k_{\mathrm{p}}^{\mathrm{s}}$, but without a maximum ${ }^{[121]}$ yielding eq. (5.5). This equation allows for a good representation of data, which can be observed in Figure 5-4.

$$
A\left(k_{\mathrm{p}}^{\mathrm{s}}\right) / \mathrm{L}^{-1} \mathrm{~mol}^{-1} \mathrm{~s}^{-1}=1.2 \cdot 10^{8}(0.063+(1-0.063) \cdot \exp (-17 \cdot W))
$$

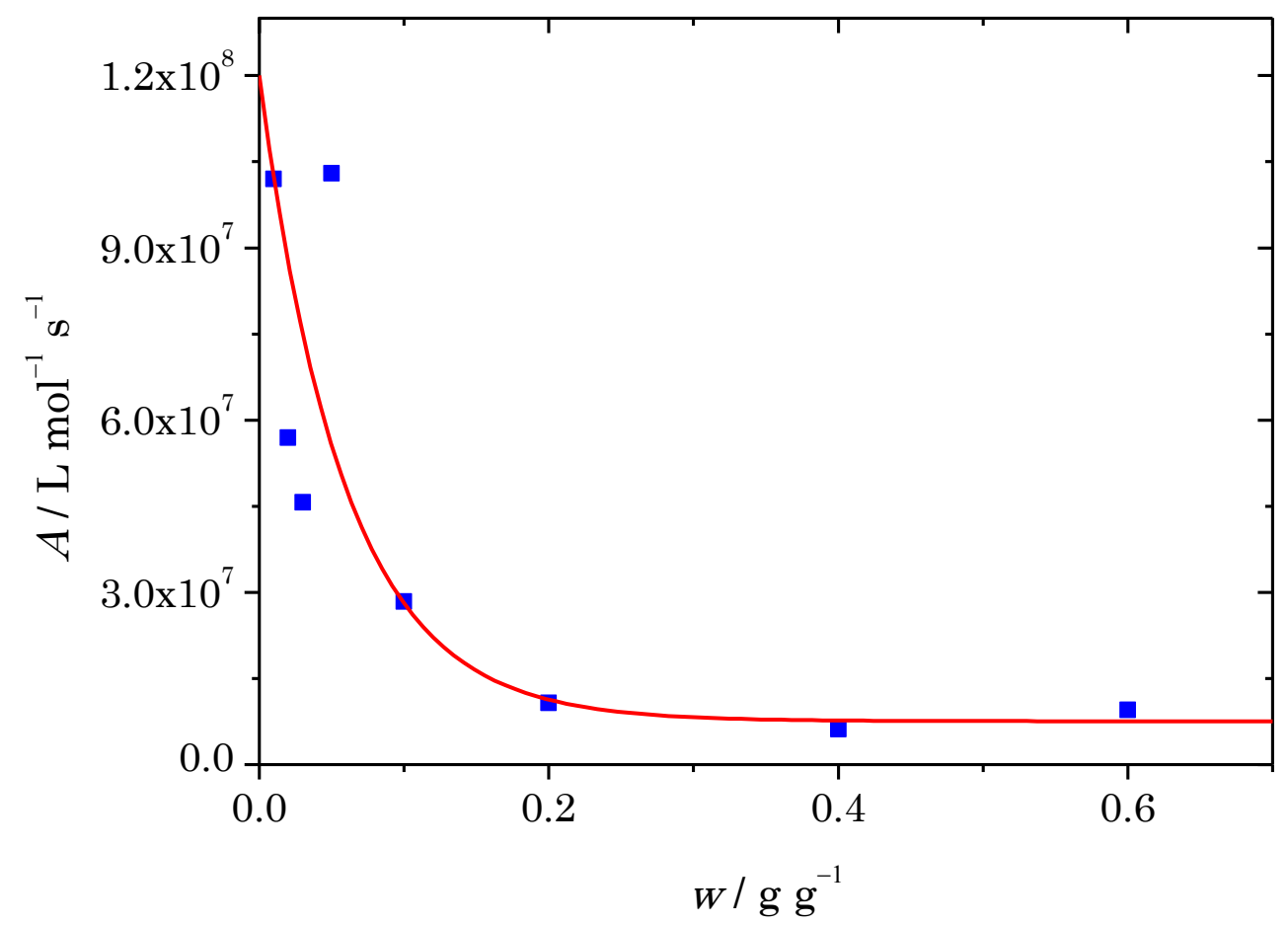

Figure 5-4 Squares: The Arrhenius pre-exponential factor shown in Figure 5-3 as a function of weight fraction of monomer. Line: Best fit, eq. (5.5). 
Although less variable than the pre-exponential factor, the activation energy seems to be a function of weight fraction of monomer as well, as is shown in Figure 5-5. The big star symbols denote $E_{\mathrm{A}}$ values from the original fitting presented in Figure 5-3. Furthermore, $E_{\mathrm{A}}$ values were calculated from measured $k_{\mathrm{p}}^{\mathrm{s}}$ values by combining eq. (5.5) for $A$ with the Arrhenius equation, eq. (2.23). This is depicted by the small star symbols, which were fitted empirically yielding eq. (5.6) plotted in Figure 5-5 as line.

$$
E_{\mathrm{A}}\left(k_{\mathrm{p}}^{\mathrm{s}}\right) / \mathrm{kJ} \mathrm{mol}^{-1}=6.7 \cdot \exp (-W \cdot 8.6)+\frac{2.6}{1+50 \cdot \exp (-W \cdot 9.9)}+10.4
$$

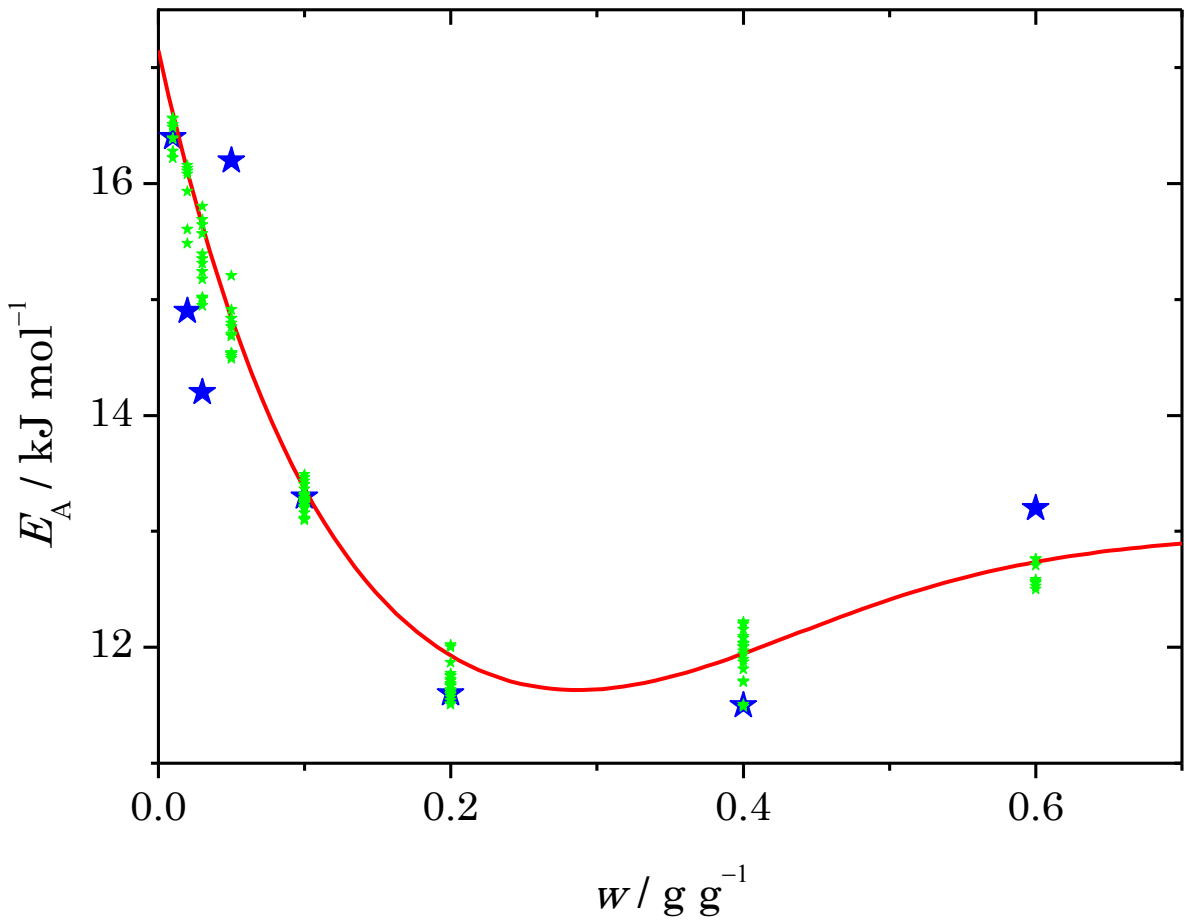

Figure 5-5 Big stars: Activation energy shown in Figure 5-3 as a function of AA weight fraction. Small stars: Activation energy derived from $k_{\mathrm{p}}$ values and eq. (5.5). Red line: Best fit of $E_{\mathrm{A}}$ (small stars), eq. (5.6). 
The combination of eq. (5.5), eq. (5.6), and eq. (2.23) yields eq. (5.7), which allows for an improved representation of the measured data and should be suitable for extrapolation.

$$
\begin{array}{r}
k_{\mathrm{p}}^{\mathrm{s}} / \mathrm{L} \mathrm{mol}^{-1} \mathrm{~s}^{-1}=\left(1.2 \cdot 10^{8}(0.063+(1-0.063) \cdot \exp (-17 \cdot W))\right) \\
\left.\cdot \exp \left(\frac{-\left(6.7 \cdot \exp (-W \cdot 8.6)+\frac{2.6}{1+50 \cdot \exp (-W \cdot 9.9)}+10.4\right)}{R /\left(\mathrm{J} \mathrm{mol}^{-1} \mathrm{~K}^{-1}\right) \cdot T / \mathrm{K}}\right)\right)
\end{array}
$$

In Figure $5-6, k_{\mathrm{p}}^{\mathrm{s}}$ is plotted as a function of temperature and AA weight fraction according to eq. (5.7). A marked dependence of $k_{\mathrm{p}}^{\mathrm{s}}$ on $W$ is seen. Moreover, a maximum of $k_{\mathrm{p}}^{\mathrm{s}}$ can be observed over the entire temperature range, which shifts towards smaller $w$ upon increasing temperature.

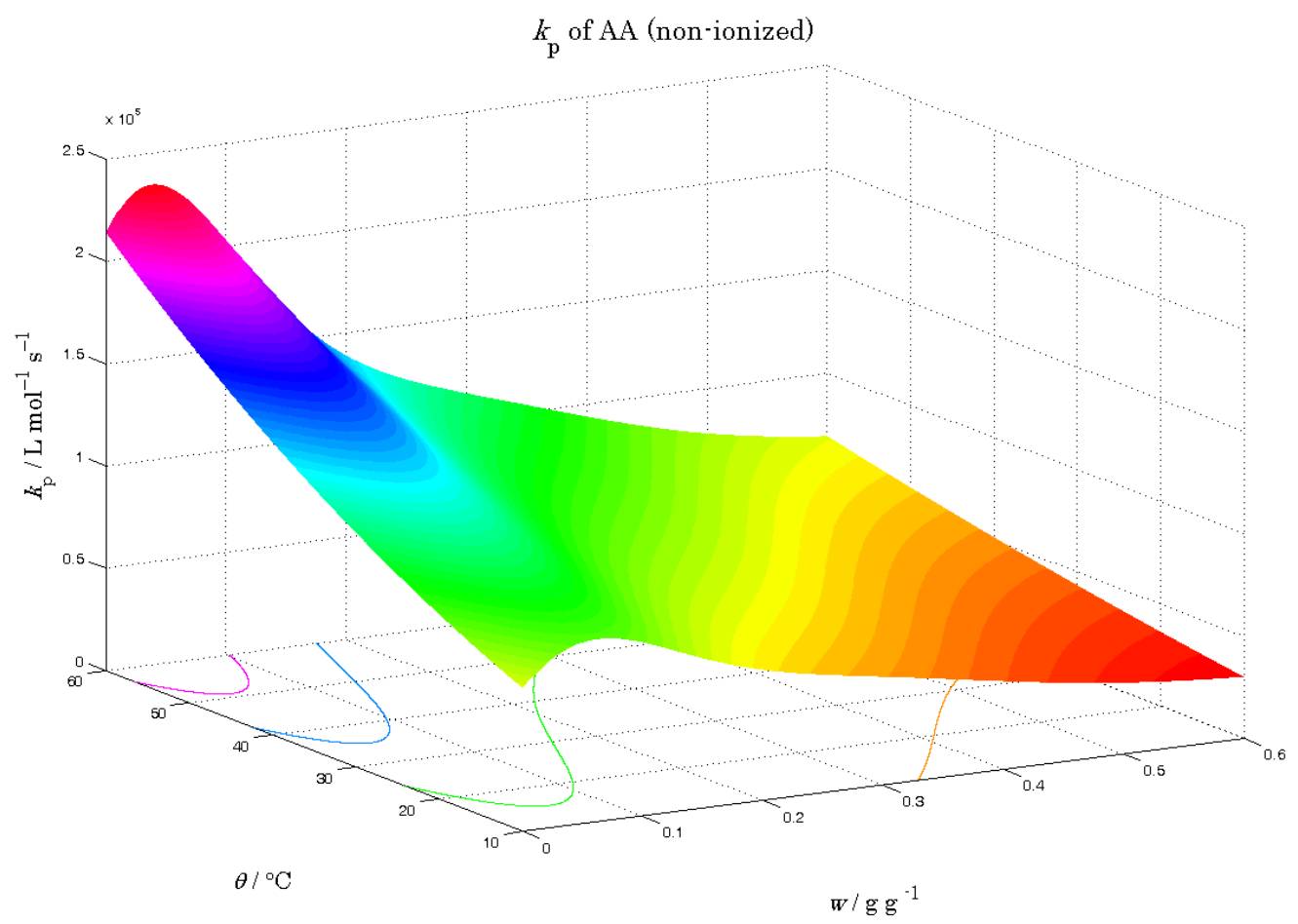

Figure 5-6 Plot of eq. (5.7) within the boundaries of strict validity. 
Eq. (5.7) is used for modeling $k_{\mathrm{p}}^{\mathrm{s}}$ up to high temperature. Therefore, the implications of the extrapolation should be considered. The plot of the equation within the temperature range of modeling is shown in Figure 5-7. Over such a broad temperature range, the dependency of $E_{\mathrm{A}}$ on $w$ has a marked effect. The values of $k_{\mathrm{p}}^{\mathrm{s}}$ at low concentration increase faster towards higher temperature than the ones at high concentration. Hence, the steepness of the increase of $k_{\mathrm{p}}^{\mathrm{s}}$ from concentrated aqueous AA solution towards higher dilution increases from $35^{\circ} \mathrm{C}$ up to $170{ }^{\circ} \mathrm{C}$. Interestingly, the maximum fades out and is predicted to disappear above approximately $150^{\circ} \mathrm{C}$.

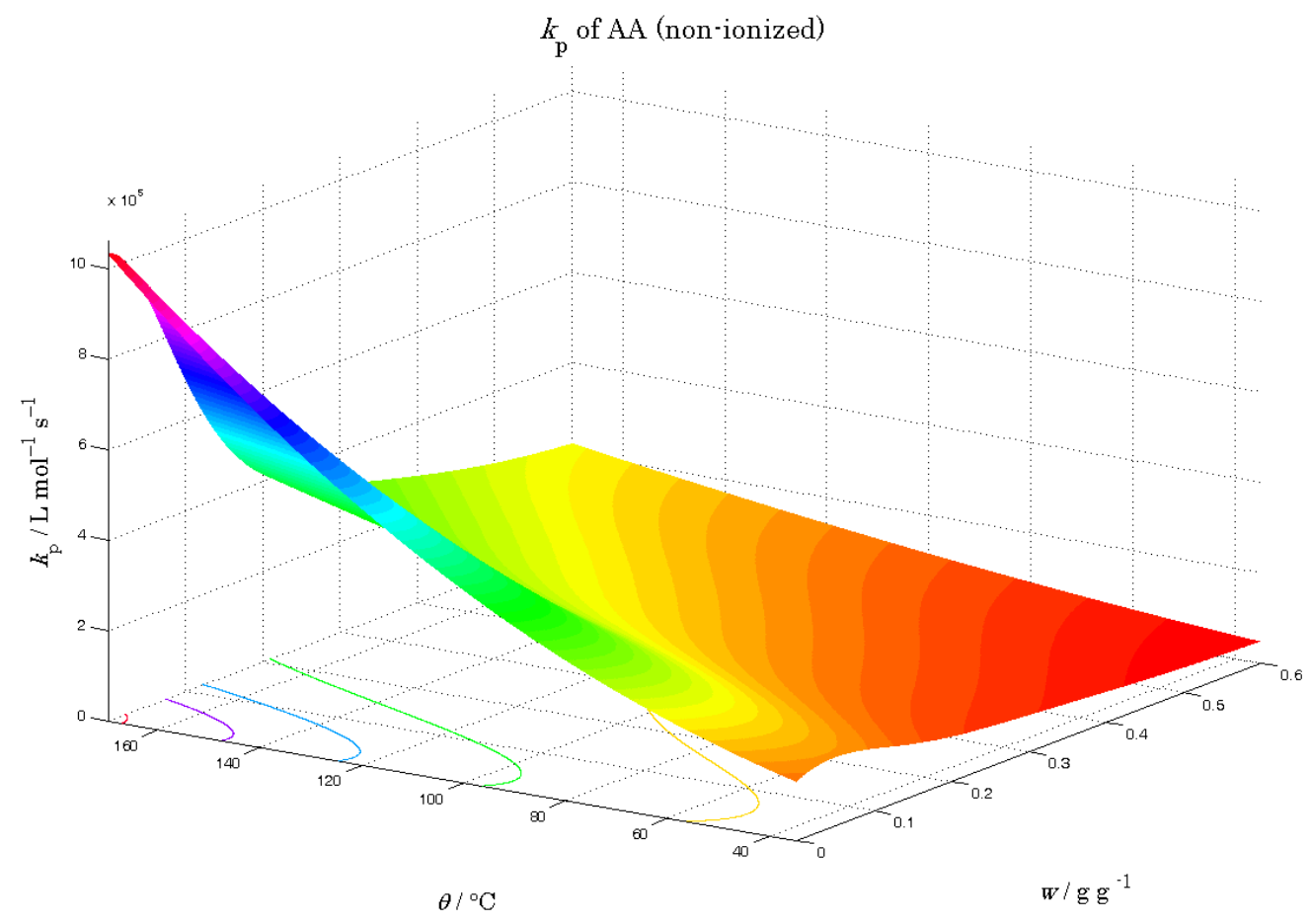

Figure 5-7 Plot of eq. (5.7) within the temperature range used in this work (including extrapolation).

\section{Discussing the behavior of $k_{p}$ in aqueous medium}

At infinite dilution, the environment of a growing chain consists of water molecules only. Towards higher monomer concentration, water molecules are replaced by monomer molecules. Hindrance of internal rotation of the transition-state structure for $k_{\mathrm{p}}$ increases. Finally, the environment of a growing chain in bulk consists exclusively of monomer molecules. The alteration in surroundings of the radical centre is not linear, but is most pronounced in the beginning and declines gradually. 
Generally, this is in agreement with experimental findings of $k_{\mathrm{p}}$ in aqueous solution. However, a maximum of $k_{\mathrm{p}}$ is difficult to reconcile with this simple approach. The analysis presented here may help to unraveled the fact of a maximum of $k_{\mathrm{p}}$ being present at about $0.03 \mathrm{~g} \cdot \mathrm{g}^{-1}$. Both $A$ and $E_{\mathrm{A}}$ depend on monomer content, the former to a larger extent, but neither of the dependencies exhibits a maximum. Instead, the maximum results from the combined dependency of $k_{\mathrm{p}}$ on $A$ and $E_{\mathrm{A}}$. This elucidates that detailed analysis is crucial and a small dependency like the one of $E_{\mathrm{A}}$ (Figure 5-5) may not be ignored when trying to understand kinetic behavior. More detailed studies are needed, like, e.g., calculations of solvent fields that consist of water and monomer in different ratios. So far, only calculations with a surrounding of pure water and toluene have been carried out (and only by treating the surrounding as a continuum or one water molecule and a continuum). ${ }^{63,64,169]}$

\subsubsection{Evaluation of $k_{\mathrm{t}}$ and Viscosity data}

Because of its diffusion-controlled nature, $k_{\mathrm{t}}$ scales with inverse viscosity (fluidity, see subchapter 2.4), hence, measurements of $k_{\mathrm{t}}$ and viscosity are compared in this section.

Fluidity of water-AA mixtures and of the two pure substances are plotted in Figure 5-8 as a function of AA content at $30^{\circ} \mathrm{C}$. The data points were fitted to a cubic function, which is used in the model to account for composition dependency of $k_{\mathrm{t}}$ at zero conversion. The correction factor for the influence of different monomer content on $k_{\mathrm{t}}, \zeta_{\eta w}$, is introduced by eq. (5.8).

$$
\begin{gathered}
\zeta_{\eta W}=1.13-1.22 \cdot W_{\mathrm{AA}}-0.968 \cdot W_{\mathrm{AA}}{ }^{2}+1.76 \cdot W_{\mathrm{AA}}{ }^{3} \\
k_{\mathrm{t}}^{1,1}=k_{\mathrm{t}}^{1,1}\left(0.1 \mathrm{~g} \mathrm{~g}^{-1}\right) \cdot \zeta_{\eta W}
\end{gathered}
$$




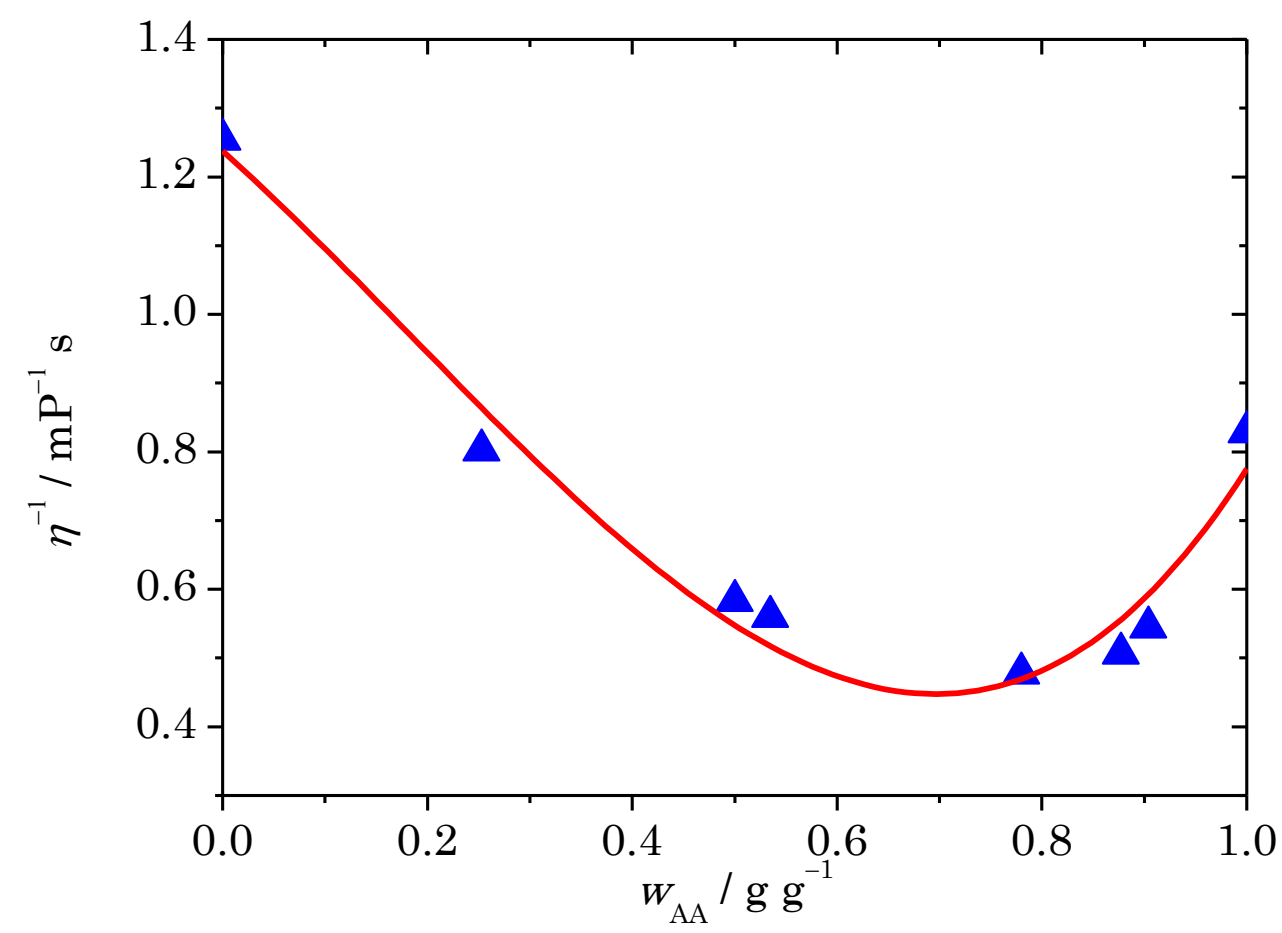

Figure 5-8 Triangles: fluidity of water/AA mixtures as a function of AA content at $30^{\circ} \mathrm{C}$. Data taken from ref. ${ }^{[171,172]}$ Line: best cubic fit used for eq. (5.9).

Experimental $k_{\mathrm{t}}^{1,1}$ values for $0.1 \mathrm{~g} \mathrm{~g}^{-1}$ and $0.5 \mathrm{~g} \mathrm{~g}^{-1}$ AA as well as the composite model (eq. (2.34)) parameters $\alpha_{\mathrm{s}}$ and $i_{\mathrm{c}}$ are available from literature. ${ }^{[36]}$ The theoretical value of $\alpha_{1}$ in good solvents, 0.16, was used in the model. ${ }^{[104-106]}$ However, this data is for SPR-SPR termination. MCR termination kinetics is discussed further below. The ratio of the $k_{\mathrm{t}}^{1,1}$ value for $0.1 \mathrm{~g} \mathrm{~g}^{-1}$ to $0.5 \mathrm{~g} \mathrm{~g}^{-1}$ AA is 6.1 , whereas the associated ratio of fluidities is $1.8 .^{[171,172]}$ The $0.1 \mathrm{~g} \mathrm{~g}^{-1}$ value was used and slightly adjusted for modeling, eq. (5.9) and eq. (5.10), as the corresponding $k_{\mathrm{p}}^{\mathrm{t}}$ and $k_{\mathrm{bb}}$ coefficients appeared to curate. The correction factor for a distribution of chain lengths (compare subchapter 4.2) is already included.

$$
k_{\mathrm{t}}^{1,1, \mathrm{ss}} /\left(\mathrm{L} \cdot \mathrm{mol}^{-1} \cdot \mathrm{s}^{-1}\right)=1.9 \cdot 10^{12} \exp \left(-\frac{1.86 \cdot 10^{3}}{(T / \mathrm{K})}\right)
$$


Chain-length dependency of termination was included in the model, because it is needed for polymerizations with CTA added to the polymerization mixture (see subchapter 4.2). Composite-model parameters have neither been measured of MCRSPR termination nor of MCR-MCR termination. Considering that $i_{\mathrm{c}}$ represents the point, at which control by center-of-mass diffusion is replaced by control via segmental diffusion, it may be smaller than the value for SPR homotermination. However, the $i_{\mathrm{c}}$ value for SPR homotermination is already rather low, so the SPR value was adopted for modeling of MCRs. For the same reason, the SPR $\alpha_{\mathrm{s}}$ value is also used for MCR termination. The theoretical value of $\alpha_{1}$ is different if radicals are located somewhere in the chain. ${ }^{[106]}$ For $\mathrm{MCR}^{\mathrm{s}}$ this is not the case, because they are located almost at the end of the chain. Furthermore, EPR experiments with BA (see subchapter 5.1.5) suggest that chain-length dependency of MCRs should be the same as for SPRs.

$$
\begin{aligned}
& \left\langle i_{\mathrm{n}}\right\rangle \leq 30: \\
& k_{\mathrm{t}, X=0} /\left(\mathrm{L} \cdot \mathrm{mol}^{-1} \cdot \mathrm{s}^{-1}\right)=k_{\mathrm{t}}^{1,1} \cdot\left\langle i_{\mathrm{n}}\right\rangle^{-0.8} \\
& \left\langle i_{\mathrm{n}}\right\rangle>30: \\
& k_{\mathrm{t}, X=0} /\left(\mathrm{L} \cdot \mathrm{mol}^{-1} \cdot \mathrm{s}^{-1}\right)=k_{\mathrm{t}}^{1,1} \cdot 30^{-0.64} \cdot\left\langle i_{\mathrm{n}}\right\rangle^{-0.16}
\end{aligned}
$$

There is no literature data for homotermination of MCRs. For crosstermination a chain-length averaged value is available, which has been derived by modeling SPPLP-EPR data under neglect of homotermination of MCRs, ${ }^{[36]}$ Under the assumption of the geometric mean, eq. (2.31), for crosstermination and the above-mentioned composite model parameters, the values from ref. ${ }^{[36]}$ yield averaged 0.18 for the ratio of the (hypothetical) $k_{\mathrm{t}}^{1,1, \mathrm{tt}}$ to $k_{\mathrm{t}}^{1,1, \mathrm{ss}}$. Slightly better representation of experimental conversion vs. time data is obtained with this coefficient being reduced (down to 0.01 ), but this low value appears far too small. Thus, 0.1 was chosen as ratio for modeling, as expressed by eq. (5.12). Because of the diffusion controlled nature of $k_{\mathrm{t}}^{1,1}$, its activation energy should be the same as for fluidity. A difference in activation energy between $k_{\mathrm{t}}^{1,1, \mathrm{ss}}$ and $k_{\mathrm{t}}^{1,1, \mathrm{tt}}$ would be difficult to reconcile with this fact. Thus, the ratio of the two coefficients is assumed to be temperature independent. Overall, for the data modeled in this work, propagation of MCRs proved to be more influential than termination. 


$$
k_{\mathrm{t}}^{1,1, \mathrm{tt}} / k_{\mathrm{t}}^{1,1, \mathrm{ss}}=0.1
$$

For the fraction of disproportionation in SPR homotermination a typical value for the polymerization of acrylate type monomers, $\kappa^{\mathrm{ss}}=0.05$, was used and for the MCR homotermination $\kappa^{\text {tt }}=0.8$ was adopted from polymerization of methacrylate-type monomers. ${ }^{[173]}$ For crosstermination an intermediate value of $\kappa^{\text {st }}=0.4$ was chosen. Temperature dependency of $\kappa$ was neglected in the model. A decrease towards higher temperature is expected. ${ }^{[173]}$ Chain-length dependency of $\kappa^{[173]}$ was neglected as well. The diffusion mean, eq. (2.31), was assumed for crosstermination yielding eq. (5.13).

$$
k_{\mathrm{t}}^{\mathrm{st}}=0.5 \cdot\left(k_{\mathrm{t}}^{\mathrm{tt}}+k_{\mathrm{t}}^{\mathrm{ss}}\right)
$$

The Norrish-Trommsdorff effect is less pronounced during polymerization of AA than for MAA. For $0.1 \mathrm{gg}^{-1}$ no conversion dependence of $k_{\mathrm{t}}$ was found, while for $0.2 \mathrm{~g} \mathrm{~g}^{-1}$ and $0.3 \mathrm{~g} \mathrm{~g}^{-1}$ a slight conversion dependence was noted in agreement with literature. ${ }^{[144,157]}$ As no measurements were done with monomer content sufficiently high to determine $C_{\mathrm{RD}}$, a reasonable value of $C_{\mathrm{RD}}=20$ was assumed. The conversion dependence of $k_{\mathrm{t}}$ was modeled by estimation of $C_{\eta}$ in the same way as described for MAA in subchapter 4.2.2 leading to eq. (5.14). As only few conversion vs. time profiles were used for estimating the relationship given by eq. (5.15), this equation should be validated by further experiments. For polymerizations of $0.1 \mathrm{~g} \mathrm{~g}^{-1} \mathrm{AA}, C_{\eta}$ can be set to zero.

$$
\begin{gathered}
k_{\mathrm{t}} /\left(\mathrm{L} \cdot \mathrm{mol}^{-1} \cdot \mathrm{s}^{-1}\right)=\frac{k_{\mathrm{t}, X=0}}{\mathrm{e}^{X \cdot C_{\mathrm{n}}}}+20 \cdot c_{\mathrm{M}} \cdot k_{\mathrm{p}} \\
C_{\eta}=360 \cdot W^{3.7} \\
0 \leq W_{\mathrm{AA}}^{0} \leq 0.3
\end{gathered}
$$


For the polymerization of $0.2 \mathrm{~g} \mathrm{~g}^{-1}$ AA at $170{ }^{\circ} \mathrm{C}$ no Norrish-Trommsdorff effect was observed $\left(C_{\eta}=0\right)$, which can be explained by the lower molar mass of polymer produced under these conditions (see subchapter 4.2).

\subsubsection{Determination $k_{b b}$ by ${ }^{13} \mathrm{C}-\mathrm{NMR}$}

The rate coefficient of backbiting during polymerization of non-ionized AA is available from modeling SP-PLP-EPR data, ${ }^{[36]}$ where a decrease by a factor of 1.9 from $0.1 \mathrm{~g} \mathrm{~g}^{-1}$ towards $0.5 \mathrm{~g} \mathrm{~g}^{-1}$ AA content was found.

In the present work, a more direct approach is used. $k_{\mathrm{bb}}$ is derived from the fraction of branching points, $x_{\mathrm{BP}}$, i.e., the ratio of monomer units that exhibit a branching point to the total number of monomer units. The method of measurement was ${ }^{13} \mathrm{C}-\mathrm{NMR}$. At this, it is important to choose parameters that allow for quantitative analysis of sprectra. These were examined as described in subchapter 3.5.2.

At negligible influence of transfer, scission, and termination by disproportionation, every backbiting event leads to a branching point. Hence, the number of branching points is given by the ratio of rate of backbiting to the rate of propagation:

$$
\boldsymbol{x}_{\mathrm{BP}}=\frac{r_{\mathrm{bb}}}{r_{\mathrm{p}}}=\frac{k_{\mathrm{bb}} \cdot c_{\mathrm{R}} \cdot \boldsymbol{x}_{\mathrm{SPR}}}{k_{\mathrm{p}}^{\mathrm{s}} \cdot c_{\mathrm{M}} \cdot c_{\mathrm{R}} \cdot \boldsymbol{x}_{\mathrm{SPR}}+k_{\mathrm{p}}^{\mathrm{t}} \cdot c_{\mathrm{M}} \cdot c_{\mathrm{R}^{\prime}} \cdot \boldsymbol{x}_{\mathrm{MCR}}}
$$

The contribution of MCRs to growth is negligible, i.e., $k_{\mathrm{p}}^{\mathrm{s}} \cdot c_{\mathrm{M}} \cdot c_{\mathrm{R}} \cdot \boldsymbol{x}_{\mathrm{SPR}} \gg k_{\mathrm{p}}^{\mathrm{t}} \cdot c_{\mathrm{M}} \cdot c_{\mathrm{R}} \cdot \boldsymbol{x}_{\mathrm{MCR}}$, which simplifies eq. (5.16) into eq. (5.17).

$$
x_{\mathrm{BP}} \simeq \frac{k_{\mathrm{bb}}}{k_{\mathrm{p}}^{\mathrm{s}} \cdot c_{\mathrm{M}}}
$$

Eq. (5.17) has the advantage of $k_{\mathrm{p}}^{\mathrm{s}}$ being accurately known. 
$0.03 \mathrm{~g} \mathrm{~g}^{-1} \mathrm{AA}$ in water were photopolymerized in a lined flask with $1.6 \cdot 10^{-4} \mathrm{~g} \mathrm{~g}^{-1}$ D1173 as the initiator. A low monomer concentration was chosen to maximize branching.

$$
k_{\mathrm{bb}} / \mathrm{s}^{-1}=4.25 \cdot 10^{9} \cdot \exp \left(\frac{-5020}{T / \mathrm{K}}\right)
$$

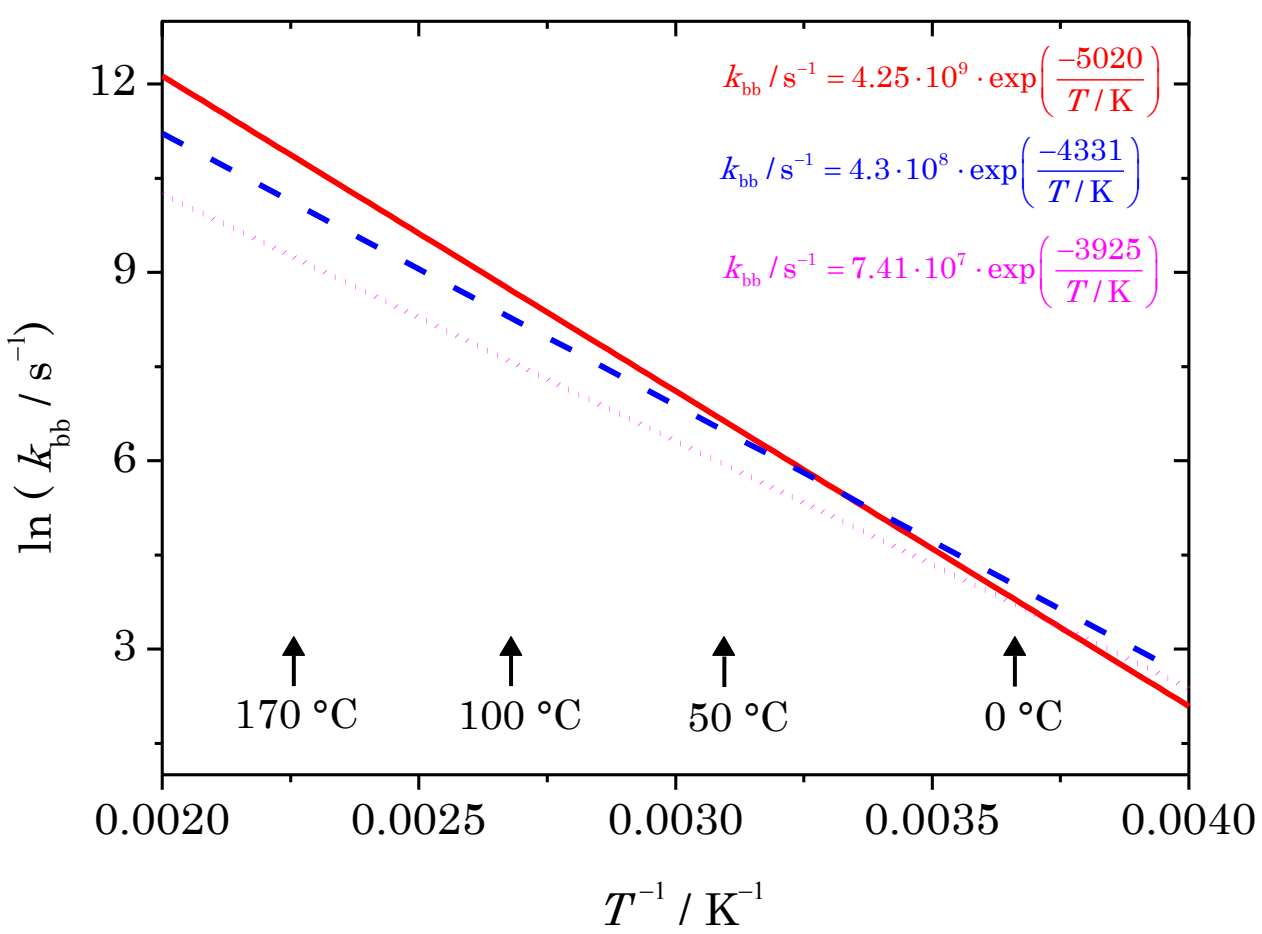

Figure 5-9 Arrhenius plot of $k_{\mathrm{bb}}$. Red, solid line: AA, ${ }^{13} \mathrm{C}-\mathrm{NMR}$, this work; blue, dashed line AA $\left(0.1 \mathrm{~g} \mathrm{~g}^{-1}\right)$, SP-PLP-EPR, from ref; ${ }^{[36]}$ dotted magenta line BA, ${ }^{13} \mathrm{C}-\mathrm{NMR}$, from ref. ${ }^{[174]}$ The corresponding equations are given in the same color.

The Arrhenius parameters were determined between 20 and $74{ }^{\circ} \mathrm{C}$ from ${ }^{13} \mathrm{C}-\mathrm{NMR}$ analysis of pAA product from low conversion (A more detailed description is given further below). The associated equation, eq. (5.18), is plotted in Figure 5-9. The Arrhenius equation from the SP-PLP-EPR data ${ }^{[36]}$ for $0.1 \mathrm{~g} \mathrm{~g}^{-1}$ AA determined between 5 and $40^{\circ} \mathrm{C}$ as well as the equation for $\mathrm{BA}^{[174]}$ are plotted for comparison. The data for $k_{\mathrm{bb}}$ of $\mathrm{AA}$ obtained by the two strategies are in good agreement. AA exhibits a slightly higher $E_{\mathrm{A}}$ than BA with also the absolute values being higher 
between 50 and $100{ }^{\circ} \mathrm{C}$. However, branching levels will be lower for pAA at identical conditions because of the higher value of $k_{\mathrm{p}}^{\mathrm{s}}$ of AA.

The EPR study indicated a decrease of $k_{\mathrm{bb}}$ by a factor of 1.9 from $0.1 \mathrm{~g} \mathrm{~g}^{-1}$ towards $0.5 \mathrm{~g} \mathrm{~g}^{-1}$ AA content. ${ }^{[36]}$ In this study, from $0.03 \mathrm{~g} \mathrm{~g}^{-1}$ towards $0.3 \mathrm{~g} \mathrm{~g}^{-1}$ AA content at $74^{\circ} \mathrm{C}$, a slight increase by the factor of 1.2 was found. The lower $k_{\mathrm{bb}}$ value at higher monomer content in the EPR study may be a consequence of the $k_{\mathrm{t}}^{1,1}$ value being relatively lower at that concentration. It was estimated to be lower than the change in fluidity. Furthermore, within the EPR-experiment, $k_{\mathrm{bb}}$ is coupled to termination and $k_{\mathrm{p}}^{\mathrm{t}}$. The latter might have been underestimated quantitatively. The result from the NMR experiment is independent of these coefficients. This supports the idea that the $k_{\mathrm{t}}^{1,1}$ of $0.1 \mathrm{~g} \mathrm{~g}^{-1} \mathrm{AA}$ should be more reliable (see subchapter 5.1.3).

The assignment of signals of the ${ }^{13} \mathrm{C}-\mathrm{NMR}$ spectrum of $\mathrm{pAA}$ is known from literature. ${ }^{[175]}$ Nonetheless, in order to ensure line assignment in addition to "normal" spectra (but with conditions allowing for quantitative evaluation, see subchapter 3.5.2) a dept-135 (distortionless enhancement by polarization transfer 135 degree) spectrum and an apt (attach proton test) spectrum were measured. In Figure 5-10 the section of the ${ }^{13} \mathrm{C}-\mathrm{NMR}$ spectrum corresponding to the backbone of the chain and the entire ${ }^{13} \mathrm{C}-\mathrm{NMR}$ spectrum (insert) are given. The carboxyl function gives signals between 170 and $190 \mathrm{ppm}$, which could in principle be used for evaluation of branching, but as line assignment was easier for the backbone and distinction of lines is more difficult for the carboxyl signals, this was not done. Around $130 \mathrm{ppm}$ the observed signal of residual monomer appears quite strong due to weaker broadening. Moreover, signals from macromonomer endgroups can be observed, but for evaluation of these endgroups ${ }^{1} \mathrm{H}-\mathrm{NMR}$ was used because of its superior S/N ratio. Towards higher field, the signals of the backbone and of endgroups appear. This region is shown enlarged in Figure 5-10. Here, in contrast to the full spectrum, an apt (red) and dept 135 (teal) spectrum are shown. For both techniques, tertiary and primary carbon atoms give negative signals and secondary ones give positive signals. The difference between the techniques is that quaternary carbons show in the apt, but not in the dept, which proves the signal at $50 \mathrm{ppm}$ to originate from quaternary carbon. It also becomes clear that the three signals between 41 and $47 \mathrm{ppm}$ belong to tertiary carbon atoms. 


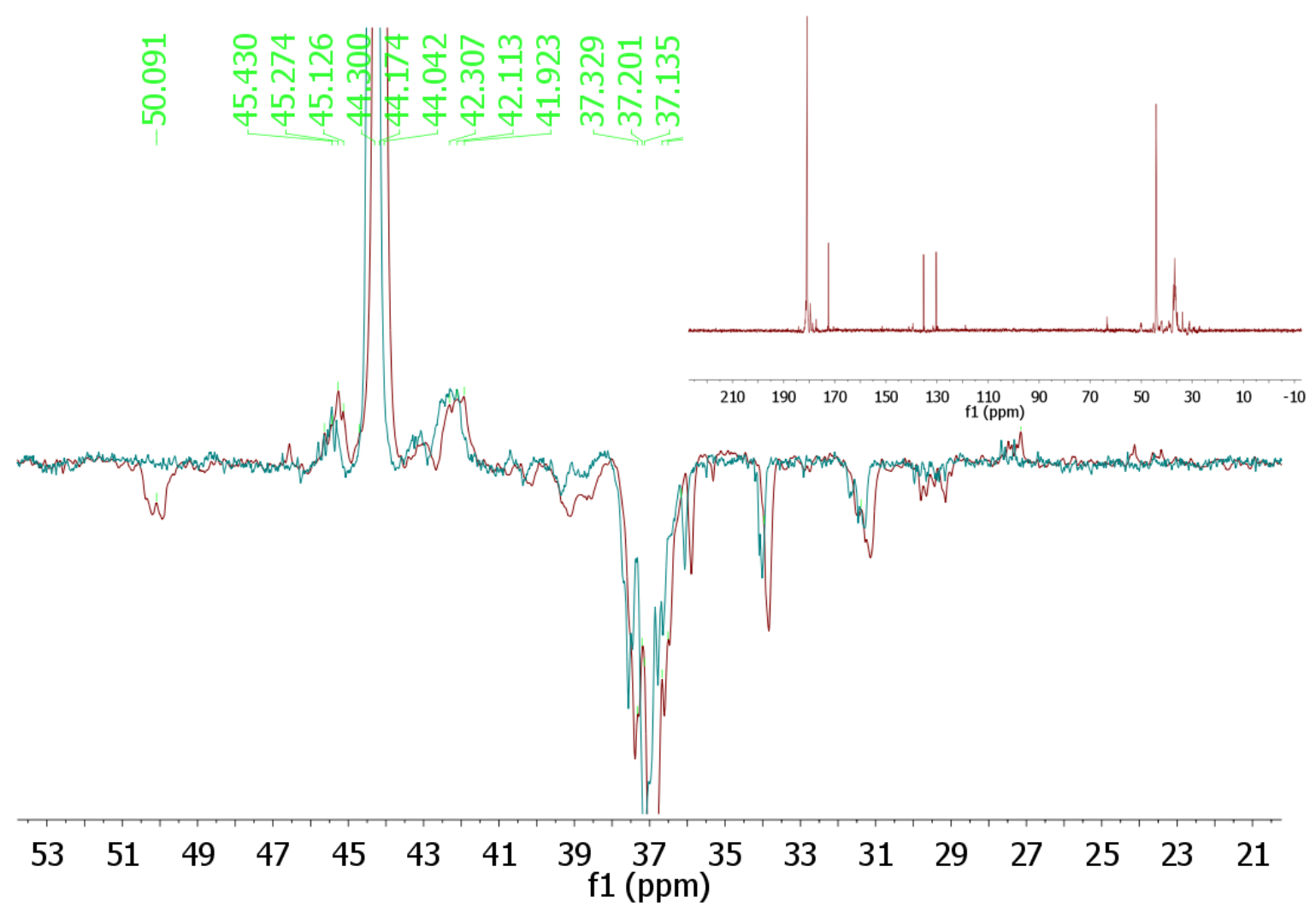

Figure 5-10 Section of the ${ }^{13} \mathrm{C}-\mathrm{NMR}$ spectrum corresponding to the backbone of the chain and the whole ${ }^{13} \mathrm{C}-\mathrm{NMR}$ spectrum (insert). The sample consists of the product of the polymerization of $0.1 \mathrm{~g} \mathrm{~g}^{-1} \mathrm{AA}$ with $0.02 \mathrm{~g} \mathrm{~g}^{-1} \mathrm{VA}-086$ in $\mathrm{H}_{2} \mathrm{O}$ at $170{ }^{\circ} \mathrm{C}$.

Insert: The carboxyl function gives signals between 170 and $190 \mathrm{ppm}$, around $130 \mathrm{ppm}$ the signal of residual monomer can be observed, appearing stronger, because lines are less broadened, as well as weak signals from macromonomer endgroups. Further towards high field the signals from the backbone of the chain as well as endgroups appear.

Large: Backbone section of the spectrum. Here, in contrast to the full spectrum, an apt (red) and dept 135 (teal) spectrum are shown. Using these techniques, tertiary and primary carbon atoms give negative signals, while secondary ones give a positive signal. The difference between the techniques is that quaternary carbons show up in the apt, but not in the dept proving the signal at $50 \mathrm{ppm}$ to originate from a quaternary carbon.

Another advantage of ${ }^{13} \mathrm{C}-\mathrm{NMR}$ is that short-chain branching and long-chain branching may be distinguished. Figure 5-11 shows the section of the ${ }^{13} \mathrm{C}-\mathrm{NMR}$ spectrum used for determination of branching. This spectrum is of the reaction polymerization product from reaction of $0.1 \mathrm{~g} \mathrm{~g}^{-1} \mathrm{AA}$ with $0.02 \mathrm{~g} \mathrm{~g}^{-1} \mathrm{VA}-086$ in $\mathrm{H}_{2} \mathrm{O}$ at $170{ }^{\circ} \mathrm{C}$. Because of the high temperature, branching is high. The pAA main chain is drawn with a short chain branch and a long chain branch. Here, peak assignment is indicated by arrows of the same color. There are the signals of the quaternary carbon atoms at a branching point, $\mathrm{C}_{\mathrm{q}}$, the tertiary carbon atoms in the chain away from branching points, $\mathrm{C}_{t} 1$, the tertiary carbon atoms in a long chain next to a branching points, $\mathrm{C}_{\mathrm{t}} 2$, and, the tertiary carbon atoms in a short chain next to a branching 
points, $\mathrm{C}_{\mathrm{t}} 3$. Assignments were taken from literature. ${ }^{[175]}$ Not for all spectra, the separation of the tertiary signals was as good as in Figure 5-11.

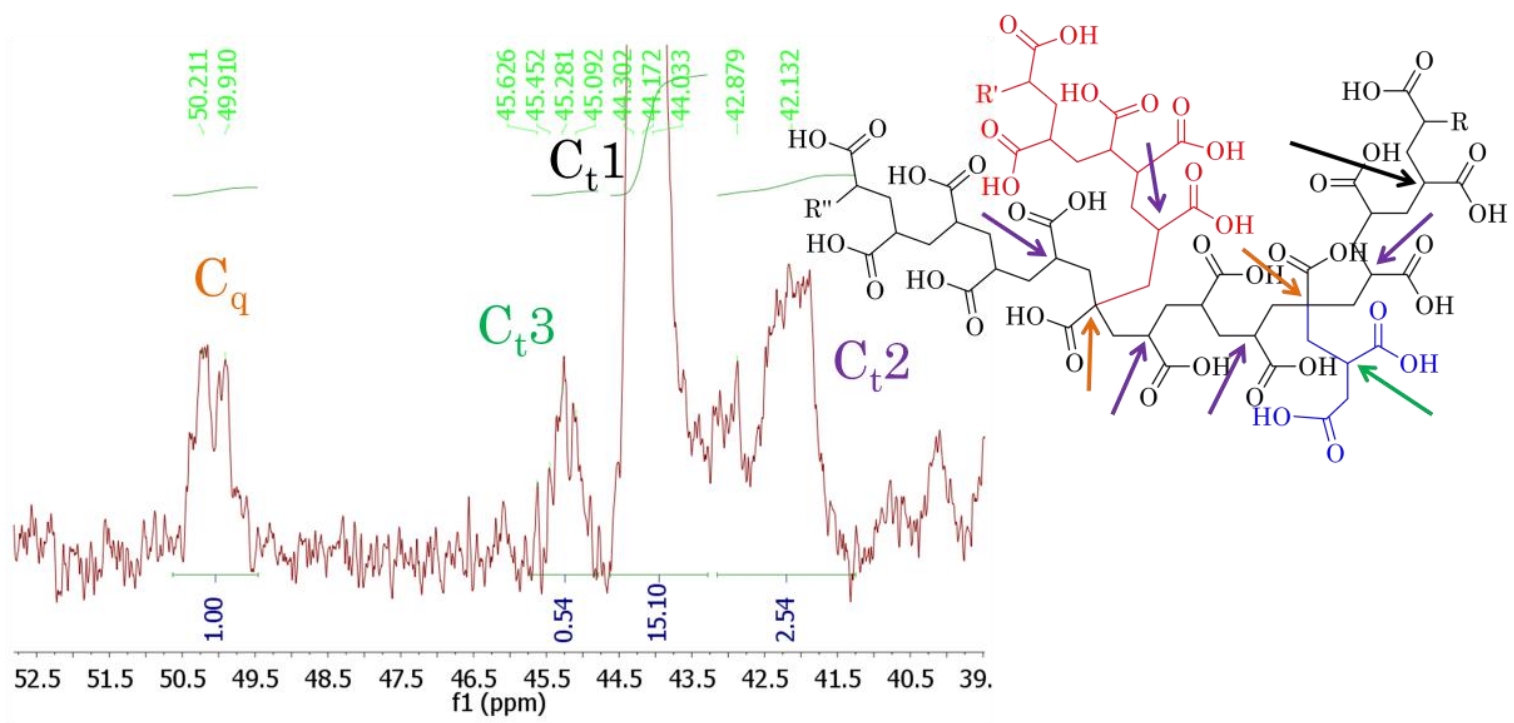

Figure 5-11 Section of the ${ }^{13} \mathrm{C}-\mathrm{NMR}$ spectrum of the reaction product of polymerization of $0.1 \mathrm{~g} \mathrm{~g}^{-1}$ AA with $0.02 \mathrm{~g} \mathrm{~g}^{-1} \mathrm{VA}-086$ in $\mathrm{H}_{2} \mathrm{O}$ at $170{ }^{\circ} \mathrm{C}$ and a Lewis structure section of a pAA molecule. The pAA is drawn consisting of the main chain (black), a short chain branch (blue), and a long chain branch (red). The signals of the quaternary carbon atoms at a branching point $\left(\mathrm{C}_{q}\right.$, orange), the tertiary carbon atoms in the chain away from branching points $\left(\mathrm{C}_{\mathrm{t}} 1\right.$, black), the tertiary carbon atoms in a long chain next to a branching points $\left(\mathrm{C}_{\mathrm{t}} 2\right.$, purple), and the tertiary carbon atoms in short chains next to branching points $\left(\mathrm{C}_{\mathrm{t}} 3\right.$, green) are marked accordingly. The corresponding positions in the polymer chain are indicated by arrows of the same color. (For $\mathrm{C}_{\mathrm{t}} 1$ only one arrow is given to keep the graph lucid.) Assignment of signals has been taken from ref. ${ }^{[175]}$

In order to calculate $x_{\mathrm{SCB}}$ from the four signals shown in Figure 5-11, three formulae were developed: eq. (5.19), eq. (5.20), and eq. (5.21). As separation of signals is difficult, it was checked that results from all three formulas agree, viz., that the evaluation of the spectrum is consistent. For the example given in Figure 5-11, eq. (5.19), eq. (5.20), and eq. (5.21) yield $x_{\mathrm{SCB}}=0.53,0.46$, and 0.52, respectively.

$$
x_{\mathrm{SCB}}=\frac{\int \mathrm{C}_{\mathrm{t}} 3}{\int \mathrm{C}_{\mathrm{q}}}
$$




$$
\begin{gathered}
x_{\mathrm{SCB}}=\frac{3 \cdot j \mathrm{C}_{\mathrm{q}}-J \mathrm{C}_{\mathrm{t}} 2}{\int \mathrm{C}_{\mathrm{q}}} \\
\boldsymbol{x}_{\mathrm{SCB}}=\frac{\int \mathrm{C}_{\mathrm{t}} 1}{\frac{\int \mathrm{C}_{\mathrm{t}} 2-2 \cdot \int \mathrm{C}_{\mathrm{t}} 1}{3}+\int \mathrm{C}_{\mathrm{t}} 1}
\end{gathered}
$$

For calculation of $x_{\mathrm{BP}}$ from the four signals shown in Figure 5-11, eq. (5.22) and eq. (5.23) were developed. Again, it was checked that results from the two formulas agree, viz., the evaluation of the spectrum is consistent. For the example given in Figure 5-11, eq. (5.22) and eq. (5.23) yield $x_{\mathrm{BP}}=0.052$ and 0.053 , respectively.

$$
\begin{gathered}
x_{\mathrm{BP}}=\frac{\mathrm{J}_{\mathrm{q}}}{\int \mathrm{C}_{\mathrm{q}}+\int \mathrm{C}_{\mathrm{t}} 3+\int \mathrm{C}_{\mathrm{t}} 1+\int \mathrm{C}_{\mathrm{t}} 2} \\
x_{\mathrm{BP}}=\frac{\frac{\int \mathrm{C}_{\mathrm{t}} 2}{\left(1-x_{\mathrm{SCB}}\right) \cdot 3+x_{\mathrm{SCB}} \cdot 2}}{\int \mathrm{C}_{\mathrm{t}} 1+\int \mathrm{C}_{\mathrm{t}} 2+\frac{\int \mathrm{C}_{\mathrm{t}} 2}{\left(1-\boldsymbol{x}_{\mathrm{SCB}}\right) \cdot 3+x_{\mathrm{SCB}} \cdot 2}+\frac{\int}{\left(1-x_{\mathrm{SCB}}\right) \cdot 3+x_{\mathrm{SCB}} \cdot 2} \cdot x_{\mathrm{SCB}}}
\end{gathered}
$$

\subsubsection{BA as a Model for AA to estimate $C_{\text {СTA }}{ }^{t}$ by EPR}

In previous work, ${ }^{[147]}$ a retardation of the AA polymerization by ME as CTA has been found for low concentrations of CTA, which was explained by a higher $k_{t}$ resulting from reducted of chain length. However, at even higher concentrations of CTA, an enhancement of rate of polymerization was found relative to the expected value considering CLDT, i.e., in going from no to high concentration of CTA, $r_{\text {polym }}$ passes through a minimum. One explanation could be that at higher CTA concentration a significant amount of MCRs undergo transfer to CTA with subsequent reinitiation of 
the CTA radical that the rate enhancing effect of transforming MCRs into SPRs notably compensates retardation by enhancement of termination.

The polymerization kinetics of BA (butyl acrylate) has been investigated with focus on propagation, termination, backbiting and high-temperature reactions. ${ }^{[40-}$ 43,49,101,107,176-181] BA polymerizations have been successfully modeled for different conditions. ${ }^{[42,43]}$ Overall BA is one of the best examined monomers. BA and AA show a similar general kinetic behavior and even kinetic coefficients are of similar size. The main difference arises from solvent, because BA is polymerized in organic solvents, while AA polymerization takes place in aqueous solution. For many monomers including AA, the rate coefficient of propagation becomes a function of monomer concentration (see subchapter 2.4.2 and 5.1.2) when polymerized in water, thus making kinetics more complex. This does not affect the comparison made in this chapter, because $C_{\mathrm{CTA}}$ is a good constant even in aqueous systems (subchapter 4.1.1).

In this subchapter investigation into the chain transfer of MCRs to CTA are described and an approximate value for $C_{\mathrm{CTA}}^{\mathrm{t}}$ is determined. On the one hand, chaintransfer constants of thiols to both methacrylate and acrylate monomers are close to each other, i.e., between 0.5 and 2. On the other hand, it was found by Junkers et al. ${ }^{[176]}$ via mass spectrometry that the amount of $\beta$-scission products is reduced a great deal even in case that small amounts of CTA $\left(0.01 \mathrm{~mol} \mathrm{~mol}^{-1}\right)$ were added to the reaction mixture. The authors concluded that the CTA reacted so fast with MCRs that transfer becomes the main reaction pathway of MCRs, i.e. the CTA "patches" MCRs thus reducing branching. So far, no experimental procedure has been developed to measure chains-transfer of MCRs to CTA directly.

Agirre et al. polymerized BA with tetrabromomethane added as CTA. ${ }^{[182]}$ Tetrabromomethane was chosen, because in contrast to thiols this CTA transfers a bromine atom instead of a hydrogen atom, and thus the "patching" product is different from the backbone of the polymer. Mass spectrometry confirmed that the amount of $\beta$-scission products was reduced, the "patching" product could not be found via ${ }^{13} \mathrm{C}-\mathrm{NMR}$ analysis, which strongly suggests that $C_{\text {CTA }}^{\mathrm{t}}$ is rather low.

As the two types of radicals, SPRs and MCRs, show different hyperfine splitting, they can be easily distinguished by EPR. In addition, absolute radical concentrations can be obtained. Unfortunately, EPR measurements of aqueous solutions are difficult and can only be carried out using special equipment (see chapter 3.4.3). Water has a rather high dipole moment of 1.855 D. ${ }^{[183]}$ In contrast, toluene is an almost ideal solvent for EPR experiments, with a dipole moment of 0.357 D. ${ }^{[183]}$ This led to the idea of using BA as a model to get a better understanding of AA kinetics. 
In previous work by Sergeeva et al. polymerization of $1.52 \mathrm{~mol} \mathrm{~L}^{-1}$ BA polymerization in toluene was investigated by EPR. ${ }^{[101]}$ Polymerization took place under constant UV initiation. Both absolute radical concentration and the fraction of MCRs were determined as a function of temperature in the range of -50 to $90^{\circ} \mathrm{C}$. As the aim of this investigation was to fathom the effect of chain transfer on MCR kinetics, the same setup and experimental procedure as in the earlier work was used, with the exception of adding different levels of ME as CTA.
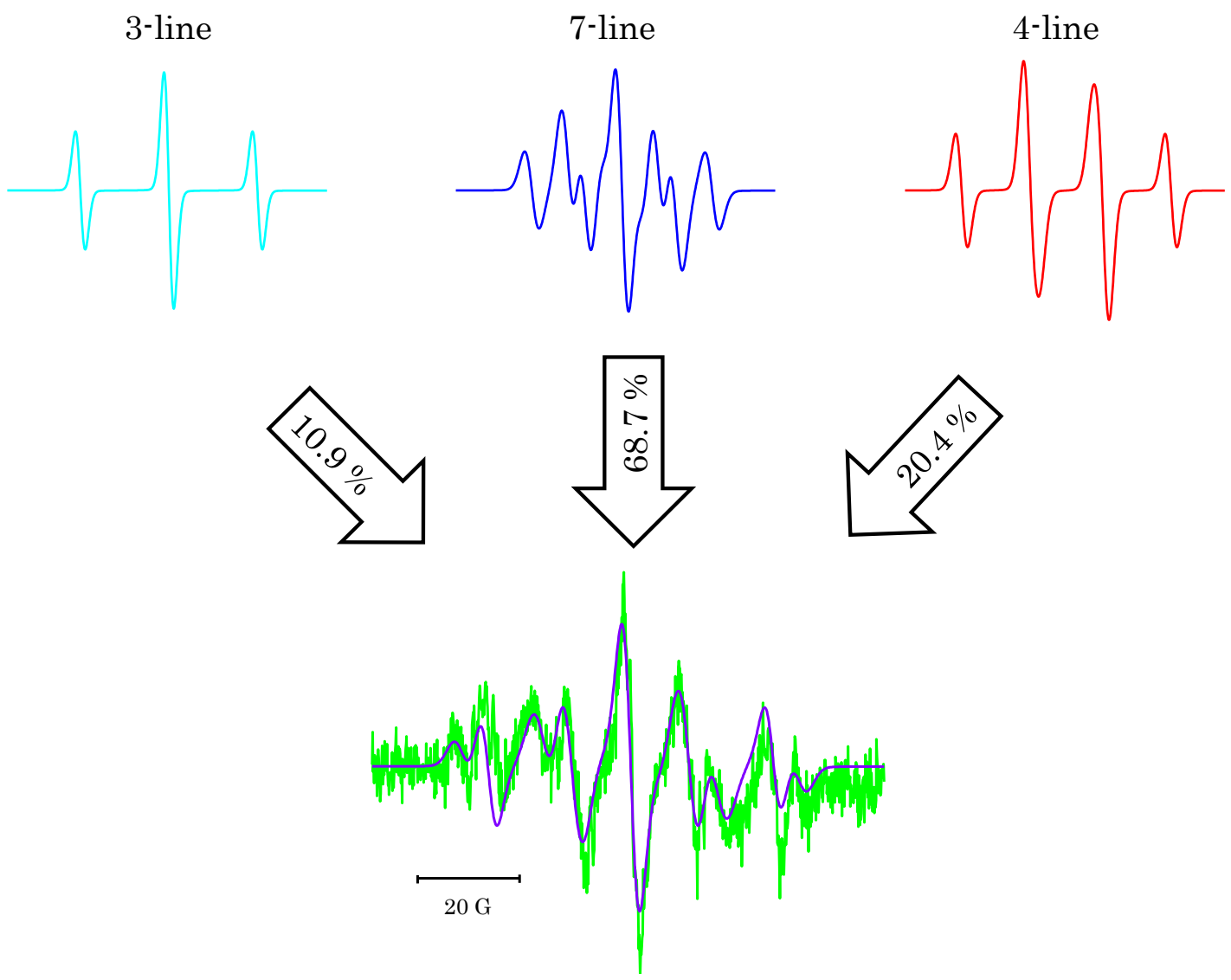

Figure 5-12 The simulated 3-line spectrum and the 7-line spectrum assigned to MCRs and the 4-line assigned to SPRs are given in the upper part. The double integrals of the 3-line, the 7 -line, and the 4 -line spectrum account for $10.9,68.7$, and $20.4 \%$, respectively, of the double integral of the combined spectrum, which best represents the measured spectrum. Conditions: $1.52 \mathrm{~mol} \mathrm{~L}^{-1} \mathrm{BA}$ in toluene, $0.02 \mathrm{eq} . \mathrm{ME}, 30^{\circ} \mathrm{C}$.

The fraction of MCRs is obtained by fitting the simulated spectra to the measured ones. The EPR spectrum of SPRs actually consists of six lines, but due to line broadening a 4-line spectrum is observed. The 7-line MCR spectrum consists intrinsically of nine lines resulting from coupling with two non-equivalent groups of 
two $\beta$-protons. The 3-line spectrum results from slow rotation of macromolecules, as discussed in detail in ref. ${ }^{[101]}$

In Figure 5-12, the three-component fit is shown consisting of the 4-line, 7-line, and 3 -line species. All three simulated spectra (top) are combined to best represent the measured spectrum. The comparison between simulated and measured spectrum (bottom) demonstrates good accuracy of the simulation. Most of the small deviation results from the baseline of the experimental spectrum being somewhat tilted. The ratio of the double integrals yields the ratio of radical concentrations and thus the fraction of MCRs is given by:

$$
x_{\mathrm{MCR}}=\frac{\iint 3 \text {-line }+\iint \text { 7-line }}{\iint 3 \text {-line }+\iint 7 \text {-line }+\iint 4 \text {-line }}
$$

Shown in Figure 5-13 is, to which extent MCRs are observed as a 3-line spectrum depending on temperature for different CTA concentrations. The 3-line specrum is associated with a hindered rotation of the macroradical. ${ }^{[101]}$ It is not observed with model species consisting of a few monomer units only. A reduction of the fraction of the 3-line spectrum may indicate that chain length is reduced into the oligomeric region. The 3-line spectrum is observed for polymerizations with $0.02 \mathrm{~mol} \mathrm{~mol}^{-1}$ CTAXXIV to the same extent as without CTA. For $0.05 \mathrm{~mol} \mathrm{~mol}^{-1}$ CTA this is approximately true as well even though the fraction of the 3-line spectrum appears to some extent reduced. On the other hand, this deviation is within experimental uncertainty.

Figure 5-14 shows the mole fraction of mid-chain radicals for BA polymerizations from low temperature, where SPRs predominate, to high temperature, where MCRs predominate. Depending on temperature, the mole fraction of MCRs is reduced slightly by CTA. For both $0.02 \mathrm{~mol} \mathrm{~mol}^{-1}$ and $0.05 \mathrm{~mol} \mathrm{~mol}^{-1}$ the reduction can only be observed between 0 and $60{ }^{\circ} \mathrm{C}$.

XxIV CTA content is given relative to monomer content. 


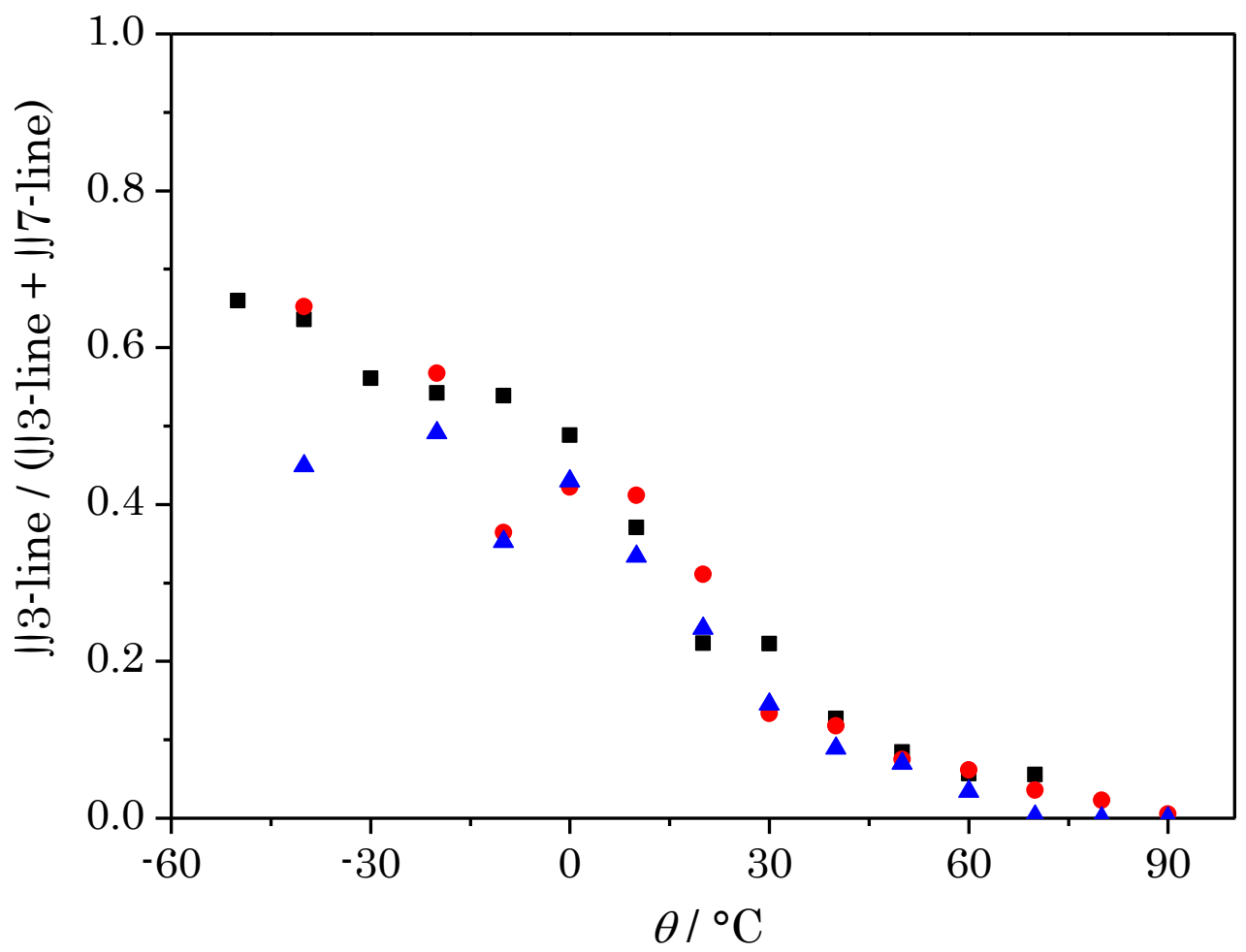

Figure 5-13 The share of the 3-line spectrum is given as a function of temperature. Comparing polymerizations without CTA (squares) taken from ref. ${ }^{[101]}$ to those with addition of $0.02 \mathrm{~mol} \mathrm{~mol}^{-1}$ CTA (circles) the 3-line spectra indicate no effect of CTA. Comparison with polymerizations, where $0.05 \mathrm{~mol} \mathrm{~mol}^{-1} \mathrm{CTA}$ (triangles) have been added, does not fully support this; there appears to be a slight lowering towards less 3-line signal.

Other than with PLP, continuous initiation ensures that radical concentration is (pseudo) stationary. Thus, eq. (2.18) can be used to analyze polymerization without CTA. For polymerizations with CTA, a transfer term, $k_{\mathrm{tr}, \mathrm{CTA}}^{\mathrm{t}} \cdot c_{\mathrm{CTA}}$, has to be added yielding eq. (5.25). Transfer to monomer may be ignored. Transfer to polymer was negligible because $\boldsymbol{x}_{\mathrm{MCR}}$ did not show any conversion dependence.

$$
x_{\mathrm{MCR}}=\frac{c_{\mathrm{MCR}}}{c_{\mathrm{SPR}}+c_{\mathrm{MCR}}}=\frac{k_{\mathrm{bb}}}{k_{\mathrm{p}}^{\mathrm{t}} \cdot c_{\mathrm{M}}+k_{\mathrm{tr}, \mathrm{CTA}}^{\mathrm{t}} \cdot c_{\mathrm{CTA}}+2 \cdot k_{\mathrm{t}}^{\mathrm{tt}} \cdot c_{\mathrm{MCR}}+2 \cdot k_{\mathrm{t}}^{\mathrm{st}} \cdot c_{\mathrm{SPR}}+k_{\mathrm{bb}}}
$$

Backbiting as well as propagation of MCRs are independent of CTA. There are two effects of CTA that influence $x_{\mathrm{MCR}}$. First, even small amounts of CTA reduce 
average chain length dramatically, which leads to higher $k_{\mathrm{t}}^{\mathrm{st}}$ and $k_{\mathrm{t}}^{\mathrm{tt}}$. At this, the former coefficient is much higher, and thus crosstermination is the dominant reaction. Second, a new reaction pathway is opened. MCRs can transfer to the CTA, which initiates as follow-up reaction, thereby converting MCRs into SPRs.

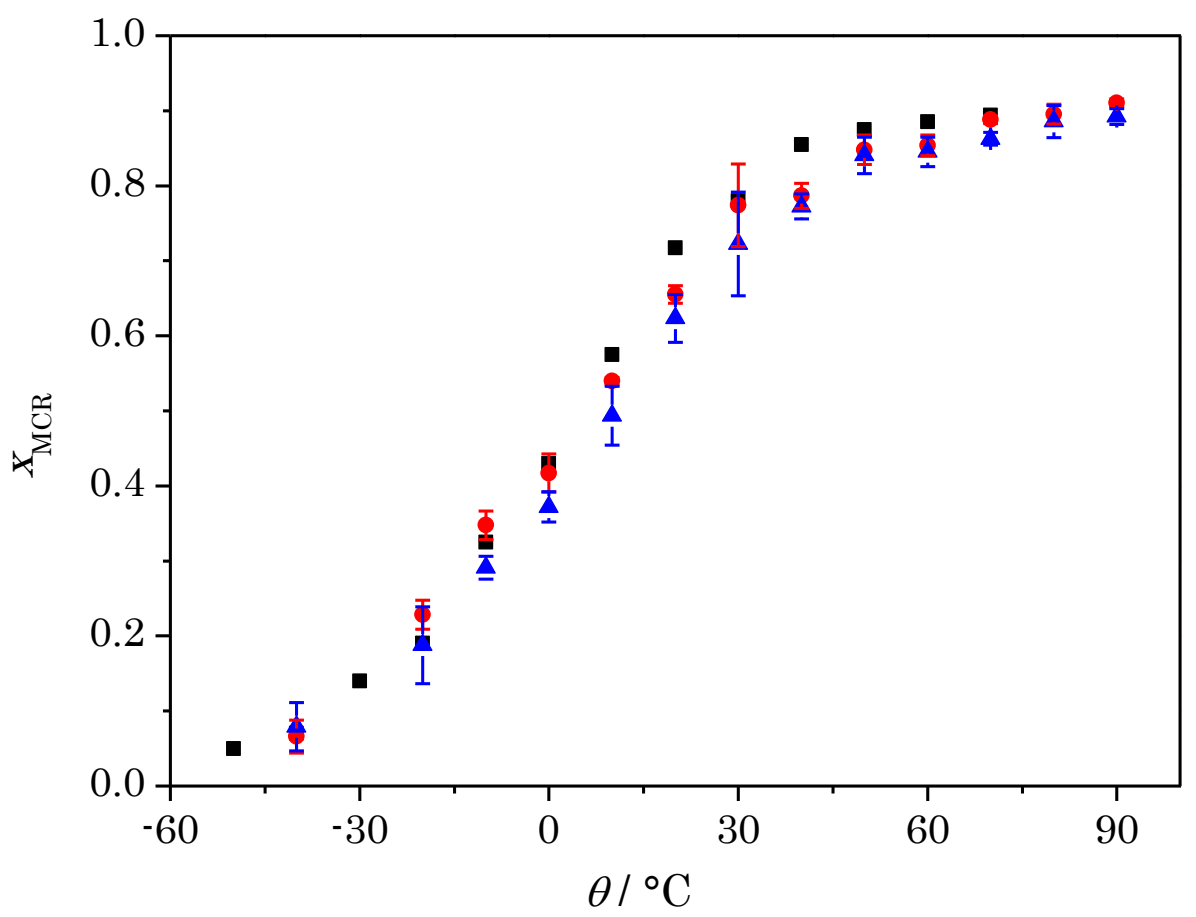

Figure 5-14 The mole fraction of mid-chain radicals during polymerization of BA (1.52 $\mathrm{mol} \mathrm{L}^{-1}$ in toluene) under continuous photoinitiation is shown as a function of temperature. The CTA reduces the fraction of MCRs; this effect increases with the amount of CTA, but the effect is small and not seen for every temperature. All data points are averages of multiple measurements. Squares: no CTA, circles: $0.02 \mathrm{~mol} \mathrm{~mol}^{-1}$, triangles: $0.05 \mathrm{~mol} \mathrm{~mol}^{-1}$. Statistical error is calculated as corrected sample standard deviation. Data of polymerizations without ME is taken from ref. ${ }^{[101]}$

At lower temperatures, backbiting and crosstermination are controlling for $x_{\mathrm{MCR}}$. As the activation energy of the former is by $27.1 \mathrm{~kJ} \mathrm{~mol}^{-1}$ higher, the fraction of MCRs increases with temperature. The higher rate of termination for polymerizations with CTA leads to the reduction of MCRs. The difference in $x_{\mathrm{MCR}}$ first increases then decreases again and disappears at ca. $60^{\circ} \mathrm{C}$. At higher temperature, termination loses importance compared to propagation of MCRs due to its $23.3 \mathrm{~kJ} \mathrm{~mol}^{-1}$ lower activation energy. ${ }^{[144,184,185]}$ Moreover, at high temperature crosstermination 
additionally loses importance, as there are only few SPRs left for termination with MCRs.

$C_{\mathrm{CTA}}^{\mathrm{s}}$ is approximately unity. In the system $\mathrm{BA}$ with thiols $C_{\mathrm{CTA}}^{\mathrm{s}}$ is 1.5 and temperature independent. ${ }^{[180]}$ In general, chain-transfer constants exhibit no or very low activation energy. That means transfer becomes important when propagation becomes important. Hence, at high temperature, a reduction of $\boldsymbol{x}_{\mathrm{MCR}}$ caused by increased transfer should be notable. This is not observed; the graphs merge at high temperature.

This phenomenon needs to be discussed in further detail. The two pathways reducing MCR concentration by transfer to CTA and by propagation are given by eq. (5.26) and eq. (5.27), respectively.

$$
\begin{gathered}
r_{\mathrm{tr}}^{\mathrm{t}}=k_{\mathrm{p}}^{\mathrm{t}} \cdot C_{\mathrm{CTA}}^{\mathrm{t}} \cdot c_{\mathrm{CTA}} \cdot x_{\mathrm{MCR}} \cdot c_{\mathrm{R}} \cdot \\
r_{\mathrm{p}}^{\mathrm{t}}=k_{\mathrm{p}}^{\mathrm{t}} \cdot c_{\mathrm{M}} \cdot x_{\mathrm{MCR}} \cdot c_{\mathrm{R}} .
\end{gathered}
$$

In order to estimate whether transfer is significant one has to look at the ratio of the two reaction rates. Doing so leads to eq. (5.28). With $0.01 \mathrm{~mol} \mathrm{~mol}^{-1} \mathrm{CTA}$, the chaintransfer constant has to be 100 to get a transfer rate as fast as propagation, which would be an uncommon value. To make transfer the dominant reaction an even higher value is needed.

$$
\frac{r_{\mathrm{tr}}^{\mathrm{t}}}{r_{\mathrm{p}}^{\mathrm{t}}}=C_{\mathrm{CTA}}^{\mathrm{t}} \frac{c_{\mathrm{CTA}}}{c_{\mathrm{M}}}
$$

Under the assumption that the long-chain approximation holds, eq. (5.29) is used to calculate the fraction of MCRs for $70^{\circ} \mathrm{C}$, a temperature, which lies in the region where termination is of less importance. Still, the differences are only a minimum value (v.i.). 


$$
x_{\mathrm{MCR}}=\frac{k_{\mathrm{bb}}}{k_{\mathrm{p}}^{\mathrm{t}} \cdot c_{\mathrm{M}}+k_{\mathrm{tr}, \mathrm{CTA}}^{\mathrm{t}} \cdot c_{\mathrm{CTA}}+k_{\mathrm{bb}}}
$$

The following Arrhenius parameters are used for calculation:[144]

$$
\begin{aligned}
& k_{\mathrm{bb}}=4.47 \cdot 10^{7} \cdot \exp \left(\frac{-31.7 \mathrm{~kJ} \mathrm{~mol}}{R \cdot T}\right) \mathrm{s}^{-1} \\
& k_{\mathrm{p}}^{\mathrm{t}}=1.52 \cdot 10^{6} \cdot \exp \left(\frac{-28.9 \mathrm{~kJ} \mathrm{~mol}}{R \cdot T}\right) \mathrm{L} \mathrm{mol}^{-1} \mathrm{~s}^{-1}
\end{aligned}
$$

In Table 5-3 the difference between $x_{\mathrm{MCR}}$ without transfer to CTA and $x_{\mathrm{MCR}}$ with transfer to CTA is given in percentage points. The log-chain approximation was used for calculation and, due to shorter chains, termination is higher for polymerization with CTA further decreasing $x_{\mathrm{MCR}}$. Thus, the differences are only a minimum value. In the experiment with the higher CTA concentration, a stronger effect is predicted. As the effect is reduced by conversion, values at zero and $50 \%$ conversion are given. . Within experimental accuracy, no conversion effect could be observed. Without transfer, a degree of monomer conversion of 0.9 would result in one percent point increase of $x_{\mathrm{MCR}}$, eq. (5.29), which is within error range of $x_{\mathrm{MCR}}$ measurement.

At $70{ }^{\circ} \mathrm{C}$, the measured difference of $x_{\mathrm{MCR}}$ between polymerization without CTA and with $0.02 \mathrm{~mol} \mathrm{~mol}^{-1}$ CTA is 0.61 percentage points and between polymerization without CTA and with $0.05 \mathrm{~mol} \mathrm{~mol}^{-1} \mathrm{CTA}$ is 3.1 percentage points. Adding experimental error of 2 percentage points, which is more than twice the corrected sample standard deviation, gives 2.6 and 5.1, respectively. These values are already very close to 1.87 and 4.53 , the calculated differences for $C_{\text {CTA }}^{\mathrm{t}}=10$ and below 2.77 and 6.62 , the calculated values for $C_{\mathrm{CTA}}^{\mathrm{t}}=15$. Besides, if the latter value of the constant were right, a notable conversion dependence would apply. Thus, $C_{\text {CTA }}^{\mathrm{t}}$ seems to be below 10 and is certainly below 15 . 
Table 5-3 The difference between $\mathrm{XMCR}$ without transfer to CTA to $\mathrm{XMCR}$ with transfer at $70^{\circ} \mathrm{C}$ is given in percentage points. Long-chain approximation is made, thus calculated numbers are the minimum values. The initial value and the one at $50 \%$ is given, because the value is a function of conversion (both monomer and CTA)

$$
0.02 \mathrm{~mol} \mathrm{~mol}^{-1} \mathrm{CTA} \quad 0.05 \mathrm{~mol} \mathrm{~mol}^{-1} \mathrm{CTA}
$$

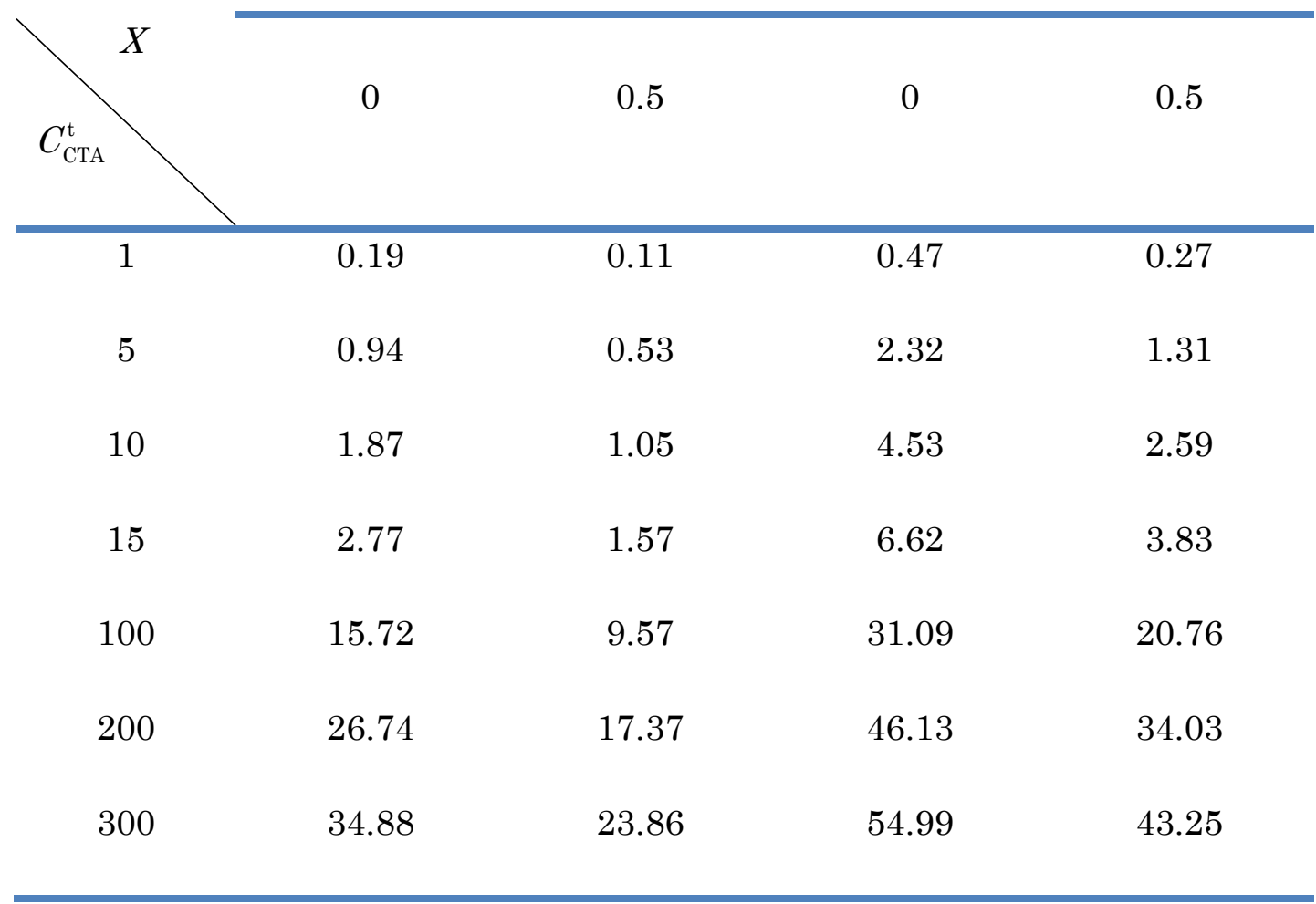

By calibration with TEMPO, absolute radical concentrations can be obtained from the double integral of the EPR signal. The overall absolute radical concentrations for different conditions are given in Figure 5-15. Throughout the polymerization reaction, high stationary radical concentrations are observed. Addition of CTA causes a reduction of the radical concentration by about a factor of 3 . The reduction does not increase linearly with CTA content. From $0.02 \mathrm{~mol} \mathrm{~mol}^{-1}$ to $0.05 \mathrm{~mol} \mathrm{~mol}^{-1}$ the reduction is only about $30 \%$.

The difference in radical concentration between the three reaction mixtures may, to some extent, be explained by the difference in $k_{\mathrm{t}}$ resulting from different chain lengths. GPC analysis of a sample from polymerization without CTA at $20^{\circ} \mathrm{C}$ gave an $M_{\mathrm{n}}$ of $3.61 \cdot 10^{4} \mathrm{~g} \mathrm{~mol}^{-1}(\mathrm{\Xi}=2.2)$. Applying the Mayo equation, eq. (2.12), and assuming $C_{\text {CTA }}^{\mathrm{s}}=1.0$, the addition of $0.02 \mathrm{~mol} \mathrm{~mol}^{-1} \mathrm{CTA}$ reduces chain length from 282 to 50. With $\alpha_{1}=0.2$ the composite model, eq. (2.34), yields an associated reduction of $k_{\mathrm{t}}$ by a factor of 1.4. According to eq. (2.6) steady-state radical concentration is proportional to the reciprocal square root of $k_{t}$. Thus, the reduction of $c_{R}$ is by a factor of 1.2 . Further reduction of the radical concentration may arise 
from termination by CTA radicals. For polymerizations with a smaller rate of initiation and therefore a lower rate of termination leading to longer chains the impact of a CTA would be stronger. It would be interesting to do the same experiment with low radical concentration $\left(10^{-8} \mathrm{~mol} \mathrm{~L}^{-1}\right)$, but these conditions are at present beyond EPR measuring range.

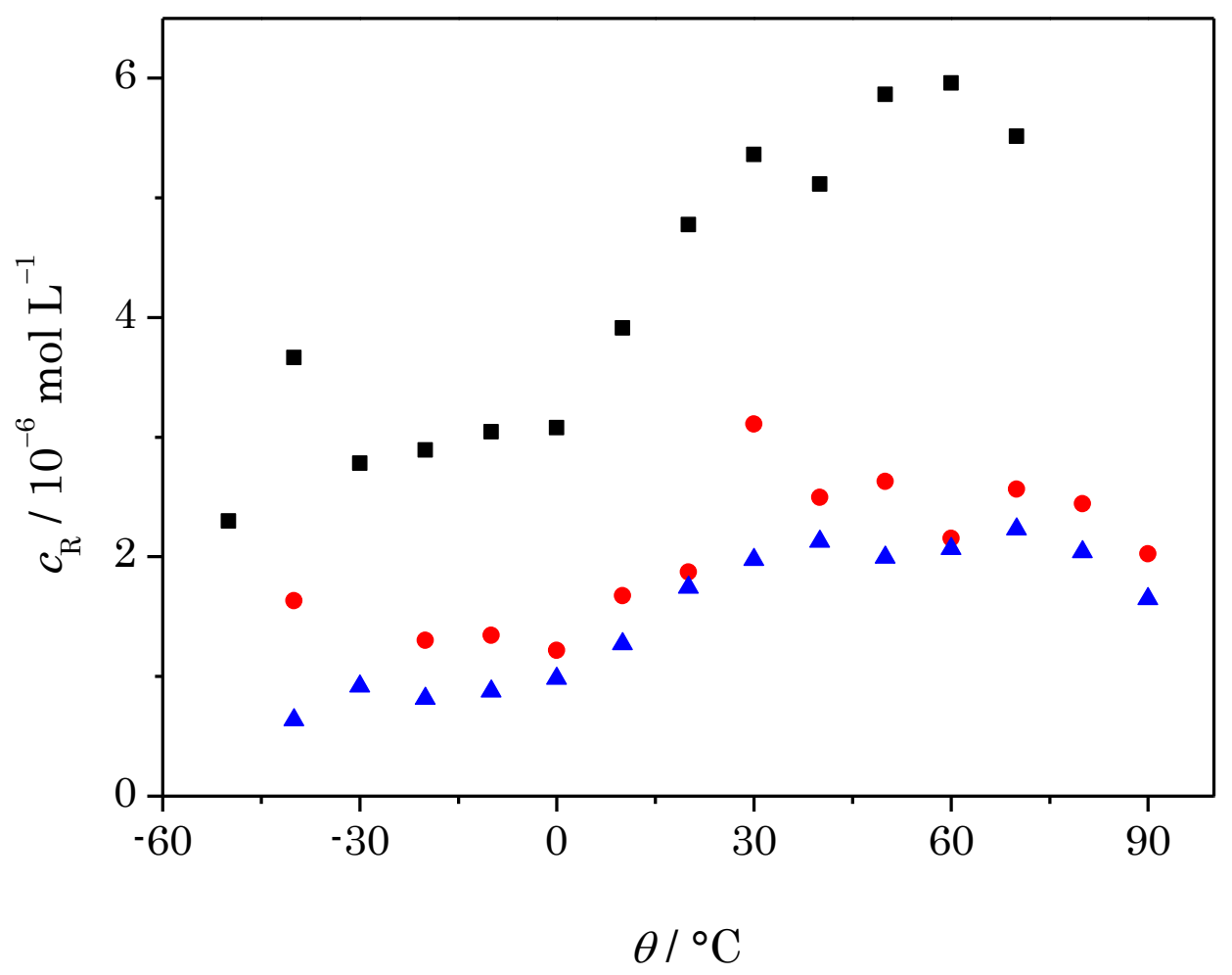

Figure 5-15 Overall radical concentrations (SPRs and MCRs) of polymerizations with different concentrations of CTA are shown as a function of temperature. Black squares: no CTA, red circles: $0.02 \mathrm{~mol} \mathrm{~mol}^{-1}$, blue triangles: $0.05 \mathrm{~mol} \mathrm{~mol}^{-1}$. Data of polymerizations without ME is taken from ref. ${ }^{[101]}$

The radical concentrations in the three reaction mixtures exhibit exactly the same temperature dependency. The radical concentration slightly increases with higher temperature. Then it increases from 0 to $30^{\circ} \mathrm{C}$, and afterwards decreases again, but slowly or stays constant.

The rate of initiation is the same, because in case of photoinitiation the energy for decomposition of initiator is not supplied thermally. Termination increases with temperature, but only slightly, as the activation energy of $k_{\mathrm{t}}$ is as low as 
$5.6 \mathrm{~kJ} \mathrm{~mol}^{-1} .{ }^{[144,184,185]}$ Hence, with a constant fraction of MCRs a slight decrease of the radical concentration with higher temperature would be expected, but this is not the case. In the temperature region under investigation a dramatic shift in $x_{\mathrm{MCR}}$ takes place. Due to MCRs terminating more slowly, a higher fraction of MCRs allows for a higher radical concentration. The fact that the inflection points of the curves in Figure 5-14 and Figure 5-15 are at the same temperature also supports MCRs kinetics as being the reason.

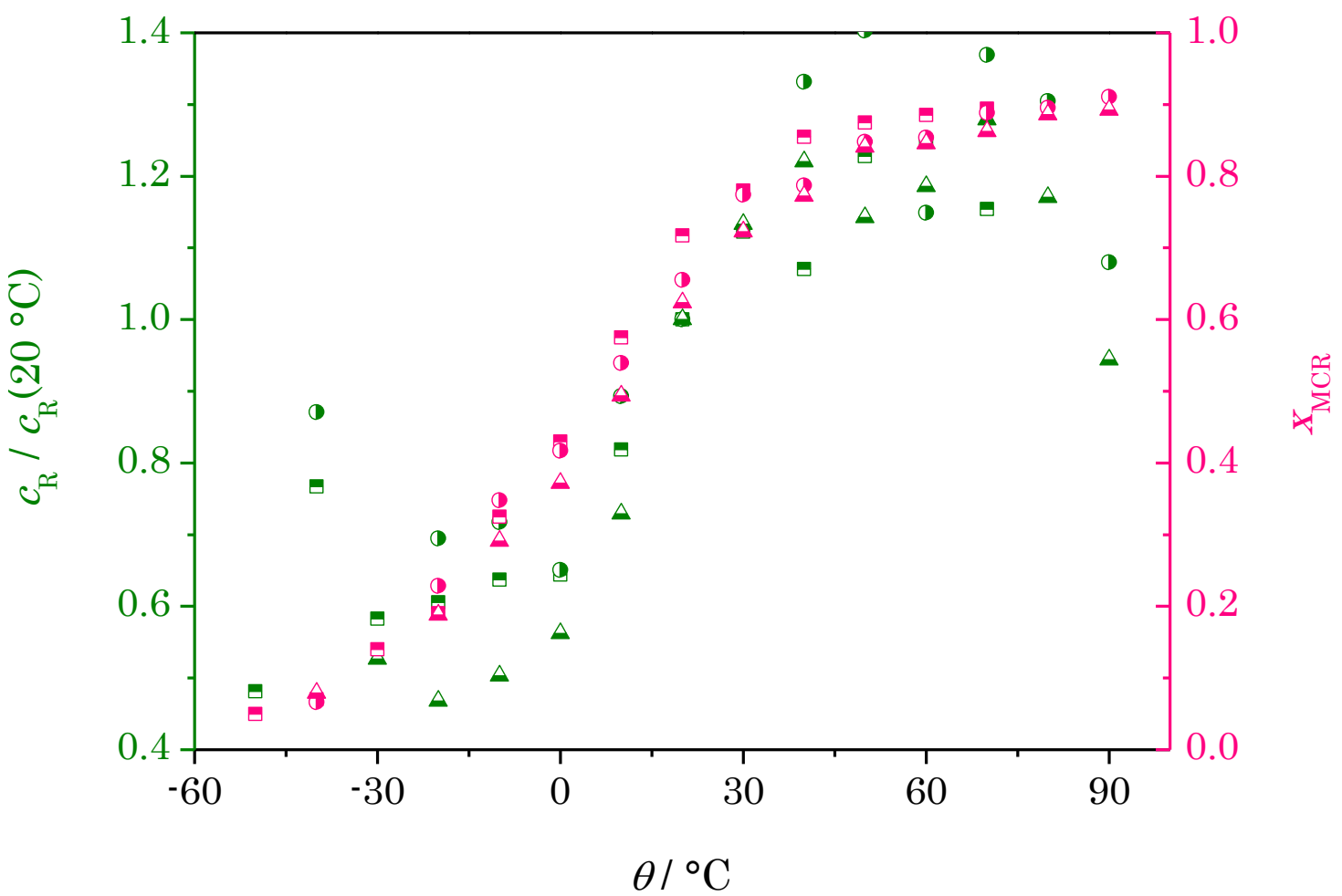

Figure 5-16 Overall stationary radical concentration (SPRs and MCRs) is plotted in olive as a function of temperature scaled to the radical concentration at $20^{\circ} \mathrm{C}$. For comparison the fraction of midchain radicals is plotted in pink. (upper half filled squares: no CTA, right half filled circles: $0.02 \mathrm{~mol} \mathrm{~mol}^{-1}$, lower half filled triangles: $0.05 \mathrm{~mol} \mathrm{~mol}^{-1}$ )

In order to better visualize both the relationship between radical concentrations during polymerization with different CTA content and how they are related to the fraction of MCRs, both quantities are plotted together in Figure 5-16. At this, radical concentration is scaled to the value at $20^{\circ} \mathrm{C}$ which is near the inflection point. The radical concentration curves of the three reaction mixtures are on top of each other 
and they are also (though somewhat more scattered) on top of the curves fir the MCR fraction.

The fact, that the three curves have, within experimental error, the same shape even though chain length of both MCRs and SPRs are quite different points towards MCRs and SPRs showing the same chain-length dependency of $k_{\mathrm{t}}$.

In this subchapter it has been shown that the chain-transfer constant of MCRs cannot be significantly higher than the chain-transfer constant of SPRs.

Even without considering higher termination rate for polymerizations with CTA, $C_{\text {CTA }}^{\mathrm{s}}$ still cannot be above 15 , which would be too low to make transfer of MCRs a major reaction pathway. Thus, effects so far attributed to high transfer of MCRs to CTA have to be explained differently, e.g., a reduction of $\beta$-scission products may be the result of increased termination. Moreover, an enhancement of rate of polymerization may result from additional initiation by the thiol-ene reaction.

Furthermore, it was demonstrated how termination increases and overall radical concentration drops by a factor of three upon addition of $0.02 \mathrm{~mol} \mathrm{~mol}^{-1}$ CTA. However, the reduction of the fractions of MCRs by higher crosstermination is only small.

\subsection{6 $\quad C_{\mathrm{CTA}}{ }^{\mathrm{s}}$ of ME with AA}

The chain-transfer constant of SPRs to ME during polymerization of AA was determined by the Mayo plot, which has been developed for systems with only one growing radical species. In the following, it will be shown that the Mayo procedure can be used for this system with MCRs being present to determine $C_{\mathrm{CTA}}^{\mathrm{s}}$. This is related to a detailed analysis of MCR kinetics by Nikitin et al. ${ }^{[38]}$

The basic idea of the Mayo equation is that the average chain length is determined by the ratio of growth of chains to the rates of termination and transfer, eq. (5.30).

$$
\left\langle i_{n}\right\rangle=\frac{r_{\mathrm{p}}}{r_{\mathrm{t}}+r_{\mathrm{tr}}}
$$


The two stopping events of the chain growth can be separated, eq. (5.58)

$$
\left\langle i_{n}\right\rangle^{-1}=\frac{r_{\mathrm{t}}}{r_{\mathrm{p}}}+\frac{r_{\mathrm{tr}}}{r_{\mathrm{p}}}
$$

In case of SPR-MCR kinetics, the first term of eq. (5.31) RHS need not be considered further, but the second has to be. Transfer to growth of both radical species may be expressed by eq. (5.32).

$$
\begin{aligned}
& \frac{r_{\mathrm{tr}}}{r_{\mathrm{p}}}=\frac{k_{\mathrm{tr}, \mathrm{CTA}}^{\mathrm{s}} \cdot c_{\mathrm{CTA}} \cdot c_{\mathrm{R}^{*}} \cdot \boldsymbol{x}_{\mathrm{SPR}}+k_{\mathrm{tr}, \mathrm{CTA}}^{\mathrm{t}} \cdot c_{\mathrm{CTA}} \cdot c_{\mathrm{R}} \cdot \boldsymbol{X}_{\mathrm{MCR}}}{k_{\mathrm{p}}^{\mathrm{s}} \cdot c_{\mathrm{M}} \cdot c_{\mathrm{R}} \cdot \boldsymbol{x}_{\mathrm{SPR}}+k_{\mathrm{p}}^{\mathrm{t}} \cdot c_{\mathrm{M}} \cdot c_{\mathrm{R}} \cdot \boldsymbol{X}_{\mathrm{MCR}}} \\
& =\frac{c_{\mathrm{CTA}} \cdot\left(k_{\mathrm{tr}, \mathrm{CTA}}^{\mathrm{s}} \cdot \boldsymbol{X}_{\mathrm{SPR}}+k_{\mathrm{tr}, \mathrm{CTA}}^{\mathrm{t}} \cdot \boldsymbol{X}_{\mathrm{MCR}}\right)}{c_{\mathrm{M}} \cdot\left(k_{\mathrm{p}}^{\mathrm{s}} \cdot \boldsymbol{x}_{\mathrm{SPR}}+k_{\mathrm{p}}^{\mathrm{t}} \cdot \boldsymbol{X}_{\mathrm{MCR}}\right)}
\end{aligned}
$$

A simplified treatment of the denominator of eq. (5.32) RHS has already been discussed in subchapter 2.3.3 (see eq. (2.17)). The same treatment can be made for the numerator, but only under the condition: $k_{\mathrm{tr}, \mathrm{CTA}}^{\mathrm{s}} \cdot \boldsymbol{x}_{\mathrm{SPR}} \gg k_{\mathrm{tr}, \mathrm{CTA}}^{\mathrm{t}} \cdot \boldsymbol{x}_{\mathrm{MCR}} \cdot$ In subchapter 5.1.5, it has been shown that the chain transfer constant of MCRs does not exceed the corresponding one for SPRs. Thus, $k_{\mathrm{tr}, \mathrm{CTA}}^{\mathrm{s}}$ is a much larger than $k_{\mathrm{tr}, \mathrm{CTA}}^{\mathrm{t}}$ and eq. (5.33) can be used for good approximation. For systems, in which eq. (5.33) is valid, the Mayo plot yields $C_{\text {CTA }}^{\text {s }}$.

$$
\frac{r_{\mathrm{tr}}}{r_{\mathrm{p}}} \simeq \frac{c_{\mathrm{CTA}} \cdot k_{\mathrm{tr}, \mathrm{CTA}}^{\mathrm{s}}}{c_{\mathrm{M}} \cdot k_{\mathrm{p}}^{\mathrm{s}}}
$$

The Mayo plot for the product of the polymerization of $0.10 \mathrm{~g} \mathrm{~g}^{-1} \mathrm{AA}$ at $50{ }^{\circ} \mathrm{C}$ in aqueous solution with $6.56 \mathrm{mmol} \mathrm{L}^{-1}$ of photoinitiator D1173 is given in Figure 5-17. 
The data points were deduced from $M_{\text {w }}$ via eq. (4.2), because this was found to give better results for the MAA/ME system (see subchapter 4.1.1).

The best fits to a straight line according to eq. (2.12) yields:

$$
C_{\mathrm{CTA}}^{\mathrm{s}}=0.28 \pm 0.04
$$

The value of $C_{\mathrm{CTA}}^{\mathrm{s}}$ of $\mathrm{AA}$ and $\mathrm{ME}$ in aqueous solution is higher than $C_{\text {CTA }}=0.12 \pm 0.01$ the value for MAA and ME in aqueous solution, which may be explained by the stability of MAA macroradicals being higher than the stability of AA macroradicals (compare subchapter 2.2.1).

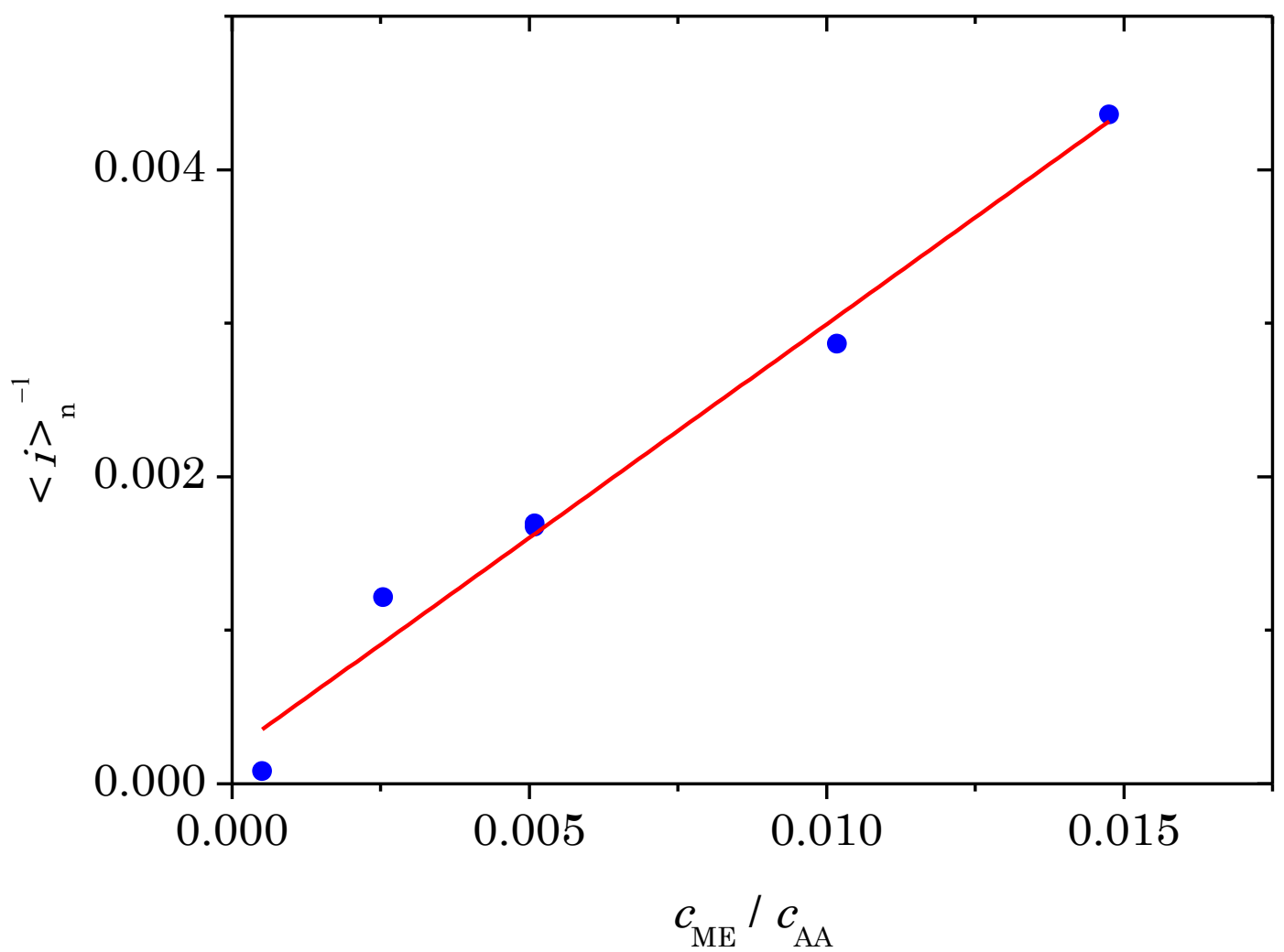

Figure 5-17 The Mayo plot, eq. (2.12), of the polymeric product of the polymerization of $0.10 \mathrm{~g} \mathrm{~g}^{-1}$ $\mathrm{AA}$ at $50^{\circ} \mathrm{C}$ in aqueous solution with $6.56 \mathrm{mmol} \mathrm{L}^{-1}$ of photoinitiator D1173 to low conversion. Data points (blue circles) were deduced from $M_{\mathrm{W}}$ via eq. (5.25). The corresponding best fits to a straight line is given in red. 


\title{
5.1.7 Determination of $k_{\mathrm{p}}$ for AA Macromonomers by ${ }^{1} \mathrm{H}-\mathrm{NMR}$
}

Above $90^{\circ} \mathrm{C}$, macromonomers, $\mathrm{MM}$, are formed during AA polymerization by backbiting and subsequent $\beta$-scission to a significant extent. They can propagate, which leads to the formation of MCRs, which may undergo $\beta$-scission again forming MMs (see subchapter 2.3.1). Hence, the separation of the corresponding coefficients is difficult. The problem was circumvented by synthesizing MM at high temperature (subchapter 3.14.3) and observing their propagation at lower temperature so that $\beta$ scission (and thus formation of MM) as follow-up reaction can be excluded.

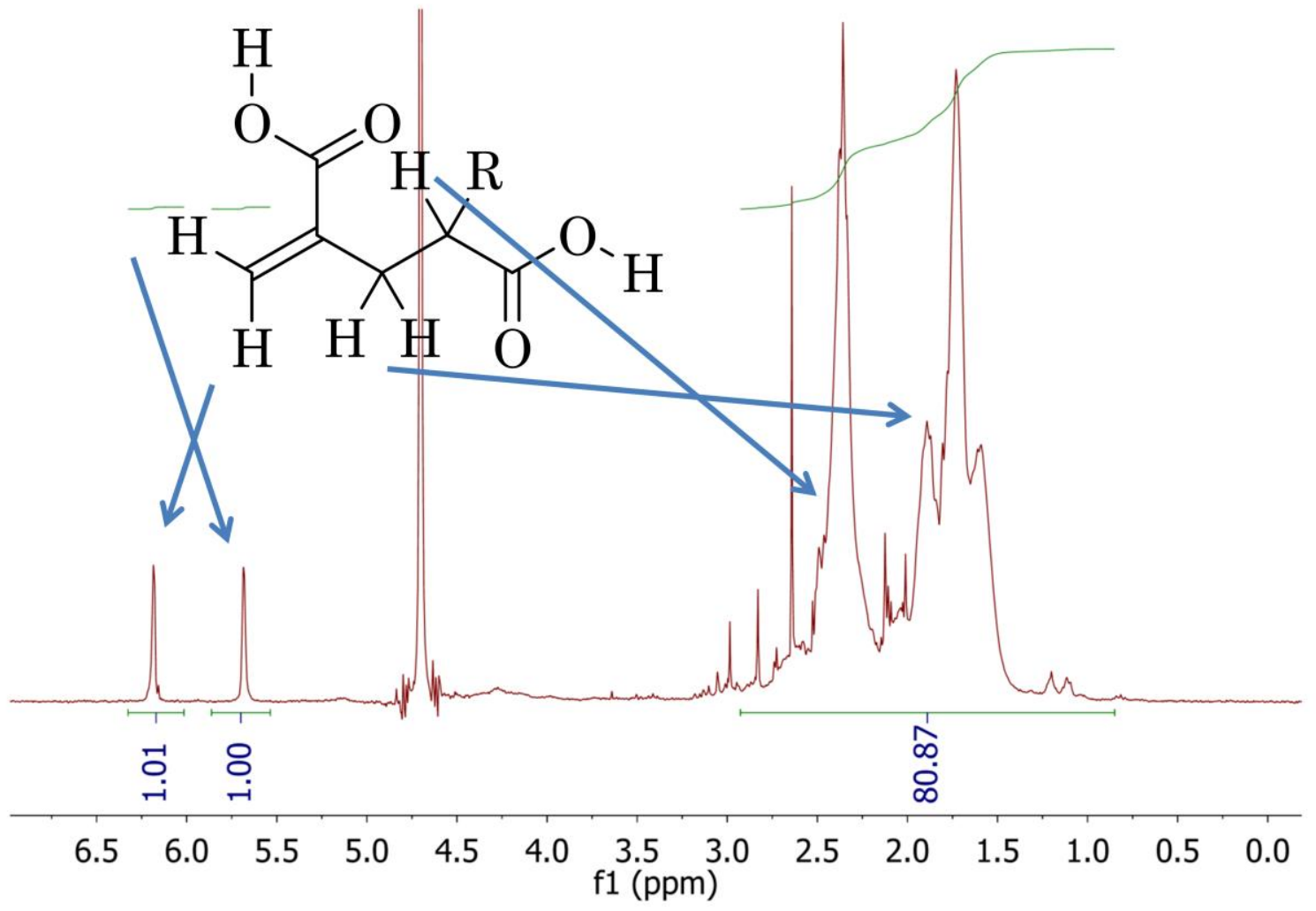

Figure 5-18

\begin{abstract}
${ }^{1} \mathrm{H}-\mathrm{NMR}$ spectrum ( $300 \mathrm{MHz}$ ) of AA macromonomers, MM(AA), synthesized in DMSO at $138^{\circ} \mathrm{C}$. The positions of the protons are attributed to the signals via blue arrows. The MM lines are: ${ }^{1} \mathrm{H}-\mathrm{NMR}\left(\mathrm{D}_{2} \mathrm{O}\right): \delta=1.73(\mathrm{bs}, 2 \mathrm{H}), 2.36(\mathrm{bs}, \mathrm{H}), 5.69(\mathrm{bs}, \mathrm{H}), 6.18(\mathrm{bs}$, $\mathrm{H})$. The prediction of the shift by ChemDraw ${ }^{\mathrm{TM}}$ is $1.75,2.35,5.95$, and 6.54 , respectively.
\end{abstract}

In Figure 5-18, the ${ }^{1} \mathrm{H}-\mathrm{NMR}$ spectrum of MM(AA) synthesized in DMSO at $138{ }^{\circ} \mathrm{C}$ is given. The positions of the protons are attributed to the signals via arrows. The chemical shift of the two protons of the terminal double bond 5.69 (bs, $1 \mathrm{H})$ and 6.18 (bs, $1 \mathrm{H}$ ) cannot be assigned to product from disproportionation, as the shift of such 
protons would be different. Moreover, vicinal coupling is typically very small, while geminal trans-coupling is always observed in high-resolution ${ }^{1} \mathrm{H}-\mathrm{NMR}$ spectroscopy

Figure 5-19 shows the ESI-MS spectrum of the sample, of which the ${ }^{1} \mathrm{H}-\mathrm{NMR}$ is depicted in Figure 5-18. The signals marked by their $\mathrm{m} / \mathrm{z}$ values are multiples of AA monomer mass. The corresponding Lewis structure of the MM(AA) is given as well. The molecule with $935.3 \mathrm{u}$ consists of eleven monomer units, thus mostly oligomeric MMs have been produced. Furthermore, no disproportionation patterns (two lines of equal height with the difference of $2.02 \mathrm{u}$ ) were found in the mass spectrum giving additional support for synthesis of MMs.

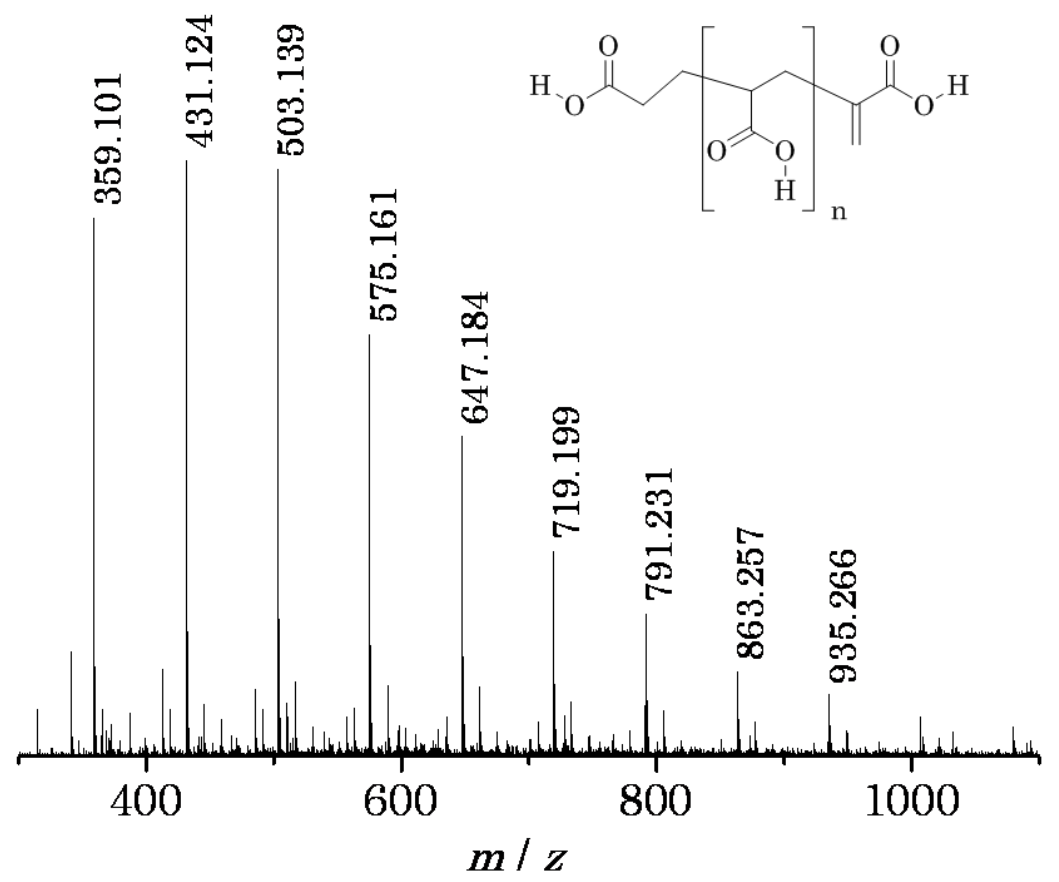

Figure 5-19 ESI-MS spectrum (negatively charged ions; methanol and water) of MM(AA) synthesized in DMSO at $138^{\circ} \mathrm{C}$. The signals marked are multiples of AA monomer mass. The structure of the MM(AA) is given as well. The molecule with $935.3 \mathrm{u}$ consists of eleven monomer units. The smaller signals in between are mostly from multible-charged polymer.

In Figure 5-20, the polymerization of $\mathrm{MM}(\mathrm{AA})$ synthesised in $\sigma$-xylene with $\mathrm{AA}$ in aqueous solution is depicted. The polymerization was carried out in NMR tubes. Both the degree of AA conversion and the ratio of MM(AA) to AA was measured via ${ }^{1} \mathrm{H}-\mathrm{NMR}$. The polymer signal is small at low conversion and in broad. Calculating $X$ 
from the ratio of monomer and polymer signal would be rather imprecise, even more so, as the MM already gives a polymer signal (compare Figure 5-18). By use of the aromatic signal of residual $o$-xylene as reference the measurement becomes more accurate.

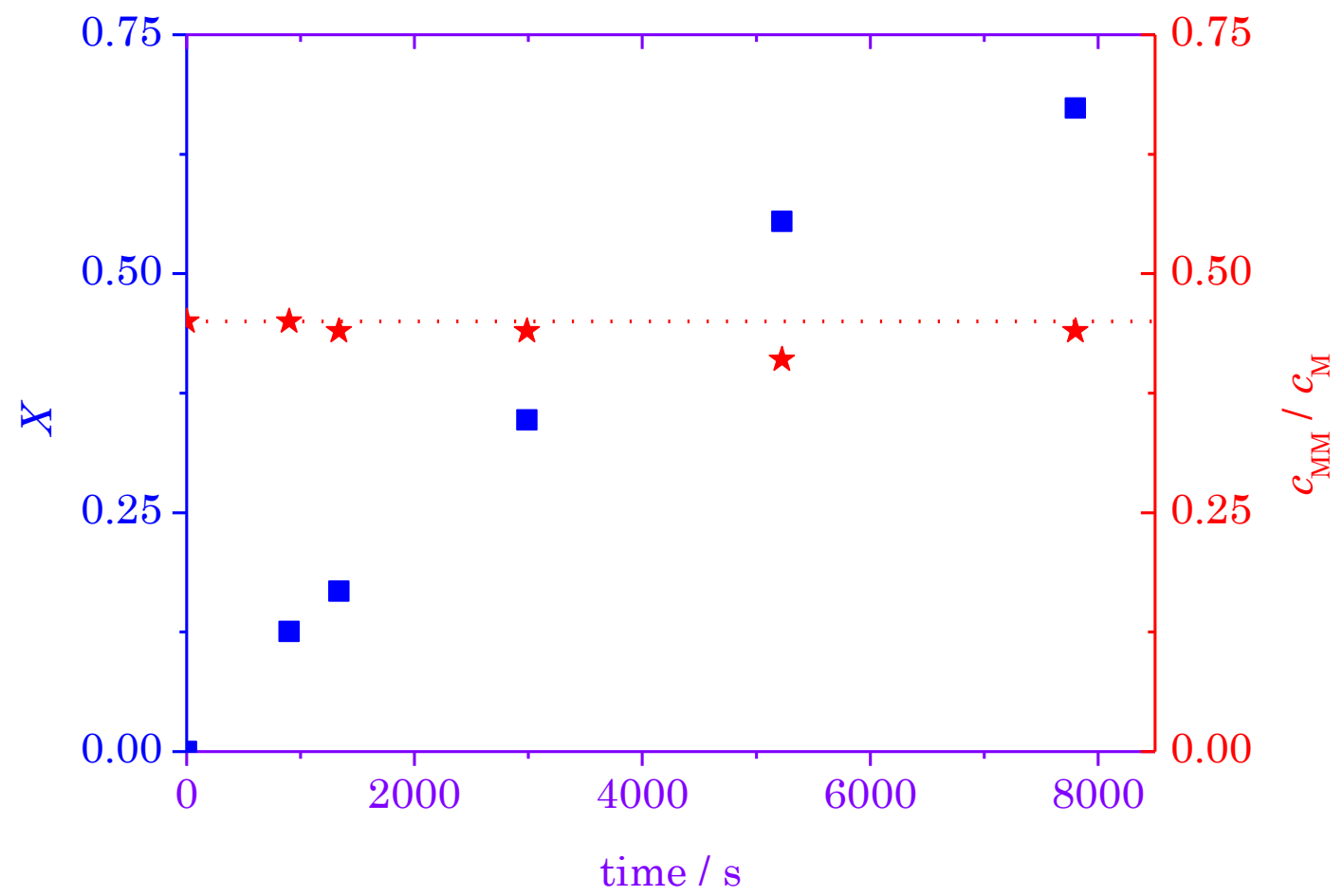

Figure 5-20 MM(AA) and AA were polymerized in NMR tubes. The degree of AA conversion was measured by ${ }^{1} \mathrm{H}-\mathrm{NMR}$ (squares), as was the ratio of $\mathrm{MM}(\mathrm{AA}$ ) to AA (stars). Consumption of monomer and macromonomer is the same, as the ratio stays constant towards higher conversion of AA. The dotted line indicates the initial ratio. The synthesis of MMs had been carried out in $\sigma$ xylene and the aromatic signal of residual $\sigma$ xylene was used as reference. $c_{\mathrm{AA}}=0.047 \mathrm{~mol} \mathrm{~L}^{-1}, c_{\mathrm{MM}(\mathrm{AA})}=0.021 \mathrm{~mol} \mathrm{~L}^{-1}$ (first moment), $c_{V}-50=0.037 \mathrm{~mol} \mathrm{~L}-1$, in aqueous solution at $50^{\circ} \mathrm{C}$.

Because of steric hindrance, propagation of MCRs with MMs was assumed negligible. Different initial ratios around 1:1 were polymerized to observe both AA and MM(AA) with similar accuracy. The coefficient of propagation of an SPR with an $\mathrm{MM}, \quad k_{\mathrm{p}, \mathrm{sM}}^{\mathrm{s}}$, was estimated via PREDICI ${ }^{\mathrm{TM}}$ simulation of profiles the $X$ and the $c_{\mathrm{MM}} / c_{\mathrm{M}}$ profiles as a function of time. The result is given by eq. (5.35). Considering the higher stability of the tertiary radical, it appears reasonable that the addition of an MM to an SPR is favored. 


$$
k_{\mathrm{p}, \mathrm{MM}}^{\mathrm{s}}=1.4( \pm 0.2) \cdot k_{\mathrm{p}}^{\mathrm{s}}
$$

In case that MCRs also add MMs, the reactivity ratios should be unity as $c_{\mathrm{MM}} / c_{\mathrm{M}}$ is independent of $X$.

\subsubsection{Modeling Polymerization at 35 to $80{ }^{\circ} \mathrm{C}$}

The previous subchapters describe the kinetic information available from independent experiments taken either from literature or from this study. The basis for modeling polymerization of AA between $35^{\circ} \mathrm{C}$ and $90{ }^{\circ} \mathrm{C}$ were mostly conversion vs. time profile. Xxv Furthermore, MMDs ${ }^{\mathrm{XxvI}}$ of polymerizations between $35^{\circ} \mathrm{C}$ and $65^{\circ} \mathrm{C}$ were used.

$k_{\mathrm{p}}^{\mathrm{t}}$ was scaled to $k_{\mathrm{p}}^{\mathrm{s}}$ and estimated for each conversion vs. time profile of polymerizations without addition of CTA. The corresponding Arrhenius plot is shown in Figure 5-21. Data from polymerizations with V-50 being the initiator are in good agreement with those from polymerizations with VA-086 being the initiator. The concatenate, best fit to a straight line yields eq. (5.36)

$$
k_{\mathrm{p}}^{\mathrm{t}} / k_{\mathrm{p}}^{\mathrm{s}}=2.41 \cdot \exp \left(-\frac{2314}{T / \mathrm{K}}\right)
$$

According to eq. (5.36) the difference in activation energy between $k_{\mathrm{p}}^{\mathrm{t}}$ and $k_{\mathrm{p}}^{\mathrm{s}}$ is $E_{\mathrm{A}}\left(k_{\mathrm{p}}^{\mathrm{t}}\right)-E_{\mathrm{A}}\left(k_{\mathrm{p}}^{\mathrm{s}}\right)=19.2 \mathrm{~kJ} \mathrm{~mol}^{-1}$, which is in good agreement with $20.5 \mathrm{~kJ} \mathrm{~mol}^{-1}$ calculated from ref. values ${ }^{[36,37]}$ and higher than $E_{\mathrm{A}}\left(k_{\mathrm{p}}^{\mathrm{t}}\right)-E_{\mathrm{A}}\left(k_{\mathrm{p}}^{\mathrm{s}}\right)=11.2 \mathrm{~kJ} \mathrm{~mol}^{-1}$ reported for BA. ${ }^{[42]}$

xxv Some of the conversion time data used for modeling in this subchapter were taken from previous work. ${ }^{[147]}$ Moreover, three conversion vs. time profiles were a courtesy of Patrick Drawe.

xxvI The Lacík group (Polymer Institute SAV, Slovakia) did the SEC analysis. 


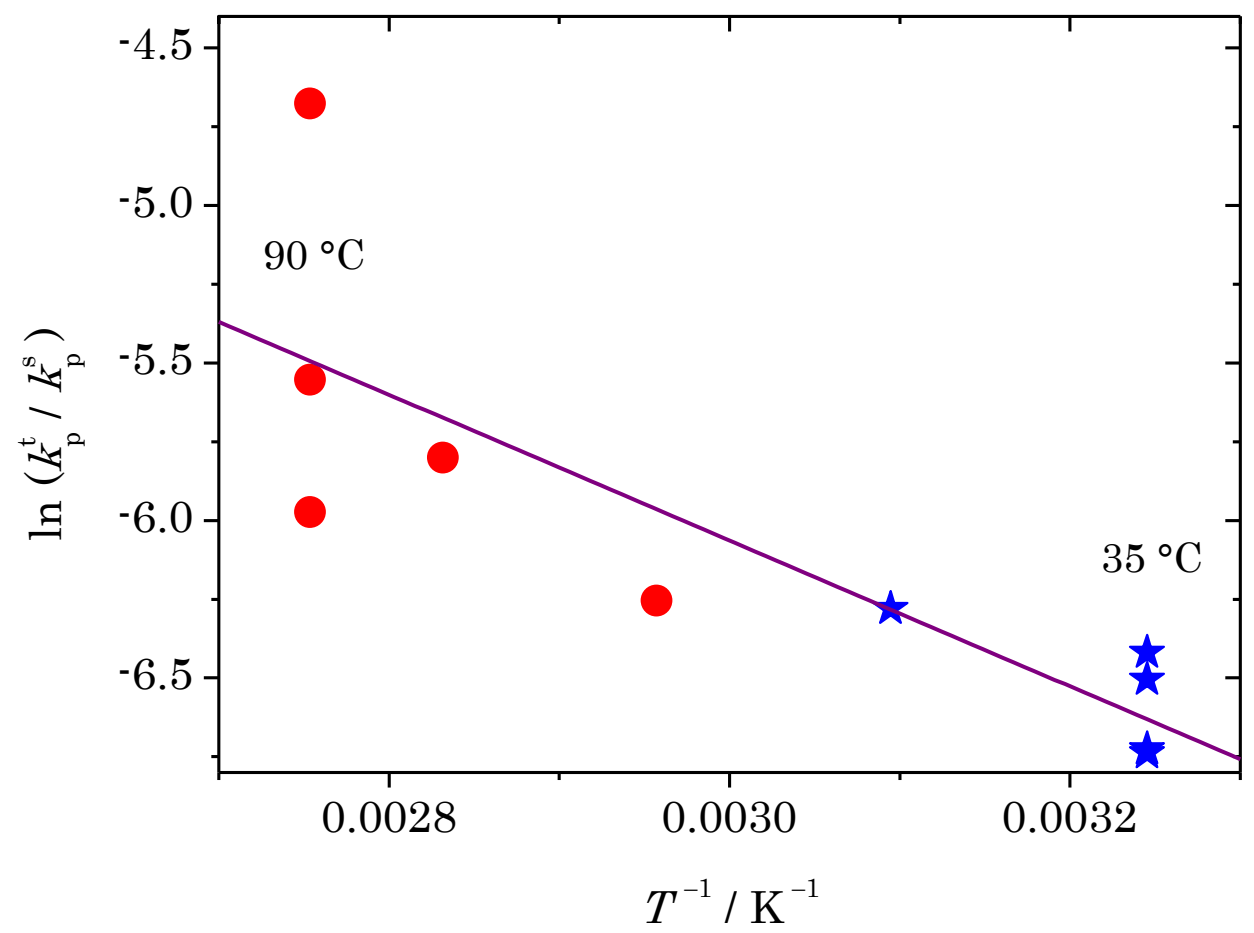

Figure 5-21 Arrhenius plot of the ratio of $k_{\mathrm{p}}{ }^{\mathrm{t}}$ to $k_{\mathrm{p}}^{\mathrm{s}}$. The values were estimated from conversion vs. time profiles by PREDICI ${ }^{\mathrm{TM}}$. Red circles: V-50 was used as initiator; blue triangles: VA-086 was used as initiator. The concatenate, best fit to a straight line is given in purple. The so-obtained difference in $E_{\mathrm{A}}$ of $k_{\mathrm{p}}^{\mathrm{t}}$ and $k_{\mathrm{p}}^{\mathrm{s}}$ is in agreement with literature. ${ }^{[36,37]}$

MMDs have been measured for different temperatures $\left(35,50\right.$, and $\left.65{ }^{\circ} \mathrm{C}\right)$ and for different levels of initiator concentration (0.01, 0.001, and $\left.0.0001 \mathrm{~g} \mathrm{~g}^{-1}\right)$. With $C_{\mathrm{tr}, \mathrm{M}}=5.37 \cdot 10^{-5}$ taken from ref. ${ }^{[90]} \mathrm{MMDs}$ were predicted too high. The deviation was systematically higher towards lower rate of initiation, i.e., low initiator concentration and low temperature. Therefore, transfer to monomer had to be increased and $C_{\mathrm{tr}, \mathrm{M}}=1 \cdot 10^{-4}$ yields simulated MMDs being in satisfying agreement experimental ones.

$0.1 \mathrm{gg}^{-1} \mathrm{AA}$ in water were polymerized at 35,50 , and $65{ }^{\circ} \mathrm{C}$ up to complete conversion with V-50 as initiator at levels of $10^{-2}, 10^{-3}$, and $10^{-4} \mathrm{~g} \mathrm{~g}^{-1}$. Molar mass was found to vary only slightly with initiator concentration. Without considering transfer to monomer MMDs were predicted too high. The deviation was systematically higher towards lower rate of initiation, i.e., low initiator concentration and low temperature. In order to illustrate the influence of $C_{\mathrm{tr}, \mathrm{M}}$, two 
measured MMDs are plotted together with the corresponding simulation not featuring transfer to monomer in Figure 5-22. At relatively high rate of initiation (left) the deviation between experiment and simulation is rather weak, but at low rate of initiation the deviation is strong. Therefore, simulation of MMDs required $C_{\mathrm{tr}, \mathrm{M}}^{\mathrm{s}}$ being:

$$
C_{\mathrm{tr}, \mathrm{M}}^{\mathrm{s}}=\frac{k_{\mathrm{tr}, \mathrm{M}}^{\mathrm{s}}}{k_{\mathrm{p}}^{\mathrm{s}}}=7.5 \cdot 10^{-5}
$$

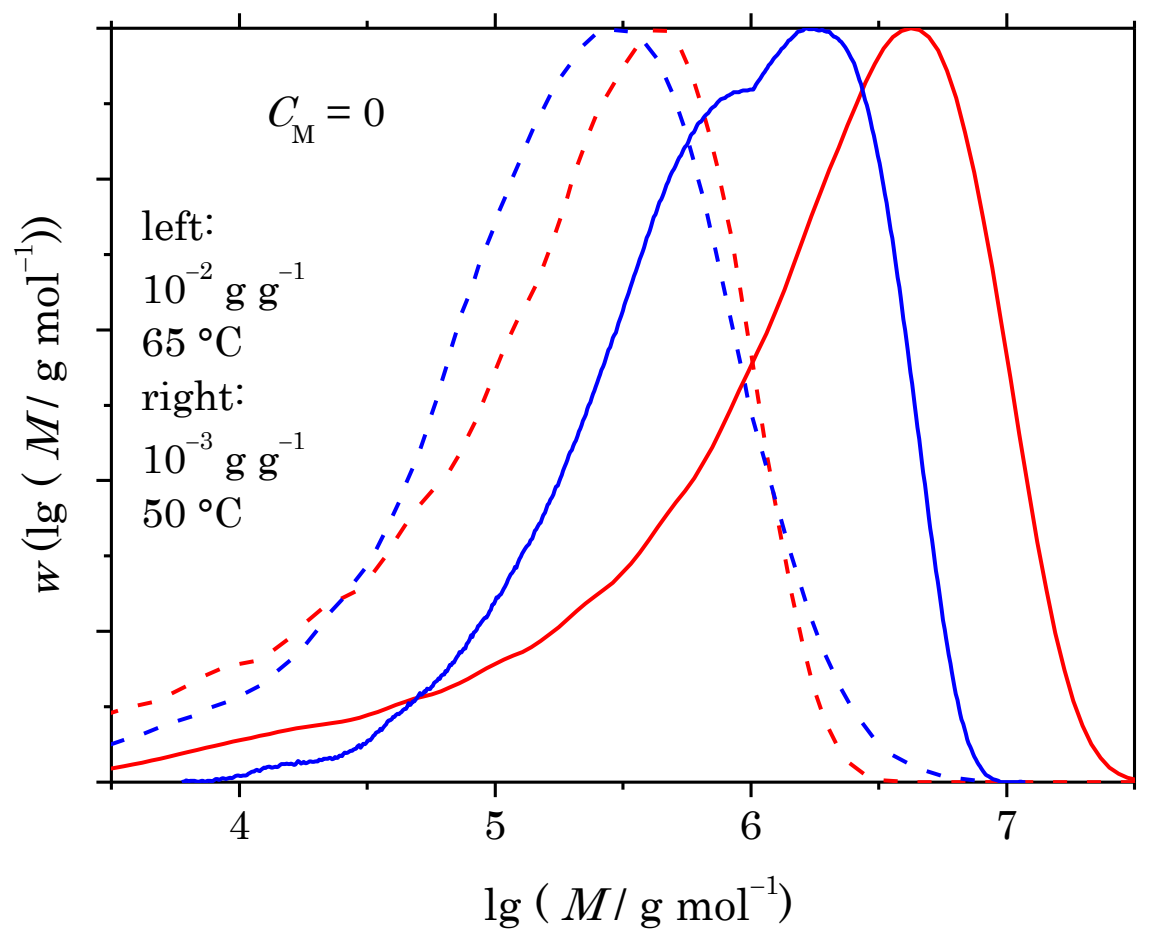

Figure 5-22 Comparison of measured and simulated MMDs without considering transfer to monomer shows deviation, which is was systematically higher towards lower rate of initiation. Blue line: polymerization of $0.1 \mathrm{~g} \mathrm{~g}^{-1} \mathrm{AA}$ to full conversion (initiator concentrations and temperatures given for each graph). Very high molar masses were reached and a significant amount of polymer was outside of SEC calibration. This resulted in a kink as calibration artifact. Red line: simulation.

In Figure 5-23, measured MMDs are compared to predictions by the PREDICI ${ }^{\mathrm{TM}}$ model. The measured MMDs feature a kink slightly above $10^{6} \mathrm{~g} \mathrm{~mol}^{-1}$. This is a calibration artifact due to end of direct calibration at this point as for MMDs from MAA polymerization (subchapter 4.2.2). With transfer to monomer being included, measured and predicted MMDs agree within experimental uncertainty, which is 
higher in this case due to said problem of MMDs being partly out of calibration range. MMDs of polymerizations with CTA are not shown as their prediction is rather trivial, provided that $k_{\mathrm{p}}$ and $C_{\mathrm{tr}, \mathrm{CTA}}$ are known with high accuracy.

For polymerizations with higher monomer content, only a few conversion vs. time profiles were measured. Eq. (5.14) leads to acceptable representation of measured data, but especially for $0.3 \mathrm{~g} \mathrm{~g}^{-1}$ AA a clear deviation in shape is seen. Probably an equation of the form eq. (4.11) (see subchapter 4.2.1) would lead to better results. For an evaluation like this, more data would be needed.

In Figure 5-24, the comparison of measured and simulated AA conversion vs. time plots of polymerization of $0.1 \mathrm{~g} \mathrm{~g}^{-1} \mathrm{AA}$ at temperatures from 35 to $80{ }^{\circ} \mathrm{C}$ demonstrates the quality of the model in this temperature range.

The agreement of measured and simulated AA conversion vs. time plots of $0.1 \mathrm{~g} \mathrm{~g}^{-1}$ AA polymerization at temperatures from 65 to $80{ }^{\circ} \mathrm{C}$ upon addition of CTA from small to very high amounts can be observed in Figure 5-24 and Figure 5-25. The quality of the prediction does not reach the level as obtained for polymerization without CTA, but is still satisfactory, especially when one considers the lower experimental reproducibility of experiments involving CTA. The conversion vs. time profiles of polymerizations with highest ME content show good agreement with the simulation (Figure 5-25). For some other polymerizations with very high ME content (not shown here) a drift towards higher rate of polymerization was found, i.e. at high CTA content further increase of CTA level did not lead to further decrease of polymerization rate but to a slight increase again. This effect might originate from additional initiation by the thiol-ene reaction, the rate of which could not be quantified. It may not be explained by high transfer of MCRs to CTA, because for the model system BA/ME it was found that $C_{\mathrm{CTA}}^{\mathrm{t}}$ cannot be much higher than $C_{\mathrm{CTA}}^{\mathrm{s}}$ (see subchapter 5.1.5).

The parameters of the model for the polymerization of non-ionized AA are summarized in Table 5-2. 


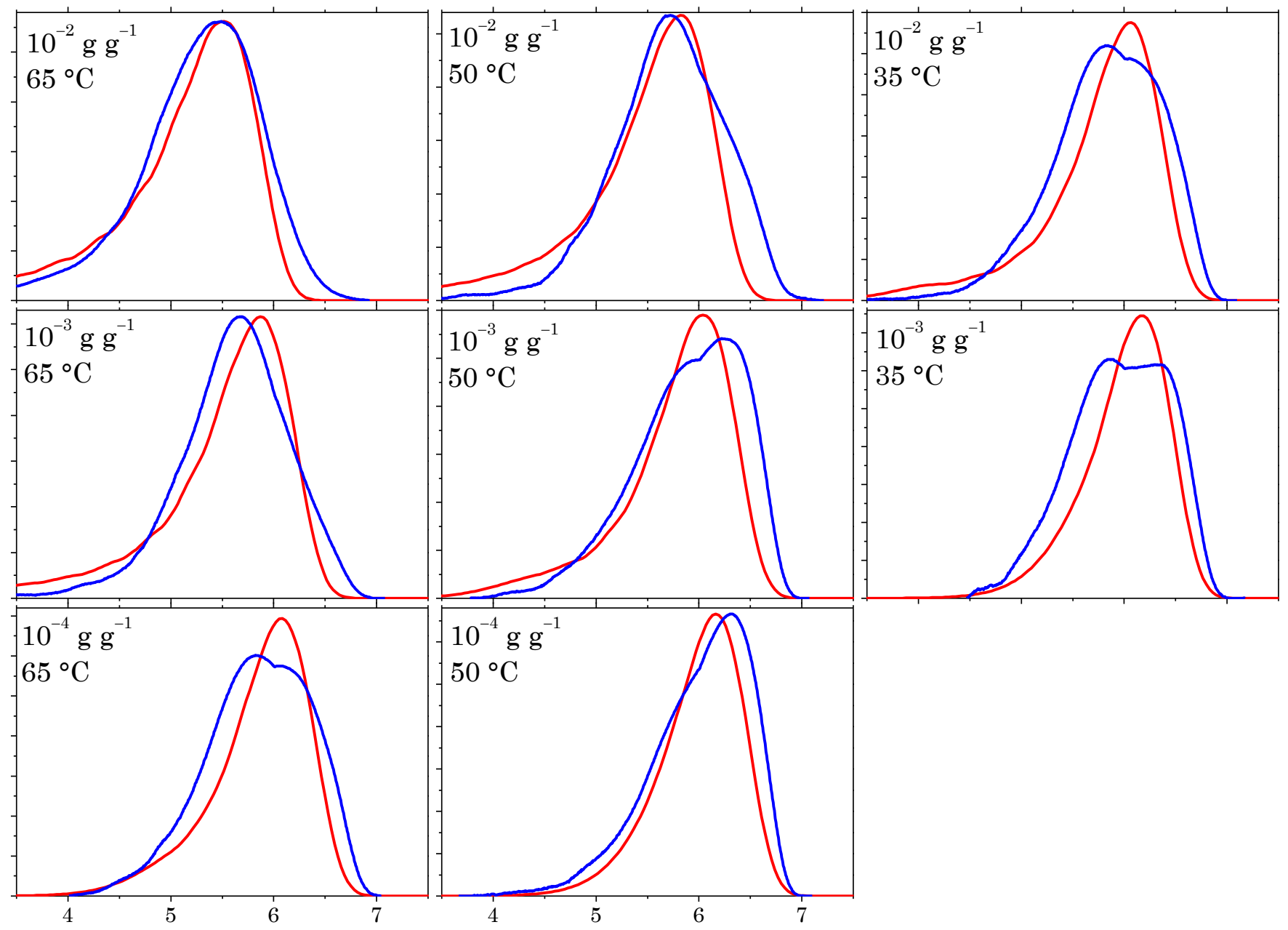




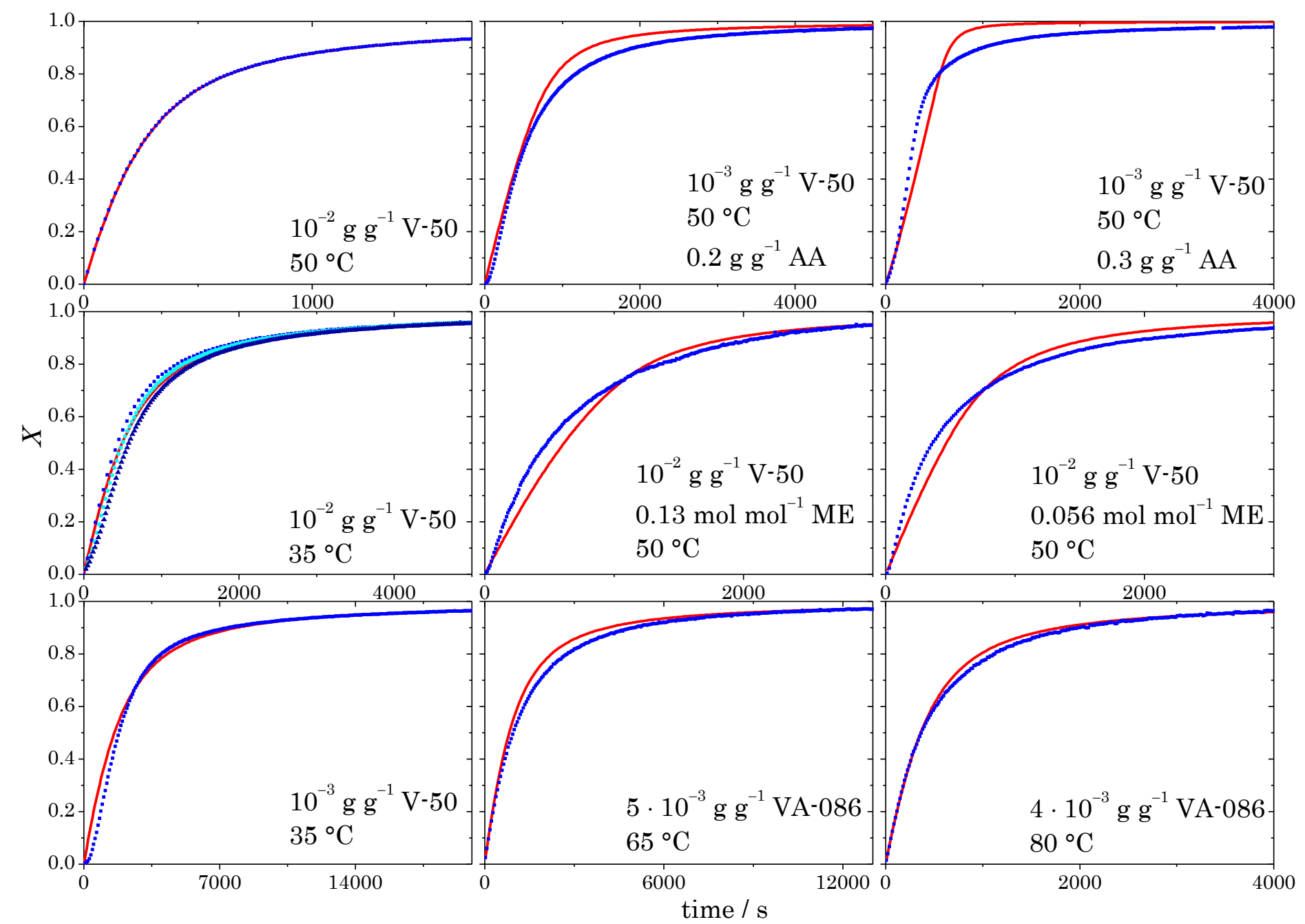

Figure 5-24 Comparison of measured and simulated AA conversion vs. time plots. Blue symbols: polymerization of $0.1 \mathrm{~g} \mathrm{~g}^{-1} \mathrm{AA}$ (if not stated otherwise) with initial CME to CMAA ratios given if applicable; initiator concentrations and temperatures are specified for each graph; the simulations are indicated by the red lines; independent repeat experiments are included (cyan and dark blue). Data for $90{ }^{\circ} \mathrm{C}$ is given in subchapter 5.1.9. 


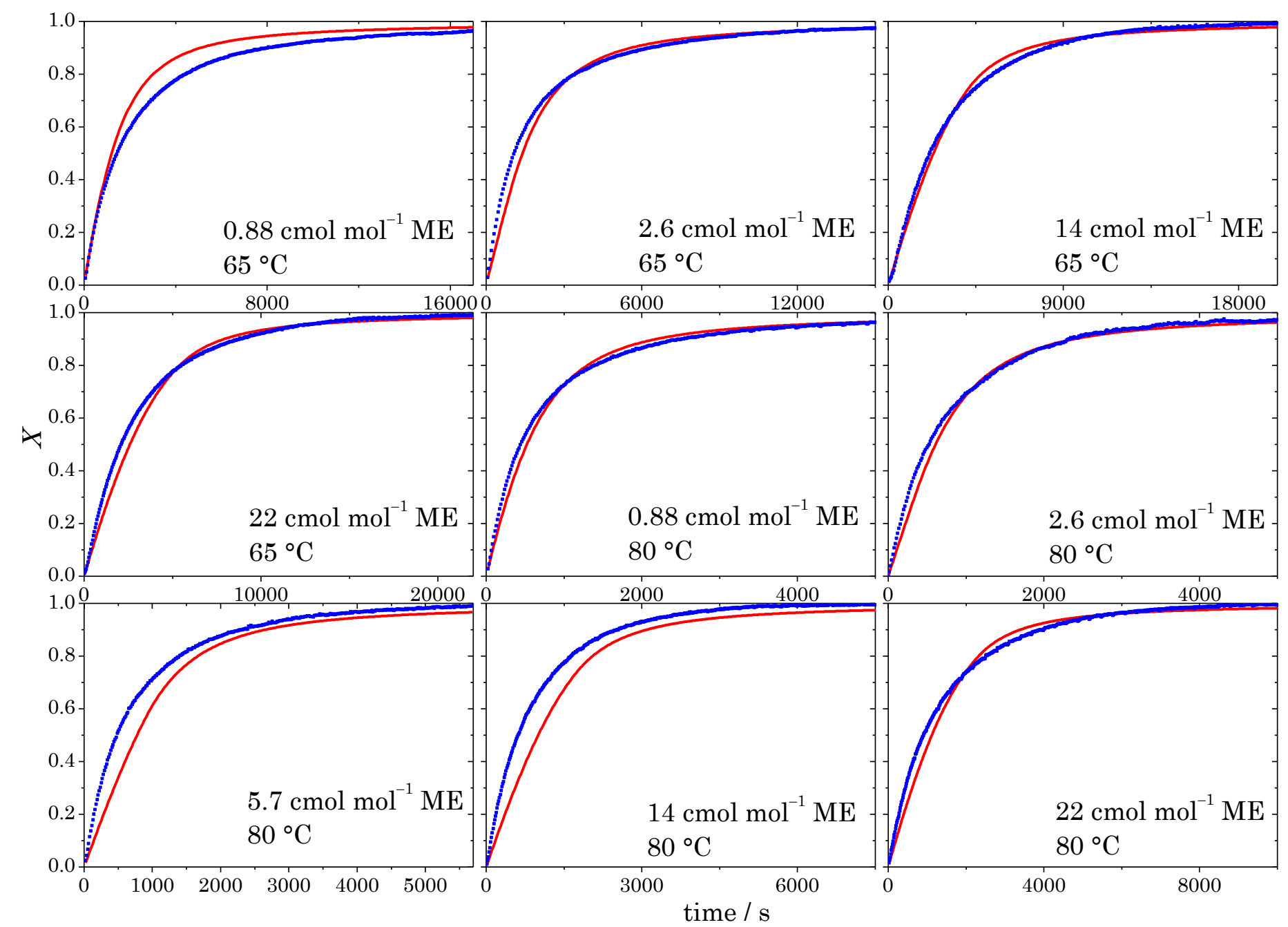

Figure 5-25 Comparison of measured and simulated AA conversion vs. time plots. Blue symbols: polymerization of $0.1 \mathrm{~g} \mathrm{~g}^{-1} \mathrm{AA}_{\text {with }} 0.005 \mathrm{~g} \mathrm{~g}^{-1}$ and $0.004 \mathrm{~g} \mathrm{~g}^{-1}$ VA-086 as the initiator at $65^{\circ} \mathrm{C}$ and $80^{\circ} \mathrm{C}$, respectively. The particular initial $c_{\text {ME }}$ to $C_{\text {MAA }}$ ratios given for each graph. The simulations are indicated by the red lines; independent repeat experiments are included (cyan). 


\subsubsection{Modeling Polymerization at High Temperature}

For polymerizations at high temperature, which means for this system above $90{ }^{\circ} \mathrm{C}$, additional reactions have to be considered leading to an even more complex reaction scheme. $\beta$-scission of MCRs may take place building macromonomers. These propagate to form $\mathrm{MCR}^{1} \mathrm{~s}$, which may add monomer and thus grow further or undergo $\beta$-scission again. The corresponding scheme is shown in Table 5-4 top.

The implementation of the additional high-temperature reactions into PREDICI ${ }^{\mathrm{TM}}$ can be simplified. Nikitin et al. ${ }^{[42]}$ modeled BA polymerization up to $170{ }^{\circ} \mathrm{C}$ with a reduced number of reactions. They condensed the reaction scheme in Table 5-4 top into the reaction scheme in Table 5-4 bottom introducing the compounded rate coefficients defined by eq.(5.38) and eq. (5.39). This strategy was adopted for the present modeling work.

$$
\begin{gathered}
k_{1}=k_{\beta} \cdot \frac{k_{\mathrm{p}, \mathrm{MM}}^{\mathrm{s}}}{2 k_{\beta}+k_{\mathrm{p}}^{\mathrm{t}} \cdot c_{\mathrm{M}}} \\
k_{2}=k_{\mathrm{p}}^{\mathrm{t}} \cdot c_{\mathrm{M}} \cdot \frac{k_{\mathrm{p}, \mathrm{MM}}^{\mathrm{s}}}{2 k_{\beta}+k_{\mathrm{p}}^{\mathrm{t}} \cdot c_{\mathrm{M}}}
\end{gathered}
$$

Table 5-4 The additional reactions that have to be taken into account for high temperature polymerization are shown in the upper part. For modeling of BA polymerization up to $170^{\circ} \mathrm{C}$ as has been shown by Nikitin et al. ${ }^{[42]}$ a simplified approach is possible to account for $\beta$-scission of $\mathrm{MCR}^{1}$, MM propagation, and $\mathrm{MCR}^{1}$ propagation. The corresponding scheme is shown in the lower part.

\begin{tabular}{lc}
\hline \multicolumn{2}{c}{ Additional high temperature reactions } \\
\hline $\mathrm{MCR}^{\mathrm{s}}$ & $\mathrm{R}_{\mathrm{MCR}^{\mathrm{s}}, i}^{\cdot} \stackrel{k_{\beta}}{\longrightarrow} \mathrm{R}_{\mathrm{SPR}, i-2}+\mathrm{MM}_{2} \dot{\vee} \mathrm{R}_{\mathrm{SPR}, 3}+\mathrm{MM}_{i-3}$ \\
MCR & $\mathrm{R}_{\mathrm{MCR}^{1}, i+j}^{\cdot} \stackrel{k_{\beta}}{\longrightarrow} \mathrm{R}_{\mathrm{SPR}, i}+\mathrm{MM}_{j} \dot{\vee} \mathrm{R}_{\mathrm{SPR}, \mathrm{j}}+\mathrm{MM}_{i}$ \\
propagation of $\mathrm{MCR}^{1}$ & $\mathrm{R}_{\mathrm{SPR}, i}^{\cdot}+\mathrm{MM}_{j} \stackrel{k_{\mathrm{p}, \mathrm{MM}}^{\mathrm{s}}}{\longrightarrow} \mathrm{R}_{\mathrm{MCR}^{1}, i+j}^{\cdot}$ \\
& $\mathrm{R}_{\mathrm{MCR}^{1}, i+j}^{\cdot}+\mathrm{M} \stackrel{k_{\mathrm{p}}^{\mathrm{t}}}{\longrightarrow} \mathrm{R}_{\mathrm{SPR}, i+j+1}^{\cdot}$
\end{tabular}


Simplified Treatment

$$
\begin{gathered}
\mathrm{R}_{\mathrm{SPR}, i}^{\cdot}+\mathrm{MM}_{j} \stackrel{k_{1}}{\longrightarrow} \mathrm{R}_{\mathrm{SPR}_{j} j}^{\cdot}+\mathrm{MM}_{i} \\
\mathrm{R}_{\mathrm{SPR}, i}^{\cdot}+\mathrm{MM}_{j} \stackrel{k_{2}}{\longrightarrow} \mathrm{R}_{\mathrm{SPR}, i+j}^{\cdot}
\end{gathered}
$$

For modeling polymerization at intermediate temperature up to $90{ }^{\circ} \mathrm{C}, k_{\mathrm{p}}^{\mathrm{t}}$ was scaled to $k_{\mathrm{p}}^{\mathrm{s}}$ with the ratio being temperature dependent (see subchapter 5.1.8). Extrapolation of this ratio up to $170{ }^{\circ} \mathrm{C}$ gave reasonable results, as will be shown below. Determining the influence of $k_{\mathrm{p}}^{\mathrm{t}}$ and $k_{\beta}$ on rate of polymerization, molar mass, and branching is not trivial. Their respective influence also depends on other parameters. Higher $k_{\mathrm{p}}^{\mathrm{t}}$ leads to higher polymerization rate, but has no direct influence on branching level. $k_{\beta}$ also increases polymerization rate, especially if $k_{\mathrm{p}}^{\mathrm{t}}$ is relatively low and $k_{\mathrm{bb}}$ high. $k_{\beta}$ reduces branching, especially if $k_{\mathrm{p}}^{\mathrm{t}}$ is low in comparison. However, the effect of $k_{\mathrm{p}}^{\mathrm{t}}$ and $k_{\beta}$ on molar masses is reverse. The final coefficients were obtained by keeping the Arrhenius expression for $k_{\mathrm{p}}^{\mathrm{t}}$ derived from moderate temperature polymerizations and adjusting $k_{\beta}$ for best representation of measured conversion vs. time profiles and molar masses. The resulting Arrhenius plot is given by eq. (5.36).

$$
k_{\beta} / \mathrm{s}^{-1}=1.9 \cdot 10^{18} \cdot \exp \left(-\frac{1.5 \cdot 10^{4}}{T / \mathrm{K}}\right)
$$

The corresponding activation energy is $127 \mathrm{~kJ} \mathrm{~mol}^{-1}$, which is close to the activation energy of $125 \mathrm{~kJ} \mathrm{~mol}^{-1}$ found for $k_{\beta}$ during thermal degradation of polyethylene. ${ }^{[186]}$ The activation energy is significantly higher than $63.9 \mathrm{~kJ} \mathrm{~mol}^{-1}$, the activation energy found for the $\beta$-scission of BA. ${ }^{[42]}$ The absolute value of $k_{\beta}$ in the considered temperature range is also bigger than the value found for BA. These comparisons suggest that this value of $k_{\beta}$ might be too high.

In order to obtain better representation of experimental data (conversion vs. time profiles, molar masses, branching level) by simulations of the model, the parameters $k_{\mathrm{p}}^{\mathrm{t}} / k_{\mathrm{p}}^{\mathrm{s}}$ and $k_{\mathrm{t}}^{1,1, \mathrm{tt}} / k_{\mathrm{t}}^{1,1, \mathrm{ss}}$ as well as composite model parameters of MCRs have been widely varied. MCRs kinetics do not have a large impact on the simulation. A slight 
variation of $k_{\mathrm{bb}}$ has been attempted, but as the prediction of the branching level is in good agreement with measured data, this coefficient appears to be accurate.

The $k_{\mathrm{t}}^{1,1, \mathrm{ss}}$ value measured for $\mathrm{AA}^{[36]}$ appears rather high in comparison to other monomers, which suggests that a lower value might lead to better modeling results. Nevertheless, lowering $k_{\mathrm{t}}^{1,1, \mathrm{ss}}$ by a factor of three the virtual dependence of $k_{\mathrm{p}}^{\mathrm{t}} / k_{\mathrm{p}}^{\mathrm{s}}$ on initiator content at $90{ }^{\circ} \mathrm{C}$ remains (see Figure 5-21). Higher and lower values for this coefficient (by a factor of 10) were attempted, but the accuracy of the simulation could not be improved.

Different values for $k_{\beta}$ and $k_{\mathrm{p}}^{\mathrm{t}} / k_{\mathrm{p}}^{\mathrm{s}}$ led to a better representation of conversion vs. time profiles, but to a prediction of higher molar masses than have been measured, which is discussed further below.

In Figure 5-26 top, the concentration of branching points, $c_{\mathrm{BP}}$, is depicted as a function of conversion and temperature. $c_{\mathrm{BP}}$ increases towards higher temperature and towards higher conversion. The branching level can be predicted very well by the model over the entire range of temperature and conversion.

In Figure 5-26 bottom, the fraction of short-chain branching is plotted as a function of conversion and temperature. The simulated values of $x_{\mathrm{SCB}}$ decrease towards higher temperature and towards higher conversion. The experimental values are much lower than simulated ones. This may be explained by further backbiting of MCRs moving the radical function backwards along the polymer chain (see subchapter 2.3.3), a reaction that is not yet included in the model. It should be noted that long-chain branching does only mean that the branch is at least one monomer unit longer than a short-chain branch of two monomer units.

Measured and simulated molar mass are compared in Figure 5-27 (top: $M_{\mathrm{n}}$; bottom: $\left.M_{\mathrm{w}}\right)$. While $M_{\mathrm{n}}$ decreases notably at high conversion, $M_{\mathrm{w}}$ stays relatively constant. Molar mass decreases towards higher temperature, which is mostly the consequence of initiator decay being more rapid. The experimental results for molar mass are somewhat scattered. The model can predict them over the whole range of temperature and conversion very well.

The conversion vs. time profiles of polymerizations between 90 and $170{ }^{\circ} \mathrm{C}$ and the according simulations are shown in Figure 5-28. Experimental results are from two different modes of operation of the tubular reactor setup (see subchapter 3.13 ). The results from the stopped flow experiments, in which the tubular reactor is bypassed 
and the high-pressure cell is used as a batch reactor, are depicted as small symbols. for 90 to $140{ }^{\circ} \mathrm{C}$. The results from polymerization in the tubular reactor are depicted as big symbols and are present from 130 to $170{ }^{\circ} \mathrm{C}$. Three different initiator concentrations were used. They are distinguished in Figure 5-28 by different colors.

At $90{ }^{\circ} \mathrm{C}$ the simulation is good agreement with experimental results for medium initiator content, for high initiator content the rate is somewhat underpredicted and for low initiator content overpredicted. For 110 and $120^{\circ} \mathrm{C}$, the rate of polymerization is predicted too low by the model. At higher temperature, the agreement of experimental conversion vs. time profiles with the simulation is satisfactory.

Comparing the simulation and experimental results from polymerization in the tubular reactor it appears that rate of polymerization is underpredicted at low conversion and over predicted at high conversion. This may not be an inaccuracy of the model but an artifact of residence time correction. The same difference can be observed comparing experimental results from batch experiments and polymerization in the tubular reactor. The model gives a good representation of experimental results from polymerization between 130 and $170{ }^{\circ} \mathrm{C}$, shown in Figure 5-28.

Table 5-5 gives the rate coefficients added for high-temperature reactions. The other rate coefficients are listed in Table 5-2, as they have also been used for modeling polymerization at moderate temperature. radical polymerization of non-ionized acrylic acid in aqueous solution at $90-170^{\circ} \mathrm{C}$. The values given in this table were obtained optimizing based on both conversion vs. time profiles and molar masses.

\begin{tabular}{lcc}
\hline reaction step & rate expression & ref. \\
\hline $\begin{array}{c}\text { propagation of } \\
\text { MMs }\end{array}$ & $k_{\mathrm{p}, \mathrm{MM}}^{\mathrm{s}}=1.4( \pm 0.2) \cdot k_{\mathrm{p}}^{\mathrm{s}}$ & this study \\
$\beta$-scission & $k_{\beta} / \mathrm{s}^{-1}=1.9 \cdot 10^{18} \cdot \exp \left(-\frac{1.5 \cdot 10^{4}}{T^{-1} / \mathrm{K}^{-1}}\right)$ & this study \\
\hline
\end{tabular}



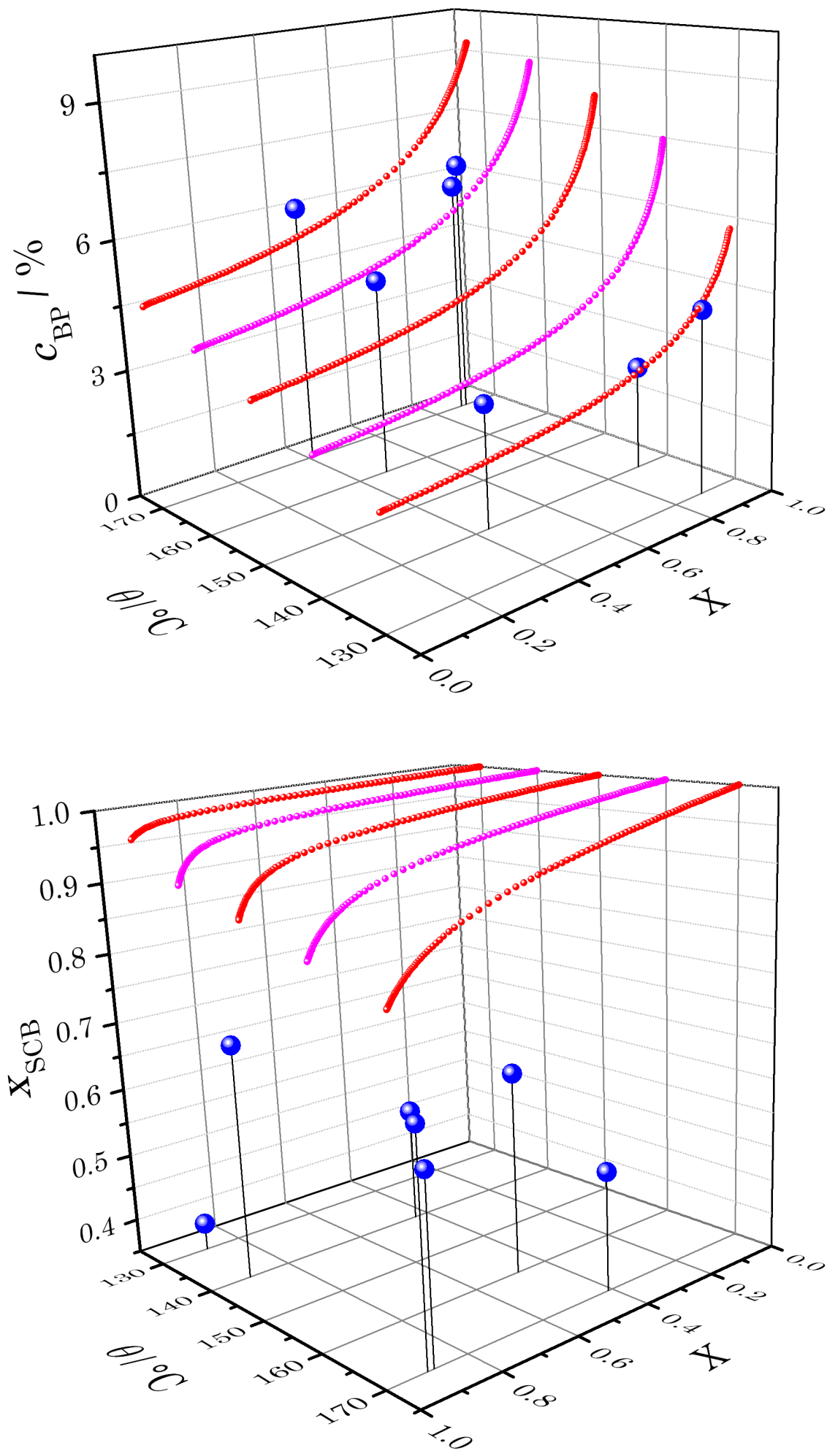

Figure 5-26

Comparison of simulated (small red and pink symbols) and measured (big blue symbols) branching level (top) and the fraction of short-chain branching (bottom) of the reaction product from $0.1 \mathrm{~g} \mathrm{~g}^{-1}$ AA with $0.02 \mathrm{~g} \mathrm{~g}^{-1} \mathrm{VA}-086$ in water. 

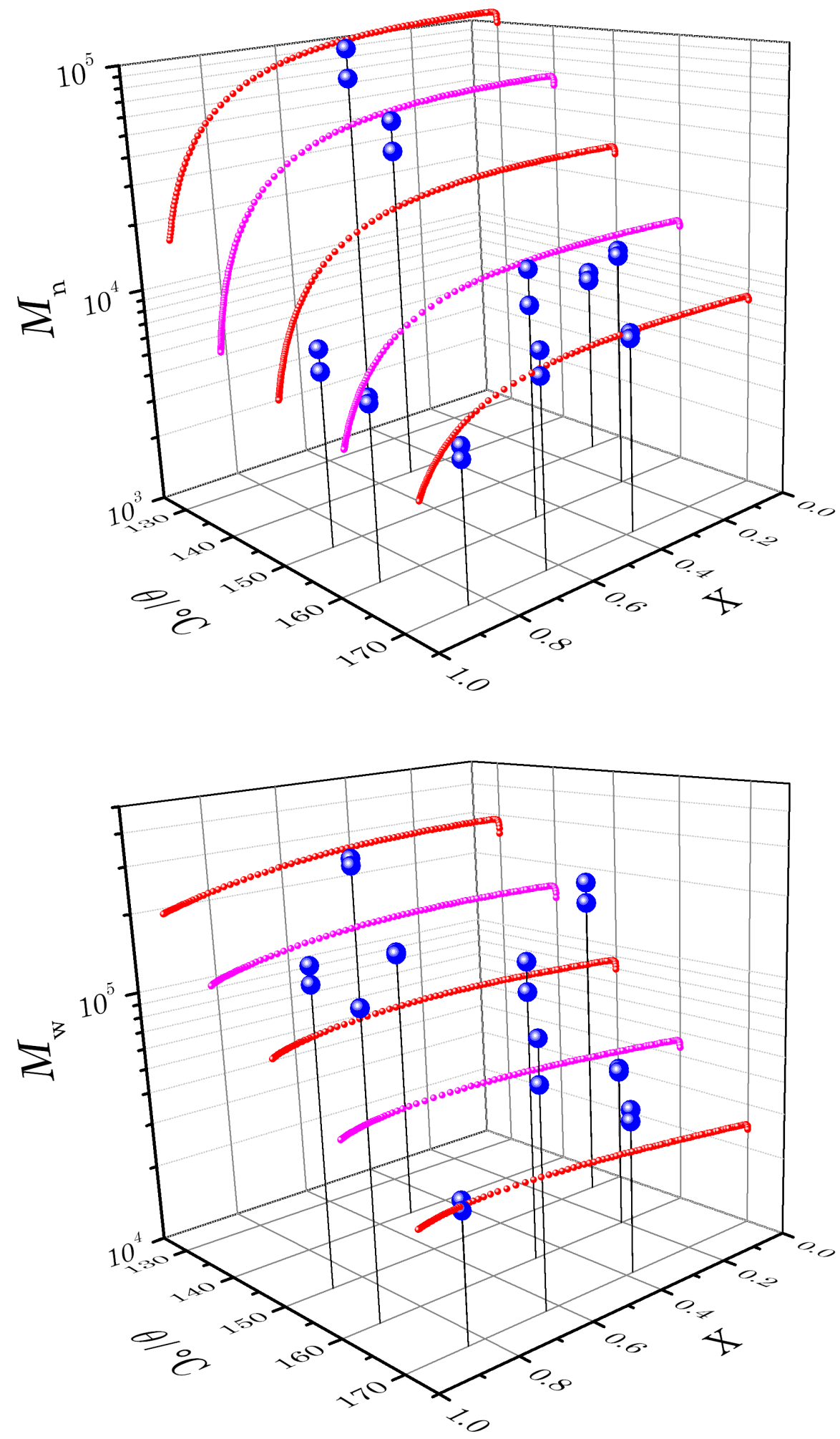

Figure 5-27 Comparison of simulated (small red and pink symbols) and measured (big blue symbols) molar mass of the reaction product from $0.1 \mathrm{~g} \mathrm{~g}^{-1}$ AA with $0.02 \mathrm{~g} \mathrm{~g}^{-1}$ VA-086 in water. 


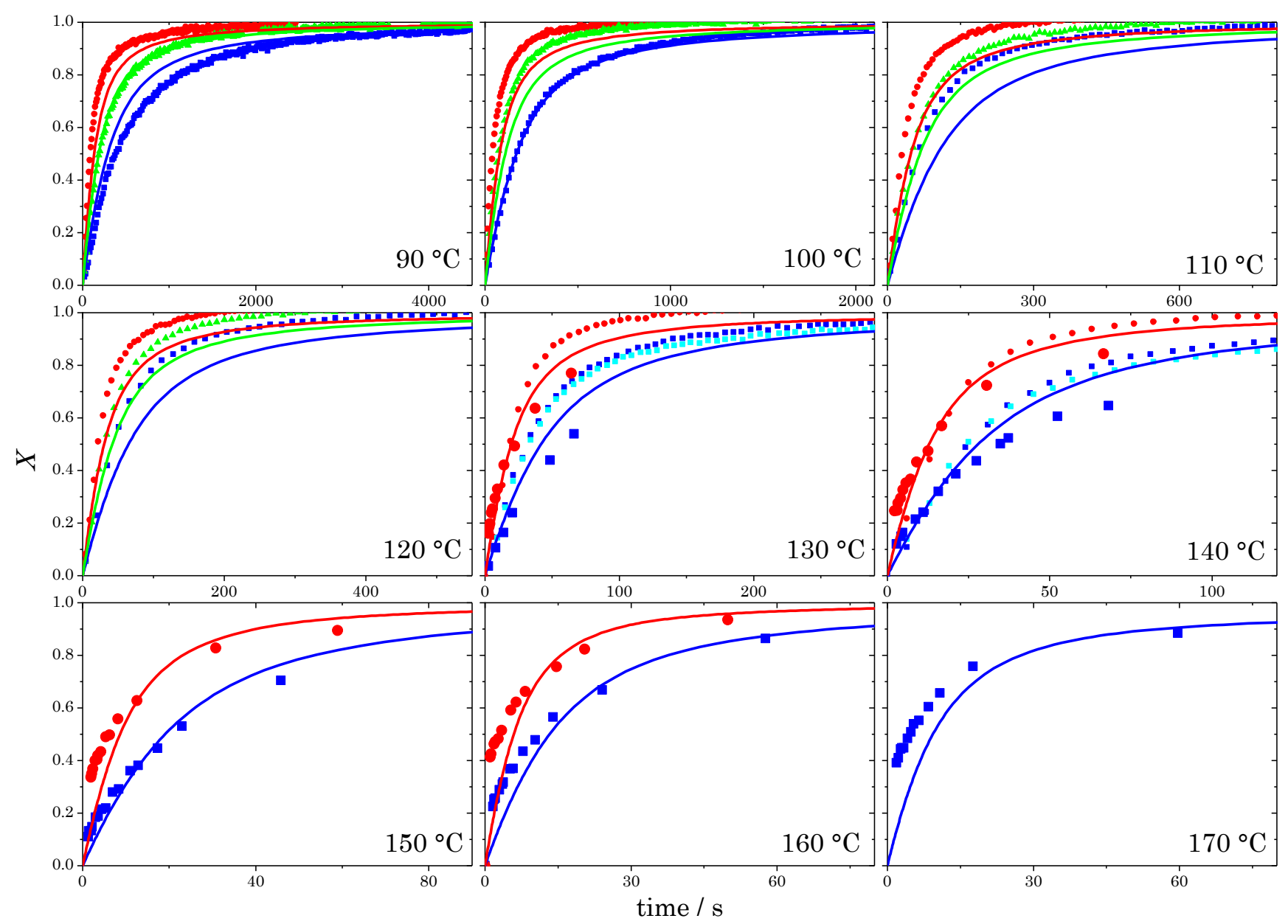

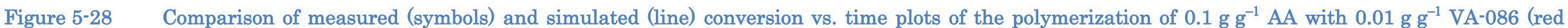
circles), $0.005 \mathrm{~g} \mathrm{~g}^{-1} \mathrm{VA}-086$ (green triangles), $0.002 \mathrm{~g} \mathrm{~g}^{-1} \mathrm{VA}-086$ (blue and cyan squares). The mode of operation was either stopped flow (batch experiment) given by small symbols or polymerization in the tubular reactor depicted as big symbols. 


\section{Alternative values for $k_{\mathrm{p}}{ }^{\mathrm{t}}$ and $k_{\beta}$}

The unexpected high value for $k_{\beta}$ and the rather low quality of polymer samples from high-temperature experiments subjected to molecular mass analysis led to the idea that molar mass may be ignored and instead conversion vs. time profiles may have priority for optimization of the model. This led a similar $k_{\beta}$ value as found for of BA and $k_{\mathrm{p}}^{\mathrm{t}} / k_{\mathrm{p}}^{\mathrm{s}}$ became even more similar to the value reported for BA than with the approach including optimization with respect to molar masses.

$$
k_{\beta} / \mathrm{s}^{-1}=1.3 \cdot 10^{9} \cdot \exp \left(-\frac{7.8 \cdot 10^{3}}{T / \mathrm{K}}\right)
$$

The corresponding activation energy is $65 \mathrm{~kJ} \mathrm{~mol}^{-1}$, which is close to the activation energy of $63.9 \mathrm{~kJ} \mathrm{~mol}^{-1}$ found for the $\beta$-scission of BA. ${ }^{[42]}$ The Arrhenius preexponential factor is slightly lower than 1.5 found for the $\beta$-scission of BA. ${ }^{[42]}$

$$
k_{\mathrm{p}}^{\mathrm{t}} / k_{\mathrm{p}}^{\mathrm{s}}=0.38 \cdot \exp \left(-\frac{1382}{T / \mathrm{K}}\right)
$$

The corresponding difference in activation energy between $k_{\mathrm{p}}^{\mathrm{t}}$ and $k_{\mathrm{p}}^{\mathrm{s}}$ is $E_{\mathrm{A}}\left(k_{\mathrm{p}}^{\mathrm{t}}\right)-E_{\mathrm{A}}\left(k_{\mathrm{p}}^{\mathrm{s}}\right)=11.5 \mathrm{~kJ} \mathrm{~mol}^{-1}$, which is lower than $20.5 \mathrm{~kJ} \mathrm{~mol}^{-1}$ calculated from ref. values, ${ }^{[36,37]}$ but surprisingly similar to $E_{\mathrm{A}}\left(k_{\mathrm{p}}^{\mathrm{t}}\right)-E_{\mathrm{A}}\left(k_{\mathrm{p}}^{\mathrm{s}}\right)=11.2 \mathrm{~kJ} \mathrm{~mol}^{-1}$ reported for BA. ${ }^{[42]}$

The agreement between simulations and experimental conversion vs. time profiles still varies with initiator concentration, but the underprediction of polymerization rate at 110 and $120^{\circ} \mathrm{C}$ is much weaker for this approach and in general the representation of experimental conversion vs. time profiles is better.

The alternative $k_{\mathrm{p}}^{\mathrm{t}} / k_{\mathrm{p}}^{\mathrm{s}}$ expression, eq. (5.42), was not developed for simulation of AA polymerization between 35 to $80^{\circ} \mathrm{C}$. 


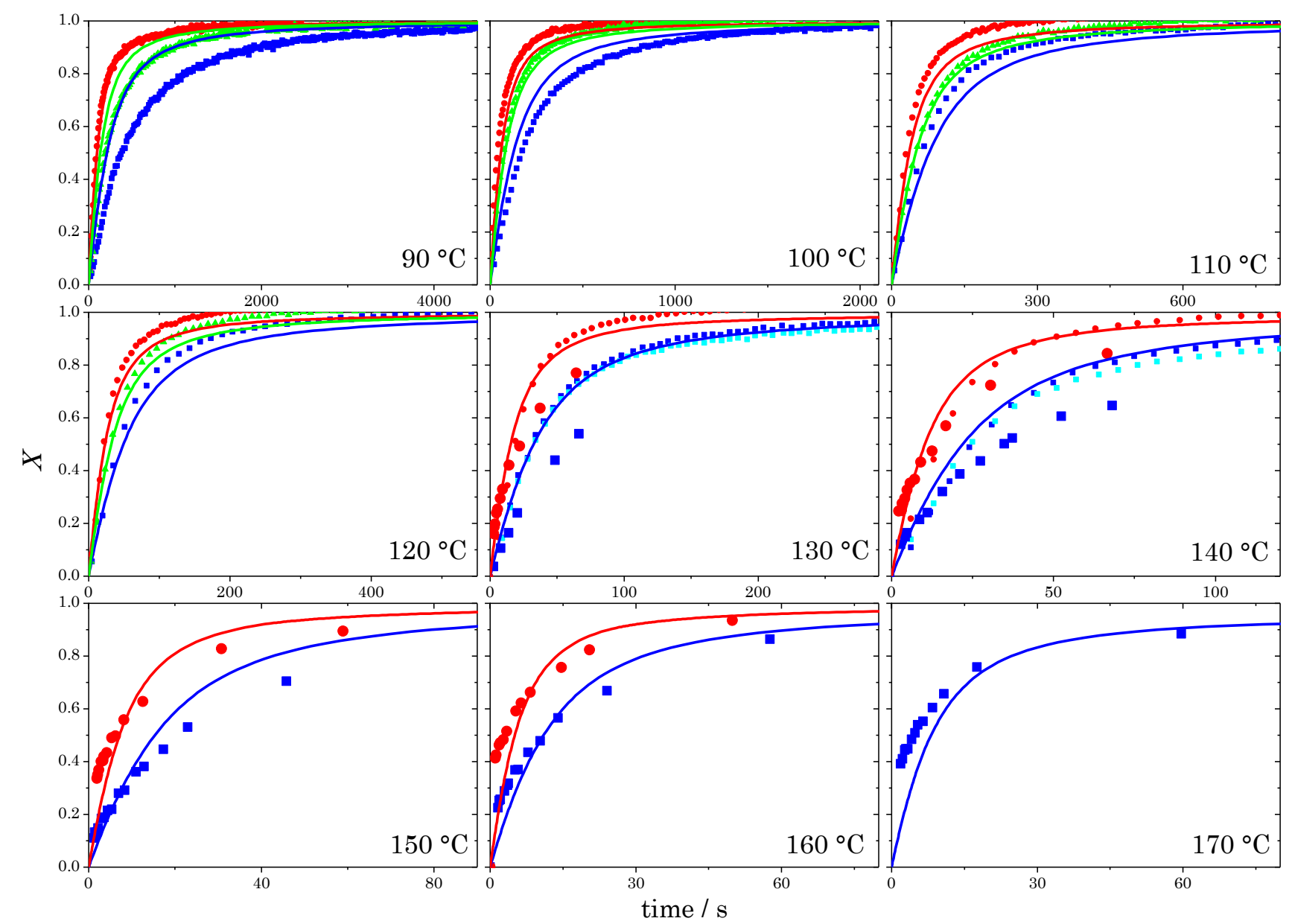

Figure 5-29

Comparison of measured (symbols) and simulated (line) conversion vs. time plots of the polymerization of $0.1 \mathrm{~g} \mathrm{~g}^{-1} \mathrm{AA}^{-1} \mathrm{with}^{0.01} \mathrm{~g} \mathrm{~g}^{-1} \mathrm{VA}-086$ (red circles), $0.005 \mathrm{~g} \mathrm{~g}^{-1}$ VA-086 (green triangles), $0.002 \mathrm{~g} \mathrm{~g}^{-1}$ VA-086 (blue and cyan squares). The simulations show here were done with different values for $k_{\mathrm{p}}{ }^{\mathrm{t}}$ and $k_{\mathrm{B}}$ than in Figure 5-28. 
Table 5-6 gives alternative values for the rate coefficients added for hightemperature reactions. The other rate coefficients are listed in Table 5-2, as they have also been used for modeling polymerization at moderate temperature.

Table 5-6 Summary of alternative rate coefficients, which were obtained by optimization based on conversion vs. time profiles only, ignoring molar masses.

\begin{tabular}{lcc}
\hline reaction step & rate expression & ref. \\
\hline $\begin{array}{c}\text { propagation of } \\
\text { MCRs }\end{array}$ & $k_{\mathrm{p}}^{\mathrm{t}} / k_{\mathrm{p}}^{\mathrm{s}}=0.38 \cdot \exp \left(-\frac{1382}{T / \mathrm{K}}\right)$ & this study \\
$\beta$-scission & $k_{\beta} / \mathrm{s}^{-1}=1.3 \cdot 10^{9} \cdot \exp \left(-\frac{7.8 \cdot 10^{3}}{T / \mathrm{K}}\right)$ & this study \\
\hline
\end{tabular}

\subsection{Model Development for Ionized Acrylic Acid}

Ionization of monomer and polymer alters rate coefficients. For polymerization of fully ionized monomer, reactions between ionized and non-ionized species need not be considered, but even in this case the level of complexity rises in comparison to polymerization of non-ionized monomer, as rate coefficients of ionized species depend on ionic strength. The effect of ionic strength on, e.g., $k_{\mathrm{p}}^{\mathrm{s}}$ is considerable, as will be shown in subchapter 5.2.1

For polymerization of partly ionized monomer complexity is further increased, because reactions of ionized with non-ionized species have to be considered and as the $\mathrm{p} K_{\mathrm{A}}$ values of monomer and polymer differ (see subchapter 5.2.10), the degree of ionization of both monomer and polymer changes with conversion.

The kinetic scheme for the polymerization of ionized AA is principally the same as for non-ionized AA, as no additional reactions occur. Thus, the kinetic scheme used for modeling consists of the kinetic scheme given in the upper part of Table $5^{-1}$ and includes kinetics of MCRs (Table 5-1 middle). High-temperature reactions of ionized AA have not been modeled. The set of rate coefficients and other variables, e.g., density, was taken from modeling of the non-ionized monomer (Table 5-2) and extended as is summarized in Table 5-7 for fully ionized AA and summarized in Table 5-8 for partly ionized AA at the end of this section. 
Some kinetic information on the effects of ionization is known from literature, but most dependencies have not yet been studied in detail. For modeling, a combination of re-evaluation of data reported by other groups and new experiments ${ }^{\mathrm{XVVII}}$ were analyzed. The level of complexity for modeling the polymerization non-ionized AA (see subchapter 5.1) already being higher than for MAA (see chapter 4) is further increased in this system. As modeling of the polymerization of fully ionized AA is easier, first a summary of the rate coefficients for this system is given with the corresponding modeling work following in subchapter 5.2.5. Afterwards the aspects of the partly ionized systems are discussed and predictions of the so-assembled model are shown in subchapter 5.2.11.

\subsection{1 $\quad k_{\mathrm{p}}$ of Fully lonized AA and dependence on lonic Strength}

$k_{\mathrm{p}}^{\mathrm{s}}$ of fully ionized AA has been measured via PLP-SEC only for one concentration $\left(0.05 \mathrm{~g} \mathrm{~g}^{-1}\right.$ at $\left.6{ }^{\circ} \mathrm{C}\right) .{ }^{[76]}$ However, the dependence of $k_{\mathrm{p}}^{\mathrm{s}}$ of fully ionized AA on monomer content should be similar to the dependence of $k_{\mathrm{p}}$ of fully ionized MAA on monomer content. ${ }^{[11]} k_{\mathrm{p}}$ of fully ionized MAA is overall less dependent on monomer content than the $k_{\mathrm{p}}$ of non-ionized MAA and towards dilute solution a decrease of $k_{\mathrm{p}}$ instead of an increase as for the non-ionized monomer was found. ${ }^{[11]}$ The dependence of $k_{\mathrm{p}}^{\mathrm{s}}$ of $\mathrm{AA}$ on $W_{\mathrm{AA}}$ and $\alpha$ is discussed in subchapter 5.2.6. The result with respect to $W_{\mathrm{AA}}$ at full ionization is given by eq. (5.43).

$$
\begin{aligned}
& k_{\mathrm{p}}^{\mathrm{s}} / \mathrm{L} \mathrm{mol}^{-1} \mathrm{~s}^{-1}=1.94 \cdot 10^{7} \cdot \exp \left(-\frac{1395}{T / \mathrm{K}}\right) \\
& \cdot(0.16+(1-0.16) \cdot \exp (-3.7 \cdot W)) \cdot(1.6 \cdot W+0.04)
\end{aligned}
$$

There is no value available for $k_{\mathrm{p}}^{\mathrm{t}}$ of partly ionized AA and only one value for $k_{\mathrm{p}}^{\mathrm{t}}$ of fully ionized AA, which was obtained by parameter estimation from SP-PLP-EPR radical concentration vs. time profiles. ${ }^{[77]}$ The literature value was estimated under the assumption that MCRs do not terminate, whereas in the present study the termination of MCRs is taken into account.

XXVII Patrick Drawe carried out the experiments with V-50 as initiator and fully ionized AA. 
It appears reasonable that $k_{\mathrm{p}}^{\mathrm{t}}$ is influenced by ionization in the same way as $k_{\mathrm{p}}^{\mathrm{s}}$. The same assumption has been made for the dependence of $k_{\mathrm{p}}^{\mathrm{t}}$ on AA content and gave good results (see subchapter 5.1). As a consequence, $k_{\mathrm{p}}^{\mathrm{t}}$ is higher than the value estimated upon neglect of MCR termination. ${ }^{[7]}$ The approach used for this modeling work leads to good representation of experimental data (v.i), whereas the lower value from ref. ${ }^{[77]}$ leads to underprediction of the rate of polymerization.

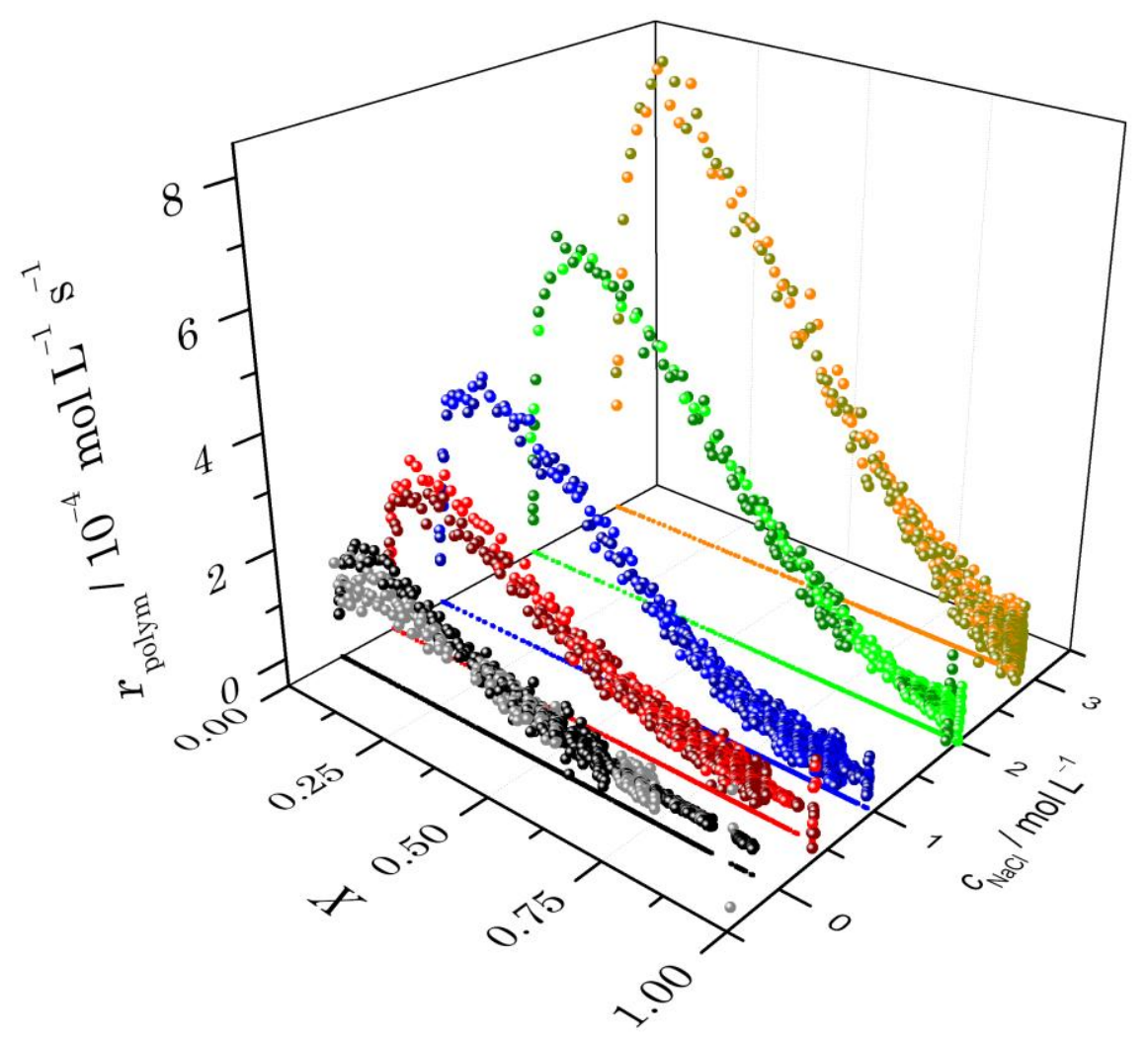

Figure 5-30 Rate of polymerization of $0.05 \mathrm{~g} \mathrm{~g}^{-1} \mathrm{AA}(\alpha=1)$ with $12 \mathrm{mmol} \mathrm{L}-1 \mathrm{~V}-50$ as the initiator in water with different amounts of added $\mathrm{NaCl}$ at $50^{\circ} \mathrm{C}$ and ambient pressure. The rate of polymerization augments linearly with amount of added salt. Repeat experiments show good agreement. This graph is a courtesy from Patrick Drawe.

Eq. (5.38) describes $k_{\mathrm{p}}^{\mathrm{s}}$ as a function of monomer content, temperature and degree of ionization, but does not consider the effect of ionic strength on polymerization kinetics. The rate of polymerization of $0.05 \mathrm{~g} \mathrm{~g}^{-1} \mathrm{AA}(\alpha=1)$ with $12 \mathrm{mmol} \mathrm{L}^{-1} \mathrm{~V}-50$ as the initiator in aqueous solution at $50{ }^{\circ} \mathrm{C}$ is shown in Figure 5-30 for different amounts of added $\mathrm{NaCl}$. The rate of polymerization augments linearly with the added amount of salt. This increase should be associated with an increase of $k_{\mathrm{p}}$, 
since variation of all other rate coefficients should result in a decrease of polymerization rate at higher $I$, as will be discussed below. Kabanov et al.[12] proposed an ion-pair mechanism as the cause for a higher $k_{\mathrm{p}}$ towards increase of $I$. Their idea was that the carboxylate unit at the chain-end of the macroradical interacts with a cation, e.g., $\mathrm{Na}^{+}$, which in turn interacts with the carboxylate moiety of a monomer molecule thus "handing it over" to the radical center and thus increases rate of propagation. These authors did not consider MCRs, as backbiting was not known for AA polymerization at the time of publication. The dependence of $k_{\mathrm{p}}^{\mathrm{s}}$ on both monomer content and degree of ionization can be explained via the transition state structure. The trends found experimentally are reproduced by calculation. ${ }^{[169]}$ So far, no attempts have been made to calculate $k_{\mathrm{p}}^{\mathrm{s}}$ for ionized acrylic acid based on transition state theory with different amounts of cations being present around the radical center.

If an ion-pair mechanism increases the rate of propagation, this effect should be stronger for MCRs - approximately twice as large - because they have carboxylate groups on both sides "handing over" monomer. Thus, the rate enhancing effect by addition of counter ions should be stronger for conditions favoring MCRs, e.g., low monomer concentration. Moreover, the rate enhancement by additional counterions should be stronger for $\mathrm{NaA}$ than for NaMA. Indeed, overtitration with sodium hydroxide, which increases the number of counter ions, results in a stronger rate enhancement of polymerization of $\mathrm{NaA}$ than of NaMA. ${ }^{[12]}$ The enhancing effect being weaker for NaMA may also explain, why it was not found in PLP-SEC experiments of NaMA (see subchapter 2.4.3).

By close inspection of the profiles given in Figure 5-30, it can be seen that the maximum of rate of polymerization slightly shifts to higher degrees of monomer conversion towards higher salt concentration. This observation supports the idea of a stronger impact on MCRs, because their fraction increases towards higher $X$. However, the variation of $x_{\mathrm{MCR}}$ is weak and more experiments under conditions, which favor MCRs to different extent, should be carried out.

As the addition of salt should have an influence on all coefficients probably with exception of initiator decay, an attempt was made to still find a good description for the influence of added salt based on data given. Additional counterions should increase the termination and backbiting rate, and thus decrease the rate of polymerization. However, as an enormous augmentation of the rate of polymerization was found, the amplifying effect on propagation seems to be dominant. For modeling purpose, the effect of higher $I$ on $k_{\mathrm{p}}$ was assumed to apply twice as strong for $k_{\mathrm{p}}^{\mathrm{t}}$. Consequently, $k_{\mathrm{p}}^{\mathrm{s}}$ is multiplied with the factor of 
enhancement of propagation, $\xi_{I}$, and $k_{\mathrm{p}}^{\mathrm{t}}$ scaled to the already enhanced $k_{\mathrm{p}}^{\mathrm{s}}$ is also multiplied with $\xi_{I}$, viz., the propagation of MCRs is augmented by $\xi_{I}{ }^{2}$.

$\xi_{I}$ was estimated via PREDICI TM from conversion vs. time profiles corresponding to the data shown in Figure 5-30 including the effect of $I$ on $k_{\mathrm{bb}}$, but ignoring the effect on $k_{\mathrm{t}}$ (see subchapter 5.2.2) resulting in eq. (5.44).

$$
\xi_{I}=\left(c_{\mathrm{Na}^{+}}^{0}-c_{A A}^{0}\right) \cdot 0.99+1
$$

\subsection{2 $\quad k_{\mathrm{t}}$ at Full Ionization}

As discussed in detail in subchapter 5.2.7, viscosity increase by ionization of monomer and Coulomb repulsion of ionized macroradicals as well as increased stiffness of the polymer chain towards ionization thereof and hindered movement of ionized macroradicals lead to a decrease of $k_{\mathrm{t}}$.

The composite-model parameters $\alpha_{\mathrm{s}}, \alpha_{1}$, and $i_{\mathrm{c}}$ at full ionization are assumed to be the same as for the non-ionized monomer. ${ }^{[77]}$ In contrast, the effect on $k_{\mathrm{t}}^{1,1}$ is large: $k_{\mathrm{t}}^{\mathrm{ss}}$ is reduced by a factor of ca. $15, k_{\mathrm{t}}^{\mathrm{st}}$ and $k_{\mathrm{t}}^{\mathrm{tt}}$ are reduced too much as to be easily measured by SP-PLP-EPR. ${ }^{[77]}$ The factor of 15 was adjusted down to 12 via PREDICI ${ }^{\mathrm{TM}}$ estimation:

$$
k_{\mathrm{t},(\alpha=1)}^{\mathrm{ss}}=\frac{k_{\mathrm{t},(\alpha=0)}^{\mathrm{ss}}}{12}
$$

$k_{\mathrm{t}}^{\mathrm{t}, \mathrm{t}}$ was obtained via PREDICI ${ }^{\mathrm{TM}}$ fitting. The decrease of $k_{\mathrm{t}}^{\mathrm{t}, \mathrm{t}}$ towards ionization is stronger than for $k_{\mathrm{t}}^{\mathrm{ss}}$, as was found experimentally (v.s.).

$$
k_{\mathrm{t},(\alpha=1)}^{\mathrm{tt}}=\frac{k_{\mathrm{t,}(\alpha=0)}^{\mathrm{tt}}}{40}
$$


It should be noted that smaller reduction of $k_{\mathrm{t}}^{\text {tt }}$ led to a less accurate representation of measured conversion vs. time profiles. $k_{\mathrm{t}}^{\text {st }}$ was calculated by the diffusion mean, eq. (2.31), as for the modeling of the non-ionized AA.

The effect of higher $I$ on $k_{\mathrm{t}}$ was not included into the model, because no directly measured data is available and analysis of conversion vs. time profiles of polymerizations with different $I$ suggests that the influence of $I$ on $k_{\mathrm{t}}$ is much weaker than the influence on $k_{\mathrm{p}}$ and $k_{\mathrm{bb}}$ (see subchapter 5.2.1). This might be explained by the effects on $k_{\mathrm{t}}$ by increasing viscosity and by screening of additional counter ions compensating each other. Otherwise, a decreased rate of polymerization would have been found with $\mathrm{NaCl}$ being added to the reaction mixture (see subchapter 5.2.6). Future work should be directed at measuring the influence of additional counterions on $k_{\mathrm{t}}$ directly, e.g., by SP-PLP-EPR.

\subsection{3 $k_{\mathrm{bb}}$ at Full Ionization and dependence on Ionic Strength}

So far, the influence of ionic strength on backbiting has not been measured. However, backbiting may be scaled to the flexibility of polymer chains (see subchapter 2.3.3), which is well understood, as the structure of pAA coils in aqueous solution has been studied as a function of both $\alpha$ and $I^{[82,84-87,163-166]}$ The dependence on $\alpha$ is discussed in subchapter 5.2.8.

The persistence chain length, $l_{\mathrm{p}}$, is a common measure for the rigidity of chains and may be subdivided into the persistence chain length without electronic influence, $l_{0}$, and the increase by ionization, the electronic persistence chain length, $l_{\mathrm{e}}$, as given by eq (5.47).

$$
l_{\mathrm{p}}=I_{0}+l_{\mathrm{e}}
$$




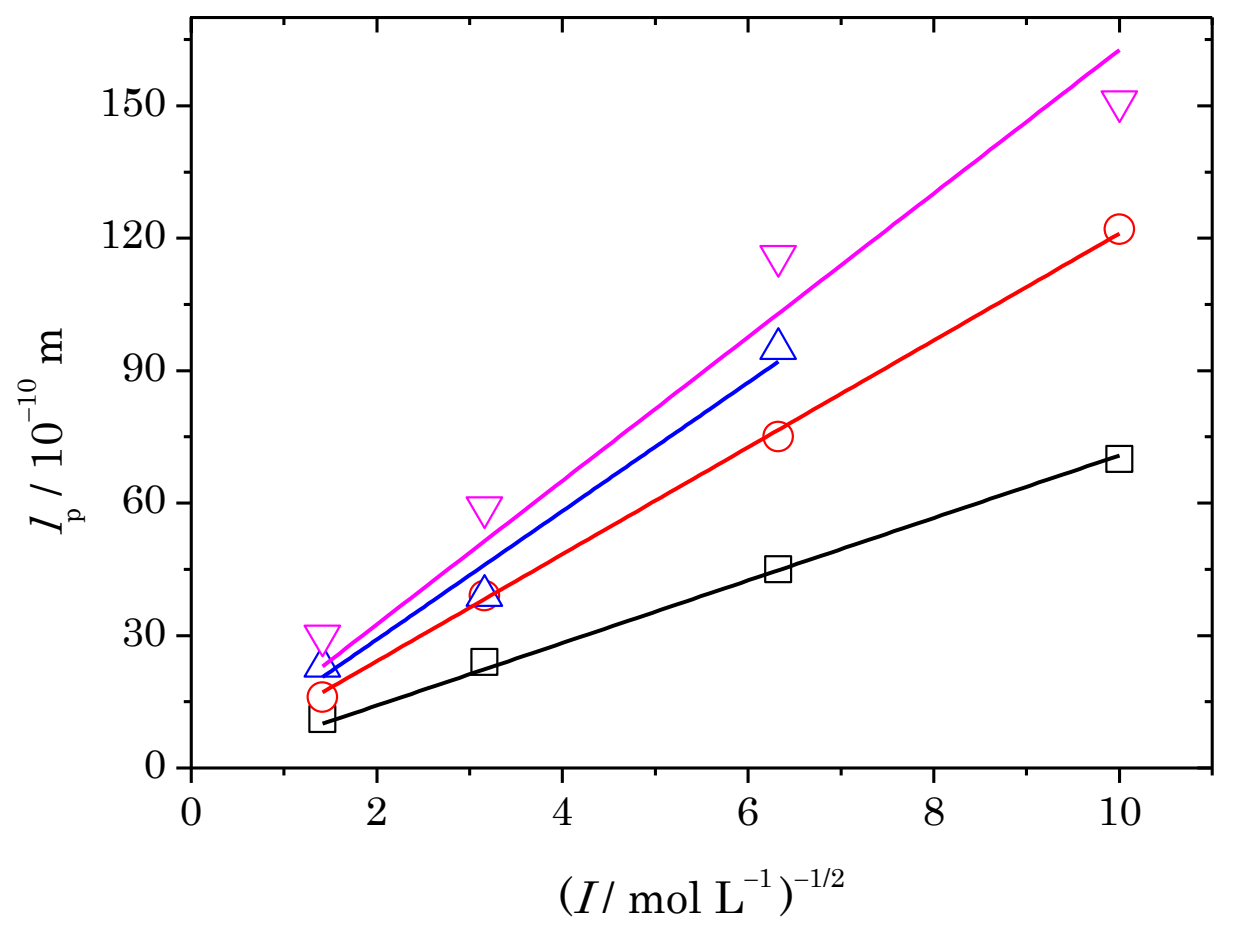

Figure 5-31 The persistence chain length of pAA plotted for $\alpha=0.6$ (downward triangles), 0.4 (upward triangles), 0.2 (circles), 0.103 (squares) as a function of the square root of ionic strength and associated straight line fits. Data was taken from ref. ${ }^{[84,187]}$

Data of the persistence chain length of pAA as a function of square root of ionic strength was taken from ref. ${ }^{[84,187]}$ and plotted in Figure 5-31. Data points for $\alpha=0.6$, $0.4,0.2$, and 0.103 were fitted to respective straight lines. The influence of $\alpha$ is discussed in subchapter 5.2.8. Considering $l_{0}=1.2 \cdot 10^{-11} \mathrm{~m}$ taken from ref. ${ }^{[84,85]}$ the so-obtained slopes were fitted as a function of $\alpha$ (stars in Figure 5-38) to develop an equation that represents the dependence of $l_{\mathrm{p}}$ on $\alpha$ and $I$. At full ionization, it simplifies into eq. (5.48).

$$
I_{\mathrm{p}}=\left(12+19.5 \cdot \sqrt{\frac{1}{I}}\right) \cdot 10^{-10} \mathrm{~m}
$$

Comparison of eq. (5.48) with the $k_{\mathrm{bb}}$ values of non-ionized and fully ionized AA yields eq. (5.49). 


$$
k_{\mathrm{bb}} / \mathrm{s}^{-1}=\frac{6.57 \cdot 10^{9} \cdot \exp \left(\frac{5020}{T / \mathrm{K}}\right)}{\left(12+19.5 \cdot \sqrt{\frac{1}{I}}\right)^{0.43}}
$$

\subsubsection{Density}

With the rate equations being formulated in terms of concentration, the density of the polymerization mixture should be known. Aqueous systems including ionization preclude the assumption of ideal mixing. The Debye-Hückel theory ${ }^{[188]}$ is commonly used to describe the behavior of ions in highly diluted solution. For simplification, ionic strength is used as reference (see eq. (2.28)).

Density can be derived from the molar volumes, $\bar{V}$, of individual components. For highly diluted solutions, the latter may be extrapolated from the molar volumes at infinite dilution, $\bar{V}^{0}$, by eq. (5.50).

$$
\bar{V}=\bar{V}^{0}+A_{\mathrm{D}-\mathrm{H}} \cdot I^{0.5}
$$

For higher concentration, a series expansion is commonly made given by eq. (5.51).

$$
\bar{V}=\bar{V}^{0}+A_{\mathrm{D}-\mathrm{H}} \cdot I^{0.5}+B_{\mathrm{D}-\mathrm{H}} \cdot I^{1}+C_{\mathrm{D}-\mathrm{H}} \cdot I^{1.5}
$$

$A_{\mathrm{D}-\mathrm{H}}$ can be derived by Debye-Hückel theory, ${ }^{[189]}$ while $B_{\mathrm{D}-\mathrm{H}}$ and $C_{\mathrm{D}-\mathrm{H}}$ are empirical. By comparison of densities of concentrated solutions with $\bar{V}^{0}$ it was found that $\bar{V}$ may be calculated for these solution by a simpler formula (v.i.). 
The apparent molar volume of pAA in aqueous solution (data taken from ref. ${ }^{[190])}$ was plotted as a function of concentration of monomer units, MU. The data was fitted to straight lines (not shown). The so-obtained values of the respective slopes were plotted in Figure 5-32 as a function of the degree of ionization of pAA (ionization agent: $\mathrm{NaOH}$ ). These data were fitted to a straight line as well, given by eq. (5.52).

$$
\mathrm{d} \bar{V} / \mathrm{d} c_{\mathrm{MU}}=1.05 \pm 0.01 \cdot \alpha
$$

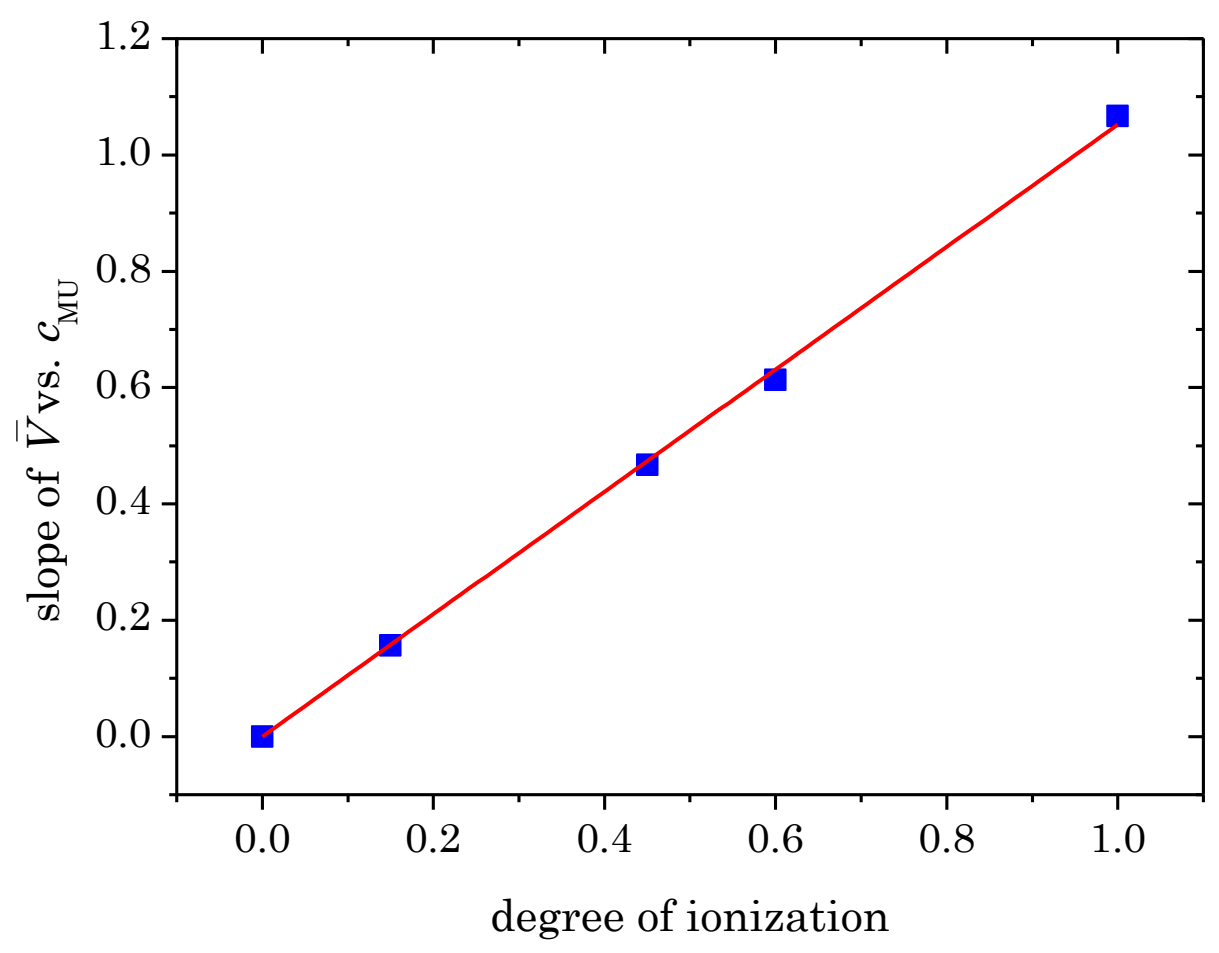

Figure 5-32 The apparent molar volume of pAA in aqueous solution plotted as a function of concentration of monomer units (ref. ${ }^{[190])}$ gives straight lines, the slopes of which (squares) increase with degree of ionization of polymer (ionization agent: $\mathrm{NaOH}$ ). The data are fitted to a straight line represented by eq. (5.52).

The slopes of the first plot (squares in Figure 5-32) increase linearly with ionization, at full ionization the slope is approximately unity. The volumes of non-ionized components are independent of $I$. Thus, molar volumes of $\mathrm{pAA}^{-}$and $\mathrm{Na}^{+}$increase with $0.5 \cdot I$ yielding eq. (5.53). 


$$
\bar{V}=\bar{V}^{0}+0.5 \cdot I
$$

The values of $\bar{V}^{0}$ for polymer and monomer at various $\alpha$ are plotted in Figure 5-33. The respective amount of sodium cations is included (from ref. [191]). One AA molecule takes up more volume than one monomer unit in pAA. Ionization decreases the molar volume of polymer (from ref. ${ }^{[192,193])}$ linearly.

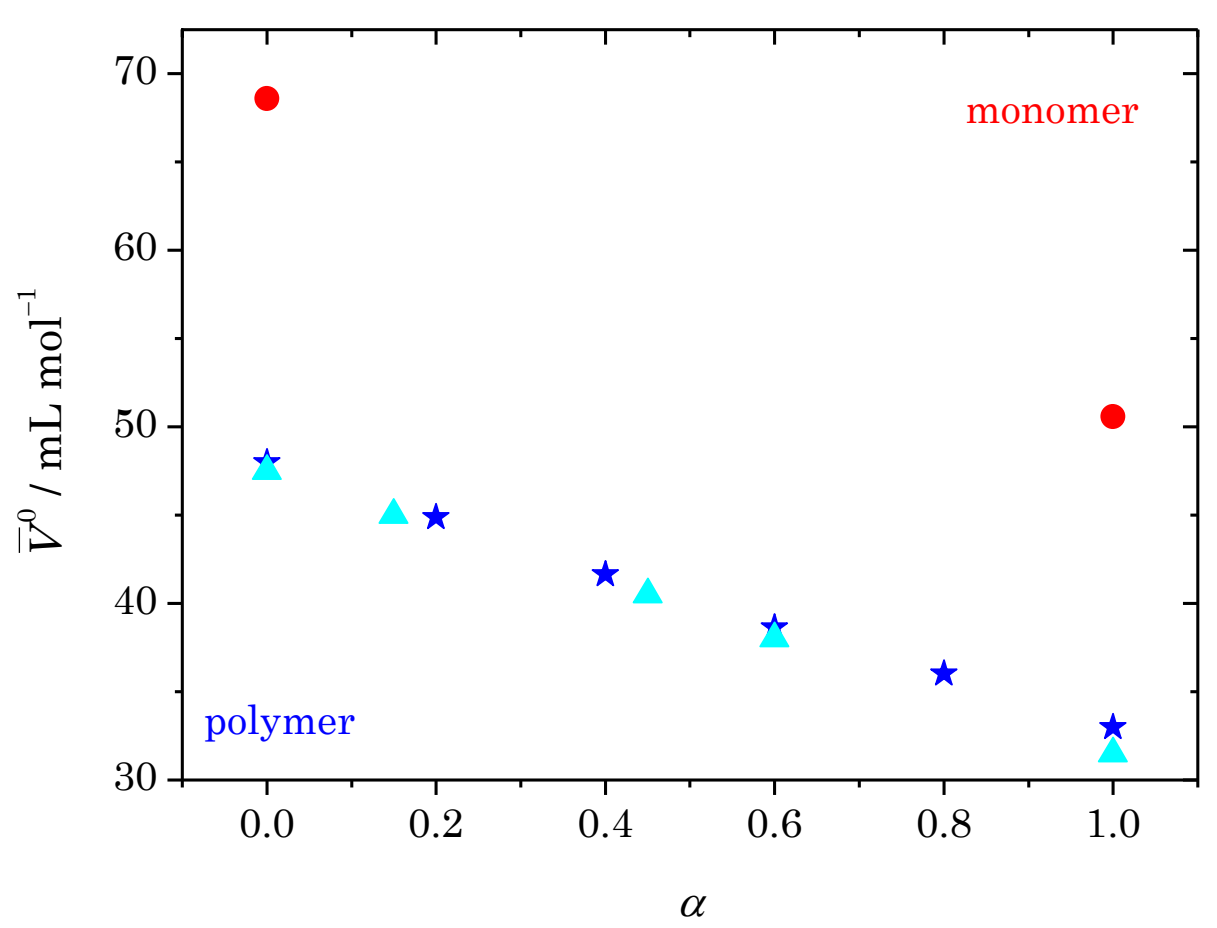

Figure 5-33 Molar volume at infinite dilution as a function of degree of ionization for MU of pAA and AA. The respective amount of sodium cations is included in the calculation, taken from ref. [191] Monomer (circles) takes up more volume than polymer (stars) and ionization decreases molar volume, data from ref. ${ }^{[192,193]}$ These values were derived from highly diluted solution by the Debye-Hückel equation, eq. (5.50). In order to check eq. (5.53) derived in this modeling study, data for highly concentrated polymer solutions (up to $18 \mathrm{~mol} \mathrm{~L}^{-1}$ )[190,194] was extrapolated to infinite dilution via this equation (cyan). It can be observed that molar volume of polymer decreases linearly towards higher degree of ionization.

In order to verify eq. (5.53) derived in this study, data for highly concentrated polymer solutions (up to $\left.18 \mathrm{~mol} \mathrm{~L}^{-1}\right)^{[190,194]}$ was extrapolated to infinite dilution via this equation. The so-obtained values for $\bar{V}^{0}$ were compared to data derived from 
measurements in highly diluted solution via the Debye-Hückel equation, eq. (5.50). As shown in Figure 5-33, values for $\bar{V}^{0}$ are in excellent agreement. Therefore, the simplified approach of eq. (5.53) seems to work well and is used for modeling. The approach is less accurate for very dilute solutions, but the absolute error is low as densities do not differ much from the one of the pure compound.

The density of AA is calculated in the model by eq. (5.54). No temperature dependency of the impact of ionization was observed in this study. Data is given in the appendix.

$$
\rho_{{\mathrm{AA} / \mathrm{AA}^{-}}^{-}}=\rho_{\mathrm{AA}} \cdot\left(\left(1-\alpha_{\mathrm{AA}}\right)+\frac{\rho_{\mathrm{AA}^{-}}}{\rho_{\mathrm{AA}}} \cdot \alpha_{\mathrm{AA}}\right)=\rho_{\mathrm{AA}} \cdot\left(\left(1-\alpha_{\mathrm{AA}}\right)+\frac{\bar{V}_{\mathrm{AA}}}{\bar{V}_{\mathrm{AA}^{-}}} \cdot \alpha_{\mathrm{AA}}\right)
$$

Because of the linear dependency of molar volume on degree of ionization, a formula of the same form as for monomer, eq. (5.54), is used to calculate the density of polymer, eq. (5.55)

$$
\rho_{\mathrm{pAA} / \mathrm{pAA}}=\rho_{\mathrm{pAA}} \cdot\left(\left(1-\alpha_{\mathrm{pAA}}\right)+\frac{\rho_{\mathrm{pAA}^{-}}}{\rho_{\mathrm{pAA}}} \cdot \alpha_{\mathrm{pAA}}\right)=\rho_{\mathrm{AA}} \cdot\left(\left(1-\alpha_{\mathrm{pAA}}\right)+\frac{\bar{V}_{\mathrm{pAA}}}{\bar{V}_{\mathrm{pAA}}} \cdot \alpha_{\mathrm{pAA}}\right)
$$

The molar volume of ions in aqueous solution is mostly negative $\mathrm{XXVIII}$ at low concentration and PREDICI ${ }^{\mathrm{TM}}$ cannot calculate with negative molar volumes. That is why, ions have the density of $1 \mathrm{~g} \mathrm{~cm}^{-3}$ in the model and their actual density is used in the calculation of density of water, which is given by eq. (5.56)

xxVIII Depending on effective charge, hydration of ions decreases the distance of surrounding water molecules to one another, which results in an overall decrease of volume. This effect is stronger for dilute solutions. 


$$
\begin{aligned}
& \rho_{\text {all }}=\frac{m_{\mathrm{H}_{2} \mathrm{O}}}{m_{\text {all }}} \cdot \rho_{\mathrm{H}_{2} \mathrm{O}}^{\text {virtual }}+\frac{m_{\text {all }}-m_{\mathrm{H}_{2} \mathrm{O}}}{m_{\text {all }}} \cdot 1 \mathrm{~g} \mathrm{~mol}^{-1} \\
& \rho_{\mathrm{H}_{2} \mathrm{O}}^{\text {virtual }}=\left(\rho_{\text {all }}-\frac{m_{\text {all }}-m_{\mathrm{H}_{2} \mathrm{O}}}{m_{\text {all }}}\right) \cdot \frac{m_{\text {all }}}{m_{\mathrm{H}_{2} \mathrm{O}}}
\end{aligned}
$$

\subsubsection{Modeling the Polymerization of Fully lonized AA}

Given the independent data (subchapter 5.2.1), as a next step conversion vs. time profiles were compared to the simulation in order to adjust the rate coefficients with highest uncertainty, as described above.

Preferably, an initiator is used, the decay of which is independent of $\mathrm{pH}$ or the presence of other substances. Otherwise, initiator kinetics are hard to separate from monomer kinetics. As discussed in subchapter 5.2.11, polymerizations withVA-086 and NaPS have been carried out, but have not been used for modeling.

V-50 has limited solubility in basic aqueous systems and its decay, which is $\mathrm{pH}$ independent at low $\mathrm{pH}$, shows some dependence on $\mathrm{pH}$ at higher $\mathrm{pH}$. The dependence is provided by the supplier given by eq. (5.57). ${ }^{[156]}$

$$
\begin{aligned}
k_{\mathrm{d}} / \mathrm{s}^{-1}= & 8.33 \cdot 10^{-6}-2.7 \cdot 10^{-8} \cdot \exp \left(\frac{\mathrm{pH}-1.9}{1.55}\right) \\
& \cdot \exp \left(1.49 \cdot 10^{4} \cdot\left(\frac{1}{\mathrm{~T} / K}-3.09 \cdot 10^{-3}\right)\right)
\end{aligned}
$$

$0.05 \mathrm{~g} \mathrm{~g}^{-1} \mathrm{AA}$ neutralized with $\mathrm{NaOH}$ to $\alpha=1$ was polymerized at different levels of $\mathrm{V}-50$ as the initiator and also under addition of $0,0.5,1,2$, and $3 \mathrm{~mol} \mathrm{~L}^{-1}$ amounts of $\mathrm{NaCl}$ at $50{ }^{\circ} \mathrm{C}$. XxIx The corresponding conversion vs. time profiles have been used for modeling. Simulated and experimental profiles are depicted in Figure 5-34. There is some deviation under variation of initiator concentration and for high amounts of added $\mathrm{NaCl}$ the initial rate of polymerization is slightly overpredicted. Nevertheless,

xxIx These experiments were carried out by Patrick Drawe. 
the overall close agreement of simulations and experimental data is gratifying to note, particularly in this case of numerous dependencies of rate coefficients.

Simulated $Ð$ values augmented a great deal towards polymerizations with addition of $\mathrm{NaCl}$. This should be checked experimentally.

The additional and modified, respectively, rate coefficients and the other parameters used for modeling radical polymerization of fully ionized acrylic acid in aqueous solution are summarized in Table 5-8. 

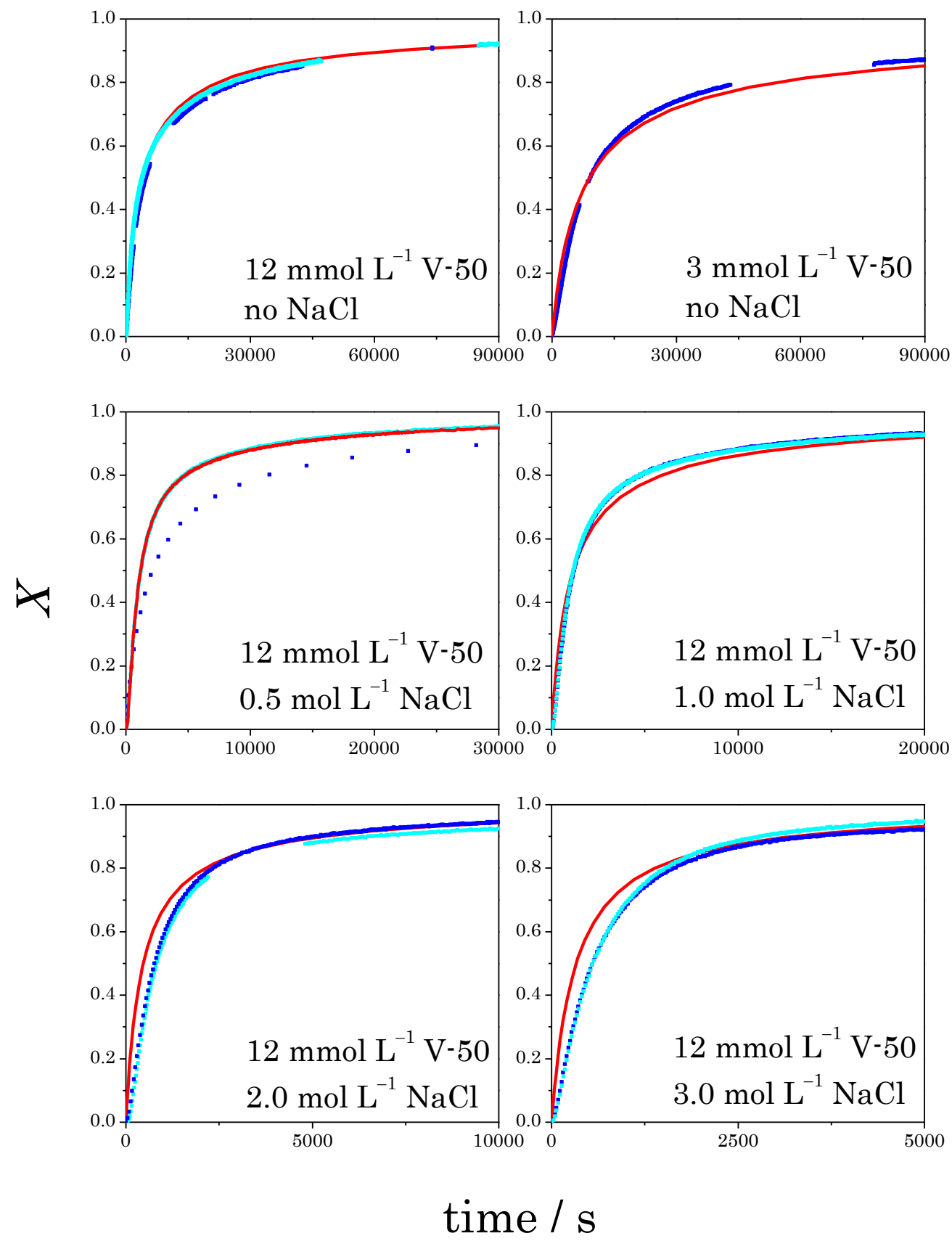

Figure 5-34 Comparison of measured (blue) and simulated (red) NaA conversion vs. time plots of polymerization of $0.05 \mathrm{~g} \mathrm{~g}^{-1} \mathrm{AA}$ neutralized with $\mathrm{NaOH}$ to $\alpha=1$ with different levels of V-50 as initiator at $50{ }^{\circ} \mathrm{C}$ and with different amounts of $\mathrm{NaCl}$ given for each graph; the simulations are indicated by the red lines; independent repeat experiments are included (cyan). The experimental part was carried out by Patrick Drawe. 
Table 5-7 Summary of modified rate coefficients and of other parameters used for modeling radical polymerization of fully ionized acrylic acid in aqueous solution complementing Table 5-2.

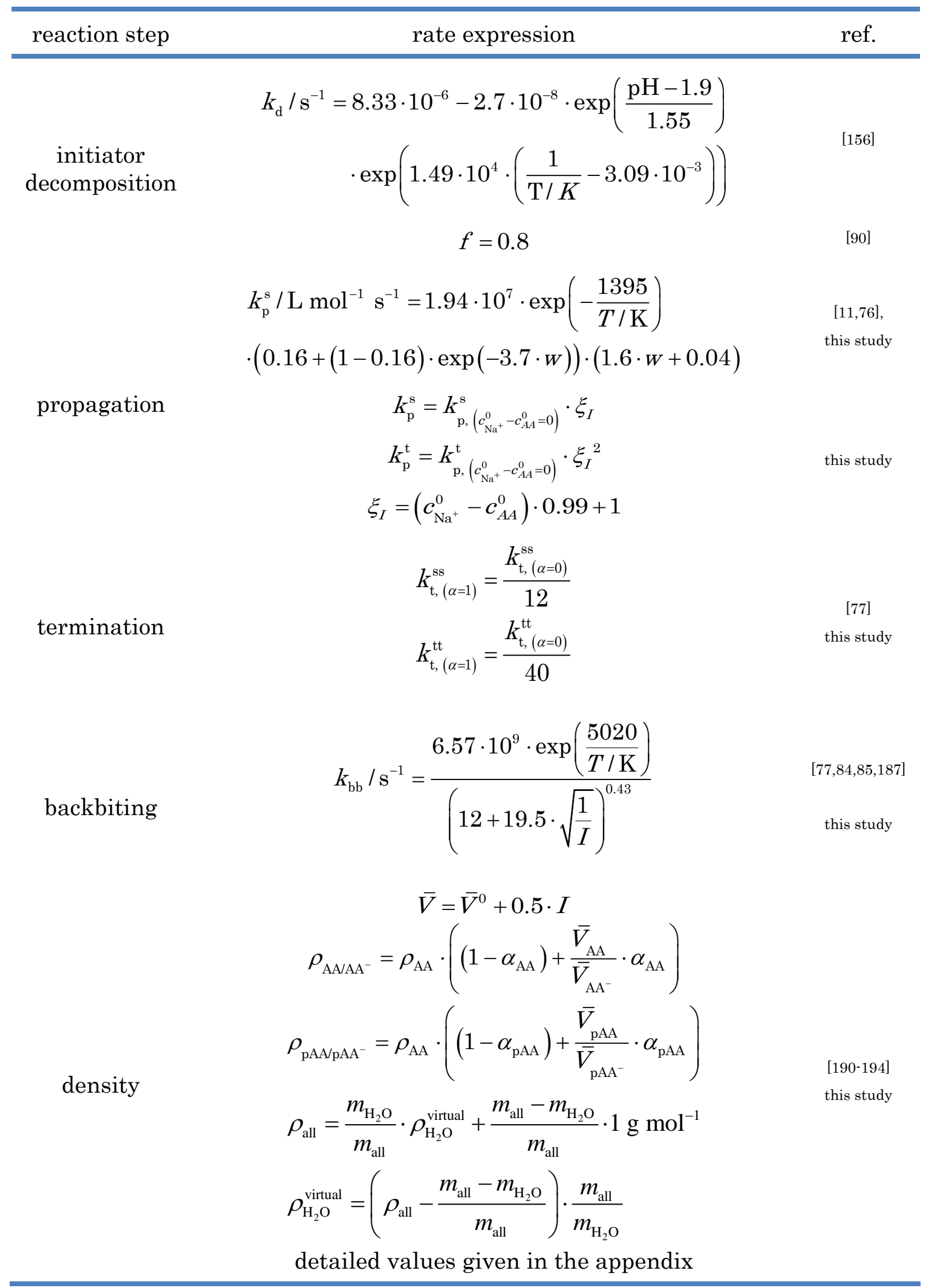




\subsubsection{The dependence of $k_{\mathrm{p}}$ on the Degree of Ionization}

Both rate of polymerization and molar mass scale with $k_{\mathrm{p}}$. Therefore accurate knowledge about $k_{\mathrm{p}}$ is particularly important. $k_{\mathrm{p}}^{\mathrm{s}}$ of $\mathrm{AA}$ at different degrees of ionization has been measured via PLP-SEC only for $0.05 \mathrm{gg}^{-1}$ at $6{ }^{\circ} \mathrm{C}$. ${ }^{[76]}$ The dependence of $k_{\mathrm{p}}^{\mathrm{s}}$ on $W$ and on $\alpha$ should be similar to the one of MAA, ${ }^{[11]}$ which is represented by eq. (2.29)[11] and holds for the range of $0.05 \leq w_{\mathrm{MAA}} \leq 0.40$. Extrapolation to low AA content at full ionization gives physicochemically unrealistic negative values (see subchapter 2.4.3, Figure 2-4).

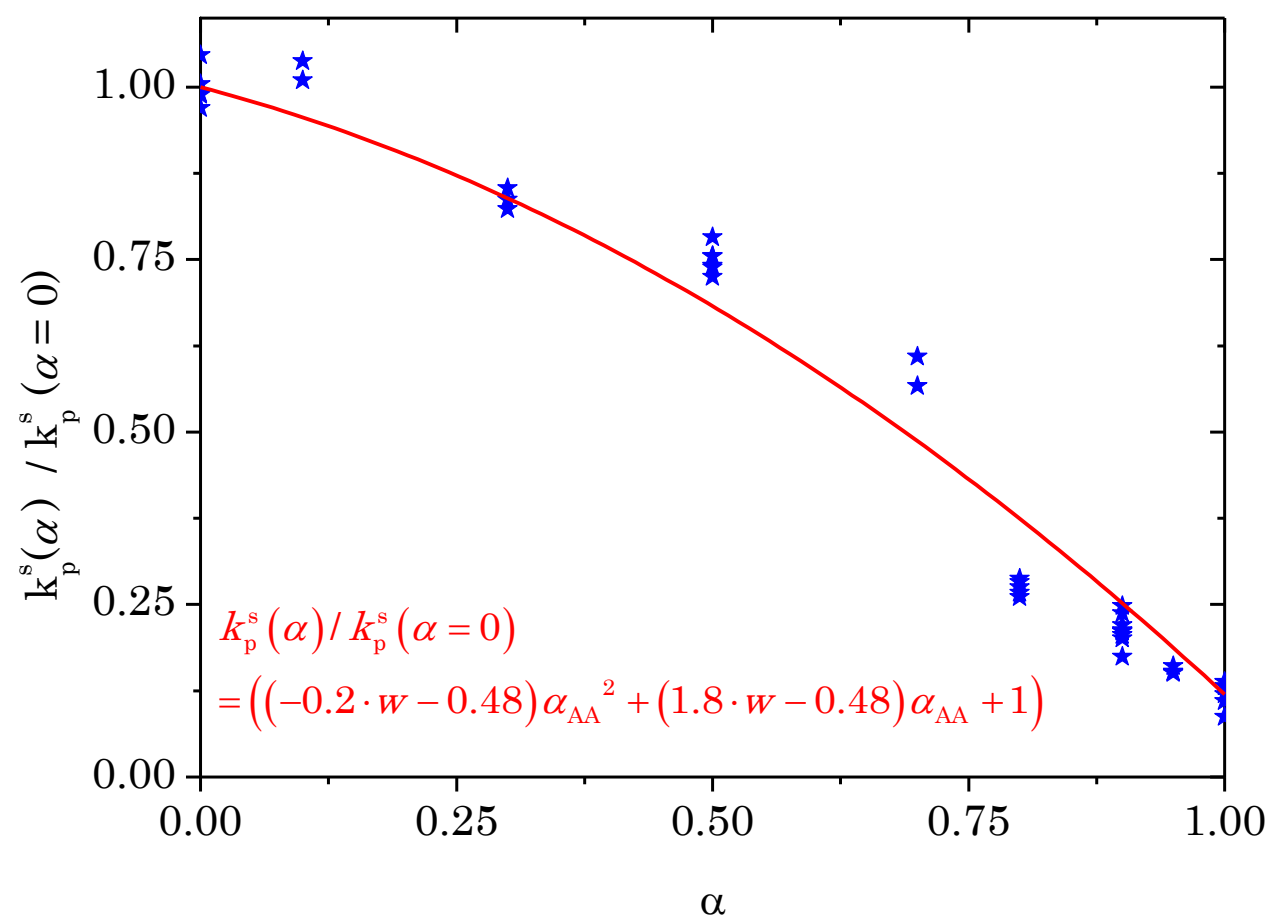

Figure 5-35 $\quad k_{\mathrm{p}}$ for a weight fraction of $0.05 \mathrm{~g} \mathrm{~g}^{-1} \mathrm{AA}$ as a function of degree of ionization (by $\left.\mathrm{NaOH}\right)$ is plotted relative to the value of the non-ionized monomer (blue stars, data taken from ref. ${ }^{[76])}$. The $3^{\text {rd }}$ factor of eq. (5.58) RHS representing the dependence of $k_{\mathrm{p}}$ on $w$ and $\alpha$ is given by the line; the corresponding formula is also given in the graph.

Eq. (2.29) consists of three parts, which are concatenated by multiplication: Firstly, $k_{\mathrm{p}}$ at $W_{\mathrm{AA}}=0$ and $\alpha=0$, secondly, a factor describing the decrease of $k_{\mathrm{p}}^{\mathrm{s}}$ towards higher $w_{\mathrm{AA}}$ at $\alpha=0$, and thirdly, a factor taking the combined dependence on $w_{\mathrm{AA}}$ and $\alpha$ into account. A modification of eq. (2.29) has been developed, in which the 
first two terms were taken from subchapter 5.1.2. In order to represent the dependence of $k_{\mathrm{p}}^{\mathrm{s}}$ on $W_{\mathrm{AA}}$ at $\alpha=0$ as simply as possible, eq. (5.4) RHS $1^{\text {st }}$ term, was used as the basis. The maximum of $k_{\mathrm{p}}^{\mathrm{s}}$ and the dependence of $E_{\mathrm{A}}$ on monomer content were not included. No data on the influence of ionization and ionic strength on $E_{\mathrm{A}}$ is available for $\mathrm{AA}$, but MAA data suggest that only a weak influence occurs. ${ }^{[11]}$ The $3^{\text {rd }}$ factor of eq. (2.29) has been modified considering both the existing PLP-SEC data and estimation via PREDICI ${ }^{\mathrm{TM}}$ for conversion-time profiles of polymerization of $0.05 \mathrm{~g} \mathrm{~g}^{-1}$ fully ionized AA at $50{ }^{\circ} \mathrm{C}$ with $\mathrm{V}-50$ as initiator at levels of 3,12 , and $36 \mathrm{mmol} \mathrm{L}^{-1}$. The general format of eq. (2.29) was not changed, but the parameters, which determine the curvature with respect to ionization, were adjusted yielding eq. (5.58). In Figure 5-35 the so-obtained $3^{\text {rd }}$ factor of eq. (5.58) is plotted together with $k_{\mathrm{p}}^{\mathrm{s}}$ values of $0.05 \mathrm{~g} \mathrm{~g}^{-1} \mathrm{AA}$ at various degrees of ionization relative to the value of the non-ionized monomer (data taken from ref. ${ }^{[66]}$ ) demonstrating the good representation of the dependence of $k_{\mathrm{p}}^{\mathrm{s}}$ on $\alpha$ for $0.05 \mathrm{~g} \mathrm{~g}^{-1} \mathrm{AA}$.

$$
\begin{aligned}
& k_{\mathrm{p}}^{\mathrm{s}} / \mathrm{L} \mathrm{mol}^{-1} \mathrm{~s}^{-1}=1.94 \cdot 10^{7} \cdot \exp \left(-\frac{1395}{T / \mathrm{K}}\right) \\
& \cdot(0.16+(1-0.16) \cdot \exp (-3.7 \cdot W)) \\
& \cdot\left((-0.2 \cdot W-0.48) \alpha_{\mathrm{AA}}{ }^{2}+(1.8 \cdot W-0.48) \alpha_{\mathrm{AA}}+1\right)
\end{aligned}
$$

The prediction of $k_{\mathrm{p}}^{\mathrm{s}}$ according to eq. (5.58) for $50{ }^{\circ} \mathrm{C}$ is depicted in Figure 5-36. The resulting plot is similar to the one for the dependence of $k_{\mathrm{p}}$ for MAA as a function of $W_{\mathrm{M}}$ and $\alpha$ (see Figure 2-4). There are, however, some important differences: Firstly, the overall $k_{\mathrm{p}}^{\mathrm{s}}$ value of AA is higher than $k_{\mathrm{p}}$ of MAA, which is represented by the first factor. Secondly, the steepness of the slope at $\alpha=0$ is higher for AA than for MAA, as represented by the second factor. Thirdly, the curvature at $w=0$ is weaker than in the MAA formula, especially, for high $\alpha$. Hence, $k_{\mathrm{p}}^{\mathrm{s}}$ does not become negative at high $\alpha$ and low $w$.

There is no value available for $k_{\mathrm{p}}^{\mathrm{t}}$ of partly ionized AA and only of one value available for $k_{\mathrm{p}}^{\mathrm{t}}$ of fully ionized AA, which was obtained by parameter estimation from SP-PLP-EPR radical concentration vs. time profiles ${ }^{[7]}$ and was not considered for modeling fully ionized AA (see subchapter 5.2.1). It appears reasonable that $k_{\mathrm{p}}^{\mathrm{t}}$ is influenced by ionization and monomer content in the same way as $k_{\mathrm{p}}^{\mathrm{s}}$. This approach was used for modeling. 


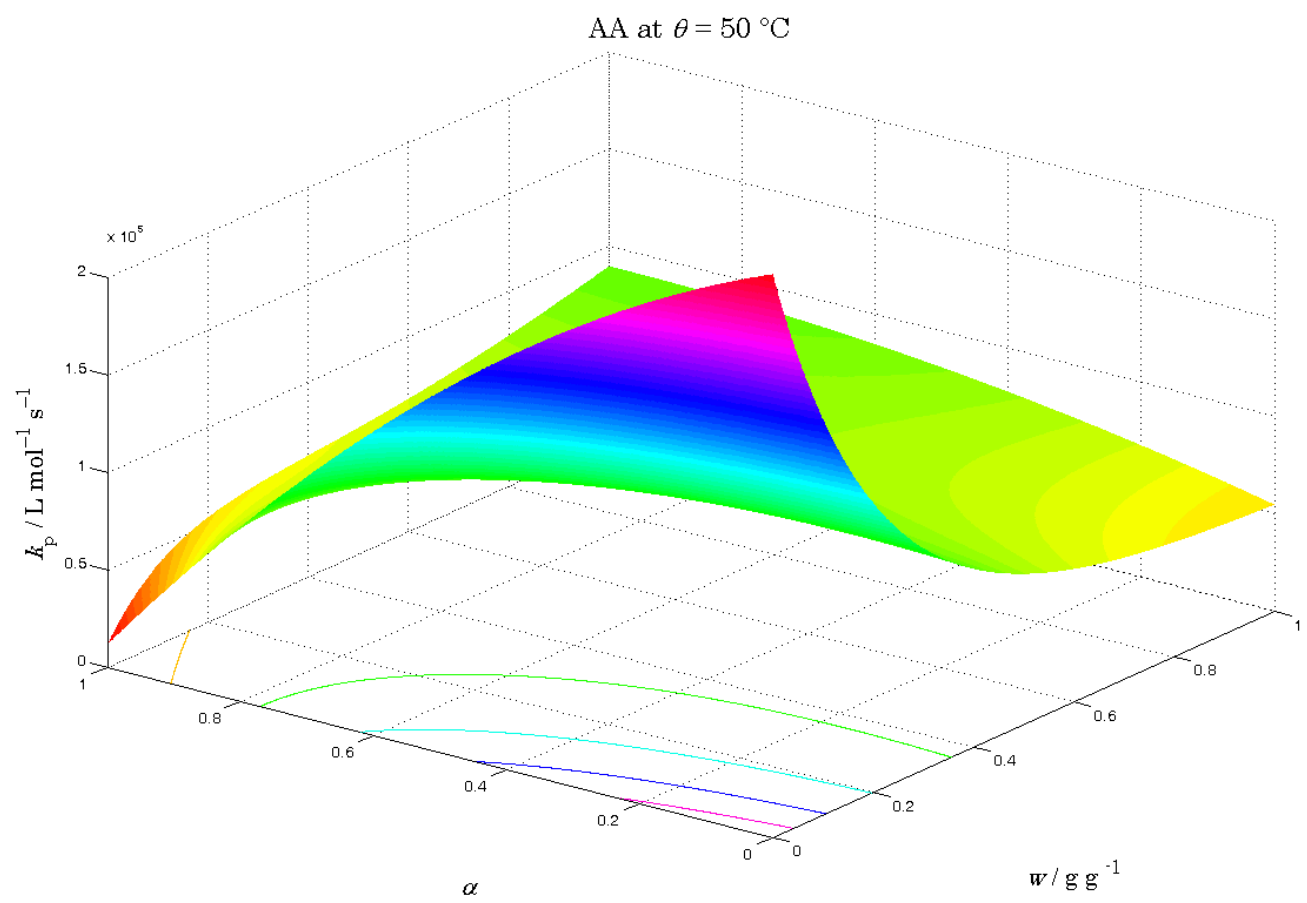

Figure 5-36

The rate coefficient of propagation of $\mathrm{AA}$ as a function of both weight fraction of monomer and degree of ionization used in the model given by eq. (2.26). The plot is similar to the one for MAA (Figure 2-4), but there are some important differences: the overall value is higher, the steepness at $\alpha=0$ is higher, and the curvature at $w=0$ is weaker.

\subsubsection{The dependence of $k_{\mathrm{t}}$ on the Degree of Ionization}

The viscosity of the reaction mixture increases towards higher $\alpha$. In addition to the so-induced lowering of $k_{\mathrm{t}}$, Coulomb repulsion of ionized macroradicals further decreases $k_{\mathrm{t}}$. It should be noted that the actual viscosity-induced reduction of $k_{\mathrm{t}}$ is even higher than the reduction of fluidity. Movement of the charged macroradicals is further decreased by larger effective size from hydration of the ionized species and repulsion by surrounding counterions. As this effect depends on degree of ionization of polymer instead of degree of ionization of monomer, its contribution is represented in the model by the Coulomb factor.

The viscosity effect is increased towards higher ionic strength, while the Coulomb contribution to decrease of $k_{\mathrm{t}}$ may be partly compensated by screening of additional counterions (see subchapter 2.4.3). Furthermore, ionization stiffens the polymer chain, which should reduce segmental diffusion, and thus decreases $k_{\mathrm{t}}$. The 
flexibility of an ionized chain is increased by screening of counter ions; at high ionic strength, the flexibility of the chain reaches the value of a non-ionized one. As discussed in subchapter 5.2.2 given data suggest that the effects from increased ionic strength on $k_{\mathrm{t}}$ mostly compensate each other.

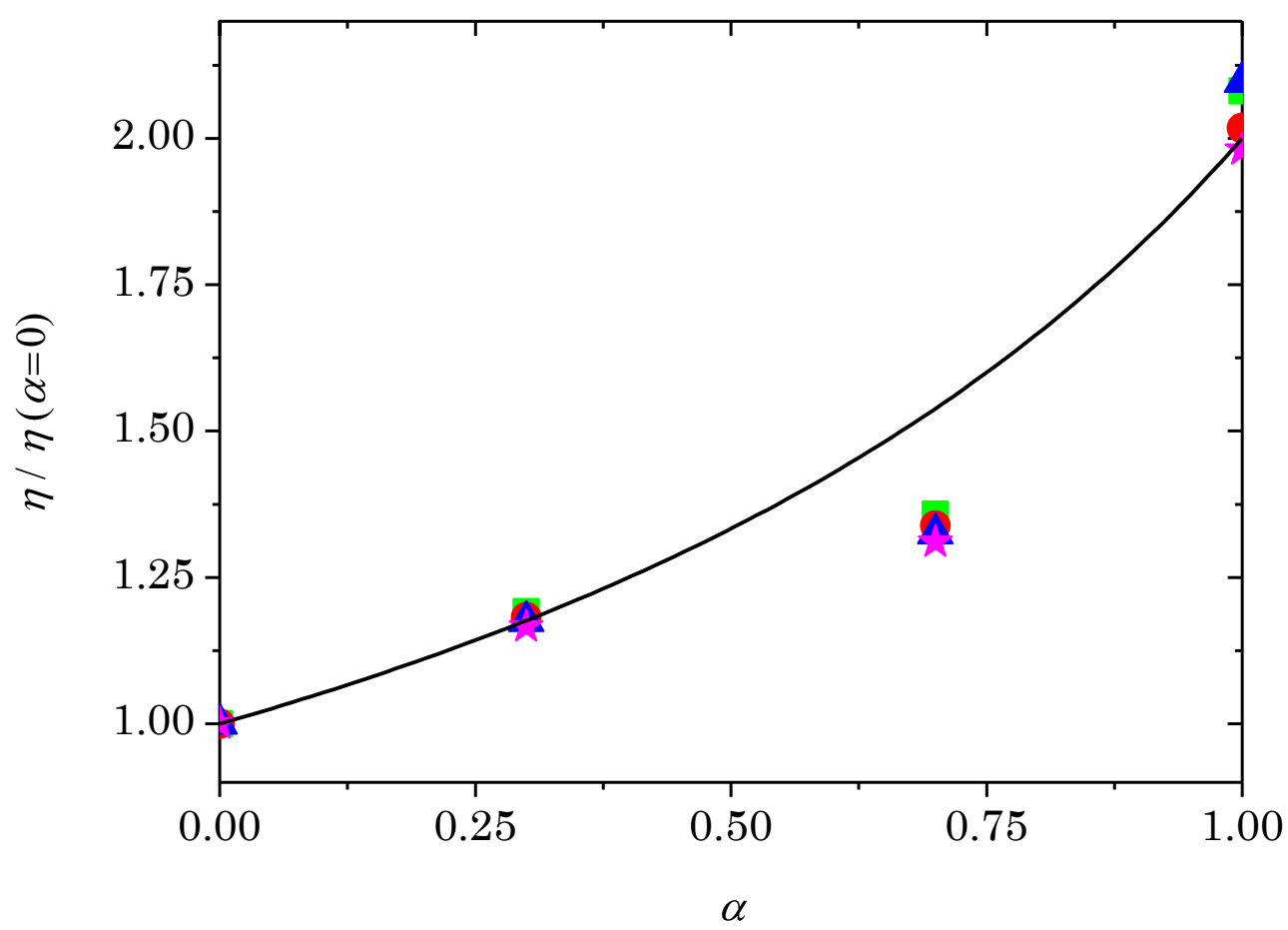

Figure 5-37 The viscosity of $0.20 \mathrm{~g} \mathrm{~g}^{-1} \mathrm{AA}$ in aqueous solution has been measured at different temperatures (square: $35^{\circ} \mathrm{C}$, circle: $65^{\circ} \mathrm{C}$, triangle: $65^{\circ} \mathrm{C}$, and star: $80^{\circ} \mathrm{C}$ ) and degrees of ionization. Plotting relative viscosity (referring to $\alpha=0$ ) shows that no temperature dependence occurs irrespective of degree of ionization. Data is approximated according to eq. (2.28) given as a line.

The viscosity of $0.20 \mathrm{~g} \mathrm{~g}^{-1} \mathrm{AA}$ in water has been measured between 35 and $80{ }^{\circ} \mathrm{C}$ for different degrees of ionization and is plotted relative to the viscosity at $\alpha=0$ in Figure 5-37. No temperature dependence could be found. For sake of simplicity, the data is approximated by eq. (5.59).

$$
\eta(\alpha) \simeq \frac{\eta(\alpha=0)}{1-0.5 \cdot \alpha_{\mathrm{AA}}}
$$


Combination of eq. (2.36) and eq. (5.59) yields the correction factor for $k_{\mathrm{t}}$ approximating the influence of viscosity under ionization, $\zeta_{\eta \alpha}$, according to eq. (5.60).

$$
\zeta_{\eta \alpha}=1-0.5 \cdot \alpha_{\mathrm{AA}}
$$

The composite-model parameters $\alpha_{\mathrm{s}}, \alpha_{1}$, and $i_{\mathrm{c}}$ are assumed to be unaffected by ionization. ${ }^{[77]}$ In contrast, the effect on $k_{\mathrm{t}}^{1,1}$ is large: $k_{\mathrm{t}}^{\mathrm{ss}}$ is reduced by a factor of ca. $15, k_{\mathrm{t}}^{\mathrm{st}}$ and $k_{\mathrm{t}}^{\mathrm{tt}}$ are reduced too much as to be easily measured by SP-PLP-EPR. ${ }^{\text {[77] }}$

The factor of 15 consists of a factor of 2 due to viscosity increase (eq. (5.60)) and a factor resulting from electrostatic repulsion and decreased flexibility, viz., the influence of ionization of monomer on $k_{\mathrm{t}}$ is represented by one factor, which applies for both SPRs and MCRs, $\zeta_{\eta \alpha}$, and a second factor that represents the influence of ionization of polymer on $k_{\mathrm{t}}$, which is different for SPRs and MCRs.

An exponential function was used for interpolation of $k_{\mathrm{t}}$ for different values of $\alpha$, as such an assumption allows for a good representation of reported $\left\langle k_{\mathrm{t}}\right\rangle$ values as a function of $\alpha,{ }^{[78]}$ although the absolute values in ref. ${ }^{[78]}$ seem to be incorrect and have not been used for modeling (see subchapter 2.4.3). The factor of 7.5 was adjusted down to 6 via PREDICI ${ }^{\mathrm{TM}}$ estimation yielding in the correction factor of Coulomb repulsion of ionized SPRs, $\zeta_{\mathrm{C} \alpha}^{\mathrm{ss}}$, defined by eq. (5.61), which leads to an overall correction given by eq. (5.62)

$$
\begin{gathered}
\zeta_{\mathrm{C} \alpha}^{\mathrm{ss}}=\exp \left(-1.8 \cdot \alpha_{\mathrm{pAA}}\right) \\
k_{\mathrm{t}}^{\mathrm{ss}}=k_{\mathrm{t},(\alpha=0)}^{\mathrm{ss}} \cdot \zeta_{\eta \alpha} \cdot \zeta_{\mathrm{C} \alpha}^{\mathrm{ss}}
\end{gathered}
$$

An exponential function was chosen for MCR homotermination as well. The exponent in eq. (5.63) was estimated from conversion vs. time profiles (shown in subchapter 5.2.5) via PREDICI TM fitting. This procedure yields the corresponding correction factor, $\zeta_{\mathrm{C} \alpha}^{\mathrm{tt}}$, given by eq. (2.32), which leads to overall correction given by 
eq. (5.64). The decrease of $k_{\mathrm{t}}^{\mathrm{t}, \mathrm{t}}$ towards ionization is stronger than for $k_{\mathrm{t}}^{\mathrm{ss}}$ as was found experimentally (v.s.).

$$
\begin{aligned}
& \zeta_{\mathrm{C} \alpha}^{\mathrm{tt}}=\exp \left(-3 \cdot \alpha_{\mathrm{pAA}}\right) \\
& k_{\mathrm{t}}^{\mathrm{t}, \mathrm{t}}=k_{\mathrm{t},(\alpha=0)}^{\mathrm{t}, \mathrm{t}} \cdot \zeta_{\eta \alpha} \cdot \zeta_{\mathrm{C} \alpha}^{\mathrm{tt}}
\end{aligned}
$$

\subsubsection{The dependence of $k_{\mathrm{bb}}$ on the Degree of Ionization}

Backbiting has only been measured for AA polymerizations without ionization (see subchapter 5.1.4) and with full ionization. ${ }^{[77]}$ The influence of ionic strength has been addressed in subchapter 5.2.3. Backbiting may be scaled to the flexibility of polymer chains (see subchapter 2.3.3), which is well understood, as the structure of pAA coils in aqueous solution has been studied as a function of both $\alpha$ and $I .^{[82,84-87,163-166]}$

Considering $I_{0}=1.2 \cdot 10^{-11} \mathrm{~m}$ taken from ref. ${ }^{[84,85]}$ the slopes from fitting presented in Figure 5-31 were fitted as a function of $\alpha$ (stars in Figure 5-38) to develop eq. (5.65), which describes $l_{\mathrm{p}}$ of pAA as a function of both $\alpha$ and $I$.

$$
l_{\mathrm{p}}=\left(12+19.5 \cdot \sqrt{\frac{\alpha_{\mathrm{pAA}}}{I}}\right) \cdot 10^{-10} \mathrm{~m}
$$

Comparison of eq. (5.65) with the $k_{\mathrm{bb}}$ values of non-ionized and fully ionized AA yields eq. (5.66). 


$$
k_{\mathrm{bb}} / \mathrm{s}^{-1}=\frac{6.57 \cdot 10^{9} \cdot \exp \left(\frac{5020}{T / \mathrm{K}}\right)}{\left(12+19.5 \cdot \sqrt{\frac{\alpha_{\mathrm{pAA}}}{I}}\right)^{0.43}}
$$

Further research should be directed towards measuring branching levels upon varying $\alpha$ and $I$ to check eq. (5.66) experimentally, provided $k_{\mathrm{p}}$ as a function of $\alpha$ and $I$ is known with high accuracy.

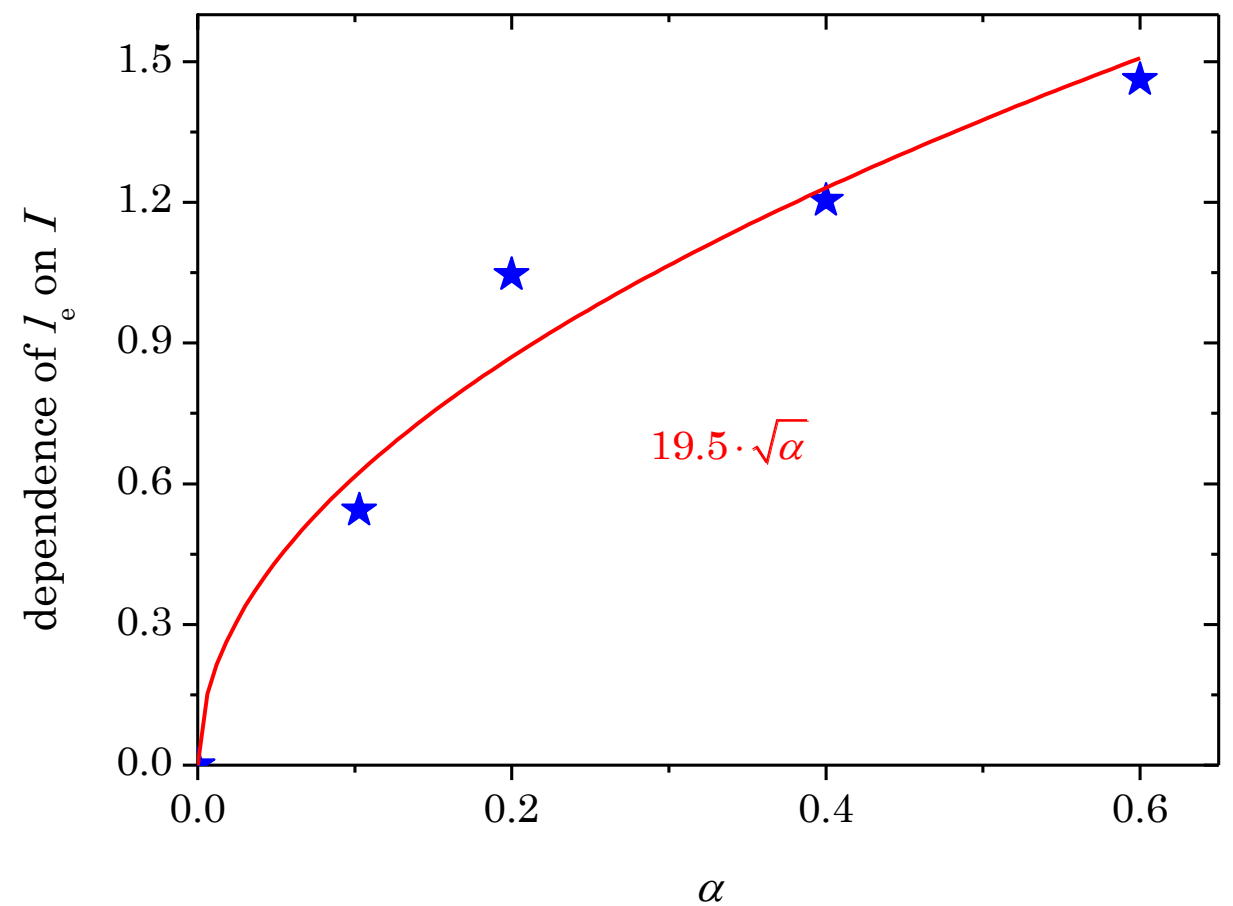

Figure 5-38 The slopes of the straight lines shown in Figure 5-31 giving the dependence of persistence chain length on $I$ are depicted as stars. The fit to a constant times the square root of $\alpha$ is depicted as line and given as formula. 


\subsection{9 $\quad \beta$-Scission}

No literature data on the influenced of ionization on the $\beta$-scission reaction of macroradicals is available. However, considerations about general radical stability (see subchapter 2.1) give a direction. A vicinal carboxylate moiety stabilizes a radical center better than a carboxyl moiety. This effect is (approximately) additive to other influences on stability, thus there need not be an influence of ionization, because both SPRs and MCRs might be stabilized to the same extent. If this stabilizing effect were slightly stronger for SPRs, $\beta$-scission would be increased.
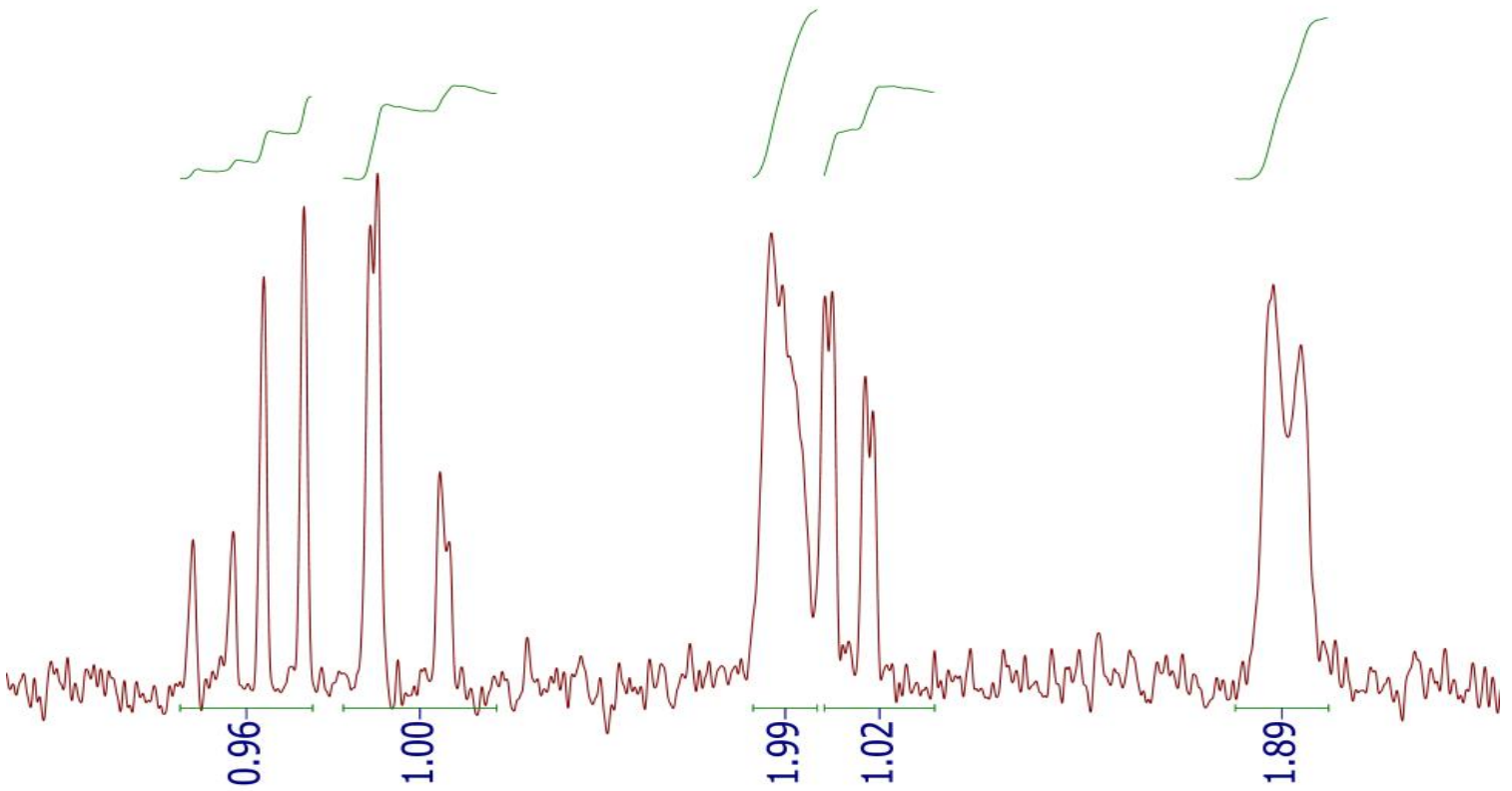

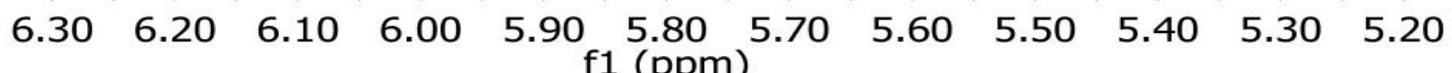

Figure 5-39 Double-bond region of the ${ }^{1} \mathrm{H}-\mathrm{NMR}$ spectrum of the product of the polymerization of
$X_{\mathrm{AA}}=0.05\left(0.12 \mathrm{~g} \mathrm{~g}^{-1}\right), \alpha=0.7$ with $0.001 \mathrm{~g} \mathrm{~g}^{-1}$ VA- 086 in water at $90{ }^{\circ} \mathrm{C}$. The two broad
signals appear to originate from MM(AA), the others from residual monomer. Double
bonds from MM exceed those from monomer residue by a factor of two. The chemical
shifts are different from the ones given in Figure $5-18$, because in this sample polymer
and monomer are partly ionized.

${ }^{1} \mathrm{H}-\mathrm{NMR}$ of the product of $0.1 \mathrm{~g} \mathrm{~g}^{-1} \mathrm{AA}$ polymerization with $\alpha=0.7$ and 1.0 at $90{ }^{\circ} \mathrm{C}$ suggests that MMs were formed, but MMs could not be detected for lower $\alpha$ under ostensibly the same conditions. Figure 5-39 shows the double-bond region of the ${ }^{1} \mathrm{H}-\mathrm{NMR}$ spectrum of the product of the polymerization of $x_{\mathrm{AA}}=0.05\left(0.12 \mathrm{~g} \mathrm{~g}^{-1}\right)$, $\alpha=0.7$ with $0.001 \mathrm{~g} \mathrm{~g}^{-1} \mathrm{VA}-086$ in water at $90{ }^{\circ} \mathrm{C}$. The two broad signals appear to 
originate from $\mathrm{MM}(\mathrm{AA})_{\mathrm{s}},{ }^{\mathrm{xxx}}$ the others from residual monomer. The chemical shifts differ from those given in subchapter 5.1.7, because pAA and AA are partly ionized.

Detection of MMs only at higher $\alpha$ may suggest an increase of $\beta$-scission by ionization. However, this finding may result from $k_{\mathrm{p}}^{\mathrm{t}}$ being reduced (see 5.2.6). A longer time span between formation of an MCR and growth thereof increases the probability of a $\beta$-scission event with the $k_{\beta}$.

The $\beta$-scission reaction was not included for modeling AA polymerization at $50{ }^{\circ} \mathrm{C}$, but has to be considered at higher temperature.

\subsubsection{The $p K_{\mathrm{A}}$ of $\mathrm{pAA}$}

The knowledge of degree of ionization of polymer is essential for calculating the dependence of rate coefficients, because, firstly, some rate coefficients, e.g., $k_{\mathrm{bb}}$ depend directly on $\alpha_{\mathrm{pAA}}$ and, secondly, $\alpha_{\mathrm{AA}}$ is linked to $\alpha_{\mathrm{pAA}}$ via the associated acidbase equilibria.

Extensive measurements on the dependence of the logarithmic acid dissociation constant, $\mathrm{p} K_{\mathrm{A}}$, of $\mathrm{pAA}$ as a function of $\alpha_{\mathrm{pAA}}$, average chain length, ionic strength (considering additionally the kind of added salt) have been carried out by De Stefano et al. ${ }^{[81]}$ The unexpected result that the $\mathrm{p} K_{\mathrm{A}}$ of polymer changes significantly with chain length, even above the oligomeric region, was also found by Laguecir et al. ${ }^{[82]}$

As the $\mathrm{p} K_{\mathrm{A}}$ value of $\mathrm{pAA}$ is a function of $\alpha$ of $\mathrm{pAA}$ and vice versa, an analytic solution to calculate $\mathrm{pH}$ is impossible. For modeling, the acid-base equilibrium of polymer is calculated together with the acid base equilibria of monomer and water by PREDICI ${ }^{\mathrm{TM}}$. The computational effort for this is high.

The protonation constant, $K^{\mathrm{H}}$, is defined by eq. (5.70).

xxx The signal at $5.3 \mathrm{ppm}$ could be either a doublet exhibiting the roof effect or two signals with almost identical chemical shift. If the signal were a doublet, it would most probably originate from a double bond belonging to disproportionation product. The other signal at $5.7 \mathrm{ppm}$ does not look like a doublet and does not exhibit a corresponding roof effect. Thus, it appears that MMs have been formed, the structure of which varies leading to slightly different chemical shifts of the signals of the terminal double bond. 


$$
K^{\mathrm{H}}=\frac{c_{\mathrm{H}-\mathrm{A}}}{c_{\mathrm{H}^{+}} \cdot c_{\mathrm{A}^{-}}}
$$

Assuming the concentration of water to remain approximately constant, leads to eq. (5.68).

$$
\mathrm{p} K_{\mathrm{A}}=-\lg \left(K_{\mathrm{A}}\right)=\lg \left(K^{\mathrm{H}}\right)
$$

One possibility to represent the relationship between $\mathrm{p} K_{\mathrm{A}}$ and $\alpha$ is the extended Henderson-Hasselbalch equation, eq. (5.69).

$$
\lg \left(K^{\mathrm{H}}\right)=\lg \left(K_{h}^{\mathrm{H}}\right)-(h-1) \cdot \lg \left(\frac{1-\alpha_{\mathrm{pAA}}}{\alpha_{\mathrm{pAA}}}\right)
$$

$h$ is an empirical parameter.

Another possibility to represent the relationship between $\mathrm{p} K_{\mathrm{A}}$ and $\alpha$ is the Högfeld equation, eq. (5.69).

$$
\lg \left(K^{\mathrm{H}}\right)=\alpha_{\mathrm{pAA}}^{2} \lg \left(K_{1}^{\mathrm{H}}\right)+2 \alpha_{\mathrm{pAA}}\left(1-\alpha_{\mathrm{pAA}}\right) \cdot \lg \left(K_{m}^{\mathrm{H}}\right)+\left(1-\alpha_{\mathrm{pAA}}\right)^{2} \cdot \lg \left(K_{0}^{\mathrm{H}}\right)
$$

The Högfeld equation was chosen for modeling, because it is more accurate especially at very high and very low degree of ionization. ${ }^{[81]}$

De Stefano et al. included the $\mathrm{p} K_{\mathrm{A}}$ value of AA for interpolation of the chain-length dependency of $K_{h}^{\mathrm{H}}$ ( extended Henderson-Hasselbalch equation) over the oligomeric region. ${ }^{\left[{ }^{[1]}\right.}$ From physicochemical perspective, a comparison with the value for the saturated monomer propanoic acid (4.87) is better, ${ }^{[195]}$ which is notably higher than the one of AA (4.25). ${ }^{[196]}$ 
<smiles>CCC(=O)O</smiles>

propanoic acid<smiles>O=C(O)CCC(=O)O</smiles>

glutaric acid<smiles>O=C(O)CCC(CCC(=O)O)C(=O)O</smiles>

pentane-1,3,5-tricarboxylic acid

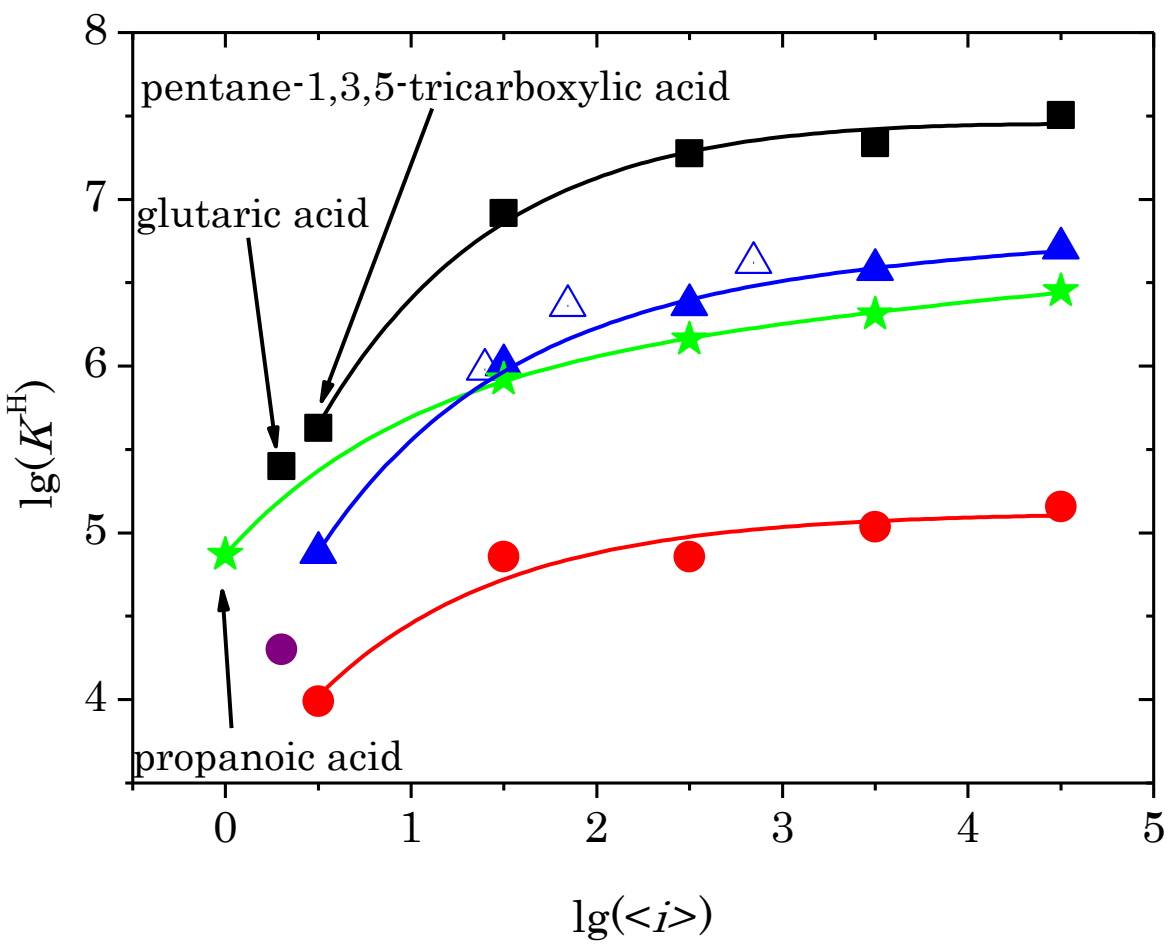

Figure 5-40 The parameters of the Högfeld equation, eq. (5.69), and the extended HendersonHasselbalch equation, eq. (5.70), are plotted for pAA at $I=0.1$ and $25^{\circ} \mathrm{C}$ as a function of chain length. Data is taken from ref. ${ }^{[81]}$ Stars: $K_{b}{ }^{\mathrm{H}}$, squares: $K_{1} \mathrm{H}\left(\mathrm{p} K_{\mathrm{A}} \alpha=1\right)$, solid triangles: $K_{\mathrm{m}}{ }^{\mathrm{H}}\left(\mathrm{p} K_{\mathrm{A}} \alpha=0.5\right)$, circles: $K_{0}^{\mathrm{H}}\left(\mathrm{p} K_{\mathrm{A}} \alpha=0\right)$. For comparison and in order to extend the chain-length region covered by eq. (5.71) into the oligomeric region, respectively, the values for propanoic acid, ${ }^{[195]}$ glutaric acid ${ }^{[199]}$ and pentane-1,3,5tricarboxylic acid ${ }^{[197]}$ are added. Lewis structures of these acids are given in the upper part. The $2^{\text {nd }} \mathrm{p} K_{\mathrm{A}}$ value of propanoic acid lies between $K_{1}{ }^{\mathrm{H}}$ and $K_{\mathrm{m}}{ }^{\mathrm{H}}$ (purple). The same fitting was done for $I=0.5,1.0,2.0$, and 3.0. For comparison, $K_{\mathrm{m}} \mathrm{H}$ for $I=0.001$ is taken from a different ref: ${ }^{[82]}$ open triangles. Data is fitted according to eq. (5.71) and depicted as solid line of corresponding color.

In this work, fitting has been carried out for the Högfeld equation parameters by adopting the procedure carried out by De Stefano et al. ${ }^{[81]}$ In order to extend the validity into the oligomeric region, the three $\mathrm{p} K_{\mathrm{A}}$ values of pentane-1,3,5tricarboxylic acid ${ }^{[197]}$ were used. It is a three-monomer-unit oligomer (short of one methyl moiety). Its first $\mathrm{p} K_{\mathrm{A}}$ value corresponds to $\lg \left(K_{0}^{\mathrm{H}}\right)$, which has the meaning of $\mathrm{p} K_{\mathrm{A}}$ of completely non-ionized polymer, its second $\mathrm{p} K_{\mathrm{A}}$ value corresponds to $\lg \left(K_{m}^{\mathrm{H}}\right)$, which has the meaning of $\mathrm{p} K_{\mathrm{A}}$ of half non-ionized polymer, and its third 
$\mathrm{p} K_{\mathrm{A}}$ value corresponds to $\lg \left(K_{1}^{\mathrm{H}}\right)$, which has the meaning of $\mathrm{p} K_{\mathrm{A}}$ of the last nonionized monomer unit. The Lewis structures of the acids are depicted in the upper part of Figure 5-40.

In order to represent the chain-length dependency of $K_{1}^{\mathrm{H}}, K_{m}^{\mathrm{H}}$, and $K_{0}^{\mathrm{H}}$, the parameters were fitted individually according to eq. (5.71). This was done for $I=0.1$, $0.5,1.0,2.0$, and 3.0, respectively. For pentane-1,3,5-tricarboxylic acid only values for $I=0.1$ are available. For higher $I$, the $\mathrm{p} K_{\mathrm{A}}$ values of the small acid molecule are possibly independent of $I .{ }^{[198]}$ Nevertheless, the $\mathrm{p} K_{\mathrm{A}}$ values of oligomers at high ionic strength calculated by the approach of this work are less certain.

$$
\lg \left(K_{x}^{\mathrm{H}}\right)=a_{0}+a_{1} \cdot\langle i\rangle^{-1}+a_{2} \cdot \lg (\langle i\rangle)
$$

The so-obtained parameters $a_{0}, a_{1}$, and $a_{2}$ were fitted by eq. (5.72), an equation of the same form as the extended Debye-Hückel equation discussed for density in subchapter 5.2.4.

$$
a_{x}=a_{x, 1}+a_{x, 2} \cdot \sqrt{I}+a_{x, 3} \cdot I
$$

The first part of the fitting process is illustrated in Figure 5-40. The parameters of the Högfeld equation, eq. (5.69), and of the extended Henderson-Hasselbalch equation, eq. (5.70), are plotted for pAA at $I=0.1$ and $25^{\circ} \mathrm{C}$ as a function of chain length. The neutralizing agent was $\mathrm{NaOH}$. Data is taken from ref. ${ }^{[81]}$ For comparison and in order to extend the chain-length range covered by eq. (5.71) into the oligomeric region, respectively, the values for propanoic acid, ${ }^{[195]}$ glutaric acid ${ }^{[199]}$ and pentane-1,3,5-tricarboxylic acid ${ }^{[197]}$ are added. Lewis structures of these acids are given in the upper part of Figure 5-40. For comparison, $K_{m}^{\mathrm{H}}$ for $I=0.001$ (blue, open triangles) is taken from ref: ${ }^{[82]}$ Data is fitted as lines according to eq. (5.71). With the so-obtained parameters, the degree of ionization of the polymer is calculated during simulation (see subchapter 5.2.5 and appendix). 


\subsubsection{Modeling the Polymerization of Partly lonized AA}

There are different possibilities of including the effects of ionization on reactivity in the model. In principle, individual reactions of non-ionized and ionized species could be modeled separately with the respective rate coefficients; e.g., the addition of an ionized monomer and a non-ionized monomer, respectively, to a macroradical with a carboxylate moiety next to the radical center as well as the addition of these monomers to a macroradical with a carboxyl moiety next to the radical center. Instead, it was decided to model the dynamic acid-base equilibria separately and to use the resulting values for (average) degree of ionization of monomer and polymer, respectively, to calculate rate coefficients corresponding to these average values. Thus, the afore-mentioned four reactions are treated as one reaction. The problem of the approach with individual reactions is that a lot of individual rate coefficients are needed, about which no information is available, and which are generally difficult to obtain; e.g., reactivity ratios of ionized polymer with non-ionized monomer and nonionized polymer with ionized monomer cannot be measured as are reactivity ratios of copolymerizations, because in this case, the resulting polymer does not yield any information about ionization of moieties at the time of monomer addition. Furthermore, the $\mathrm{p} K_{\mathrm{A}}$ value of the carboxyl group next to the radical center is different from the average $\mathrm{p} K_{\mathrm{A}}$ value of the polymer. Thus, attempts to consider the penultimate effect pose severe problems, since the $\mathrm{p} K_{\mathrm{A}}$ value of the penultimate carboxyl moiety strongly depends on the ultimate one being ionized or not. Moreover, acid-base equilibria are faster than the other reaction steps under consideration. Because of the von Grotthuss mechanism, acid-base equilibria, like other reactions of $\mathrm{H}^{+}$in water, are even faster than the "normal" diffusion limit. ${ }^{[200]}$ Hence, protonation and deprotonation of a monomer may happen several time during one addition step.

The dynamic acid-base equilibria are calculated by PREDICI ${ }^{\mathrm{TM}}$, which is implemented into the model by means of counter species (more details are given in the appendix). For each calculation step, $\mathrm{p} K_{\mathrm{pAA}}$ of polymer is calculated. Together with the equilibria of AA and water, this yields $\alpha_{\mathrm{AA}}$ and $\alpha_{\mathrm{AA}}$ for each calculation step.

After implementation of independent data into the model, conversion vs. time profiles are the basis of optimization. At best an initiator should be used, the decay of which is independent of $\mathrm{pH}$ or other substances in the reaction mixture. Otherwise, initiator kinetics are hard to separate from the monomer kinetics. 
VA-086 meets the requirement of the decay being independent of $\mathrm{pH}$ (see subchapter 5.1.1), and thus appears to be an ideal initiator for this system. Conversion vs. time profiles have been measured for polymerization of $0.16 \mathrm{~g} \mathrm{~g}^{-1} \mathrm{AA}$ $\left(x_{\mathrm{M}}=0.05\right)$ at $90^{\circ} \mathrm{C}$ with $10^{-2}, 10^{-3}$, and $10^{-4} \mathrm{~g} \mathrm{~g}^{-1} \mathrm{VA}-086$ at $\alpha=0,0.3,0.7$, and 1 (ionizing agent $\mathrm{NaOH}$ ) given in the appendix. At the lowest initiator concentration, dead-end polymerization is reached long before the putative half-live of the initiator at $2.57 \cdot 10^{4} \mathrm{~s}$ given by eq. (5.3). It was checked experimentally that a dead-end polymerization took place by resuming the polymerization by addition of initiator. Moreover, polymerization of ionized and partly ionized monomer is faster than polymerization of non-ionized monomer, which is contradictory to the expectation on the basis of the rate coefficients described above. With higher initiator concentration, the rate of polymerization is in the beginning almost independent of AA ionization. Then a dead-end polymerization is reached again even before the putative half-life of the initiator. The deviation from the expected behavior is outside experimental uncertainty and reproducibility of the experiments is good, as repeat experiments show. At this stage, the only possible explanation for this behavior is that the decay of VA-086 is independent of $\mathrm{pH}$, but depends heavily on the content of ionized AA. Accelerated initiator decay by ionized AA is supported by the fact that unexpected low molar mass was found for the polymerization of fully ionized AA with VA-086. Thus, initiator kinetics seem to dominate overall kinetics and the conversion-time profiles of ionized AA initiated by VA-086 have not been modeled.

Furthermore, polymerizations of $0.16 \mathrm{~g} \mathrm{~g}^{-1} \mathrm{AA}\left(\mathrm{xM}_{\mathrm{M}}=0.05\right)$ at $90^{\circ} \mathrm{C}$ initiated by $10^{-3}$, $10^{-4}$, and $10^{-5} \mathrm{~g} \mathrm{~g}^{-1}$ sodium persulfate, NaPS, at $\alpha=0,0.3,0.7$, and 1 (ionizing agent $\mathrm{NaOH}$ ) were carried out (conversion vs. time profiles given in the appendix). NaPS as initiator has the advantage of good solubility. Furthermore, it is of industrial relevance for initiating polymerizations in aqueous systems. Its decay is influenced directly by $\mathrm{pH}^{[201]}$ and also by $\mathrm{AA}$ depending on the degree of ionization. ${ }^{[56]}$ The latter influence is alleged to be higher towards lower concentration of monomer, ${ }^{[56]}$ which was interpreted as rather high uncertainty of $k_{\mathrm{d}}$ measurements. Modeling with constant $k_{\mathrm{d}}$ gave reasonable results, but as the exact dependency of $k_{\mathrm{d}}$ is unknown, the conversion vs time profiles have not be used for optimization of the model.

The model should be capable of predicting conversion vs. time profiles for the polymerization at other degrees of ionization and other monomer concentrations. In Figure 5-41, simulated conversion vs. time profiles of $0.1 \mathrm{~g} \mathrm{~g}^{-1}$ and $0.2 \mathrm{~g} \mathrm{~g}^{-1} \mathrm{AA}$ at $\alpha=0,0.2,0.4,0.6,0.8$, and 1 are shown. The rate of initiation is identical for all polymerizations. A low degree of ionization is almost without influence on rate of 
polymerization. The highest difference in polymerization rate changing $\alpha$ by 0.2 is observed between $\alpha=0.6$ and $\alpha=0.8$. For $0.1 \mathrm{gg}^{-1} \mathrm{AA}$ the influence of $\alpha$ on polymerization rate is more pronounced than for $0.2 \mathrm{~g} \mathrm{~g}^{-1} \mathrm{AA}$, which may be explained by the difference of $k_{\mathrm{p}}$ at $\alpha=0$ and at $\alpha=1$ becoming larger towards lower monomer content. For $0.2 \mathrm{~g} \mathrm{~g}^{-1} \mathrm{AA}$ and low $X$, the decrease of $k_{\mathrm{p}}$ towards higher $\alpha$ may be compensated by decrease of $k_{\mathrm{t}}$. Simulated molar masses (not shown) decrease similarly to simulated polymerization rates.

Cutié et al. found a higher difference in rate of polymerization already for a low degree of ionization, ${ }^{[80]}$ but in that study NaPS was used as initiator, the decay of which is increased by the presence non-ionized AA. ${ }^{[56]}$

Future work should be direct towards checking of the model by actual measurement of these as well as MMD varying $w$ and $\alpha$.

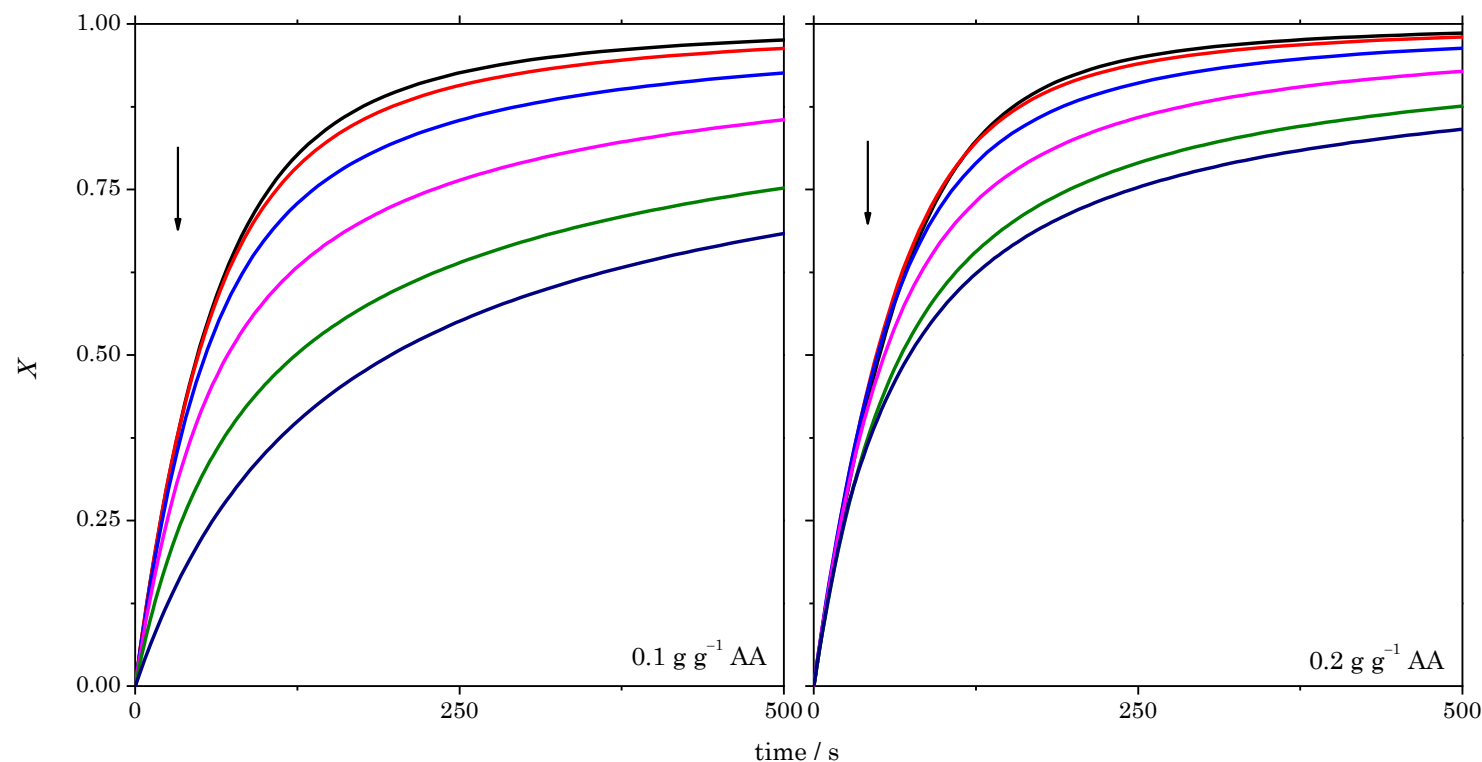

Figure 5-41 Simulated conversion vs. time profiles of $0.1 \mathrm{~g} \mathrm{~g}^{-1} \mathrm{AA}$ (left) and $0.2 \mathrm{~g} \mathrm{~g}^{-1} \mathrm{AA}$ (right) at $\alpha=0,0.2,0.4,0.6,0.8$, and 1 . The arrow indicates the direction of higher $\alpha$. The recipes contain $0.02 \mathrm{~g} \mathrm{~g}^{-1} \mathrm{D} 1173$ as the initiator, the decay of which was set to the value of the setup described in subchapter 3.14.4, which results in a half-live of $1625 \mathrm{~s}$. The rate of initiation is identical for all polymerizations. 


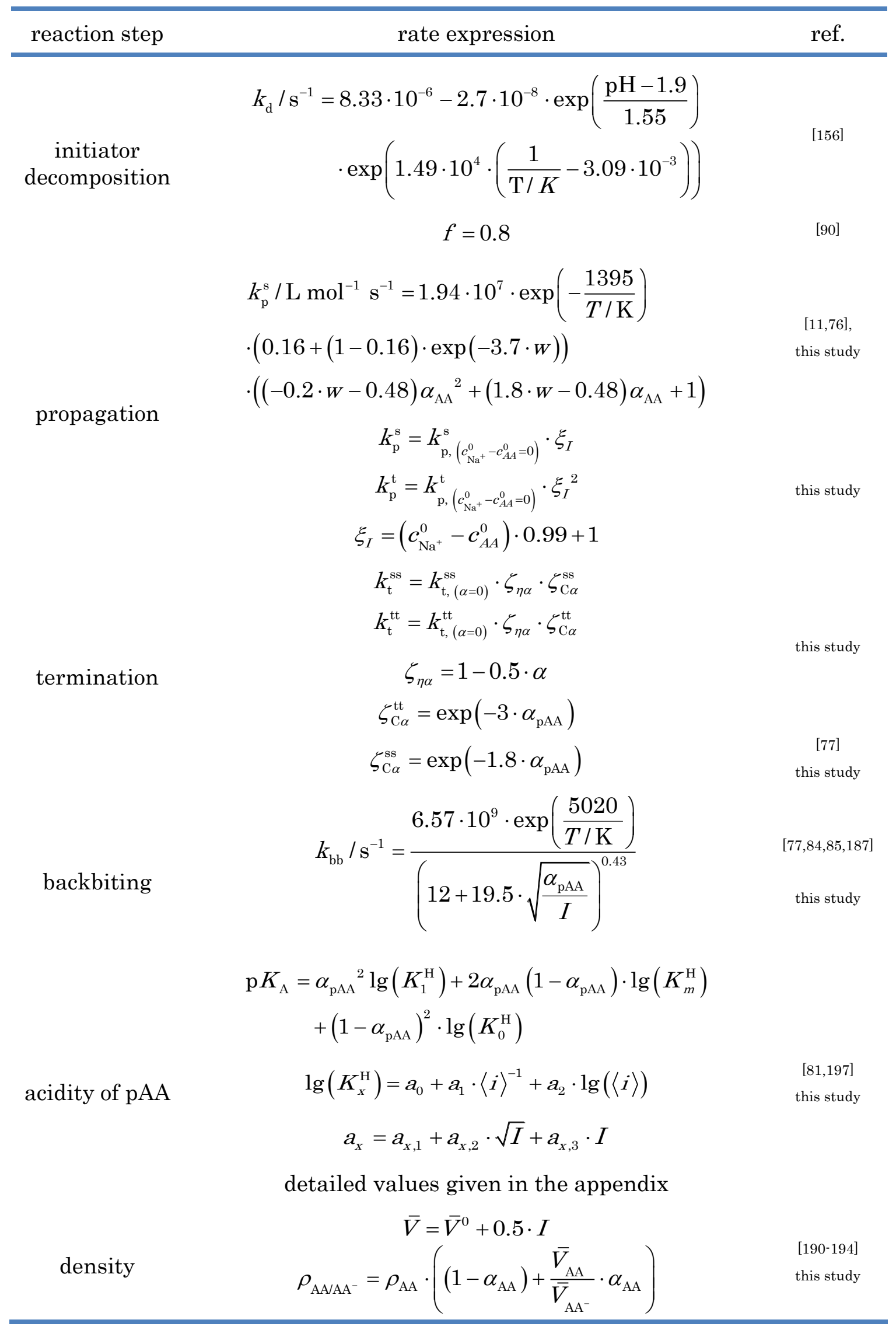




$$
\begin{aligned}
& \rho_{\mathrm{pAA} / \mathrm{pAA}}=\rho_{\mathrm{AA}} \cdot\left(\left(1-\alpha_{\mathrm{pAA}}\right)+\frac{\bar{V}_{\mathrm{pAA}}}{\bar{V}_{\mathrm{pAA}^{-}}} \cdot \alpha_{\mathrm{pAA}}\right) \\
& \rho_{\mathrm{all}}=\frac{m_{\mathrm{H}_{2} \mathrm{O}}}{m_{\text {all }}} \cdot \rho_{\mathrm{H}_{2} \mathrm{O}}^{\text {virtual }}+\frac{m_{\mathrm{all}}-m_{\mathrm{H}_{2} \mathrm{O}}}{m_{\mathrm{all}}} \cdot 1 \mathrm{~g} \mathrm{~mol}^{-1} \\
& \rho_{\mathrm{H}_{2} \mathrm{O}}^{\text {virtual }}=\left(\rho_{\text {all }}-\frac{m_{\mathrm{all}}-m_{\mathrm{H}_{2} \mathrm{O}}}{m_{\mathrm{all}}}\right) \cdot \frac{m_{\mathrm{all}}}{m_{\mathrm{H}_{2} \mathrm{O}}}
\end{aligned}
$$$$
\text { detailed values given in the appendix }
$$ 



\section{6 \\ Acrylamide}

The polymerization of AAm (acrylamide, IUPAC: 2-prop-eneamide) is of industrial interest as the product is widely used as thickener in, e.g., wastewater treatment, gel electrophoresis, paper production, and mining. The polymerization of AAm has been studied overall. ${ }^{[155,202]}$ The propagation has been examined separately by PLPSEC ${ }^{[145,167,203,204] X X X I}$ and by theoretical calculation, which gave that water as a solvent (compared to vacuum and toluene) reduces activation energy of $k_{\mathrm{p}}$. ${ }^{[65]}$ The termination reaction and transfer to monomer have been studied by $\gamma$-radiolysis relaxation $^{[205]}$ and SP-PLP-NIR as well as chemical initiation. ${ }^{[145]}$ Copolymerization, e.g., with AA has been studied and a pronounced dependence on $\mathrm{pH}$ was found. Both reactivity ratios vary with degree of ionization, as well as the rate of polymerization $^{[75,168]}$ and chain transfer to thiols. ${ }^{[152]}$ The kinetic behavior of AAm and its methylated derivates (at the $\alpha$-carbon or one or two times at the nitrogen) has been investigated and extensively discussed. ${ }^{[145]}$ Backbiting during homopolymerization of AAm has not been reported. This subchapter describes how backbiting during polymerization of AAm has been verified.

The most sensitive and most direct method to detect midchain radicals (MCRs) is EPR measurement. MCRs can be formed during polymerization of AAm only by backbiting and transfer to polymer. The latter can only occur after polymer is formed i.e. at significant conversion and especially for higher initial concentration of monomer. Hence, by comparison of experiments with different initial monomer concentrations and considering how conversion influences the fraction of MCRs, its

xxxI The reader may note that both Pascal et al. and Seabrook et al. describe a now widely discarded explanation for concentration dependence. 
source may be identified. Accordingly, EPR is the method of choice to prove that backbiting occurs. It can also be used to quantify backbiting i.e. measure the corresponding rate coefficient. By measuring under continuous initiation, concentration and fraction of MCRs can be obtained. Another method to detect backbiting is ${ }^{13} \mathrm{C}-\mathrm{NMR}$. As both propagation and termination (in case of combination only) of an MCR lead to a branching point, the concentration of branching points allows for inference on backbiting. The results from both methods are presented hereafter.

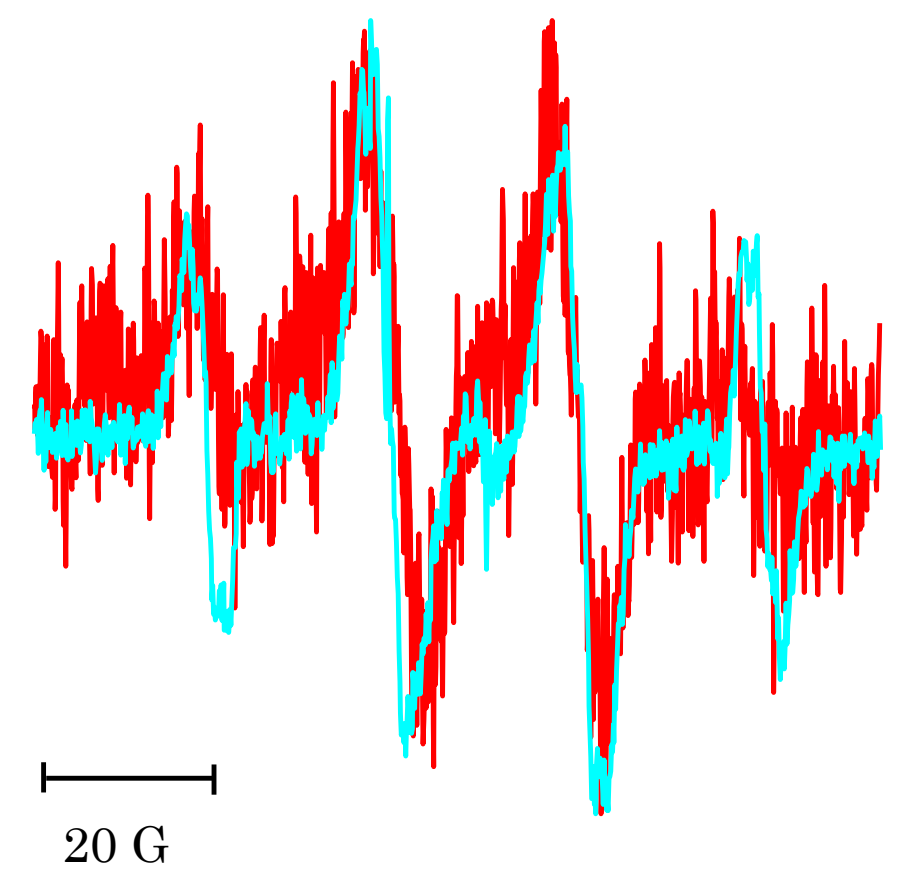

Figure 6-1 The EPR spectra of BA (aqua line) and AAm (red line) are quite similar. Shown are spectra from conditions at which there are almost solely SPRs. The AAm spectrum results from addition of four spectra to improve signal-to-noise ratio. Conditions AAm: photoinitiation, $0.200 \mathrm{~g} \mathrm{~g}^{-1}$ monomer in $\mathrm{H}_{2} 0,273 \mathrm{~K}$; $\mathrm{BA}: 0.223 \mathrm{~g} \mathrm{~g}^{-1}$ monomer in toluene, $223 \mathrm{~K}$. The BA spectrum is taken from ref. ${ }^{[101]}$

The almost pure SPR spectra of AAm and BA are compared in Figure 6-1. The lines are nearly on top of each other. Hence, coupling constants and line broadening only deviate slightly. The similarity of the spectra makes signal assignment easy, as the assignment for BA can be adapted. For details of the assignment of lines to SPRs and MCRs see subchapter 5.1.5. 
For other acrylate type monomers, e.g., BA, $k_{\mathrm{bb}}$ has a notably higher activation energy than the other rate coefficients with which the fraction of MCRs, $x_{\mathrm{MCR}}$, decreases $\left(k_{\mathrm{p}}^{\mathrm{t}}, k_{\mathrm{t}}^{\mathrm{st}}\right.$, and $k_{\mathrm{t}}^{\mathrm{tt}}$; compare eq. (2.18)). This is why, $\boldsymbol{x}_{\mathrm{MCR}}$ increases with temperature. Given this, polymerization temperature was increased to check for changes in the spectrum resulting from an augmentation of $x_{\mathrm{MCR}}$. The most prominent new line appears in the center and additional smaller lines appear next to the small outermost lines of the SPR spectrum. For BA this is discussed in detail here. ${ }^{[101]}$

In Figure 6-2, spectra from measurements that had polymer added to the reaction mixture in order to make critical coupling easier and improve S/N-ratio. In Figure 62 a the spectrum for $273 \mathrm{~K}$ (red) looks the same as the one in Figure 6-1. This shows that transfer to the added polymer is not significant, as indicated in additional experiments at higher temperature by measuring a reaction mixture out of the polymer, initiator, and water without the monomer and not finding a signal.
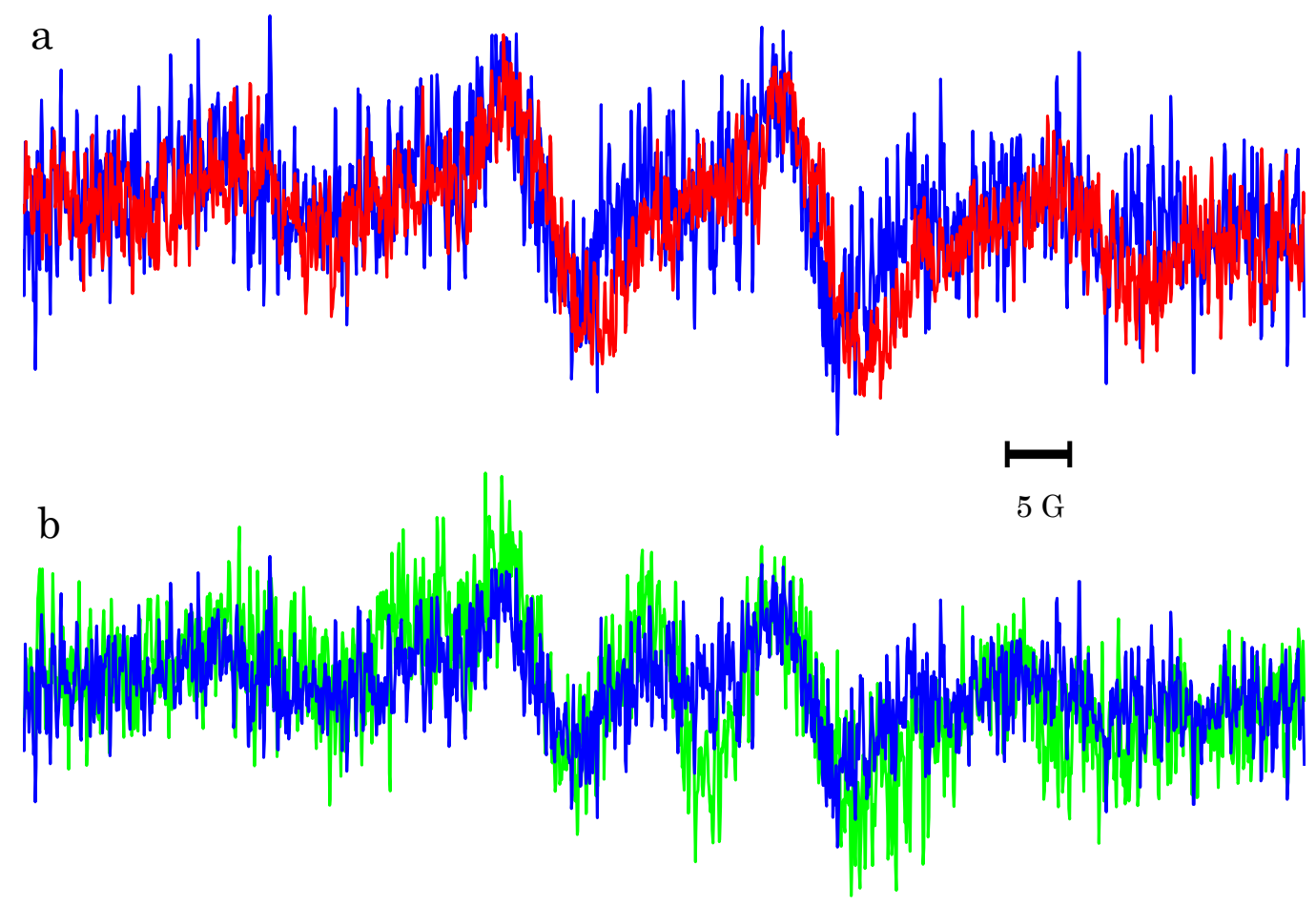

Figure 6-2 The EPR spectra of AAm polymerization under continuous UV initiation at different temperature are compared. a: $273 \mathrm{~K}$ (red) and $298 \mathrm{~K}$ (blue); b: $298 \mathrm{~K}$ (blue, same as a) and $323 \mathrm{~K}$ (green) This demonstrates how the fraction of MCRs increases with temperature. Conditions: $0.140 \mathrm{~g} \mathrm{~g}^{-1}$ monomer in $\mathrm{H}_{2} 0,0.300 \mathrm{~g} \mathrm{~g}^{-1}$ polyvinylpyrrolidone, addition of two spectra to improve signal-to-noise ratio. 
Comparing the spectrum recorded at $273 \mathrm{~K}$ (red) with the spectrum measured at $298 \mathrm{~K}$ (blue) in Figure 6-2 a reveals the changes expected for an increase of MCRs: A new line appears in the middle of the spectrum and the outer lines seem to broaden. The latter results from the fact that the resolution of the spectrum is not sufficient to observe the two lines separately. Increasing temperature further leads to an enhancement of the effects mentioned above. In Figure $6-2 \mathrm{~b}$ the spectrum recorded at $298 \mathrm{~K}$ (blue) is compared to the spectrum measured at $323 \mathrm{~K}$ (green). The middle line gets even more intense towards $323 \mathrm{~K}$ and has the same intensity as the inner lines of SPR spectrum which means the fraction of MCRs is ca. 0.4, which has been calculated for BA for this ratio. ${ }^{[101]}$ Moreover, the outer lines broaden more.

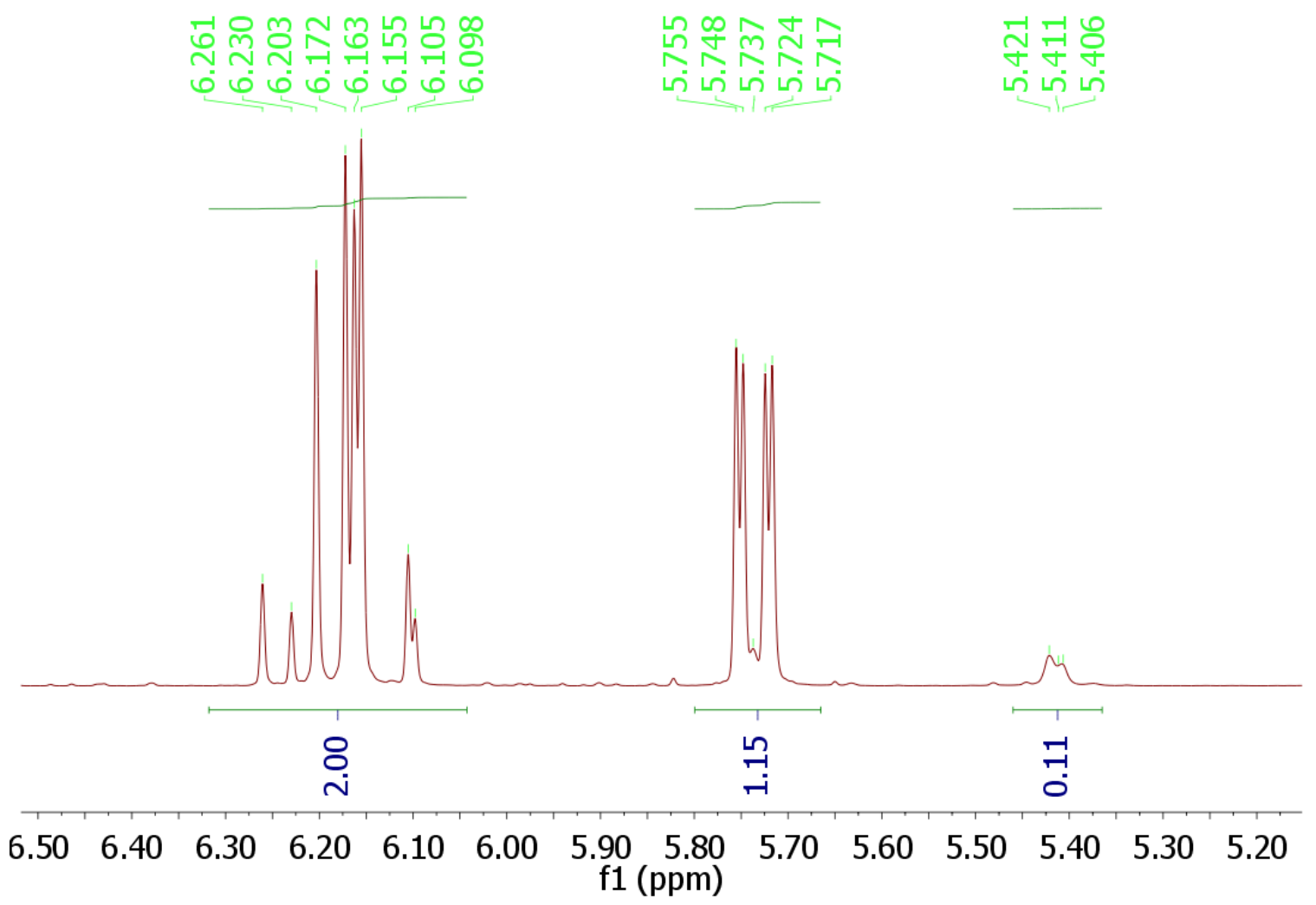

Figure 6-3 Double-bond region of the ${ }^{1} \mathrm{H}-\mathrm{NMR}$ spectrum of the reaction product of the polymerization of $0.05 \mathrm{~g} \mathrm{~g}^{-1} \mathrm{AAm}$ with $0.02 \mathrm{~g} \mathrm{~g}^{-1} \mathrm{VA}-086$ in $\mathrm{H}_{2} \mathrm{O}$ at $170{ }^{\circ} \mathrm{C}$ is given. The MM lines are: ${ }^{1} \mathrm{H}-\mathrm{NMR}\left(\mathrm{D}_{2} \mathrm{O}\right): \delta=5.41(\mathrm{bs}, \mathrm{H}), 5.74(\mathrm{bs}, \mathrm{H})$. The prediction of the shift by ChemDraw ${ }^{\mathrm{TM}}$ is 5.49 and 5.89 , respectively. The other lines result from monomer residue.

In order to verify further the finding of backbiting happening during radical polymerization of AAm an independent, different experiment, NMR, was carried out. Subject to the $k_{\mathrm{p}}^{\mathrm{t}}$ to $k_{\mathrm{p}}^{\mathrm{s}}$ ratio, ${ }^{13} \mathrm{C}-\mathrm{NMR}$ is a less sensitive method than EPR to 
detect backbiting. Such being the case, it was performed on a polymer sample for which a relative high concentration of branching points was expected. The polymerization was carried out with a low monomer content $\left(0.05 \mathrm{~g} \mathrm{~g}^{-1}\right)$, as well as low initiator content $\left(0.02 \mathrm{~g} \mathrm{~g}^{-1}\right)$, and at a high temperature $\left(170^{\circ} \mathrm{C}\right)$ to full conversion. At this high temperature, $\beta$-scission as follow-up reaction to backbiting is expected to occur at a significant rate. Therefore, ${ }^{1} \mathrm{H}-\mathrm{NMR}$ was carried out to find macromonomers.

Figure 6-3 shows the lines of monomer and macromonomer in the ${ }^{1} \mathrm{H}-\mathrm{NMR}$. The similarity to AA is obvious (see Figure 5-18 and Figure 6-3). The two lines associated with macromonomer $5.41(\mathrm{bs}, \mathrm{H})$ and 5.74 (bs, H) cannot be assigned to the disproportionation product as the shift of its protons would be different and the trans coupling would be stronger. Furthermore, no disproportionation patterns (two lines of equal height with the difference of $2.02 \mathrm{u}$ ) were found in the mass spectrum (v.i.). The origin of the macromonomers can only be transfer to polymer with subsequent $\beta$-scission. Since initial monomer concentration is very low, intermolecular transfer to polymer should be insignificant. Thus, the finding of macromonomers confirms a high backbiting rate of AAm at this elevated temperature.

The ${ }^{13} \mathrm{C}$-NMR spectrum of pAAm is very similar to the corresponding pAA spectrum (see Figure 5-10). Nevertheless, in order to ensure line assignment a normal spectrum and a dept-135 (distortionless enhancement by polarization transfer 135 degree) spectrum were measured. In spectra measured with this technique, tertiary and primary carbon atoms give negative signals, secondary ones give a positive signal, and quaternary carbons do not show. The spectrum is given in Figure 6-4. The small peak of the quaternary carbon atom of the branching point is shown enlarged in Figure 6-5. The quaternary nature is confirmed by the dept-135 spectrum. Compared to pAA the line of the branching point is shifted slightly towards high field. 


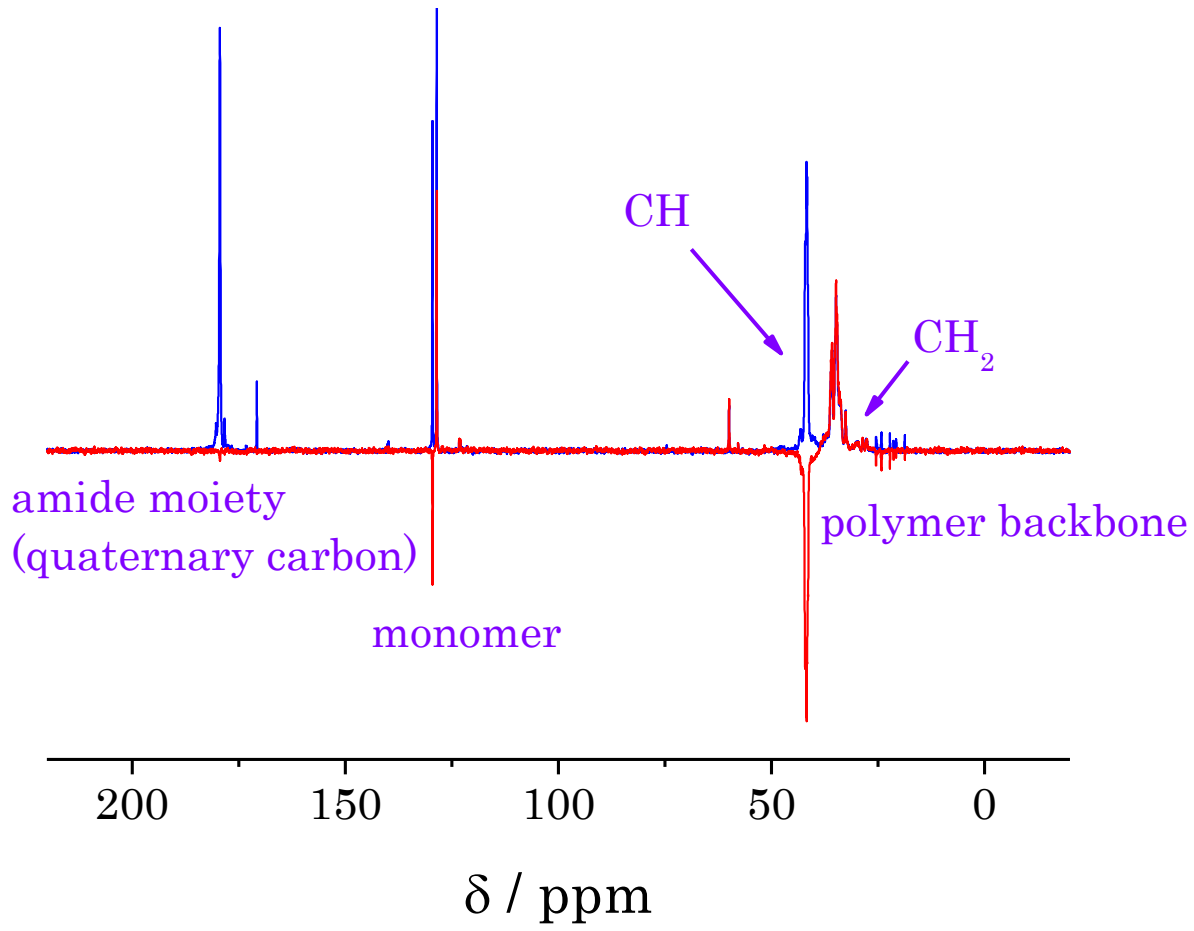

Figure 6-4 The full ${ }^{13} \mathrm{C}-\mathrm{NMR}$ spectrum of the reaction product of the polymerization of $0.05 \mathrm{~g} \mathrm{~g}^{-1}$ AAm with $0.02 \mathrm{~g} \mathrm{~g}^{-1} \mathrm{VA}-086$ in $\mathrm{H}_{2} \mathrm{O}$ at $170^{\circ} \mathrm{C}$ is given. The spectrum is very similar to the corresponding $\mathrm{AA} / \mathrm{pAA}$ spectrum. In order to ensure line assignment a normal spectrum (blue) and a dept-135 spectrum (red) are compared. In the latter tertiary and primary carbon atoms give negative signals, secondary ones give a positive signal, and quaternary carbons do not show. Peaks are assigned accordingly. The signal of the quaternary carbon atom associated with branching is amplified in Figure 6-5.

The concentration of branching points can be derived by integrating ${ }^{13} \mathrm{C}-\mathrm{NMR}$ peaks of the quaternary carbon and the CH-backbone peaks (see subchapter 5.1.4, eq. (5.22)). The spectrum acquired with standard parameters gives $1.5 \%$ branching which can be considered as a minimum value. A spectrum with the same parameters as for quantitative ${ }^{13} \mathrm{C}-\mathrm{NMR}$ analysis of pAA (see subchapter 3.5.2) leads to $2.9 \%$ branching. The polymer of the latter measurement had already been degraded; however, degradation towards acrylic acid should not distort the result, as the NMR spectra are very similar and cyclization ${ }^{\mathrm{XXXI}}$ does not directly involve the carbons used for calculation.

xxxII pAAm is known to undergo cyclization by elimination of water and ammonia or by forming imido cycles. 


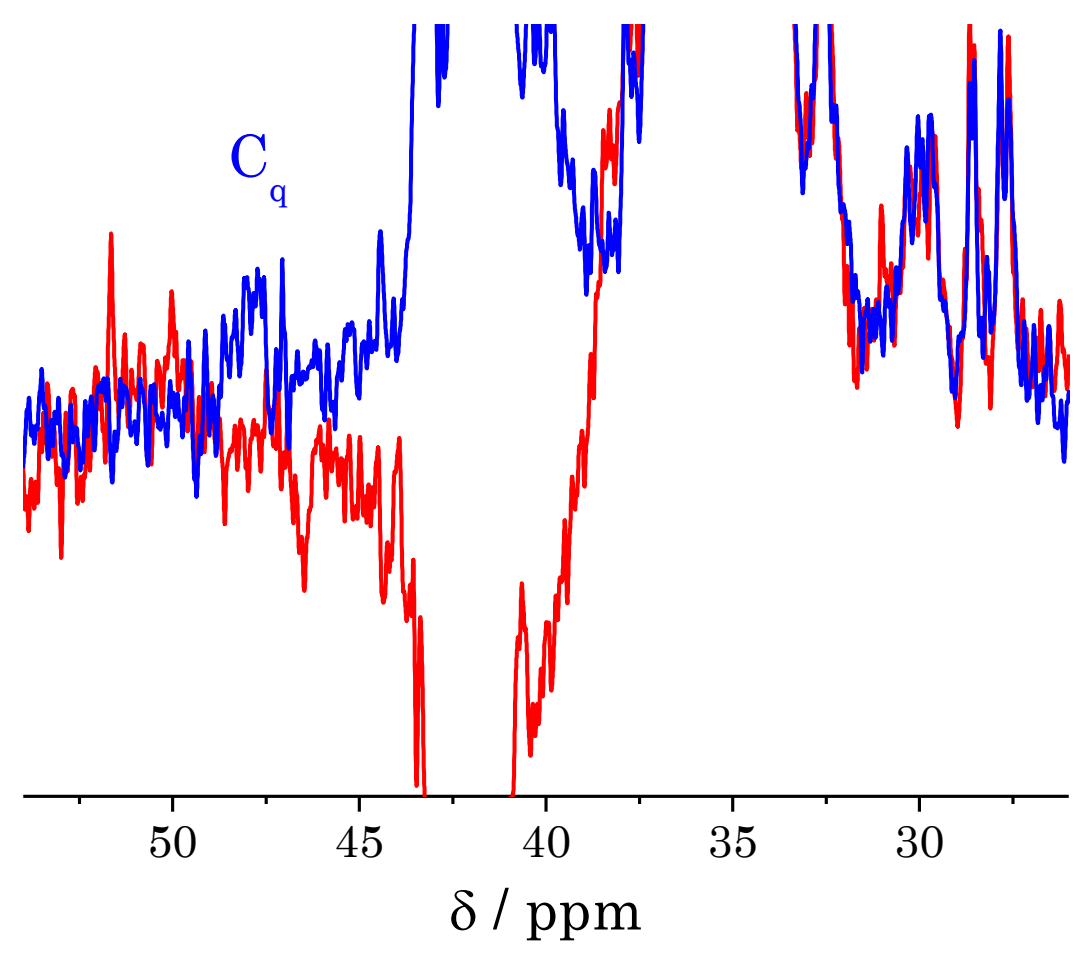

Figure 6-5 Enlargement of the two spectra shown in Figure 6-4. The small peak of the quaternary carbon $\left(\mathrm{C}_{\mathrm{q}}\right)$ shows in the normal spectrum (blue), but not in the dept-135 spectrum (red).

Under the assumptions that $\beta$-scission has only marginal influence on branching, and branching doubles from low to full conversion, taking $k_{\mathrm{p}}^{\mathrm{s}}$ from ref. ${ }^{[145]}$ the following calculation gives the backbiting coefficient during AAm polymerization at $170{ }^{\circ} \mathrm{C}$ as rough estimate:

$$
\begin{aligned}
& k_{\mathrm{p}}^{\mathrm{s}}\left(5 \mathrm{cg} \mathrm{g}^{-1}\right)=1.04 \cdot 10^{8} \cdot \exp \left(\frac{18.6 \mathrm{~kJ} \mathrm{~mol}^{-1}}{R \cdot T}\right) \mathrm{L} \mathrm{mol}^{-1} \mathrm{~s}^{-1} \\
& \Rightarrow k_{\mathrm{p}}^{\mathrm{s}}\left(5 \mathrm{cg} \mathrm{g}^{-1}, 170{ }^{\circ} \mathrm{C}\right)=6.68 \cdot 10^{5} \mathrm{~L} \mathrm{~mol}^{-1} \mathrm{~s}^{-1} \\
& k_{\mathrm{bb}} \simeq x_{\mathrm{BP}} \cdot 0.5 \cdot k_{\mathrm{p}}^{\mathrm{s}} \cdot c_{\mathrm{M}}=0.029 \cdot 6.68 \cdot 10^{5} \mathrm{~L} \mathrm{~mol}^{-1} \mathrm{~s}^{-1} \cdot 0.62 \mathrm{~mol} \mathrm{~L}^{-1}=6.0 \cdot 10^{3} \mathrm{~s}^{-1}
\end{aligned}
$$

This $k_{\mathrm{bb}}$ value is by a factor of 8.5 smaller than the according value for AA. If a high amount of MCRs is terminated by disproportionation, the so-obtained $k_{\mathrm{bb}}$ will be too 
low. However, disproportionation product could be found neither by ${ }^{1} \mathrm{H}-\mathrm{NMR}$ nor by ESI-MS (v.i.).

The typical high molar mass of pAAm is not well suited for analysis by ESI-MS. Nontheless, the polymer from high-temperature polymerization, which has been used for NMR analysis (v.s.), has shorter chains. Still, much polymer has a molar mass that is too high for ESI-MS. This is why signal-to-noise ratio is rather inferior. The ESI-MS analysis does not allow for confirmation of branching. The molar mass is not altered by backbiting if it is followed by propagation.

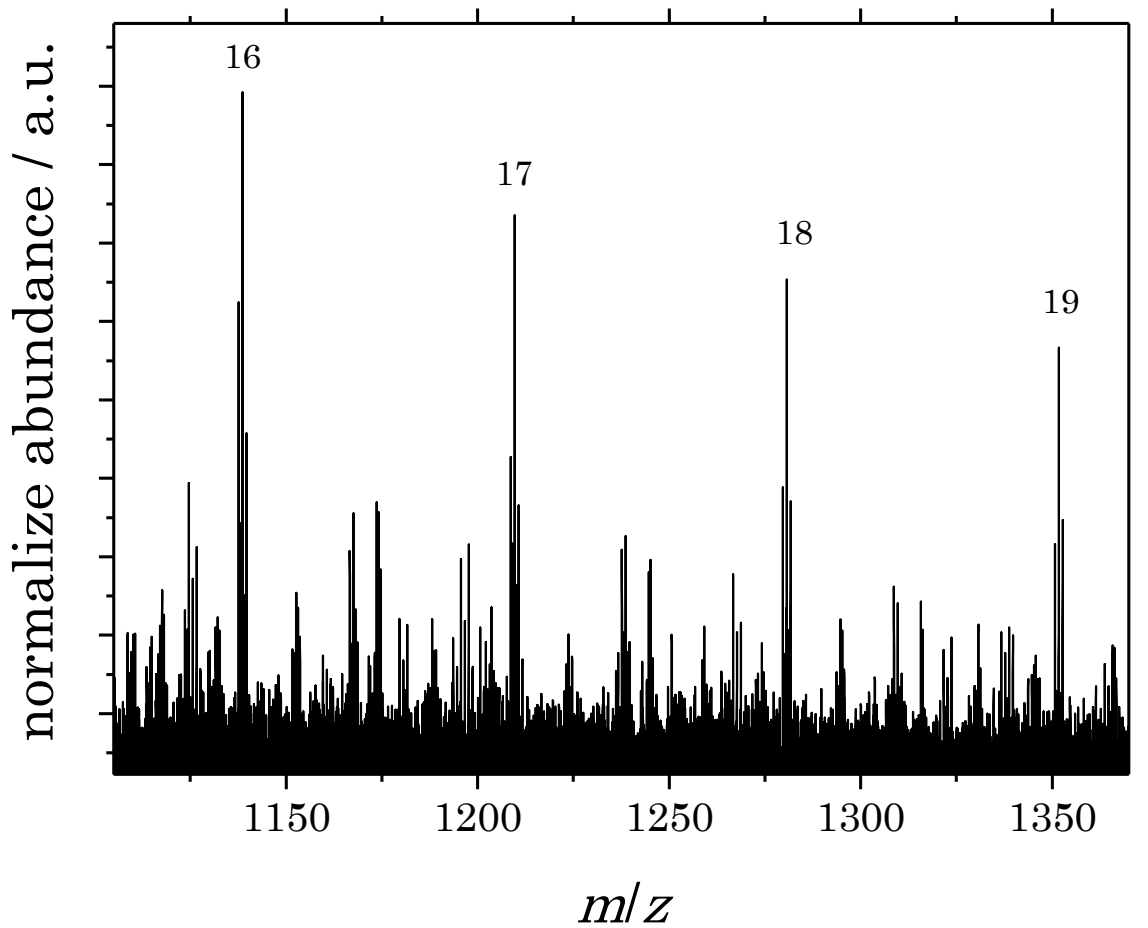

Figure 6-6 Section of an ESI-MS spectrum (acetonitrile, water and a small bit of formic acid) of the product of the polymerization of $0.05 \mathrm{~g} \mathrm{~g}^{-1} \mathrm{AAm}$ in water at $170{ }^{\circ} \mathrm{C}$ with $0.02 \mathrm{~g} \mathrm{~g}^{-1}$ VA-086. The number of monomer units is given for the main signals. They are multiples of monomer mass. The signals inbetween are mostly from more than one time ionized polymer.

The main polymer line is a mutiple of the monomer mass plus a sodium cation or a proton in case acid was added to the solution. The latter gives a better signal-to-noise ratio and a typical spectrum is shown in Figure 6-6. Both the 
$\beta$ scission product and the transfer to monomer product exhibit a molar masses that fit the major signals. Considering the low molar mass of the corresponding polymer, it may be excluded that this main signal is the $\beta$-scission product. Thus, it may be assumed that transfer to monomer is the dominant chain-stopping event at this high temperature. Signals that correspond to polymer with one or two initiator-fragment endgroups could not be identified. Most probably the endgroups undergo various sidereactions.

Another interesting finding is that the apparent isotope peak is much higher than expected (Figure 6-7). This can be explained by degradation of the polymer. The nucleophilic attack of water or hydroxide transforms an amide moiety into a carboxyl group, i.e. an acrylamide monomer unit is converted into an acrylic acid monomer unit effectively changing the homopolymer into a copolymer. Naturally this may also happen to monomer in the reactuion mixture consequently making it a copolymerization. The difference in $\mathrm{m} / \mathrm{z}$ of the first line of the two monomer units is $1.0256 \mathrm{u}$. The rightmost peak in Figure 6-6 is shown enlarged in Figure 6-7. Details of the peak and calculated values for the 19 monomer unit homopolymer and the corresponding one with one moiety hydrolyzed is given in Table 6-1. In this example, more than $5 \%$ of the acrylamide monomer units are hydrolyzed.

The next biggest peak found has an $\mathrm{m} / \mathrm{z}$ ratio, which is $34.0 \mathrm{u}$ smaller than the main peak. This can be explained by cyclization under elimination of water and ammonia. This reaction is known to occur with pAAm depending on temperature and $\mathrm{pH}$. Again, the apparent isotope peak is too big. This can be ascribed to hydrolysis of the amide function as discussed above. Furthermore, lines from double and multiple ionization are found. 


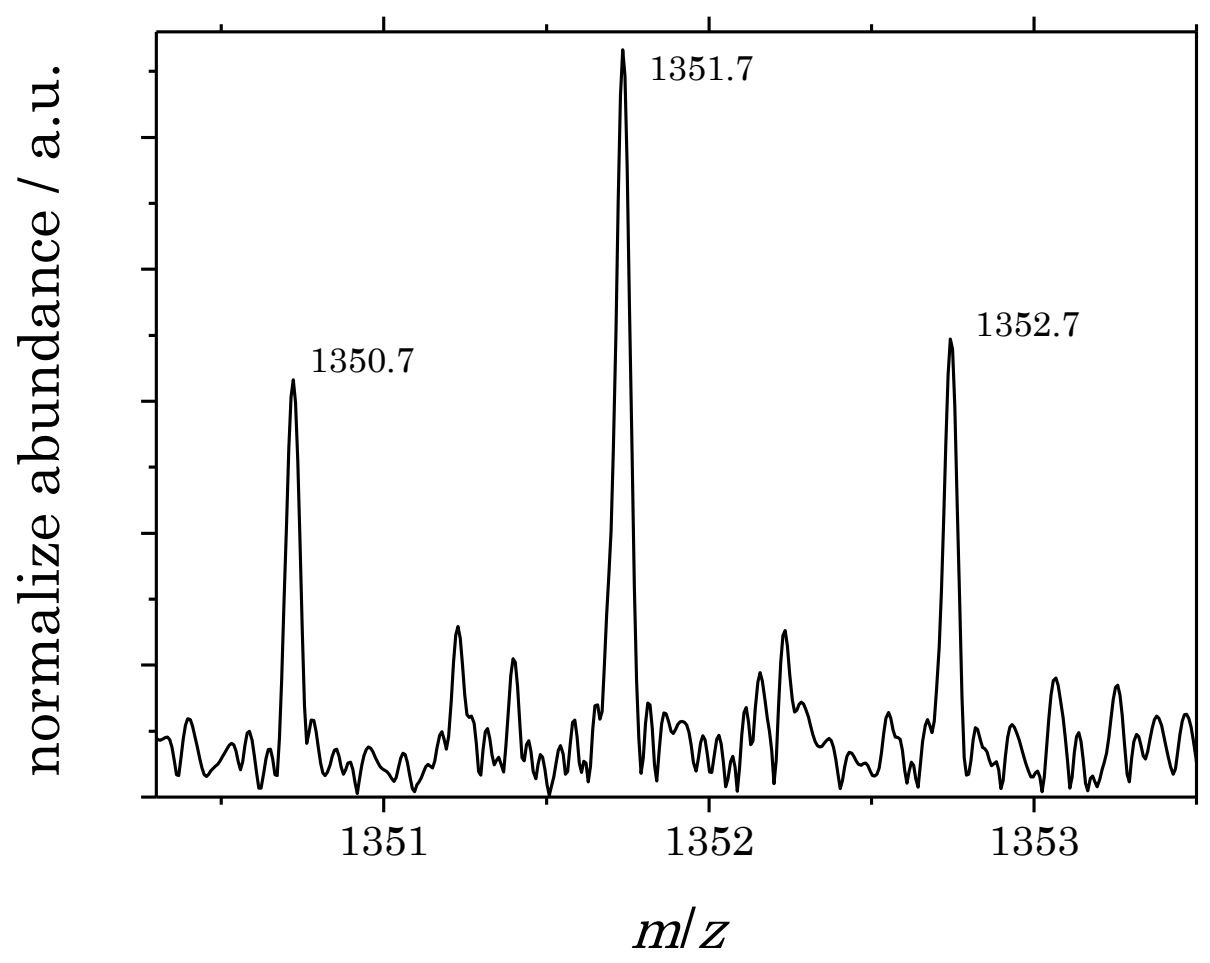

Figure 6-7 Enlargement of the 19 monomer unit peak given in Figure 6-6. m/z of the peak center is given next to the related peak.

Table 6-1 Comparison between the lines shown in Figure 6-6 and calculation for corresponding polymer.

\begin{tabular}{cccccc}
\hline & \multicolumn{2}{c}{ found } & \multicolumn{2}{c}{$\begin{array}{c}\text { calculated: } \\
(\mathrm{AAm})_{19}\left[\mathrm{H}^{+}\right]\end{array}$} & \multicolumn{2}{c}{$\begin{array}{c}\text { calculated: } \\
(\mathrm{AAm})_{18}(\mathrm{AA})\left[\mathrm{H}^{+}\right]\end{array}$} \\
\hline $\mathrm{m} / \mathrm{z}$ & $\begin{array}{c}\text { normalized } \\
\text { intensity }\end{array}$ & $\mathrm{m} / \mathrm{z}$ & $\begin{array}{c}\text { normalized } \\
\text { intensity }\end{array}$ & $\mathrm{m} / \mathrm{z}$ & $\begin{array}{c}\text { normalized } \\
\text { intensity }\end{array}$ \\
\hline 1350.7 & 1.00 & 1350.7 & 1.00 & & 1.00 \\
1351.7 & 1.79 & 1351.7 & 0.62 & 1351.7 & 0.62 \\
1352.7 & 1.10 & 1352.7 & 0.19 & 1352.7 & \\
\hline
\end{tabular}





\section{7 \\ Closing Remarks}

The radical polymerization of methacrylic acid, acrylic acid and acrylamide in aqueous solution has been investigated by several different methods and setups. Kinetic models for both acrylic acid and methacrylic acid have been developed applying the program PREDICI ${ }^{\mathrm{TM}}$. Good representation of experimental conversion vs. time profiles and molar mass distributions as well as the branching level in case of acrylic acid could be achieved.

Both the Mayo and the CLD procedure reveal that $C_{\mathrm{CTA}}$ of MAA to ME is independent of monomer content. As $k_{\mathrm{p}}$ exhibits an increase upon decreasing MAA concentration from bulk to dilute solution by about one order of magnitude, a constant ratio of $k_{\mathrm{p}}$ to $k_{\mathrm{tr}, \mathrm{CTA}}$ thus means $k_{\mathrm{tr}, \mathrm{CTA}}$ also varies by one order of magnitude. This observation may be understood as the genuine kinetic effect associated with hindrance to rotational motion in the transition state structure by the molecular environment being primarily due to characteristics of the radical chain-end and thus being more or less identical for propagation and for transfer to the CTA. This finding probably holds for other transfer reactions as well, which should be checked by measuring the transfer constants to different molecules in aqueous solution. Thereby water as a solvent has the advantage of transfer to water always being negligible.

$k_{\mathrm{t}}$ values from chemically initiated polymerization of MAA at negligible monomer conversion were evaluated for a broad range of average chain lengths. Excellent agreement with the composite model was found applying parameters from literature. The influence of conversion on composite-model parameters is still a controversial question. This procedure may give low cost, low afford access to composite-model 
parameters, at least for the long-chain region, for polymerizations at intermediate and higher conversion. Experiments could be carried out by mixing polymer of different molar masses with monomer, solvent, initiator, and CTA. The predicament would be that the structure of polymer coils in solution is different for dry polymer dissolved and polymer solution directly after being formed by polymerization. A possibly better approach would be to produce the polymer in a first step, add CTA to different extent, and polymerize further now with different chain lengths depending on the amount of CTA. For the second approach special care has to be taken to ensure accurate knowledge of initiator and monomer concentration. The so-obtained initial $k_{\mathrm{t}}$ values give composite-model parameters for the corresponding $X$.

Polymerizing under different levels of CTA with polymer added at the beginning may also give deeper insight into the Norrish-Trommsdorff effect, which is especially pronounced in case of MAA polymerization in aqueous solution. The intensity of the Norrish-Trommsdorff effect decreasing towards higher CTA content in the polymerization mixture could be correlated to molar mass of polymer in solution. The chain length of macroradicals and of dead polymer in solution were not independent, as both were correlated to CTA content. It would be interesting to check the dependence of $k_{\mathrm{t}}$ on molar mass of polymer in solution for different chain lengths of macroradicals, which could be achieved by the above-described polymerizations with polymer added to the reaction mixture.

The relationship between $k_{\mathrm{t}}^{1,1}$ and viscosity of the reaction mixture is well understood as long as no polymer is present, i.e., a zero conversion. The relationship between $k_{\mathrm{t}, \mathrm{TD}}$ and viscosity resulting from the presence of polymer sometimes referred to as macroscopic viscosity in contrast to the microscopic viscosity "felt" by the macroradicals is not well understood. The influence of polymer in the reaction mixture on viscosity is more pronounced than the influence on $k_{\mathrm{t}}$, but the general correlation is unknown. It would be desirable to gain a deeper understanding of this relationship. Attempts to correlate $k_{\mathrm{t}}$ and viscosity require accurate knowledge of viscosity at a given conversion. The polymerization mixture that has reached the desired conversion should be measured directly and without delay, because the viscosity of the reaction mixture changes with time. As viscosity can be measured in a glass capillary, it is possible to photopolymerize stepwise with analysis of degree of monomer conversion, e.g., by NIR and viscosity for every step.

The polymerization of $0.3 \mathrm{~g} \mathrm{~g}^{-1}$ MAA exhibits an initial plateau region of $k_{\mathrm{t}}$ up to $X=0.22$, while the polymerization of $0.1 \mathrm{~g} \mathrm{~g}^{-1} \mathrm{MAA}$ exhibits a decline of $k_{\mathrm{t}}$ for the same range of polymer content. It could be shown that addition of isobutyric acid as saturated monomer analogue to the polymerization of $0.1 \mathrm{~g} \mathrm{~g}^{-1}$ MAA eliminates the 
Norrish-Trommsdorff effect, which points to both monomer and polymer content being important for $k_{\mathrm{t}}$ of a polymerization mixture. This elimination of the NorrishTrommsdorff effect may be interesting for industrial applications, as more constant reaction conditions may lead to better product properties. The data indicates that a higher MAA content in the reaction mixture, and thus in the solvent-swollen polymer coils, enhances segmental mobility and permeability of the polymer-water solution for macroradicals. The influence of other small molecules on $k_{\mathrm{t}}$ at different $X$ should be investigated systematically. The above-described approach of stepwise polymerization may also help to understand this phenomenon, as an influence of these molecules on the viscosity of the polymer solution might be correlated to their influence on the Norrish-Trommsdorff effect.

Aqueous solutions of MAA exhibit very pronounced rheopecty, i.e. sheer force applied to the solution results in an increase of viscosity. It appears rewarding to investigate, whether sheer forces also decrease $k_{\mathrm{t}}$, and thus the rate of polymerization and the molar mass of polymeric product can be increased by intense stirring.

Detailed analysis of $k_{\mathrm{p}}$ data of AA revealed that the maximum found at about $0.03 \mathrm{~g} \cdot \mathrm{g}^{-1}$ monomer content may be explained by both $A$ and $E_{\mathrm{A}}$ depending on monomer content. Taking the measured, weak dependence of $E_{\mathrm{A}}$ on AA content into account, extrapolation to high temperature suggests that the maximum disappears above $150^{\circ} \mathrm{C}$. For many monomers, the low monomer content region has not been studied by PLP-SEC, but for MAA no maximum was found. It would be interesting to see whether other monomers than AA exhibit a maximum in the dependence of $k_{\mathrm{p}}$ on the monomer content as well.

The influence of monomer content on $k_{\mathrm{t}}^{1,1}$ was implemented into the model by correlation to the effect of monomer content on viscosity. For an even more detailed analysis, viscosity should be measured as a function of monomer content up to high temperature.

There are some open questions about termination kinetics in general that should be answered. The correlation between $k_{\mathrm{t}}^{\mathrm{tt}}$ and $k_{\mathrm{t}}^{\text {ss }}$ as well as the way these coefficients are combined to yield $k_{\mathrm{t}}^{\text {st }}$ should be studied in more detail. It appears promising to measure pure MCR termination in a model system, e.g., by SP-PLP-EPR, which consists of MCR precursors of defined chain lengths that decay to MCRs (and a very rapidly terminating species that disappears quickly) after UV-irradiation. Such an approach, although experimentally challenging, may as well help to settle another question. Composite-model parameters of MCRs have never been measured. Nevertheless, simulation of conversion vs. time profiles and radical concentrations at 
different chain lengths in combination with associated MCR fractions suggest that MCR exhibit the same chain-length dependency as SPRs, which should be verified in an independent experiment.

A difference in activation energy for $k_{\mathrm{t}}^{\mathrm{tt}}$ and $k_{\mathrm{t}}^{\mathrm{ss}}$ has been reported in literature. Different activation energies for two diffusion-controlled reactions in the same system appear unlikely and could only be explained by steric hindrance of MCRMCR termination being reduced towards higher temperature. In this study, no indication for a difference in activation energy could be found, however, the reaction conditions under investigation made propagation of MCRs, but not termination, the important reaction path. A setup to measure $k_{\mathrm{t}}^{\mathrm{tt}}$ should be used to check whether activation energy of $k_{\mathrm{t}}^{\mathrm{tt}}$ is higher than activation energy of fluidity.

Additional information about MCR kinetics may be derived from detailed analysis, including simulation, of continuously initiated EPR experiments, as both absolute radical concentration and the fraction of MCRs can be obtained.

Utilizing a precursor of two different chain lengths may help to answer the question whether the diffusion mean, the geometric mean, or the harmonic mean is the optimum procedure for calculating the termination between two chains of different chain length. Most probably, this question can only be answered by direct measurement of the termination between radicals of two different chain lengths.

The Norrish-Trommsdorff effect is weaker for AA polymerization than for MAA polymerization. The conversion dependence of $k_{\mathrm{t}}$ was modeled in the same way as described for MAA but, as only a few conversion vs. time profiles were used for deriving the expression, validation thereof, by measuring more conversion vs. time profiles of polymerizations with high AA content, e.g., by ${ }^{1} \mathrm{H}-\mathrm{NMR}$ or NIR is desirable. Care has to be taken to keep temperature constant, as high monomer content leads to high heat of reaction.

By measuring the fraction of MCRs during butyl acrylate polymerization via EPR, it could be shown that the transfer of MCRs to CTA is not an important reaction path. Extension of these experiments towards higher temperature, where the influence of termination is less important for $\boldsymbol{x}_{\mathrm{MCR}}$, viz., the long-chain approximation gives a better estimate, would be desirable. EPR measurements of acrylate and CTA at high temperature are possible with the combination of, e.g., naphthalene as solvent (boiling point: $218^{\circ} \mathrm{C}$ ), dodecanthiol as CTA (boiling range: $266-283^{\circ} \mathrm{C}$ ), and octadecyl acrylate (boiling point: $400.1^{\circ} \mathrm{C}$ ). Additionally, EPR measurement of 
acrylate and CTA in highly viscous system, e.g., polyethylene glycol, would also lead to the long-chain approximation being a better estimate.

The backbiting reaction of AA was quantified via ${ }^{13} \mathrm{C}-\mathrm{NMR}$. A dependence of $k_{\mathrm{bb}}$ on monomer-to-solvent ratio reported in literature, comparing $0.1 \mathrm{~g} \mathrm{~g}^{-1}$ and $0.5 \mathrm{~g} \mathrm{~g}^{-1} \mathrm{AA}$, could not be found in this study for $0.03 \mathrm{~g} \mathrm{~g}^{-1}$ and $0.3 \mathrm{~g} \mathrm{~g}^{-1}$ AA. A detailed study of $k_{\mathrm{bb}}$ over a broad range of monomer concentrations should be carried out.

The polymerization of macromonomers synthesized at high temperature has been studied at $50{ }^{\circ} \mathrm{C}$ to separate propagation of macromonomers from $\beta$-scission. This approach should also be used to check the line assignment of short-chain branching and long-chain branching in ${ }^{13} \mathrm{C}-\mathrm{NMR}$.

The AA model developed for the temperature range of 35 to $90^{\circ} \mathrm{C}$ was extended towards high-temperature polymerization up to $170^{\circ} \mathrm{C}$, where $\beta$-scission and propagation of macromonomers need to be considered. Quantification of $\beta$-scission proved to be difficult because of the rather low quality of polymer samples from hightemperature experiments subjected to molar mass analysis. It is highly desirable to compare the simulation to a second data set of molar masses of product from hightemperature AA polymerization.

A model for the polymerization of ionized AA was developed, which takes numerous dependencies of rate coefficients on ionization and ionic strength into account. This model should be checked by comparison to conversion vs. time profiles and MMDs from polymerization of AA under variation of degree of ionization, ionic strength, monomer, and initiator content.

Furthermore, the reduced termination rate found for polymerization of fully ionized AA should lead to radical concentration during chemically initiated polymerization being high enough for EPR measurement.

A more pronounced enhancement of propagation by increased ionic strength for MCRs than for SPRs could be observed by its influence on $\boldsymbol{x}_{\mathrm{MCR}}$. Preferably, low concentrations of monomer should be investigated to maximize $x_{\mathrm{MCR}}$ and thus radical concentration, to ensure that no gel effect applies, and to avoid problems of solubility.

Coefficients used in the model for partly ionized AA should be measured by independent experiments. PLP-SEC experiments yielding $k_{\mathrm{p}}^{\mathrm{s}}$ should be carried out under variation of degree of ionization for higher weight fraction of monomer to check the equation used in the model. Furthermore, the influence of counterions on 
$k_{\mathrm{p}}^{\mathrm{s}}$ should be investigated systematically. Thereby the ratio of counterions to monomer should be varied. It needs to be examined whether the influence of ionic strength on $k_{\mathrm{p}}^{\mathrm{s}}$ is higher than on $k_{\mathrm{p}}^{\mathrm{t}}$. A possible influence of the counterion on $k_{\mathrm{p}}^{\mathrm{s}}$ should be investigated as well. An ion-pair effect as a possible explanation for the increase of $k_{\mathrm{p}}^{\mathrm{s}}$ should vary with the strength of coordination, size of the counterion, and charge number. It would be particularly interesting, whether non-coordinating cations also lead to an augmentation of $k_{\mathrm{p}}^{\mathrm{s}}$. Moreover, an ion-pair effect should apply for other ionic monomers as well; it should be checked whether the polymerization rate of other ionic monomers can be manipulated by addition of salt.

As the dependence of $k_{\mathrm{p}}^{\mathrm{s}}$ on both monomer content and degree of ionization can be explained via the transition state structure, $k_{\mathrm{p}}^{\mathrm{s}}$ should be calculated for ionized acrylic acid with different amounts of cations being present around the radical center based on transition state theory.

SP-PLP-EPR should be used to measure $k_{\mathrm{t}}$ at intermediate degrees of ionization to check the interpolation used in the model. Apparently, the effect of ionic strength on $k_{\mathrm{t}}$ is weak. An increase of $k_{\mathrm{t}}$ caused by screening of counterions may be partly compensated by increased viscosity. Nevertheless, for a more accurate picture, $k_{\mathrm{t}}$ should be measured at full ionization under variation of ionic strength. In case of screening, the temperature dependence of $k_{\mathrm{t}}$ cannot be expected to be the same as the temperature dependence of fluidity.

$k_{\mathrm{bb}}$ should be quantified by ${ }^{13} \mathrm{C}-\mathrm{NMR}$ analysis of low conversion product of the polymerization of AA under variation of degree of ionization and of ionic strength. Accurate knowledge of $k_{\mathrm{p}}^{\mathrm{s}}$ at given $\alpha, w$, and $I$ is essential for the analysis. The mechanism of termination has to be checked by ${ }^{1} \mathrm{H}-\mathrm{NMR}$, to examine whether all backbiting events lead to branching points.

It should be examined whether the $\mathrm{p} K_{\mathrm{A}}$ of $\mathrm{AA}$ is independent of ionic strength by measuring the $\mathrm{pH}$ value of a dilute solution of $\mathrm{AA}$ at $\alpha=0.5$ under variation of ionic strength, e.g., by addition of $\mathrm{NaCl}$.

MCRs were found during acrylamide homopolymerization in aqueous solution via EPR revealing the backbiting reaction to take place. Modeling the polymerization of AAm is a logical follow-up of the modeling of AA as the kinetic scheme is the same. 



\section{Appendix}

$\underline{\text { Data of density and viscosity measurements of MAA solutions }}$

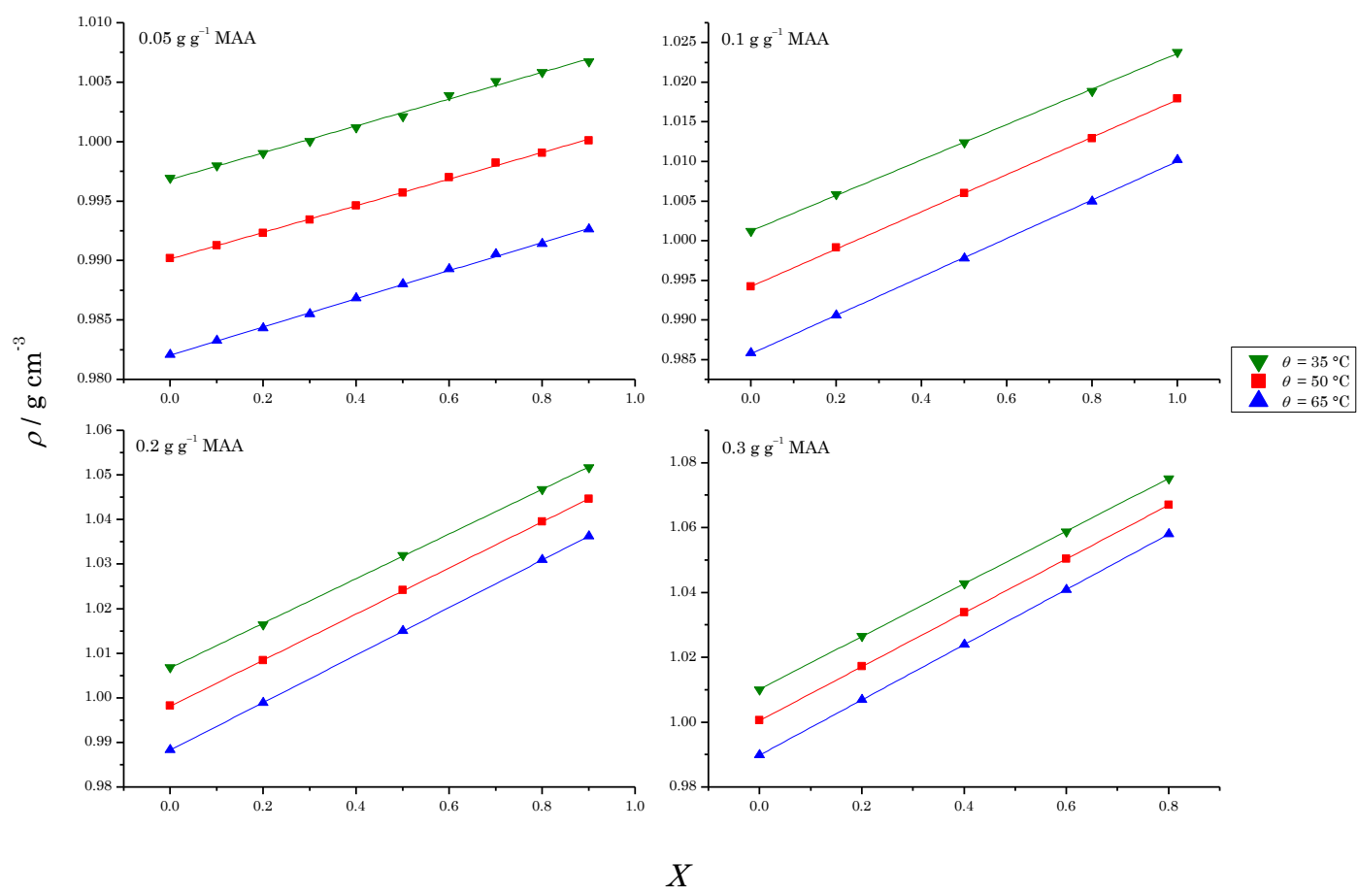

0-1 Density of solutions of MAA in water at different temperature. 


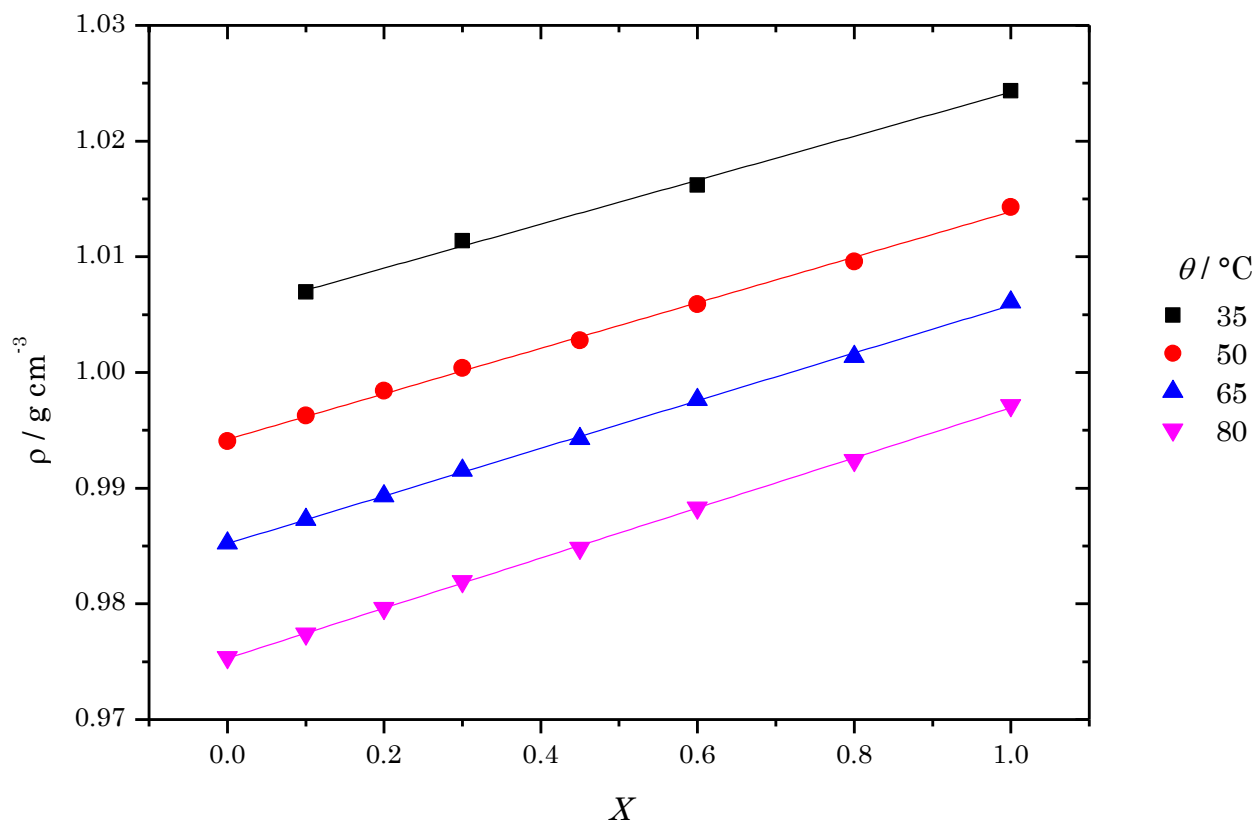

Figure 0-2 Density of $0.1 \mathrm{~g} \mathrm{~g}^{-1} \mathrm{MAA} / \mathrm{pMAA}$ in water. Different degrees of monomer conversion were simulated by mixing monomer, polymer, and solvent.

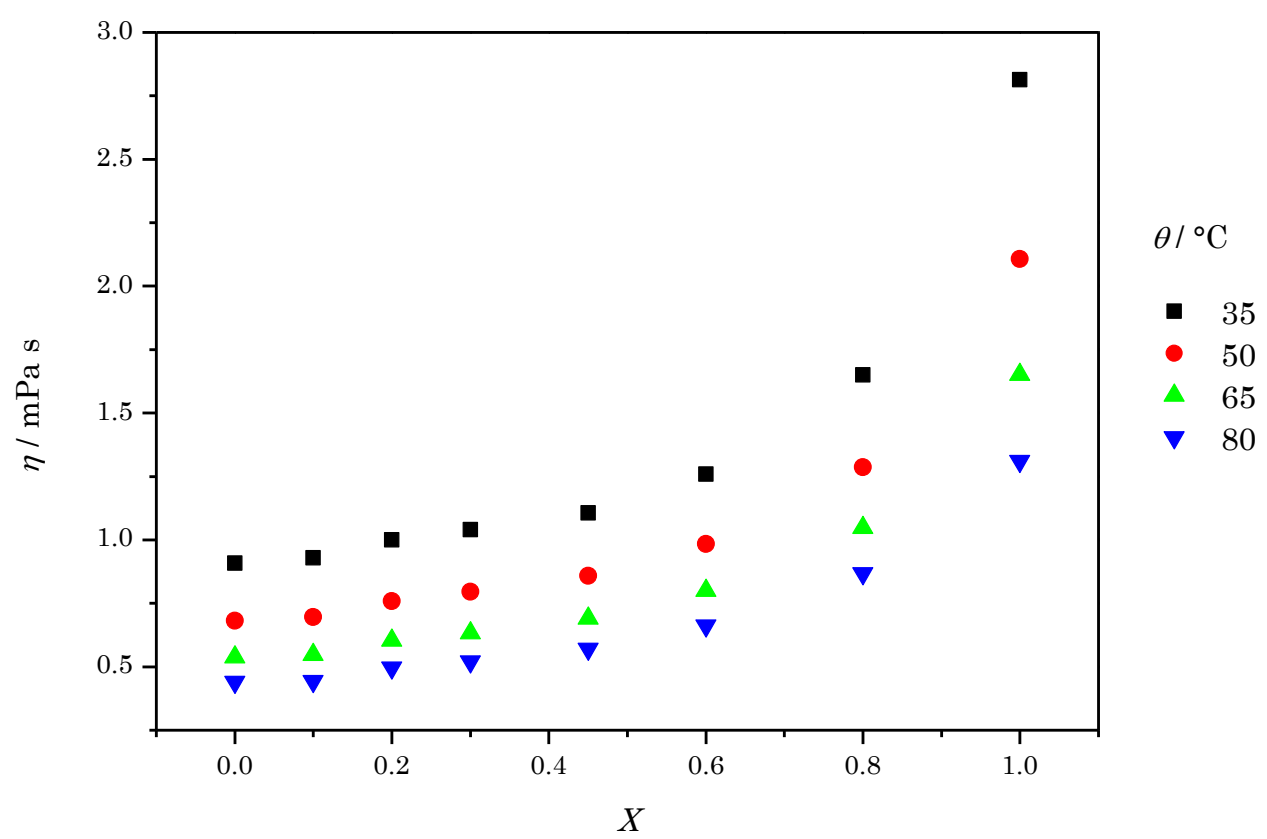

Figure 0-3 Viscosity of $0.1 \mathrm{~g} \mathrm{~g}^{-1} \mathrm{MAA} / \mathrm{pMAA}$ in water. Different degrees of monomer conversion were simulated by mixing monomer, polymer, and solvent. 


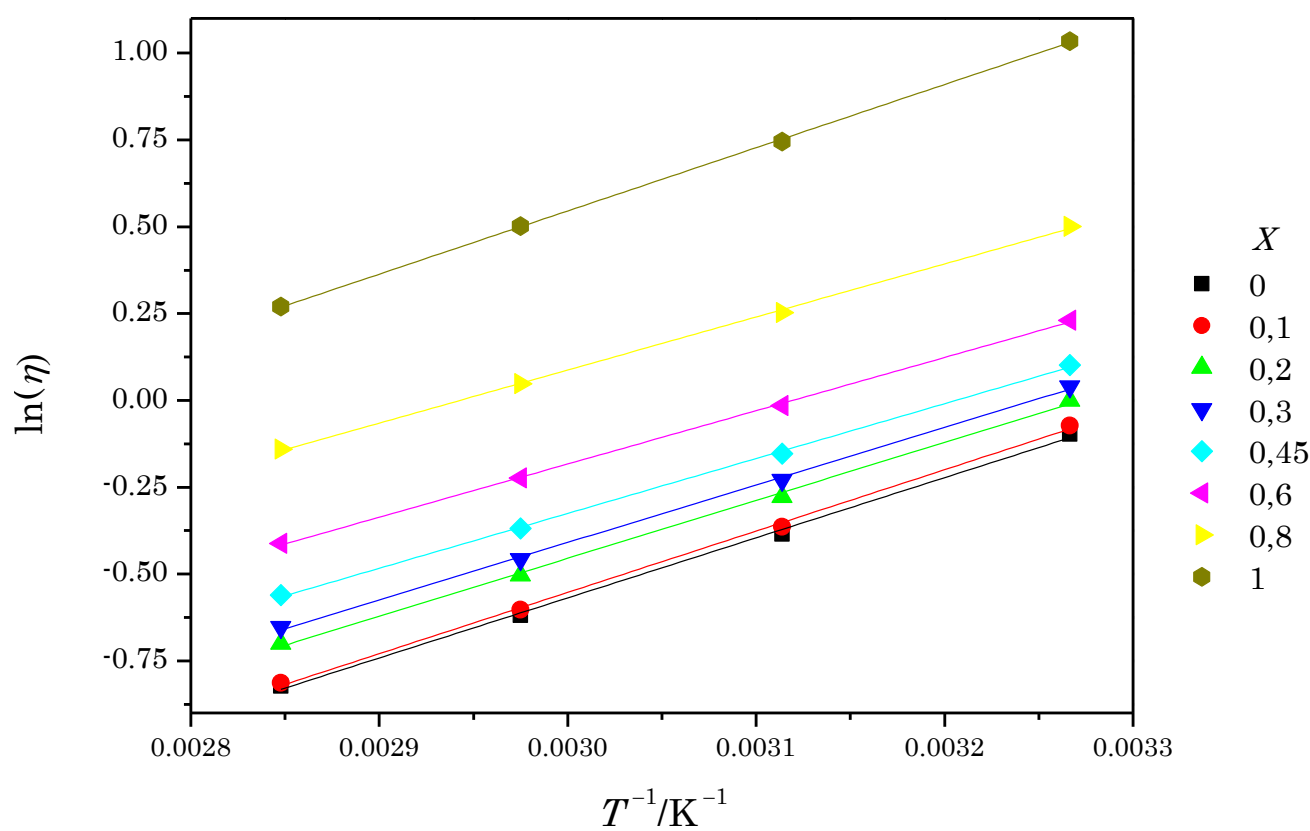

Figure 0-4 Arrhenius plot of the data presented in Figure 0-3.

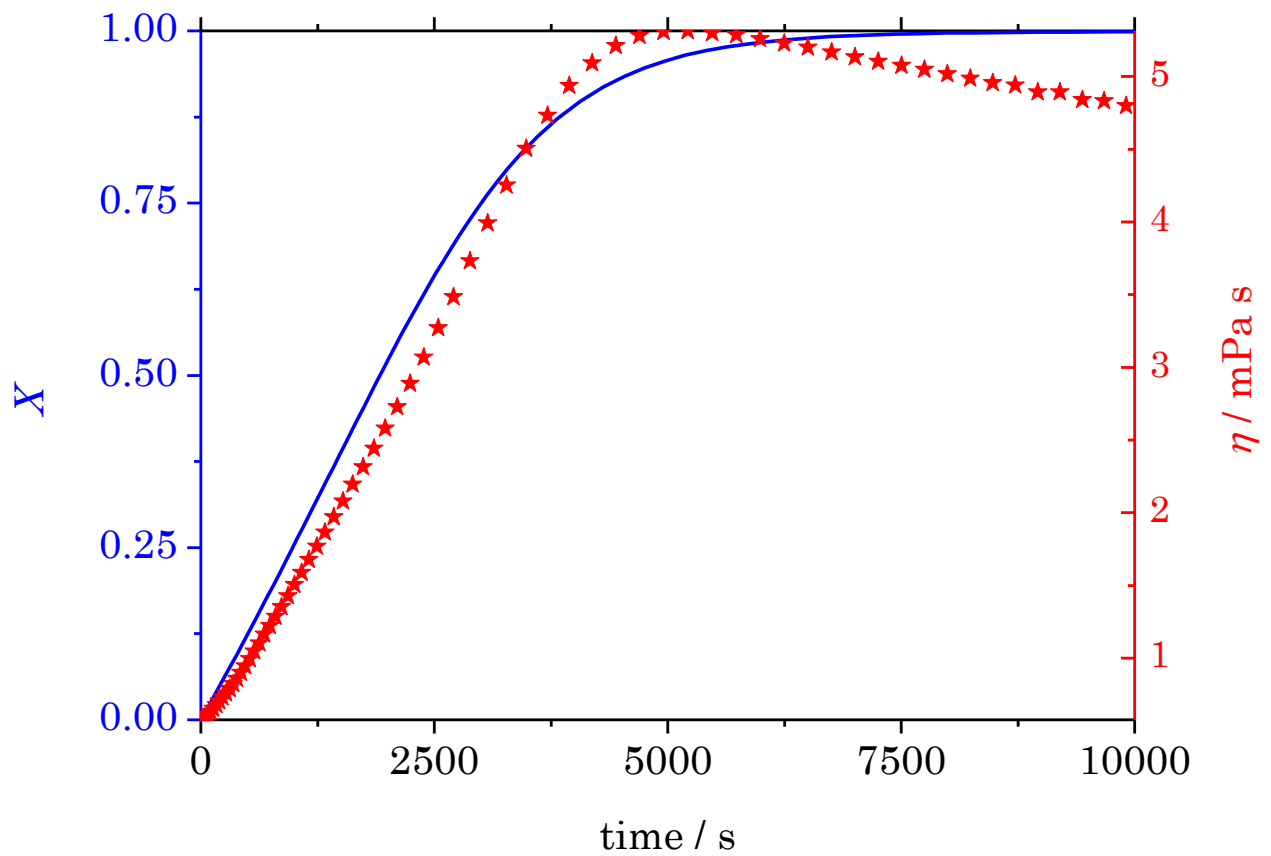

Figure 0-5 $\quad 0.045 \mathrm{~g} \mathrm{~g}^{-1} \mathrm{MAA}$ were polymerized in water with $0.005 \mathrm{~g} \mathrm{~g}^{-1} \mathrm{NaPS}$ as initiator at $50{ }^{\circ} \mathrm{C}$ inside a viscosity measuring capillary. Red stars give the constantly measured viscosity; for comparison, the blue line gives the corresponding conversion-time profile (simulation). 
Figure 0-5 compares the development of viscosity during a polymerization of $0.045 \mathrm{~g} \mathrm{~g}^{-1} \mathrm{MAA}$ in water with $0.005 \mathrm{~g} \mathrm{~g}^{-1} \mathrm{NaPS}$ as initiator at $50{ }^{\circ} \mathrm{C}$ with the development of degree of monomer conversion. The corresponding conversion-time profile was simulated. They increase almost in parallel. The maximum in viscosity occurs slightly before full conversion is reached; this might be inaccuracy of the model. Interestingly, viscosity decrease after the point of approximately full conversion. This may originate from the fact that polymer structure in solution changes with time. Directly after the polymer has been formed, the polymer-water matrix is not in its most stable form. This effect can also be found when preparing polymer solutions from dry polymer and solvent. Here, again viscosity is a function of time.

Branching level and fraction of short-chain branching

Table 0-1 Measured branching levels and fractions of short-chain branching.

\begin{tabular}{ccccccc}
\hline$\theta /{ }^{\circ} \mathrm{C}$ & $W_{1} / \%$ & $X$ & $\mathrm{DB} \_$exp $\%$ & BP_exp/\% & $x_{\text {SCB_exp }}$ & sample \\
\hline 130 & 0.2 & 0.885 & 0.5 & 4.2 & 0.39 & $120320-10$ \\
130 & 1 & 0.166 & 8.57 & & & $120326-02$ \\
130 & 1 & 0.420 & 5.08 & & & $120326-11$ \\
130 & 1 & 0.967 & 0.6 & 6.4 & 0.42 & $120326-15$ \\
140 & 0.2 & 0.436 & 0.5 & 2.8 & 0.52 & $120313-14$ \\
140 & 0.2 & 0.9 & 0.85 & 2.4 & 0.69 & $120313-16$ \\
150 & 0.2 & 0.134 & 2.75 & & & $120314-03$ \\
150 & 1 & 0.349 & 4.92 & 5.3 & 0.76 & $120327-02$ \\
150 & 1 & 0.894 & 1.37 & & & $120327-14$ \\
160 & 0.2 & 0.478 & 1.34 & 4.6 & 0.64 & $120315-11$ \\
160 & 1 & 0.935 & 1.63 & 5.8 & 0.45 & $120329-13$ \\
160 & 1 & 0.413 & 3.7 & 4.1 & 0.97 & $120329-01$ \\
160 & 1 & 0.424 & 5.26 & & & $120329-02$ \\
170 & 0.2 & $\max$ & 1.32 & 6.3 & 0.67 & 120427 \\
170 & 0.2 & 0.884 & 1.56 & 5.8 & 0.61 & $120319-15$ \\
170 & 0.2 & 0.410 & 0.55 & 6.1 & 0.52 & $120319-02$
\end{tabular}


Density and viscosity of AA solutions at different degrees of ionization and temperatures

Table 0-2 Density in $\mathrm{g} \mathrm{cm}^{-3}$ of $0.2 \mathrm{~g} \mathrm{~g}^{-1} \mathrm{AA}$ in water.

\begin{tabular}{rcccc}
\hline & & & & \\
& 0 & 0.3 & 0.7 & 1 \\
\hline 35 & 1.0174 & 1.0367 & 1.0551 & 1.1071 \\
50 & 1.0095 & 1.0306 & 1.0474 & 1.1004 \\
65 & 0.9970 & 1.0186 & 1.0342 & 1.0868 \\
\hline
\end{tabular}

Table 0-3 Viscosity in $\mathrm{mPa}$ s of $0.2 \mathrm{~g} \mathrm{~g}^{-1} \mathrm{AA}$ in water.

\begin{tabular}{rcccc}
\hline & & & & \\
& 0 & 0.3 & 0.7 & 1 \\
\hline 35 & 0.97077 & 1.15637 & 1.31790 & 2.01999 \\
50 & 0.71404 & 0.84385 & 0.95575 & 1.44071 \\
65 & 0.55292 & 0.64987 & 0.73260 & 1.15896 \\
80 & 0.44845 & 0.52296 & 0.58762 & 0.88798 \\
\hline
\end{tabular}

Modeling Acid-Base Equilibria

The counter species for non-ionized,_AA, and ionized,_AA ${ }^{-}$, monomer respectively, are set at time zero by the schedule function according to initial concentration.

For each propagation step a monomer-to-polymer counter species, p+_AA, is generated, which transforms one monomer counter into a polymer counter,_pAA, in a fast reaction: 


$$
\begin{aligned}
& \_\mathrm{AA}+\mathrm{p}+\_\mathrm{AA} \longrightarrow \_\mathrm{pAA} \\
& { }_{-} \mathrm{AA}^{-}+\mathrm{p}+\_\mathrm{AA} \longrightarrow \mathrm{pAA}^{-}
\end{aligned}
$$

The equilibria are calculated by PREDICI ${ }^{\mathrm{TM}}$ as three different equilibrium reactions, which are coupled as they all involve the $\mathrm{H}^{+}$species.

$$
\begin{aligned}
& \mathrm{OH}^{-}+\mathrm{H}^{+} \stackrel{K_{\mathrm{w}}}{\rightleftharpoons} \mathrm{H}_{2} \mathrm{O} K_{\mathrm{AA}} \\
& -\mathrm{AA}^{-}+\mathrm{H}^{+} \stackrel{K_{\mathrm{AA}}}{\rightleftharpoons} \_\mathrm{AA} \\
& \mathrm{pAA}^{-}+\mathrm{H}^{+} \stackrel{K_{\mathrm{AA}}}{\rightleftharpoons} \_\mathrm{pAA}
\end{aligned}
$$

At this, autoprotolysis of water is given by $K_{\mathrm{w}}$, the equilibrium constants of AA and pAA are denoted by $K_{\mathrm{AA}}$ and $K_{\mathrm{pAA}}$, respectively. The values for $K_{\mathrm{AA}}$ was taken from ref. ${ }^{[196]}$ and $K_{\mathrm{pAA}}$ is calculated by PREDICI ${ }^{\mathrm{TM}}$ (v.i.).

As the dynamic acid-base equilibria are calculated by PREDICI ${ }^{\mathrm{TM}}$, which is implemented into the model by means of counter species (v.s.). These monomer and polymer counters do not have mass, so $\mathrm{H}^{+}$has to be without mass as well, because otherwise mass would be created by deprotonation or vanish by protonation. Consequently, non-recipe components must have an "incorrect" mass. Mass and charge balance and all masses in recipes are correct. The actual masses and masses used in the model are summarized in Table 0-4. 
Table 0-4 In order to keep mass balance correct, molar mass of $\mathrm{H}^{+}$was set to zero (bold). Molar masses of non-recipe components were adjusted accordingly (in italics). All molar masses in the recipes are correct.

actual molar mass $/ \mathrm{g} \mathrm{mol}^{-1}$ molar mass in model $/ \mathrm{g} \mathrm{mol}^{-1}$

\begin{tabular}{lll}
\hline $\mathrm{H}_{2} \mathrm{O}$ & 18.02 & 18.02 \\
$\mathrm{H}^{+}$ & 1.01 & 0 \\
$\mathrm{OH}^{-}$ & 17.01 & 18.02 \\
$\mathrm{Na}^{+}$ & 23.00 & 21.99 \\
$\mathrm{Cl}^{-}$ & 35.45 & 36.46 \\
$\mathrm{AA}$ & 72.06 & 72.06 \\
$\mathrm{NaA}$ & 94.05 & 94.05 \\
$\mathrm{NaOH}$ & 40.01 & 40.01 \\
$\mathrm{NaCl}$ & 58.45 & 58.45 \\
\hline
\end{tabular}

Values complementing Table 5-8:

$$
\begin{aligned}
& \bar{V}_{\mathrm{AA}}^{0} / \mathrm{cm}^{3} \mathrm{~mol}^{-1}=68.6 \\
& \bar{V}_{\mathrm{AA}^{-}}^{0} / \mathrm{cm}^{3} \mathrm{~mol}^{-1}=50.59 \\
& \bar{V}_{\mathrm{pAA}}^{0} / \mathrm{cm}^{3} \mathrm{~mol}^{-1}=47.5 \\
& \bar{V}_{\mathrm{pAA}^{-}}^{0} / \mathrm{cm}^{3} \mathrm{~mol}^{-1}=31.5 \\
& \bar{V}_{\mathrm{Na}^{+}}^{0} / \mathrm{cm}^{3} \mathrm{~mol}^{-1}=-6.61 \\
& \bar{V}_{\mathrm{H}^{+}}^{0} / \mathrm{cm}^{3} \mathrm{~mol}^{-1}=-5.4 \\
& \bar{V}_{\mathrm{Cl}^{-}}^{0} / \mathrm{cm}^{3} \mathrm{~mol}^{-1}=-5.4 \\
& \bar{V}_{\mathrm{OH}^{-}}^{0} / \mathrm{cm}^{3} \mathrm{~mol}^{-1}=-5.4
\end{aligned}
$$




$$
\begin{aligned}
& \mathrm{p} K_{\mathrm{A},(\mathrm{pAA})}=\alpha^{2} \lg \left(K_{1}^{\mathrm{H}}\right)+2 \alpha(1-\alpha) \cdot \lg \left(K_{m}^{\mathrm{H}}\right)+(1-\alpha)^{2} \cdot \lg \left(K_{0}^{\mathrm{H}}\right) \\
& \lg \left(K_{1}^{H}\right)=a_{0}+a_{1} \cdot\langle i\rangle^{-1}+a_{2} \cdot \lg (\langle i\rangle) \\
& a_{0}=7.18137-7.18137 \cdot \sqrt{I}+0.92341 \cdot I \\
& a_{1}=-3.04382-4.38437 \cdot \sqrt{I}-1.35496 \cdot I \\
& a_{2}=-0.02289+0.28888 \cdot \sqrt{I}-0.14243 \cdot I \\
& \lg \left(K_{0}^{H}\right)=b_{0}+b_{1} \cdot\langle i\rangle^{-1}+b_{2} \cdot \lg (\langle i\rangle) \\
& b_{0}=4.60602-0.50455 \cdot \sqrt{I}+0.20921 \cdot I \\
& b_{1}=-1.15644+0.78001 \cdot \sqrt{I}-0.34245 \cdot I \\
& c_{2}=0.04168+0.0465 \cdot \sqrt{I}-0.34245 \cdot I \\
& \lg \left(K_{m}^{H}\right)=c_{0}+c_{1} \cdot\langle i\rangle^{-1}+c_{2} \cdot \lg (\langle i\rangle) \\
& c_{0}=5.62787-2.6009 \cdot \sqrt{I}+1.04678 \cdot I \\
& c_{1}=-1.78404+4.00311 \cdot \sqrt{I}-1.56342 \cdot I \\
& c_{2}=0.10223+0.26537 \cdot \sqrt{I}-0.14252 \cdot I
\end{aligned}
$$


$\underline{\text { Conversion vs. time profiles for AA at various degree of ionization, which have not }}$ been used for modeling
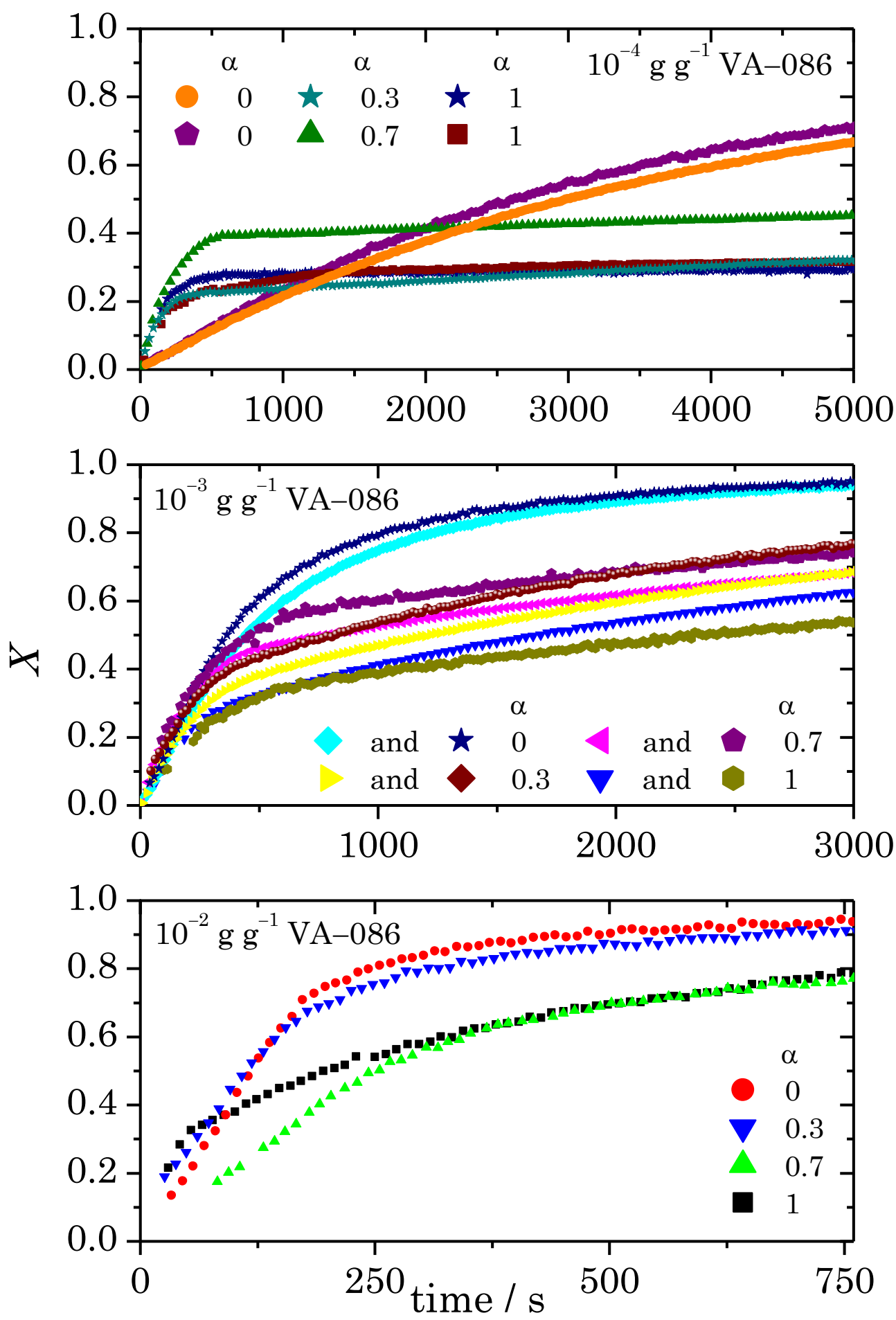

Figure 0-6 Measured conversion vs. time profiles from polymerization of $0.2 \mathrm{~g} \mathrm{~g}^{-1} \mathrm{NaA}(\mathrm{xM}=0.05)$ at $90^{\circ} \mathrm{C}$ with different levels of VA-086 as initiator (indicted in the graph) at different degrees of ionization of monomer (given in the graph as well). 

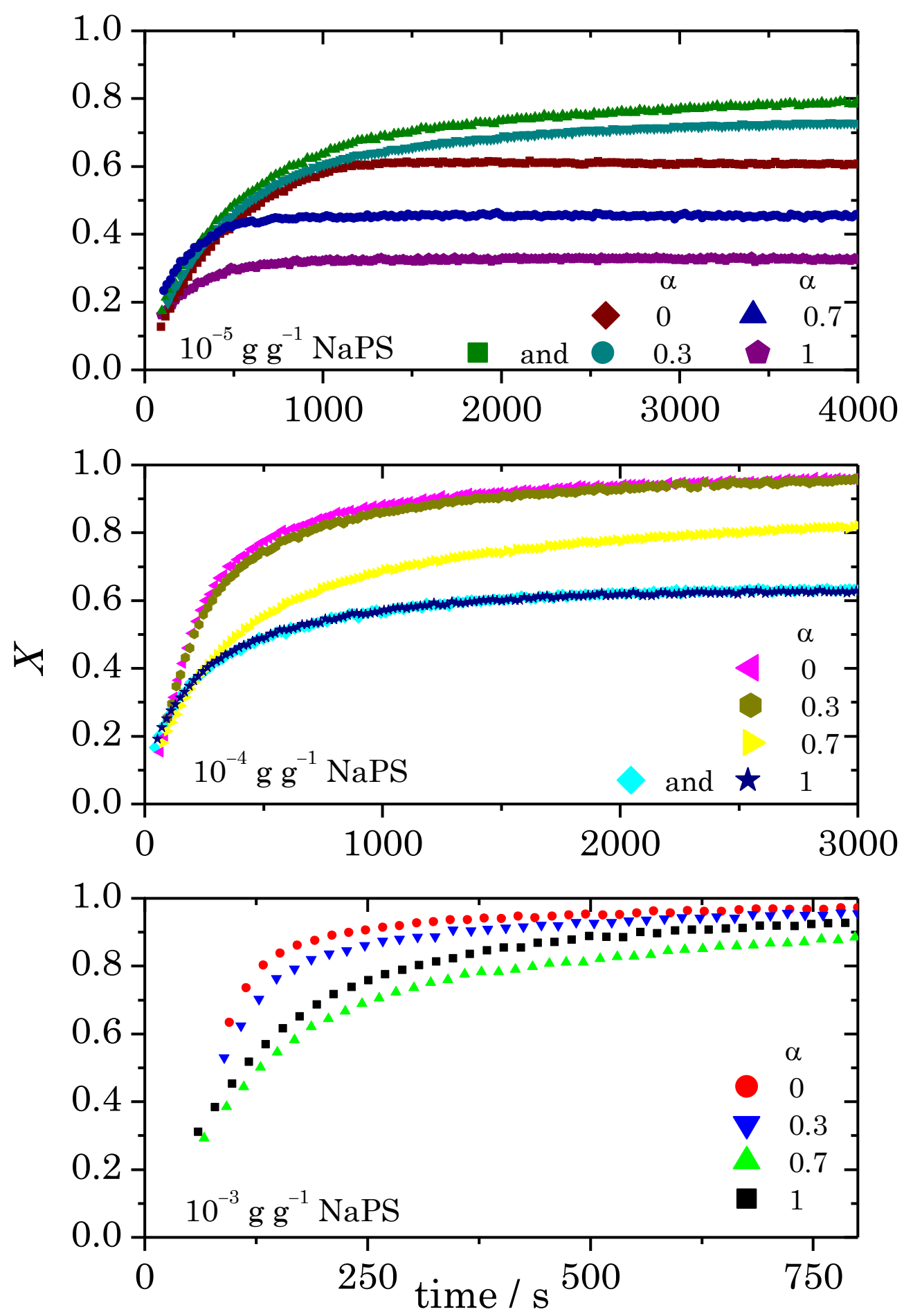

Figure 0-7 Measured conversion vs. time profiles from polymerization of $0.2 \mathrm{~g} \mathrm{~g}^{-1} \mathrm{NaA}\left(\mathrm{x}_{\mathrm{M}}=0.05\right)$ at $90^{\circ} \mathrm{C}$ with different levels of NaPS as initiator (indicted in the graph) at different degrees of ionization of monomer (given in the graph as well). 



\section{Abbreviations and Symbols}

$A \quad$ Arrhenius pre-exponential factor

$A^{\prime} \quad$ pressure-independent Arrhenius pre-exponential factor

$A_{\mathrm{D}-\mathrm{H}} \quad 1^{\text {st }}$ Debye-Hückel parameter (not empirical)

$\AA \quad 10^{-10} \mathrm{~m}$

AA acrylic acid

AAm acrylamide

apt attached proton test (NMR technique)

at acquisition time

$B_{\mathrm{D}-\mathrm{H}} \quad 2^{\text {nd }}$ Debye-Hückel parameter (empirical)

BA butyl acrylate

bb backbiting

BP branching point

bs broad singlet

bt broad triplet

$\mathrm{C}_{\mathrm{x}} \quad$ carbon, index p: primary, $\mathrm{s}:$ secondary, $\mathrm{t}$ : tertiary, q:quaternary

$C_{\mathrm{x}}^{\mathrm{y}} \quad$ chain transfer constant (x giving species to which transfer occurs, y giving of type of radical: $\mathrm{s}$ - secondary, $\mathrm{t}$ - tertiary)

$C_{\text {D-H }} \quad 3^{\text {rd }}$ Debye-Hückel parameter (empirical)

$c_{\mathrm{x}}^{\mathrm{y}} \quad$ concentration ( $\mathrm{x}$ giving species, $\mathrm{y}$ giving time)

ca. circa, Latin: around/about

CLD chain-length distribution 
CLDP chain-length-dependent propagation

CLDT chain-length-dependent termination

$D^{\mathrm{X}} \quad$ diffusion coefficient of species $\mathrm{X}$

$Ð \quad$ dispersity

D1173 2-hydroxy-2-methyl-1-phenylpropan-1-one

dept-135 distortionless enhancement by polarization transfer 135 degree (NMR technique)

DiAA diacrylic acid (2-(acryloyloxy)acetic acid)

DMSO dimethyl sulfoxide

DTBP di-tert-butyl peroxide

$E \quad$ energy (an index of A refers to activation, an index of $\mathrm{P}$ to laser pulse, an index of $\lambda$ to molar energy of photons at given laser wavelength)

e.g. $\quad$ exempli gratia, Latin: for example

EPR electron paramagnetic resonance

et al. et alii / et aliae, Latin: and others

eq. equation

$f \quad$ fraction of initiator fragments available for initiation

FID free induction decay

FT Fourier transform

H-A acid (general)

HPLC high-performance liquid chromatography

$h \quad$ empirical parameter of the extended Henderson-Hasselbalch equation

I ionic strength

i molecular species

$i \quad$ chain length or running index; chevron indicates: averaged, i.e. degree of polymerization, index of $\mathrm{n}$ indicates: nuber-averaged 
IBA iso-butyric acid

i.e. id est, Latin: that is

j $\quad$ chain length or running index

$K^{\mathrm{H}} \quad$ protonation constant, subscript indicates corresponding degree of ionization of polymer

$k_{\mathrm{B}} \quad$ Boltzmann constant

$k_{\mathrm{x}}^{\mathrm{y}} \quad$ rate coefficient ( $\mathrm{x}$ giving reaction: bb - backbiting, d - (initiator) dissociation, i - initiation, $\mathrm{p}$ - propagation, $\mathrm{t}-$ termination $<$ may be further specified after comma $\mathrm{D}$ : diffusion, $\mathrm{C}$ : chemical reaction>, $\operatorname{tr}, \mathrm{X}-$ transfer to species $\mathrm{X}$; $\mathrm{y}$ giving time or chain length of involved polymer species or of type of radical(s): $\mathrm{s}$ - secondary, $\mathrm{t}$ - tertiary), chevron indicate: chain-length averaged, superscript zero denotes: at time zero/zero conversion

$1 \quad$ length, index p: persistence (chain) length, 0: persistence (chain) length without electronic influence, e: electronic persistence (chain) length

lg decadic logarithm

LHS left-handed side

M monomer

$M_{\mathrm{n}} \quad$ number average molar mass

$M_{\text {w }} \quad$ weight average molar mass

$M_{\mathrm{x}} \quad$ molar mass of species x

MAA methacrylic acid

MALDI matrix-assisted laser desorption ionization

MCR midchain radical (s: formed by an 1,5-hydrogen shift; 1: not (directly) formed by an 1,5 -hydrogen shift )

ME 2-mercaptoethanol

MeHQ hydroquinone monomethyl ether

MM macromonomer 
MMA methyl methacrylate

MMD molar-mass distribution

MMMP 2-methyl-4-(methylthio)-2-morpholino-propiophenone

MS mass spectrometry

MU monomer unit

$n_{\mathrm{x}}^{\mathrm{y}} \quad$ quantity (amount) of species $\mathrm{x}$ at time $\mathrm{y}$

$\mathrm{NaA} \quad$ sodium acrylate $=$ fully ionized acrylic acid with sodium counter ion

NaMA sodium methacrylate $=$ fully ionized methacrylic acid with sodium counter ion

NaPS sodium persulfate

NIR near infrared

NOE nuclear Overhauser effect

$\mathrm{P}_{i} \quad$ dead polymer chain with a chain length of $i$

$P \quad$ radiant power (index 0 means: in front of cell)

p propagation

$p \quad$ pressure

pAA poly(acrylic acid)

pAAm poly(acrylamide)

pBA poly(butyl acrylate)

PLP pulsed laser polymerization

pMAA poly(methacrylic acid)

polym polymerization (used as index)

pVP polyvinylpyrrolidone

$Q_{\text {x }} \quad$ partition function of species X (index of ${ }^{\ddagger}$ indicates activated complex) 
$\mathrm{R}_{i}^{*} \quad$ growing polymer chain with a chain length of $i$ (dot indicates radical species)

$\mathrm{R} \quad$ rest of the molecule not depicted for reasons of space available (only used in drawings of molecules)

$R \quad$ universal gas constant

$r \quad$ rate

rd relaxation delay

RHS right-handed side

$\mathrm{RD} \quad$ reaction diffusion

SEC size exclusion chromatography

$\mathrm{S} / \mathrm{N} \quad$ signal-to-noise

SD segmental diffusion

SP single pulse

SPR secondary propagating radical

$T \quad$ absolute temperature (in kelvin)

$t \quad$ time

TEMPO 2,2,6,6-tetramethylpiperidine 1-oxyl

TD translational diffusion

ToF time-of-flight

$\bar{V} \quad$ molar volume, index 0 indicates extrapolated to infinite dilution

V-50 2-[(E)-2-(1-carbamimidoyl-1-methylethyl)diazen-1-yl]-2-methylpropanimidamide dihydrochloride

VA-086 2,2'-Azobis[2-methyl- $N$-(2-hydroxyethyl)propionamide]

v.i. vide infra, Latin: see below

viz. videre licit, Latin: it is permitted to see (namely)

v.s. vide supra, Latin: see above 
$W_{\mathrm{x}} \quad$ weight fraction of $\mathrm{x}$

X arbitrary species (with dot: arbitrary radical species)

$X_{\mathrm{Y}} \quad$ degree of conversion, index gives species; without index it refers to monomer

$x_{\mathrm{x}} \quad$ mole fraction of $\mathrm{x}$

Y arbitrary species (with dot arbitrary radical species)

Z $\quad$ charge number

$\alpha \quad$ degree of ionization

$\alpha_{1} \quad$ exponent of chain-length dependency in region of long chains

$\alpha_{\mathrm{s}} \quad$ exponent of chain-length dependency in region of short chains

$\Delta^{\ddagger} V \quad$ activation volume

$\delta \quad$ kinetic chain length

$\delta \quad$ chemical shift (only if referring to NMR)

$\varepsilon \quad$ molar decadic absorption coefficient

$\zeta \quad$ correction factor for $k_{\mathrm{t}}$; superscript: $\mathrm{s}$ stands for secondary radical, $\mathrm{t}$ stands for tertiary radical; subscript: $d$ stands for distribution (correcting a value for one chain length to use it for a distribution, which average chain length is the former one), $\eta w$ stands for correction of viscosity change with different monomer content, $\eta \alpha$ stands for correction of viscosity change with ionization, $\mathrm{C} \alpha$ stands for correction of Coulomb repulsion of ionized macroradicals

$\eta \quad$ dynamic viscosity, subscript $\mathrm{r}$ : relative, superscript $X$ : at conversion $X$

$\theta \quad$ temperature in ${ }^{\circ} \mathrm{C}$

$\kappa \quad$ fraction of disproportionation, index gives type of radical terminating: $\mathrm{s}:$ secondary, t:tertiary

$\lambda \quad$ slope of first CLD plot

$\tilde{v} \quad$ wavenumber 
$v \quad$ kinematic viscosity

$\xi_{I} \quad$ correction factor for influence of additional salt on $k_{\mathrm{p}}$

$\rho \quad$ density

$\sigma \quad$ transmission coefficient

$\tau \quad$ residence time, index: calc: calculated from mass flow, density of the reaction mixture and geometric volume of the reactor.

$\Phi \quad$ radiant power (intensity) at a certain wavenumber 



\section{References}

[1] Staudinger, H.; Fritschi, J. Helv. Chim. Acta. 1922, 5, 785-806.

[2] Staudinger, H. Ber. dtsch. Chem. Ges. A/B 1920, 53, 1073-1085.

[3] Baekeland, L. H., US patent No. 942699, 1909.

[4] Verband der Chemischen Industrie e.V., Chemiewirtschaft in Zahlen 2013, 2013.

[5] Olaj, O. F.; Bitai, I.; Hinkelmann, F. Makromol. Chem. 1987, 188, 1689-1702.

[6] Buback, M.; Hippler, H.; Schweer, J.; Voegele, H. P. Makromol. Chem. Rapid Commun. 1986, 7, 261-265.

[7] Buback, M.; Egorov, M.; Junkers, T.; Panchenko, E. Macromol. Rapid Commun. 2004, 25, 1004-1009.

[8] Beuermann, S.; Buback, M.; Hesse, P.; Kuchta, F.-D.; Lacík, I.; van Herk, A. M. Pure Appl. Chem. 2007, 79, 1463-1469.

[9] Beuermann, S.; Buback, M.; Hesse, P.; Kukučková, S.; Lacík, I. Macromol. Symp. 2007, 248, 41-49.

[10] Beuermann, S. Macromol. Rapid Commun. 2009, 30, 1066-1088.

[11] Lacík, I.; Učňovaá, L.; Kukučková, S.; Buback, M.; Hesse, P.; Beuermann, S. Macromolecules 2009, 42, 7753-7761.

[12] Kabanov, V. A.; Topchiev, D. A.; Karaputadze, T. M. J. Polym. Sci. Pol. Sym. 1973, 42, 173-183.

[13] Giese, B. Angew. Chem. 1983, 95, 771-782. 
[14] IUPAC, http://goldbook.iupac.org/, accessed: February 2013.

[15] Russell, K.; Tobolsky, A. J. Am. Chem. Soc. 1953, 75, 5052-5054.

[16] Pryor, W. A.; Lasswell, L. D. Adv. free-radical chem. 1975, 5, 27.

[17] Kharasch, F. R., M. S.; Mayo Chem. Ind. 1938, 57, 752.

[18] Bouguer, P. Essai d'Optique, sur la gradation de la lumiere; Claude Jombert: Paris, 1729; pp 63-67.

[19] Lambert, J. H. Photometria sive de Mensura et Gradibus Luminis, Colorum et Umbrae; Eberhard Klett: Augsburg, 1760; p 391.

[20] Beer, A. Ann. Physik Chemie 1852, 86, 78-88.

[21] Buback, M.; $\quad$ Gilbert, R. G.; $\quad$ Russell, G. T.; $\quad$ Hill, D. $\quad$ J. T.; $\quad$ Moad, G.; O'Driscoll, K. F.; Shen, J.; Winnik, M. A. J. Polym. Sci. A Polym. Chem. 1992, 30, 851-863.

[22] Hutchinson, R. A.; Paquet, D. A.; McMinn, J. H. Macromolecules 1995, 28, $5655-5663$.

[23] Heuts, J. P. A.; Davis, T. P.; Russell, G. T. Macromolecules 1999, 32, 60196030

[24] Loubat, C.; Boutevin, B. Polym. Int. 2001, 50, 375-380.

[25] Bordwell, F. G.; Zhang, X.-M.; Satish, A. V.; Cheng, J.-P. J. Am. Chem. Soc. 1994, 116, 6605-6610.

[26] Mayo, F. R. J. Am. Chem. Soc. 1943, 65, 2324-2329.

[27] Whang, B. Y. C.; Ballard, M. J.; Napper, D. H.; Gilbert, R. G. Aust. J. Chem. 1991, 44, 1133.

[28] O’Brien, J. L.; Gornick, F. J. Am. Chem. Soc. 1955, 77, 4757-4763.

[29] Moad, G.; Moad, C. L. Macromolecules 1996, 29, 7727-7733.

[30] Suddaby, K. G.; Maloney, D. R.; Haddleton, D. M. Macromolecules 1997, 30, 702-713.

[31] Buback, M.; Laemmel, R. A. Macromol. Theory Simul. 1997, 6, 145-150.

[32] Roedel, M. J. J. Am. Chem. Soc. 1953, 75, 6110-6112. 
[33] Toh, J. S. S.; Huang, D. M.; Lovell, P. A.; Gilbert, R. G. Polymer 2001, 42, $1915-1920$.

[34] Cuccato, D.; Mavroudakis, E.; Dossi, M.; Moscatelli, D. Macromol. Theory Simul. 2013, 22, 127-135.

[35] Staudinger, H.; Schulz, G. V. Ber. dtsch. Chem. Ges. A/B 1935, 68, 23202335 .

[36] Barth, J.; Meiser, W.; Buback, M. Macromolecules 2012, 45, 1339-1345.

[37] Lacík, I.; Beuermann, S.; Buback, M. Macromolecules 2003, 36, 9355-9363.

[38] Nikitin, A. N.; Hutchinson, R. A. Macromolecules 2005, 38, 1581-1590.

[39] Cuccato, D.; Dossi, M.; Moscatelli, D.; Storti, G. Macromol. React. Eng. 2012, $6,330-345$.

[40] Liang, K.; Hutchinson, R. A. Macromol. Rapid Commun. 2011, 32, 1090-1095.

[41] Liang, K.; Hutchinson, R. A.; Barth, J.; Samrock, S.; Buback, M. Macromolecules 2011, 44, 5843-5845.

[42] Nikitin, A. N.; Hutchinson, R. A.; Wang, W.; Kalfas, G. A.; Richards, J. R.; Bruni, C. Macromol. React. Eng. 2010, 4, 691-706.

[43] Wang, W.; Nikitin, A. N.; Hutchinson, R. A. Macromol. Rapid Commun. 2009, 30, 2022-2027.

[44] Engler, C.; Weißberg, J. Kritische Studien über die Vorgänge der Autoxydation; 1904; p 179.

[45] Schulz, V. G. V.; Henrici, G. Makromol. Chem. 1956, 18, 437-454.

[46] Gladyshev, G. Proc. Acad. Sci. USSR 1974, 215, 898-901.

[47] Kern, V. W. Makromol. Chem. 1948, 1, 199-208.

[48] Russell, G. T.; Napper, D. H.; Gilbert, R. G. Macromolecules 1988, 21, 21332140 .

[49] Nikitin, A. N.; Grady, M. C.; Kalfas, G. A.; Hutchinson, R. A. Macromol. React. Eng. 2008, 2, 422-435.

[50] von Smoluchowski, M. Z. Phys. Chem 1917 92, 129-168. 
[51] Einstein, A. Ann. Phys. 1905, 17, 549-560.

[52] Van't Hoff, J. H. Études de dynamique chimique; Frederik Muller \& Co: Amsterdam, 1884; pp 114-118.

[53] Arrhenius, S. Z. Phys. Chem. 1889, 4, 226-248.

[54] Ewell, R. H.; Eyring, H. J. Chem. Phys. 1937, 5, 726-737.

[55] Powell, R. E.; Roseveare, W. E.; Eyring, H. Ind. Eng. Chem. 1941, 33, 430435.

[56] Henton, D. E.; Powell, C.; Reim, R. E. J. Appl. Polym. Sci. 1997, 64, 591-600.

[57] Kuchta, F.-D.; van Herk, A. M.; German, A. L. Macromolecules 2000, 33, 3641-3649.

[58] Beuermann, S.; Paquet, D. A.; McMinn, J. H.; Hutchinson, R. A. Macromolecules 1997, 30, 194-197.

[59] Beuermann, S.; Buback, M.; Hesse, P.; Lacík, I. Macromolecules 2006, 39, 184-193.

[60] Eyring, H. J. Chem. Phys. 1935, 3, 107-115.

[61] Eyring, H.; Polanyi, M. Z. Phys. Chem. B 1931, 12, 279-311.

[62] Heuts, J. P. A.; Gilbert, R. G.; Radom, L. Macromolecules 1995, 28, 87718781.

[63] Thickett, S. C.; Gilbert, R. G. Polymer 2004, 45, 6993-6999.

[64] Degirmenci, I.; Ozaltin, T. F.; Karahan, O.; Van Speybroeck, V.; Waroquier, M.; Aviyente, V. J. Polym. Sci. A Polym. Chem. 2013, 51, 20242034.

[65] De Sterck, B.; $\quad$ Vaneerdeweg, R.; $\quad$ Du Prez, F.; $\quad$ Waroquier, M.; Van Speybroeck, V. Macromolecules 2009, 43, 827-836.

[66] Stach, M.; Lacík, I.; Chorvákt, D.; Buback, M.; Hesse, P.; Hutchinson, R. A.; Tang, L. Macromolecules 2008, 41, 5174-5185.

[67] Stach, M.; $\quad$ Lacík, I.; $\quad$ Kasák, P.; $\quad$ Chorv/'akt, D.; $\quad$ Saunders, A. J.; Santanakrishnan, S.; Hutchinson, R. A. Macromol. Chem. Phys. 2010, 211, 580-593. 
[68] Ito, H.; Shimizu, A. J. Chem. Soc. Japan, Ind. Chem. Sect 1955, 58, 194.

[69] Kabanov, V.; Topchiev, D.; Karaputadze, T.; Mkrtchian, L. Eur. Polym. J. 1975, 11, 153-159.

[70] Manickam, S.; Venkatarao, K.; Subbaratnam, N. Eur. Polym. J. 1979, 15, 483-487.

[71] Plochocka, K. J. Macromol. Sci., Rev. Macromol. Chem. Phys. 1981, 20, 67148 .

[72] Pinner, S. H. J. Polym. Sci. 1952, 9, 282-285.

[73] Blauer, G. J. Polym. Sci. 1953, 11, 189-192.

[74] Scott, R. A.; Peppas, N. A. AIChE Journal 1997, 43, 135-144.

[75] Rintoul, I.; Wandrey, C. Polymer 2005, 46, 4525-4532.

[76] Lacík, I.; Beuermann, S.; Buback, M. Macromol. Chem. Phys. 2004, 205, 1080-1087.

[77] Barth, J.; Buback, M. Macromolecules 2012, 45, 4152-4157.

[78] Anseth, K. S.; Scott, R. A.; Peppas, N. A. Macromolecules 1996, 29, 83088312 .

[79] Shoaf, G. L.; Poehlein, G. W. J. Appl. Polym. Sci. 1991, 42, 1239-1257.

[80] Cutié, S. S.; Smith, P. B.; Henton, D. E.; Staples, T. L.; Powell, C. J. Polym. Sci. B Polym. Phys. 1997, 35, 2029-2047.

[81] De Stefano, C.; Gianguzza, A.; Piazzese, D.; Sammartano, S. React. Funct. Polym. 2003, 55, 9-20.

[82] Laguecir, A.; Ulrich, S.; Labille, J.; Fatin-Rouge, N.; Stoll, S.; Buffle, J. Eur. Polym. J. 2006, 42, 1135-1144.

[83] Katchalsky, A.; Blauer, G. Trans. Faraday Soc. 1951, 47, 1360-1370.

[84] Tricot, M. Macromolecules 1984, 17, 1698-1704.

[85] Mylonas, Y.; Staikos, G.; Ullner, M. Polymer 1999, 40, 6841-6847.

[86] Adamczyk, Z.; Bratek, A.; Jachimska, B.; Jasinski, T.; Warszynski, P. J. Phys. Chem. B 2006, 110, 22426-22435. 
[87] Dobrynin, A. V.; Rubinstein, M. Prog. Polym. Sci. 2005, 30, 1049-1118.

[88] Breslau, B. R.; Miller, I. F. J. Phys. Chem. 1970, 74, 1056-1061.

[89] Olaj, O.; Schnöll-Bitai, I. Eur. Polym. J. 1989, 25, 635-641.

[90] Buback, M.; Hesse, P.; Hutchinson, R. A.; Kasak, P.; Lacík, I.; Stach, M.; Utz, I. Ind. Eng. Chem. Res. 2008, 47, 8197-8204.

[91] Lacík, I.; Beuermann, S.; Buback, M. Macromolecules 2001, 34, 6224-6228.

[92] Beuermann, S.; Buback, M.; Hesse, P.; Kukučková, S.; Lacík, I. Macromol. Symp. 2007, 248, 23-32.

[93] Piton, M. C.; Gilbert, R. G.; Chapman, B. E.; Kuchel, P. W. Macromolecules 1993, 26, 4472-4477.

[94] De Gennes, P.; Leger, L. Annu. Rev. Phys. Chem. 1982, 33, 49-61.

[95] Tsvetkov, V.; Kolomiets, I.; Lezov, A. Soedin., Ser. A 1968, 10, 74.

[96] Smith, G. B.; Heuts, J. P.; Russell, G. T. Macromol. Symp. 2005, 226, 133146.

[97] Smith, G. B.; Russell, G. T.; Heuts, J. P. Macromol. Theory Simul. 2003, 12, 299-314.

[98] Buback, M.; Egorov, M.; Junkers, T.; Panchenko, E. Macromol. Chem. Phys. 2005, 206, 333-341.

[99] Buback, M.; Müller, E.; Russell, G. T. J. Phys. Chem. A 2006, 110, 3222-3230.

[100] Barth, J.; Buback, M.; Hesse, P.; Sergeeva, T. Macromolecules 2009, 42, 481488.

[101] Barth, J.; Buback, M.; Hesse, P.; Sergeeva, T. Macromol. Rapid Commun. 2009, 30, 1969-1974.

[102] Barth, J.; Buback, M.; Schmidt-Naake, G.; Woecht, I. Polymer 2009, 50, 57085712.

[103] Barth, J.; Buback, M. Macromol. Rapid Commun. 2009, 30, 1805-1811.

[104] Khokhlov, A. R. Makromol. Chem. Rapid Commun. 1981, 2, 633-636.

[105] Olaj, O. F.; Zifferer, G. Makromol. Chem. Rapid Commun. 1982, 3, 549-556. 
[106] Friedman, B.; O’Shaughnessy, B. Macromolecules 1993, 26, 5726-5739.

[107] Barner-Kowollik, C.; Russell, G. T. Prog. Polym. Sci. 2009, 34, 1211-1259.

[108] Buback, M.; Junkers, T. Macromol. Chem. Phys. 2006, 207, 1640-1650.

[109] Taylor, D. R.; van Berkel, K. Y.; Alghamdi, M. M.; Russell, G. T. Macromol. Chem. Phys. 2010, 211, 563-579.

[110] Schuh, H.; Fischer, H. Helv. Chim. Acta. 1978, 61, 2130-2164.

[111] Buback, M.; Busch, M.; Kowollik, C. Macromol. Theory Simul. 2000, 9, 442452.

[112] Theis, A.; Davis, T. P.; Stenzel, M. H.; Barner-Kowollik, C. Macromolecules 2005, 38, 10323-10327.

[113] Johnston-Hall, G.; $\quad$ Stenzel, M. H.; $\quad$ Davis, T. P.; $\quad$ Barner-Kowollik, C.; Monteiro, M. J. Macromolecules 2007, 40, 2730-2736.

[114] Johnston-Hall, G.; Monteiro, M. J. J. Polym. Sci. A Polym. Chem. 2008, 46, 3155-3173.

[115] Johnston-Hall, G.; Monteiro, M. J. Macromolecules 2008, 41, 727-736.

[116] Olaj, O. F.; Vana, P.; Zoder, M.; Kornherr, A.; Zifferer, G. Macromol. Rapid Commun. 2000, 21, 913-920.

[117] Willemse, R. X. E.; Staal, B. $\quad$ B. P.; van Herk, A. M.; Pierik, S. C. J.; Klumperman, B. Macromolecules 2003, 36, 9797-9803.

[118] Smith, G. B.; Russell, G. T.; Yin, M.; Heuts, J. P. Eur. Polym. J. 2005, 41, $225-230$.

[119] Johnston-Hall, G.; Theis, A.; Monteiro, M. J.; Davis, T. P.; Stenzel, M. H.; Barner-Kowollik, C. Macromol. Chem. Phys. 2005, 206, 2047-2053.

[120] Barth, J.; Buback, M. Macromolecules 2011, 44, 1292-1297.

[121] Beuermann, S.; Buback, M.; Hesse, P.; Hutchinson, R. A.; Kukučková, S.; Lacík, I. Macromolecules 2008, 41, 3513-3520.

[122] Benson, S. W.; North, A. M. J. Am. Chem. Soc. 1959, 81, 1339-1345.

[123] Benson, S. W.; North, A. M. J. Am. Chem. Soc. 1962, 84, 935-940. 
[124] R. G. W. Norrish, R. R. S. Nature 1942, 150, 336-337.

[125] Trommsdorff, V. E.; Köhle, H.; Lagally, P. Makromol. Chem. 1948, 1, 169198.

[126] Soh, S.; Sundberg, D. J. Polym. Sci. Polym. Chem. Ed. 1982, 20, 1315-1329.

[127] Buback, M.; Schweer, J. Z. Phys. Chem. 1989, 161, 153-165.

[128] Allen, P.; Patrick, C. Makromol. Chem. 1964, 72, 106-118.

[129] Buback, M.; Degener, B. Makromol. Chem. 1993, 194, 2875-2883.

[130] Buback, M.; Huckestein, B.; Russell, G. T. Macromol. Chem. Phys. 1994, 195, $539-554$.

[131] Ballard, M. J.; Napper, D. H.; Gilbert, R. G. J. Polym. Sci. Polym. Chem. Ed. 1984, 22, 3225-3253.

[132] Carswell, T.; Hill, D.; Londero, D.; O’Donnell, J.; Pomery, P.; Winzor, C. Polymer 1992, 33, 137-140.

[133] Zetterlund, P. B.; Yamazoe, H.; Yamada, B.; $\quad$ Hill, D. J.; $\quad$ Pomery, P. J. Macromolecules 2001, 34, 7686-7691.

[134] Zetterlund, P. B.; Yamazoe, H.; Yamada, B. Macromol. Theory Simul. 2003, $12,379-385$.

[135] Zetterlund, P. B.; Yamauchi, S.; Yamada, B. Macromol. Chem. Phys. 2004, $205,778-785$.

[136] Buback, M. Makromol. Chem. 1990, 191, 1575-1587.

[137] Wulkow, M. Macromol. Theory Simul. 1996, 5, 393-416.

[138] Wampler III, F. M. Plant/Operations Progress 1988, 7, 183-189.

[139] Acrylic Acid Dimerization; Technical Report, 1997, Technical Data Sheet.

[140] Buback, M. Angew. Chem. 1991, 103, 658-670.

[141] Beuermann, S.; Buback, M.; Hesse, P.; Junkers, T.; Lacík, I. Macromolecules 2006, 39, 509-516.

[142] NIST, Webbook, 2008. webbook.nist.gov/chemistry/.

[143] Barth, J. Ph.D. thesis, Georg-August-Universität Göttingen, 2011. 
[144] Hesse, P. Ph.D. thesis, Universität Göttingen, 2008.

[145] Schrooten, J. Ph.D. thesis, Georg-August-Universität Göttingen, 2013.

[146] Handbook of Chemistry and Physics - 84th Edition ; Lide, D. R., Ed.; CRC press, 2003; pp 8/-43.

[147] Wittenberg, N. F. G. Diplomarbeit, Georg-August-Universität Göttingen, 2009 .

[148] Weiß, D. M.Sc. thesis, Georg-August-Universität Göttingen, 2012.

[149] van Herk, A. M. J. Chem. Educ. 1995, 72, 138-.

[150] Van Herk, A. M.; Dröge, T. Macromol. Theory Simul. 1997, 6, 1263-1276.

[151] Beuermann, S.; Buback, M.; Davis, T. P.; Gilbert, R. G.; Hutchinson, R. A.; Olaj, O. F.; Russell, G. T.; Schweer, J.; van Herk, A. M. Macromol. Chem. Phys. 1997, 198, 1545-1560.

[152] Henríquez, C.; Bueno, C.; Lissi, E. A.; Encinas, M. V. Polymer 2003, 44, 5559 -5561 .

[153] Nikitin, A. N.; Castignolles, P.; Charleux, B.; Vairon, J.-P. Macromol. Rapid Commun. 2003, 24, 778-782.

[154] Hutchinson, R. A.; Aronson, M. T.; Richards, J. R. Macromolecules 1993, 26, 6410-6415.

[155] Husain, M. M.; Misra, S. N.; Miss, A. G. Makromol. Chem. 1976, 177, 29192926.

[156] Information provided by Wako Chemicals GmbH on request.

[157] Kukučková, S. Ph.D. thesis, Bratislava/Göttingen, 2006.

[158] Olaj, O. F.; Zifferer, G.; Gleixner, G. Makromol. Chem. Rapid Commun. 1985, $6,773-784$.

[159] Mahabadi, H. K. Macromolecules 1985, 18, 1319-1324.

[160] Olaj, O. F.; Zifferer, G.; Gleixner, G. Macromolecules 1987, 20, 839-850.

[161] Mahabadi, H. K. Macromolecules 1991, 24, 606-609. 
[162] Wittenberg, N. F. G.; Buback, M.; Stach, M.; Lacík, I. Macromol. Chem. Phys. 2012, 213, 2653-2658.

[163] Sarraguça, J.; Pais, A. Chem. Phys. Lett. 2004, 398, 140-145.

[164] Masiak, M.; Hyk, W.; Stojek, Z.; Ciszkowska, M. J. Phys. Chem. B 2007, 111, 11194-11200.

[165] Sakurai, M.; Imai, T.; Yamashita, F.; Nakamura, K.; Komatsu, T. and Nakagawa, l. T. Polym. J. 1993, 25, 1247-1255.

[166] Taylor, T. J.; Stivala, S. S. J. Polym. Sci. B Polym. Phys. 2003, 41, 1263-1272.

[167] Gromov, V.; Galperina, N.; Osmanov, T.; Khomikovskii, P.; Abkin, A. Eur. Polym. J. 1980, 16, 529-535.

[168] Paril, A.; Alb, A.; Giz, A.; Çatalgil Giz, H. J. Appl. Polym. Sci. 2007, 103, 968974.

[169] Deglmann, P.; Müller, I.; Becker, F.; Schäfer, A.; Hungenberg, K.-D.; Weiß, H. Macromol. React. Eng. 2009, 3, 496-515.

[170] Torii, H.; Fujimoto, K.; Kawaguchi, H. J. Polym. Sci. A Polym. Chem. 1996, $34,1237-1243$.

[171] BASF Corporation, Acrylic Acid Glacial (Technical Data Sheet); Technical Report, 2001.

[172] The Dow Chemical Company, Acrylic Acid, Glacial (Technical Data Sheet); Technical Report.

[173] Günzler, K. F. Ph.D. thesis, Georg-August-Universität Göttingen, 2007.

[174] Nikitin, A. N.; Hutchinson, R. A.; Kalfas, G. A.; Richards, J. R.; Bruni, C. Macromol. Theory Simul. 2009, 18, 247-258.

[175] Loiseau, J.; Doërr, N.; Suau, J. M.; Egraz, J. B.; Llauro, M. F.; Ladavière, C.; Claverie, J. Macromolecules 2003, 36, 3066-3077.

[176] Junkers, T.; Koo, S. P. S.; Davis, T. P.; Stenzel, M. H.; Barner-Kowollik, C. Macromolecules 2007, 40, 8906-8912.

[177] Castignolles, P.; Graf, R.; Parkinson, M.; Wilhelm, M.; Gaborieau, M. Polymer 2009, 50, 2373-2383. 
[178] Barth, J.; Buback, M.; Hesse, P.; Sergeeva, T. Macromolecules 2010, 43, 4023-4031.

[179] Ahmad, N. M.; Heatley, F.; Lovell, P. A. Macromolecules 1998, 31, 2822-2827.

[180] de la Fuente, J. L.; López Madruga, E. Macromol. Chem. Phys. 2001, 202, 375-381.

[181] Plessis, C.; Arzamendi, G.; Alberdi, J. M.; van Herk, A. M.; Leiza, J. R.; Asua, J. M. Macromol. Rapid Commun. 2003, 24, 173-177.

[182] Agirre, A.; Santos, J. I.; Etxeberria, A.; Sauerland, V.; Leiza, J. R. Polym. Chem. 2013, 4, 2062-2079.

[183] Handbook of Chemistry and Physics - 84th Edition -; Lide, D. R., Ed.; CRC press, 2003; pp 9/-51.

[184] Beuermann, S.; Buback, M. Prog. Polym. Sci. 2002, 27, 191-254.

[185] Nikitin, A. N.; Hutchinson, R. A. Macromol. Theory Simul. 2006, 15, 128-136.

[186] Gao, Z.; Amasaki, I.; Kaneko, T.; Nakada, M. Polym. Degrad. Stab. 2003, 81, $125-130$.

[187] Noda, I.; Tsuge, T.; Nagasawa, M. J. Phys. Chem. 1970, 74, 710-719.

[188] Debye, P.; Hückel, E. Physik. Z. 1923, 24, 185-206.

[189] Archer, D. G.; Wang, P. J. Phys. Chem. Ref. Data 1990, 19, 371-412.

[190] Hiraoka, K.; Yokoyama, T. J. Polym. Sci. B Polym. Phys. 1986, 24, 769-778.

[191] Conway, B. E.; Laliberte, L. H. Trans. Faraday Soc. 1970, 66, 3032-3047.

[192] Gianni, P.; Lepori, L. J. Solution Chem. 1996, 25, 1-42.

[193] Barta, L.; Hepler, L. G. J. Phys. Chem. 1989, 93, 5588-5595.

[194] Hiraoka, K.; Shin, H.; Yokoyama, T. Polym. Bull. 1982, 8, 303-309.

[195] Smith, M. Organic Chemistry: An Acid Base Approach; CRC Press, 2011; p 214.

[196] Handbook of Chemistry and Physics - 84th Edition ; 84th ed.; Linde, D. R., Ed.; CRC Press, 2004; pp 8/-47. 
[197] Arnold, R.; Ticktin, S.; Monseair, G.; Stephen, A. M. J. Chem. Soc. 1963, O, $5810-5815$.

[198] Soni, M. L.; Kapoor, R. C. Int. J. Quantum Chem. 1981, 20, 385-391.

[199] Dewick, P. M. Essentials of Organic Chemistry: For Students of Pharmacy, Medicinal Chemistry and Biological Chemistry; John Wiley \& Sons, 2013; p 144.

[200] Pines, E.; Magnes, B.-Z.; Lang, M. J.; Fleming, G. R. Chem. Phys. Lett. 1997, $281,413-420$.

[201] Kolthoff, I. M.; Miller, I. K. J. Am. Chem. Soc. 1951, 73, 3055-3059.

[202] Mahdavian, A.; Abdollahi, M.; Bijanzadeh, H. R. J. Appl. Polym. Sci. 2004, 93, 2007-2013.

[203] Seabrook, S. A.; Tonge, M. P.; Gilbert, R. G. J. Polym. Sci. A Polym. Chem. 2005, 43, 1357-1368.

[204] Pascal, P.; Winnik, M. A.; Napper, D. H.; Gilbert, R. G. Macromolecules 1993, $26,4572-4576$.

[205] Seabrook, S. A.; Pascal, P.; Tonge, M. P.; Gilbert, R. G. Polymer 2005, 46, 9562-9573. 


\section{Danksagung/Acknowledgements}

Mein außerordentlicher Dank gilt Herrn Prof. Dr. M. Buback für die interessante Themenstellung sowie die stete Diskussionsbereitschaft und Förderung dieser Arbeit.

Außerdem bedanke ich mich bei Prof. Dr. P. Vana, MBA für die Übernahme des Korreferats.

Special thanks go to Prof. Robin A. Hutchinson (Queen's University, Canada) for inviting me to Canada and introducing me to modeling, lots of helpful discussions and suggestions, and altogether a very fruitful cooperation.

I thank Igor Lacík, DSc, and Marek Stach, PhD, (Polymer Institute SAV, Slovakia) for discussions providing insight into challenging SEC analyses, and determination of many MMDs. Contributions of further coworkers at the Polymer Institute of the Slovak Academy of Sciences in Bratislava are also gratefully acknowledged. Ďakujem.

I thank Sandhya Santanakrishnan, PhD, for help with Predici and showing me around at Queen's.

I'd like to thank Calista Preusser (Queen's University, Canada) for interesting discussion and for sharing results.

Ein besonderer Dank gebührt Dr. Jens Schrooten, Dr. Johannes Barth, Hendrik Kattner, Henrik Schröder und Patrick Drawe für ihre unermüdliche Diskussionsbereitschaft und viele hilfreiche Anregungen. 
Weiterer Dank gilt Herrn Dr. H.-P. Vögele für die ständige Hilfsbereitschaft bei technischen Fragen, besonders zu den IR-Spektrometern.

Bei Herrn R. Machinek bedanke ich mich für Ratschläge zur NMR-Messung und tatkräftige Hilfe beim Finden der richtigen Messbedingungen, sowie bei der gesamten NMR-Abteilung für die Messung von NMR-Proben.

Herrn Dr. H. Frauendorf danke ich für die MS-ESI und HPLC Messungen sowie hilfreiche Anmerkungen.

I wish to thank Dr. Tatiana Sergeeva for discussions and support regarding evaluation of EPR spectra as well as for sharing results.

Ich danke Patrick Drawe und Dr. Johannes Barth für das Teilen von Ergebnissen.

Großer Dank gebührt Dr. Johannes Buback für das Programm Predici Batch, eine exzellente Ergänzung des Programms PREDICI ${ }^{\mathrm{TM}}$.

Ich danke Daniel Weiß, dessen Masterarbeit ich mitbetreut habe, für die gute Zusammenarbeit und das Teilen von Ergebnissen.

Ich danke Justus B. Söllner und Roman Kremring, deren Bachelorarbeiten ich betreut habe, für die Zusammenarbeit.

I thank Dr. Hugo Vale (BASF SE) for helpful discussions and suggestions.

Herrn Prof. Dr. F. Meyer möchte ich für die Bereitstellung des ESR-Spektrometers danken. 
Thanks are given to Prof. Dr. Alex M. van Herk (Technische Universiteit Eindhoven, the Netherlands) for providing the program Contour.

Den Mitarbeitern der Institutswerkstätten danke ich für ihre Hilfsbereitschaft und zuverlässige Arbeit.

Ich möchte mich bei Patrick Drawe, Henrik Schröder, Julia Möhrke, Hendrik Kattner, Nicolai Sörensen, Dennis Hübner und Jan-Hendrik Schütz für das Korrekturlesen dieser Arbeit bedanken.

Die Hilfe von Dr. Jens Schrooten bei der Bedienung des Lasers für PLP soll an dieser Stelle dankend erwähnt werden.

Ich danke Cathrin Conrad und Björn Sölter dafür, dass sie Kaffeebohnen und Milch für die Abteilungen eingekauft haben - ohne den Kaffee zwischendurch hätte ich es nicht geschafft ;-)

Bei allen Mitgliedern der Arbeitskreise Buback und Vana bedanke ich mich für die angenehme Arbeitsatmosphäre und die stete Hilfsbereitschaft, nicht zu vergessen zahlreiche Frühstücke, Kuchen, Eis, aber auch gemeinsame Ausflüge zum Kanufahren oder Wandern.

Bei der BASF SE möchte ich mich für die finanzielle Unterstützung dieser Arbeit bedanken.

Ich danke dem Deutschen Akademischen Austauschdienst (DAAD) für die finanzielle Unterstützung meines Aufenthaltes an der Queen's University in Kanada. 
Abschließend möchte ich meinen Freunden, speziell der „Reisegruppe“, für die moralische Unterstützung und die zahlreichen willkommenen Ablenkungen danken.

Ich danke meiner Freundin, dass sie meistens ;-) an mich geglaubt hat und immer für mich da ist.

Mein größter Dank gilt meiner Familie. Für ihre Begleitung und Bestärkung, ihre Geduld und ihr Vertrauen in mich bin ich ihnen zutiefst dankbar. 


\section{CURRICUlum VitaE}

\section{Personal Details}

Name

Date and Place of Birth

Citizenship

\section{Education}

2009/Oct-present

2010/May-2010/Jul

2004/Oct-2009/Aug

2009/Aug/12

2008/Oct-2009/Apr

2010/May-2010/Jul

2003/Jun/26

\section{Scholarships}

2010/May-2010/July
Nils Friedrich Gunter Wittenberg 1983/Nov/06 in Hamburg

German

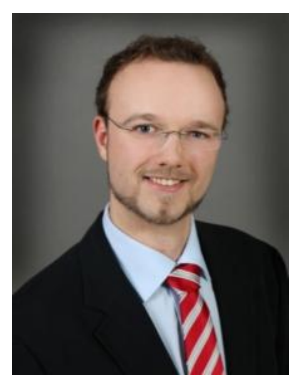

doctoral dissertation research at Georg-AugustUniversität Göttingen, workgroup Prof. M. Buback, "Kinetics and Modeling of the Radical Polymerization of Acrylic Acid and of Methacrylic Acid in Aqueous Solution"

research stay at Queen's University in Kingston, ON, Canada, workgroup Prof. R. Hutchinson

diploma studies in chemistry at Georg-AugustUniversität Göttingen

Chemie-Diplom (graduation in chemistry)

diploma thesis, workgroup Prof. M. Buback, "Initiierung und Regelung bei radikalischer (Meth)Acrylsäurepolymerisation in wässriger Lösung” research stay at University of New South Wales in Kensington, NSW, Australia, workgroup Prof. R. Read Abitur at the Gymnasium Rissen, Hamburg

DAAD (Deutscher Akademischer Austauschdienst) for a research stay at Queen's University in Kingston, ON, Canada 